\title{
Unternehmenszusammenschlüsse in der Milch- und Zuckerindustrie unter wettbewerbsrecht- lichen und ökonomischen Gesichtspunkten
}

\author{
Dissertation \\ zur Erlangung des Doktorgrades \\ der Fakultät für Agrarwissenschaften \\ der Georg-August-Universität Göttingen
}

Christian Ulrich Janz

Göttingen, Dezember 2002 


\section{Danksagung}

Die vorliegende Arbeit wurde am Institut für Agrarökonomie der GeorgAugust-Universität Göttingen angefertigt. Die Anfertigung dieser Arbeit und inre Veröffentlichung wurde durch eine Vielzahl von Personen und Institutionen unterstützt, denen ich an dieser Stelle herzlich danken möchte.

Für die Überlassung des Themas und die wissenschaftliche Betreuung bedanke ich mich bei Herrn Prof. Dr. S. von Cramon-Taubadel. Seine konstruktiven Anregungen waren für die Konzeption und Durchführung der Arbeit äußerst hilfreich. Für die Übernahme des Korreferates danke ich Herrn Prof. Dr. W. Brandes. Herrn Dr. W. Winkler danke ich für die Hilfestellungen in den juristischen Bereichen. Mein ganz besonderer Dank gilt den Mitarbeiterinnen und Mitarbeitern des Instituts für Agrarökonomie. Ihre ständige Hilfsbereitschaft, der fachliche und private Gedankenaustausch und das angenehme Arbeitsklima haben einen großen Beitrag zur Vollendung der vorliegenden Arbeit geleistet. An dieser Stelle möchte ich Frau Christa Körber ganz besonders für Ihre Unterstützung in allen technischen und logistischen Fragestellungen danken.

Zur Fertigstellung dieser Arbeit war ich auf die Auskunftbereitschaft einer Vielzahl von Personen angewiesen. Besonderer Dank gilt in diesem Zusammenhang den Mitarbeitern des Bundeskartellamtes sowie den Vertretern der befragten Unternehmen.

Der größte Dank gilt aber meiner Familie, die mich in jeder Phase der Arbeit tatkräftig unterstützt und mir viel Rückhalt gegeben hat. Meinen Eltern danke ich dafür, dass sie mir diese Ausbildung ermöglicht und mich besonders zu Beginn der Arbeit finanziell unterstützt haben. Meinem Bruder Martin Janz gilt besonderer Dank für seine kritischen Anmerkungen und konstruktiven Beiträge, die besonders in der Endphase dieser Arbeit äußerst hilfreich waren. 


\section{Gliederung}

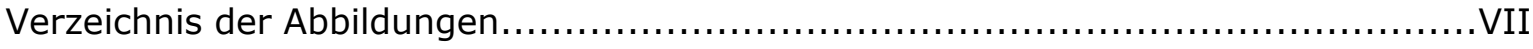

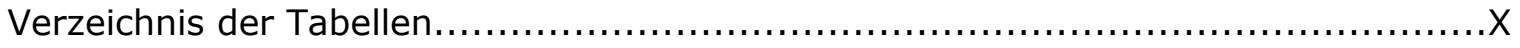

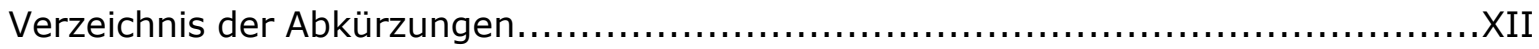

1 EINLEITUNG, ZIELSETZUNG UND AUFBAU DER STUDIE ........................ 1

2 ENTWICKLUNG VON UNTERNEHMENSZUSAMMENSCHLÜSSEN ............. 4

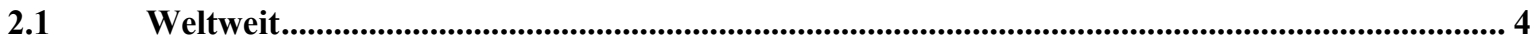

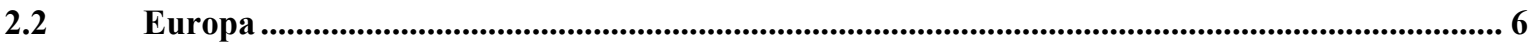

$2.3 \quad$ Deutschland................................................................................................................................................... 8

$2.4 \quad$ Ernährungsindustrie ............................................................................................................................. 9

$2.5 \quad$ Zuckerindustrie ...................................................................................................................................... 14

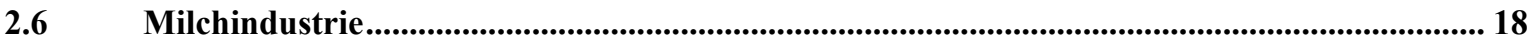

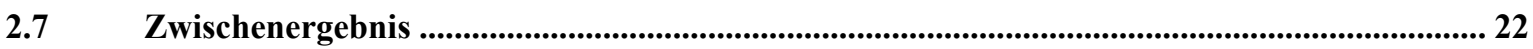

3 MOTIVE UND AUSWIRKUNGEN VON UNTERNEHMENS-

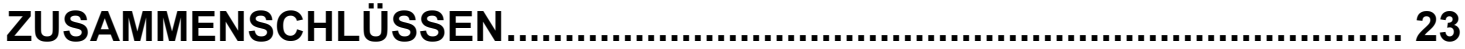

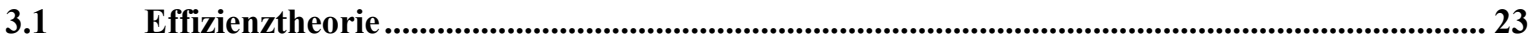

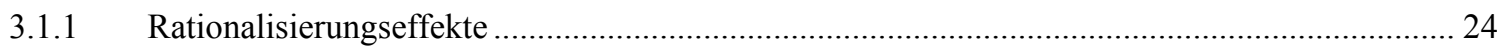

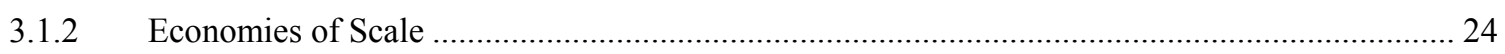

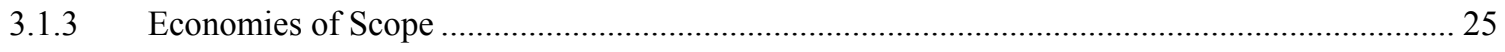

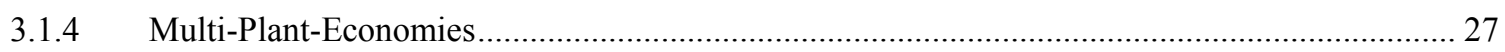

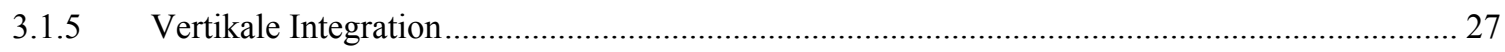

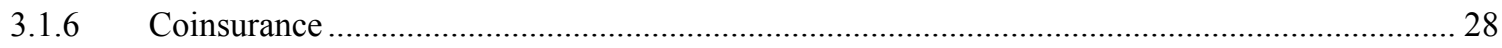

Konzept des überlegenden Managements............................................................................. 28

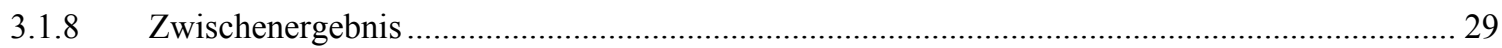

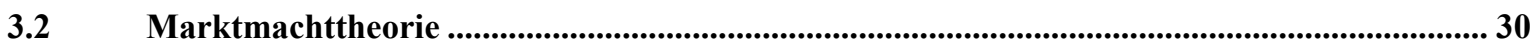

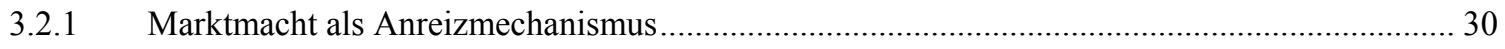

3.2.2 Praktische Auswirkungen von Marktmacht................................................................. 31

3.2.2.1 Absprachen zwischen Unternehmern innerhalb einer Branche ........................................ 32

3.2.2.2 Absprachen zwischen Unternehmern mehrerer Branchen ............................................. 32

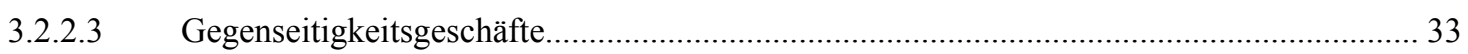

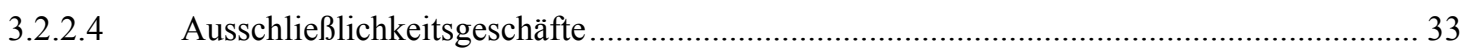

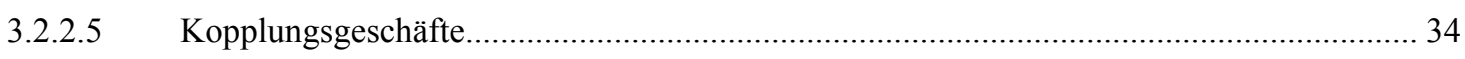




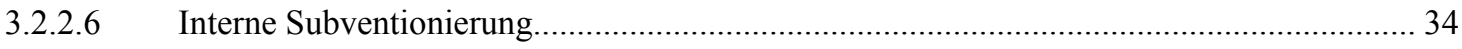

3.2.2.7 Abschreckung möglicher Herausforderer ...................................................................... 34

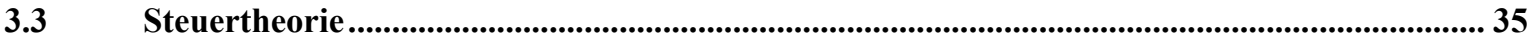

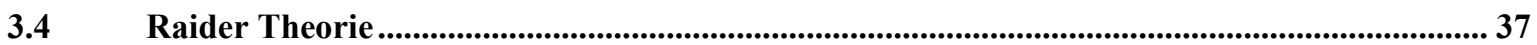

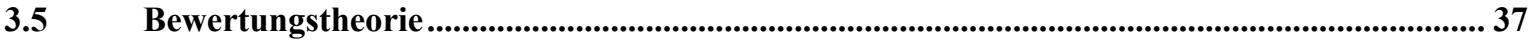

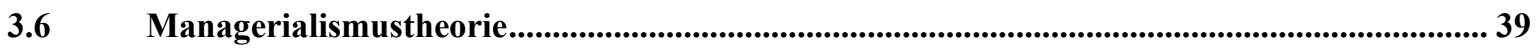

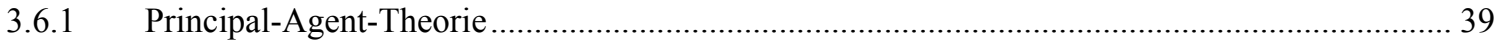

Weitere Zusammenschlusstheorien .....................................................................................43

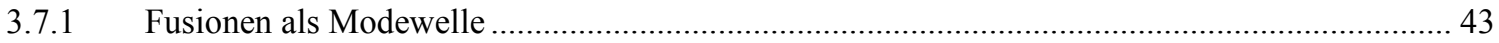

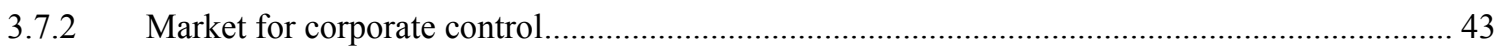

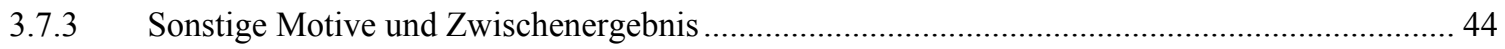

\section{INDUSTRIEÖKONOMISCHE UNTERSUCHUNGEN VON FUSIONEN ......... 46}

Theoretische Studien zu horizontalen Fusionen ..................................................................46

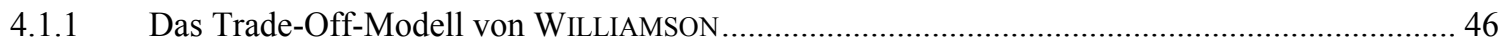

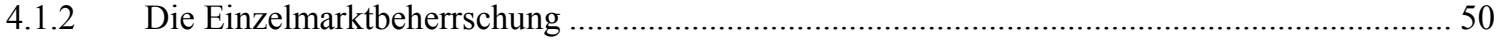

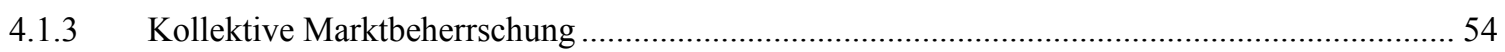

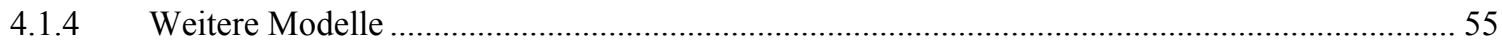

4.1.5 Erkenntnisse industrieökonomischer Fusionsmodelle ......................................................... 57

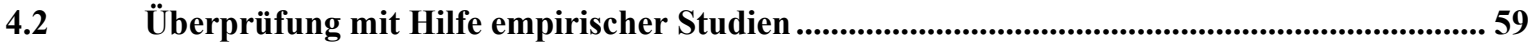

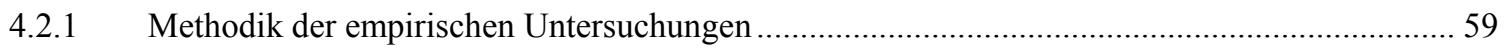

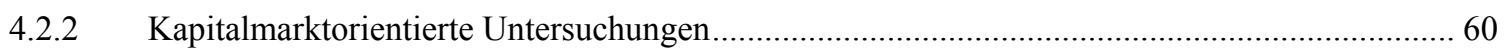

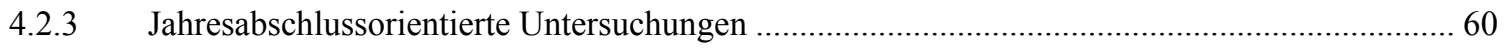

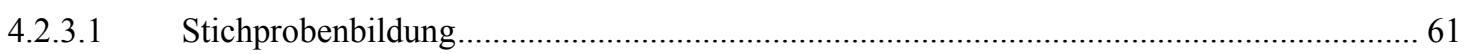

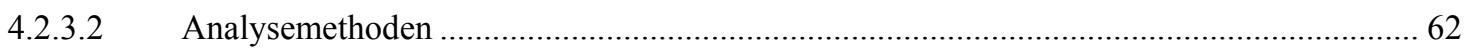

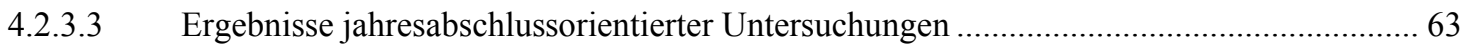

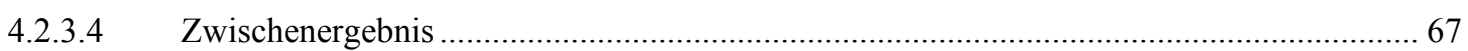

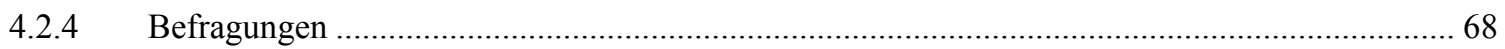

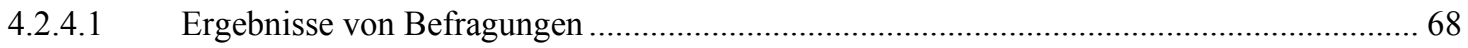

4.2.5 Weitere Untersuchungen zur Beurteilung von Unternehmenszusammenschlüssen ...................69

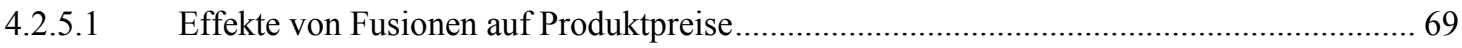

4.2.5.2 Auswirkungen von Fusionen auf Marktanteile ............................................................... 71

4.2.5.3 Weitere betriebswirtschaftliche Untersuchungen ......................................................... 72

4.3 Schlussfolgerung: Theoretische Analyse und empirische Überprüfung der Wohlfahrtseffekte von Fusionen 
5.1 Die Zusammenschlusskontrolle im deutschen Recht...................................................................... 76

5.1.1 Hintergrund des deutschen Wettbewerbsrechts .......................................................................... 76

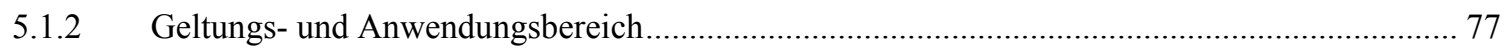

5.1.2.1 Der fusionsrechtliche Unternehmensbegriff ................................................................ 77

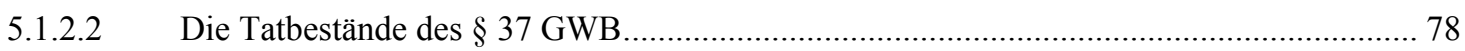

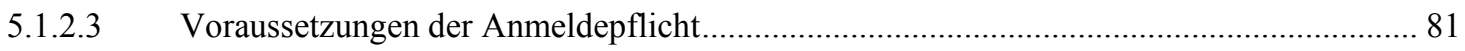

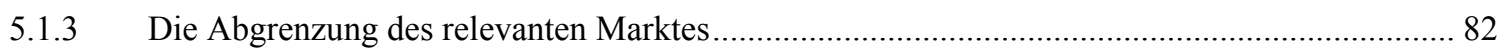

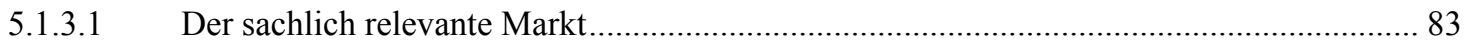

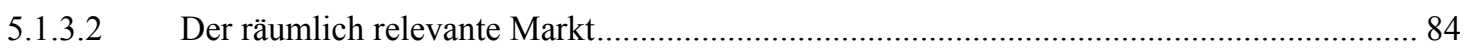

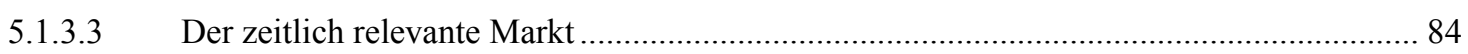

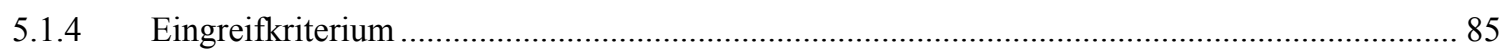

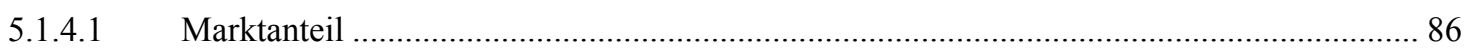

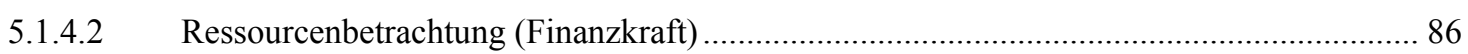

5.1.4.3 Zugang zu den Beschaffungs- und Absatzmärkten.......................................................... 87

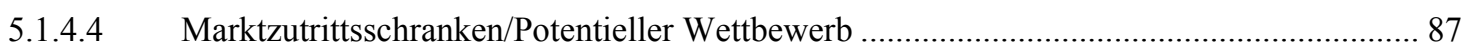

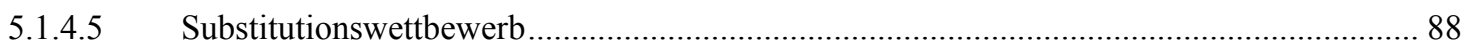

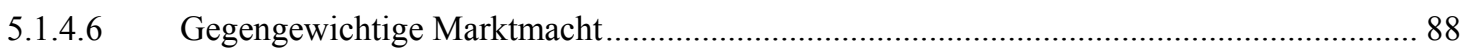

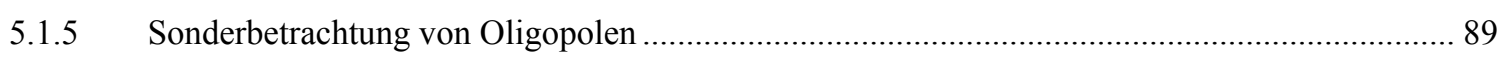

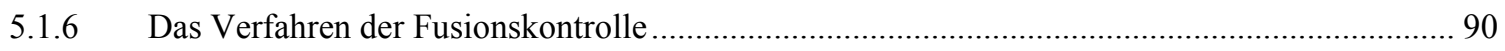

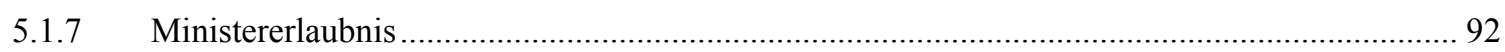

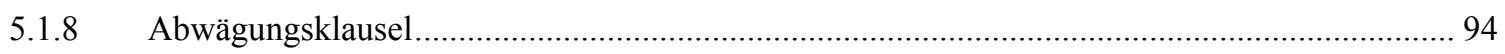

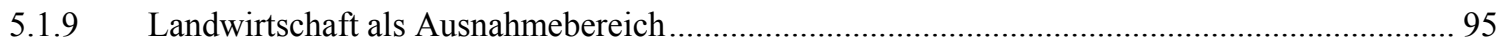

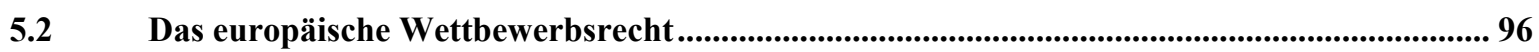

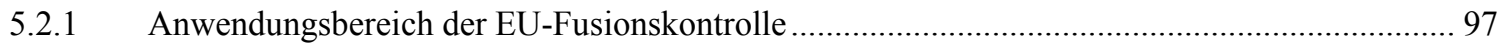

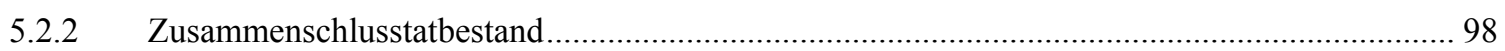

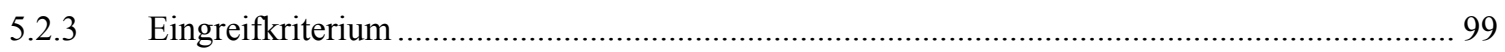

5.2.4 Der relevante Markt in der europäischen Fusionskontrolle ...................................................... 100

5.2.5 Das Verfahren der europäischen Fusionskontrolle .................................................................. 101

5.2.6 Sonstige Anmerkungen zum europäischen Wettbewerbsrecht................................................. 102

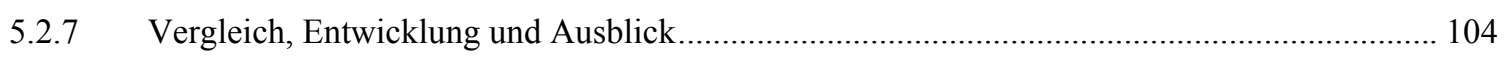

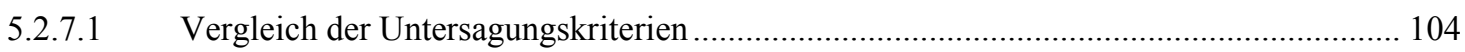

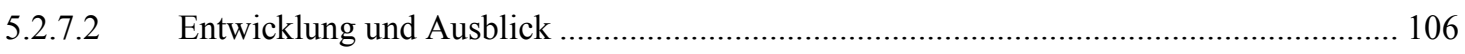

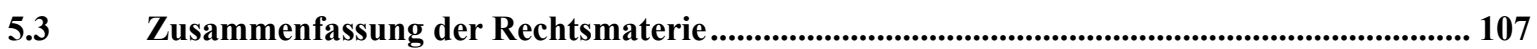

6 BEURTEILUNG AUSGEWÄHLTER FUSIONEN IN DER 
6.2 Zusammenschlussbeurteilung ausgewählter Fälle durch die Wettbewerbsbehörden

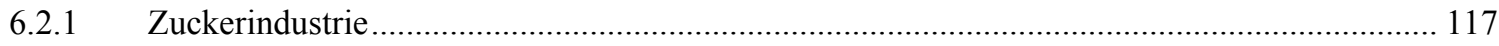

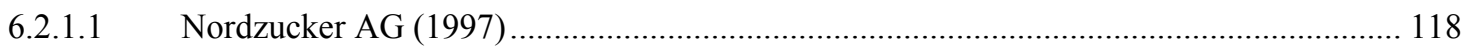

6.2.1.2 Nordzucker AG und Union-Zucker (2002) .............................................................. 118

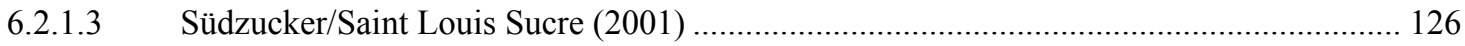

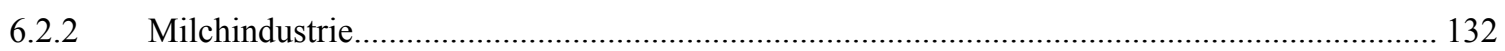

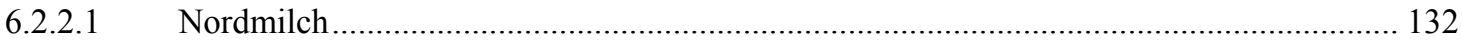

6.2.2.2 EU-Kommission: Friesland Coberco/Nutricia (2001) ................................................... 136

7 BERÜCKSICHTIGUNG UND WERTUNG WICHTIGER KOMPONENTEN DER ZUSAMMENSCHLUSSKONTROLLE............................................. 139

Konzepte der Marktabgrenzung und ihre Problematik ........................................................ 139

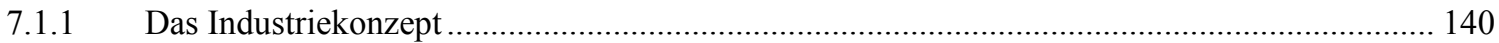

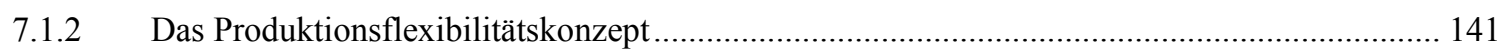

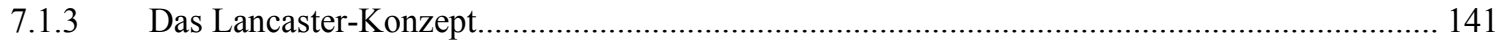

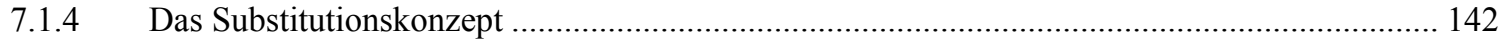

7.1.4.1 Substitutionslücken als Basis für die räumliche Marktabgrenzung ................................ 143

7.1.4.2 Elastizitäten als Maß für Substitutionslücken......................................................... 146

7.1.5 Empirische Tests zur räumlichen Marktabgrenzung relevanter Märkte ................................ 147

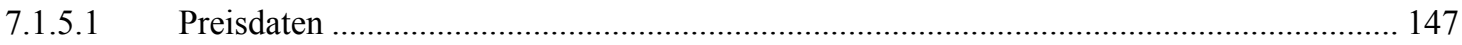

7.1.5.2 Handelsströme und Marktanteile ............................................................................... 149

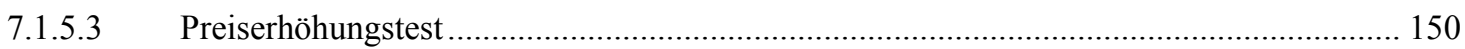

7.1.6 Praktische Implementierung im deutschen und europäischen Recht.................................. 152

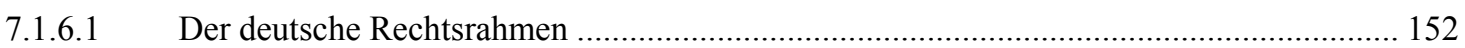

7.1.6.2 Abgrenzung des relevanten Marktes in der EU ......................................................... 153

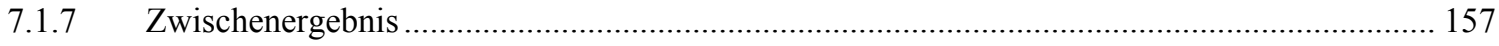

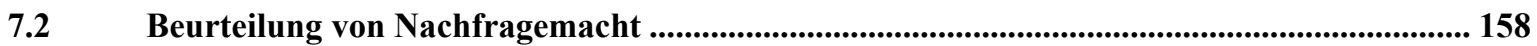

7.2.1 Marktmacht im LEH: Problemstellung und Stand der Literatur......................................... 159

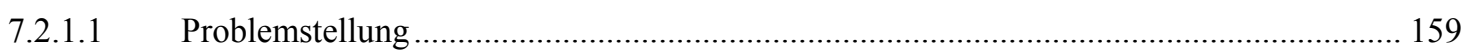

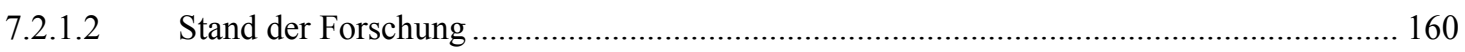

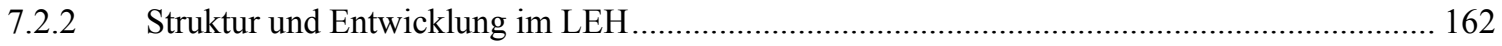

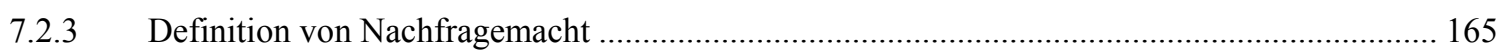

7.2.4 Entstehung von Nachfragemacht zwischen Hersteller und Handel ..................................... 167

7.2.4.1 Begrenzter Wettbewerb auf der horizontalen Ebene des Handels ................................... 168

7.2.4.2 Vertikale Ebene: Thesen zur Marktmacht des Handels gegenüber den Lieferanten.......... 169

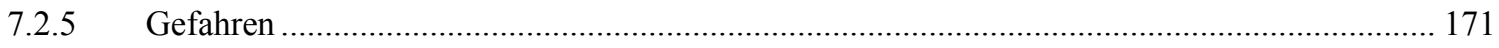

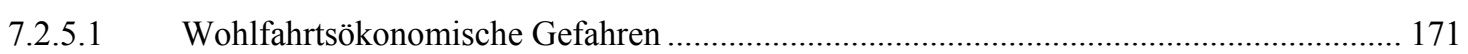




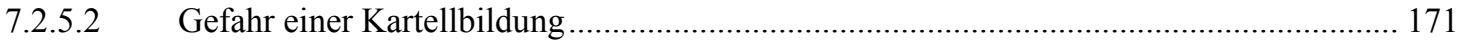

7.2.6 Praktische Auswirkungen und Indikatoren von Nachfragemacht......................................... 172

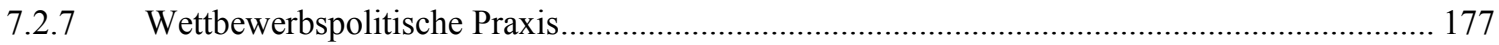

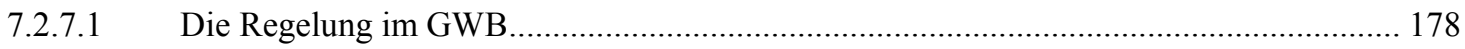

7.2.7.2 Die Berücksichtigung von Nachfragemacht auf europäischer Ebene............................. 179

7.2.7.3 Vorgehensweise des Bundeskartellamtes ................................................................. 179

7.2.7.4 Beurteilung der Lebensmitteleinzelhandelkonzentration durch die Monopolkommission 180

7.2.8 Zwischenergebnis 181

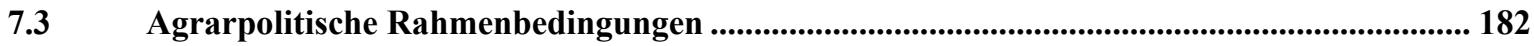

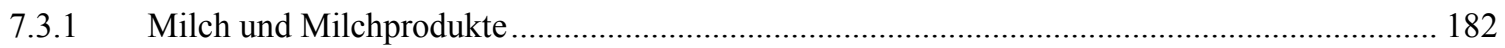

7.3.1.1 EG-Marktordnung für Milch und Milcherzeugnisse ............................................. 183

7.3.1.2 Garantiemengenregelung für Milch............................................................................... 187

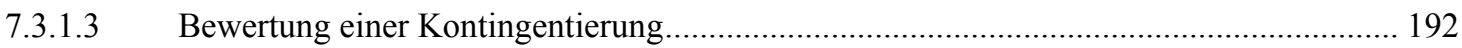

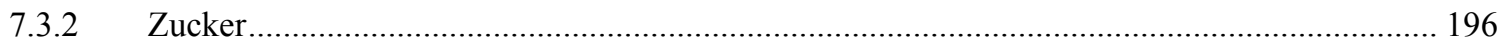

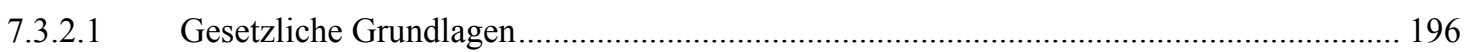

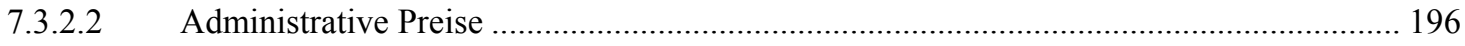

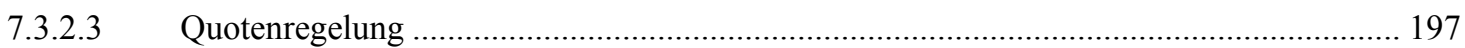

7.3.2.4 Regelungen des EU-Binnenmarktes ........................................................................ 200

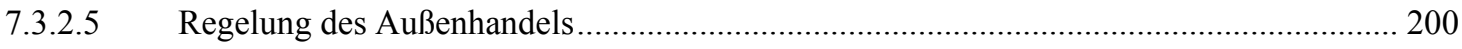

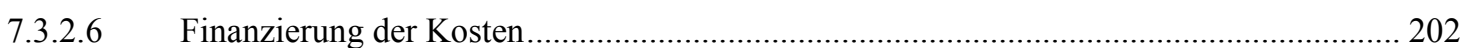

7.3.2.7 Exkurs: Garantierte Verarbeitungsspanne ............................................................. 203

7.3.2.8 Kosten der Zuckermarktordnung ......................................................................... 204

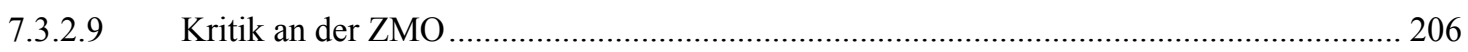

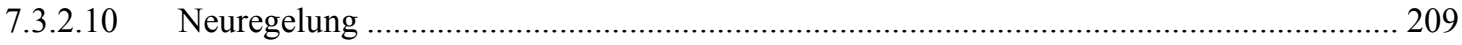

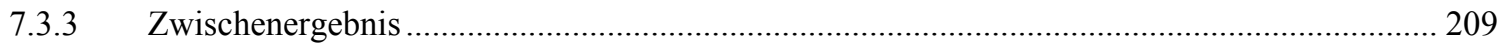

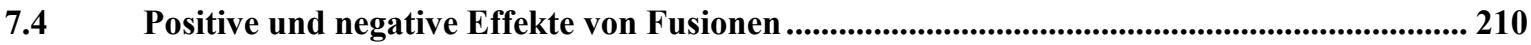

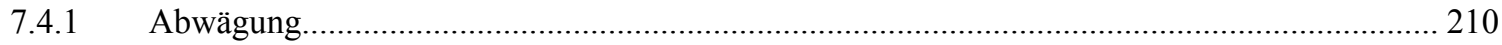

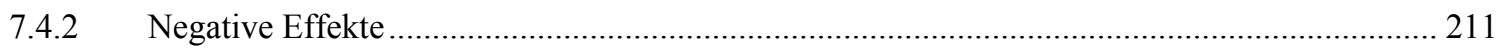

7.4.2.1 Ökonomische Instrumente zur Ermittlung negativer Effekte ..................................... 213

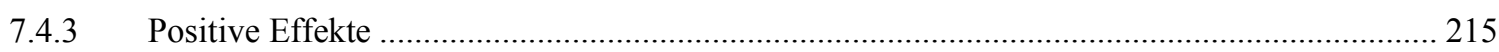

\section{PRAKTISCHE ANWENDUNG ÖKONOMISCHER INSTRUMENTE ZUR ERMITTLUNG VON MARKTMACHTEFFEKTEN UND EFFIZIENZVORTEILEN}

\subsection{Kosten der Zuckerproduktion und mögliche Einsparungspotentiale: Erkenntnisse aus}

Expertengutachten .............................................................................................................................. 217

8.1.1 Vorüberlegungen zur Standortbestimmung der Zuckerrübenverarbeitung............................. 218

8.1.2 Erfassungskosten versus Fixkostendegression ........................................................... 219

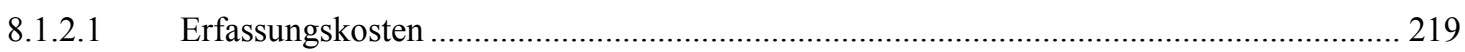




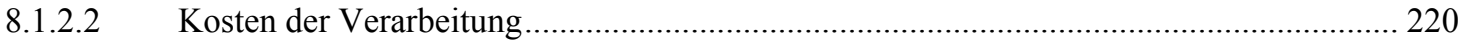

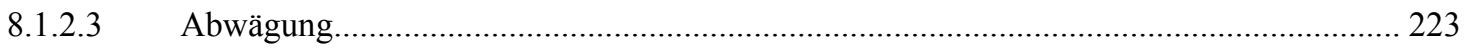

8.1.3 Veränderung der Kosten der Zuckerverarbeitung.......................................................... 225

8.1.3.1 Versuch der Ermittlung einzelner Komponenten ..................................................... 225

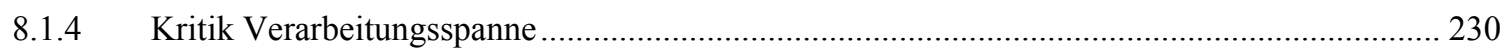

8.1.5 Erlöse: Rückschlüsse mit Hilfe von Preisbeobachtungen ................................................. 233

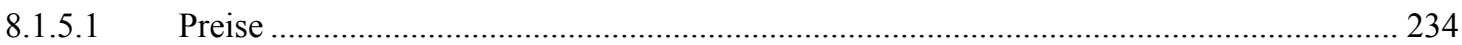

8.1.6 Bilanzanalyse: Rückschlüsse von vergangenheitsbezogenen Beobachtungen auf zukünftiges

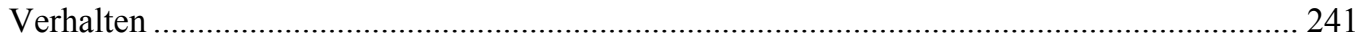

8.1.6.1 Vorüberlegungen zur Bilanzanalyse ........................................................................... 241

8.1.6.2 Bilanzanalyse in der Zuckerindustrie - Beurteilung der Nordzucker AG ...................... 243

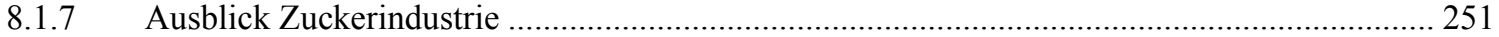

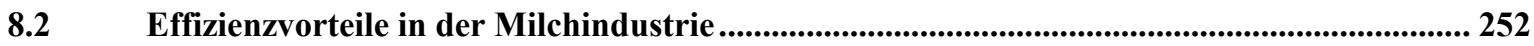

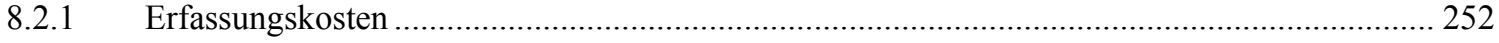

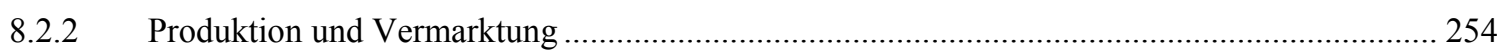

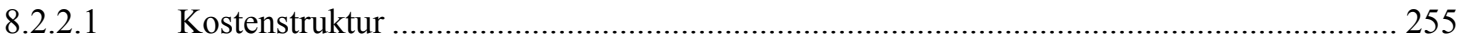

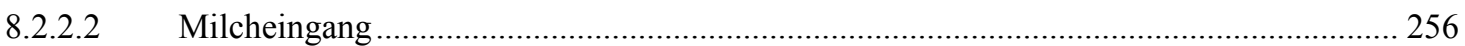

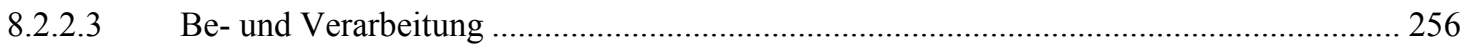

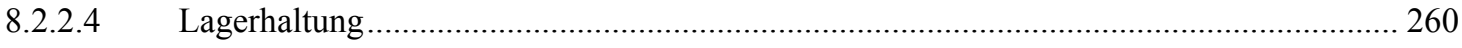

8.2.2.5 Transport/Marketing/Absatz ....................................................................................... 261

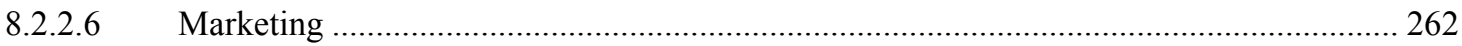

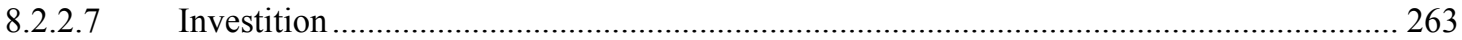

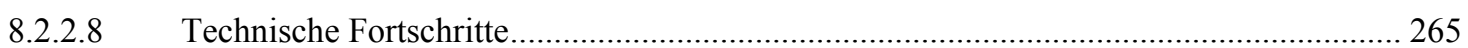

8.2.3 Optimale Betriebsgröße insgesamt: eine Abwägung ...................................................... 266

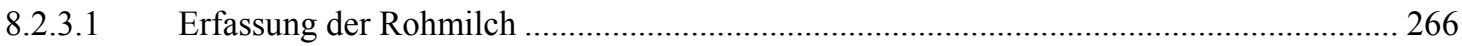

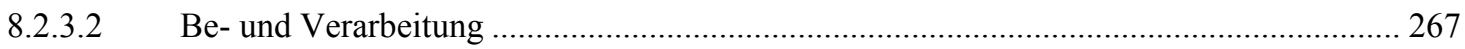

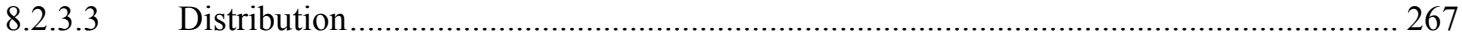

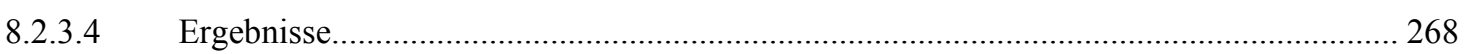

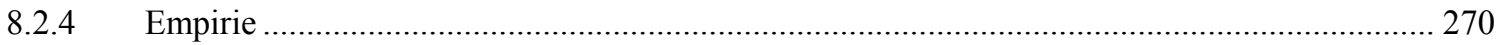

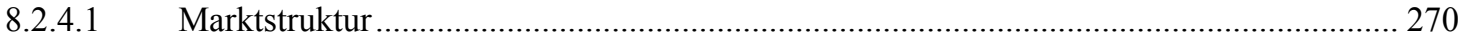

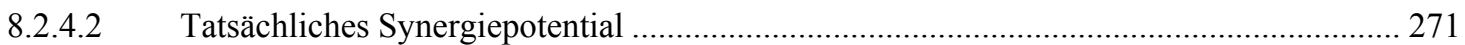

8.2.5 Rückschlüsse auf Marktmacht mit Hilfe von Preisbeobachtungen...................................... 273

8.2.6 Bilanzanalyse ausgewählter Unternehmen der Milchindustrie: Nordmilch e.G..................... 278

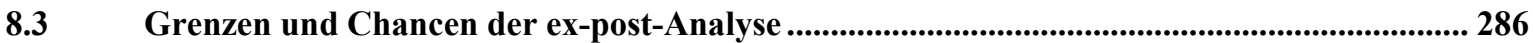

9 ZUSAMMENFASSUNG UND EMPFEHLUNG …................................... 289

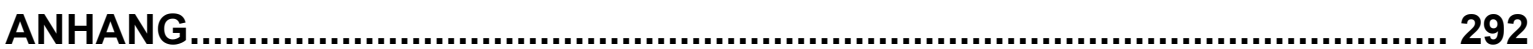




\section{VERZEICHNIS DER ABBILDUNGEN}

Abbildung 1: Aufbau und Fragestellungen der Untersuchung .................................................................. 3

Abbildung 2: Anzahl und Rahmenbedingungen von Fusionen ........................................................................ 4

Abbildung 3: Transaktionsvolumen weltweiter Fusionen (Angaben in Mrd. US \$) ................................... 5

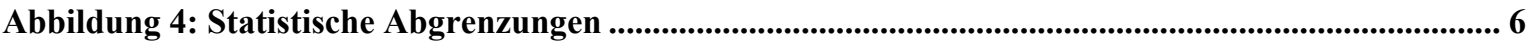

Abbildung 5: Transaktionen mit europäischer Beteiligung von 1991-2001 ............................................ 6

Abbildung 6: Transaktionsvolumen und Anzahl von Fusionen innerhalb Europas .................................... 7

Abbildung 7: Beim Bundeskartellamt angezeigte vollzogene Fusionen .....................................................8 8

Abbildung 8: Anzahl der Arten von Unternehmenszusammenschlüssen...............................................9

Abbildung 9: Struktur und Komponenten der Wertschöpfungskette in der deutschen

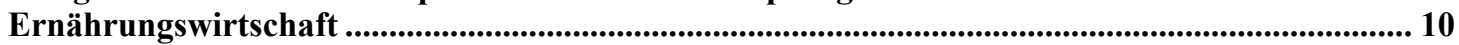

Abbildung 10: Fusionen und Akquisitionen in Deutschland 1998 und 1999....................................... 11

Abbildung 11: Umsatzanteil der 10 größten Unternehmen (in Prozent)............................................. 13

Abbildung 12: Zukünftige Akquisitionsziele der deutschen Ernährungsindustrie ................................... 13

Abbildung 13: Entwicklung der Verarbeitungsmenge und Anzahl der Fabriken bzw. Unternehmen der Fabriken

Abbildung 14: Entwicklung der Anzahl der Molkereibetriebe bzw. Unternehmen sowie der durchschnittlichen (jährlichen) Verarbeitungsmenge pro Betrieb ................................................ 18

Abbildung 15: Mögliche Ursachen von economies of scale..................................................................25

Abbildung 16: Keine Marktmacht vor dem Zusammenschluss .............................................................4 47

Abbildung 17: Marktmacht vor einem Zusammenschluss .....................................................................4 48

Abbildung 18: Veränderung des Cournot-Nash-Gleichgewichts durch einen Zusammenschluss .......... 51

Abbildung 19: Gewinne der Insider nach einem Zusammenschluss in Abhängigkeit der Reaktionen des

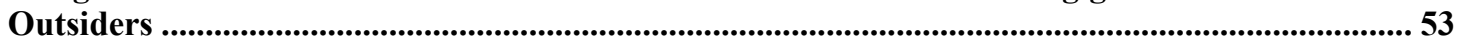

Abbildung 20: Aufbau des Wettbewerbsrechts .............................................................................................. 77

Abbildung 21: Verfahren der Zusammenschlusskontrolle...................................................................... 92

Abbildung 22: Übersicht über das EG- Fusionskontrollverfahren................................................... 102

Abbildung 23: Standorte der ,Nordmilch““-Molkereien .......................................................................... 133

Abbildung 24: Der sachlich relevante Markt Coberco/Nutricia .............................................................. 137

Abbildung 25: Abgrenzung des sachlich relevanten Produktmarktes ................................................... 139

Abbildung 26: Substitutionslücke bei räumlich verteilten Marktteilnehmern ....................................... 144

Abbildung 27: „Chain relationship“ bei räumlich verteilten Marktteilnehmern................................... 145

Abbildung 28: Unternehmenskonzentration - Ernährungsindustrie und Einzelhandel im Vergleich 1998

Abbildung 29: Konzentration im deutschen Lebensmittelhandel................................................... 163

Abbildung 30: Konzentration im europäischen LEH (Top 5) .......................................................163 
Abbildung 32: Wettbewerb im Handel: Produktspezifische Beurteilung ............................................... 167

Abbildung 33: Bedeutung von Rabatten und Konditionen............................................................................ 173

Abbildung 34: Bedeutung von Handelsmarken in Europa 1999.............................................................. 176

Abbildung 35: Vertriebsform von Lebensmitteln.......................................................................177

Abbildung 36: Kosten der Marktorganisation (nach Kategorie aufgelistete Ausgaben) ..................... 193

Abbildung 37: Prinzip der europäischen Quotenregelung ................................................................... 199

Abbildung 38: Mögliche negative Effekte einer Fusion ......................................................................212

Abbildung 39: Wirkungszusammenhänge kostenrelevanter Faktoren bei der Zuckerproduktion ..... 218

Abbildung 40: Interdependenzen bei der Standort- und Betriebsgrößenbestimmung in der Zuckerindustrie

Abbildung 41: Die durchschnittlichen Zuckerrübenverarbeitungs- und erfassungskosten in den kalkulierten Weißzuckerfabriken in Abhängigkeit von der Kapazität und Produktionsdichte . 223

Abbildung 42: Spezifischer Energiebedarf in der Zuckerindustrie 1953/54 bis 2005 in $\quad \mathrm{kWh} / 100 \mathrm{~kg}$

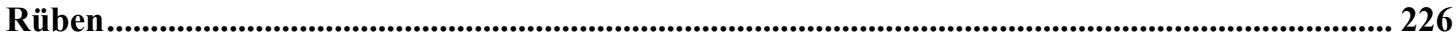

Abbildung 43: Entwicklung des Stromverbrauchs pro kg Zuckererzeugung ........................................ 227

Abbildung 44: Entwicklung der Preisindices verschiedener Inputfaktoren (Basis 1995) ..................... 227

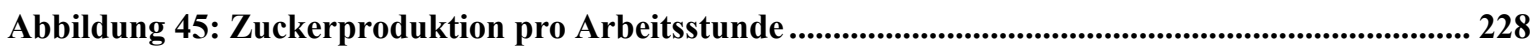

Abbildung 46: Personalkosten in der Zuckerindustrie ....................................................................... 229

Abbildung 47: Personalkosten pro kg Zuckererzeugung........................................................................ 229

Abbildung 48: Entwicklung der Industriezuckerpreise in Deutschland und Europa ........................... 235

Abbildung 49: Verfügbarkeit verschiedener Hersteller........................................................................ 237

Abbildung 50: Standorte der Zuckerindustrie ................................................................................... 238

Abbildung 51a: Preise innerhalb Deutschlands für Südzucker Raffinade (1000g) ............................... 239

Abbildung 52: Entwicklung von Jahresüberschuss und Umsatz der Nordzucker AG .......................... 244

Abbildung 53: Entwicklung von Anlagevermögen und Abschreibungen der Nordzucker.................... 245

Abbildung 54: Entwicklung der Mitarbeiterzahl und des Personlaufwandes (pro Mitarbeiter) der

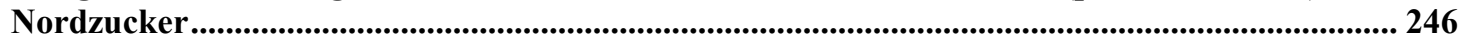

Abbildung 55: Rübenverarbeitung pro Mitarbeiter .................................................................................. 246

Abbildung 56: Entwicklung des Umsatzes pro Mitarbeiter ............................................................... 247

Abbildung 57: Aufwand pro Mitarbeiter im Vergleich mit anderen Unternehmen ................................ 247

Abbildung 58: Umsatz pro kg Zucker ................................................................................................................ 248

Abbildung 59: Anlagevermögen pro Tonne Rübenverarbeitung.......................................................... 248

Abbildung 60: Entwicklung des Umlaufvermögens pro Tonne Rübenverarbeitung ............................. 249

Abbildung 61: Entwicklung des theoretischen Gewinns pro kg Zucker .............................................. 249

Abbildung 62: Eigenkapitalrentabilität in der Zuckerbranche (vor Steuern)...................................... 250

Abbildung 63: Unterschiedliche Bedeutung einzelner Standortfaktoren für die befragten Molkereien 
Abbildung 64: Schnittkäseproduktion.

Abbildung 65: ZMP-Vergleichspreise

Abbildung 66: Milchmarktpreisentwicklungen.

Abbildung 67: Entwicklung von ausgewählten Frisch- und Kondensmilcherzeugnissen und

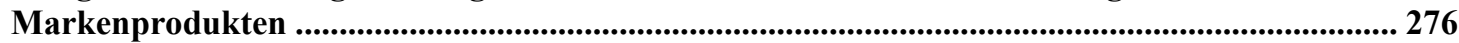

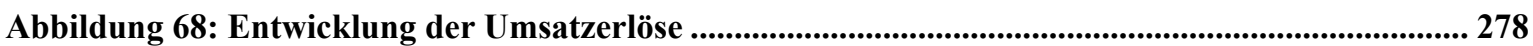

Abbildung 69: Entwicklung der Jahresüberschüsse/-unterschüsse ........................................................ 279

Abbildung 70: Entwicklung des Anlagevermögens und der Abschreibungen ....................................... 279

Abbildung 71: Entwicklung des Personalaufwandes und der Mitarbeiterzahl ...................................... 280

Abbildung 72: Personalaufwendungen verschiedener Unternehmen pro Mitarbeiter ........................... 281

Abbildung 73a: Entwicklung der Produktivität der Mitarbeiter (Umsatz pro Mitarbeiter) ................ 281

Abbildung 74: Durchschnittlicher Erlös pro kg Milch .................................................................. 283

Abbildung 75: Entwicklung des Anlagevermögens pro t Milchverarbeitung...................................... 283

Abbildung 76: Entwicklung des Umlaufvermögens pro kg Milchverarbeitung ...................................... 284

Abbildung 77: Entwicklung des theoretischen Gewinns pro kg Milch (vor Steuer) .............................. 284 


\section{VERZEICHNIS DER TABELLEN}

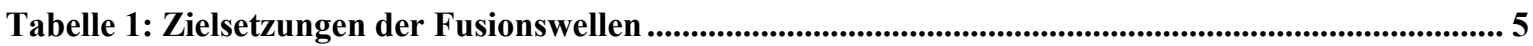

Tabelle 2: Unter der Fusionskontrolle erfasste Fälle ............................................................................... 7

Tabelle 3: Strukturdaten der Ernährungsindustrie im Jahr 2000.......................................................... 10

Tabelle 4: Wichtige Zusammenschlüsse in der Ernährungsindustrie ................................................... 12

Tabelle 5: Anzahl der Zuckerunternehmen (Zuckerfabriken) in Europa ............................................... 14

Tabelle 6: Aufteilung der Zuckerquoten und der Unternehmen in den jeweiligen Mitgliedsstaaten .... 16

Tabelle 7: Anteil der Unternehmen an den Höchstquoten.............................................................................. 17

Tabelle 8: Unternehmen und Produktionsrichtung .......................................................................... 19

Tabelle 9: Durchschnittsbetriebsgröße verschiedener Produktionsrichtungen (jährlich) in Tonnen ... 19

Tabelle 10: Konzentrationsraten in der deutschen Milchwirtschaft 2000............................................ 20

Tabelle 11: Europa Top 25 Milchverarbeiter (2001 basierend auf Daten von 1999) ................................. 21

Tabelle 12: Notwendige prozentuale Kosteneinsparung bei gegebener Nachfragelastizität und

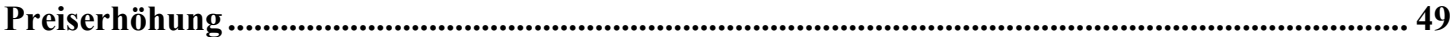

Tabelle 13: Zusammenstellung wichtiger Oligopolmodelle ..................................................................5 56

Tabelle 14: Ergebnisse einzelner jahresabschlussorientierter Studien........................................................ 63

Tabelle 15: Auswirkungen eines Zusammenschlusses auf die Profitabilität.............................................. 66

Tabelle 16: Beurteilungskriterien in der Fusionskontrolle (nach Maßgabe der gesetzlichen

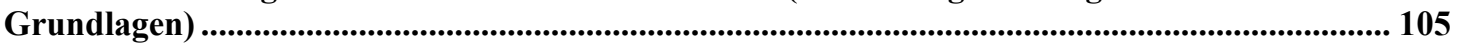

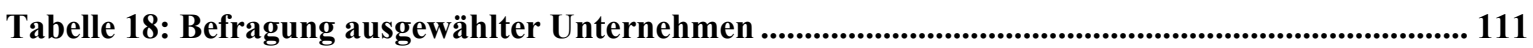

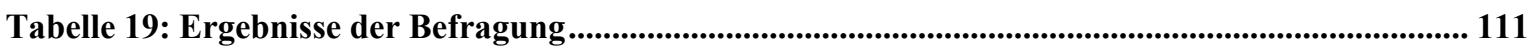

Tabelle 20: Kennzahlen der Zusammenschlusspartner 1996/97 ................................................... 119

Tabelle 21: Marktstruktur des Zuckermarktes in Deutschland ........................................................... 123

Tabelle 22: Marktstruktur des Zuckermarktes in Europa.......................................................................... 128

Tabelle 23: Kennzahlen der Zusammenschlusspartner ................................................................... 133

Tabelle 24: Marktanteile der Zusammenschlusspartner (v.H.) ..................................................... 137

Tabelle 25: Überblick verschiedener Elastizitätsmaße ........................................................................ 146

Tabelle 26: Bedeutende Fusionen des Handels ................................................................................ 164

Tabelle 27: Verteilung nach Branchen ..................................................................................................................... 174

Tabelle 28: Verteilung nach Umsatzkonzentration und horizontaler Marktposition ............................ 174

Tabelle 29: Entwicklung wichtiger Milchpreise ................................................................................................... 184

Tabelle 30: Gesamtreferenzmenge gemäß EG-VO 1256/1999 Artikel 3 Absatz 2 in Tonnen............... 189

Tabelle 31: Entwicklung der Direktzahlungen ............................................................................................ 192

Tabelle 32: Marktordnungspreise für Zuckerrüben in ECU bzw. €/dt................................................. 197

Tabelle 33: Aktuelle Aufteilung der Quoten ........................................................................................................ 198 
Tabelle 34: EU-Kosten der Marktorganisation für Zucker (in Mio. Euro) .

Tabelle 35: Erfassungskosten einer Zuckerfabrik in DM/t Weißzucker

Tabelle 36: Kostendegression der Zuckerproduktion in Abhängigkeit von der Kapazitätsauslastung in DM/t Weißzucker

Tabelle 37: Durchschnittliche Kampagnelänge

Tabelle 38: Kosten der Zuckerverarbeitung (DM je Tonne Weißzucker)

Tabelle 39: Entwicklung der tatsächlichen Betriebsgrößenstrukturen

Tabelle 40: Entwicklung der Verarbeitungskosten in $€$ pro Tonne

Tabelle 41: Gewinnmarge der Zuckerindustrie in ECU bzw. €/Tonne

Tabelle 42: Differenz zwischen theoretischer und praktischer Bewertung

Tabelle 43: Unternehmenskennzahlen (Angaben in Tausend Tonnen)

Tabelle 44: Gesamtauswertung untersuchter Zuckerprodukte anhand d. MADAKOM Datensatzes 240

Tabelle 45: Wettbewerbsfähigkeit der internationalen Zuckerproduktion: Durchschnittskosten der Zuckererzeugung in US \$/Tonne.

Tabelle 46: Kostenstruktur in Molkereien (ohne Rohstoff)

Tabelle 47: Herstellkosten ohne Rohstoff und statische Skaleneffekte in der Modellabteilung allgemeine Milchbehandlung

Tabelle 48: Herstellkosten einschließlich Rohstoff und statische Skaleneffekte in ausgewählten Modellabteilungen

Tabelle 49: Gesamte und entscheidungsrelevante Herstellkosten für Schnittkäse (ohne Rohstoff) bei verschiedenen Abteilungsgrößen und Kapazitätsauslastungsgeraden........................................... 258

Tabelle 50: Modellkosten für die Herstellung von Goudakäse................................................................ 260

Tabelle 51: Materialindizes verschiedener Molkereiprodukte.......................................................... 262

Tabelle 52: Relativer Finanzbedarf in Abhängigkeit von der investierten Abteilungsgröße ................ 264

Tabelle 53: Beispielh. Auswirkungen der Automatisierung auf den Personalbedarf und die Kosten 265

Tabelle 54: Struktur der Milcherzeugung in Deutschland sowie unterstellte Modellannahmen .... 267

Tabelle 55: Modellspezifische Kosten der Erfassung, Be- und Verarbeitung und Distribution in Abhängigkeit unterschiedlicher Betriebsstättengrößen

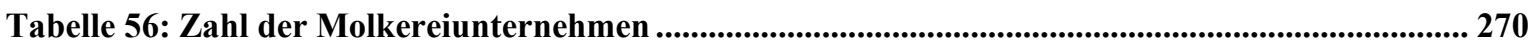

Tabelle 57: Molkereiunternehmen und durchschnittliche Herstellung von Milchprodukten............... 271

Tabelle 58: Rohstoffkostenanteile an den Verbraucherpreisen bei Milch und Milcherzeugnissen ..... 273

Tabelle 59: Mögliche Anwendbarkeit ökonomischer Analysverfahren 


\section{VERZEICHNIS DER ABKÜRZUNGEN}

- $\quad$ a.BL. = alte Bundesländer

- $\quad A G=\quad$ Aktiengesellschaft

- $\quad A K P=\quad$ Afrika, Karibik, Pazifik

- $\quad \mathrm{BE}=\quad$ Betriebsergebnis

- $\quad \mathrm{BGH}=$ Bundesgerichtshof

- $\quad B G H Z=\quad$ Entscheidungen des Bundesgerichtshofs in Zivilsachen

- $\quad$ BkartA $=$ Bundeskartellamt

- $\quad$ BMWi $=\quad$ Bundesministerium für Wirtschaft

- $\quad$ BT-Drs = Drucksache des Deutschen Bundestages

- $\quad$ BVE $=\quad$ Bundesverband der Ernährungsindustrie

- CAOBISCO = Association des Industries des Chocolaterie, Biscuiterie et Confiserie de I'UE

- $\quad$ CEFS $=\quad$ Comité Européan des Fabricants de Sucre

- $\quad$ CIUS $=\quad$ Committee of Industrial Users of Sugar

- c.p. $=\quad$ ceteris paribus

- $\quad \mathrm{DK}=\quad$ Durchschnittskosten

- $\quad \mathrm{EBA}=\quad$ Everything but arms

- $\quad$ ECR $=\quad$ Efficiency Consumer Response

- $\quad$ EGV $=\quad$ Vertrag zur Gründung der Europäischen Gemeinschaft

- $\quad \mathrm{EK}=\quad$ Eigenkapital

- $\quad \mathrm{F}+\mathrm{E}=\quad$ Forschung und Entwicklung

- $\mathrm{FK}=\quad$ Fremdkapital

- $\quad$ fob $=\quad$ free on board

- GATT $=$ General Agreement on Tarifs and Trade

- $\quad \mathrm{GbR}=\quad$ Gesellschaft bürgerlichen Rechts

- $\mathrm{GK}=\quad$ Grenzkosten

- $\mathrm{GmbH}=$ Gesellschaft mit beschränkter Haft

- GWB $=$ Gesetz gegen Wettbewerbsbeschränkungen

- $\quad$ GZT $=\quad$ Gemeinsamer Zolltrarif

- $\quad \mathrm{HHI}=\quad$ Hirschmann-Herfindahl-Index

- $\quad \mathrm{HGB}=$ Handelsgesetzbuch

- $\quad$ IVP $=\quad$ Interventionspreis

- $\quad \mathrm{IZZ}=\quad$ Informationszentrum Zuckerverwender

- JuS = Juristische Schulung 
- $\quad$ JÜ $=\quad$ Jahresüberschuss

- $\mathrm{KG}=\quad$ Kammergericht

- $\quad K G=\quad$ Kommanditgesellschaft

- $\mathrm{KgaA}=$ Kommanditgesellschaft auf Aktien

- $\quad$ LBS $=\quad$ London Business School

- $\quad$ LDC $=\quad$ Least Developed Countries

- $\quad$ LEH $=\quad$ Lebensmitteleinzelhandel

- $\quad$ LM $=\quad$ Nachschlagewerk des Bundesgerichtshof in Zivilsachen, herausgegeben von Lindenmaier und Möhring

- $\quad$ LZ $=\quad$ Lebensmittelzeitung

- $\quad M \& A=\quad$ Mergers and Acquisitions

- $\mathrm{MB}=\quad$ Marktbeherrschende Stellung

- $\quad$ MIV $=\quad$ Milchindustrieverband

- $\mathrm{MMP}=$ Magermilchpulver

- $\quad$ n.BL. $=$ neue Bundesländer

- $\quad$ NEI $=\quad$ Netherlands Institute of Economics

- $\quad$ NJW $=\quad$ Neue Juristische Wochenschrift

- OECD = Organisation for Economic Cooperation and Development

- $\mathrm{OHG}=\quad$ Offene Handelsgesellschaft

- OLG $=$ Oberlandesgericht

- $\quad P=\quad$ Preis

- $\quad$ PWC $=$ PriceWaterhouseCoopers

- $\quad$ RegE $=$ Regierungsentwurf

- $\quad$ RES $=$ Rohstoffeinsatz

- $\quad$ RWI $=\quad$ Rheinisch-Westfälisches Institut für Wirtschaftsforschung

- $\quad$ SLC $=\quad$ Substantial Lessening of Competition

- $\quad$ SLS = Saint Louis Sucre

- $\quad \mathrm{TB}=\quad$ Tätigkeitsbericht

- $\quad$ UNCTAD = United Nations Conference on Trade and Development

- $\quad$ UWG $=\quad$ Gesetz gegen den unlauteren Wettbewerb

- $\quad$ WTO $=$ World Trade Organization

- WuW $=$ Zeitschrift für Wirtschaft und Wettbewerb

- $\quad W V Z=$ Wirtschaftliche Vereinigung Zucker

- $\quad$ ZAG $=\quad$ Zucker Aktiengesellschaft Uelzen-Braunschweig

- $\quad$ ZMO $=$ Zuckermarktordnung

- $\quad$ ZMP $=\quad$ Zentrale Markt- und Preisberichtstelle 


\section{Einleitung, Zielsetzung und Aufbau der Studie}

"Auch diesmal warfen die Richter Montis Kontrollbehörde verschiedene Analysemängel, Begründungsfehler und mangelnde Beweise vor" ist dem Leitartikel der Frankfurter Allgemeinen Zeitung bzgl. der Entscheidung des Europäischen Gerichtshofs über das Fusionsvorhaben von Tetra Laval und Sidel zu entnehmen ${ }^{1}$. Der europäische Wettbewerbskommissar nahm zu den Vorwürfen folgendermaßen Stellung: „Wir müssen alle aus den Fehlern lernen und vor allem in der wirtschaftlichen Analyse besser überzeugen"2.

Diese Studie soll neben der Verschaffung eines Überblickes über das Forschungsfeld von Fusionen im wesentlichen einen Beitrag zu der Diskussion um den grundlegenden Trade Off bei Fusionen im Agrar- und Ernährungsbereich leisten, der sich aus Gefahren für den Wettbewerb und aus positiven Effizienzwirkungen ergibt.

Die weltweite Fusionswelle, die Mitte der neunziger Jahre einsetzte und weiterhin andauert, ließ auch den Agrar- und Ernährungsindustriesektor nicht unberührt und hat der wettbewerbspolitischen Diskussion neuen Auftrieb gegeben (z.B. Südzucker/Saint Louis Sucre; Nordzucker/Union-Zucker; MZO/NM/Bremerland/Hansano). Insbesondere die Fusionskontrolle ist wieder in den Blickpunkt gerückt. Von Vertretern aus der Wirtschaft, der Wissenschaft und den Entscheidungsinstitutionen werden die Fusionsaktivitäten höchst unterschiedlich beurteilt. Hinter der Bandbreite der Standpunkte verbergen sich nicht nur unterschiedliche ordnungspolitische Grundpositionen sondern auch Unsicherheiten darüber, welche Ursachen und Motive die gesteigerten Fusionsaktivitäten der letzten Jahre begründen.

Es gibt eine Vielzahl von wissenschaftlichen Untersuchungen, die bei einer Beurteilung der Wettbewerbspolitik berücksichtigt und auf ihre Anwendbarkeit für Unternehmensfusionen geprüft werden sollten. Neben der Vermittlung von rechtlichen Grundlagen und ihren ökonomischen Auswirkungen sollen in dieser Studie die Besonderheiten von Unternehmensfusionen im Zucker- und Milchsektor hervorgehoben werden, um Erkenntnisse zu gewinnen, ob die vom Bundeskartellamt bzw. der Europäischen Kommission angewandten juristischen Aspekte zur Überprüfung von Fusionen im Einklang mit ökonomischen Kriterien stehen.

\footnotetext{
${ }^{1}$ Vgl. Frankfurter Allgemeine Zeitung vom 25.10.02: „Europarichter kippen drittes Fusionsverbot von Wettbewerbskommissar Monti", S. 14; vgl. auch FINANCIAL TIMES DEUTSCHLAND: www.ftd.de/pw/eu/1035389220549.html

${ }^{2}$ Ebenda
} 
Konkret wird zunächst die Bedeutung von Unternehmenszusammenschlüssen und -beteiligungen weltweit wie auch national verdeutlicht. Der Schwerpunkt wird auf die Milch- und Zuckerbranche in Europa gelegt. Darauf aufbauend soll die Motivation von Fusionen aus Sicht der Unternehmen näher ergründet und mit Hilfe modelltheoretischer Untersuchungen auf Wohlfahrtswirkungen geschlossen werden. Dabei wird vor allem auf den Zielkonflikt zwischen Effizienz und Marktmacht hingewiesen und die Frage nach dem Schutz vor negativen Effekten von Zusammenschlüssen aufgegriffen. Nach Vorstellung des Rechtsrahmens sowohl auf europäischer als auch nationaler Ebene wird die praktische Umsetzung der Kartellrechtspraxis für ausgewählte Zusammenschlüsse überprüft und die Kritik der betroffenen Unternehmen beurteilt. Kontroverse Aspekte werden detailliert analysiert und mit Hilfe ökonomischer Erkenntnisse diskutiert. Anhand von empirischen Forschungsergebnissen und modernen betriebswirtschaftlichen Analysemethoden werden Möglichkeiten einer präziseren Zusammenschlussbeurteilung vorgestellt. Abschließend werden die Ergebnisse zusammengefasst und im Zuge der Schlussfolgerung mögliche Modifikationen aufgezeigt.

Die Erkenntnisse werden durch folgende Methodik gewonnen: Zunächst werden wichtige Entwicklungen der industrieökonomischen wie auch der betriebswirtschaftlichen Literatur bzgl. der Wirkung von Fusionen dargelegt und die Vorgaben des Rechtsrahmens sowie der tatsächlichen Rechtspraxis untersucht. Anschließend werden die schriftlichen und mündlichen Ergebnisse der Unternehmensbefragungen vorgestellt, um die praktischen Motive, Interessenskonflikte und mögliche Verbesserungspotentiale aufzunehmen. Durch Gespräche und Akteneinsicht bei den Kartellbehörden konnten entsprechende Stellungnahmen sowie die verfahrensrechtliche Vorgehensweise studiert werden. Theoretische Erkenntnisse werden anschließend anhand einer ex-post Analyse überprüft und bewertet. In Kombination mit den vorigen Erkenntnissen werden daraus mögliche Verbesserungsvorschläge für eine Zusammenschlusskontrolle erarbeitet. Abbildung 1 verdeutlicht den Aufbau und die Fragestellungen der Arbeit. 


\section{Abbildung 1: Aufbau und Fragestellungen der Untersuchung}

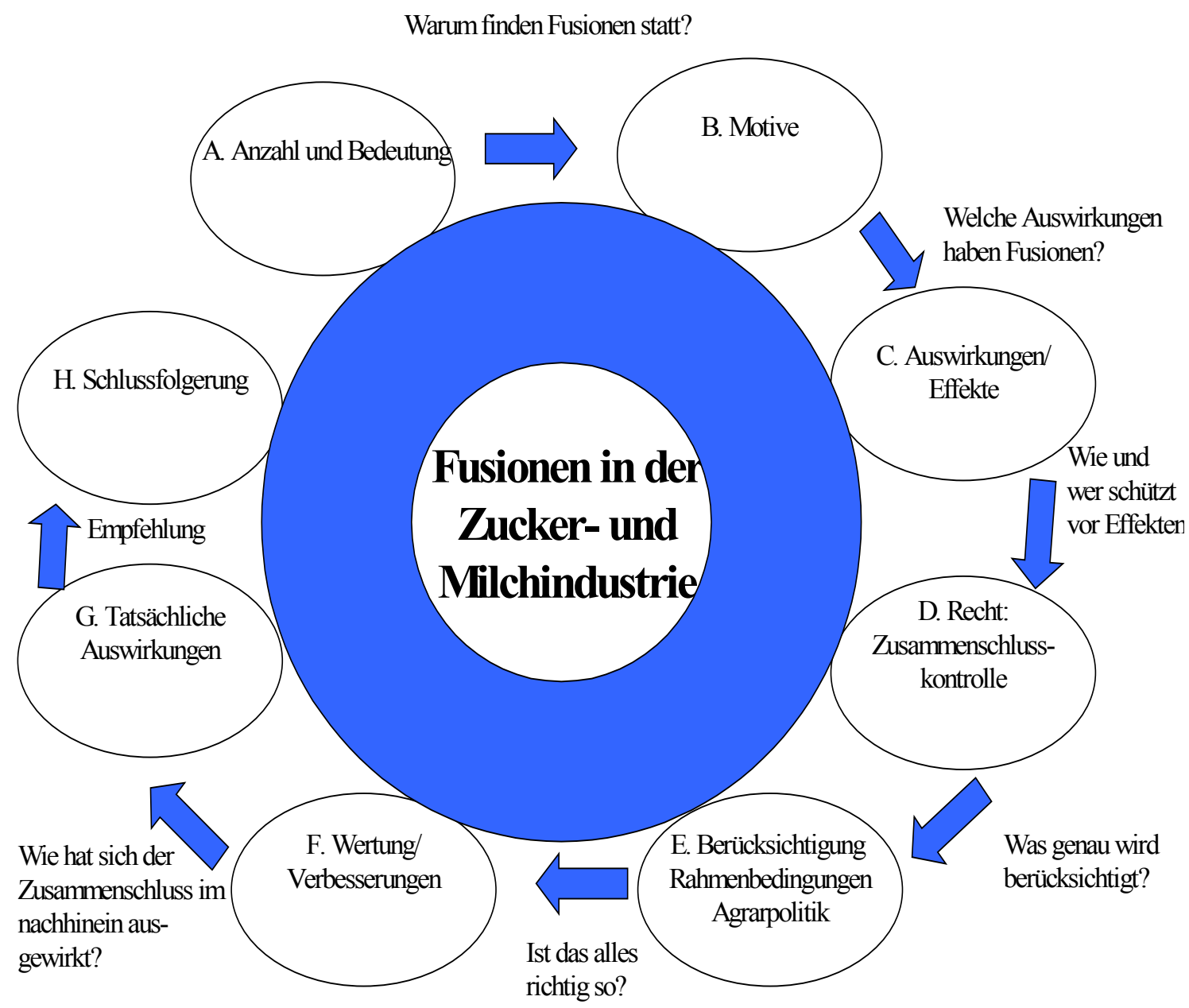

Quelle: eigene Darstellung 


\section{Entwicklung von Unternehmenszusammenschlüssen}

Zu Beginn dieser Untersuchung wird die Bedeutung von Fusionen und Unternehmenszusammenschlüssen dargestellt. Dabei werden zunächst als grober Überblick die welt-, europa- und deutschlandweiten Fusionsaktivitäten herausgestellt. Im weiteren Verlauf der Untersuchung liegt der Fokus im Bereich der Ernährungsindustrie, wobei abschlieBend speziell die Zucker- und Milchbranche näher analysiert werden.

\subsection{Weltweit}

Zunächst sei ein historischer Exkurs über sogenannte Fusionswellen vollzogen. Es scheint, als verlaufen Fusionen in Zyklen ${ }^{3}$ (vgl. Abbildung 2). Die Ursachen können vielfältig sein und basieren z.B. auf veränderte Rechtsrahmen, wechselnde Führungsideologien, sinkende Transaktionskosten oder intuitives Handeln mit der Folge, dass ähnlich wie bei Aktienmärkten Fusionsbooms und -crashs eintreten. Einzelne Fusionsmotive werden im folgenden Kapitel (vgl. Kapitel 1) näher vorgestellt. Gleichzeitig wird der Versuch unternommen, einige wichtige Rahmenbedingungen aufzuzeigen, die in der Literatur für das Zustandekommen der Wellen herangezogen werden.

Abbildung 2: Anzahl und Rahmenbedingungen von Fusionen

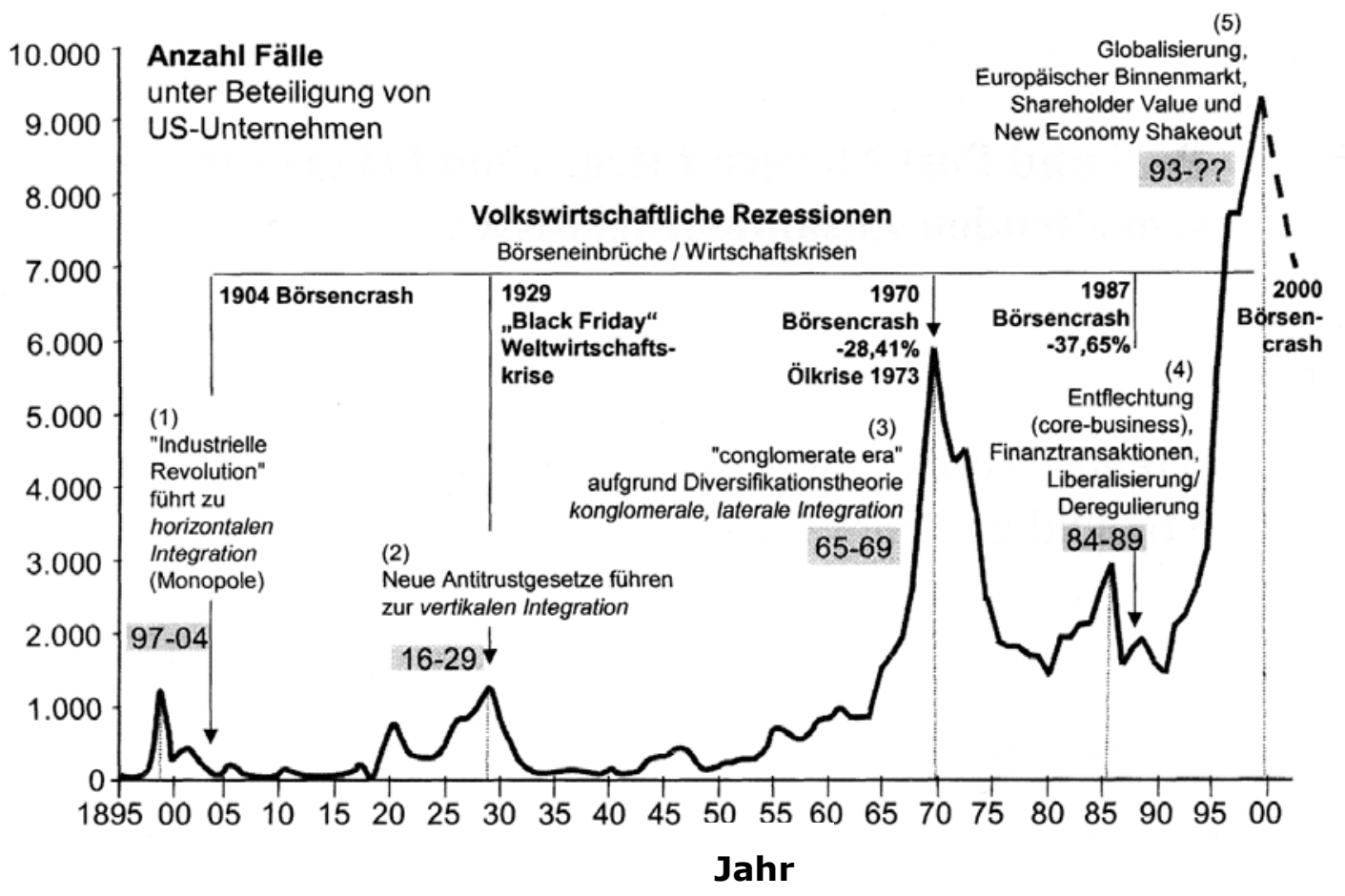

Quelle: JANSEN/MÜLLER-STEWENS 2001, S. 3

${ }^{3}$ Vgl. u.a. JASNIK 2002, S. 51 
Die managementorientierte Literatur leitet aus den Fusionswellen unterschiedliche strategische Zielsetzungen ab, die in Tabelle 1 zusammenfassend dargestellt sind.

Tabelle 1: Zielsetzungen der Fusionswellen

\begin{tabular}{|l|l|l|}
\hline Zeitraum & & Strategische Zielsetzung \\
\hline $1897-1904$ & 1. Welle & $\begin{array}{l}\text { Vermeidung von Überkapazitäten und Preisverfall durch horizontale Zusam- } \\
\text { menschlüsse: Aufbau von Trusts }\end{array}$ \\
\hline $1916-1929$ & 2. Welle & $\begin{array}{l}\text { Vertikale Integration; Streben nach marktbeherrschender Position und Kon- } \\
\text { trolle des gesamten Produktlebenszyklus }\end{array}$ \\
\hline $1965-1969$ & 3. Welle & $\begin{array}{l}\text { Entstehung großer Konglomerate durch Aufnahme von Targets in Portfolio: } \\
\text { vorrangig in den USA }\end{array}$ \\
\hline $1984-1990$ & 4. Welle & $\begin{array}{l}\text { "Merger Mania" - Strategische „Mergers- and Acquisitions"-Transaktionen: } \\
\text { Konzentration auf Kerngeschäft, Erschließung neuer Synergien }\end{array}$ \\
\hline $1993-?$ & 5. Welle & $\begin{array}{l}\text { Globale Mega-Deals: Fortschreitende digitale Globalisierung, Schaffung des } \\
\text { europäischen Binnenmarktes, zunehmend wertorientierte Unternehmensfüh- } \\
\text { rung }\end{array}$ \\
\hline
\end{tabular}

Quelle: www.mergers-and-acquisitions.de/fakten1010.htm; 21.08.2002

Wie zuvor skizziert, sind die letzten Jahre erneut von einer zunehmenden Anzahl von Unternehmenszusammenschlüssen gekennzeichnet. Weltweit wurde im Jahr 2000 ein neues Rekordtransaktionsvolumen von 3.500 Mrd. US \$ erreicht. Abbildung 3 verdeutlicht die Entwicklung:

Abbildung 3: Transaktionsvolumen weltweiter Fusionen (Angaben in Mrd. US \$)

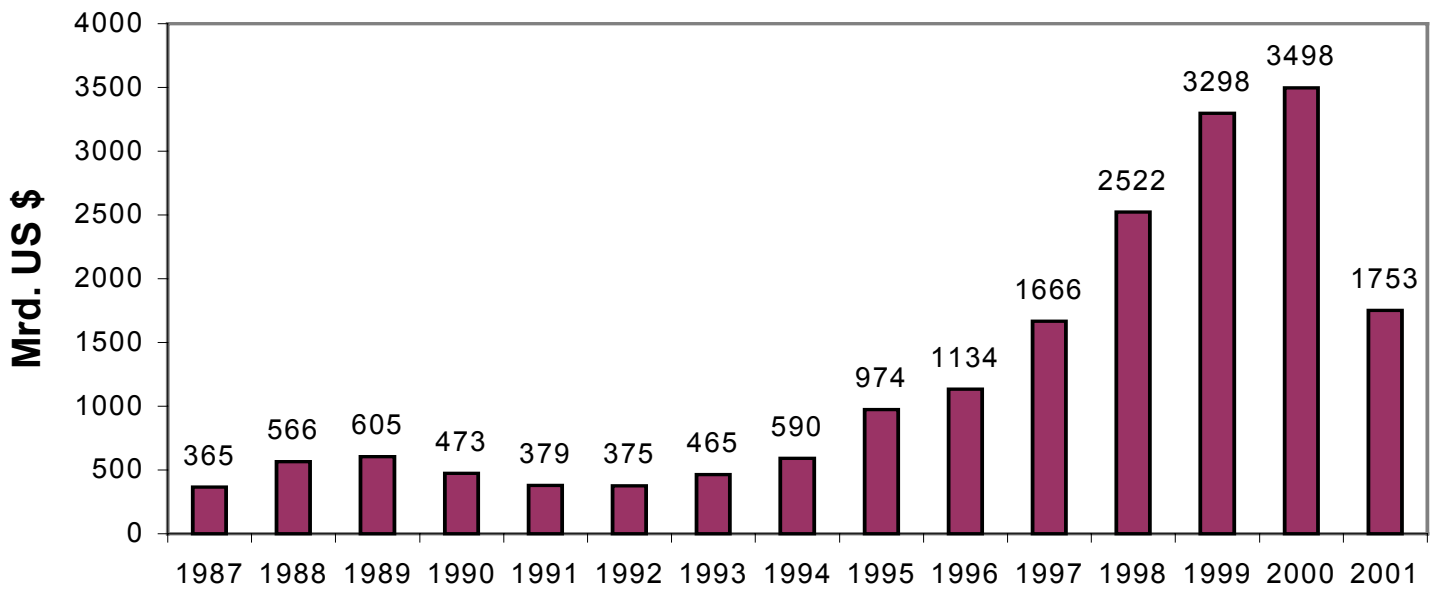

Quelle: THOMSON FINANCIAL 2002

Im Jahr 2001 sowie in der ersten Jahreshälfte des Jahres 2002 wurde ein Rückgang der Fusionstätigkeit festgestellt, wobei dies größtenteils auf eine eher pessimistisch 
geprägte Weltkonjunktur und dem damit verbundenen Investitionsklima zurückzuführen ist ${ }^{4}$.

\subsection{Europa}

Nachdem ein Überblick über das globale Geschehen am "Mergers and Acquisitions"Markt vermittelt wurde, steht in diesem Abschnitt die europäische Dimension im Mittelpunkt der Betrachtung. Beim Vergleich von Statistiken werden vielfach unterschiedliche Zahlen für Anzahl und Volumen von Transaktionen angeführt. Neben Unterschieden bei der Datenerfassung ist dies durch die Definitions- und Abgrenzungsproblematik zu erklären. Folgende Zusammenschlüsse (Abbildung 4) wurden bei der Erstellung von Abbildung 5 berücksichtigt.

Abbildung 4: Statistische Abgrenzungen

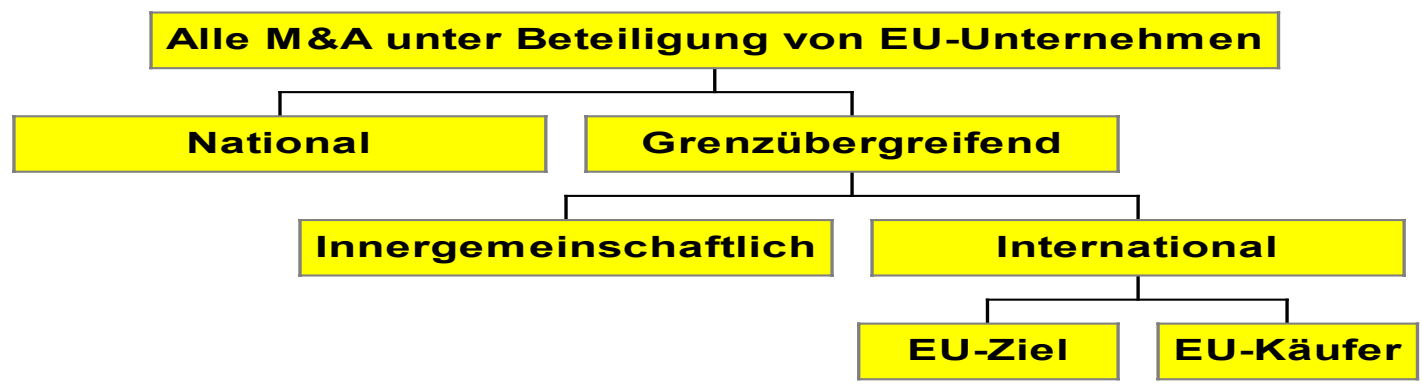

Quelle: EUROPÄISCHE KOMMISSION 2000a, S. 8

Danach ist innerhalb der letzten 10 Jahre folgende Entwicklung (Abbildung 5) zu beobachten. Auch hier scheint ein Rückgang der Fusionsaktivität stattzufinden.

Abbildung 5: Transaktionen mit europäischer Beteiligung von 1991-2001

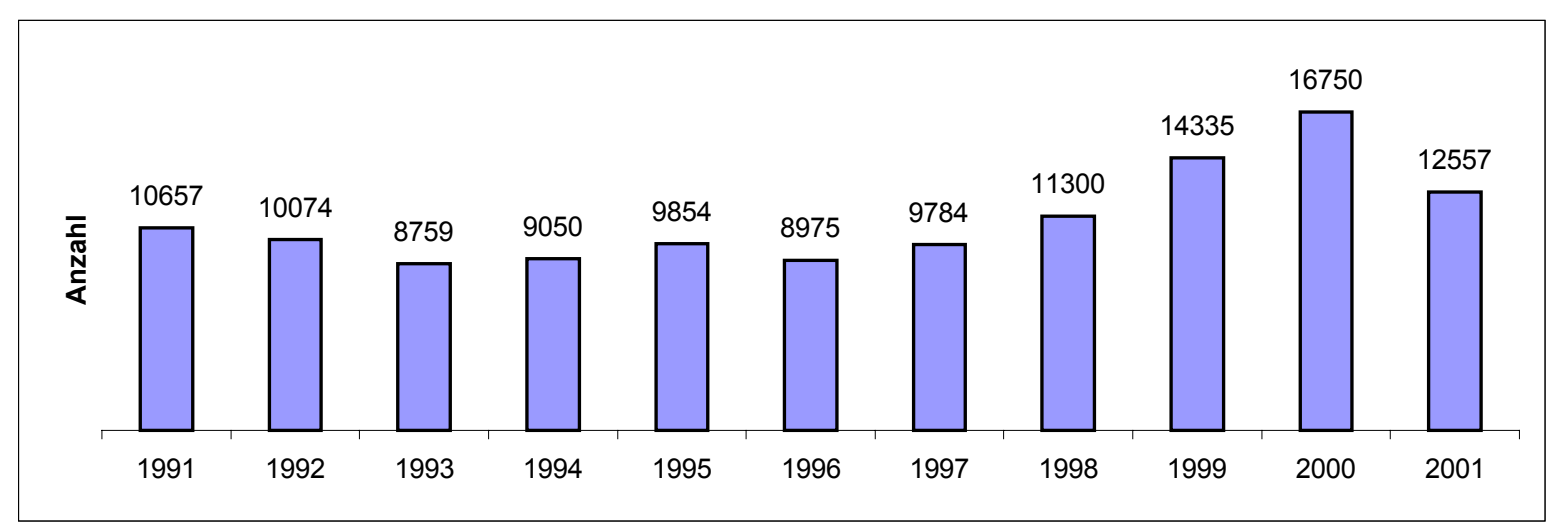

Quelle: EUROPEAN COMMISSION 2001, S. 3

\footnotetext{
${ }^{4}$ Vgl. Financial Times DeUtSChLAND vom 10.01.2002 (online); Welt vom 25.06.2002 (online); KPMG 2002
} 
In der obigen Abbildung sind alle Varianten von Unternehmenstransaktionen mit europäischer Beteiligung enthalten. Vielfach handelt es sich jedoch um Transaktionen mit geringem Volumen oder um Beteiligungen außerhalb Europas, die kaum Beachtung in der Öffentlichkeit finden. Innerhalb Europas kann folgende Entwicklung des Transaktionsvolumens bzw. der Anzahl beobachtet werden (Abbildung 6).

\section{Abbildung 6: Transaktionsvolumen und Anzahl von Fusionen innerhalb Europas}

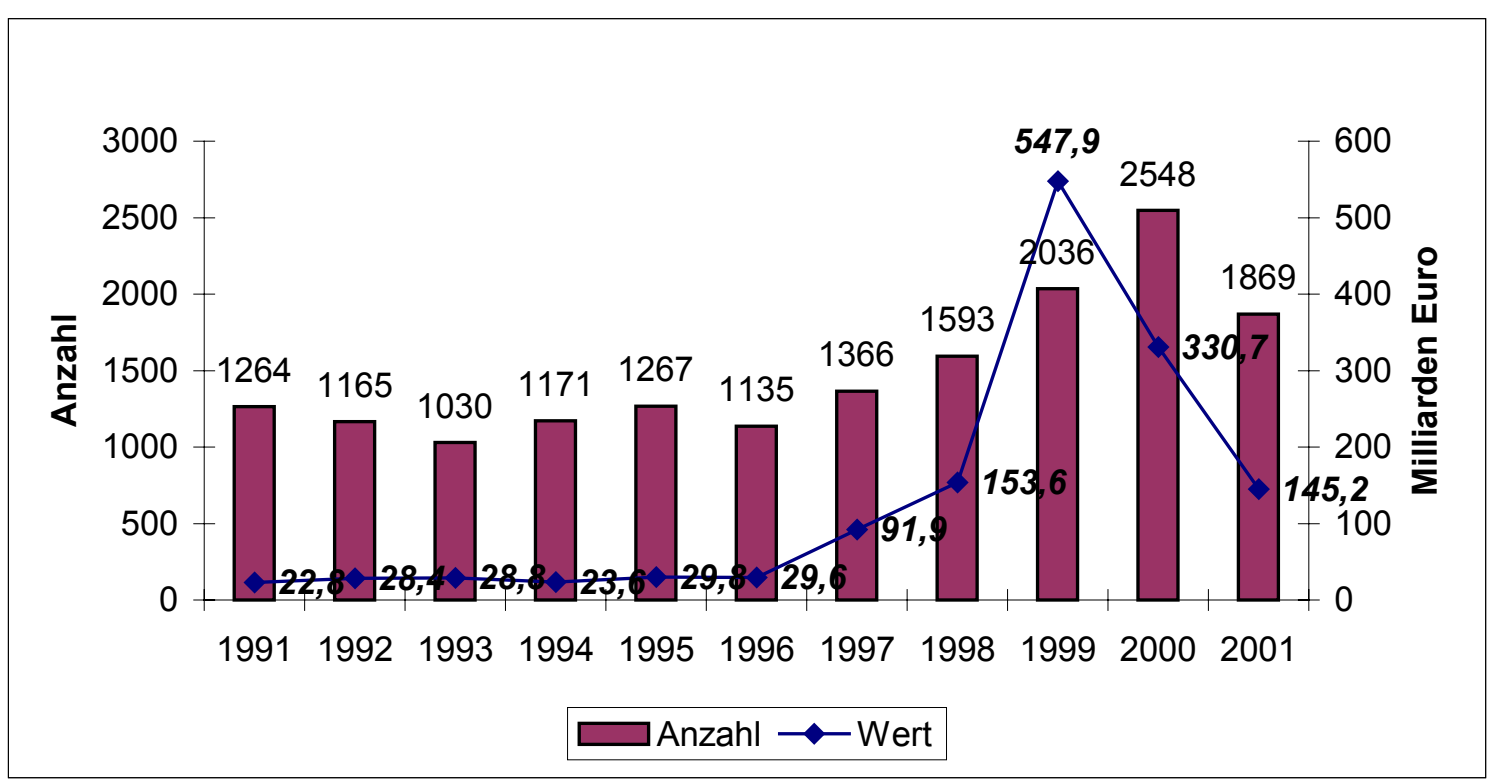

Quelle: EUROPEAN COMMISSION 2001, S. 7

Der Schwerpunkt dieser Untersuchung liegt in der Beurteilung der Wettbewerbspraxis der Europäischen Kommission sowie des Bundeskartellamtes (auf nationaler Ebene). Aufgrund der Aufgreifkriterien (vgl. Kapitel 5) liegt die Anzahl der von der europäischen Wettbewerbsbehörde erfassten und untersuchten Fälle weitaus geringer. Nach der amtlichen Statistik ergeben sich danach folgende Werte (vgl. Tabelle 2).

Tabelle 2: Unter der Fusionskontrolle erfasste Fälle

\begin{tabular}{|l|l|l|l|l|l|l|l|l|l|}
\hline & $\begin{array}{l}\mathbf{1 9 9 0} \\
\mathbf{1 9 9 4}\end{array}$ & $\mathbf{1 9 9 5}$ & $\mathbf{1 9 9 6}$ & $\mathbf{1 9 9 7}$ & $\mathbf{1 9 9 8}$ & $\mathbf{1 9 9 9}$ & $\mathbf{2 0 0 0}$ & $\mathbf{2 0 0 1}$ & $\begin{array}{l}\text { Insge- } \\
\text { samt }\end{array}$ \\
\hline Angemeldet & 288 & 110 & 131 & 172 & 235 & 292 & 345 & 335 & 1908 \\
\hline Zurückgezogen & 11 & 4 & 6 & 9 & 9 & 12 & 14 & 12 & 77 \\
\hline Entscheidungen & 274 & 109 & 125 & 134 & 235 & 268 & 341 & 334 & 1820 \\
\hline \begin{tabular}{l} 
Darunter (FKVO) \\
\hline $\begin{array}{l}\text { Kein Anwendungsbe- } \\
\text { reich (Art. 6.1a) }\end{array}$
\end{tabular} 25 & 9 & 6 & 4 & 6 & 1 & 1 & 1 & 53 \\
\hline $\begin{array}{l}\text { Phase 1: keine Beden- } \\
\text { ken (Art. 6.1b) }\end{array}$ & 222 & 90 & 109 & 118 & 207 & 236 & 293 & 299 & 1574 \\
\hline
\end{tabular}


Tabelle 2 (Fortsetzung)

\begin{tabular}{|l|l|l|l|l|l|l|l|l|l|}
\hline & $\begin{array}{l}\mathbf{1 9 9 0} \\
\mathbf{1 9 9 4}\end{array}$ & $\mathbf{1 9 9 5}$ & $\mathbf{1 9 9 6}$ & $\mathbf{1 9 9 7}$ & $\mathbf{1 9 9 8}$ & $\mathbf{1 9 9 9}$ & $\mathbf{2 0 0 0}$ & $\mathbf{2 0 0 1}$ & $\begin{array}{l}\text { Insge- } \\
\text { samt }\end{array}$ \\
\hline $\begin{array}{l}\text { Phase 1: Bedenken } \\
\text { (Art. 6.1b) }\end{array}$ & 9 & 3 & 0 & 2 & 12 & 19 & 28 & 13 & 86 \\
\hline $\begin{array}{l}\text { Verweisung an Mit- } \\
\text { gliedstaat (Art. 9) }\end{array}$ & 1 & 0 & 3 & 1 & 1 & 3 & 2 & 1 & 12 \\
\hline $\begin{array}{l}\text { Phase 2: keine Aufla- } \\
\text { gen (Art. 8.2) }\end{array}$ & 5 & 2 & 1 & 1 & 3 & 0 & 3 & 5 & 20 \\
\hline $\begin{array}{l}\text { Phase 2: mit Auflagen } \\
\text { (Art. 8.2) }\end{array}$ & 10 & 3 & 3 & 7 & 4 & 8 & 12 & 10 & 57 \\
\hline Untersagung (Art. 8.3) & 2 & 2 & 3 & 1 & 2 & 1 & 2 & 5 & 18 \\
\hline
\end{tabular}

Quelle: EU-Kommission, Bericht über die Wettbewerbspolitik, Ifd. Jgg.

\subsection{Deutschland}

Auf nationaler Ebene wurde dem Bundeskartellamt die unten angeführte Anzahl von Zusammenschlüssen gemeldet (vgl. Abbildung 7). Dabei ist zu beachten, dass ein gewisser Fusionsboom in den 90er Jahren in erster Linie auf die Effekte der Wiedervereinigung Deutschlands zurückzuführen ist ${ }^{5}$.

\section{Abbildung 7: Beim Bundeskartellamt angezeigte vollzogene Fusionen}

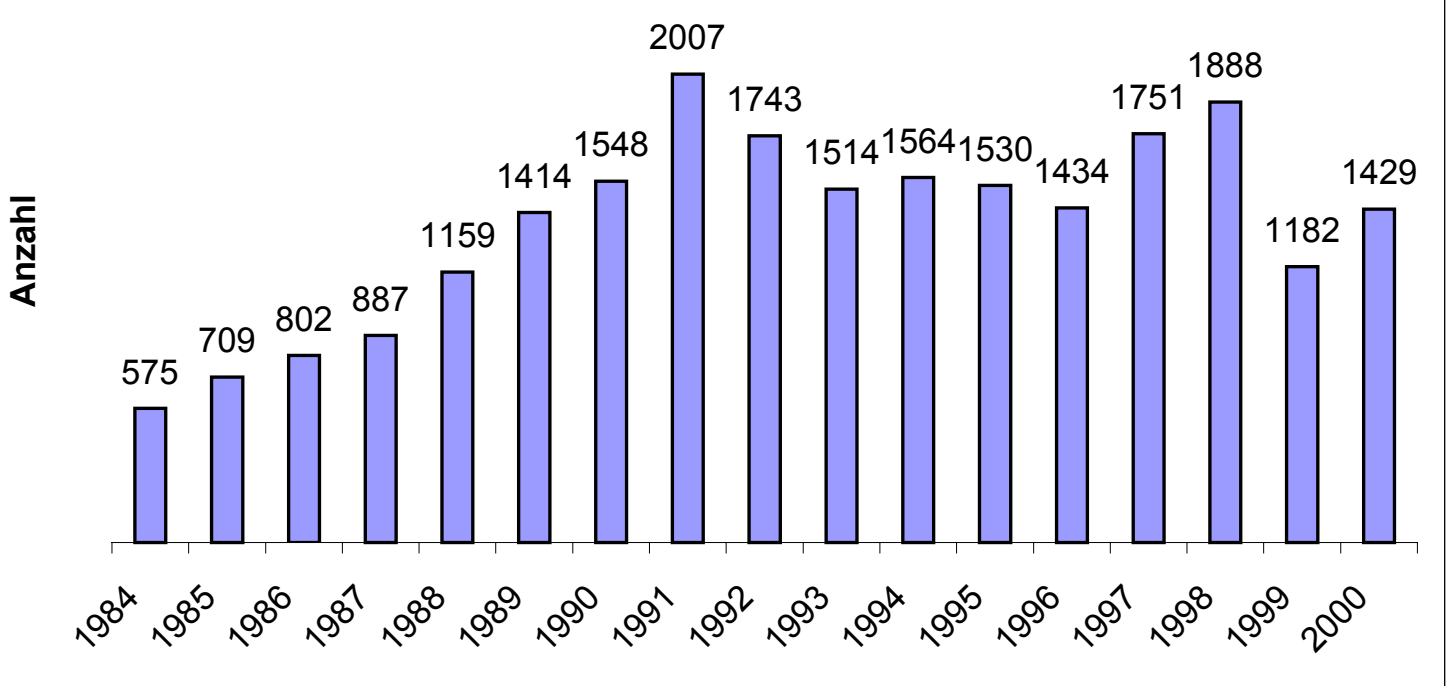

Quelle: BundeSKARTELLAMT, Tätigkeitsbericht, Ifd. Jgg.

${ }^{5} \mathrm{Vgl.} \mathrm{BUNDESKARTELLAMT} 1999$ 
Die reale Zahl der Zusammenschlüsse und Beteiligungen liegt weitaus höher, denn ein Großteil von Unternehmensverbindungen, die unter der Aufgreifschwelle des Wettbewerbsrechts liegen, werden nicht erfasst. Ebenso sind Unternehmenszusammenschlüsse mit deutscher Beteiligung, die unter das europäische oder Drittlandrecht fallen, nicht berücksichtigt.

Unternehmenszusammenschlüsse können in horizontale (Zusammenschlüsse innerhalb einer Branche), vertikale (Zusammenschlüsse mit vor- oder nachgelagerten Branchen) oder konglomerate (Zusammenschlüsse mit branchenfremden Unternehmen) unterteilt werden. In dieser Untersuchung wird der Schwerpunkt auf horizontale Unternehmenszusammenschlüsse gelegt, da diese quantitativ den größten Anteil an Fusionen ausmachen (vgl. Abbildung 8) und auch den Großteil der wettbewerblichen Bedenken seitens der Kartellbehörden auf sich ziehen ${ }^{6}$.

\section{Abbildung 8: Anzahl der Arten von Unternehmenszusammenschlüssen}

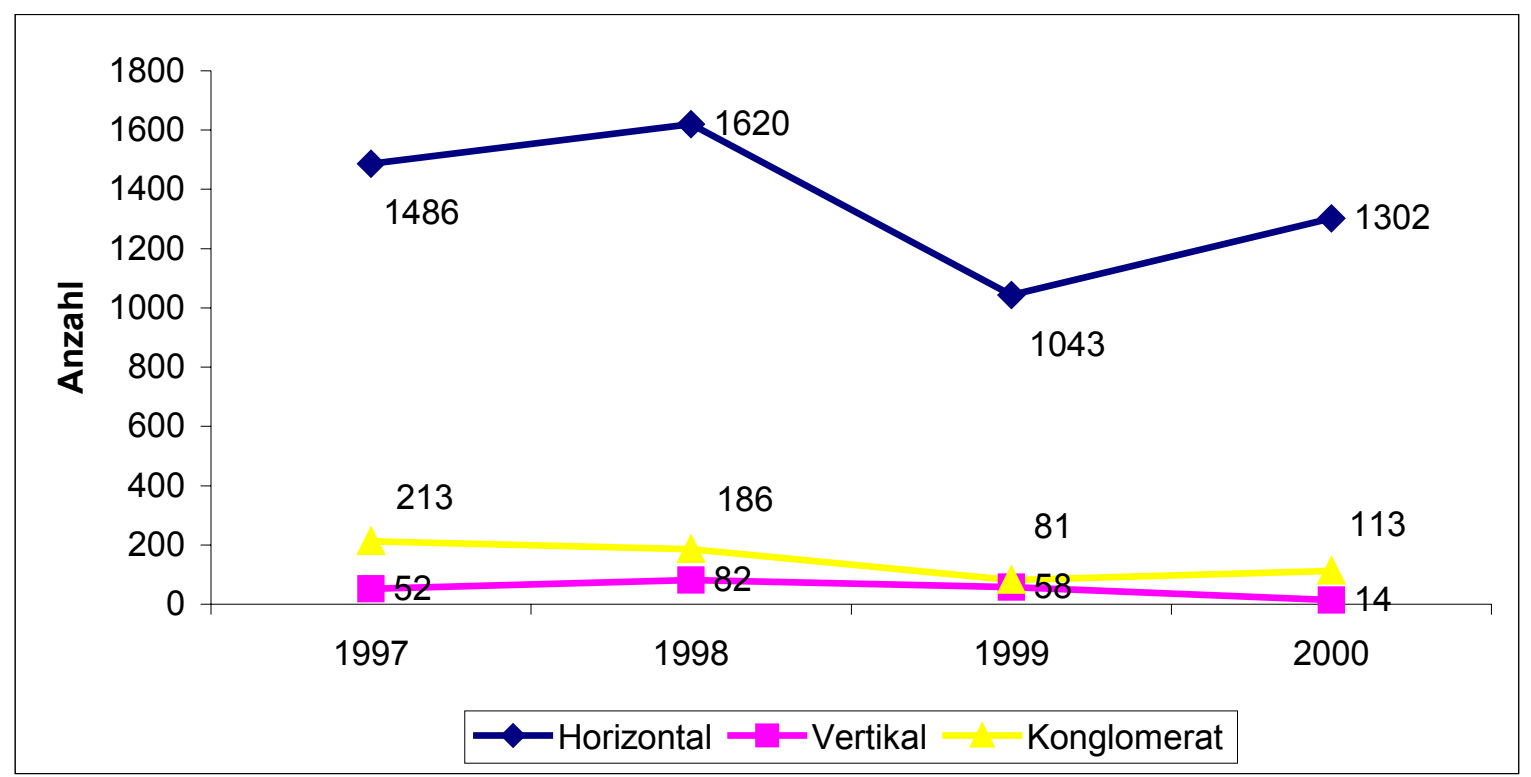

Quelle: BUNDESKARTELLAMT, Tätigkeitsbericht, Ifd. Jgg.

\subsection{Ernährungsindustrie}

Im Jahr 2001 wurde in der Ernährungsindustrie ein Umsatz von 126,6 Mrd. € erwirtschaftet ${ }^{7}$. Darüber hinaus ist die Ernährungsindustrie mit anderen Wirtschaftszweigen verkettet, auf die ein Unternehmenszusammenschluss zumindest indirekte Auswirkun-

\footnotetext{
${ }^{6}$ Vgl. dazu EU-KOMmISSION: Bericht über die Wettbewerbspolitik, laufende Jahrgänge und BUNDESKARTELLAMT: Tätigkeitsbericht, laufende Jahrgänge

7 Vgl. BMVEL 2002, Ernährungswirtschaft
} 
gen ausübt. Die nachfolgende Abbildung (Abbildung 9) vermittelt die Vernetzung und Größenordnung der betroffenen Branchen.

Abbildung 9: Struktur und Komponenten der Wertschöpfungskette in der deutschen Ernährungswirtschaft

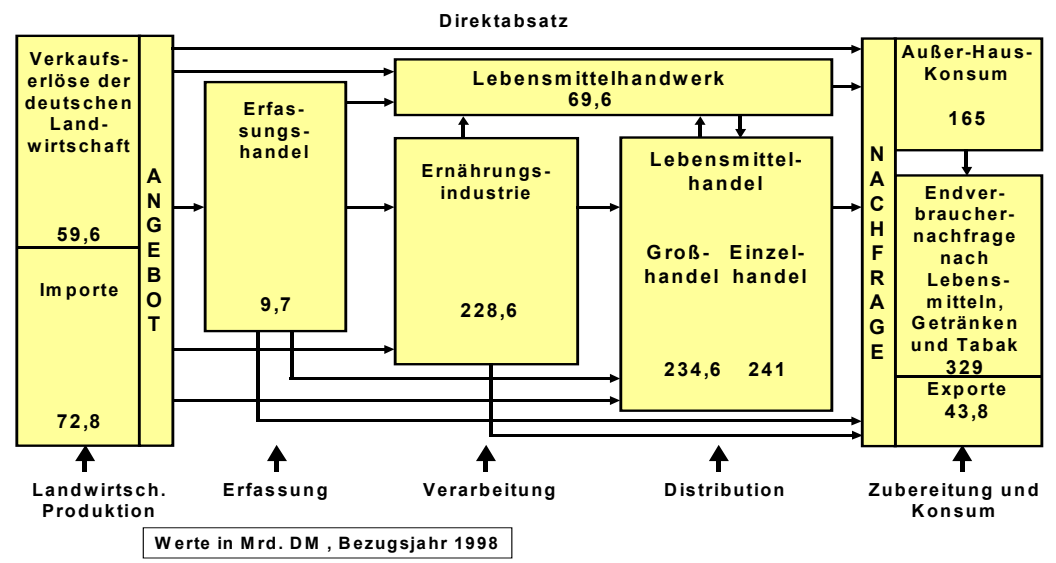

Quelle: WeIndLMAIER 2001

Innerhalb der Ernährungsindustrie teilen sich die Kennziffern auf verschiedene Branchen auf (vgl. Tabelle 3).

Tabelle 3: Strukturdaten der Ernährungsindustrie im Jahr 2000

\begin{tabular}{|l|l|l|l|}
\hline Ausgewählte Branche & Umsatz (Mio. DM) & Beschäftigte & Betriebe \\
\hline Schlachten und Fleischverarbeitung & 42455 & 112677 & 1363 \\
\hline Fischverarbeitung & 3634 & 10637 & 99 \\
\hline Obst- und Gemüseverarbeitung & 14553 & 28246 & 322 \\
\hline $\begin{array}{l}\text { Herst. v. pflanzl. u. tierischen Ölen und } \\
\text { Fetten }\end{array}$ & 8270 & 4972 & 34 \\
\hline Milchverarbeitung & 41024 & 41142 & 286 \\
\hline $\begin{array}{l}\text { Mahl- und Schälmühlen, Herst. v. } \\
\text { Stärke }\end{array}$ & 6829 & 10336 & 108 \\
\hline Herstellung v. Backwaren & 18846 & 164246 & 2384 \\
\hline Herstellung v. Dauerbackwaren & 5895 & 19923 & 105 \\
\hline Zuckerindustrie & 6219 & 6684 & 38 \\
\hline Herstellung v. Süßwaren & 14535 & 30710 & 150 \\
\hline Herstellung v. Teigwaren & 907 & 2446 & 32 \\
\hline
\end{tabular}


Tabelle 3 (Fortsetzung)

\begin{tabular}{|l|l|l|l|}
\hline Ausgewählte Branche & Umsatz (Mio. DM) & Beschäftigte & Betriebe \\
\hline Verarbeit. v. Kaffee, Tee, Kaffeeersatz & 7776 & 6269 & 44 \\
\hline Herstellung von Würzen und Soßen & 6629 & 14585 & 73 \\
\hline $\begin{array}{l}\text { Herst. v. homog. u. diätet. Nahrungs- } \\
\text { mitteln }\end{array}$ & 1682 & 3662 & 14 \\
\hline Herstellung von Spirituosen & 5638 & 4420 & 91 \\
\hline Herst. von Bier & 18062 & 37618 & 367 \\
\hline $\begin{array}{l}\text { Mineralbrunnen, Herst. v. Erfri- } \\
\text { schungsgetränken }\end{array}$ & 12469 & 25113 & 229 \\
\hline Ernährungsindustrie & $\mathbf{2 3 5 4 4 5}$ & $\mathbf{5 5 4 6 3 3}$ & $\mathbf{6 1 3 6}$ \\
\hline
\end{tabular}

Quelle: BMVEL 2001, Statistisches Jahrbuch

Unternehmenszusammenschlüsse prägten in den vergangenen Jahren das Bild der Ernährungsindustrie und werden auch zukünftig an Bedeutung gewinnen ${ }^{8}$.

1998 und 1999 gehörte der Lebensmittelbereich zu der zweitgrößten transaktionsaktivsten Branche in Europa (gemessen an der Anzahl).

Abbildung 10: Fusionen und Akquisitionen in Deutschland 1998 und 1999

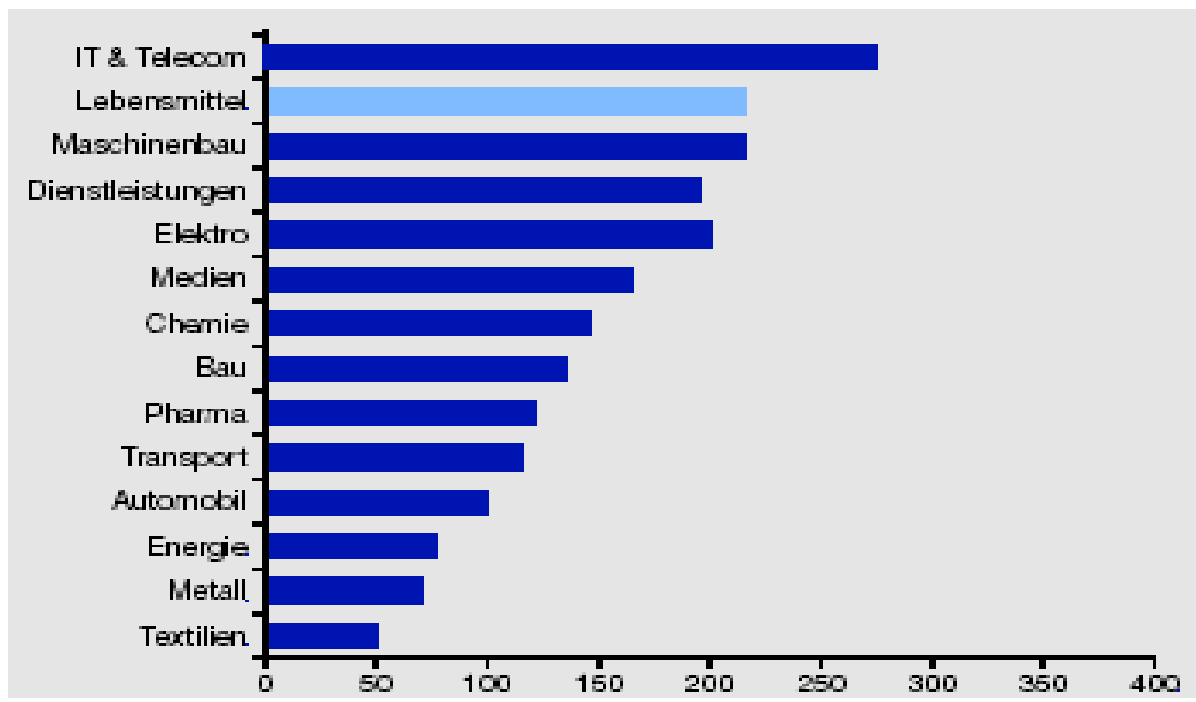

Anzahl der Transaktionen

Quelle: Deutsche Genossenschaftsbank 2000, S. 89

${ }^{8}$ Vgl. Deutsche GenossenschaftSBank 2000, S. 59 ff. 
Auf europäischer Ebene sind in den Jahren 2000 und 2001 8\% aller Unternehmenszusammenschlüsse in der Industrie im Bereich der Ernährungsindustrie getätigt worden ${ }^{9}$. Für besondere Aufmerksamkeit sorgten in den vergangenen Jahren folgende Zusammenschlüsse (Tabelle 4).

Tabelle 4: Wichtige Zusammenschlüsse in der Ernährungsindustrie

\begin{tabular}{|c|c|c|c|}
\hline Jahr & beteiligte Unternehmen (Europa) & Jahr & beteiligte Unternehmen (Europa) \\
\hline \multirow[t]{11}{*}{1998} & Coca-Cola/Cadbury-Schweppes & 2000 & Müller/Weihenstephan \\
\hline & Nestlé/San Pellegrino & & Bongrain/Boursault \\
\hline & United Biscuits/Delacre & & Unilever/Bestfoods \\
\hline & Südzucker/Hjems & & Danone/Scottish Newcastle \\
\hline & Numico/Szabolcsteij & & General Mills/Pillsbury/Diageo \\
\hline & Parmalat/Pauls & & Nabisco/United Biscuits \\
\hline & Parmalat/Clesa & 2001 & Südzucker/Saint Louis Sucre \\
\hline & Parmalat/Farmland Dairies & & Nutricia/Baxter \\
\hline & Besnier/Locatelli & & Cargill/Cerestar \\
\hline & Nestlé/Kim Bordon & & Heineken/Bayrische Brauholding \\
\hline & Campina/MW Köln-Wuppertal & & Becks/Interbrew \\
\hline \multirow[t]{10}{*}{1999} & Danone/Vilavicenio & & Friesland Coberco/Nutricia \\
\hline & Danone/Griesson & & Nestlé/Ralston Purina \\
\hline & Danone/Bolshevik & & Pernod Ricard/ Diageo/ Seagram \\
\hline & Unigate/ M.Surgeles/G.Traiteur & & Pepsi Co./Quaker \\
\hline & EQT/Findus & & Heinz/CSM \\
\hline & Parmalat/Cirio & & Cargill/Agribrands \\
\hline & Danone/Bakoma & & ABN/Perkins Food \\
\hline & Unilever/Mountain Cream & 2002 & Danish Crown/Steff Houlberg \\
\hline & Nordmilch/MZO/Bremerland/Hansano & & Barilla/Kamps \\
\hline & Barilla/Wasa & & Karlsberg/Brauholding International \\
\hline \multirow[t]{4}{*}{2000} & Danone/McKesson & & Nestlé/Schöller \\
\hline & Henninger/Odenwald/Oppacher & & Masterfoods/Royal Canin \\
\hline & Philip Morris/Nabisco & & Nordzucker/Union-Zucker \\
\hline & Tuffi-Campina/Emzett & & \\
\hline
\end{tabular}

Quelle: eigene Zusammenstellung nach EU-KoмmIssIon, Generaldirektion Wettbewerb: http://europa.eu.int/comm/competition/mergers/cases sowie BUNDESKARTELLAMT: http://www.bundeskartellamt.de/archiv.html

Die Folge von Zusammenschlüssen ist eine weitere Konzentration innerhalb der Branche. Die Konzentrationsrate (1998) der 10 größten Unternehmen $\left(C_{10}\right)$ sind in der

${ }^{9}$ Vgl. EU-COMMISSION 2001, S. 17 
folgenden Darstellung aufgelistet (Abbildung 11). Bei den im Fokus stehenden Zuckerund Milchbranchen handelt es sich demnach um Sektoren, die von stark unterschiedlicher Konzentrationsrate geprägt sind.

Abbildung 11: Umsatzanteil der 10 größten Unternehmen (in Prozent)

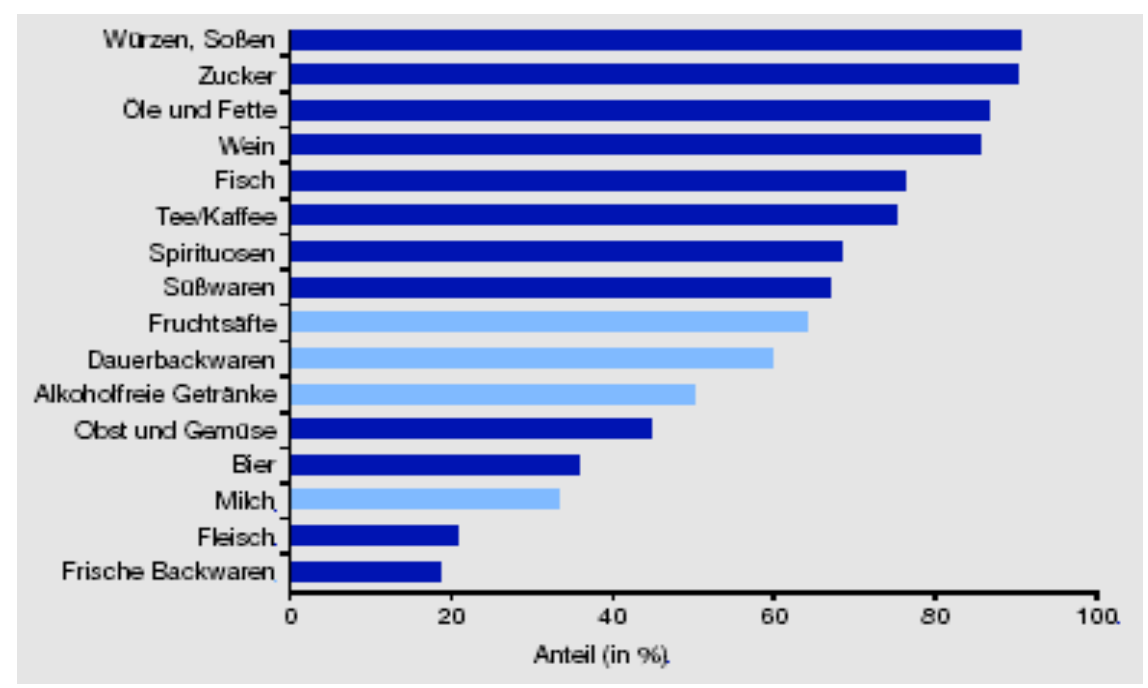

Quelle Deutsche Genossenschaftsbank 2000, S. 54

Auch zukünftig scheint der Trend zu weiteren Unternehmenszusammenschlüssen anzuhalten. In der Branchenuntersuchung vom Bundesverband der Ernährungsindustrie (BVE) und der Beratungsgesellschaft PriceWaaterhouseCoopers (PWC) verneinten nur $34 \%$ der befragten Unternehmer die Fusionsbereitschaft ${ }^{10}$ (vgl. Abbildung 12). Besonders innerhalb Europas bzw. in den Beitrittsstaaten wird voraussichtlich der Großteil der Zusammenschlüsse stattfinden.

Abbildung 12: Zukünftige Akquisitionsziele der deutschen Ernährungsindustrie

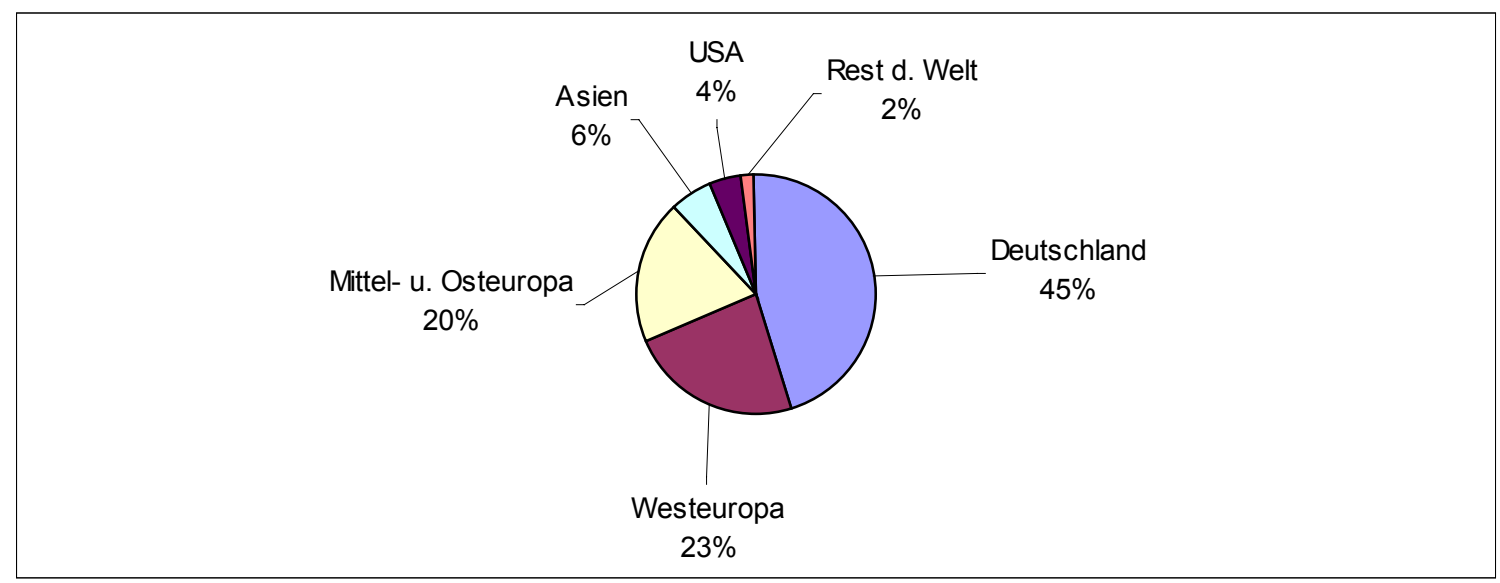

Quelle: Bundesverband der ERnÄHRUngsindustrie/PriceWAaterhouseCoOPers 1998, S. 13

${ }^{10} \mathrm{Vgl.} \mathrm{BVE/PWC} 1998$, S. 13 
Diese Ergebnisse werden von vorläufigen Ergebnissen einer Befragung von 119 Unternehmern der Ernährungsindustrie aus dem Jahre 2002 gestützt ${ }^{11}$.

\subsection{Zuckerindustrie}

Auch in der europäischen Zuckerindustrie schreitet der Strukturwandel voran (vgl. Tabelle 5 sowie Abbildung 13). Die Zuckerindustrie wurde in der Vergangenheit von zahlreichen Werksschließungen, Unternehmenszusammenschlüssen und somit einer zunehmenden Konzentration geprägt. Als Folge von "fixierten" Produktionsquoten stieg die durchschnittliche Verarbeitungsmenge der Fabriken an (vgl. dazu Kapitel 7.3.2 sowie Kapitel 8.1).

Tabelle 5: Anzahl der Zuckerunternehmen (Zuckerfabriken) in Europa

\begin{tabular}{|c|c|c|c|c|c|c|c|c|c|c|c|c|}
\hline & 1991 & 1992 & 1993 & 1994 & 1995 & 1996 & 1997 & 1998 & 1999 & 2000 & 2001 & 2002 \\
\hline A & $1(3)$ & $1(3)$ & $1(3)$ & $1(3)$ & $1(3)$ & $1(3)$ & $1(3)$ & $1(3)$ & $1(3)$ & $1(3)$ & $1(3)$ & $1(3)$ \\
\hline B & $11(11)$ & $11(11)$ & $9(9)$ & $7(9)$ & $7(9)$ & $7(9)$ & $5(8)$ & $5(8)$ & $5(8)$ & $6(9)$ & $6(9)$ & $6(9)$ \\
\hline DK & $1(5)$ & $1(5)$ & $1(4)$ & $1(4)$ & $1(4)$ & $1(4)$ & $1(4)$ & $1(4)$ & $1(4)$ & $1(4)$ & $1(3)$ & $1(3)$ \\
\hline SF & $1(4)$ & $1(3)$ & $1(3)$ & $2(3)$ & $2(3)$ & $2(3)$ & $2(3)$ & $2(3)$ & $2(3)$ & $1(3)$ & $1(3)$ & $1(3)$ \\
\hline $\mathrm{F}$ & $\begin{array}{l}30 \\
(50)\end{array}$ & $\begin{array}{l}30 \\
(48)\end{array}$ & $\begin{array}{l}29 \\
(48)\end{array}$ & $\begin{array}{l}26 \\
(46)\end{array}$ & $\begin{array}{l}26 \\
(46)\end{array}$ & $\begin{array}{l}22 \\
(45)\end{array}$ & $\begin{array}{l}22 \\
(45)\end{array}$ & $\begin{array}{l}22 \\
(42)\end{array}$ & $\begin{array}{l}18 \\
(40)\end{array}$ & $\begin{array}{l}18 \\
(39)\end{array}$ & $\begin{array}{l}18 \\
(39)\end{array}$ & $\begin{array}{l}18 \\
(37)\end{array}$ \\
\hline D & $\begin{array}{l}12 \\
(37)\end{array}$ & $\begin{array}{l}16 \\
(60)\end{array}$ & $\begin{array}{l}14 \\
(52)\end{array}$ & $\begin{array}{l}15 \\
(43)\end{array}$ & $\begin{array}{l}15 \\
(42)\end{array}$ & $\begin{array}{l}15 \\
(39)\end{array}$ & $\begin{array}{l}14 \\
(36)\end{array}$ & $\begin{array}{l}13 \\
(34)\end{array}$ & $\begin{array}{l}13 \\
(34)\end{array}$ & $\begin{array}{l}9 \\
(32)\end{array}$ & $\begin{array}{l}9 \\
(31)\end{array}$ & $\begin{array}{l}9 \\
(29)\end{array}$ \\
\hline GR & $1(5)$ & $1(5)$ & $1(5)$ & $1(5)$ & $1(5)$ & $1(5)$ & $1(5)$ & $1(5)$ & $1(5)$ & $1(5)$ & $1(5)$ & $1(5)$ \\
\hline IRL & $1(2)$ & $1(2)$ & $1(2)$ & $1(2)$ & $1(2)$ & $1(2)$ & $1(2)$ & $1(2)$ & $1(2)$ & $1(2)$ & $1(2)$ & $1(2)$ \\
\hline ITA & $\begin{array}{l}12 \\
(31)\end{array}$ & $\begin{array}{l}12 \\
(29)\end{array}$ & $\begin{array}{l}12 \\
(25)\end{array}$ & $\begin{array}{l}12 \\
(24)\end{array}$ & $\begin{array}{l}11 \\
(23)\end{array}$ & $\begin{array}{l}10 \\
(23)\end{array}$ & $\begin{array}{l}9 \\
(23)\end{array}$ & $\begin{array}{l}9 \\
(23)\end{array}$ & $\begin{array}{l}10 \\
(23)\end{array}$ & $\begin{array}{l}9 \\
(22)\end{array}$ & $\begin{array}{l}9 \\
(21)\end{array}$ & $\begin{array}{l}9 \\
(20)\end{array}$ \\
\hline NL & $2(7)$ & $2(7)$ & $2(6)$ & $2(6)$ & $2(6)$ & $2(6)$ & $2(5)$ & $2(5)$ & $2(5)$ & $2(5)$ & $2(5)$ & $2(5)$ \\
\hline$P$ & 2 & 2 & 2 & 2 & 2 & & & & & $4(4)$ & $4(4)$ & $4(4)$ \\
\hline$E$ & $6(24)$ & $5(24)$ & $5(22)$ & $4(21)$ & $4(21)$ & $4(20)$ & $4(18)$ & $4(17)$ & $3(15)$ & $3(16)$ & $3(16)$ & $3(16)$ \\
\hline S & $1(6)$ & $1(6)$ & $1(6)$ & $1(6)$ & $1(5)$ & $1(5)$ & $1(5)$ & $1(5)$ & $1(5)$ & $1(4)$ & $1(4)$ & $1(3)$ \\
\hline UK & $2(12)$ & $2(10)$ & $2(10)$ & $2(10)$ & $2(9)$ & $2(9)$ & $2(9)$ & $2(9)$ & $2(9)$ & $2(10)$ & $2(10)$ & $2(8)$ \\
\hline $\begin{array}{l}\text { EU- } \\
15\end{array}$ & $\begin{array}{l}87 \\
(197)\end{array}$ & $\begin{array}{l}86 \\
(213)\end{array}$ & $\begin{array}{l}82 \\
(194)\end{array}$ & $\begin{array}{l}80 \\
(180)\end{array}$ & $\begin{array}{l}76 \\
(177)\end{array}$ & $\begin{array}{l}73 \\
(172)\end{array}$ & $\begin{array}{l}65 \\
(165)\end{array}$ & $\begin{array}{l}64 \\
(159)\end{array}$ & $\begin{array}{l}64 \\
(154)\end{array}$ & $\begin{array}{l}59 \\
(158)\end{array}$ & $\begin{array}{l}59 \\
(155)\end{array}$ & $\begin{array}{l}59 \\
(147)\end{array}$ \\
\hline
\end{tabular}

Quelle: CEFS, sugar statistics; BARTENS Ifd. Jgg.

\footnotetext{
${ }^{11}$ Vgl. Befragung in der Studie: NeumanN (im Druck): Wettbewerbsmonitor der deutschen Ernährungsindustrie
} 
Die Abnahme der Zahl der Fabriken, wie auch Unternehmen führt - bedingt durch die Zuckermarktordnung (ZMO) - bei konstanter Gesamtproduktion, zu einer Erhöhung der durchschnittlichen Verarbeitungsmenge pro Fabrik (Unternehmen). Exemplarisch wird dies in Abbildung 13 veranschaulicht. Für die weiteren EU-Mitgliedsstaaten sei auf Daten im Anhang (vgl. Anhang C) verwiesen.

Abbildung 13: Entwicklung der Verarbeitungsmenge und Anzahl der Fabriken bzw. Unternehmen der Fabriken

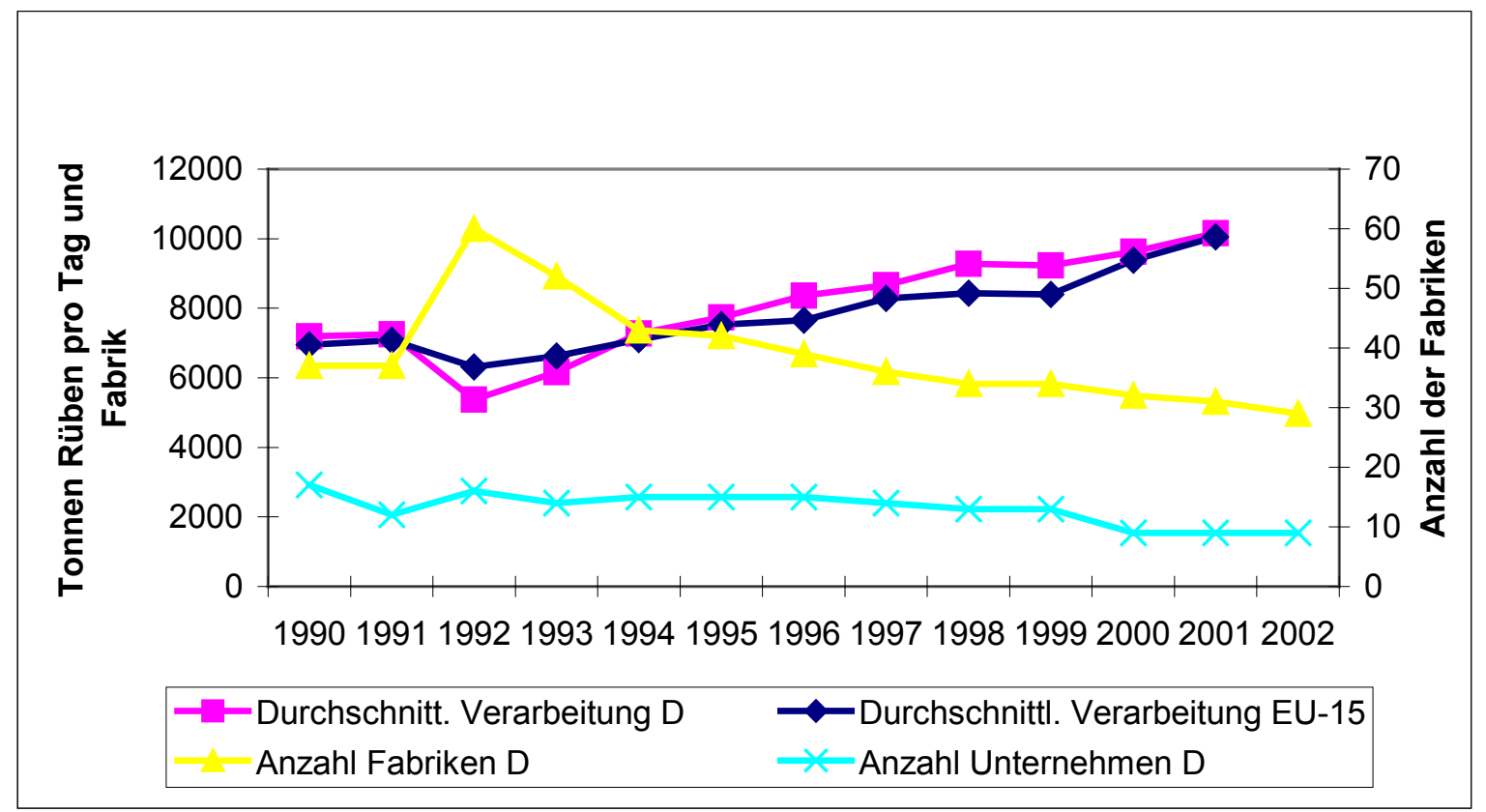

Quelle: eigene Darstellung auf Grundlage CEFS ; BARTENS, Ifd. Jgg.

Ferner ist bei der Betrachtung der Unternehmensanzahl zu beachten, dass die Zahl der "tatsächlich" unabhängig voneinander agierenden Unternehmen noch wesentlich geringer als die Anzahl der in der Statistik ausgewiesenen Unternehmen ist. Grund dafür sind die zahlreichen Konzernverflechtungen zwischen den Unternehmen auf nationaler und europäischer Ebene. So besteht beispielsweise die Südzucker-Gruppe derzeit aus sechs einzeln ausgewiesenen Zuckerunternehmen (Südzucker AG, Südzucker GmbH, Südzucker International, Agrana, RT, Saint Louis Sucre) ${ }^{12}$, obwohl die Lenkung über den Südzucker Konzern stattfindet.

Aktuell agieren folgende Unternehmen am europäischen Markt (Tabelle 6).

12 Vgl. dazu KRICK 2001 
Tabelle 6: Aufteilung der Zuckerquoten und der Unternehmen in den jeweiligen Mitgliedsstaaten

\begin{tabular}{|c|c|c|c|}
\hline Zuckerunternehmen & Höchstquote & $\begin{array}{c}\% \text { der nationalen } \\
\text { Quote }\end{array}$ & $\%$ der EU-Quote \\
\hline RT (Südzucker) & 620343,6 & 75,1 & 4,25 \\
\hline Groupe Sucrier & 151485,6 & 18,3 & 1,04 \\
\hline Fontenoy & 54170,8 & 6,6 & 0,37 \\
\hline B- insgesamt & 826000 & 100 & 5,66 \\
\hline DK- Danisco Sugar & 424629 & 100 & 2,91 \\
\hline GR- HSI & 319000 & 100 & 2,19 \\
\hline Südzucker AG/GmbH & 1375818 & 39,9 & 9,43 \\
\hline Nordzucker AG & 1070548 & 31 & 7,34 \\
\hline Pfeifer\&Langen/Diamant & 617783 & 17,9 & 4,23 \\
\hline Jülich AG & 145782 & 4,2 & 1 \\
\hline Danisco Sugar GmbH & 126565 & 3,7 & 0,87 \\
\hline $\begin{array}{l}\text { Union-Zucker (Nordzu- } \\
\text { cker) }\end{array}$ & 112817 & 3,3 & 0,77 \\
\hline D- insgesamt & 3449313 & 100 & 23,64 \\
\hline $\begin{array}{l}\text { Azzucarera Ebro Agrico- } \\
\text { las }\end{array}$ & 782474 & 78,2 & 5,36 \\
\hline ACOR & 147794 & 14,8 & 1,01 \\
\hline ARJ & 69732 & 7 & 0,48 \\
\hline E- insgesamt & 1000000 & 100 & 6,85 \\
\hline Eridania Beghin Say & 1179156 & 35,5 & 8,08 \\
\hline $\begin{array}{l}\text { Saint Louis Sucre (Süd- } \\
\text { zucker) }\end{array}$ & 730479 & 22 & 5,01 \\
\hline SVI & 352155 & 10,6 & 2,41 \\
\hline USDA & 342456 & 10,3 & 2,35 \\
\hline Cristal Union & 307595 & 9,3 & 2,11 \\
\hline PCA & 892900 & 26,9 & 6,11 \\
\hline Andere & 164543 & 5 & 1,13 \\
\hline $\begin{array}{l}\text { F insgesamt (Fest- } \\
\text { land) }\end{array}$ & 3319233 & 100 & 22,75 \\
\hline IRL- Greencore & 200200 & 100 & 1,37 \\
\hline Eridania Beghin Say & 794225,1 & 50,6 & 5,44 \\
\hline SFIR & 295472,4 & 18,8 & 2,02 \\
\hline Sadam & 280619,7 & 17,9 & 1,92 \\
\hline Co.Pro.A\&B & 110521,9 & 7,1 & 0,76 \\
\hline Molise & 87410,9 & 5,6 & 0,6 \\
\hline I - insgesamt & 1568250 & 100 & 10,75 \\
\hline Consun & 54400 & 62,4 & 3,73 \\
\hline
\end{tabular}


Tabelle 6 (Fortsetzung)

\begin{tabular}{|l|c|c|c|}
\hline Zuckerunternehmen & Höchstquote & $\begin{array}{c}\text { \% der nationalen } \\
\text { Quote }\end{array}$ & \% der EU-Quote \\
\hline CSM & 328000 & 37,6 & 2,25 \\
\hline NL insgesamt & $\mathbf{8 7 2 0 0 0}$ & $\mathbf{1 0 0}$ & $\mathbf{5 , 9 8}$ \\
\hline A- Agrana (Südzucker) & 390410 & 100 & 2,68 \\
\hline DAI (Kontinent) & 70000 & 87,5 & 0,48 \\
\hline SINAGA (Azoren) & 10000 & 12,5 & 0,07 \\
\hline P-insgesamt & $\mathbf{8 0 0 0 0}$ & $\mathbf{1 0 0}$ & $\mathbf{0 , 5 5}$ \\
\hline S- Danisco Sugar AB & 370000 & 100 & 2,54 \\
\hline FIN- Danisco Finland & 146776 & 100 & 1,01 \\
\hline Oy & 1144000 & 100 & 7,84 \\
\hline UK- British Sugar & $\mathbf{1 4 5 9 2 4 1 1}$ & & $\mathbf{1 0 0}$ \\
\hline EU- insgesamt & & & \\
\hline
\end{tabular}

Quelle: KRICK 2000, S. 429

Daraus können die Konzentrationsraten an der europäischen Zuckerquote errechnet werden (Tabelle 7).

Tabelle 7: Anteil der Unternehmen an den Höchstquoten

\begin{tabular}{|l|c|c|}
\hline Zuckerunternehmen & Höchstquote in t & In \% der EU Quote \\
\hline Südzucker & 3134049,4 & 21,5 \\
\hline Eridania Beghin Say & 1973381 & 13,5 \\
\hline British Sugar & 1144000 & 7,8 \\
\hline Danisco & 1068970 & 7,3 \\
\hline Nordzucker & 1183365 & $\mathbf{8 , 1}$ \\
\hline Die 5 größten $\left.\mathbf{( C R}_{5}\right)$ & $\mathbf{8 5 0 3 7 6 5 , 5}$ & $\mathbf{5 8 , 3}$ \\
\hline AEA & 782474 & 5,4 \\
\hline Pfeifer \& Langen & 613972 & 4,2 \\
\hline Consun & 541000 & 3,7 \\
\hline SVI & 347420,1 & 2,4 \\
\hline USDA & 339500,2 & 2,3 \\
\hline CSM & 331000 & $\mathbf{8 6 , 7 6}$ \\
\hline HSI & 319000 & 2,2 \\
\hline Cristal Union & 307595 & 2 \\
\hline SFIR & 295472,4 & 2 \\
\hline Sadam & $\mathbf{1 2 6 6 0 7 1 9}$ & 1,92 \\
\hline Die 15 großen $\left.\mathbf{C R}_{15}\right)$ & $\mathbf{2 8 0 6 1 9 , 7}$ & \\
\hline
\end{tabular}

Quelle: KRICK 2000, S. 430; ergänzt und aktualisiert um eigene Angaben 


\subsection{Milchindustrie}

In diesem Abschnitt werden einige wichtige Entwicklungen innerhalb des Milchsektors näher dargelegt. Allgemein lässt sich auch hier der Trend zu weiteren Zusammenschlüssen und Kooperationen mit der Folge einer ansteigenden Konzentration beobachten.

In Deutschland ist der Strukturwandel wie auch im übrigen Europa ${ }^{13}$ weiter vorangeschritten. Abbildung 14 gibt einen Überblick über Anzahl der Unternehmen bzw. Betriebe sowie durchschnittlicher Milchverarbeitung (pro Betrieb), wobei ähnlich wie bei den Unternehmen der Zuckerindustrie darauf hingewiesen wird, dass die tatsächliche Anzahl der unabhängig voneinander agierenden Unternehmen unter den ausgewiesenen Unternehmenszahlen liegt, da zahlreiche Konzernverflechtungen und Beteiligungen das Bild der Milchindustrie prägen.

Abbildung 14: Entwicklung der Anzahl der Molkereibetriebe bzw. Unternehmen sowie der durchschnittlichen (jährlichen) Verarbeitungsmenge pro Betrieb

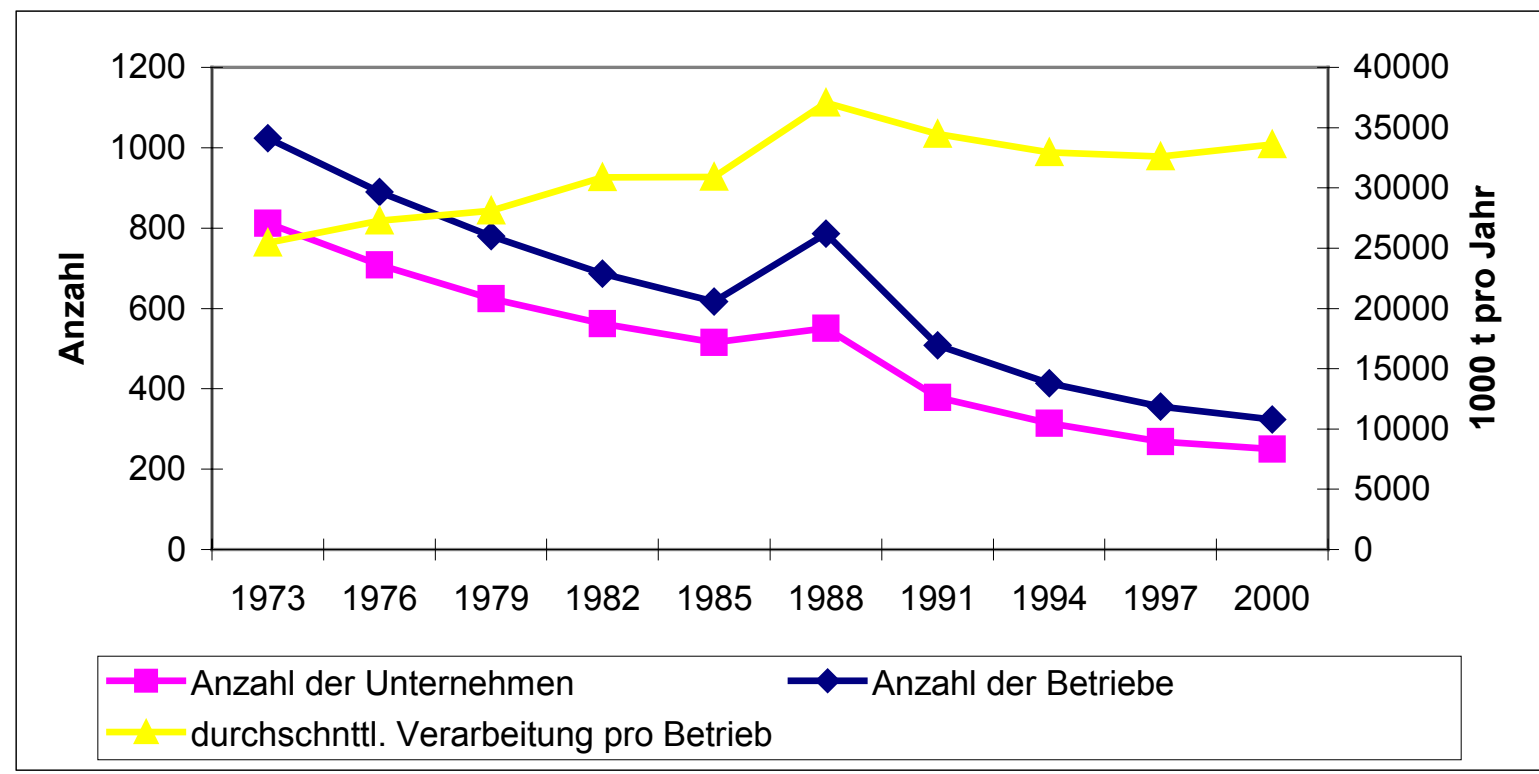

Anm: Datengrundlage im Anhang E

Quelle : eigene Darstellung auf Grundlage von ZMP Marktbilanz Milch, Ifd. Jgg.

Auf der Verwendungsseite war folgende Anzahl von Unternehmen in unterschiedlichen Bereichen aktiv (Tabelle 8).

${ }^{13}$ Nähere Daten der Molkereibranche in den einzelnen Mitgliedstaaten sind dem Anhang E zu entnehmen. 
Tabelle 8: Unternehmen und Produktionsrichtung

Anzahl der Unternehmen nach der Produktionsrichtung

\begin{tabular}{|l|c|c|c|c|c|c|c|c|}
\hline & $\mathbf{1 9 7 9}$ & $\mathbf{1 9 8 2}$ & $\mathbf{1 9 8 5}$ & $\mathbf{1 9 8 8}$ & $\mathbf{1 9 9 1}$ & $\mathbf{1 9 9 4}$ & $\mathbf{1 9 9 7}$ & $\mathbf{2 0 0 0}$ \\
\hline Frischmilcherzeugnisse & 589 & 527 & 464 & 476 & 329 & 245 & 220 & 198 \\
\hline Kondensmilch & 16 & 14 & 15 & 26 & 20 & 17 & 16 & 16 \\
\hline Milchpulver & 101 & 90 & 78 & 98 & 72 & 51 & 48 & 43 \\
\hline Butter & 516 & 462 & 415 & 404 & 263 & 177 & 157 & 135 \\
\hline Hartkäse & 72 & 66 & 62 & 62 & 51 & 48 & 48 & 51 \\
\hline Schnittkäse & 121 & 108 & 107 & 145 & 101 & 90 & 84 & 84 \\
\hline Weichkäse & 78 & 66 & 63 & 80 & 58 & 60 & 48 & 37 \\
\hline $\begin{array}{l}\text { Sauermilch- und } \\
\text { Kochkäse }\end{array}$ & 50 & 46 & 36 & 73 & 44 & 37 & 30 & 25 \\
\hline Frischkäse & 323 & 283 & 244 & 256 & 173 & 125 & 112 & 94 \\
\hline Schmelzkäse & 18 & 20 & 20 & 23 & 23 & 22 & 23 & 20 \\
\hline Kasein & 7 & 9 & 8 & 9 & 7 & 5 & 3 & 4 \\
\hline
\end{tabular}

Quelle : ZMP, Ifd. Jgg.

Daraus ergeben sich die in Tabelle 9 genannten durchschnittlichen (jährlichen) Produktionsmengen in den verschiedenen Bereichen je Unternehmen.

Tabelle 9: Durchschnittsbetriebsgröße verschiedener Produktionsrichtungen (jährlich) in Tonnen

\begin{tabular}{|l|c|c|c|}
\hline Produktionsrichtung & $\mathbf{1 9 9 4}$ & $\mathbf{1 9 9 7}$ & $\mathbf{2 0 0 0}$ \\
\hline Frischmilcherzeugnisse & 32119 & 37750 & 42670 \\
darunter Konsummilch & 18002 & 28188 & 31676 \\
Kondensmilch & 31053 & 35388 & 35438 \\
Milchpulver & 10394 & 10256 & 11081 \\
Butter & 2606 & 2818 & 3147 \\
Hartkäse & 3744 & 3817 & 3680 \\
Schnittkäse & 4314 & 5958 & 6106 \\
Weichkäse & 1632 & 2540 & 3062 \\
Pasta filata Käse & - & - & 5075 \\
Sauermilch- und Kochkäse & 922 & 1130 & 1260 \\
Frischkäse & 5594 & 6703 & 8078 \\
Käse insgesamt & 5642 & 7328 & 8736 \\
Schmelzkäse & 7459 & 6526 & 8535 \\
Kasein & 1680 & 3533 & 3350 \\
\hline
\end{tabular}

Quellen: eigene Zusammenstellung auf Basis BML, Ifd Jgg.; ZMP, Ifd. Jgg. 
Um einige Kennzahlen transparenter hervorzuheben, werden nachfolgend einige Konzentrationsziffern der deutschen und europäischen Ernährungsindustrie basierend auf der aktuellsten Erfassung der Deutschen Milchwirtschaft ${ }^{14}$ vorgestellt (Tabelle 10).

Tabelle 10: Konzentrationsraten in der deutschen Milchwirtschaft 2000

\begin{tabular}{|c|c|c|c|c|}
\hline $\begin{array}{l}\text { > bis Rang } \\
\left(\mathrm{CR}_{x}\right)\end{array}$ & $\begin{array}{c}\text { Milchverarbeitung } \\
\text { Mio. kg }\end{array}$ & $\begin{array}{c}\text { Milchverarbeitung \% } \\
\text { der Gesamtverarbei- } \\
\text { tung }\end{array}$ & $\begin{array}{c}\text { Umsatz Mio. } \\
\text { DM }\end{array}$ & $\begin{array}{c}\text { Umsatz \% des } \\
\text { Branchenumsatzes }\end{array}$ \\
\hline 5 & 10481 & 38,5 & 13309 & 34 \\
\hline 10 & 14134 & 51,9 & 17850 & 45,7 \\
\hline 15 & 16622 & 61,1 & 21627 & 55,3 \\
\hline 20 & 18625 & 68,5 & 24459 & 62,6 \\
\hline 25 & 20244 & 74,4 & 26786 & 68,5 \\
\hline 30 & 21763 & 80 & 28601 & 73,2 \\
\hline 35 & 23080 & 84,8 & 30327 & 77,6 \\
\hline 40 & 24144 & 88,7 & 31896 & 81,6 \\
\hline 45 & 25018 & 91,9 & 33206 & 84,9 \\
\hline 50 & 25768 & 94,7 & 34206 & 87,5 \\
\hline 55 & 26416 & 97,1 & 35072 & 89,7 \\
\hline \multicolumn{3}{|c|}{ Milchverarbeitung gesamt: 27211 Mio. kg } & \multicolumn{2}{|c|}{ Branchenumsatz 39100 Mio. DM } \\
\hline
\end{tabular}

Quelle: Deutsche Milchwirtschaft SPEZIAL 2001

Im europäischen Vergleich sind es im wesentlichen die heutigen Großmolkereien die das Fusionsgeschehen der letzten Jahre bestimmten. Unter den Europa Top 25 befinden sich bereits vier deutsche Unternehmen (vgl. Tabelle 11) - dennoch ist die Milchverarbeitungsmenge von beispielsweise "Arla Food" noch bei weitem höher als bei dem größten deutschen Unternehmen.

${ }^{14}$ Vgl. Deutsche Milchwirtschaft SpeZial 2001 
Tabelle 11: Europa Top 25 Milchverarbeiter (2001 basierend auf Daten von 1999)

\begin{tabular}{|c|c|c|c|}
\hline Rang & Unternehmen & Land & Milchmenge Mio. kg \\
\hline 1 & Arla Foods & DK/ S & 7100 \\
\hline 2 & Lactalis & $F$ & 6540 \\
\hline 3 & Friesland Coberco & NL & 5900 \\
\hline 4 & Campina Melkunie & NL & 4700 \\
\hline 5 & Nordmilch & D & 4500 \\
\hline 6 & Bongrain & $F$ & 3700 \\
\hline 7 & Dairy Crest & GB & 3100 \\
\hline 8 & Danone & $F$ & 2900 \\
\hline 9 & Nestlé & $\mathrm{CH}$ & 2800 \\
\hline 10 & Humana & D & 2540 \\
\hline 11 & Sodiaal & $F$ & 2400 \\
\hline 12 & Glanbia & IRL & 2150 \\
\hline 13 & Express Dairies & GB & 2100 \\
\hline 14 & Entrement & $F$ & 1900 \\
\hline 15 & Valio & FIN & 1660 \\
\hline 16 & Laita & $F$ & 1650 \\
\hline 17 & Müller & $D$ & 1500 \\
\hline 18 & Parmalat & I & 1270 \\
\hline 19 & Lactogal & $P$ & 1100 \\
\hline 20 & Fromageries Bel & $F$ & 1000 \\
\hline 21 & Ebro-Puleva & $E$ & 1000 \\
\hline 22 & Berglandmilch & $A$ & 920 \\
\hline 23 & $3 A$ & $F$ & 900 \\
\hline 24 & Dairygold & IRL & 870 \\
\hline 25 & Omira & D & 850 \\
\hline
\end{tabular}

Quelle: Deutsche Milchwirtschaft 2001, RABOBANK 2000, S. 36 


\subsection{Zwischenergebnis}

In den vorigen Ausführungen konnte die Bedeutung von Fusionen herausgestellt werden. Sowohl global als auch national lässt sich in den letzten Jahren eine zunehmende Fusionsaktivität beobachten. Auch die Ernährungsindustrie ist von dieser Entwicklung betroffen. Bei den hier im Mittelpunkt stehenden Branchen konnte gezeigt werden, dass es sich bei der Zuckerindustrie um einen Sektor mit bereits hoher Konzentration handelt, während die Milchindustrie weit niedrigere Konzentrationsraten aufweist. Im nächsten Kapitel wird hinterfragt, wie es zu dieser Entwicklung gekommen ist und warum überhaupt Unternehmen fusionieren? In den anschließenden Kapiteln wird dann analysiert, inwieweit Konzentrationsraten Erkenntnisse über Wettbewerbsverhalten liefern und inwiefern die in diesem Kapitel vorgestellten Strukturdaten bei der Beurteilungspraxis berücksichtigt werden. 


\section{Motive und Auswirkungen von Unternehmenszusam- menschlüssen}

In diesem Kapitel werden mögliche Fusionsmotive näher vorgestellt. Dabei lässt sich feststellen, dass Unternehmenszusammenschlüsse oftmals nicht von einer Zielsetzung allein dominiert werden, sondern in der Praxis häufig eine Kombination mehrerer Aspekte zur Entscheidungsfindung beitragt. Im folgenden werden Erkenntnisse aus der Literatur erörtert.

\subsection{Effizienztheorie}

Die Effizienztheorie basiert auf der Annahme, dass Unternehmenszusammenschlüsse durchgeführt werden, um vorhandene Unternehmensressourcen mit möglichst hoher Effizienz in Outputs umzuwandeln. Diesem Ansatz zufolge ist das durch den Zusammenschluss entstandene Unternehmen erfolgreicher als die einzelnen Unternehmenseinheiten vor der Fusion, da Ressourcen ökonomischer eingesetzt werden können und damit Kostenersparnisse einhergehen. Die Effizienztheorie erlangte in der USamerikanischen Wettbewerbspolitik erhöhte Aufmerksamkeit und wird von den Anhängern der Chicago School vertreten. Danach sind Unternehmenszusammenschlüsse eher von Effizienzstreben als von Marktmachtkalkülen motiviert. Effizienzvorteile können durch folgende ökonomische Phänomene erklärt werden:

- Rationalisierungseffekte,

- Economies of Scale und Scope,

- Multi-Plant-Economies,

- Vertikale Integration,

- Coinsurance,

- Konzept des überlegenden Managements.

Diese Phänomene werden im folgenden näher erläutert. 


\subsubsection{Rationalisierungseffekte}

Produktionsanlagen zweier Unternehmen sind oftmals mit unterschiedlichen Kosten verbunden. Beispielsweise kann eine Anlage des Unternehmens A bereits an der Kapazitätsgrenze arbeiten und wegen des hohen Auslastungsgrads mit hohen Grenzkosten assoziiert sein, während eine ähnliche Anlage des Unternehmens B nicht entsprechend beansprucht ist. Durch Fusion beider Unternehmen lassen sich durch eine teilweise Verlagerung der Produktion von Unternehmen A hin zu Unternehmen B Kostenvorteile erzielen. Diese Verlagerung kann auch die Form annehmen, dass sich Unternehmensteile der beiden Unternehmen jeweils auf bestimmte Produkte spezialisieren ${ }^{15}$.

\subsubsection{Economies of Scale}

Mit steigender Betriebsgröße können betriebliche Größenvorteile (economies of scale) realisiert werden. Größenvorteile können in fast allen Bereichen des Unternehmens, beispielsweise in Produktion, Finanzierung, Verwaltung, Lagerhaltung oder im Einkauf, vorliegen. Die Ursachen solcher Größenvorteile sind vielfältig und können z.B. durch die Beseitigung von doppelten Fixkosten entstehen ${ }^{16}$. So lassen sich beispielsweise Absatz- und Beschaffungswege in einem Unternehmen zentral kostengünstiger aufbauen und pflegen als bei einer dezentralen Verwaltung. Doppelaufwendungen für Forschungen sind ein weiteres Beispiel für Synergieeffekte, die durch Zusammenlegung von Unternehmensaktivitäten realisiert werden können. Produktionsanlagen sind meist auf ein bestimmtes Produktionsvolumen ausgelegt. Für die jeweiligen Unternehmen $A$ und $B$ ist die Anschaffung einer neuen Anlage trotz hoher variablen Kosten der alten Anlagen oftmals nicht vorteilhaft. Bei einem Zusammenschluss hingegen lassen sich die Anschaffungskosten für eine neue größere Anlage auf ein größeres Produktionsvolumen verteilen ${ }^{17}$. Schließlich lassen sich aufgrund des höheren Produktionsvolumens bei der Beschaffung von Vorprodukten oftmals günstigere Konditionen erzielen. Economies of Scale sind unternehmens- und branchenspezifisch. In Abbildung 15 wird der Versuch einer Schematisierung genereller Ursachen von economies of scale vorgenommen:

\footnotetext{
${ }^{15}$ Vgl. FeHL/OBERENDER 1989, S. $142 \mathrm{ff}$.

${ }^{16}$ Nach AlbreCht 1994, S. 6 ff.

17 Vgl. PAPROTTKA 1996, S. 49 ff.
} 
Abbildung 15: Mögliche Ursachen von economies of scale

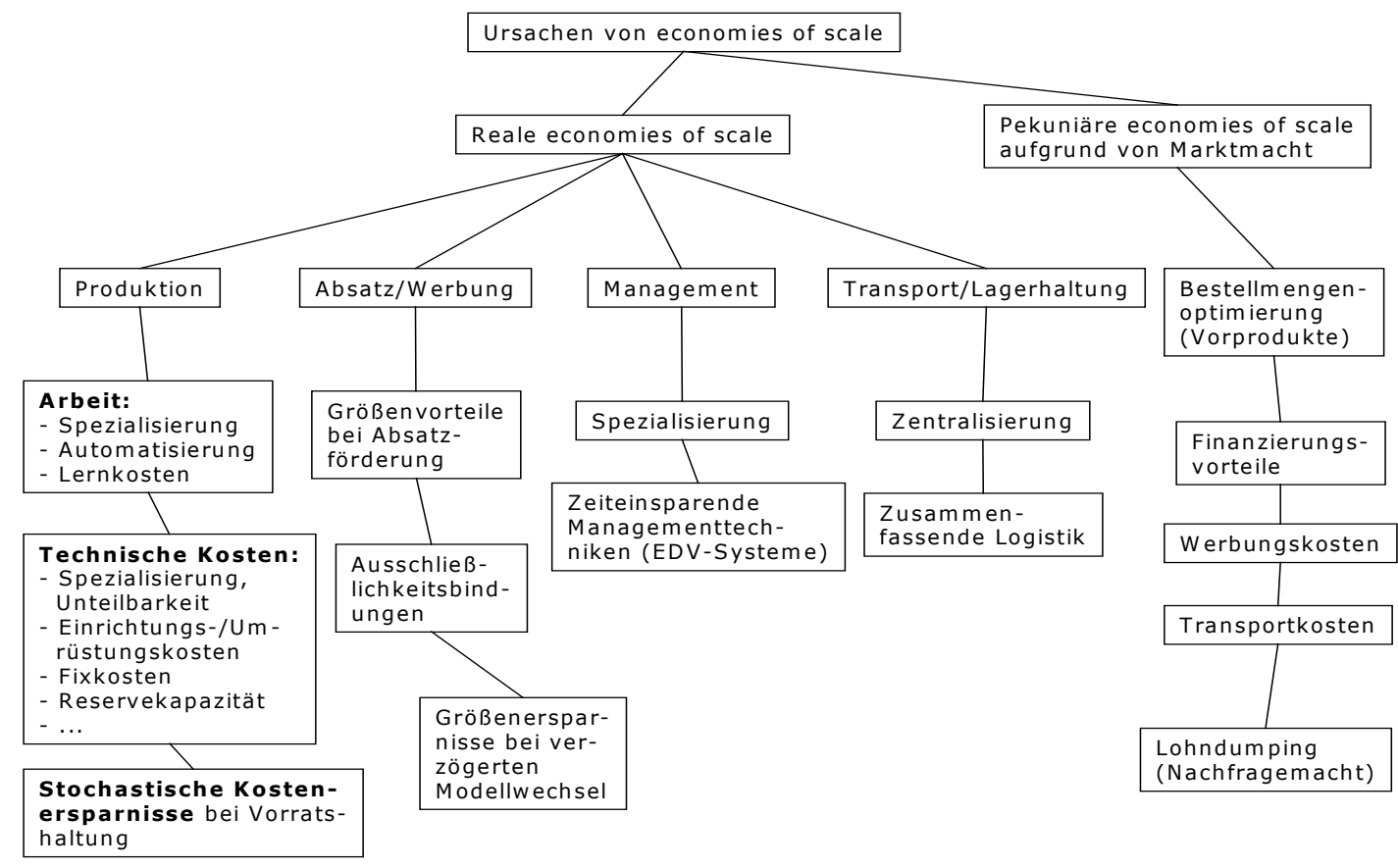

Quelle: eigene Darstellung in Anlehnung an SCHмIDт 1999, S. 86

\subsubsection{Economies of Scope}

Oftmals können neben produktionsabhängigen Kostenvorteilen auch Kostenersparnisse durch gemeinsame Produktion von mehreren verschiedenen Produkten realisiert werden ${ }^{18}$. Diese Verbundvorteile entstehen formal, wenn die Durchschnittskosten bei gemeinsamer Produktion geringer sind als die Summe der Durchschnittskosten bei separater Herstellung. Im Gegensatz zu den economies of scale erwachsen die Kostenersparnisse aus der Reichweite der unternehmerischen Tätigkeiten (scope) ${ }^{19}$. Economies of scope beruhen auf gemeinsam genutzten Produktionsfaktoren (shared inputs) für die Herstellung verschiedener Güter ${ }^{20}$. Es kann sich dabei um unteilbare Produktionsfaktoren handeln, die durch die Produktion nur eines Guts nicht ausgelastet sind, oder um Produktionsfaktoren, die bei der Verwendung für ein Gut unentgeltlich auch für ein zweites Gut zur Verfügung stehen. Verbundvorteile resultieren dann entweder aus niedrigeren Durchschnittskosten oder aus der Erlössteigerung infolge verbesserter Produktqualität.

\footnotetext{
${ }^{18} \mathrm{Vgl.} \mathrm{SETH} \mathrm{1990,} \mathrm{S.} 101$

${ }^{19}$ Vgl. KinNe 2000, S. 28
} 
Ein Beispiel für Verbundvorteile auf der Produktionsseite kann eine Maschine sein, die für mehrere Erzeugnisse nutztbar ist und bei Produktion eines Erzeugnisses alleine nicht an ihre Kapazitätsgrenze stößt.

Synergetische Effekte sind im Falle der verbundenen Produktion von zwei oder mehreren Gütern - insbesondere beim Einsatz von finanziellen, technologischen oder unternehmerischen Ressourcen - möglich ${ }^{21}$ :

- Finanzvorteile infolge des geringeren Konkursrisikos von Konglomeraten und damit niedrigeren Fremdkapitalzinsen sowie erweiterten Kreditrahmen,

- Marketingvorteile durch Verwendung eines Markennamens für verschiedene Produkte (z.B. „Porsche" für Autos und Brillen),

- Kostenersparnisse in Forschung und Entwicklung im Falle sog. Abfallentwicklungen; Ersparnis von Such- und Informationskosten bei den Verwendungen von Erfindungen (shared input),

- Kostenvorteile beim Einsatz des dispositiven Faktors durch gemeinsame Stabsabteilungen sowie bessere Ausnutzung der Führungsqualitäten des Managements für verschiedene Produkte (public input).

Formell können die economies of scope folgendermaßen dargestellt werden:

$$
\mathrm{SC}=\frac{\mathrm{K}(\mathrm{x}, 0)+\mathrm{K}(0, \mathrm{y})-\mathrm{K}(\mathrm{x}, \mathrm{y})}{\mathrm{K}(\mathrm{x}, \mathrm{y})} \times 100
$$

$K(x, 0)$ bzw. $K(0, y)$ stellen die Produktionskosten für die jeweiligen Güter $x$ und $y$ in zwei unabhängigen Unternehmen dar, während $\mathrm{K}(x, y)$ die entsprechenden Produktionskosten in einem zusammengeschlossenen Unternehmen repräsentieren. Ist die Messzahl $^{22}$ SC positiv, liegen economies of scope vor. Der Index misst den prozentual eingesparten Kostenanteil, der entsteht, sobald beide der Güter in einem Unternehmen hergestellt werden. Negative Indexwerte hingegen deuten auf das Vorhandensein von Kostennachteilen (diseconomies of scope) bei gemeinsamer Produktion hin. Ursache hierfür kann beispielsweise Bürokratie in Großunternehmen und die damit verbundene mangelnde Motivation von Mitarbeitern $\operatorname{sein}^{23}$.

\footnotetext{
${ }^{20} \mathrm{Vgl.} \mathrm{PANZAR/WiLlig} \mathrm{1981,} \mathrm{S.} 268 \mathrm{ff}$.

${ }^{21}$ In Anlehnung an SCHMIDT 1999, S. 101

${ }^{22}$ Ausmaß der Kostenvorteile
} 


\subsubsection{Multi-Plant-Economies}

Analog können in der mehrbetrieblichen gegenüber der einbetrieblichen Produktion Kostenvorteile beobachtet werden ${ }^{24}$. Vorteile der Mehrbetrieblichkeit (multi-planteconomies) bestehen, wenn eine Gütermenge in einem Unternehmen mit mehreren Betrieben zu geringeren Gesamtkosten als in mehreren Unternehmen mit jeweils einem Betrieb hergestellt werden kann. "Zum Tragen" kommen diese Kostenvorteile, sobald die Bedingungen auf den Beschaffungs- und Absatzmärkten gegen eine Realisierung der technisch optimalen Betriebsgröße sprechen. Dies ist der Fall, wenn der Ausschöpfung betrieblicher Größenvorteile steigende Transportkosten, ein erhöhtes Risiko von Produktionsausfällen oder hohe Durchschnittskosten bei Kapazitätsunterauslastungen gegenüberstehen ${ }^{25}$.

\subsubsection{Vertikale Integration}

Vorteile der vertikalen Integration liegen bei mehrstufigen Produktionsprozessen dann vor, wenn die Organisationskosten bei unternehmensinterner Koordination von aufeinanderfolgenden Produktionsstufen in einem Unternehmen geringer sind als die Transaktionskosten bei marktlicher Koordination ${ }^{26}$. Allerdings können Kostenvorteilen der vertikalen Integration Produktionskostennachteile durch nicht ausgeschöpfte economies of scale gegenüberstehen ${ }^{27}$. Dies geschieht, sobald aufeinanderfolgende Fertigungsstufen unterschiedlich optimale Produktionskapazitäten aufweisen. Betriebliche Größenvorteile können nur dann auf allen integrierten Produktionsstufen realisiert werden, wenn das Unternehmen über eine mindestoptimale Betriebsgröße in Höhe des kleinsten gemeinsamen Vielfachen verfügt. Andernfalls können Kostennachteile auf einzelnen Produktionsstufen auftreten. In solchen Fällen ist abzuwägen, ob die Kostenvorteile betrieblicher Größe oder die Kostenersparnisse hierarchischer Koordination überwiegen.

\footnotetext{
${ }^{23}$ Vgl. SCHMIDT 1999, S. 102

24 Vgl. SCHERER 1975

${ }^{25}$ In Anlehnung an KINNE 2000, S. 29

${ }^{26}$ Vgl. Williamson 1989, S. 135 ff. und die dort genannten Quellen

${ }^{27} \mathrm{Vgl}$. SCHERER/ROSS 1990, S. 109
} 


\subsubsection{Coinsurance}

Eine rein finanzielle Begründung für Zusammenschlüsse zwischen Unternehmen, deren Einkommensströme nicht vollständig korrelieren, lieferten HigGINS und SCHALL (1975) sowie LEWELLEN (1971) in der sogenannten "Coinsurance Hypothesis".

Wenn die Einkommensströme zwei sich zusammenschließender Unternehmen nicht vollständig korrelieren, z.B. aufgrund unterschiedlicher Abhängigkeit vom Konjunkturzyklus, führt das am Gesamtunternehmen zu einer Stabilisierung der Einkommensströme und zu einer Reduktion der zeitlichen Gewinnschwankungen ${ }^{28}$.

Ein wesentlicher Gesichtspunkt dabei ist die Möglichkeit der internen Subventionierung. Gewinne, die von einem Unternehmen erzielt werden, können zur Tilgung von Verlusten in einem anderen Unternehmen verwendet werden ${ }^{29}$. Dementsprechend wird das Risiko der Zahlungsunfähigkeit verringert. WESTON und CHUNG (1983) sprachen in diesem Zusammenhang von einer verminderten Konkurswahrscheinlichkeit und einem daraus resultierenden niedrigeren Gegenwartswert der Konkurskosten. Infolge dessen kann es zu einer Reduktion der Kapitalkosten kommen ${ }^{30}$.

In der Literatur finden sich zahlreiche empirische Untersuchungen, die auf die Existenz des Coinsurance-Effekts - zumindest bei konglomeraten Zusammenschlüssen - hinweisen $^{31}$. AMIT und LIVNAT (1988) hingegen kamen in ihrer Untersuchung zu dem Ergebnis, dass eine „pure financial diversification" zwar mit stabileren Cashflows, aber auch mit niedrigeren Rentabilitäten verbunden ist ${ }^{32}$.

\subsubsection{Konzept des überlegenden Managements}

Einen weiteren Erklärungsansatz verbesserter Effizienz liefert das Konzept des überlegenden Managements. In der Literatur werden auch häufig die Bezeichnungen „inefficient management hypothesis", "improved management hypothesis" oder "internal efficiency hypothesis" verwendet ${ }^{33}$.

Ausgangspunkt dieser Überlegungen sind Unternehmen, die aufgrund ineffizienten Managements ihre Ressourcen nicht optimal nutzen. Erhalten potentielle Käufer In-

\footnotetext{
${ }^{28}$ Vgl. BÜHNER 1990, S. 14

${ }^{29} \mathrm{Vgl.} \mathrm{BÜHNER/SPINDLER} \mathrm{1986,} \mathrm{S.} 603$

${ }^{30} \mathrm{Vgl.}$ Weston/Chung 1983, S. 4

31 SETH 1990, S. 431, nennt mehrere solcher Untersuchungen und kommt selbst auch zu diesem Ergebnis.

32 Vgl. Amit/LiVNAT 1988, S. 99

${ }^{33} \mathrm{Vgl}$. ALBRECHT 1994, S. 10
} 
formationen über ein solch ineffizient geführtes Unternehmen, so wird das Unternehmen demnach Ziel eines Übernahmeversuches. Der Käufer setzt voraus, dass das übernommene Unternehmen nach dem Zusammenschluss besser geführt werden kann.

Dem Konzept des überlegenen Managements zufolge geht also einem Zusammenschluss eine Periode ineffizienter Unternehmensleitung beim Kaufobjekt voraus. Der Zusammenschluss beendet diese Periode und unterstellt die Ressourcen des Kaufobjektes der Kontrolle des überlegenden Managements. Zusammenschlüsse können damit als Antwort auf eine suboptimale Unternehmensstrategie des Kaufobjektes angesehen werden ${ }^{34}$.

Die Relevanz des Konzepts des überlegenden Managements in der Praxis konnte CLARKE (1987) bestätigen. In Diskussionen mit potentiellen Käufern wurden die eigenen überlegenden Managementfähigkeiten oft als einziger Grund für eine verbesserte Effizienz unter neuer Kontrolle genannt ${ }^{35}$.

\subsubsection{Zwischenergebnis}

In Presse- und Geschäftsberichten werden Unternehmenszusammenschlüsse in erster Linie durch Effizienzpotentiale begründet. Der hohe Anteil gescheiterter Fusionen (vgl. Kapitel 4.2) lässt aber vermuten, dass das Effizienzstreben zumindest nicht das alleinige Motiv zur Durchführung von Unternehmenszusammenschlüssen ist ${ }^{36}$. PORTER (1987) gelangte in diesem Zusammenhang zu der Überzeugung, dass "die wenigsten Manager, die von Synergie reden, eine klare Vorstellung haben, was sie damit meinen". „Damit ist Synergie oftmals nichts weiter als ein gedankenloses Lippenbekenntnis oder die nachträgliche Rechtfertigung einer Diversifikation, die aus ganz anderen Gründen vorgenommen wurde ${ }^{37 " . ~ T R A U T W E I N ~(1990) ~ k a m ~ b e i ~ s e i n e n ~ e m p i r i s c h e n ~}$ Überprüfungen zu dem gleichen Resultat. Er nannte eine Reihe von Fällen, in denen Manager Synergien zur Rechtfertigung ihrer Zusammenschlüsse angeführt haben, und vergleicht diese Darstellung mit den Aussagen unabhängiger Beobachter. Die festgestellten Unterschiede bestätigten PORTERS These ${ }^{38}$. Im weiteren werden zusätzliche relevante Motive von Unternehmenszusammenschlüssen näher analysiert.

\footnotetext{
${ }^{34}$ Vgl. MALATESTA 1983 , S. 157

${ }^{35}$ Vgl. CLARKE 1987, S. 13

${ }^{36} \mathrm{Vgl}$. DEMMER 2002

${ }^{37}$ Vgl. PORTER 1987, S. 38-41 nach AlbRECHT 1994, S. 10

38 Vgl. Trautwein 1990, S. 285
} 


\subsection{Marktmachttheorie}

Das Streben nach Marktmacht ist neben dem Effizienzkriterium ein weiteres wesentliches Motiv, Unternehmenszusammenschlüsse durchzuführen. Aus wettbewerbspolitischer Sicht kann Marktmacht positiven und negativen Einfluss auf den Markt ausüben.

Marktmacht wird in der ökonomischen Theorie in Abgrenzung zur vollkommenen Konkurrenz definiert. Unter den idealtypischen Bedingungen der vollkommenen Konkurrenz können Anbieter, welche homogene Güter am Markt tauschen, nicht den Marktpreis beeinflussen. Freier Marktzugang und -austritt stellen sicher, dass Preise und Grenzkosten (bzw. Durchschnittskosten) übereinstimmen und alle Unternehmen, die zu einem über den Grenzkosten liegenden Preis anbieten, ihre Nachfrager sofort vollständig verlieren. Anbieter unter vollständiger Konkurrenz haben somit keinen Einfluss auf den Handlungsspielraum der übrigen Teilnehmer. Ökonomische Macht kann daher nur bei Marktformen der unvollkommenen Konkurrenz auftreten. Marktmacht drückt sich dann in der Fähigkeit aus, dauerhaft ein über den langfristigen Grenzkosten liegenden Preis zu erzielen, ohne dabei beträchtliche Umsatzeinbußen zu erleiden ${ }^{39}$.

\subsubsection{Marktmacht als Anreizmechanismus}

In den meisten Märkten bieten Unternehmen nicht identische Produkte an. Die Produkte unterscheiden sich durch Qualität, Preis und andere Eigenschaften. U.a. bedingt durch die Heterogenität, entsteht bei den Kunden eine unterschiedliche Wertschätzung gegenüber den Produkten, woraus den Unternehmen eine gewisse Marktmacht zuteil wird. Daraus kann resultieren, dass ein mit einem Produkt eines Unternehmens zufriedener Kunde trotz Preissenkung eines ähnlichen Konkurrenzproduktes seine Kaufentscheidung unverändert lässt. Ein nicht zu vernachlässigender Grund für die Produkttreue sind Informationskosten, die mit einem Wechsel zum Konkurrenzprodukt verbunden sind. Auch bei Wegfall des Informationskostenargumentes wird häufig durch moderate Preisänderung die Kaufentscheidung nicht beeinflusst, wenn der empfundene Wertunterschied zu einem Konkurrenzprodukt groß genug ist ${ }^{40}$. Daraus ergibt sich ein gewisser Preisspielraum, der als Ausdruck von Marktmacht von Unternehmen angesehen werden kann. Es ist dabei zu beachten, dass die Wertschätzung von Kunden auch für Unternehmen nicht kostenlos ist. Kunden müssen Produkte - speziell neue Produkte - kennenlernen und Gefallen an ihnen finden. Zur Erreichung eines großen Kundenkreises wird ein

\footnotetext{
${ }^{39}$ Vgl. UTTON 1995, S. 10

${ }^{40}$ Vgl. VARIAN 1995, S. $589 \mathrm{ff}$.
} 
Kundenkreises wird ein Unternehmen zumindest einen Teil der Informationskosten der Konsumenten tragen müssen. Diese Marketingaufwendungen haben teilweise Fixkostencharakter. Je höher die Aufwendungen zur Entwicklung und Einführung eines Produktes ausfallen, desto weniger Unternehmen werden in dem Markt tätig sein ${ }^{41}$.

Aus Informationskosten- und Fixkostenansatz lässt sich eine bedeutende Erkenntnis gewinnen: Die mit Marktmacht verbundenen Gewinnmöglichkeiten stellen einen Anreiz dar, die mit der Versorgung dieser Güter verbundenen Fixkosten zu decken. Marktmacht stellt somit ein wichtiges Anreizargument in Wettbewerbsprozessen dar. Betont wird an dieser Stelle, dass Marktmacht per se wettbewerbspolitisch nicht negativ gesehen wird, wenn ihr die eben beschriebene Rolle zugesprochen werden kann ${ }^{42}$.

\subsubsection{Praktische Auswirkungen von Marktmacht}

In der Theorie wird davon ausgegangen, dass Marktmacht zu überdurchschnittlichen Gewinnen einzelner Unternehmen oder Unternehmensvereinigungen führen kann. In der praktischen Anwendung ist es nicht immer einfach, den theoretischen "Spielraum" zwischen Preis und Grenzkosten zu ermitteln. Marktmacht äußert sich dagegen in vielen Fällen nicht in direkt erhöhten Preisforderungen gegenüber den Konsumenten sondern in anderen Formen, die indirekt zu überdurchschnittlichen Gewinnen führen (dazu ausführlich Kapitel 7.2). Dabei stehen die unten genannten Quellen des Gewinns aus Marktmacht im Vordergrund ${ }^{43}$ :

- Absprachen zwischen Unternehmern, die sich in einer Branche treffen,

- Absprachen zwischen Unternehmern, die sich in mehr als einer Branche treffen,

- Gegenseitigkeitsgeschäfte,

- Ausschließlichkeitsgeschäfte,

- Kopplungsgeschäfte,

- interne Subventionierung,

- Abschreckung möglicher Herausforderer.

\footnotetext{
${ }^{41}$ Vgl. BeSter 2000, S. 50

${ }^{42} \mathrm{Vgl.} \mathrm{Kapitel} 6.2$

${ }^{43}$ In Anlehnung an ALBRECHT 1994, S. 11
} 


\subsubsection{Absprachen zwischen Unternehmern innerhalb einer Branche}

Mit der Übernahme eines direkten Konkurrenten verringert sich die Anzahl der Unternehmen in der betrachteten Branche. Damit steigt die gegenseitige Abhängigkeit zwischen den verbleibenden Unternehmen. Das wiederum erhöht die Wahrscheinlichkeit stillschweigender oder auch offener Absprachen zwischen den Anbietern einer Branche und damit die Fähigkeit zur Durchsetzung höherer Preise ${ }^{44}$.

Empirische Untersuchungen über die Existenz solcher gewinnsteigernder AntiWettbewerbseffekte in Folge von Unternehmenszusammenschlüssen kamen nicht zu einheitlichen Ergebnissen.

Während Jensen (1984), ECKBo (1983) und Stillman (1983) keine Hinweise für Preisund in deren Folge Gewinnsteigerungen finden konnten, kam CHATTERJEE (1986) zu dem Resultat, dass "collusive synergies" mit höheren Wertsteigerungen verbunden sind als andere Synergien ("financial and operational").

\subsubsection{Absprachen zwischen Unternehmern mehrerer Branchen}

Mit der Übernahme eines Unternehmens aus einer anderen Branche vergrößert sich für den Erwerber die Wahrscheinlichkeit, dass er auf einen Konkurrenten in mehr als einer Branche treffen wird ("multimarket contracts").

In einer solchen Situation wird der Konkurrent auf jede Aktion des Unternehmens mit Reaktionen in allen anderen Branchen, in denen beide agieren, antworten ${ }^{45}$. Diese gesteigerte gegenseitige Abhängigkeit erhöht die Wahrscheinlichkeit von Absprachen zwischen den Konkurrenten.

Da die Kontakte zwischen den Unternehmern in mehreren Märkten vorliegen, sind Absprachen in einem oder auch in mehreren der gemeinsamen Märkte möglich. Diese "mutual forbearance theory of conglomerate behavior" wurde 1955 von EDWARDS entwickelt ${ }^{46}$.

\footnotetext{
${ }^{44}$ Vgl. WESTON/CHUng 1983, S. 6. Diese Überlegungen wurden aus verschiedenen Modellen abgeleitet (Oligopoltheorie von StIGLER, "dominant firm Modelle", Cournot-Nash Modelle).

${ }^{45} \mathrm{Vgl.}$ FeinBerg 1985, S. 226

${ }^{46}$ Vgl. EdWARDS 1955, S. 331
} 


\subsubsection{Gegenseitigkeitsgeschäfte}

Gegenseitigkeitsgeschäfte liegen vor, wenn zwei oder mehr Unternehmer stillschweigend oder offen eine Übereinkunft über die gegenseitige Abnahme von Produkten getroffen haben. Zwei Unternehmer, die jeweils potentielle Käufer der Produkte des anderen sind, gehen in diesem Fall eine Art zweiseitiger "Verknüpfung" ein ${ }^{47}$. Gegenseitigkeitsgeschäfte können demnach immer dann abgeschlossen werden, wenn ein Zulieferer gleichzeitig Abnehmer ist oder umgekehrt.

Die Existenz von Gegenseitigkeitsgeschäften ist jedoch noch kein Indikator der Ausnutzung von Marktmacht, denn Gegenseitigkeitsgeschäfte können schlichtweg das Resultat einer Einkaufspolitik sein, die den billigsten Anbieter auswählt oder die anfallenden Suchkosten nach möglichen Anbietern minimiert. Die im Rahmen der Marktmachttheorie relevanten Gegenseitigkeitsgeschäfte beruhen auf der Annahme, dass ein Unternehmer die Macht besitzt, einen anderen Unternehmer zu Gegenseitigkeitsgeschäften zu zwingen. Die Macht des Unternehmers kann sich dabei auf der Angebotsseite (Monopol) oder der Nachfrageseite (Monopson) äußern ${ }^{48}$ (ausführlich in Kapitel 7.2).

Unternehmenszusammenschlüsse können die Voraussetzung für Gegenseitigkeitsgeschäfte schaffen, denn sie vergrößern die Macht des Verkäufers und damit die Möglichkeit, einen anderen Unternehmer zu Gegenseitigkeitsgeschäften zu zwingen.

Darüber hinaus können sie das Potential für die notwendigen zweiseitigen Verknüpfungen schaffen oder vergrößern. Theoretische Überlegungen kommen zu dem Ergebnis, dass hauptsächlich konglomerate Zusammenschlüsse zu Gegenseitigkeitsgeschäften führen können ${ }^{49}$.

\subsubsection{Ausschließlichkeitsgeschäfte}

Ausschließlichkeitsgeschäfte ("exclusive dealing") liegen vor, wenn ein Unternehmen ein anderes zur zusätzlichen Abnahme von Produkten zwingt. Mit Hilfe seiner Marktmacht könnte ein Produzent Handelsunternehmen dazu zwingen, den Markenartikel des gekauften Unternehmens in deren Handelsprogramm aufzunehmen. Unter Umständen könnten die Handelsunternehmen sogar dazu aufgefordert werden, vergleichbare Produkte von Konkurrenten des erworbenen Unternehmens aus dem Sortiment zu eliminieren.

\footnotetext{
${ }^{47}$ Vgl. MUELLER 1979, S. 3

${ }^{48} \mathrm{Vgl}$. ALBRECHT 1994, S. 13
} 


\subsubsection{Kopplungsgeschäfte}

Die Überlegungen bezüglich der Kopplungsgeschäfte („tie-in-sales) sind sehr eng mit denen der Ausschließlichkeitsgeschäfte verbunden ${ }^{50}$. Besitzt ein Unternehmen das Angebotsmonopol bei einem bestimmten Produkt, so kann ein Teil der daraus resultierenden Marktmacht auch auf andere Produkte übertragen werden. Kopplungsgeschäfte verbinden also den Absatz zweier oder mehrerer Produkte. Diese spezielle Form der Ausübung von Marktmacht steht nur Mehrproduktunternehmen zur Verfügung. Konglomerate Zusammenschlüsse vergrößern damit das Potential für Kopplungsgeschäfte.

\subsubsection{Interne Subventionierung}

Interne Subventionierung ${ }^{51}$ liegt vor, wenn ein Unternehmen den Gewinn einer Geschäftseinheit nutzt, um die Verluste einer anderen Sparte zu kompensieren. Die Gewinne aus dem Verkauf eines Produktes können dabei vor allem für das Anbieten eines anderen Produktes zu einem nicht kostendeckenden Preis verwendet werden. Die Mischkalkulation innerhalb eines Mehrproduktunternehmens ermöglicht also Unterkostenverkäufe einzelner Produkte.

Ein kleines, wenig diversifiziertes Konkurrenzunternehmen wird nicht über die notwendigen Ressourcen verfügen, einem solchen Preiskampf standzuhalten, so dass es auf diesem Wege zur Aufgabe gezwungen werden kann.

Wenn ein Unternehmen darauf abzielt, einen Konkurrenten durch Unterkostenverkäufe vom Markt zu verdrängen, dürfte seine Fähigkeit dies zu erreichen durch einen kongIomeraten Zusammenschluss mit einem anderen Unternehmen deutlich vergrößert werden $^{52}$.

\subsubsection{Abschreckung möglicher Herausforderer}

Jedes Unternehmen kann durch Angriffe von Konkurrenten geschädigt werden, wobei zwischen zwei Typen von Konkurrenten differenziert wird: neue Anbieter in der Bran-

\footnotetext{
${ }^{49}$ Vgl. LORIE/HALPERN 1970, S. 151

${ }^{50} \mathrm{Vgl}$. LORIE/HALPERN 1970 , S. $154 \mathrm{f}$.

${ }^{51}$ In der englischsprachigen Literatur werden die Begriffe „predatory pricing" oder "cross-subsidizing" verwendet.

52 Vgl. LORIE/HALPERN 1970, S. 156
} 
che und etablierte Konkurrenten, die sich um eine Umpositionierung bemühen. Für beide Typen wird im folgenden der Begriff "Herausforderer" benutzt.

Herausforderer bringen in den meisten Fällen neue Kapazitäten, den Wunsch nach (zusätzlichem) Gewinn und erhebliche Mittel in den Wettbewerb innerhalb einer Branche ein. Dadurch können die Preise "gedrückt" oder die Kosten erhöht werden. In beiden Fällen sind negative Auswirkungen auf die Rentabilität der etablierten Wettbewerber zu erwarten. Ein Unternehmen muss deshalb darauf bedacht sein, die Gefahr des Angriffs von Konkurrenten zu minimieren. Dieses Risiko hängt im wesentlichen von den existierenden strukturbedingten Markteintritts- und Mobilitätsbarrieren sowie von den zu erwartenden Vergeltungsmaßnahmen $a b^{53}$.

Sind die Barrieren hoch und/oder muss der Herausforderer scharfe Gegenmaßnahmen befürchten, so ist die Angriffsgefahr gering. Da Marktmacht mit hohen strukturbedingten Barrieren und mit der Fähigkeit zu massiver Vergeltung verbunden ist, erschwert sie den Angriff von potentiellen Herausforderern und ermöglicht damit dauerhaft hohe Gewinne. Einige wichtige Maßnahmen zur Erhöhung strukturbedingter Barrieren, die im Zuge von Unternehmenszusammenschlüssen realisiert werden können, sind ${ }^{54}$ :

- Schließung von Produkt- oder Positionslücken,

- Abriegelung von Zugängen zu Vertriebskanälen,

- Steigerung von größenbedingten Kostendegressionen,

- Verhinderung alternativer Technologien,

- Anbindung von Lieferanten.

\subsection{Steuertheorie}

Bei der Steuertheorie steht bei Unternehmenszusammenschlüssen das Motiv der Ausnutzung von Steuervorteilen im Vordergrund. Die Steuergesetzgebung bietet eine Reihe möglicher Zusammenschlussmotive:

- Übernahme des Verlustvortrages,

\footnotetext{
${ }^{53}$ Vgl. PORTER 1990, S. 609 ff.

${ }^{54}$ In Anlehnung an PORTER 1989, S. $610 \mathrm{ff}$.
} 
- Erhöhung der abschreibungsfähigen Vermögensgegenstände,

- Steuerersparnis durch Unternehmenskauf statt Dividendenzahlung ${ }^{55}$.

Ein denkbares Szenario geht von einem Unternehmen mit großen steuerabzugsfähigen Verlusten aus, dessen Kauf einem Unternehmen mit ausreichend großen Gewinnen Steuerersparnisse ermöglicht ${ }^{56}$. Ein weiteres Szenario basiert auf der Behauptung, dass steuerlich abgeschriebene, aber weiterhin nutzbare Vermögensgegenstände die Attraktivität eines möglichen Kaufobjektes steigern ${ }^{57}$.

DeRTOuzous und THORPE (1982) kamen zu dem Ergebnis, dass dieses Szenario in Verbindung mit Erbschaftssteuern eine Vielzahl der Zusammenschlüsse innerhalb der USamerikanischen Zeitungsindustrie erklären kann. Der potentielle Käufer ist demnach bereit, einen Aufpreis auf den Marktwert des Kaufobjektes zu zahlen und somit die Basis der abschreibungsfähigen Vermögensgegenstände zu erhöhen. Dem vereinigten Unternehmen stehen nach dem Zusammenschluss Abschreibungsmöglichkeiten und damit Steuervorteile zur Verfügung, die dem Kaufobjekt alleine nicht zur Verfügung gestanden hätten.

Ein weiteres Szenario geht von einem Unternehmen mit frei verfügbarem Cashflow aus, den anstelle der möglichen Dividendenzahlung ein anderes Unternehmen erwirbt, vorausgesetzt "that there is an effective personal tax differential between dividends and capital gains" 58 .

Die Meinungen in der Literatur über die Relevanz der Steuertheorie gehen auseinander. Mueller (1979) ist der Ansicht, dass sich besonders zum Ende der 70er Jahre mögliche Steuervorteile als Motiv für Zusammenschlussaktivitäten herausgestellt haben $^{59}$. Andererseits kam KAPLAN (1987) zu dem Ergebnis, dass Steuervorteile nur einen geringen Anteil des normalerweise gezahlten Aufpreises auf den Marktwert des Kaufobjektes erklären können ${ }^{60}$. Steuerliche Beweggründe werden häufig auch als Grund für eine Zunahme internationaler Unternehmenszusammenschlüsse angeführt

\footnotetext{
${ }^{55}$ In Deutschland besonders aktuell durch die Neuregelung mit Wirkung zum 01. Januar 2002 im Einkommensteuergesetz bzgl. Veräußerungsgewinnen von Beteiligungen; dazu BAULIG und PRELLBERG 2001

${ }^{56} \mathrm{Vgl}$. RoLl 1988, S. 247

57 Vgl. RoLl 1988, S. 247

$58 \mathrm{Vgl}$. Roll 1988, S. 248

${ }^{59}$ Vgl. MUELLER 1979, S. 6

${ }^{60} \mathrm{Vgl}$. TRautwein 1990, S. 284
} 
(WEICHENRIEDER 1998), jedoch dürfte es sich dabei eher um Mitnahmeeffekte als um das Motiv der Fusion handeln ${ }^{61}$.

\subsection{Raider Theorie}

Die Raider Theorie basiert auf der Annahme, dass der Raider Anteile eines Unternehmens erwirbt, um dieses Unternehmen dann zu Lasten der anderen Eigentümer auszuplündern, indem das Vermögen dieses Unternehmens zum eigenen Unternehmen transferiert wird.

Der Begriff "Raider" (zu deutsch: Plünderer) wurde hauptsächlich von der USamerikanischen Wirtschaftspresse geprägt. Aus den unterschiedlichen Definitionen der Wirtschaftspresse leiteten HOLDERNESS und SHEEHAN (1985) ein übergreifendes Konzept $a b$, das die Reduzierung des Vermögens der anderen Eigentümer des gekauften Unternehmens in den Vordergrund stellt. Damit ist ein Raider eine "Person", die einen Vermögenstransfer von den anderen Eigentümern des gekauften Unternehmens zu sich selbst bewirkt.

Eine Möglichkeit, einen solchen Vermögenstransfer durchzuführen, besteht darin, dass der Raider seine Einflussmöglichkeiten im erworbenen Unternehmen nutzt, um sich selbst übermäßige Vergütungen und Vergünstigungen zu bewillligen ${ }^{62}$.

\subsection{Bewertungstheorie}

Die Bewertungstheorie stellt auf Unterschiede in der Unternehmensbewertung bei Zusammenschlüssen $\mathrm{ab}$. Unterschiede in der Unternehmensbewertung entstehen durch unterschiedliche Erwartungen bezüglich der zukünftigen finanziellen Erträge (Entnahmeerwartungen), die aus dem Unternehmensbesitz resultieren, sowie dem anzusetzenden Kapitalisierungszinssatz. Wenn diese Unterschiede in der Form vorliegen, dass potentielle Käufer den Unternehmenswert höher einschätzen als die Eigentümer, werden Zusammenschlüsse möglich.

Ausgangspunkt der Bewertungstheorie sind Unterschiede in der Unternehmensbewertung. Damit stellt sich die Frage, wie solche Unterschiede entstehen können. In der

\footnotetext{
${ }^{61}$ So auch KLEINERT/KLODT 2000, S. 45

${ }^{62} \mathrm{Vgl.} \mathrm{HoldeRNeSS/SHeEHAN} \mathrm{1985,} \mathrm{S.} 556$
} 
Literatur werden vor allem die "Economic Disturbance Theory of Mergers" und "Asymmetrien bzgl. der vorhandenen Informationen" als Begründung genannt.

Die "Economic Disturbance Theory" von GORT ${ }^{63}$ erklärt Unterschiede in der Unternehmensbewertung durch ökonomische Störungen. Dabei sind grundsätzlich zwei Erklärungsansätze zu unterscheiden: Erstens verändern diese Störungen zufällig die Gewichtung der Erwartungen der einzelnen Marktteilnehmer. Zweitens erschweren ökonomische Störungen Vorhersagen über die Zukunft und erhöhen damit die Varianz der Unternehmensbewertungen (z.B. schneller Technologiewandel, Markteintrittsbarrieren $)^{64}$.

Das Vorhandensein von Asymmetrien bzgl. der vorhandenen Informationen bzw. von Informationsdefiziten führt ebenfalls zu Unterschieden in der Unternehmensbewertung und damit eventuell zu Zusammenschlüssen. Wenn der potentielle Käufer über andere Informationen als der Eigentümer verfügt und aus diesen Informationen einen höheren Unternehmenswert als der Eigentümer ableitet, dann werden Zusammenschlüsse möglich.

RoLL (1986) untersuchte in seiner "Hybris Hypothesis of Corporate Takeovers" die Rolle von Selbstüberschätzung der einzelnen Entscheidungsträger des kaufenden Unternehmens als eine wesentliche Erklärung für das Zustandekommen von Unternehmenszusammenschlüssen ${ }^{65}$ und gelangte zu dem Ergebnis, dass die "Hybris Hypothesis" aufgrund der vorliegenden empirischen Untersuchung über Unternehmenszusammenschlüsse nicht verworfen werden kann.

In der Literatur finden sich zahlreiche Hinweise, die darauf schließen lassen, dass der Bewertungstheorie beim Entstehen von Zusammenschlüssen eine entscheidende Bedeutung zukommt.

RAVENSCRAFT und SCHERER (1987) nannten eine Reihe von Interviews, aus denen ersichtlich wurde, dass potentielle Käufer aktiv auf der Suche nach (ihrer Meinung) unterbewerteten Unternehmen sind ${ }^{66}$. Auch die in den letzten Jahren zunehmende Diskussion um die Einführung internationaler Rechnungslegungsgrundsätze (US-GAAP,

\footnotetext{
${ }^{63}$ Vgl. GORT 1969, S. 624

${ }^{64} \mathrm{Vgl.}$ ALBRECHT 1994, S. 21

${ }^{65}$ Vgl. RoLl 1986, S.197 ff.

${ }^{66}$ Vgl. RAVENSCRAFT/SCHERER 1987, S. 9
} 
IAS etc.) dürfte zusätzliche Anreize für eine Fusionsaktivität durch Um- und Unterbewertung von Unternehmen schaffen ${ }^{67}$.

\subsection{Managerialismustheorie}

Kernbestand der Managerialismustheorie ist, dass Unternehmenszusammenschlüsse von Managern zur Maximierung ihres eigenen Nutzens durchgeführt werden ${ }^{68}$. Es wird also bezweifelt, ob bei der Übernahmeentscheidung des Managements die Interessen der Anteilseigner Berücksichtigung finden.

Ausgangspunkt dieser Überlegungen bildet die Arbeit von BERLE und MEANS (1932) über die Trennung von Eigentum und Leitung in einem Unternehmen. Entscheidend dabei ist, dass die Leitung (das Management) häufig andere Interessen verfolgt als die Eigentümer, und somit hohes Konfliktpotential entstehen kann.

\subsubsection{Principal-Agent-Theorie}

Der grundlegende Gedanke der Trennung von Eigentum und Leitung wurde in verschiedenen "Managerial Models of the Firm" aufgenommen (BAUMOL (1967), MARRIS (1964), WiLLIAMSON (1964)). Diese manageriellen Modelle liefern einen Erklärungsansatz, der suggeriert, dass Manager zum Schaden der Eigentümer eigene Ziele stärker verfolgen als die Gewinnmaximierung ${ }^{69}$.

In BAUMOLS Modell verfolgten Manager das Ziel der Umsatzmaximierung. Dabei wird dem Gewinn lediglich die Funktion einer Nebenbedingung beigemessen, welche erfüllt werden muss, um die Eigentümer zufriedenzustellen ${ }^{70}$.

In MARRIS Modell hingegen verfolgten die Manager das Ziel der Wachstumsmaximierung. MARRIS ist der Auffassung, dass den eigenen Nutzen maximierende Manager die Wachstumsrate als Zielvariable ansehen ${ }^{71}$.

\footnotetext{
${ }^{67} \mathrm{Vgl}$. SCHMUSCH 2002

${ }^{68}$ Vgl. WiCKeLgRen 2001; vgl. AlbreCHT 1994, S. 22

${ }^{69} \mathrm{Vgl}$. ALBRECHT 1994, S. 23

$70 \mathrm{Vgl.} \mathrm{BaUMOL} \mathrm{1976,} \mathrm{S.} 46 \mathrm{ff}$.

${ }^{71} \mathrm{Vgl.} \mathrm{MARRIS} \mathrm{1964,} \mathrm{S.} 49 \mathrm{ff}$.
} 
Bei WilLIAMSON verfolgen Manager das Ziel der Maximierung präferierter Ausgaben. Dabei wurden präferierte Ausgaben als eine zusammengesetzte Variable modelliert, die persönliche Bedürfnisse und Ziele von Managern berücksichtigt ${ }^{72}$. Als Managementeigeninteressen ${ }^{73}$ werden in der Literatur im wesentlichen folgende Zielsetzungen genannt:

- Streben nach Macht und Prestige,

- Streben nach höherem Einkommen,

- Streben nach vermindertem persönlichen Anstellungsrisiko.

Macht und Prestige von Managern sind eng mit Größe und Wachstum des Unternehmens verbunden. Die Rentabilität spielt in diesem Zusammenhang häufig eine untergeordnete Rolle ${ }^{74}$. Dementsprechend steht das Streben nach Macht unmittelbar mit der Vergrößerung des eigenen Einflussbereiches in Verbindung.

Unter höherem Einkommen verstand WILLIAMSON einerseits die Erhöhung der Gehälter, andererseits aber auch solche Ausgaben der Unternehmung, die den Managern unmittelbar zugute kommen (Ausgaben für Größe und Ausstattung von Arbeitsräumen, Dienstwagen, Chauffeur etc. ${ }^{75}$ ). Williamson (1964) und PenRose (1959) argumentierten, dass der signifikante positive Zusammenhang zwischen Größe der Unternehmung und Vergütung des Managements einen Wachstumsanreiz für das Top-Management bietet, auch wenn aus diesen Wachstumsaktivitäten keine Gewinne für die Eigentümer zu erwarten sind.

Manager sind daran interessiert, das Risiko des Arbeitsplatzverlustes zu vermindern. Da eine Reduzierung des Unternehmensrisikos durch Diversifikation mit einer Reduktion des eigenen persönlichen Anstellungsrisikos einhergeht, ist die Unternehmensdiversifikation eine beliebte Managermaßnahme ${ }^{76}$.

Von entscheidender Bedeutung für die Argumentation der Managerialisten ist, dass die obigen Managementinteressen eng mit der Größe des Unternehmens zusammenhängen. Den eigenen Nutzen maximierende Manager streben hiernach ein schnelles Un-

\footnotetext{
72 Vgl. Williamson 1964, S. 32 ff.

${ }^{73}$ Die Eigentümer verfolgen in erster Linie das Ziel der Sicherheit der Verzinsung ihrer Kapitalanlage

${ }^{74} \mathrm{Vgl}$. Mueller 1969, S. 644

${ }^{75} \mathrm{Vgl.}$ Williamson 1964, S. 35

${ }^{76} \mathrm{Vgl}$. BÜHNER 1990, S. 8
} 
ternehmenswachstum an. Da Unternehmenszusammenschlüsse in der Praxis den schnellsten Wachstumsweg darstellen, kann an dieser Stelle ein direkter Zusammenhang zwischen Zusammenschlussaktivität und manageriellen Eigeninteresse bei der erwerbenden Unternehmung hergestellt werden. Dementsprechend behauptete MUELLER (1969) in seiner "managerialistischen" Erklärung für Zusammenschlüsse, dass Unternehmen Managern die Möglichkeit bieten, deren Machtbereich schnell und umfassend auszudehnen. MUELLER folgerte daraus, dass Manager aus Eigeninteresse Zusammenschlüsse durchführen ${ }^{77}$.

Ausgehend von der Trennung von Eigentum und Leitung und dem daraus resultierenden Agency-Konflikt haben sich zwei weitere Zusammenschlusstheorien entwickelt:

- die „Free Cash Flow Theory of Takeovers",

- die "Marktkontrolltheorie”

JENSEN brachte durch seine "Free Cash Flow Theory" zum Ausdruck, dass Manager bemüht sind, Auszahlungen an die Eigentümer so gering wie möglich zu halten, da Auszahlungen die Ressourcen ihrer Kontrolle beeinträchtigen und damit ihre Macht reduzieren $^{78}$.

Zusammenschlüsse stellen eine Möglichkeit für die Verwendung von Finanzmitteln innerhalb des Unternehmens dar. Die "Free Cash Flow Theory” besagt, dass Manager von Unternehmen mit hohen „Free Cash Flows" eher Zusammenschlüsse mit niedrigeren Erfolgsaussichten durchführen ${ }^{79}$. Die Verwendung von „Free Cash Flows" im Interesse der Manager liefert also eine weitere Erklärung für das Entstehen von Unternehmenszusammenschlüssen.

Bei der Marktkontrolltheorie sind Unternehmen als ein externer Kontrollmechanismus anzusehen, der die Abweichungen der Manager von den Eigentümerinteressen begrenzt ${ }^{80}$. Manager, die in erster Linie ihre Eigeninteressen verfolgen, nutzen die Unternehmensressourcen im Sinne der Eigentümer nicht optimal. Die sich aus einer optimalen Ressourcenverwendung ergebende Gewinnchance veranlasst die Teilnehmer des Marktes zur Übernahme des Unternehmens, Auswechselung des Managements und auf diese Weise Etablierung des im Interesse der Eigentümer geführten Unternehmens.

\footnotetext{
77 Vgl. Mueller 1969, S. 644 ff.

78 Vgl. JENSEN 1986, S. 323

79 Vgl. JeNSEN 1988, S. 34

80 Vgl. Trautwein 1990, S. 285; SCherer 1988, S. 69 f.; Stillman 1983, S. 226
} 
Diese Theorie interpretiert Zusammenschlüsse also als eine Methode zur Umgehung des Agency-Problems. Die Managerialismustheorie argumentiert dementsprechend, dass das Agency-Problem nicht gelöst wird und die Zusammenschlussaktivität eine Manifestation des Agency-Problems ist.

Empirisch wird in der Wirtschaftspresse häufig das Vorliegen von Managementinteressen bei Zusammenschlüssen unterstellt ${ }^{81}$. Neben diesen eher spekulativen Aussagen finden sich auch in der wissenschaftlichen Literatur einige direkte Hinweise, die die Managerialismustheorie untermauern. So kamen z.B. RAVENSCRAFT und SCHERER (1987) aufgrund mehrerer studienbegleitender Fallstudien zu dem Ergebnis, dass „empirebuilding-motives" bei vielen Zusammenschlüssen präsent sind ${ }^{82}$.

You et al. konnten zeigen, dass die Verluste der Eigentümer des erwerbenden Unternehmens am größten sind, wenn Manager ein Motiv sowie die Möglichkeit hatten, den eigenen Nutzen zu mehren. Diese Bedingungen sind gegeben, wenn die Manager und Direktoren nur einen geringen Anteil der Aktien des kaufenden Unternehmens halten, oder wenn der Anteil der Direktoren, die gleichzeitig Teil des Managements sind, relativ groß ist. Ein signifikanter Einfluss der Bedeutung der Anteile des kaufenden Unternehmens am Vermögen seiner Manager und Direktoren konnte dagegen nicht festgestellt werden ${ }^{83}$.

FAULI-OlLER und MotTA (1996) zeigten theoretisch in Abhängigkeit von der Entlohnungsstruktur sowohl für den Cournot- als auch für den Bertrand-Wettbewerb den Anreiz zur Durchführung von auch unprofitablen Fusionen für Manager. Je stärker die Bezahlung des Managements umsatzabhängig statt gewinnabhängig ist, desto größer ist der Anreiz für die Durchführung von auch unprofitablen Fusionen. Für das Vorliegen eines Prinzipal-Agent-Problems gibt es außerdem auch Hinweise aus ökonometrischen Studien (MORCK und YEUNG 1997).

\footnotetext{
81 Vgl. Demmer 2002; Wirtschaftswoche Nr. 6 vom 01.02.2001, S. 47-53: "Ende des Wahns";

LeBENSMITTELZEITUNG vom 22. Juni 2001, S. 29: „Ernüchterung nach dem Fusionstraum”; TRAUTWEIN 1990, S. 288

82 Vgl. RAVENSCRAFT/SCHERER 1987, S. 214

${ }^{83} \mathrm{Vgl}$. You et al. 1986, S. 217
} 


\subsection{Weitere Zusammenschlusstheorien}

\subsubsection{Fusionen als Modewelle}

SCHENK (1996) erklärte das Entstehen von Fusionswellen als Folge einer defensiven Unternehmensstrategie ${ }^{84}$. In einer Welt mit unvollkommenen Informationen beinhaltet eine Aktion des Konkurrenten eine Information, die für die eigene Entscheidung genutzt werden kann. Steht ein Unternehmen vor einer Alternative mit zwei gleich wahrscheinlichen Ergebnissen, kann die Entscheidung eines anderen Unternehmens, das vor einer ähnlichen Ausgangssituation steht, ausschlaggebend für die eigene Entscheidungsfindung sein. Denn es kann daraus abgeleitet werden, dass das andere Unternehmen seine Schlussfolgerungen rational getroffen hat und vielleicht über andere (zusätzliche) Informationen verfügt. Während CAPLIN und LEAHY (1994) "Bubbles" und die darauf folgenden Crashs auf diese Art erklärten, wendete SCHENK (1996) diese Theorie auf Fusionswellen an. Dabei geht SCHENK - Ergebnisse der Experimente von KAHNEMAN und TVERSKY (1979) zugrundelegend - davon aus, dass sich Individuen bei Entscheidungen unter Unsicherheit eher von der Minimax-Regel leiten lassen, statt Erwartungswerte zu maximieren. Die Minimax-Regel besagt, dass aus einem Set von verschiedenen Strategien unter Unsicherheit diejenige ausgewählt wird, bei der das „Bedauern" im Fall des Misserfolges am geringsten ist. Hierbei ist zu beachten, dass dies nicht zwangsläufig die Strategie mit dem höchsten Erwartungswert darstellt.

\subsubsection{Market for corporate control}

Eine weitere These zur Erklärung von Fusionen beinhaltet das Konzept des "markets for coporate control" (DEWEY 1961; MANNE 1965). Danach sind die meisten Unternehmenszusammenschlüsse nicht durch Marktmachtkalküle oder Skalenerträge motiviert, sondern es handelt sich vielmehr um eine "zivilisierte Form" des Bankrottes des übernommenen Unternehmens, d.h. um eine freiwillige Liquidation, die das Vermögen von schrumpfenden auf wachsende Unternehmen umschichtet ${ }^{85}$. Die Übernahme unterdurchschnittlich rentabler und damit schlechter geführter Unternehmen durch Unternehmen mit fähigerem Management stellt damit den Grund für eine Fusionsaktivität dar. Unternehmensübernahmen dienen somit dem Austausch unfähigen Managements. Die Existenz eines solchen "markets for corporate control" wirkt deshalb als

\footnotetext{
${ }^{84}$ Vgl. KLEINERT/KLODT 2000, S. 42

${ }^{85} \mathrm{Vgl}$. KLEINERT/KLODT 2000, S. 64
} 
eine Motivation für jedes Management, den Unternehmenswert zu mehren, da sonst eine Übernahme und mit ihr der eigene Arbeitsplatzverlust droht. Wettbewerbspolitisch sind solche Fusionen wohlfahrtsfördernd einzuordnen, sobald die Effizienzgewinne die Wohlfahrtseinbußen durch die größere Marktmacht des fusionierten Unternehmens übersteigen. Die Welle der Konglomeratbildungen in den Vereinigten Staaten Ende der siebziger Jahre wurde als Bestätigung dieser These angesehen. Neuere empirische Ergebnisse geben keine eindeutigen Hinweise auf die Rolle solch eines „markets for corporate control"86.

\subsubsection{Sonstige Motive und Zwischenergebnis}

Neben den oben vorgestellten Unternehmenszusammenschlusstheorien werden in der Literatur vereinzelt weitere Zusammenschlussmotive genannt. Der Erwerb von Vermögenswerten wie Kundenstamm, Patente, Humankapital oder auch Firmennamen können einen sehr großen Fusionsanreiz bieten (letztendlich ist dies Folge einer Unterbewertung). MoтTA (1996) zeigte das modelltheoretisch für die Internationalisierungsstrategie eines Unternehmens. Für ein Unternehmen ist das Besitzen von spezifischen Vorteilen danach nicht unbedingt erforderlich, um im Ausland Investitionen zu tätigen. Vielmehr kann der Erwerb eben jener Vorteile ein Grund sein, die Aktivitäten auf das Ausland auszudehnen. Nach einer Studie der Beratungsgesellschaft KPMG (1998) zielen 12 v.H. der Fusionen hauptsächlich auf den Erwerb neuer Technologie bzw. Produkte und Dienstleistungen ${ }^{87}$. Für spezifische Branchen können zusätzlich weitere Faktoren ausschlaggebend für eine Fusionsaktivität sein:

- Eintritt in neue Märkte,

- schnelle Marktpräsenz,

- Sicherung des Absatzes oder der Beschaffung,

- Vervollständigung des Produktprogramms,

- Fehlen notwendiger eigener Kenntnisse und Fähigkeiten,

- Zugang zu neuen Technologien,

- Marktbereinigung durch Stillegung des erworbenen Unternehmens,

\footnotetext{
${ }^{86}$ Vgl. dazu KLEINERT/KLODT 2000, S. 65 und die dort zitierten Studien

${ }^{87}$ Vgl. KPMG 1998: www.kpmg.co.uk/uk/service/manage/research/mergers
} 
- Abwehrmaßnahme gegen feindliche Übernahmeversuche,

- Kapitalanlage,

- Anpassung an sinkende Transaktionskosten.

Festzuhalten bleibt, dass Motive von Unternehmensfusionen vielfältig sein können und empirisch oftmals schwierig nachweisbar sind. Unterschiedliche Rahmenbedingungen der verschiedenen Jahrzehnte und Branchen erschweren einen Direktvergleich von Fusionsmotiven, so dass aus den vorigen Ausführungen nur bedingt allgemeine Aussagen zu Fusionsaktivitäten spezifischer Branchen getroffen werden können.

Auch wenn die Frage der Intention zu diesem Zeitpunkt nicht eindeutig geklärt werden kann, lassen sich Erkenntnisse über das wettbewerbliche Verhalten gewinnen. Dies ist Gegenstand des folgenden Kapitels. 


\section{Industrieökonomische Untersuchungen von Fusionen}

Es gibt eine Vielzahl von wissenschaftlichen Arbeiten, die sich mit den Auswirkungen von Konzentration ${ }^{88}$ und Fusionen beschäftigen. Dabei ist das Forschungsfeld von unterschiedlichen Zielsetzungen bestimmt. Einerseits werden in den vorwiegend industrieökonomischen Ansätzen die Auswirkungen von Unternehmenszusammenschlüssen auf die Marktstruktur untersucht und daraus wohlfahrtsökonomische Folgerungen aus modifizierten Oligopolmodellen abgeleitet. Andererseits ergründen betriebswirtschaftliche Untersuchungen in erster Linie Erfolge, Motivationen und Zusammenhänge von Unternehmensgröße und Rentabilität. Das Hauptaugenmerk dieser Arbeit wird tendenziell auf die wohlfahrtsökonomische Analyse von Fusionen gerichtet; wichtige Erkenntnisse der betriebswirtschaftlichen Forschung werden jedoch ergänzend aufgenommen.

\subsection{Theoretische Studien zu horizontalen Fusionen}

In der ökonomischen Literatur werden verschiedene theoretische Modelle zu dieser Problematik vorgestellt. Die Industrieökonomik liefert generelle Konditionen, unter denen Fusionen zu Preissteigerungen und/oder Kostenreduktionen oder zu Wohlfahrtssteigerungen führen. Zunächst wird das Modell von WiLLIAMSON vorgestellt, der bereits 1968 auf den Zielkonflikt zwischen Effizienzvorteil und Marktmachteffekt hinwies. Anschließend wird auf Erkenntnisse aus weiterentwickelten Oligopolmodellen eingegangen, wobei zwischen der Gefahr der Einzelmarktbeherrschung und der kollektiven Marktbeherrschung unterschieden wird. Der theoretische Überblick endet mit einer Zusammenstellung weiterer wichtiger Studien und hält anschließend die Erkenntnisse aus der theoretischen Analyse fest.

\subsubsection{Das Trade-Off-Modell von WILLIAMSON}

Der meist genutzte Ansatz für die Bewertung von Wohlfahrtsauswirkungen bei Fusionen ist das Trade-Off-Modell von WilLIAMSON (1968). Ökonomische und rechtliche Übereinstimmung bzgl. der Effizienz von Fusionen wurde maßgeblich durch den Beitrag "Economics as an Antitrust Defence" (1968) erreicht. In einem einfachen Partialgleichgewichtsmodell erläuterte WiLLIAMSON mögliche Trade-Offs zwischen zunehmender Marktmacht und Effizienzvorteilen durch Fusionen. Wenngleich das Modell die größte Anwendung auf horizontale Fusionen findet, kann es auch auf vertikale und konglomerate Fusionen ausgedehnt werden.

\footnotetext{
${ }^{88}$ Gründe, Messung und Erfassung von Konzentration werden detaillierter im Anhang B beschrieben
} 
Das WiLliamSon Modell ist statischer Natur, stützt sich auf die neoklassische Ökonomie und folgt somit den Erklärungsansätzen der früheren Monopol-Wohlfahrtsanalysen von HARBERGER (1954) ${ }^{89}$. In dem Grundmodell unterstellte Williamson, dass es sich um einen Zusammenschluss eines Duopols bei homogenen Gütern handelt, welches durch den Zusammenschluss in ein Monopol konvertiert wird ${ }^{90}$. WiLLIAMSON nutzte ein Modell, das auf dem Konzept der Gesamtwohlfahrt beruht. Um die Wohlfahrtseffekte zu demonstrieren, werden zwei Szenarien abgebildet: zum einen eine Situation mit und zum anderen eine Situation ohne Marktmacht vor dem Zusammenschluss (vgl. Abbildung 16 und Abbildung 17). Vereinfachend werden lineare Nachfragefunktionen sowie konstante Grenz- und Durchschnittskosten unterstellt ${ }^{91}$.

Abbildung 16: Keine Marktmacht vor dem Zusammenschluss

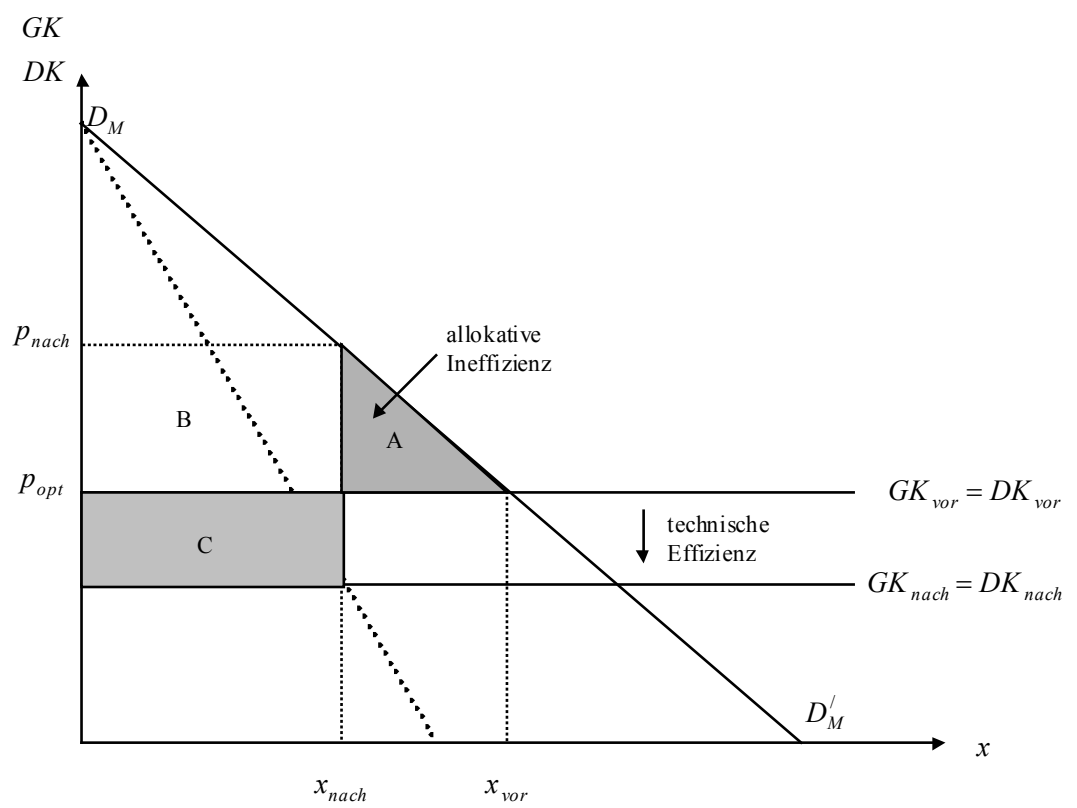

Quelle: WiLLIAMSON 1968, S. 21

\footnotetext{
${ }^{89} \mathrm{Vgl}$. CoWLing et al. 1980 , S. 16

90 Vgl. Röller/Stennek/Verboven 2000, S. $30 \mathrm{ff}$.

${ }^{91}$ Der Modellansatz lässt sich auch auf anders verlaufende Nachfrage- und Kostenfunktionen übertragen. Beispielhaft in WATERSON 1983, S. $223 \mathrm{ff}$.
} 
Abbildung 17: Marktmacht vor einem Zusammenschluss

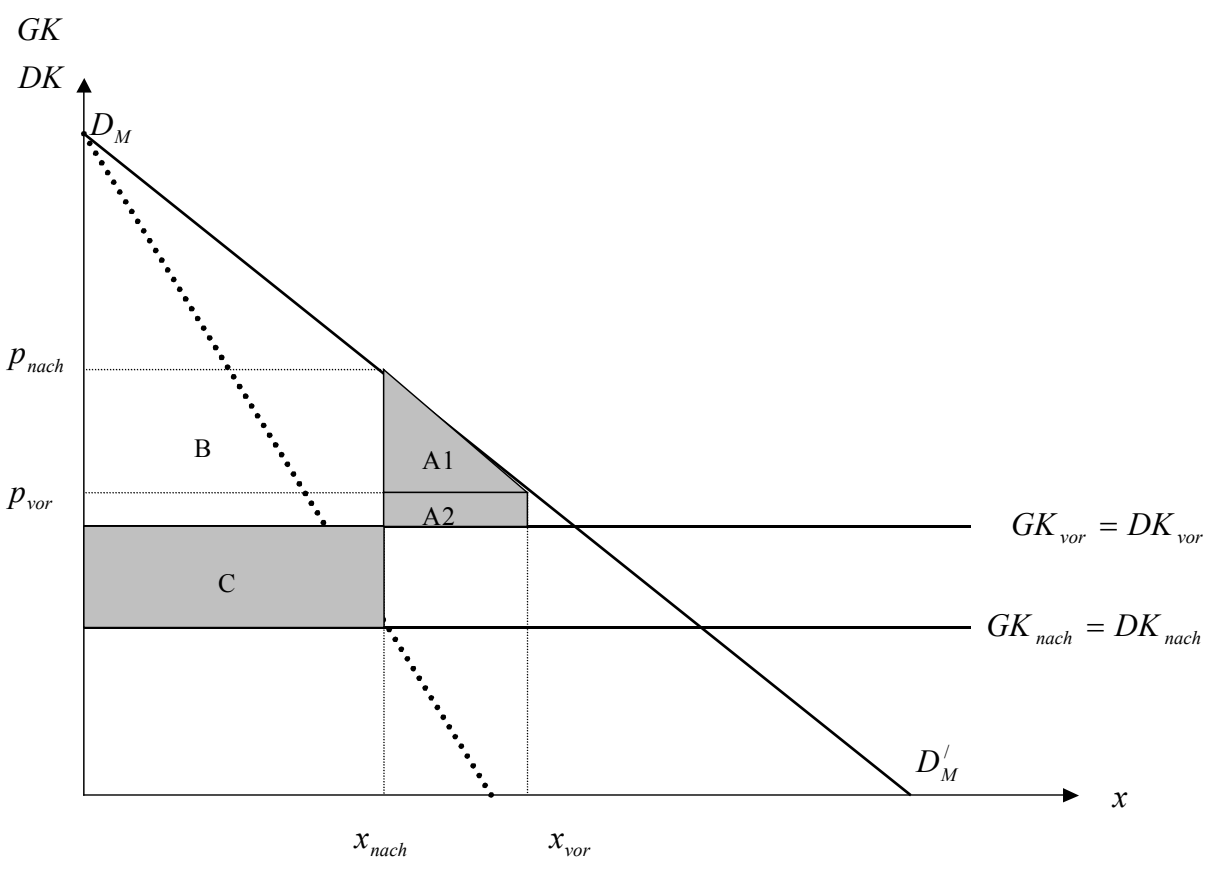

Quelle: Cowling et al. 1980, S. 17

Beiden Grafiken kann ein Wohlfahrtsverlust nach einem hypothetischen Unternehmenszusammenschluss in Höhe der Flächen $A$ bzw. $A 1$ entnommen werden (sogenannte allokative Ineffizienz) $)^{92}$. Bei Existenz von Marktmacht vor dem Zusammenschluss beträgt das Ausmaß der allokativen Ineffizienzen nach der Fusion die Summe der Flächeninhalte $A 1$ und $A 2$. Dem stehen Produzentengewinne durch Verbesserung der technischen Effizienz in Höhe der Fläche $C$ gegenüber. Die Wohlfahrtsgewinne, die in Folge der Rentenumlenkung von Konsument zu Produzent (Fläche $B$ ) entstehen, werden von WiLLIAMSON im Gegensatz zum Konsumentenwohlfahrtskonzept beim Gesamtwohlfahrtskonzept vernachlässigt ${ }^{93}$.

WiLLIAMSON bestimmte den sozialen Nettowohlfahrtseffekt auf "naive" Art, indem er die beiden Flächeninhalte graphisch gegeneinander aufrechnete $(C-A$ bzw. $C-(A 1+$ A2)). Danach resultieren positive Gesamteffekte, wenn das Ausmaß der technischen Effizienzen gegenüber den entstehenden allokativen Ineffizienzen überwiegt $(C>A$

\footnotetext{
92 Durch den Zusammenschluss steigt der Preis von $p_{\text {vor }}$ auf $p_{\text {nach }}$ und entsprechend die angebotene Menge von $x_{\text {vor }}$ auf $x_{\text {nach }}$.

${ }^{93} \mathrm{Vgl}$. CRAMPTON 1994, S. 66
} 
bzw. $C>(A 1+A 2)$. Seine wettbewerbspolitische Empfehlung beinhaltete daher, einen Zusammenschluss freizugeben, sobald der Nettowohlfahrtseffekt positiv ausfällt ${ }^{94}$.

In der praktischen Anwendung stößt das Modell jedoch auf Grenzen. Vor allem die Ermittlung von Macht- und Kosteneffekten sowie die zeitgleiche Umsetzung von Preiserhöhungen und Kostensenkungen sind in der Realität schwer nachzuweisen. Zudem werden nur diejenigen Effekte ermittelt, die unmittelbar die fusionierenden Unternehmen betreffen. Reaktionen der nicht beteiligten Unternehmen sowie dynamische Aspekte werden in dem Modell nicht erfasst ${ }^{95}$. Trotz umfangreicher Kritik an der Operationalität des Ansatzes ist es WiLLIAMSON anhand seines Modells gelungen, auf den grundsätzlichen Zielkonflikt zwischen allokativer und technischer Effizienz aufmerksam gemacht zu haben ${ }^{96}$.

Bei Kenntnissen über Wettbewerbsbedingungen und Marktstrukturen kann das Ausmaß der nach dem Zusammenschluss erforderlichen Kostensenkung zur Übertretung des preissteigernden Marktmachteffekts ermittelt werden. In Tabelle 12 werden die Grenzkostenersparnisse exemplarisch dargestellt:

Tabelle 12: Notwendige prozentuale Kosteneinsparung bei gegebener Nachfragelastizität und Preiserhöhung

\begin{tabular}{|c|c|c|c|c|c|c|}
\hline \multirow{2}{*}{$\begin{array}{l}\text { Nachfrageelastizität } \\
\text { Marktmachtniveau } \\
\left(=P^{\text {vor }} / G K^{\text {vor }}\right)\end{array}$} & \multicolumn{2}{|c|}{2} & \multicolumn{2}{|c|}{1} & \multicolumn{2}{|c|}{0,5} \\
\hline & 1,00 & 1,05 & 1,00 & 1,05 & 1,00 & 1,05 \\
\hline Preiserhöhung & & & & & & \\
\hline 5 & 0,26 & 0,78 & 0,12 & 0,38 & 0,06 & 0,19 \\
\hline 10 & 1,05 & 2,15 & 0,5 & 1,03 & 0,25 & 0,5 \\
\hline 20 & 4,4 & 6,82 & 2,0 & 3,1 & 0,95 & 1,48 \\
\hline 30 & 10,35 & 14,28 & 4,5 & 6,21 & 2,1 & 2,9 \\
\hline
\end{tabular}

Quelle: WILLIAMSON 1969, S. 957

Die hergeleiteten Ergebnisse beruhen auf recht vereinfachten Annahmen ${ }^{97}$ und gelten nur im statischen Modellrahmen. Trotz dieser Kritik vermittelt Tabelle 12, dass die Grenzkostenersparnisse mit zunehmender Nachfragelastizität und steigenden Preiserhöhungen zunehmen müssen, um negative Gesamtwohlfahrtseffekte zu neutralisieren.

\footnotetext{
94 Vgl. Williamson 1968, S. $22 \mathrm{ff}$.

95 Williamson hat selbst auf die Schwächen seines "Trade-Off-Modells" hingewiesen. Vgl. hierzu WiLLIAMSON 1968, S. 23; WILLIAMSON 1977, S. 247

96 Vgl. CAMESASCA 2000, S. 58

97 Z.B. keine Berücksichtigung von Fixkostenersparnissen
} 
Völlig unberücksichtigt bleiben zunächst jedoch die Reaktionen unbeteiligter Unternehmen sowie dynamische Aspekte.

\subsubsection{Die Einzelmarktbeherrschung}

WILLIAMSON betrachtete bei seiner Untersuchung eine Konstellation, bei der sich auf einem Markt zwei Unternehmen zu einem Monopol zusammenschlossen. Wie später im rechtlichen Teil erläutert wird, ist ein solcher Zusammenschluss in der Realität selten anzutreffen. Vielmehr werden Oligopolstrukturen vorgefunden, wie es auch in der Zucker- und Milchindustrie der Fall ist (vgl. Kapitel 2). Bei einem Zusammenschluss entstehen Reaktionen der Insider (am Zusammenschluss beteiligt) und der Outsider (Marktteilnehmer, die nicht am Zusammenschluss beteiligt sind). Es kommt somit innerhalb eines Oligopolmodells zu Veränderungen des marktlichen Gleichgewichts (vgl. Abbildung 18). Zur Illustration wird das Cournot-Nash-Gleichgewicht vor und nach einem Zusammenschluss in einem homogenen Modell mit drei Unternehmen $(A+B$ schliessen sich zusammen, $C$ verbleibt alleine) miteinander verglichen ${ }^{98}$.

Jeder Anbieter betrachtet die Angebotsmenge der beiden Konkurrenten als gegeben und maximiert seinen Gewinn entsprechend der Restnachfragefunktion ${ }^{99}$, wobei annahmegemäß alle Unternehmen identische Kostenfunktionen aufweisen. Daraus folgt, dass die beide sich zusammenschließenden Unternehmen die gleichen Ausbringungsmengen im Gleichgewicht herstellen. Für die beiden Insider kann daher eine gemeinsame Reaktionsfunktion $\left(R_{i}^{\text {vor }}\left(x_{o}\right)\right)$ in einem zweidimensionalen Koordinatensystem unterstellt werden. Der Ousider wählt bei gegebenen (gleichen) Absatzmengen der Insider die gewinnmaximale Menge, die sich aus der Reaktionsfunktion $R_{o}\left(x_{i}\right)$ ergibt. Im Gegenzug dazu gibt die Reaktion der Insider dann an, welche (gleichen) Mengen die Insider bei gewählter Menge des Outsiders anbieten. Vor einem Zusammenschluss liegt das Marktgleichgewicht im Schnittpunkt der Reaktionskurven der Insider und des Outsiders (Punkt $C^{\text {vor }}$ ).

\footnotetext{
98 Beschreibung in Anlehnung an KINNE 2000, S. 43 ff.. SALANT/SWITZER/REYNOLDS (SSR) unterstellen bei Unternehmenszusammenschlüssen ein symmetrisches Cournot-Modell mit konstanten Grenzkosten und Mengenvariation. Die Symmetriebedingungen setzen das Vorliegen von gleich großen Unternehmen vor und nach einem Zusammenschluss voraus. Zur Erfüllung dieser Restriktion legen SSR eine komplette Einstellung der Produktion eines Fusionspartners zugrunde (Vgl. SALANT/SWITZER/REYNOLDS 1983, S. 191). PERRY UND PORTER hingegen berücksichtigen in ihrem Modell Fixkosten mit den damit verbundenen potentiellen Einsparungseffekten. Asymmetrische Unternehmensgrößen werden danach durch eine unterschiedliche Ausstattung mit dem fixen Faktor möglich (Vgl. PERRY/PORTER 1985, S. 218 f.; siehe auch die Arbeit von FARRELL/SHAPIRO 1990).

${ }^{99}$ Die Restnachfrage bzw. Residualnachfrage ergibt sich aus der Gesamtnachfrage abzüglich der Angebotsmengen der beiden anderen Anbieter.
} 


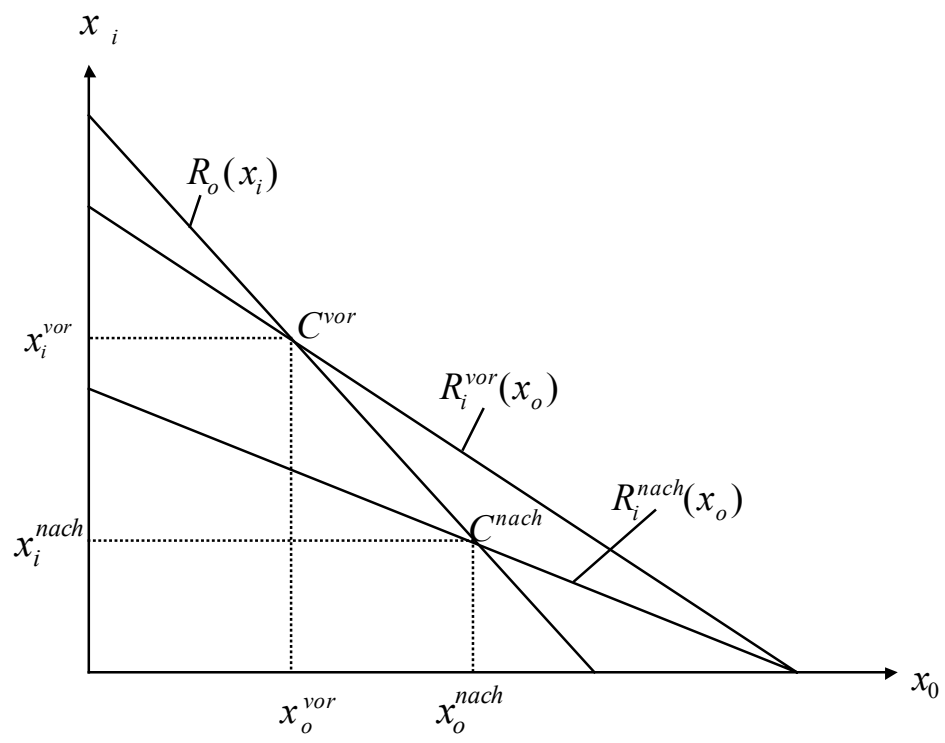

Quelle: KinNe 2000, S. 47 (angelehnt an SALANT/SWITZER/ReYNOLDS 1983, S. 188)

Nach der Fusion verändern sich Gewinnmaximierungs- sowie Reaktionsfunktion der Insider. Sie werden versuchen, ihre gesamte Ausbringungsmenge zu beschränken (Monopolverhalten), um höhere Preise am Markt durchzusetzen, wobei eine Verschiebung der Reaktionsfunktion $R_{i}^{\text {nach }}\left(x_{0}\right)$ nach innen bewirkt wird ${ }^{100}$. Die Reaktionskurve des Outsiders $\left(R_{o}\left(x_{i}\right)\right)$ ändert sich dagegen nicht, da die angebotene Menge des Outsiders weiterhin von der abgesetzten Menge der Konkurrenten abhängt und es irrelevant ist, welches Unternehmen das Gut herstellt. Der Outsider betrachtet die von den Insidern nicht mehr bediente Nachfrage als den ihm verbleibenden Teil der Nachfrage und wird entsprechend seine Produktion ausdehnen, soweit ausreichend verfügbare Produktionskapazitäten vorhanden sind. Der neue Marktgleichgewichtszustand stellt sich bei Punkt $C^{\text {nach }}$ ein. Es zeigt sich, dass nach dem Zusammenschluss die Produktionsmenge der Insider im Vergleich zum Ausgangsgleichgewicht geringer $\left(x_{i}^{\text {nach }}<x_{i}^{\text {vor }}\right)$ und die des Outsiders höher $\left(x_{0}{ }^{\text {nach }}>x_{0}{ }^{\text {vor }}\right)$ ist.

Treffen Unternehmen mehrmals aufeinander (wie es in der Realität der Fall ist), müssen strategische Erwägungen und Einschätzungen des Verhaltens der Marktteilnehmer berücksichtigt werden (dynamische Modelle). Berücksichtigen Insider die Reaktions-

${ }^{100}$ Siehe dazu formalisierte Darstellung bei WiLlig 1991, S. 295 f. sowie SALANT/SWITZER/ReYNOLDS 1983, S. $190 \mathrm{ff}$. 
vermutung des Outsiders (modelltheoretisch mit Hilfe konjekturaler Variationen), verändern sich die Reaktionskurven entsprechend. Dies führt aber zu keinen gravierend abweichenden Ergebnissen, wie MCELROY zeigen konnte ${ }^{101}$. Mengenreduktionen und damit einhergehende Preissteigerungen sind demnach auch im dynamisierten Cournot-Modell das Resultat von Unternehmenszusammenschlüssen ${ }^{102}$.

Die Mengenausdehnung kann durch den Verlauf der Reaktionskurve des Outsiders dargestellt werden, wobei die Mengenexpansion durch die Angebotselastizität (des Outsiders) bestimmt wird. Eine "flache" (elastische) Reaktionskurve des Outsiders deutet somit auf eine "aggressive" Mengenausdehnung als Reaktion auf Beschränkungen der Angebotsmenge der Insider $\mathrm{hin}^{103}$. Durch die (im dynamischen CournotModell) Berücksichtigung dieses Zusammenhanges werden die Insider dementsprechend weniger stark die Angebotsmenge reduzieren. Eine relativ "steile" (unelastische) Reaktionskurve des Outsiders impliziert hingegen, dass dieser nur mit geringen Mengenausdehnungen auf die Mengenreduktion der Insider antwortet. In diesem Fall wird es den Insidern ermöglicht, die Angebotsmenge relativ stark zu verringern, ohne einen negativen Mengeneffekt des Outsiders befürchten zu müssen.

Da der erzielbare Marktpreis von der Gesamtmenge des Marktes determiniert wird, ist der zunächst erhoffte, höher liegende Preis der Insider (durch entsprechende Mengenreduktion) aufgrund der Outsiderreaktion längerfristig nicht durchsetzbar. Das Ausmaß der Mengenänderung des Outsiders auf die Gewinnsituation der Insider wird durch Abbildung 19 veranschaulicht, wobei die Summe der Gewinne der Insider vor dem Zusammenschluss ${ }^{104}\left(G_{i}^{\text {vor }}\right)$ und ihr Gesamtgewinn nach dem Zusammenschluss ${ }^{105}$ $\left(G_{i}^{\text {nach }}\right)$ (beider Industrieunternehmen) als lineare Funktion der Ausbringungsmenge des Outsiders abgetragen wird.

\footnotetext{
${ }^{101} \mathrm{Vgl.} \mathrm{MCELROY} \mathrm{1991,} \mathrm{S.} 333 \mathrm{ff}$.

102 In den Bertrand-Modellen lassen sich analog Zusammenschlusseffekte bei Preiswettbewerb analysieren. Auch hier zeigt sich, dass der zusammmenschlussbedingte Zuwachs an Marktmacht mit Preissteigerungen verbunden ist, wobei der Preisanstieg für die In- und Outsider allerdings unterschiedlich hoch ausfallen kann. Vgl. dazu DENECKERE/DAVIDSON 1985, S. $473 \mathrm{ff}$.

103 Die aktuellen bzw. potentiellen Konkurrenten verfügen somit über eine hohe Angebotselastizität

${ }^{104}$ Vor dem Zusammenschluss = premerger
} 
Abbildung 19: Gewinne der Insider nach einem Zusammenschluss in Abhängigkeit der Reaktionen des Outsiders

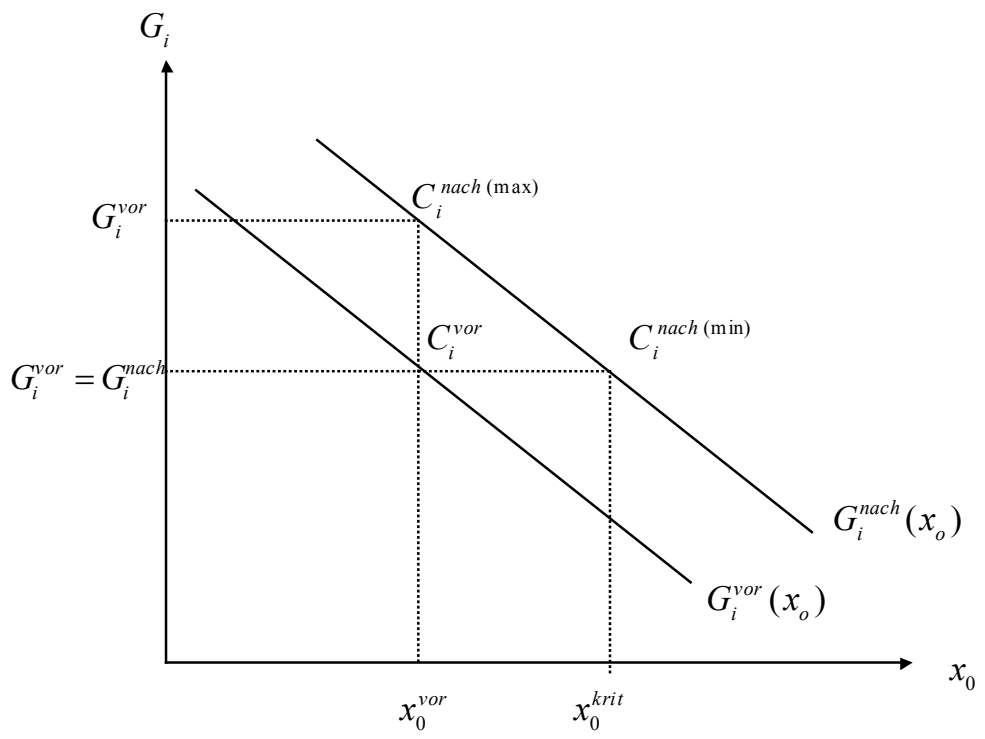

Quelle: KInNe 2000, S. 50 (angelehnt an SALANT/SWITZER/REYNOLDS 1983, S. 189)

Die postmerger Gesamtgewinne der Insider können somit aufgrund der Mengenreaktion der Outsider höher oder niedriger als die Summe der premerger Gewinne ausfallen. Die Insider werden solange höhere Gewinne realisieren können $\left(G_{i}^{\text {vor }}>G_{i}^{\text {nach }}\right)$, wie die Mengenbeschränkung (der Insider) größer als die Mengenexpansion (des Outsiders) ist. Dabei erzielen die Insider einen maximalen postmerger Gewinn bei konstanter Ausbringungsmenge des Outsiders (Punkt $C_{i}^{\text {nach(max)}}{ }^{\text {) }}$, und den gleichen Gewinn wie

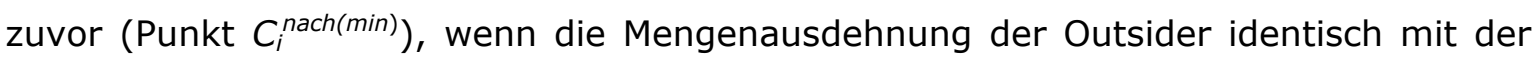
Mengenbeschränkung der Insider ist (auch als kritische Ausbringungsmenge des Outsiders bezeichnet $)^{106}$. Verluste bzw. geringere Gewinne $\left(G_{i}^{\text {nach }}<G_{i}^{\text {vor }}\right)$ resultieren bei allen Mengenausweitungen, die jenseits der kritischen Ausbringungsmenge $\left(x_{O}>x_{0}{ }^{k r i t}\right)$ liegen.

Die Mengenanpassung des Outsiders beeinflußt somit den Erfolg eines Zusammenschlusses. Solange Produktionskapazitäten unbeschränkt sind wird der Outsider seine Menge soweit ausdehnen, wie die Aussicht auf höhere Gewinne besteht. Durch die skizzierte Reaktionsverbundenheit kann die paradoxe Situation eintreten, dass der

\footnotetext{
105 Nach dem Zusammenschluss = postmerger

${ }^{106}$ Analytische Bestimmung der kritischen Ausbringungsmenge in GAUDET/SALANT 1993, S. 143
} 
Outsider stärker als die Insider von dem Zusammenlegen der Unternehmensressourcen profitiert. Bei Vorhersehbarkeit der Reaktionen (strategische Erwägungen) von Inund Outsidern kämen dann in solchen Fällen Unternehmenszusammenschlüsse gar nicht erst zustande ${ }^{107}$. Für die Wettbewerbsbeurteilung resultiert daraus, dass den nicht am Zusammenschluss beteiligten Unternehmen eine große Bedeutung bei der Erzielung marktbeherrschender Stellungen zukommen kann. Dies gilt gleichermaßen für neu in den Markt eintretende Unternehmen ${ }^{108}$.

\subsubsection{Kollektive Marktbeherrschung}

Eine Realisierung eines höheren Preises durch Einschränkung der produzierten Menge ist, wie zuvor dargestellt, abhängig von der Reaktion der übrigen Produzenten. Ist es möglich, die Verhaltensweise der Marktakteure zu koordinieren, so wird in der wettbewerbsrechtlichen Literatur von Kollusion ${ }^{109}$ gesprochen, und das Kartell wird sich dem Marktverhalten eines Monopolisten annähern, da aus Sicht der Beteiligten die Produzentenrente erhöht werden kann. Die Mitglieder eines Kartells versuchen, eine abgestimmte Strategie zu praktizieren, durch die ein höherer Gewinn als im regulären Wettbewerb erzielt werden kann ${ }^{110}$. Die am Kartell beteiligten Unternehmen können sich wie ein Monopolist verhalten und versuchen, den gemeinsamen Gewinn zu maximieren. Für ein einzelnes Unternehmen kann es rational sein, durch Unterbietung des Kollusionspreises seine Menge auszuweiten und dadurch kurzfristige Gewinnvorteile zu erzielen (Cheating). Reagieren die anderen Kollusionspartner ebenfalls mit Preissenkungen (Vergeltung), verschlechtern sich die Gewinnaussichten für jedes einzelne Unternehmen in der Gruppe, so dass weitere Anreize für Cheating entstehen und die KolIusion schließlich zerbricht ${ }^{111}$. Antizipieren die Unternehmen mögliche Vergeltungs-

107 Diese Überlegungen sind Gegenstand zahlreicher (jüngerer) theoretischer Arbeiten. SALANT/SWITZER/REYNOLDS kamen zu dem Ergebnis, dass Zusammenschlüsse im Cournot-Oligopol mit Ausnahme des Dyopols immer aufgrund der Mengenreaktion der Outsider zu Verlusten führten (SAlANT/SWitzer/ReYnolds 1983, S. 190 ff). Siehe auch Perry/PorTer 1985; GAudet/SAlent 1993. Letztere gingen sogar soweit, dass sie ein sogenanntes „Profitabilitätskriterium" herleiteten. Danach erhöhte ein Zusammenschluss nur dann die postmerger Gewinne der Insider, wenn die Anzahl der am Zusammenschluss beteiligten Unternehmen die Anzahl der Outsider um mehr als ein Unternehmen überstieg.

${ }^{108}$ In Anlehnung an KINNE 2000, S. 50

${ }^{109}$ Kollusion = Absprache, Kartell, aufeinander abgestimmte Verhaltensweisen. Unter Kollusion wird wettbewerbsbeschränkendes Verhalten zur Erzielung kollektiver Vorteile verstanden. Vgl. KANTZENBACH/KRUSE 1989, S. 26. Dabei wird zwischen expliziter Kollusion, die ausdrückliche Vereinbarungen zwischen Unternehmen beinhaltet, und impliziter Kollusion, die keine bewusste Vereinbarung erfordert, unterschieden. Da die Unterscheidung eher für die wettbewerbspolitische Praxis relevant ist, wird im folgenden nicht weiter zwischen den beiden Kollusionsformen differenziert.

110 Vgl. NeUmann 2000, S. 78

${ }^{111}$ Vgl. dazu Cheating-Anreize und Vergeltungsmaßnahmen, KRUSE 1994, S. 5 ff. 
maßnahmen, kann es aus Unternehmersicht jedoch rational sein, auf kurzfristige positive Effekte zu verzichten, um die längerfristigen Vorteile der Kollusion realisieren zu können. Die Stabilität einer Kollusionslösung wird somit von der Wirkungskette zwischen Kollusionsvereinbarungen, Cheating, Aufdecken des Cheating und Bestrafung des Cheating bestimmt ${ }^{112}$.

Die Kontrollbildung wird daher häufig aus Interessendivergenz entstehender Rivalitäten erschwert. Unternehmenszusammenschlüsse verringern die Anbieterzahl im Markt und zugleich die Anzahl der in Frage kommenden Kollusionspartner. Dadurch sinken die Transaktionskosten der Kollusionsbildung. Bei oligopolistischen Marktstrukturen führt ein Zusammenschluss dazu, dass sich die Reaktionsverbundenheit der Oligopolisten untereinander erhöht. Die Spürbarkeit von Nachfrageverschiebungen steigt und zugleich wachsen die Aufdeckungs- und Sanktionsmöglichkeiten von Cheating. Dies verringert die Cheating-Anreize für das einzelne Unternehmen. Damit verstärken Zusammenschlüsse zwischen Unternehmen c.p. die Kollusionsanreize und reduzieren zugleich die Gefahr des Cheating. Die Kollusionsvorteile durch Zusammenschlüsse sind dabei um so ausgeprägter, je kleiner die Anbieterzahl vor einem Zusammenschluss ist $^{113}$.

Zusammenschlüsse können zudem auf Bestimmungsfaktoren wie Kostenstrukturen, Produktheterogenität, Markttransparenz etc. Einfluss ausüben. Allgemein gilt: Legen sich Unternehmen durch Zusammenschlüsse stärker auf einen Markt fest (irreversible Investitionen hoch) oder treffen Unternehmen mit einem ähnlichen Produktsortiment (homogene Güter) aufeinander, steigt die Kollusionswahrscheinlichkeit ${ }^{114}$.

\subsubsection{Weitere Modelle}

Darüber hinaus gibt es noch eine Vielzahl anderer Modelle, die sich mit der Wohlfahrtsauswirkung von Fusionen beschäftigen. LEVIN benutzte beispielsweise ein Modell, in dem die Unternehmen in einem Markt mit homogenen Gütern nicht den Preis, sondern die Menge als Variable nutzen ${ }^{115}$. Als Ergebnis lässt sich festhalten, dass Fusionen mit einem Marktanteil von unter $50 \%$ stets wohlfahrtssteigernd sind - unabhängig

\footnotetext{
112 Zu den Einflussfaktoren stabiler Kollusionen siehe die Wirkungskette in KANTZENBACH/KOTTMANN/KRÜGER 1996 , s. 36 ff.

${ }^{113}$ In Anlehnung an KINNE 2000, S. 52; vgl. auch MARTIN 2001, S. 47 ff.

${ }^{114}$ Vgl. KinNe 2000, S. 53

${ }^{115}$ Vgl. LeVIN 1990, S. $1238 \mathrm{ff}$.
} 
von einer Ausdehnung oder Reduktion der Produktionsmenge ${ }^{116}$. WERDEN und FrOEB (1998) untersuchten den Effekt von Marktzutrittsschranken. Geringere Markteintrittsbarrieren reduzieren die Möglichkeit der Preisanhebung und die damit einhergehenden Gewinnmöglichkeiten der fusionierenden Unternehmen. Um mögliche neueintretende Konkurrenten abzuschrecken, werden diese Unternehmen unter Umständen sogar den Preis nach Zusammenschlüssen reduzieren ${ }^{117}$.

Nicht alle Modelle können in dieser Studie wiedergegeben werden. Einige wichtige und aktuelle Arbeiten werden abschließend in Tabelle 13 zusammengefasst. Die Ergebnisse der Modelle können z.T. erheblich aufgrund der Modelltypen varieren. So ist zwischen endogenen und exogenen, Cournot und Bertrand, statischen und dynamischen, homogenen und heterogenen, kooperativen und nicht kooperativen Modellen zu unterscheiden, sowie die Berücksichtigung von Fixkosten und evtl. unterschiedlicher Grenzkosten mit heranzuziehen. Unter Bestimmung des Modellrahmens kann dann die "best response function" der Marktteilnehmer bestimmt werden. Der Endzustand spiegelt sich dann im Nash-Equilibrium wider, bei dem kein Teilnehmer einen Anreiz sieht, seine Situation zu verändern.

Tabelle 13: Zusammenstellung wichtiger Oligopolmodelle

\begin{tabular}{|c|c|c|}
\hline Autor (Jahr) & Eigenschaften & Hauptaussagen \\
\hline WILLIAMSON (1968) & $\begin{array}{l}\text { Marktmacht versus Effizienzvor- } \\
\text { teile, statisch }\end{array}$ & $\begin{array}{l}\text { Trotz Preissteigerung kann Fusion wohlfahrts- } \\
\text { erhöhend sein }\end{array}$ \\
\hline $\begin{array}{l}\text { SALANT/SWITZER/ } \\
\text { REYNOLDS (1983) }\end{array}$ & $\begin{array}{l}\text { Statisch, Cournot-Modell, linea- } \\
\text { re Nachfrage- und Kostenfunkti- } \\
\text { on, exogen }\end{array}$ & $\begin{array}{l}\text { Unter } 80 \% \text { Marktanteil (beteiligte Unternehmen) } \\
\text { nicht profitabel }\end{array}$ \\
\hline $\begin{array}{l}\text { DENECKERE/ } \\
\text { DAVIDSON (1985) }\end{array}$ & $\begin{array}{l}\text { Exogen, differentierte Produkte, } \\
\text { Preissetzer (Bertrand) }\end{array}$ & $\begin{array}{l}\text { Nur profitabel, wenn Kostenreduktion möglich, an- } \\
\text { sonsten Vergeltung durch Outsider }\end{array}$ \\
\hline $\begin{array}{l}\text { PERRY/PORTER } \\
(1985)\end{array}$ & $\begin{array}{l}\text { EXogen, baut auf DENECKE/ } \\
\text { DAVIDSON auf }\end{array}$ & $\begin{array}{l}\text { Fusionierende Unternehmen beschränken aktiv die } \\
\text { Rentabilität der Outsider }\end{array}$ \\
\hline $\begin{array}{l}\text { FARREL/SHAPIRO } \\
(1990)\end{array}$ & $\begin{array}{l}\text { Exogen, Cournot-Modell, ho- } \\
\text { mogen }\end{array}$ & $\begin{array}{l}\text { Positive Wohlfahrtseffekte (trotz Preissteigerung), } \\
\text { sobald kombinierte Marktanteile vor Fusion < als } \\
\text { der gewichtete Durchschnittsmarktanteil; alle Fu- } \\
\text { sionen, die mit dem größten (oder zu dem größ- } \\
\text { ten) Unternehmen stattfinden, führen zu Preis- } \\
\text { steigerungen (falls keine Synergieeffekte) = } \\
\text { wohlfahrtsmindernd }\end{array}$ \\
\hline $\begin{array}{l}\text { KAMIEN/ZANG } \\
(1990)\end{array}$ & Endogen, homogen, dynamisch & $\begin{array}{l}\text { Monopoleffekt kann nur ex ante durch geringe An- } \\
\text { zahl von Marktteilnehmern verhindert werden }\end{array}$ \\
\hline LEVIN (1990) & $\begin{array}{l}\text { Konstante aber möglicherweise } \\
\text { unterschiedliche Grenzkosten, } \\
\text { statisch }\end{array}$ & $\begin{array}{l}\text { Jede (profitable) Fusion mit kombiniertem Marktan- } \\
\text { teil < } 50 \% \text { ist wohlfahrtsfördernd; Fusionen mit } \\
\text { größtem Unternehmen (oder die zu größtem U. } \\
\text { führen) sind wohlfahrtsmindernd }\end{array}$ \\
\hline
\end{tabular}

\footnotetext{
${ }^{116}$ Vgl. RöLLER/StEnNEK/VERBoven 2000, S. 34
}

${ }^{117}$ Vgl. WeRden/Froeb 1998, S. 525 
Tabelle 13 (Fortsetzung)

\begin{tabular}{|l|l|l|}
\hline Autor (Jahr) & Eigenschaften & Hauptaussagen \\
\hline $\begin{array}{l}\text { GAUDET/SALANT } \\
(1992)\end{array}$ & $\begin{array}{l}\text { Endogen, untersuchen generelle } \\
\text { Konditionen }\end{array}$ & Allgemein: nur profitable Fusionen finden statt \\
\hline $\begin{array}{l}\text { MCAFEE/WILLIAMSON } \\
(1992)\end{array}$ & Statisch, Cournot-Nash & $\begin{array}{l}\text { Fusionen, die zu einer symmetrischen Marktstruktur } \\
\text { führen, sind wohlfahrtsfördernd }\end{array}$ \\
\hline BAIK (1995) & $\begin{array}{l}\text { Statisch (2 Stufen): 1. Wahl der } \\
\text { Outputmenge, 2. Preiswettbe- } \\
\text { werb }\end{array}$ & $\begin{array}{l}\text { Fusionen können zu Preissenkungen führen (wohl- } \\
\text { fahrtssteigernd) }\end{array}$ \\
\hline $\begin{array}{l}\text { POLASKY/MASON } \\
(1998)\end{array}$ & $\begin{array}{l}\text { 2-Perioden Modell, Unterschei- } \\
\text { dung kurz- und langfristiger } \\
\text { Effekte, homogen }\end{array}$ & $\begin{array}{l}\text { Langfristig fast alle Zusammenschlüsse wohlfahrts- } \\
\text { fördernd - kurzfristig nicht unbedingt: hängt von } \\
\text { Möglichkeit der Outsider zur Mengenexpansion ab: } \\
\text { Marktschranken wichtig }\end{array}$ \\
\hline $\begin{array}{l}\text { GOWRISANKARAN } \\
(1999)\end{array}$ & $\begin{array}{l}\text { Endogen, dyanmisch, Investiti- } \\
\text { onskosten, ex ante Entschei- } \\
\text { dung vor Mengenwettbewerb }\end{array}$ & $\begin{array}{l}\text { Markov-Perfect Nash Equilibrium, zunehmende } \\
\text { Markteintrittskosten führen zu steigender Konzentra- } \\
\text { tion }\end{array}$ \\
\hline $\begin{array}{l}\text { DOCKNER/ } \\
\text { GAUNERSDORFER } \\
\text { (2001) }\end{array}$ & $\begin{array}{l}\text { Dynamisches Cournot-Modell, } \\
\text { Markov-Strategie }\end{array}$ & $\begin{array}{l}\text { Fusionen sind immer profitabel, Synergien sind nicht } \\
\text { erforderlich }\end{array}$ \\
\hline RODRIGUES (2001) & $\begin{array}{l}\text { Endogen, 2-Perioden, Festkos- } \\
\text { ten, (1) begrenzte Nummer von } \\
\text { Marktteilnehmern, (2) voraus- } \\
\text { sichtlicher Markteintritt anderer } \\
\text { Unternehmen, (3) Fixkostende- } \\
\text { gression }\end{array}$ & $\begin{array}{l}\text { (1): Anreiz zu fusionieren sinkt, somit sinkt auch } \\
\text { Konzentration im Marktgleichgewicht } \\
\text { (2)+(3): beides steigt } \\
\text { endogen generierte Fusionen sind wohlfahrtsstei- } \\
\text { gernd, solange die Wettbewerbsintensität zuvor } \\
\text { gering war oder ex ante hoch konzentriert ist. }\end{array}$ \\
\hline
\end{tabular}

Quelle: eigene Zusammenstellung

\subsubsection{Erkenntnisse industrieökonomischer Fusionsmodelle}

Zunächst ist aus wettbewerbspolitischer Sicht das Phänomen der Marktmacht ( $p>G K$ ) negativ zu bewerten. Verschiedene Oligopolmodelle demonstrieren, welche Faktoren das Entstehen von Marktmacht begünstigen ${ }^{118}$ :

1. eine geringe Anzahl von Unternehmen in einem Markt (hohe Marktanteile und Marktkonzentration);

2. Produktdifferenzierung: wenn die Güter unterschiedlich homogen sind, schwenkt ein Konsument erst bei größeren Preisunterschieden auf ein anderes Produkt um;

3. Kapazitätsbeschränkungen: wenn die fusionierenden Unternehmen ihre Menge einschränken und somit der Preis ansteigt, müssen die verbleibenden Unter-

118 Vgl. StenneK 2002, S. 7; MottA 2000, S. 7 ff. ; MARTin 2001, S. 47 
nehmen die Fähigkeit besitzen, die entsprechende Differenz produzieren zu können;

4. Information des Verbrauchers: nur wenn der Verbraucher über Preise und Konkurrenten informiert ist, kann er einen niedrigeren Preis eines Herstellers bei einem vergleichbaren Produkt wahrnehmen. Falls dies nicht der Fall ist, besteht kein Anreiz für die Outsider den Preis zu unterbieten;

5. Switching costs (Kosten der Umstellung) für Abnehmer: müssen gering sein, damit kein monopolistischer Spielraum entsteht;

6. Kartellverhalten;

7. gegengewichtige Marktmacht.

Bei der Analyse von Fusionen muss festgehalten werden, dass eine Fusion positive wie auch negative Effekte hervorrufen kann ${ }^{119}$. Positiv sind die Effekte einzuordnen, die den Unternehmen erlauben, Synergieeffekte zu realisieren, welche sich in niedrigeren Produktionskosten (Einsparung von Ressourcen) niederschlagen. Negative Effekte beruhen auf einer Verringerung der Wettbewerbsfähigkeit, was zu ökonomischen Ineffizienzen führen kann (dead-weight-loss) oder eine Umschichtung der Konsumentenzur Produzentenrente fördert ${ }^{120}$.

Bei den negativen Effekten ist besonders zwischen Koordinationseffekten (Kollusion) und der Verringerung des Wettbewerbs (internalisation oder unilateral effect) zu unterscheiden. Die Koordinationseffekte (Kartell) führen zu einem monopolähnlichen Verhalten der Unternehmen. Mit Verringerung der Anzahl der Marktteilnehmer steigt die Gefahr einer Kartellbildung. Dies hängt neben der Teilnehmeranzahl auch von Faktoren wie Transparenz des Marktes, Gewinn- und Vergeltungsfaktoren ${ }^{121}$, Diskontierungsfaktor (zukünftiger Gewinne) oder der Symmetrie des Marktes ab ${ }^{122}$. Der unilaterale Effekt besteht in der mehrfach gezeigten Tendenz eines Monopolisten den Preis dem Grenzerlös gleichzusetzen, wodurch die Nachfrage verringert und ein höherer Preis realisiert wird, als es in der Wettbewerbssituation der Fall wäre. Eine Preiserhöhung hat jedoch nicht nur Einfluss auf den Gewinn der an der Fusion beteiligten Un-

\footnotetext{
${ }^{119}$ Vgl. STenNeK 2002, S. 28; auch Buccirossi et al. (2002), S. 375

${ }^{120} \mathrm{Vgl}$. STENNEK 2002, S. 28

${ }^{121}$ Vgl. MOTTA 2000, S. 15

122 Unterschiedliche Grenzkosten spiegeln sich bei sonst gleichen Bedingungen in der Größe des Marktanteils wider.
} 
ternehmen (Insider) sondern ebenso auf den der Outsider ${ }^{123}$. Eine Betrachtung der Wettbewerbsbedingungen für die verbleibenden Unternehmen ist somit unabdingbar.

Die Kartellbehörden sollten daher als Schlussfolgerung zu den Erkenntnissen aus den industrieökonomischen Modellen Fusionen auf zweierlei Eigenschaften prüfen: a) Erzielung einer dominanten Position und b) Reduzierung des Wettbewerbs (auch Fusionen, die keine dominante Stellung einnehmen, können wettbewerbsfeindlich sein, sofern keine Synergievorteile vorliegen ${ }^{124}$ ). Außerdem müssten aus wohlfahrtsökonomischer Betrachtung Effizienzvorteile (positive Effekte) ermittelt und mit den negativen Auswirkungen saldiert werden (vgl. auch Kapitel 7.4.2).

\section{2 Überprüfung mit Hilfe empirischer Studien}

\subsubsection{Methodik der empirischen Untersuchungen}

BAUMOLS These, dass größere Unternehmen eine höhere Rentabilität erzielen als kleinere ${ }^{125}$, hat eine Vielzahl von empirischen Untersuchungen ausgelöst, die sich mit der Frage nach dem Zusammenhang zwischen Rentabilität und Unternehmensgröße (bzw. Unternehmenswachstum) auseinandergesetzt haben. Die industrieökonomische Forschung kam allerdings zu keinem eindeutigen Ergebnis: Das Spektrum der erarbeiteten empirischen Ergebnisse reicht von einer positiven über eine negative Korrelation bis hin zu dem Resultat, dass überhaupt kein Zusammenhang zwischen den beiden Variablen existiert ${ }^{126}$.

Damit Ergebnisse überhaupt vergleichbar sind, ist eine Kenntnis über die zugrundegelegten Rahmenbedingungen und Methoden erforderlich. Da auch im empirischen Teil dieser Studie verschiedene Analysemethoden gewählt werden, sollen im folgenden mögliche Forschungsvorgangsweisen und dazugehörige Ergebnisse vorgestellt werden.

Für Unternehmen aus dem deutschen Raum liegen nur wenige empirische Untersuchungen zum Erfolg von Unternehmenszusammenschlüssen vor. Der geschilderte Literaturüberblick über Erfolgsanalysen bezieht sich deshalb in erster Linie auf Ergebnisse

\footnotetext{
${ }^{123}$ Vgl. MOTTA 2000, S. 5

${ }^{124}$ Vgl. MotTA 2000, S. 23 (Beispiel)

${ }^{125} \mathrm{Vgl}$. BAUMOL 1967 , S. $42 \mathrm{ff}$.

${ }^{126}$ Vgl. SCHNeider 1993, S. 12 ff.; IRSCH 1988, S. 519ff.; AlbaCh/BOCK/WARNKE 1985, S. 33 ff; BöBel 1984, S. $56 \mathrm{ff}$.
} 
von Arbeiten aus dem internationalem Bereich, primär auf Analysen über Zusammenschlüsse in den Vereinigten Staaten. Die Ansätze lassen sich wie folgt unterteilen:

- Event-Studien oder sog. kapitalmarktorientierte Untersuchungen, die sich auf die Reaktion des Kapitalmarktes (Aktienmarktes) auf Unternehmenszusammenschlüsse beziehen;

- jahresabschlussorientierte Analysen, die sich auf die Änderungen der durch Rechnungslegung ermittelbaren Situationen der Unternehmen beziehen;

- Erfolgsuntersuchungen durch Befragungen, die die unternehmensinterne Beurteilung eines Zusammenschlusses analysieren und somit auch Wirkungen, die durch extern verfügbare Daten nicht erkennbar sind, einbeziehen.

\subsubsection{Kapitalmarktorientierte Untersuchungen}

Bei den kapitalmarktorientierten Explorationen wird der Versuch unternommen, Vermögensänderungen der Aktionäre bzw. Anteilseigner bei dem erwerbenden und dem erworbenen Unternehmen zu erfassen und zu interpretieren. Aus den Untersuchungsergebnissen lassen sich Erkenntnisse über den Verlauf von Aktienkursen vor und nach dem Zusammenschluss ableiten; Aktienkurse reflektieren somit die Erwartungen und Erfolge von Unternehmenszusammenschlüssen am Kapitalmarkt. Diese Methode kann dementsprechend für Unternehmen herangezogen werden, die an einer Börse notiert sind, und somit eine akquisitionsbedingte Wertermittlung der Aktien zu beobachten ist ${ }^{127}$. Dieser Ansatz soll hier jedoch nicht näher herangezogen werden, da es sich bei den zu untersuchenden Unternehmen der Milch- und Zuckerbranche nicht ausschließlich um börsennotierte Unternehmen handelt und deshalb Schlussfolgerungen nur begrenzt aussagefähig wären. Der interessierte Leser sei jedoch auf die angeführte Literatur verwiesen ${ }^{128}$. Allerdings ist die Aussagefähigkeit der Event-Studien über Wettbewerbsauswirkungen von Fusionen umstritten ${ }^{129}$.

\subsubsection{Jahresabschlussorientierte Untersuchungen}

Jahresabschlussorientierte Analysen gehen im Gegensatz zu kapitalmarktorientierten Analysen die Wirkungen von Zusammenschlüssen auf der Unternehmensebene nach. Grundlage dafür sind extern verfügbare Daten, die der Rechnungslegung oder sonsti-

\footnotetext{
127 Vgl. GERPOTt 1993, S. 197-206

${ }^{128}$ Insbesondere BÜHNER 1990 liefert eine Überblick über verschiedene Ansätze von event studies und deren empirische Überprüfung

${ }^{129} \mathrm{Vgl.} \mathrm{MCAfFEe/WiLLiAMS} \mathrm{1988;} \mathrm{FridolfSSON/StENNEK} 1999$
} 
gen öffentlichen Publikationen des Geschäftsverlaufs entnommen werden (zur Veröffentlichungspflicht s. Anhang F). Jahresabschlussorientierte Untersuchungen beziehen sich ausschließlich auf übernehmende Unternehmen, welche die akquirierten nach einem Zusammenschluss eingegliedert haben ${ }^{130}$.

\subsubsection{Stichprobenbildung}

Bei den jahresabschlussorientierten Untersuchungen der Wirkung von Unternehmenszusammenschlüssen können zwei Arten der Stichprobenbildung unterschieden werden:

- fusionsbezogene Stichproben,

- unternehmensbezogene Stichproben.

Fusionsbezogene Stichproben beziehen sich direkt auf Unternehmenszusammenschlüsse und berücksichtigen den Zeitpunkt ihrer Durchführung. Die ausgewählten Unternehmen werden dann unter konkreter Bezugnahme auf die ausgewählten Zusammenschlüsse analysiert. Beispiele für fusionsbezogene Stichproben finden sich bei DEWING (1922) und KUMAR (1981). DEWING untersuchte diversifizierte Konzerne, die sich in dem von ihm untersuchten Zeitraum zusammengeschlossen hatten. Er verglich die Gewinne, die das zusammengeschlossene Unternehmen nach der Fusion erzielte, mit den Gewinnen der einzelnen Unternehmen vor der Fusion. KUMAR untersuchte die Bruttoinvestitionen übernehmender Unternehmen vor und nach einem Zusammenschluss.

Unternehmensbezogene Stichproben lassen die Zeitpunkte der Fusion unberücksichtigt. Zusammenschlüsse werden lediglich für die Auswahl der Stichprobenunternehmen herangezogen. Die Analysen beziehen sich dann auf die ausgewählten Unternehmen und nicht mehr direkt auf deren Zusammenschlüsse. Für die Bildung unternehmensbezogener Stichproben werden unterschiedliche Kriterien herangezogen. So untersuchte beispielsweise HOPKINS (1987) nur fusionsaktive Unternehmen. Darunter versteht er solche, die im ausgewählten Zeitraum mehr als zwei Zusammenschlüsse durchgeführt hatten. KELLY (1967) bildete seine Stichprobe aus Unternehmen, deren durch Zusammenschlüsse bedingtes Umsatzwachstum in einem festgelegenen Zeit-

${ }^{130}$ Vgl. BÜHNER 1990, S. 84 
raum mehr als 20 v.H. betrug. GIMPEL-ISKE (1973) analysierte Unternehmen, die mindestens ein anderes übernommen hatten ${ }^{131}$.

\subsubsection{Analysemethoden}

Bei den jahresabschlussorientierten Studien der Wirkung von Unternehmenszusammenschlüssen können zwei grundsätzliche Analysemethoden unterschieden werden:

- Vorher-nachher-Analysen,

- komparative Objektanalysen.

Mit Vorher-nachher-Analysen werden die durch Unternehmenszusammenschlüsse ausgelösten Veränderungen der ausgewählten Untersuchungsvariablen analysiert. Die Länge der Untersuchungszeiträume und deren Lage zum Zeitpunkt des Zusammenschlusses sind dabei sehr unterschiedlich. So analysierte zum Beispiel Kelly (1967) die Marktwert- und Rentabilitätsentwicklung akquirierender Unternehmen anhand einer Zeitreihe, die sich von fünf Jahren vor bis fünf Jahre nach dem Fusionszeitpunkt erstreckte. KUMAR (1981) verglich die Bruttoinvestitionen der Käuferunternehmen in den acht Jahren nach der Fusion mit dem Wert im Jahr vor der Fusion.

Bei komparativen Objektanalysen wird der Erfolg von Unternehmenszusammenschlüssen durch eine Gegenüberstellung der fusionierenden Unternehmen mit Kontrollgruppen von Unternehmen, Branchendurchschnittswerten oder Durchschnittswerten des gesamten industriellen Sektors beurteilt.

Bei der Kontrollgruppenbildung werden zumeist Unternehmen herangezogen, die keine Fusion durchgeführt haben. Dabei bestehen unterschiedliche Anforderungen an die Vergleichbarkeit. LEV und MANDELKEN (1972) untersuchten beispielsweise Unternehmen, die gleiche Bilanzsummen und Tätigkeitsbereiche wie die Stichprobeunternehmen aufwiesen. KeLLY (1967) stellte seinen Stichprobeunternehmen, die durch Fusion ein Umsatzwachstum von mehr als 20 Prozent erzielten, solche Kontrollgruppen gegenüber, deren Wachstum weniger als fünf Prozent betrug. Seine Kontrollgruppen konnten somit durchaus auch Unternehmen enthalten, die ihrerseits (kleinere) Zusammenschlüsse durchgeführt hatten.

Bei der Verwendung von Branchendurchschnittswerten als Vergleichobjekt werden die zu analysierenden Unternehmen zunächst ihren Haupttätigkeitsbereichen zugeordnet

${ }^{131}$ In Anlehnung an BÜHNER 1990, S. 84 ff. 
(z.B. STICH 1974). Dann werden Durchschnittswerte der gesamten Branche mit denen der Stichprobeunternehmen verglichen.

Die letzte Möglichkeit komparativer Objektanalysen besteht darin, die Ergebnisse der Stichprobeunternehmen mit Durchschnittswerten des gesamten industriellen Sektors zu vergleichen (z.B. STICH 1974).

\subsubsection{Ergebnisse jahresabschlussorientierter Untersuchungen}

Jahresabschlussorientierte Explorationen analysieren den Erfolg von Unternehmenszusammenschlüssen anhand unterschiedlichster Variablen.

Folgende jahresabschlussorientierte Variablen werden im allgemeinen zur Beurteilung des Erfolges von Unternehmenszusammenschlüssen herangezogen:

- Größenmaße: Umsatz, Vermögen, Kapital, Gewinn

- Rentabilitätsmaße: Eigen-, Fremd-, Gesamtkapitalrentabilität

- Aktionärsbezogene Maße: Börsenkurs, Dividende, Auszahlungsraten

Dabei werden Absolutwerte, Wachstums- und Änderungsraten entweder als Zeitreihe vor und nach einem Zusammenschluss oder objektbezogen komparativ verglichen. Die sich daraus ergebende Fülle unterschiedlicher Untersuchungsmaße verhindern detaillierte Ergebnisvergleiche.

Tabelle 14: Ergebnisse einzelner jahresabschlussorientierter Studien

\begin{tabular}{|l|l|c|}
\hline Autor (Jahr) & Untersuchungsgegenstand & $\begin{array}{l}\text { Situation nach } \\
\text { Fusion }\end{array}$ \\
\hline DEWING (1922) & Gewinnentwicklung & - \\
\hline NATIONAL INDUSTRIAL CONFERENCE BOARD (1929) & Gewinnentwicklung & $+/-$ \\
\hline LIVERMORE (1935) & Langfristige Gewinnentwicklung & $+/-$ \\
\hline KELLY (1967) & Marktwert u. Rendite & - \\
\hline REID (1968) & Größenwachstum/Erträge & - \\
\hline HOGARTY (1970) & Eigenkapitalrentabilität & + \\
\hline Lorie/Halpern (1970) & Rendite & - \\
\hline Ansoff/Brandenburger/Portner/Radosevich (1971) & 21 Maßgrößen & - \\
\hline Singh (1971) & Rentabilität & - \\
\hline REID (1971) & Rentabilität & $+/-$ \\
\hline WeStON/MANSINGHKA (1971) & Wachstum & \\
\hline
\end{tabular}


Tabelle 14 (Fortsetzung)

\begin{tabular}{|c|c|c|}
\hline Autor (Jahr) & Untersuchungsgegenstand & $\begin{array}{l}\text { Situation nach } \\
\text { Fusion }\end{array}$ \\
\hline BHASKAR (1972) & Gewinne & - \\
\hline KURANDT (1972) & 8 Kennziffern & $+/-$ \\
\hline LEV/MANDELKER (1972) & 7 Erfolgsvariablen & - \\
\hline RYDEN (1972) & Umsatz/Aktienpreis & $+/-$ \\
\hline GIMPEL-ISKE (1973) & Beteiligungsbuchwerte/Umsätze & + \\
\hline STICH (1974) & 11 Erfolgsvariablen & $+/-$ \\
\hline UTTON (1974) & Sachanlagevermögen & $+/-$ \\
\hline KUMPS (1975) & Gesamtkapitalrentabilität & $+/-$ \\
\hline MEEKS (1977) & Gesamtkapitalrentabilität & $+/-$ \\
\hline RYDEN/EDBERG (1980) & Gewinn & - \\
\hline CABLE/PALFREY/RUNGE (1980) & Rendite & + \\
\hline KUMAR (1981) & Bruttoinvestitionen & + \\
\hline MUELLER (1983) & Marktanteile & - \\
\hline MUELLER (1986) & Gesamtkapitalrentabilität & $+/-$ \\
\hline HOPKINS (1987) & Marktposition & + \\
\hline RAVENSCRAFT/SCHERER (1987) & Gesamtkapitalrentabilität & - \\
\hline COLEY/REINTON (1988) & Erfolg/Produktprogramm & $+/-$ \\
\hline BALDWIN/GORECKI (1990) & Marktanteile & $+/-$ \\
\hline HeALY (1992) & Jahresüberschuss & + \\
\hline STEWERT/KIM (1993) & Preisentwicklung & $+/-$ \\
\hline COSH/HUGHES (1995) & Rentabilität & - \\
\hline KLEINERT/KLODT (2000) & Rentabilität & $+/-$ \\
\hline
\end{tabular}

Quelle: eigene Darstellung, z.T. aus BüHNER 1990, S. 89 übernommen

Tabelle 14 gibt einen generellen Überblick von jahresabschlussorientierten Untersuchungen und fasst die Auswirkungen auf Erfolgsvariablen von Unternehmenszusammenschlüssen zusammen. Auffallend ist, dass es zu keinen einheitlichen Aussagen der Ergebnisse kommt.

Der Darstellung zufolge finden nur wenige Studien positive Wirkungen von Unternehmenszusammenschlüssen auf die Profitabilität. Healy et al. (1992) fanden eine Erhöhung des Jahresüberschusses nach Fusionen für eine Stichprobe mit den 50 größten US-amerikanischen Fusionen von 1979 bis 1984. Besonders umfangreich und aufwendig war die Untersuchung von RAVENSCRAFT und SCHERER (1987), die 5966 Fusionen auf deren Profitabilitätswirkungen auf Ebene der Produktlinie überprüften. Sie decken einen negativen Einfluss von Fusionen auf die Profitabilität auf. Zum gleichen Ergebnis 
kamen auch MeEks (1977) sowie COSH und Hughes (1995) in umfangreichen Untersuchungen für das Vereinigte Königreich.

Die Analysen zeigen ein eher gemischtes Bild der Wirkungen von Unternehmensübernahmen. Die Ursache kann auch darin liegen, dass sich Umfang, Zeiträume, Raum, Rahmenbedingungen aber auch Untersuchungsgegenstand voneinander unterscheiden. Eine detaillierte Beschreibung aller aufgelisteten Untersuchungen würde den Rahmen dieser Studie sprengen. Wichtige Ergebnisse der jüngsten Untersuchungen im Rahmen der jahresabschlussorientierten Analysen sollen jedoch kurz vorgestellt werden.

Smith und Hershman (1997) ${ }^{132}$ nutzten die als Datensatz in der Zeitschrift "Mergers and Acquisitions" bekannt gegebenen Übernahmen und fanden in ihrer Untersuchung von Fusionen der neunziger Jahre eine Erfolgsquote von 52 v.H. gemessen an der Profitabilität gegenüber dem Branchendurchschnitt drei Jahre nach dem Zusammenschluss $^{133}$. Damit schnitten die Fusionen der neunziger etwas besser ab als ihre Vorgänger in den 80er Jahren (37 v.H.). Von entscheidender Bedeutung war die Größe der fusionierenden Unternehmen. Standen die Chancen einer erfolgreichen Übernahme bei einer Fusion mit einem kleineren oder mittleren Partner $(<30 \mathrm{v} . \mathrm{H}$. der eigenen Größe) noch 50:50, so sanken sie bei einem größeren Partner auf 25 v.H. Drei Viertel dieser Fusionen in den neunziger Jahren waren nicht erfolgreich! Die Höhe der Prämienzahlungen oder die strategische Ausrichtung waren dagegen dieser Studie zufolge für die Profitabilität des Unternehmens nach der Fusion nicht ausschlaggebend. Entscheidende Bedeutung kommt der Zeit nach dem Zusammenschluss zu. In über der Hälfte der Fälle ist die Nutzung von Synergien während des Zusammenschlusses laut SMITH und HERSHMAN (1997) einfach vergessen worden, da sich die beteiligten Unternehmen allzu sehr auf Rationalisierung konzentrierten und das Zusammenführen unterschiedlich strukturierter Organisationen knappe Managementressourcen band ${ }^{134}$.

KLEINERT und KLODT (2000) überprüften 103 von der EU-Kommission zwischen 1991 bis 1996 untersuchte Fälle (vgl. Tabelle 15). Die Rentabilität der Übernahmen wurde mittels Vergleich der Produktivität des übernehmenden Unternehmens, gemessen als Gewinn aus operativem Geschäft dividiert durch das eingesetzte Kapital, vor und nach dem Unternehmenszusammenschluss (jeweils gewichtet nach dem Branchendurchschnitt) ermittelt.

\footnotetext{
132 Vgl.: www.mercermc.com/news/mercerandhoredart.html

${ }^{133} \mathrm{Vgl.} \mathrm{KLEINERT/KLODT} \mathrm{2000,} \mathrm{S.} 65$

${ }^{134}$ In Anlehnung an KLEINERT/KLODT 2000, S. 64 ff.
} 
Die Profitabilität von 62 der 103 Unternehmen in dieser Stichprobe erhöhte sich infolge einer Fusion, während die übrigen 41 Unternehmen negative Auswirkungen erlitten. Erfolg wird dabei als Veränderung des Ergebnisses gegenüber dem Branchendurchschnitt gemessen. Überraschend ist, dass sich die übernehmenden Unternehmen in den meisten Fällen nicht als überdurchschnittlich rentabel vor der Fusion herausstellten. 61 übernehmende Unternehmen (59 v.H.) waren vor der Fusion nur unterdurchschnittlich rentabel. Nur ca. 50 v.H. der untersuchten Fälle schnitten drei Jahre nach einem Unternehmenszusammenschluss besser ab als der Branchendurchschnitt. Fusionen mit einem Fusionsvolumen von über $1 \mathrm{Mrd}$. Dollar erreichten schlechtere Ergebnisse als der Durchschnitt aller Unternehmen.

Tabelle 15: Auswirkungen eines Zusammenschlusses auf die Profitabilität

A: Anteil der übernehmenden Unternehmen mit höherer und niedrigerer Profitabilität relativ zum Branchendurchschnitt vor und nach einer Fusion (v.H.)

\begin{tabular}{|l|c|c|}
\hline & $\begin{array}{c}\text { Höher als der Branchendurch- } \\
\text { schnitt (3-Jahres Schnitt) }\end{array}$ & $\begin{array}{c}\text { Niedriger als der Branchendurch- } \\
\text { schnitt }\end{array}$ \\
\hline Profitabilität vor der Fusion & 40,8 & 59,2 \\
\hline Profitabilität nach der Fusion & 49,5 & 50,5 \\
\hline
\end{tabular}

Anmerkung: Proftitabilität $=$ Rohgewinn relativ zum eingesetzten Kapital (Drei-Jahres-Durchschnitt)

B: Veränderung der Profitabilität im Zuge von Unternehmenszusammenschlüssen (v.H.)

\begin{tabular}{|l|c|c|}
\hline & Profitabilitätssteigerung & Profitabilitätsreduzierung \\
\hline Alle Fusionen & 60,2 & 39,8 \\
\hline Volumen: < 1. Mrd. Dollar & 64,1 & 35,9 \\
\hline $\begin{array}{l}\text { Volumen: } 1 \text { Mrd. Dollar < } \\
\text { Mrd. Dollar }\end{array}$ & 50,0 & 50,0 \\
\hline Volumen: $>$ 5 Mrd. Dollar & 46,2 & 53,8 \\
\hline
\end{tabular}

Quelle: KLEINERT/KLODT 2000, S. 67

Unterschiedliche Muster ergeben sich auch, wenn die übernommenen Unternehmen hinsichtlich ihres Tätigkeitsfelds in rohstoffnahe Industrien, arbeitsintensive Bereiche, Sektoren mit hohen Skalenerträgen, Sektoren mit starker Produktdifferenzierung und einen Bereich mit hohem Technologieanteil eingeteilt werden. Der durchschnittliche Effekt von Fusionen auf die Produktivität der Unternehmen in rohstoffnahen und arbeitsintensiven Branchen ist negativ in dieser Stichprobe. Geringe Gewinne erzielen Unternehmen in Industrien mit hohen Skalenerträgen; in Industrien mit hohem Technologieanteil und starker Produktdifferenzierung können die fusionierenden Unternehmen größere Gewinne verbuchen. Das spräche für Synergieeffekte, die auf eine stär- 
kere Ausnutzung unternehmensinterner Skalenerträge, etwa in Forschung und Entwicklung oder im Marketing, zurückzuführen sind ${ }^{135}$.

\subsubsection{Zwischenergebnis}

Ein eindeutiges Ergebnis lässt sich aus den jahresabschlussorientierten Untersuchungen nicht ableiten.

Die Ursache der unterschiedlichen Ergebnisse kann vielerei Natur sein. Das schlimmste Szenario besteht, wenn trotz zunehmender Marktmachteffekte interne Ineffizienten überwiegen, die die Gesamtrentabilität verschlechtern. Eine weitere Möglichkeit besteht darin, dass es zu keiner Einsparung der Durchschnittskosten kommt und Konkurrenten als Reaktion auf die Fusion ihre Produktionsmenge ausdehnen. Dies wäre ein überraschendes Ergebnis und lässt erneut die Eingangsfrage offen, warum Unternehmen auch bei nicht besonders vielversprechenden Aussichten überhaupt fusionieren?

Wichtig ist, dass der Begriff des Erfolges einer Fusion unterschiedlich definiert werden kann. Empirische Überprüfungen sind von der Methodik her keineswegs homogen. Beispielsweise untersuchen einige Wissenschaftler die Profitabilität von Unternehmenszusammenschlüssen vor und nach der Fusion. Andere dagegen vergleichen die Fusion mit anderen Unternehmen der gleichen Branche, um Umwelteffekte auszuschalten. FridolfsSSON und STENNEK (1999) wiesen jedoch darauf hin, dass diese um Ausschaltung externer Effekte bemühten Untersuchungen nicht unproblematisch sind. Als Grund hierfür kann angeführt werden, dass eine Fusion generell auch Effekte auf die Outsider ausübt, d.h., bei Fusion zweier Unternehmen profitieren davon auch die anderen Unternehmen. Dieses Profitieren der Outsider kann zeitlebens den Nutzen für die Insider übertreffen (stark positive externe Effekte). Eine Erklärung hierfür ist das Teilhaben an einem höheren Preis bei konstanten Produktionsmengen (vgl. dazu auch Kapitel 4.1). Was also aus Sicht eines einzelnen Unternehmens als Misserfolg erachtet wird, kann auf Branchenebene durchaus erfolgreich sein. Umgekehrt kann sich ein erfolgreicher Unternehmenszusammenschluss branchentechnisch als Misserfolg mit stark negativen Effekten herausstellen. Diese Problematik deutet darauf hin, die Ergebnisse der Studien mit Vorsicht zu interpretieren.

${ }^{135} \mathrm{Vgl.} \mathrm{KLEINERT/KLODT} \mathrm{2000,} \mathrm{S.} 68$ 


\subsubsection{Befragungen}

MöLLER (1983) führte eine umfassende Befragung zu Zusammenschlüssen deutscher Unternehmen durch und nahm auch ausführlich zur Eignung einer Befragung für die Datenermittlung Stellung. Dabei sind besonders Probleme der Validität und Reliabilität von Interviews bedeutsam.

Validität ist die Eindeutigkeit eines Ergebnisses. Dabei ist zwischen interner und externer Validität zu unterscheiden. Interne Validität betrifft die Gültigkeit des Untersuchungsergebnisses für die analysierten Teilaspekte. Bei der Erfolgsermittlung von Unternehmenszusammenschlüssen können dabei Probleme der Zurechenbarkeit entstehen. Wenn die umfassende Unternehmenssituation als "erfolgreich" bezeichnet wird, so muss dies nicht auf einen gelungenen Zusammenschluss schliessen lassen. Andere, nicht separat erfragte Faktoren können so positive Auswirkungen haben, dass ein Zusammenschluss trotz eines Misserfolgs nicht dementsprechend beurteilt wird.

Externe Validität betrifft die Allgemeingültigkeit von Stichprobenergebnissen. Dabei besteht für Analysen von Unternehmenszusammenschlüssen die Gefahr einer Verzerrung, wenn über nicht erfolgreiche Zusammenschlüsse keine Auskunft erteilt wird.

Reliabilität ist die Reproduzierbarkeit von Ergebnissen bei wiederholten Befragungen. Sie ist bei Analysen zum Erfolg von Unternehmenszusammenschlüssen in doppelter Hinsicht problematisch. Zunächst können Zusammenschlüsse von verschiedenen Interviewpartnern unterschiedlich gewertet werden. Weiterhin besteht die Gefahr einer zeitlichen Änderung der Beurteilung. Dies gilt einerseits für länger zurückliegende Unternehmenszusammenschlüsse, die anhand einer eventuell veränderten Situation zum Befragungstermin anders beurteilt werden, und andererseits könnte bei nicht lange vor dem Interview zurückliegenden Zusammenschlüssen eine noch nicht abgeschlossene Integration zu vorschnellen Einschätzungen führen.

\subsubsection{Ergebnisse von Befragungen}

KITCHING (1967) analysierte 69 amerikanische Unternehmenszusammenschlüsse und kam zu folgendem Gesamtergebnis: 68 v.H. wurden als Erfolg, 32 v.H. als Misserfolg angesehen. In einer zweiten Studie explorierte KITCHING (1974) die Übernahme europäischer Unternehmen durch amerikanische oder andere europäische Unternehmen. Dabei wurden 53 Prozent der Zusammenschlüsse als erfolgreich und 47 Prozent als nicht erfolgreich eingestuft. Das Ergebnis wird jedoch dadurch verzerrt, dass NichtAntworten als Misserfolg gewertet wurden. In einer umfassenden Befragung zum Erfolg von 100 bundesdeutschen Unternehmenszusammenschlüssen durch MöLLER (1983) wurden 62 Prozent als Voll- oder Teilerfolg und 38 v.H. als Misserfolg beurteilt. 
Die Ergebnisse wurden in den einzelnen Studien anhand unterschiedlicher Kriterien differenziert. Vergleiche können anhand der Diversifikation oder des Größenverhältnisses der am Zusammenschluss beteiligten Unternehmen getroffen werden (KITCHING (1974); MöLLER (1983)) $)^{136}$.

Nach dem Kriterium der Diversifikation sind horizontale und vertikale Unternehmenszusammenschlüsse am erfolgreichsten. Horizontale Zusammenschlüsse wurden bei den Befragungen von KITCHING zu 59 (Untersuchung von 1974) und 89 Prozent (Untersuchung von 1967) als Erfolg beurteilt. Bei MöLLER fielen insgesamt 63,4 Prozent in die Kategorien „voller Erfolg" oder zumindest „Teilerfolg". Für vertikale Zusammenschlüsse ergab sich bei Kitching eine Erfolgsquote von 53,3 v.H. (1974) und 100 Prozent (1967) der Fälle. Bei MöLlER wurden 80 Prozent der vertikalen Zusammenschlüsse als erfolgreich eingeschätzt. Für konglomerate Zusammenschlüsse bestehen nach den Befragungen die geringsten Erfolgsaussichten. Während in den Untersuchungen nach KITCHING noch 35,4 (1974) beziehungsweise 58 Prozent (1967) der Fusionen als Erfolge beurteilt wurden, waren bei MöLLER ausschließlich Misserfolge zu verzeichnen.

Bei einer nach dem Größenverhältnis differenzierten Beurteilung der Zusammenschlüsse zeigte sich, dass die Übernahme großer Unternehmen in den meisten Fällen als Erfolg eingeschätzt wurde. Wenn das übernommene Unternehmen mehr als die Hälfte des Umsatzes (bei KITCHING) oder der Beschäftigten (bei MöLLER) in Relation zum übernehmenden Unternehmen aufwies, wurden die Zusammenschlüsse in 80 bzw. 87,5 Prozent als erfolgreich eingeschätzt. Bei einem Verhältnis von weniger als 5 Prozent sank die Erfolgsquote auf 38,7 bzw. 52,7 Prozent.

\subsubsection{Weitere Untersuchungen zur Beurteilung von Unternehmenszu- sammenschlüssen}

\subsubsection{Effekte von Fusionen auf Produktpreise}

\subsection{Direkte Studien}

Es gibt überraschend wenige Untersuchungen, die Effekte von Fusionen auf Produktpreise analysieren. In den einzelnen Studien konnten Preissteigerungen als Folge einer Fusion nicht eindeutig belegt werden. Hinzu kommt das generelle Problem bei diesen Studien, dass Umweltbedingungen wie Faktorpreise, Nachfrageeinbrüche etc. ebenfalls Veränderungen unterliegen.

${ }^{136}$ Ausführlich in BÜHNER 1990, S. 100 
BARTON und SHERMAN (1984) untersuchten den Preiseffekt zweier Fusionen der Firma Xidex im Mikrofilmbereich. Die erste Fusion führte zu einem Preisanstieg von 11 v.H., während die zweite eine Preiserhöhung von 23 v.H. zur Folge hatte ${ }^{137}$. KIM und SINGAL (1993) betrachteten Flugpreise zwischen 1985 bis 1989 in den USA. Aus einem umfangreichen Datenpool stellte sich heraus, dass nach einer Fusion im Durchschnitt eine Preissteigerung von 9 v.H. im Vergleich zu der Kontrollgruppe ermittelt wurde. Als zentrales Ergebnis dieser Studie konnte ein signifikant positiver Zusammenhang zwischen Flugpreisänderungen und Unternehmenskonzentration nachgewiesen werden.

CotTERILL (1990) untersuchte den Effekt sechs horizontaler Fusionen von Supermärkten in den USA. Zunächst stellte CoTTERILL die Marktanteile vor und nach der Fusion fest und berechnete die Veränderung der Marktkonzentration (anhand HHI-Index ${ }^{138}$ ). Durch Zuhilfenahme der zuvor geschätzten Beziehung zwischen Marktkonzentration und Marktpreis prognostizierte COTTERILL die Preisentwicklung nach der Fusion. Schließlich errechnete er die implizierte Zunahme in den Endverbraucherausgaben und verglich diese mit den realen Endverbraucherausgaben, wobei sich eine Preisdifferenz von 1 bis 3 v.H. ergab ${ }^{139}$.

STEWART und KIM (1993) untersuchten den partiellen Einfluss von Fusionen auf Preise, Preisaufschläge und Nachfrageelastizitäten für 117 US-amerikanische Unternehmen der Jahre 1985 und 1986 mit dem Ziel, daraus Wohlfahrtsveränderungen durch Fusionstätigkeit messen zu können. Der Einfluss von Unternehmenszusammenschlüssen ist nach ihrer Untersuchung nur in Industriezweigen mit mittleren Konzentrationen bedeutsam ${ }^{140}$. Von den 59 Branchen dieser Kategorie war lediglich in einer ein positiver Wohlfahrtseffekt durch Fusionen zu verzeichnen, und die zusätzlichen Gewinne der Unternehmen konnten den Rückzug der Konsumentenrente nur hier überkompensieren $^{141}$. Die Konsumentenrente ging aufgrund von Unternehmenszusammenschlüssen in sämtlichen 59 Industriezweigen zurück, während die Gewinne in 51 Industriezweigen stiegen. Die Gesamtwohlfahrtseinbuße durch Fusionen betrug in dieser Stichprobe für die Jahre 1985 und 1986 ca. 4,6 Mrd. Dollar. Die Autoren folgerten daraus, dass die wenig strenge Fusionskontrolle der Reagan-Jahre den Vereinigten Staaten erhebliche Wohlfahrtseinbußen verursacht hatte ${ }^{142}$.

\footnotetext{
137 Vgl. RöLler/STENNEK/VERBOVEn 2000, S. 43

${ }^{138}$ Erläuterung des HHI-Indexes in Anhang B

139 In Anlehnung an Röller/StenneK/Verboven 2000, S. 45; basiert auf CotTERILL 1989

140 Vgl. STEWART/KIM 1993, S. 712

${ }^{141} \mathrm{Vgl.} \mathrm{STEWART/KIM} \mathrm{1993,} \mathrm{S.} 717 \mathrm{f}$.

142 Vgl. STEWART/KIM 1993, S. $701 \mathrm{ff}$.
} 


\subsection{Indirekte Studien}

Eine weitere Möglichkeit die Preiseffekte einer Fusion zu analysieren bieten die sogenannten indirekten Studien, die geographisch abgegrenzte Märkte unterschiedlich starker Konzentration miteinander vergleichen. Wenn hohe Preise mit hoher Konzentration korrelieren, ist dies ein indirekter Beweis für Marktmachteffekte von Fusionen.

SCHMALENSEe (1989), der eine positive Korrelation zwischen Marktkonzentration und Preisniveau nachwies, wurde von BRESNAHAN (1989) anhand ausgewerteter "crosssection" Literatur in seinen Ergebnissen bekräftigt.

LAMM (1981) kam bei Untersuchungen des US-Nahrungsmittelhandels zwischen 1974 und 1977 zu dem Ergebnis, dass zunehmende Konzentration auch zu Preisanstiegen führt, sobald nicht mehr als drei Zusammenschlusspartner beteiligt sind. Bei vier oder mehr Fusionspartnern führte der Zusammenschluss dagegen zu Preisreduzierungen, die LAMM auf Skalenerträge (Lagerkapazität) ab einer bestimmten Unternehmensgröße zurückführte.

MARVEL (1978) beschäftigte sich mit Benzinpreisen in den USA zwischen 1964 und 1971 und konnte eine signifikante Beziehung zwischen Konzentration und Preis feststellen. Vor allem im unteren Preissegment waren in seiner Analyse höhere Preise das Resultat von Konzentration.

Geithman, MARVEL und Weiss (1981) hinterfragten kritische Grenzwerte in verschiedenen Industriebranchen und fanden u.a. heraus, dass in einigen Branchen zwar Grenzwerte vorlagen ${ }^{143}$ (z.B. $\mathrm{CR}_{4}=50$ ), in anderen Branchen hingegen nicht festgestellt werden konnten (z.B. Supermärkte). Herauszustellen bleibt, dass es keine einheitliche kritische Grenze (z.B. für Wettbewerbsrecht) gibt.

In Europa untersuchte VERBOVEN (1996) Preisunterschiede in der Automobilindustrie und folgerte, dass tendenziell die Preis-Kosten-Marge in Märkten mit hohen Marktanteilen größer ist. In den Heimatländern der Unternehmen (z.B. FIAT in Italien) ist der Preissetzungsspielraum generell größer.

\subsubsection{Auswirkungen von Fusionen auf Marktanteile}

Wie aus den theoretischen Modellen (vgl. Kapitel 4.1) hervorgeht, hat eine Steigerung von Marktmacht tendenziell zur Folge, dass mit einem Zusammenschluss ein Preisan-

${ }^{143}$ Marktmachteffekte überwiegen Effizienzeffekte 
stieg und eine Reduzierung der Produktionsmengen einhergehen. Beim Überwiegen von Effizienzvorteilen äußert sich das in einer Gewinnerhöhung durch Angebotsausdehnung und Preisreduzierung, so dass über die Entwicklung der Marktanteile auf Marktmacht- bzw. Effizienzeffekte rückgeschlossen werden kann.

Es gibt wenige Untersuchungen, die die Auswirkungen von Fusionen auf Marktanteile untersuchen, und auch diese Ergebnisse lassen keine eindeutigen Schlussfolgerungen $\mathrm{zu}$.

GOLDBERG (1973) untersuchte die Marktanteile von 44 Unternehmen über einen durchschnittlichen Zeitraum von dreieinhalb Jahren nach einer Fusion. Er fand keine signifikante Änderung der Marktanteile und damit auch keine Hinweise auf eine abnehmende Wettbewerbsintensität auf den betreffenden Märkten.

MUELLER (1986) hingegen ermittelte einen signifikant schrumpfenden Marktanteil nach Unternehmenszusammenschlüssen in einer Stichprobe von 209 Unternehmen des verarbeitenden Gewerbes in den elf Jahren nach einer Fusion. Die Marktanteile reduzierten sich aber weitaus stärker, als mit Marktmachteffekten allein zu erklären wäre. Deshalb müssen laut MUELLER Ineffizienzen hinzukommen, um einen Erklärungsansatz für die relativ starke Verminderung der Marktanteile zu liefern. In seiner Untersuchung ergeben sich also nicht zwei gegenläufige Effekte von Fusionen, sondern zwei gleichgerichtete Einflüsse in Richtung einer Wohlfahrtsminderung ${ }^{144}$.

Auch BALDWIN und GORECKI (1990) stellten in einer Untersuchung bei in horizontalen Fusionen übernommenen Werken eine Verminderung des Marktanteils fest. Für Werke, die bei einer nicht-horizontalen Fusion übernommen wurden, konnte hingegen kein Rückgang des Markteanteiles ermittelt werden. Den Unterschied bei der Entwicklung der Marktanteile beider Gruppen erklärten BALDWIN und GORECKI mit Marktmachteffekten, die stärker nach horizontalen als nach vertikalen oder konglomeraten Fusionen auftreten ${ }^{145}$.

\subsubsection{Weitere betriebswirtschaftliche Untersuchungen}

Auf einen Grund für die Schwierigkeiten, Gewinnsteigerungen durch das Ausnutzen von fusionsbedingten Synergien zu realisieren, wies SCHENK (1996) hin. Unternehmensübernahmen binden finanzielle Mittel und Managementkapazitäten. Andere langfristige Ziele werden vernachlässigt. Besonders negativ wirkt sich eine Übernahme demnach aus, wenn Forschung und Entwicklung vernachlässigt werden. Eine Abnahme

\footnotetext{
${ }^{144}$ In Anlehnung an KLEINERT/KLODT 2000, S. 58

${ }^{145}$ In Anlehnung an KLEINERT/KLODT 2000, S. 58
} 
der Wettbewerbsfähigkeit ist die Folge. SCHENK führte das Aufholen japanischer Unternehmen im internationalen Wettbewerb in den sechziger, siebziger und achtziger Jahren auch auf die geringe Anzahl der Unternehmenszusammenschlüsse in Japan zurück. Fusionen vernichten nach dieser Studie - verglichen mit internem Wachstum Wert $^{146}$.

Auch Übernahmen, die mit dem Ziel der Akquisition von technischem Wissen unternommen wurden, bilden laut PIEPER (1998) keine Ausnahme. In einer Wirkungsuntersuchung von Unternehmensübernahmen auf Forschung und Entwicklung kam sie zu dem Schluss, dass gemessen an der Zahl der Patentanmeldungen eine externe Akquisition von technischem Wissen im Durchschnitt weniger erfolgreich als die Eigenentwicklung ist. Ursache dafür ist ihrer Ansicht nach der finanzielle Substitutionseffekt: Für Zins- und Tilgungszahlungen werden langfristige Investitionen - auch Forschungsund Entwicklungsinvestitionen - gekürzt. Auch ein Zusammenführen der ehemals separaten Forschung stellt die Unternehmen vor Probleme (technologische Substitutionseffekte). Die Akquisition technischen Wissens wird zwar immer wieder als relevanter Grund für Unternehmensübernahmen ins Feld geführt, scheint jedoch wenig erfolgreich zu sein ${ }^{147}$.

\subsection{Schlussfolgerung: Theoretische Analyse und empirische Überprüfung der Wohlfahrtseffekte von Fusionen}

Motive und Auswirkungen von Unternehmenszusammenschlüssen sind schon seit längerem Gegenstand von Wissenschaft und Wettbewerbspolitik. Dabei gibt es eine Reihe unterschiedlicher Forschungsansätze zur Ableitung einer normativen Wettbewerbspolitik, in denen der Versuch unternommen wird, reale Vorgänge in Modelle abzubilden. Modelltheoretische Ergebnisse verdeutlichen, dass Zusammenschlüsse durchaus positive Effizienzwirkungen haben können. Die unter den Bezeichnungen Rationalisierungs-, Größen- und Verbundvorteile vorgestellten Einsparungspotentiale kommen nicht nur den Unternehmen zugute, sie verringern auch den notwendigen Ressourcenverbrauch auf gesamtwirtschaftlicher Ebene. Dies gilt ebenso für eine evtl. erhöhte Innovationstätigkeit sowie des Austauschs eines ineffizienten Managements.

Andererseits geht mit Unternehmenszusammenschlüssen ein effizienzminderndes Potential einher. Dies äußert sich in der erhöhten Wahrscheinlichkeit einer Kollusion, ei-

\footnotetext{
${ }^{146} \mathrm{Vgl}$. SCHENK 1996, S. $255 \mathrm{ff}$.

147 Vgl. Pieper 1998, S. 242 ff.
} 
ner Verringerung der Vielfalt von Produkten, einer Errichtung von strategischen Markteintrittsbarrieren und schließlich in der zuvor diskutierten Ausübung von Marktmacht. Für Unternehmen positiv besetzte Motive für einen Zusammenschluss können unter Effizienzgesichtspunkten negativ zu beurteilen sein. Die vorgestellten industrieökonomischen Modelle deuten darauf hin, dass Preise nach einem Zusammenschluss steigen, wenn Synergiepotentiale nicht in erheblichen Maßen ausgenutzt werden können. Die Ausnutzung von Rationalisierungsvorteilen allein ist dabei oftmals nicht ausreichend. Zwar disziplinieren die verbleibenden Wettbewerber die Preiserhöhung zu einem gewissen Grad, ähnlich wie in der Situation eines partiellen Kartells, aber auch die verbleibenden Konkurrenten profitieren von dem verminderten Wettbewerbsdruck und haben die Möglichkeit zu Preiserhöhungen. Bei der Beurteilung von Zusammenschlüssen sollte somit die Wettbewerbsbehörde folgenden potentiellen Gefahren besondere Aufmerksamkeit schenken:

- Erzielung einer dominanten Position (Insider),

- Verringerung des Restwettbewerbes (Outsider),

- Kartellbildungsgefahr (Reduzierung der Marktteilnehmerzahl).

Für die Wettbewerbspolitik stellt sich die Frage, wie die empirischen Ergebnisse zu interpretieren sind. In einem optimistischen Szenario sind fehlgeschlagene Zusammenschlüsse ein Teil eines Suchprozesses für profitable Strategien, die auf Erfahrungen beruhen, welche sich als unzutreffend herausstellten. Ist bei diesem Versuch keine Marktmacht verstärkt worden, so gehörte bei einem fehlgeschlagenen Zusammenschluss der Ressourcenverbrauch zu den Kosten des Wettbewerbsprozesses selbst. Zusammenschlüsse wären dann unbedenklich. Im pessimistischen Szenario ist jedoch durch den Zusammenschluss Marktmacht, die grundsätzlich die Gewinnmöglichkeiten erhöht, verstärkt worden. Jedoch sind durch den Zusammenschluss Kostensteigerungen bewirkt worden, die den Gewinnanstieg überkompensieren. Unter Berücksichtigung der Konsumentenrentenreduktion treten erhebliche Wohlfahrtsverluste auf. Zusammenschlüsse sind in diesem Szenario deutlich kritischer zu beurteilen.

Werden Effizienzgesichtspunkte in den Vordergrund der Wettbewerbspolitik gestellt, müssen effizienzsteigernde (Synergieeffekte) und effizienzmindernde (Marktmacht) Potentiale gegeneinander abgewogen werden. Aus den theoretischen wie auch empirischen Ergebnissen ergibt sich, dass ein Zusammenschluss ab einer kritischen Grenze (z.B. Marktanteil > 50 v.H.) nicht generell zu untersagen ist. Es ergibt sich vielmehr daraus, dass Unternehmenszusammenschlüsse fallweise analysiert werden müssten. 
Im nächsten Kapitel wird daher der deutsche und europäische Rechtsrahmen näher erläutert, um im Anschluss daran die praktische Umsetzung der Entscheidungsträger zu analysieren. 


\section{Grundzüge des Wettbewerbsrechts}

Unternehmensfusionen in Deutschland werden vom Bundeskartellamt bzw. der Europäischen Kommission beobachtet und kontrolliert. Diese Institutionen handeln nach dem Wettbewerbsrecht und haben die Befugnis, Fusionen mit oder ohne Auflagen zu gestatten oder zu untersagen. Um der Argumentation der Rechtspraxis zu folgen, werden in diesem Kapitel zunächst wichtige Komponenten der Zusammenschlusskontrolle, die zugrundeliegenden Paragraphen und deren Anwendung in der Rechtsprechung kommentiert.

\subsection{Die Zusammenschlusskontrolle im deutschen Recht}

\subsubsection{Hintergrund des deutschen Wettbewerbsrechts}

Am 1. Januar 1958 trat das Gesetz gegen Wettbewerbsbeschränkungen (GWB) in Deutschland in Kraft und löste damit die Dekartellierungsbestimmungen von 1947 ab. Es wurde einerseits durch ordnungspolitische Vorstellungen des Neoliberalismus und anderseits durch das US-amerikanische Antitrustrecht geprägt ${ }^{148}$. Seit Inkrafttreten des GWB wurde das Gesetz fünfmal novelliert. Bereits 1955 war eine Zusammenschlusskontrolle im Regierungsentwurf vorgesehen, wurde jedoch im Bundestag mit der Begründung, dass dadurch die wünschenswerte Entwicklung zu optimalen Betriebsgrößen behindert würde, abgelehnt ${ }^{149}$. Erst im Zuge der 2. GWB-Novelle von 1973 wurde eine vorbeugende Fusionskontrolle eingeführt. Seither wurde insbesondere die Fusionskontrollverordnung ausgeweitet, konkretisiert und im Zuge der sechsten Novelle im Jahre 1998 mit dem Europäischen Recht harmonisiert ${ }^{150}$.

Der Begriff des Wettbewerbsrechts umfasst ein breites Spektrum von Gesetzesnormen, die das gemeinsame Ziel zum Schutz gegen wettbewerbsfeindliche Verhaltensweisen beinhalten. Das deutsche Recht gliedert sich in das Recht gegen den unlauteren Wettbewerb (UWG), in welchem Regelungen über Geschäftspraktiken, Patentwesen, Werbung, Bezeichnungen usw. vermittelt werden, und das Recht gegen Wettbewerbsbeschränkungen (GWB), welches u.a. Gesetzesnormen über Preis- und Mengenabsprachen, Missbrauch von Marktmacht und die Zusammenschlusskontrolle beinhal-

\footnotetext{
148 Vgl. SCHMIDT 1999, S. 162

${ }^{149} \mathrm{Vgl.}$ AHRNS/FESER 1997, S. 75

${ }^{150} \mathrm{Vgl.} \mathrm{SCHMIDT} 1999$, S. 165
} 
tet. Die folgende Abbildung (Abbildung 20) gibt einen Überblick mit den jeweils zugrundeliegenden Paragraphen.

Abbildung 20: Aufbau des Wettbewerbsrechts

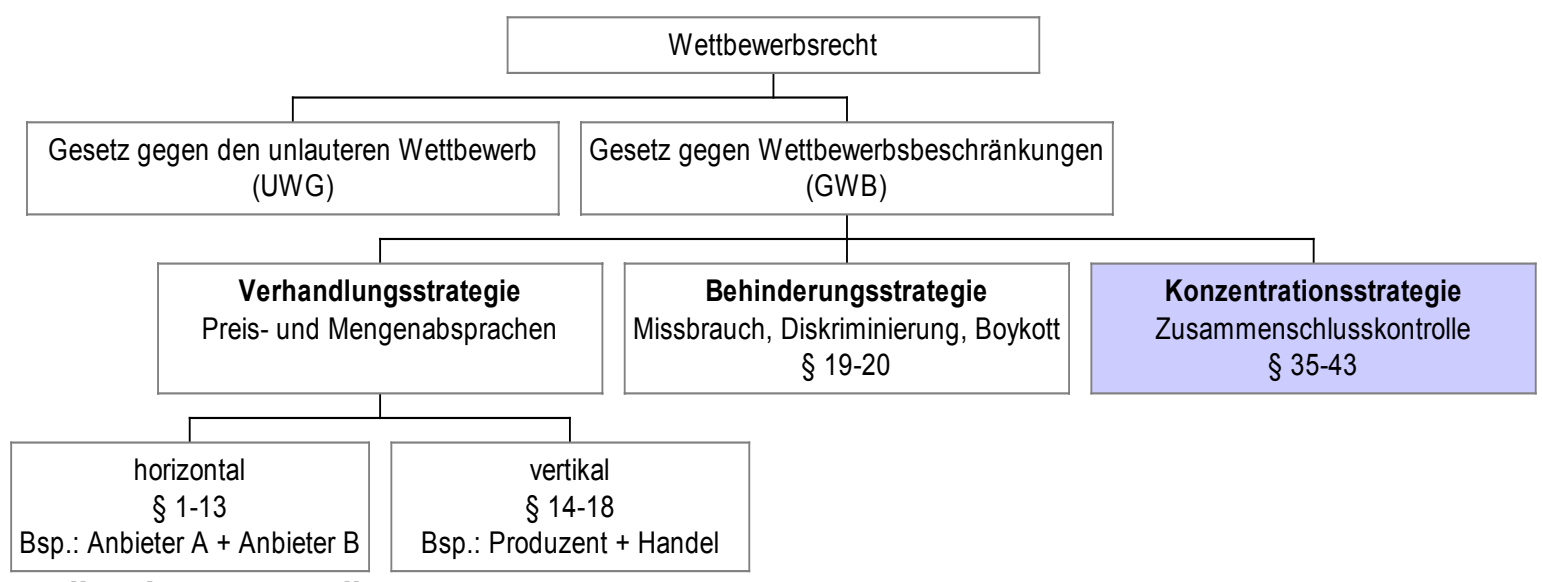

Quelle: eigene Darstellung

\subsubsection{Geltungs- und Anwendungsbereich}

\subsubsection{Der fusionsrechtliche Unternehmensbegriff}

Der Unternehmensbegriff im Sinne der Zusammenschlusskontrolle ist funktional zu verstehen. Grundsätzlich genügt jede selbständige, nicht rein private und außerhalb des Erwerbslebens liegende Tätigkeit einer Person in der Erzeugung oder Verteilung von Waren oder gewerblichen Leistungen ${ }^{151}$. Jede natürliche oder juristische Person kann Unternehmen sein, solange sie nur am wirtschaftlichen Verkehr teilnimmt. Weder die Rechtsform, noch eine Gewinnerzielungsabsicht sind von Belang. Selbst gemeinnützige Unternehmen sind an das GWB gebunden ${ }^{152}$. Die umstrittene Frage, ob Konzerne Unternehmenseigenschaft im Innenverhältnis zu den Konzerntochtergesellschaften besitzen, wird nach heutiger allgemeiner Auffassung bejaht ${ }^{153}$. Werden mehrere Rechtsträger zusammen mit anderen unternehmerisch tätig, ohne dass dadurch eine juristische Person entsteht, z.B. bei der Gesellschaft bürgerlichen Rechts, der Offenen

\footnotetext{
${ }^{151}$ Entscheidung des Bundesgerichtshofs in Zivilsachen (BGHZ) 19, 72 (79 f.) = Neue Juristische Wochenschrift (NJW) 1956, 341; BGHZ 36, 91 (102 ff.) = NJW 1962, 196; BGHZ 52, 65 (66) = NJW 1969, 1716; BGHZ 64, 232 (234 f.) = NJW 1975, 1223 = JuS 1975, 662 Nr. 8; BGHZ (GS) 67, 81 (84) = NJW 1976, 1941; BGHZ 69, 59 (60) = NJW 1977, 2121; BGHZ 74, 359 (354 f.) = NJW 1979, $2401=$ JuS 1980, 227 Nr. 11; BGHZ 137, 297 (304) = NJW 1998, 756 = Juristische Schulung (JuS) 1998, 461 Nr. 10 "DFB"

152 Grdlg. BGHZ 137, 297 (311 f.) = NJW 1998, 756 = JuS 1998, 461 Nr. 10 "DFB"

153 Vgl. IMMENGA/MeSTMÄCKER 1997, § 1, Rdnr. 65
} 
Handelsgesellschaft oder der Kommanditgesellschaft, dann ist im Zweifel die Gesellschaft als Teilnehmer am Wirtschaftsleben das Unternehmen, wenn sich die Gesellschaft selbständig am Geschäftsverkehr beteiligt ${ }^{154}$. Der oder die Gesellschafter sind für sich nur dann Unternehmer, wenn sie in ihrer Person die Voraussetzungen erfüllen ${ }^{155}$.

\subsubsection{Die Tatbestände des $§ 37$ GWB}

1. Vermögenserwerb: Als Zusammenschluss im Sinne des Gesetzes gilt der Erwerb von Vermögen eines anderen Unternehmens ganz oder zu einem wesentlichen Teil durch Verschmelzung, Umwandlung oder sonstige Weise, wobei es keine Rolle spielt, ob der Vermögenserwerb auf Vertrag oder Gesetz beruht, so dass in der Regel zugleich ein Kontrollerwerb im Sinne Nr. 2 des $\S 37$ I vorliegen dürfte ${ }^{156}$. Neben objektiven, geldwerten Gütern und Rechten gehören subjektive Rechte und Chancen zum Vermögen, sofern sie nur im Verkehr gehandelt werden ${ }^{157}$. Als Erwerb werden neben dem Verkauf von Unternehmensteilen die Verschmelzung, die überragende Umwandlung sowie die Vermögensübertragung im Wege der Liquidation oder bei Auflösung eines Gemeinschaftsunternehmens ${ }^{158}$ verstanden. Der Begriff "wesentlicher Teil" ( $\S 37$ Abs. 1 Nr. 1 GWB) beinhaltet nicht nur Vermögensteile, die in ihrem Verhältnis zum Gesamtvermögen des Veräußerers quantitativ ausreichend hoch sind. Wesentlich ist ein Vermögensteil vielmehr stets dann, wenn ihm im Hinblick auf die Produktion, die Vertriebsziele und die jeweiligen Marktverhältnisse eine eigenständige Bedeutung zukommt und er deshalb als ein vom übrigen Vermögen des Veräußerers abtrennbarer einheitlicher Teil erscheint ${ }^{159}$. Das können beispielsweise eine Betriebsstätte (z.B. Filiale eines Lebensmittelhandelsunternehmens ${ }^{160}$ ), ein Geschäftsbereich (z.B. der Bereich „Tiefkühlkost" eines Nahrungsmittelherstellers), ein

\footnotetext{
${ }^{154}$ Vgl. IMMENGA/MESTMÄCKER 1997, § 1, Rdnr. 69

155 Vgl. LANGEN/BunTE 2001, § 1, Rdnr. 6, zitiert nach Burkhardt 1995, S. 30

${ }^{156}$ BGHZ 119, 117 (125f.) = LM § 23 GWB Nr. 17 = Die AG 1993, 36 = NJW 1993, 264 „Melitta/Kraft (Frapan)"

157 Vgl. EMMERICH 1999, S. 282

${ }^{158}$ Vgl. KG, WuW/E OLG $2007 \mathrm{f}$.

159 in Anlehnung an: BundeSKARTELLAMt 2000, Merkblatt Fusionskontrolle, S. 11

160 Vgl. KG, WuW/E OLG 3591 = Die AG 1986, 230 „Coop SH/Deutscher Supermarkt”; BkartA, Die AG 1984, 159
} 
„Tiefkühlkost" eines Nahrungsmittelherstellers), ein Warenzeichen ${ }^{161}$ oder die Verlagsund Titelrechte einer Zeitung sein ${ }^{162}$.

2. Kontrollerwerb: Der Begriff des Kontrollerwerbes wurde in Anlehnung an Art. 3 der europäischen Fusionskontrollverordnung durch die 6. Novelle in das Gesetz eingeführt. Kontrolle liegt danach vor, wenn auf die Tätigkeit eines anderen Unternehmens ein bestimmter Einfluss ausgeübt werden $k a n n^{163}$. Durch diese Generalklausel sollen sämtliche Konstruktionen erfasst werden, mit denen ein steuernder unternehmerischer Einfluss erreicht wird - einschließlich Minderheitsbeteiligungen ${ }^{164}$ (Reaktion des Gesetzgebers auf mögliche Umgehungsstrategien in der früheren Gesetzgebung). Dabei kann die Kontrolle durch ein oder mehrere Unternehmen erworben werden; die Erwerber brauchen nicht miteinander verbunden zu sein. Für die gemeinsame Kontrolle reicht es aus, dass Unternehmen aufgrund einer gemeinsamen Unternehmenspolitik die eigenen Wettbewerbsinteressen im Verhältnis zueinander und gegenüber dem abhängigen Unternehmen abstimmen und durchsetzen können, z.B. aufgrund von Poolverträgen, erhöhten Zustimmungserfordernissen in der Gesellschafterversammlung, oder weil aufgrund einer auf Dauer angelegten Interessensgleichheit eine einheitliche Einflussnahme gesichert ist. Begründen mehrere Unternehmen die gemeinsame Kontrolle über ein anderes Unternehmen, so sind alle mitkontrollierenden Unternehmen und die Zielgesellschaft am Zusammenschluss beteiligt. Beispiel: Fünf Unternehmen mit je 150 Mio. $€$ Umsatz gründen ein Gemeinschaftsunternehmen, an dem sie sich mit jeweils $20 \%$ beteiligen, und sehen vor, dass alle wichtigen Entscheidungen mit der Mehrheit von 81 \% getroffen werden. Hier liegt ein kontrollpflichtiger Zusammenschluss vor ${ }^{165}$. Ein weiterer Tatbestand ist der Übergang von gemeinsamer zu alleiniger Kontrolle. Beispielsweise sind an einem Unternehmen Gesellschafter $A$ mit $60 \%$ und Gesellschafter $B$ mit $40 \%$ beteiligt. Alle Entscheidungen werden mit Zweidrittelmehrheit getroffen ( $A$ und $B$ kontrollieren somit gemeinsam). Erwirbt $A$ nun die restlichen $40 \%$ der Anteile, ist der Zusammenschluss kontrollpflichtig. Entsprechendes gilt bei dem Übergang von der Kontrolle durch drei auf zwei Unternehmen ${ }^{166}$. Ein weiterer Aspekt betrifft den Erwerb einer gesicherten Hauptversammlungsmehrheit. So genügt

\footnotetext{
${ }^{161}$ Vgl. WUW/E BGH 2783 „Warenzeichenerwerb"

162 Vgl. WUW/E OLG 4637 „Bote vom Grabfeld/Main-Post”

${ }^{163}$ Vgl. BUNDESKARTELLAMT 2000, Merkblatt Fusionskontrolle, S. 12

${ }^{164}$ Begr. zum RegE, BT-Dr. 13/9720, S. 43

${ }^{165}$ Erklärung und Beispiel weitgehend aus BundESKARTELLAMT 2000, Merkblatt Fusionskontrolle, S. 12 übernommen

${ }^{166}$ Vgl. BUndeSKARTELLAMT 2000, Merkblatt Fusionskontrolle, S. 12
} 
beispielsweise eine Beteiligung von $35 \%$ an einer AG, wenn die Hauptversammlungsmehrheit nachhaltig nur bei unter $70 \%$ liegt (Streubesitz) ${ }^{167}$.

3. Anteilserwerb: Der Anteilserwerb ist in der Praxis der wichtigste Zusammenschlusstatbestand ${ }^{168}$. Seit der 5. GWB Novelle (1989) werden Stimmrechts- und Kapitalanteilserwerb durchgehend gleich behandelt. Anteile im Sinne dieser Vorschriften sind solche an Unternehmen in Gesellschaftsform, d.h. in der Regel nicht an Einzelunternehmen ${ }^{169}$. Der Erwerb einer Option dagegen gilt nicht als Zusammenschlusstatbestand ${ }^{170}$. Erfasst werden neben den Kapitalgesellschaften ( $A G, K G a A, G m b H$ ) auch Personengesellschaften ( $O H G, K G, G b R$ ) sowie Genossenschaften, Versicherungsvereine auf Gegenseitigkeit oder - wenn sie Unternehmen sind - Vereine des bürgerlichen Rechts. Auch stille Beteiligungen können erfasst sein, wenn der stille Gesellschafter an der Geschäftsführung maßgeblich beteiligt ist oder im Innenverhältnis über Stimmrechte verfügt ${ }^{171}$. In Ergänzung zum Kontrollerwerb greift der Anteilserwerb ab $25 \%$ der Stimmrechts- oder Kapitalbeteiligung anderer Unternehmen. Additiv werden neben den eigenen Anteilen des Unternehmens auch Anteile hinzugerechnet, die einem anderen auf Rechnung dieses Unternehmens gehören und, wenn der Inhaber des Unternehmens ein Einzelkaufmann ist, auch die Anteile, die sonstiges Vermögen des Inhabers sind ${ }^{172}$. Erwerben mehrere Unternehmen gleichzeitig oder nacheinander im vorbezeichnetem Umfang Anteile an einem anderen Unternehmen, gilt dies hinsichtlich der Märkte, auf denen das andere Unternehmen tätig ist, auch als Zusammenschluss der sich beteiligten Unternehmen untereinander (Gemeinschaftsunternehmen) ${ }^{173}$.

4. Sonstige Verbindungen: Ziel dieser Generalklausel ist es, alle sonstigen Möglichkeiten der Einflussnahme von Unternehmen zu erfassen, die durch die o.g. Tatbestände nicht erfasst werden. So gab es in der Vergangenheit beispielsweise Beteiligungen, die von Umgehungskonstruktionen (24,9\%-Fälle $\left.{ }^{174}\right)$ geprägt wurden, so dass wettbewerblich erheblicher Einfluss auf ein anderes Unternehmen ausgeübt werden konnte. Hierbei sind sämtliche faktischen und rechtlichen Elemente des Sachverhalts zu be-

\footnotetext{
167 Vgl. BosCH/SCHÜTz 2000, § 37, Rdnr. 27

${ }^{168}$ Vgl. MÖSCHEL 1983, S. 470

${ }^{169}$ Vgl. MÜLLER-URI, Rdnr. 274

170 WUW/E BGH 2276, 2283 "Süddeutscher Verlag/Donau-Kurier"

171 Vgl. BURKHARDT 1995, S. 179

172 Vgl. Zurechnungsklausel in EMMERICH 1999, S. 291

${ }^{173}$ Vgl. Teilfusion der Mütter in EMMERICH 1999, S. 294

${ }^{174}$ Begr. zum RegE von 1989 (BT-Dr. 11/4610), S. 18 f.
} 
rücksichtigen ${ }^{175}$. Beispiele sind die Aufteilung des Beteiligungserwerbs auf mehrere Unternehmen, die auch sonst kooperieren ${ }^{176}$, oder auf sonst verbundene und befreundete Unternehmen oder Familienangehörige, so dass die Gruppe insgesamt über eine Mehrheit verfügt ${ }^{177}$.

\subsubsection{Voraussetzungen der Anmeldepflicht}

Nach dem deutschen Wettbewerbsrecht werden kontrollpflichtige und nicht kontrollpflichtige Zusammenschlüsse unterschieden. Kontrollpflichtig sind diejenigen Kooperationen, in denen die beteiligten Unternehmen insgesamt auf weltweite Umsatzerlöse von mehr als 500 Millionen Euro kommen und außerdem mindestens ein beteiligtes Unternehmen im Inland Umsatzerlöse von mehr als 25 Millionen Euro erzielt hat ( $\S 35$ Abs. 1). Nicht kontroll- und anzeigepflichtig sind Zusammenschlüsse dann, wenn erstens der Zusammenschluss keine Inlandsauswirkung zur Folge hat ${ }^{178}$, zweitens die zuvor genannten Umsatzschwellen nicht erreicht werden, drittens die de minimisKlause ${ }^{179}$ erfüllt ist oder viertens die Bagatellmarktklausel anwendbar ist.

1. Inlandsauswirkung: Angewendet wird das Gesetz, sobald sich der Zusammenschluss auf dem Inland auswirkt, auch wenn er außerhalb des Geltungsbereiches realisiert wurde ( $\S 130$ Abs. 2). Von einer Auswirkung ist dann auszugehen, wenn der Inlandsumsatz der beteiligten Unternehmen 25 Millionen Euro überschreitet ( $\S 35$ Abs. 1 Nr. 2). Dabei werden verschiedene Fallkonstruktionen erfasst:

a) Ein Zusammenschluss wird im Inland realisiert. Dabei ist belanglos, ob ein ausländischer Konzern beteiligt ist oder nicht. Beispiel: Nordzucker AG erwirbt sämtliche Anteile der Union Zucker.

b) Ein Zusammenschluss wird im Ausland realisiert. Inlandsauswirkung entsteht, sobald mindestens ein Unternehmen im Inland tätig war oder aber auch wenn durch den Zusammenschluss die inländische Marktstruktur verändert wird. Beispiel: Südzucker AG Deutschland erwirbt Mehrheit an Saint Louis Sucre in Frankreich.

2. Umsatzschwellen: Es gelten die Umsatzerlöse des letzten Geschäftsjahres. Die genaue Ermittlung erfolgt nach $\S 38$ Abs. 1 GWB bzw. $\S 277$ Abs. 1 HGB. Die Mehrwert-

\footnotetext{
175 in Anlehnung an EMMERICH 1999, S. 299

176 BundesKARTELLAMT, TB 1991/92, S. 130

177 BUNDESKARTELLAMT, TB 1991/92, S. 24, 139 "Allianz/Dresdener Bank"

${ }^{178}$ Es gilt das Auswirkungsprinzip eines Zusammenschlusses im Sinne von § 130 Abs. 2 GWB.

${ }^{179}$ Begriff de minimis-Klausel ersetzt den bisherigen Begriff der Anschlussklausel
} 
und Verbrauchssteuer werden nicht berücksichtigt. Darüber hinaus bleiben bei Konzernen Innenumsatzerlöse außer Betracht. Zu beachten sind außerdem die Sonderregelungen einzelner Branchen ${ }^{180}$, die unter $\S 38$ Abs. 2 ff. beschrieben werden.

3. De minimis-Klausel: Die de minimis-Klausel ist erfüllt, soweit ein Unternehmen nicht im Sinne des $\S 36$ Abs. 2 GWB abhängig ${ }^{181}$ ist und im letzten Geschäftsjahr weltweite Umsatzerlöse von weniger als zwanzig Millionen Deutsche Mark erzielt hat. Obwohl es bei einem Zusammenschluss mit einem Großunternehmen zum Überschreiten der in $\S 35$ Abs. 1 genannten Umsatzschwellen käme, soll kleineren und mittleren Unternehmen ein zusätzlicher Weg eröffnet werden, sich ungehindert Großunternehmen anzuschließen, um so Strukturwandel zu ermöglichen ${ }^{182}$.

4. Bagatellmarktklausel: Die Fusionskontrolle findet keine Anwendung, sobald es sich um einen Bagatellmarkt handelt: Ein Markt ist betroffen, auf dem seit mindestens fünf Jahren ${ }^{183}$ Waren oder gewerbliche Leistungen angeboten werden und im letzten Kalenderjahr weniger als 15 Millionen Euro umgesetzt wurden. Das Bundeskartellamt reagiert auf wettbewerbsbeschränkende Gesamtstrategien von Großunternehmen wie z.B. die künstliche Marktaufteilung in Teilmärkte unter 15 Mio. Euro ${ }^{184}$ mit der Bündeltheorie, wonach im Rahmen der Bagatellmarktklausel benachbarte, im wesentlichen übereinstimmende Märkte zusammenzurechnen sind ${ }^{185}$.

\subsubsection{Die Abgrenzung des relevanten Marktes}

Besonders schwierig erweist sich die Abgrenzung des relevanten Marktes. Die Abgrenzung des relevantes Marktes ist von entscheidender Bedeutung, denn erst die Bestimmung des Marktes lässt Aussagen über beispielsweise Größe und Anzahl der Anbieter oder die Höhe der jeweilige Marktanteile der betroffenen Unternehmen und der daraus evtl. resultierenden marktbeherrschenden Stellung $\mathrm{zu}^{186}$ (dazu ausführlich in Kapitel 7.1).

\footnotetext{
${ }^{180}$ Geregelt sind hier der Handel mit Waren, Versicherungsunternehmen, Kredit- und Finanzinstitute, Bausparkassen, Zeitungen und Rundfunk.

${ }^{181}$ Klausel bezieht sich lediglich auf selbständige Unternehmen. Eine Inanspruchnahme durch Konzernkonstruktionen wird abgelehnt. Vgl.: EMMERICH 1999, S. 278

182 Begr. zum RegE (BT-Dr. VI/2520), S. 32

${ }^{183}$ In der Regel ist innerhalb dieser Zeitspanne die Entwicklungsphase abgeschlossen. Vgl.: BUNDESKARTELLAMT, TB 1987/88, S.11, 73f. „Bertelsmann/IBM"

184 BGHZ 81, 56 (62f.) = NJW 1981, 2699 "Transportbeton Sauerland"

185 Vgl. EMMERICH 1999, S. 280

${ }^{186}$ Vgl. Berg 1999, S. 346
} 
Die Abgrenzung des für die Beurteilung des Zusammenschlusses relevanten Marktes erfolgt nach dem Wettbewerbsrecht in sachlicher, räumlicher und mitunter auch zeitlicher Hinsicht.

\subsubsection{Der sachlich relevante Markt}

Die Rechtsprechung orientiert sich vornehmlich an dem Bedarfsmarktkonzept, dem Kriterium der funktionellen Austauschbarkeit aus Sicht der Marktgegenseite ${ }^{187}$. Angebotsmacht wird demnach unter anderem durch die Ausweichmöglichkeiten der Abnehmer, Nachfragemacht durch die Ausweichmöglichkeit der Anbieter bestimmt ${ }^{188}$. Bei der Prüfung von Angebotsmacht sind Waren oder Leistungen einer bestimmten Art einzubeziehen, die entweder gleichwertig sind oder Substitutionscharakter besitzen ${ }^{189}$. Nicht die physikalische oder chemische Identität - also objektive Eigenschaften - sondern subjektive Austauschbarkeit bestimmt den sachlich relevanten Markt ${ }^{190}$. Die Judikative bedient sich u.a. folgender Kriterien ${ }^{191}$ : Umsatz, Eigenschaft und Verwendungszweck aber auch Preisunterschiede, so dass z.B. Luxusartikel einen getrennten Markt gegenüber Standardprodukten bilden, obwohl qualitative Unterschiede nicht feststellbar sind ${ }^{192}$. Bei Bestimmung der Nachfragemacht wird ebenfalls auf das Bedarfsmarktkonzept zugegriffen, jedoch ist hier die Austauschbarkeit aus Sicht der Anbieter entscheidend. Aus Angebotsperspektive gehören also sämtliche Nachfrager und Produkte, auf die ohne übermäßige Schwierigkeiten ausgewichen werden kann, einschließlich der Vertriebswege und Absatzmärkte ${ }^{193}$, zu demselben Markt. Sobald eine Produktion ohne wesentlichen Aufwand umgestellt werden kann, zählen solche Produkte zum sachlich relevanten Markt ${ }^{194}$. Exemplarisch sind nachfolgend Beispiele für eigenständige sachlich relevante Märkte aufgeführt:

- Weizen- und Roggenmehl ${ }^{195}$,

- Schnittblumen und Zierpflanzen ${ }^{196}$,

\footnotetext{
187 Vgl. RITTNER 1989, S. 169

188 Vgl. LANGEN/BUNTE, § 22, Rdnr. 11

189 WuW/E BGH 1435, 1440 „Vitamin B-12"

190 WuW/E BKartA 1189, 1190 „Handpreisaufzeichnungsgerät"

${ }^{191} \mathrm{Vgl.} \mathrm{BURKHARDT} 1995$, S. 113

192 WuW/E OLG 3577, 3584 „Hussel-Mara”; WuW/E OLG 4167, 4169 „Kampffmeyer-Plange"

193 Vgl. BURKHARDT 1995, S. 114

194 Vgl. LANGEN/BUNTE, § 22, Rdnr. 23

195 WuW/E BGH 2575, 2577 „Kampffmeyer/Plange”
} 
- Bauaufträge oberhalb und unterhalb eines bestimmten Auftragsvolumens ${ }^{197}$,

- Kali als Einzeldünger gegenüber Industriekali ${ }^{198}$,

- Transportbeton gegenüber Ortbeton ${ }^{199}$.

\subsubsection{Der räumlich relevante Markt}

Die räumlich relevante Marktabgrenzung bezieht sich im Gegensatz zu der sachlichen Marktabgrenzung auf ein bestimmtes Gebiet und berücksichtigt die Austauschmöglichkeiten aus Sicht der Abnehmer ${ }^{200}$. Strittig ist die größtmögliche Abgrenzung des räumlichen Marktes. So erkannte die Rechtspraxis das Bundesgebiet als grundsätzlich größte territoriale Einheit an, während Auslandsmärkte, selbst wenn sie mit dem deutschen Markt bereits eine Einheit bildeten, nicht berücksichtigt wurden ${ }^{201}$. Mit der sechsten GWB-Novelle öffnete sich der Gesetzgeber den globalen Anforderungen und erweiterte den maximalen räumlich relevanten Markt auf den Weltmarkt ${ }^{202}$. Der Markt für Zivilflugzeuge mit den Duopolisten Airbus und Boeing induziert die Notwendigkeit dieser Neuregelung ${ }^{203}$. Die Untergrenzen für kleinstmögliche, räumlich relevante Märkte werden meist durch Transportkosten der Produkte ${ }^{204}$, regionale Präferenzen (Lokalzeitung ${ }^{205}$ ) oder Nachfrageverhalten (Einzelhandel ${ }^{206}$ ) bestimmt.

\subsubsection{Der zeitlich relevante Markt}

Die zeitlich relevante Marktabgrenzung findet in der Fusionskontrolle nur eine geringe Anwendung. Sie ist heranzuziehen, wenn Unternehmen aufgrund vorübergehender Umstände eine ebenso temporäre Machtposition erlangen können. Als Beispiele sind

\footnotetext{
196 WUW/E OLG 2862f. "Rewe/Florimex"

197 WuW/E DE-R 94 „Hochtief/Philipp Holzmann"

198 WuW/E BKartA 2885 „Kali + Salz/PCS"

199 WuW/E BKartA 1771, 1774 "Transportbeton"

200 Vgl. MÜLLER-URI, Rdnr. 217

${ }^{201}$ BGHZ 131, 107 = NJW 1996, 595

202 Vgl. dazu BundeSKARTELLAMT (2001): Tätigkeitsbericht, S. 20

203 Begr. zum RegE, BT-Dr. 13/9720, S. 56

${ }^{204}$ WuW/E BGH 1655 (1658) „Zementmehlanlagen II"

205 WuW/E OLG 4547 „Lübecker Nachrichten/Stormarner Tageblatt”

206 WuW/E OLG 4657 „Kaufhof/Saturn"
} 
Messen, Volksfeste oder sonstige Veranstaltungen wie eine Olympiade oder Fußballländerspiele ${ }^{207}$ zu nennen.

\subsubsection{Eingriffskriterium}

Ein Zusammenschluss wird vom Bundeskartellamt untersagt, wenn zu erwarten ist, dass er eine marktbeherrschende Stellung begründet oder verstärkt ( $\S 36$ GWB). Der Begriff der Marktbeherrschung wird in § 19 Abs. 2 GWB definiert: "Ein Unternehmen ist marktbeherrschend, soweit es als Anbieter oder Nachfrager einer bestimmten Art von Waren oder gewerblichen Leistungen (1.) ohne Wettbewerber ist oder (2.) keinem wesentlichen Wettbewerb ausgesetzt ist oder (3.) eine im Verhältnis zu seinen Wettbewerbern überragende Marktstellung hat".

In der Fusionskontrollpraxis ist der ersten Variante eine nur geringe Bedeutung beizumessen $^{208}$. Hierunter fallen Zusammenschlüsse, wodurch das Fehlen jeglichen Wettbewerbs (kein weiterer konkurrierender Anbieter bzw. Nachfrager) hervorgerufen wird $^{209}$. Die zweite Variante - kein wesentlicher Wettbewerb - erfasst hauptsächlich Oligopole und Einzelmarktbeherrschungen mit hohen Marktanteilen ${ }^{210}$. Bislang gab es Anwendungen im Bereich der Energieversorgung und der Pressewirtschaft ${ }^{211}$. Die weitaus größte Relevanz in der Anwendung der Fusionskontrolle erlangt die dritte Variante $^{212}$ : eine im Verhältnis zu den Wettbewerbern überragende Marktstellung. Dabei wird in erster Linie geprüft, ob aus einem Zusammenschluss eine derartige Verschlechterung der Marktstruktur resultiert, dass für die fusionierenden Unternehmen ein übermäßiger, vom Wettbewerb nicht mehr hinreichend kontrollierter Verhaltensspielraum entsteht ${ }^{213}$. Nachfolgend werden die Prüfungskriterien erläutert, die das Bundeskartellamt bei der Beurteilung von Unternehmenszusammenschlüssen berücksichtigt ${ }^{214}$. Hervorzuheben ist, dass bei der Beurteilung der Marktstellung eines Unternehmens nicht allein ein Strukturkriterium ausschlaggebend ist, sondern eine Gesamt-

\footnotetext{
207 Vgl. EMMERICH 1999, S. 184 und der dort zitierten Literatur

208 Vgl. BUNDESKARTELLAMT 2000: Auslegungsgrundsätze, S. 5

${ }^{209}$ Vgl. BUNDESKARTELLAMT 2000: Auslegungsgrundsätze, S. 5

${ }^{210} \mathrm{Vgl.}$ BUNDESKARTELLAMT 2000: Auslegungsgrundsätze, S. 6

${ }^{211}$ WUW/E OLG 4835, 4855 „WAZ/Iserlohner Kreisanzeigen” (1991), WuW/E BkartA 2701, 2707 „Stadtwerke Garbsen" (1994), WuW/E BkartA 2591 „Fresenius/Schiwa” (1993)

212 Vgl. EMMERICH 1999, S. 305

${ }^{213}$ BGHZ 79, 62 = NJW 1981, 1786 „Klöckner/Becorit”, KG WuW/E OLG 3759 „Pillsbury/SonnenBassermann"

214 in Anlehnung an: BundeSKARTELLAMT 2000: Auslegungsgrundsätze, S. $11 \mathrm{ff}$.
} 
betrachtung aller relevanten Umstände durchgeführt wird ${ }^{215}$. Das kann dazu führen, dass Unternehmenszusammenschlüsse trotz hoher Marktanteile nicht untersagt werden, weil andere Faktoren wie z.B. potentieller Wettbewerb aus dem Ausland keine unkontrollierten wettbewerblichen Verhaltensspielräume erwarten lassen ${ }^{216}$.

\subsubsection{Marktanteil}

Marktanteile sind besonders bei horizontalen Zusammenschlüssen das ausschlaggebende Kriterium bei der Entscheidungsfindung ${ }^{217}$ und werden auf Grundlage des relevanten Marktes ermittelt. Ein erheblicher ${ }^{218}$ Marktanteil deutet auf eingeschränkte Ausweichmöglichkeiten der Marktgegenseite und einen erhöhten Verhaltensspielraum eines Unternehmens hin. Neben der absoluten Höhe sind der Marktanteilsabstand zum nächsten Wettbewerber, die Verteilung der Marktanteile im übrigen und die Entwicklung der Marktanteile über mehrere Perioden zu berücksichtigen ${ }^{219}$. Die Berechnung der Marktanteile orientiert sich am in Umsatz ausgedrückten Wertanteil und nur in Einzelfällen an anderen Parametern ${ }^{220}$.

\subsubsection{Ressourcenbetrachtung (Finanzkraft)}

Durch überlegende Finanzkraft können beispielsweise bei den Wettbewerbsparametern Preis, Investition, Forschung und Werbung Verhaltensspielräume erlangt werden. Neben dem Aufbau eines breiten Produktionsprogramms ${ }^{221}$ und der Ausnutzung von technologischen Ressourcen ${ }^{222}$ kann vor allem bei konglomeraten Fusionen ein Entmutigungs- und Abschreckungseffekt (z.B. aufgrund der Möglichkeit von Gewinntransfer und Verlustausgleich über verschiedene Märkte hinweg ${ }^{223}$ ) entstehen. Als Parameter gelten hier in erster Linie Umsätze, Cash-Flows, Gewinne, liquide Mittel, Jahresüber-

\footnotetext{
${ }^{215}$ BT-Drs. VI/2520, S.21 f., grundlegend WuW/E BGH 1504 "GKN-Sachs" (1978) und WuW/E BGH 3037, 3041 „Raiffeisen" (1995)

${ }^{216}$ Vgl. BUNDESKARTELLAMT, Beschluss v. 18.01.1999 "Babcock/Steinmüller"

217 Vgl. BURKHARDT 1995, S. 201

${ }^{218}$ Es gibt keine absolute Schwelle im deutschen Kartellrecht. Grundsätzlich ist jedoch ein Marktanteil von $33 \%$ und mehr als bedenklich einzustufen (Marktbeherrschungsvermutung § 19 Abs. 3 Satz 1 GWB)

${ }^{219}$ Vgl. auch BOSCH/SCHÜTZ 2000, § 36, Rdnr. 79

${ }^{220}$ z.B. Passagieraufkommen beim Luftverkehr: WuW/E BKartA 2335, 2345ff. "Daimler/MBB" (1989)

${ }^{221}$ WuW/E BGH 2521, 2530 f. "Zahnradfabrik Friedrichshafen/Allison" 1993

222 BKartA, Beschluss v. 20.09.1999 "Henkel/Luhns", S. 27 ff.

${ }^{223}$ WuW/E BKartA 1753, 1758 „bituminöses Mischgut" (1978)
} 
schüsse, aber auch der Zugang zu nationalen und internationalen Kapitalmärkten sowie getätigte Investitionen und Gewinnmöglichkeiten ${ }^{224}$.

\subsubsection{Zugang zu den Beschaffungs- und Absatzmärkten}

Diesem Merkmal wird erhöhte Aufmerksamkeit bei der Beurteilung von vertikalen Kooperationen geschenkt. Eine marktbeherrschende Stellung ist dadurch zu begründen, dass durch eine vertikale Fusion für Mitbewerber der Zugang zu Beschaffungs- und Absatzmärkten erschwert oder gar verschlossen werden kann (Erhöhung der Marktzutrittsschranken). Im Zuge der vertikalen Integration wird die Wettbewerbsfähigkeit hauptsächlich bei knappen Ressourcen/Rohstoffen vermindert ${ }^{225}$. Auch eine Verflechtung mit Lieferanten oder Abnehmern sowie der Ausbau bestehender Lieferbeziehungen können marktrelevante Wirkungen entfalten, indem im Zuge einer Fusion die Abnahme der eigenen Produkte sichergestellt und die Nachfrage des Erwerbers dem Markt dauerhaft entzogen wird ${ }^{226}$. Einen weiteren Wettbewerbsvorteil kann ein Unternehmen dadurch ausnutzen, indem es durch vertikale Fusion ein umfassenderes Sortiment anbietet und somit als Komplettanbieter auftreten kann bzw. die Möglichkeit zur Systementwicklung ${ }^{227}$ (vor allem bei komplementären Gütern) im Sinne einer Verdrängungsstrategie nutzt. Weitere Vorteile werden darin gesehen, dass durch eine vertikale Fusion eine hohe Reputation oder eine besondere Markengeltung ${ }^{228}$, ein eigenes Filialnetz ${ }^{229}$, eine etablierte Vertriebslogistik ${ }^{230}$ oder eine gesicherte Vorproduktbeschaffung ${ }^{231} \mathrm{zu}$ Lasten der Mitbewerber ausgenutzt werden kann.

\subsubsection{Marktzutrittsschranken/Potentieller Wettbewerb}

Der Prüfung von Marktzutrittschancen bzw. potentiellem Wettbewerb kommt in der Zusammenschlusskontrolle große Bedeutung zu. Ein Unternehmen verfügt nur über einen geringen kontrollierbaren Verhaltensspielraum, wenn die Marktzutrittsschranken niedrig sind und somit potentieller Wettbewerb sehr wahrscheinlich ist ${ }^{232}$. Es wird in

\footnotetext{
224 BundeskartelLamt Beschluss v. 03.03.2000 "Cisco/IBM", S. 21

${ }^{225}$ Vgl. RuPPELt in: LANGEN/BUNTE, § 24, Rdnr. 30

${ }^{226}$ WuW/E BKartA 2829, 2837f. „Kolbenschmidt” (1995), WuW/E BGH 1949, 1952 f. „Braun-Almo” (1982)

227 BundeSKARTELLAMT, Beschluss v. 27. Mai 1999 „Federal Mogul/Alcan”.

${ }^{228}$ WuW/E BKartA 2370, 2375 „Melitta-Kraft” (1989)

229 WuW/E OLG 2849, 2859 "Lufthansa/first" (1982)

230 BKartA, Beschluss v. 25. Februar 1999 "Lekkerland/Tobaccoland"

231 WuW/E BGH 2575, 2581 „Kampfmeyer-Plange"

232 Vgl. BUNDESKARTELLAMT 1997, Tätigkeitsbericht 1995/96, S. 102
} 
der Literatur zwischen drei Formen von Marktzutrittschancen differenziert: a) gesetzliche Marktzutrittschancen: Hierunter werden Rechtsnormen oder Verwaltungsvorschriften verstanden, die den Marktzutritt oder Parametereinsatz von Unternehmen beschränken und dadurch potentiellen Wettbewerb zugunsten der etablierten Unternehmen verringern ${ }^{233}$ (z.B. Patentschutz), b) strukturelle Marktzutrittsschranken: Sie beruhen auf zutrittsbehindernden Wirkungen, die durch folgende Faktoren hervorgerufen werden können: Ressourcen (Abschreckungswirkung gegenüber potentiellen Wettbewerbern), Marktentwicklung (Marktphase), Transportkosten/ Abnehmernähe, Economies of scale, Economies of scope, technische Marktzutrittsschranken, c) strategische Marktzutrittsschranken: Hier versuchen etablierte Unternehmen durch bewusstes Marktverhalten, Zutrittsschranken aufzubauen. Beispiele hierfür wären langfristige Lieferverträge mit Abnehmern, Ausschließlichkeitsverträge, Industriestandards für komplementäre Güter oder die Schaffung von Nachfragepräferenzen. Zudem können bei ausländischen Märkten weitere Markzutrittsschranken bestehen, die durch Handelshemmnisse, Sprachbarrieren, Präferenz für inländische Anbieter, Beschaffungsverhalten staatlicher Abnehmer usw. hervorgerufen werden ${ }^{234}$.

\subsubsection{Substitutionswettbewerb}

Der preisliche Verhaltensspielraum eines Unternehmen wird auch dadurch eingeschränkt, dass seitens der Konsumenten Ausweichmöglichkeiten auf nicht identische aber funktionell ähnliche Substitutionsprodukte bestehen (z.B. Butter und Margarine). In der fusionsrechtlichen Bewertung hat der Substitutionswettbewerb insofern Bedeutung, als dass es bei einem Zusammenschluss mit einem Substitutionsgüter produzierenden Unternehmen zu einer Verstärkung einer bestehenden marktbeherrschenden Stellung kommen kann ${ }^{235}$ und somit Ausweichmöglichkeiten auf der Nachfrageseite geringer werden.

\subsubsection{Gegengewichtige Marktmacht}

Umstritten ist die wettbewerbliche Einordnung der gegengewichtigen Marktmacht ${ }^{236}$. Wenn der Zugang zu alternativen Absatz- oder Beschaffungsmärkten durch die Struktur der Marktgegenseite eingeschränkt ist, ist auch die Möglichkeit der Marktgegenseite, auf andere Unternehmen auszuweichen, limitiert. Zu prüfen sind alle wettbewerbli-

\footnotetext{
233 BUNDESKARTELLAMT 2000, Auslegungsgrundsätze, S. 26

234 BUNDESKARTELLAMT 2000, Auslegungsgrundsätze, S. 31

235 WuW/E BGH 1536 f. "Erdgas Schwaben” (1978), BKartA Beschluss v. 15.04.1999 „Oberbayrisches Volksblatt/RFR Regionales Fernsehen", S. 5
} 
chen Reaktionsmöglichkeiten der Marktgegenseite gegenüber dem marktbeherrschenden Unternehmen, wie beispielsweise die Möglichkeit einer Umstellung der Vertriebsstrategie oder einer Erschließung von Absatz- und Bezugsalternativen durch andere unternehmerische Dispositionen ${ }^{237}$ (ausführlich in Kapitel 7.2).

\subsubsection{Sonderbetrachtung von Oligopolen}

Gemäß § 19 Absatz 2 Satz 2 GWB sind zwei oder mehrere Unternehmen marktbeherrschend, soweit zwischen innen für eine bestimmte Art von Waren oder gewerblichen Leistungen kein wesentlicher Wettbewerb besteht. Eine Gesamtheit von Unternehmen gilt als marktbeherrschend, wenn sie

1. aus drei oder weniger Unternehmen besteht, die zusammen einen Marktanteil von 50 von Hundert erreichen, oder

2. aus fünf oder weniger Unternehmen besteht, die zusammen einen Marktanteil von zwei Dritteln erreichen,

es sei denn, die Unternehmen weisen nach, dass die Wettbewerbsbedingungen zwischen ihnen wesentlichen Wettbewerb erwarten lassen oder die Gesamtheit der Unternehmen im Verhältnis zu den übrigen Wettbewerbern keine überragende Marktstellung hat.

Wie die aufgeführten Paragraphen verdeutlichen, schenkt der Gesetzgeber den Oligopolisten besondere Beachtung. Oligopole werden per se nicht als schädlich angesehen, vielmehr verfolgt die Fusionskontrollverordnung des GWB einen strukturorientierten Ansatz zur Verhinderung von Gefährdungslagen durch Oligopole ${ }^{238}$. Eine Wettbewerbsprüfung erfolgt in dreifacher Hinsicht: a) Der relevante Markt weist Wettbewerbsbedingungen auf, welche den Unternehmen erlauben von Wettbewerbshandlungen abzusehen, und wettbewerbsbeschränkendes Verhalten begünstigen (Prüfung des Binnenwettbewerbs), b) sofern neben dem Oligopol weitere Unternehmen auf dem Markt tätig sind, besteht zwischen diesen und dem Oligopol kein wesentlicher Wettbewerb, und es kommt zu einer überragenden Marktstellung des Oligopols (Prüfung des AuBenwettbewerbs), c) Einsatz der Wettbewerbsparameter zur Erfüllung der Wettbewerbsfunktionen (Wettbewerbsgeschehen).

\footnotetext{
${ }^{236}$ BUNDESKARTELLAMT 2000, Auslegungsgrundsätze, S. 33

237 Vgl. BURKHARDT 1995, S. 206

${ }^{238}$ Vgl. BUNDESKARTELLAMT 2000, Auslegungsgrundsätze, S. 43
} 
Die Prüfungskriterien entsprechen im wesentlichen den Kriterien der Einzelmarktbeherrschung, dennoch erweist sich eine Beurteilung als außerordentlich schwierig. So ist beispielsweise der Nachweis fehlenden Binnenwettbewerbs beim Erwerb durch einen Oligopolaußenseiter schwer begründbar, wenn vor dem Zusammenschluss erhebliche Investitionen in langfristige Wachstumsstrategien getätigt wurden ${ }^{239}$ oder wenn in der Vergangenheit ein Preisverfall zu beobachten war, der zu Marktanteilsrückgängen der Oligopolisten zugunsten der Oligopolaußenseiter führte ${ }^{240}$. Sobald dagegen im Zeitverlauf ein gleichförmiges Preissetzungsverhalten (Parallelverhalten) nachweisbar ist, entspricht dies keinem wesentlichen Wettbewerb und ein Zusammenschluss ist zu untersagen ${ }^{241}$.

\subsubsection{Das Verfahren der Fusionskontrolle}

Das Verfahren der Fusionskontrolle wird in den $\S \S 40$ und 41 GWB geregelt und kann in Vor- und Hauptprüfverfahren unterteilt werden (vgl. Abbildung 21). Im Vorprüfverfahren entscheidet das Bundeskartellamt innerhalb eines Monats, ob das Zusammenschlussvorhaben als unbedenklich einzustufen ist und deshalb ohne weiteres freigegeben werden kann oder eine intensive Prüfung notwendig ist, und somit das Hauptprüfverfahren eröffnet wird ( $\S 40$ Abs. 1). In diesem Fall werden die beteiligten Unternehmen durch einen sogenannten „Monatsbrief" benachrichtigt ${ }^{242}$. Erst im Hauptprüfverfahren, für das dem Bundeskartellamt höchstens vier Monate seit Eingang der vollständigen Anmeldeunterlagen zur Verfügung stehen (§ 40 Abs. 2 Satz 2 GWB), entscheidet das Amt endgültig über die Freigabe oder Untersagung des Zusammenschlus$\operatorname{ses}^{243}$. Eine Fristverlängerung kann entweder mit Zustimmung der "anmeldenden" Unternehmen erfolgen oder wenn entgegen dem Vollzugsverbot ${ }^{244}$ ( $\S 41$ Abs. 1) der Zusammenschluss vorzeitig vollzogen wird. Neben der Alternative, einen Zusammenschluss freizugeben bzw. vollständig zu untersagen, kann das Bundeskartellamt eine Freigabe auch mit Bedingungen und Auflagen versehen. Es darf sich allerdings nur um strukturelle Bedingungen oder Auflagen handeln ${ }^{245}$. Mögliche Gegenstände von Bedin-

\footnotetext{
${ }^{239}$ BKartA, Beschluss v. 02.07.1999 "Corning/BICC", S.17 f.

240 BKartA, Beschluss v. 18.04.2000 "Gemplus/ODS", S. 13

${ }^{241}$ Vgl. BOSCH/SCHÜTZ 2000, § 36, Rdnr. 106

242 Vgl. BOSCH/SCHÜTZ 2000, § 40, Rdnr. 1

${ }^{243}$ Vgl. EMMERICH 1999, S. 334

244 Das Bundeskartellamt kann auf Antrag eine Befreiung vom Vollzugsverbot erteilen, wenn wichtige Gründe geltend gemacht werden, insbesondere um schweren Schaden von einem beteiligten Unternehmen oder von Dritten abzuwenden ( $\$ 41$ Abs. 2 GWB)

${ }^{245}$ Eine detaillierte Darstellung verschafft die Dissertation von UHLIG 1996
} 
gungen oder Auflagen können u.a. die Abgabe von Beteiligungen, die Auflösung von bzw. der Austritt aus wettbewerbsbeschränkenden Vereinbarungen, die Veränderung der Organisation oder der Willensbildung beteiligter Unternehmen sowie Änderungen in der Art und Weise der Ausübung ihrer wirtschaftlichen Tätigkeiten oder aber auch die Aufnahme eines Mitbewerbers bei der Gründung eines Gemeinschaftsunternehmens sein ${ }^{246}$. Um den Bedenken der Kartellbehörde entgegenzukommen, besteht die Möglichkeit, sogenannte Vorfristzusagen zu gewähren. Als Vorfristzusagen werden Zusagen bezeichnet, die die beteiligten Unternehmen schon während der Prüfungsverfahren zugestehen und deren Erfüllung mithin schon vor dem Zeitpunkt der Bundeskartellamtentscheidung in Kraft tritt. Umstritten sind dagegen die Zulässigkeit von Nachfristzusagen, d.h. von Zusagen, die erst nach Ablauf der verschiedenen Untersagungsfristen (z.B. Veräußerung von Beteiligungen binnen einer Frist von zwei Jahren $^{247}$ ) umgesetzt werden ${ }^{248}$. Bevor ein Zusammenschluss von der Kartellbehörde untersagt bzw. mit Bedingungen oder Auflagen freigegeben wird, werden den Unternehmen Anhörungen zur Gegenargumentation gewährt ${ }^{249}$.

Gegen Verfügungen des Bundeskartellamtes kann vor dem Oberlandesgericht in Düsseldorf ${ }^{250}$ Beschwerde eingelegt werden. Gegen die Entscheidung des Oberlandesgerichtes ist Rechtsbeschwerde beim Bundesgerichtshof möglich. Mit der sechsten GWBNovelle ist außerdem erstmals eine Klage Dritter gegen Freigabeentscheidungen möglich, sobald eine Betroffenheit begründet werden kann ${ }^{251}$.

Eine Entflechtungsregelung kennt das GWB nicht. Anders als im US-Recht kann das Bundeskartellamt somit kein Unternehmen, das durch internes Wachstum eine marktbeherrschende Stellung erlangt hat, entflechten. Ein vollzogener Zusammenschluss, den das Bundeskartellamt untersagt hat oder dessen Freigabe widerrufen wurde, ist aufzulösen ( $\S 41$ Abs. 3 GWB). Insoweit werden durch das Bundeskartellamt die zur Auflösung erforderlichen Maßnahmen angeordnet ${ }^{252}$.

\footnotetext{
246 Vgl. EMMERICH 1999, S.341 f.

247 WuW 1997, 883; 1998, 356, 358, 566

248 Vgl. EMMERICH 1999, S. 336

249 Vgl. BUNDESKARTELLAMT 1999, Tätigkeitsbericht, S. 13

250 Vor dem Umzug von Berlin nach Bonn war das Kammergericht Berlin zuständig

${ }^{251}$ Begr. zum RegE, BT-Dr. 13/9720, S. 44

252 Vgl. BundeSKARTELLAMT 1999, Tätigkeitsbericht, S. 16
} 
Abbildung 21: Verfahren der Zusammenschlusskontrolle

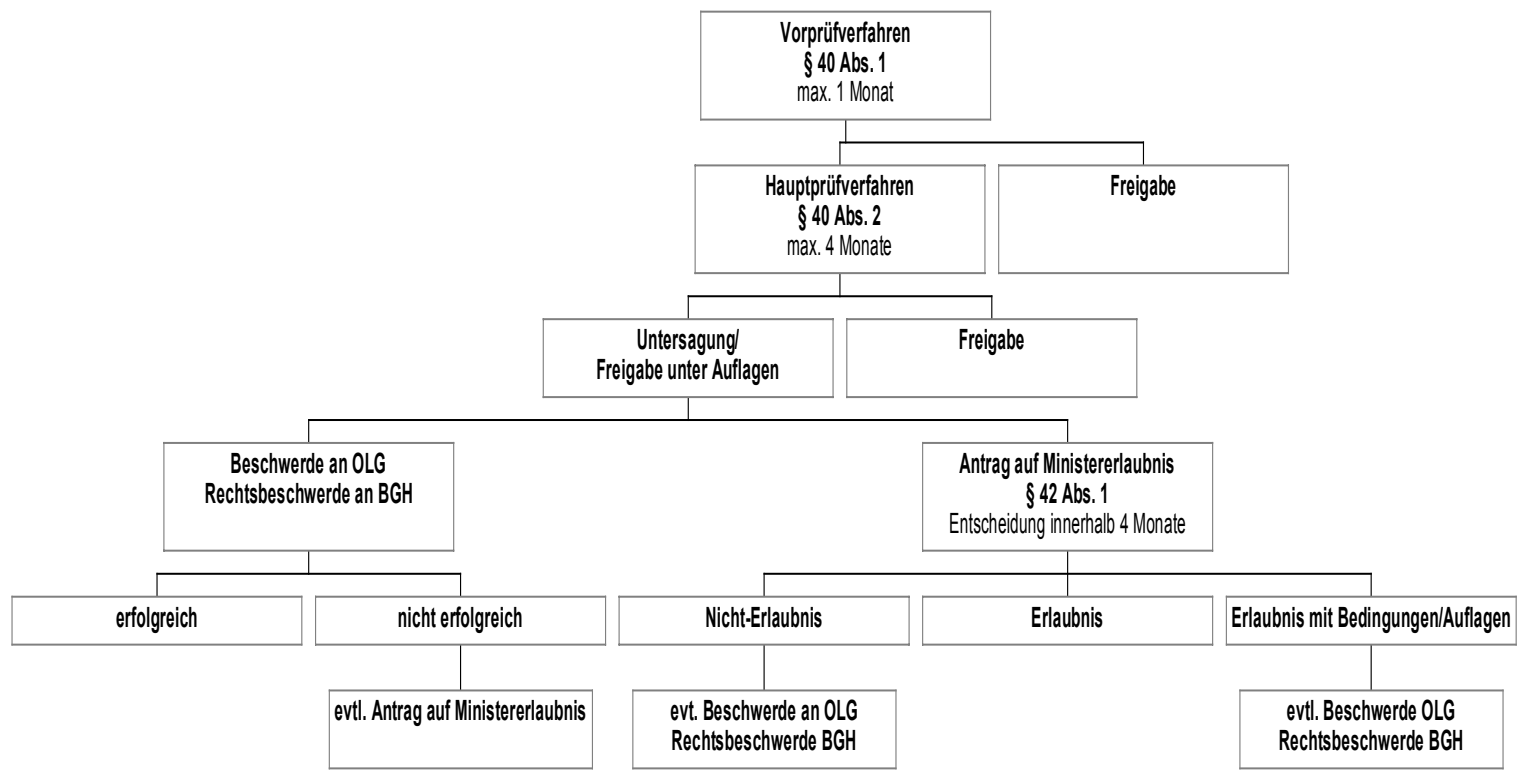

Quelle: eigene Darstellung

\subsubsection{Ministererlaubnis}

Der Bundesminister für Wirtschaft kann auf Antrag einen vom Bundeskartellamt untersagten Zusammenschluss erlauben, wenn im Einzelfall die Wettbewerbsbeschränkung infolge des Zusammenschlusses von gesamtwirtschaftlichen Vorteilen überwogen wird oder der Zusammenschluss durch ein Interesse der Allgemeinheit gerechtfertigt ist ${ }^{253}$ (§ 42). Der Minister kann allerdings eine Fusion nur erlauben, wenn sie aus staats-, wirtschafts- oder gesellschaftspolitischen Gründen großen Gewichts gerechtfertigt ist. Diese Gründe müssen konkret nachgewiesen werden. Einzelwirtschaftliche Vorteile des Zusammenschlusses für die beteiligten Unternehmen finden nur dann Berücksichtigung, wenn es sich dabei zugleich um "gesamtwirtschaftliche" Vorteile handelt, an deren Erzielung ein öffentliches Interesse besteht ${ }^{254}$. In der Rechtspraxis gab es bislang folgende Beispiele, die gesamtwirtschaftliche Vorteile bzw. Interessen der Allgemeinheit begründeten ${ }^{255}$ :

- Erhaltung des vollen technischen Potentials oder Know-hows, das ohne Zusammenschluss verloren ginge; Erhaltung eines Teams hochspezialisierter Fachkräfte ${ }^{256}$,

\footnotetext{
253 Vgl. MÖSCHEL 1983, S. 567

254 BMWi, WuW/E BWM 149 „Kapal”; WuW/E BWM 213 = Die AG 1993, 238 „Baywa/WLZ"

255 Vgl. BosCH/SCHÜTZ 2000, § 42, Rdnr. 9

${ }^{256}$ BMWi, Verfügung vom 01.08.1977, WuW/E 159 ff., 162/163 „Thyssen/Hüller"
} 
- Beseitigung von Strukturkrisen ${ }^{257}$,

- Sanierung von Unternehmen, wenn deren Fortbestand im Gemeinwohl liegt ${ }^{258}$,

- Sicherung, Schaffung oder Zuwachs gesamtwirtschaftlich notwendiger Wirtschaftsbereiche, beispielsweise Energieversorgung ${ }^{259}$,

- Rationalisierungsvorteile (ausnahmsweise berücksichtigt) ${ }^{260}$,

- die Stärkung der internationalen Wettbewerbsfähigkeit ${ }^{261}$,

- die Entlastung öffentlicher Haushalte durch Privatisierung (Subventionsrisiken $)^{262}$,

- Sicherung von Arbeitsplätzen ${ }^{263}$,

- militärpolitische Gründe ${ }^{264}$.

Ebenso wie bei der Beurteilung durch das Bundeskartellamt kann das Bundesministerium für Wirtschaft bei Anwendung der Ministererlaubnis den Zusammenschluss mit Auflagen und Bedingungen freigeben ${ }^{265}$.

\subsubsection{Abwägungsklausel}

§ 36 Abs. 1 GWB erlaubt Zusammenschlüsse trotz Begründung oder Verstärkung einer marktbeherrschenden Stellung, wenn den beteiligten Unternehmen der Nachweis gelingt, dass mit dem Zusammenschluss Verbesserungen der Wettbewerbsbedingungen verbunden sind, die die Nachteile der Marktbeherrschung nicht nur ausgleichen sondern überkompensieren. Berücksichtigt werden dabei, anders als bei der Anwendung

\footnotetext{
${ }^{257}$ BMWi, Verfügung vom 22.07.1997, WuW/E 225, 226/227 „Kali + Salz/PCS"

${ }^{258}$ BMWi, Verfügung vom 17.10.1976, WuW/E 155, 157 „Babcock/Artos"

${ }^{259}$ BMWi, Verfügung vom 05.03.1979, WuW/E 165, 171 „VEBA/BP”; BMWi, Verfügung vom 01.02.1974, WuW/E 147 "VEBA/Gelsenberg"; jeweils im Energiebereich; abgelehnt für den genossenschaftlichen Bereich in BMWi, Verfügung vom 16.06.1992, WuW/E213, 216/217 „BayWa AG/WLZ Raiffeisen AG"

${ }^{260}$ BMWi, Verfügung vom 24.01.1990, WuW/E 207, 210 „MAN/Sulzer"; BMWi, Verfügung vom 06.09.1989, WuW/E 191, 198 „Daimler/MBB"

${ }^{261}$ BMWi, Verfügung vom 06.09.1989, WuW/E 191, 197 „Daimler/MBB”; BMWi, Verfügung vom 09.12.1981, WuW/E 177ff., 179/180 „IBH/Wibau"

262 BMWi, Verfügung vom 06.09.1989, WuW/E 191, 195ff. „Daimler/MBB"

263 BMWi, Verfügung vom 09.12.1981, WuW/E 177, 180, 182 „IBH/Wibau”; BMWi, Verfügung vom 22.07.1997, WuW/E 225, 228 „Kali + Salz/PCS"

264 BMWi, Verfügung vom 06.09.1989, WuW/E 191 ff., 198, „Daimler/MBB"

${ }^{265}$ Vgl. BURKHARDT 1995, S. 217, Rdnr. 608
} 
der Ministererlaubnis, nur Verbesserungen der Wettbewerbsbedingungen; sonstige wirtschaftliche oder allgemeine Interessen sind nicht in Betracht zu ziehen ${ }^{266}$. Grundsätzlich sind bei der Anwendung der Abwägungsklausel drei Vorraussetzungen zu erfüllen: Es sind a) eine vermutliche Verbesserung der Wettbewerbsbedingungen, b) eine Kausalität zwischen den Vorteilen und dem Zusammenschluss und c) ein Überwiegen der Vorteile gegenüber den Nachteilen des Zusammenschlusses nachzuweisen. Besonderes Augenmerk gilt der Auswirkung des Zusammenschlusses auf die Mitbewerber, da die Fusionskontrolle primär die Zielsetzung verfolgt, Märkte offen zu halten und Raum für die Entfaltung von Wettbewerbsprozessen zu schaffen ${ }^{267}$. Einer Sonderstellung im Wettbewerbsrecht kommt der Sanierungsfunktion zu. Unter Sanierungsfunktion wird die Übernahme eines vor dem finanziellen Zusammenbruch stehenden Unternehmens verstanden ${ }^{268}$. Auch bei der Sanierungsfunktion müssen die Vorteile gegenüber den mit innen verbundenen Nachteilen überwiegen ${ }^{269}$. Dies ist nur der Fall, wenn bei Zusammenbruch des gefährdeten Unternehmens eine noch schwerwiegendere Verschlechterung der Wettbewerbsbedingungen als infolge der Sanierungsfusion droht oder wenn durch den Zusammenschluss eine größere Zahl selbständiger Unternehmen erhalten werden kann als im Falle einer Untersagung der Fusion ${ }^{270}$. Die Beurteilungspraxis zeigt dagegen, dass eine Gegenrechnung positiver Effekte kaum stattfindet (vgl. Kapitel 6.2). Begründet wird dies damit, dass, sobald ein Verhaltensspielraum unkontrolliert ist (marktbeherrschende Stellung), die Weitergabe von Vorteilen an die Verbraucher nicht garantiert werden kann ${ }^{271}$.

Es ist in erster Linie dem Ausschuss für Ernährung, Landwirtschaft und Forsten zu verdanken, dass die Abwägungsklausel ( $\S 36$ Abs. 1) überhaupt in die sechste Novelle des GWB eingefügt wurde ${ }^{272}$. Folgende Argumente waren zur Begründung angeführt ${ }^{273}$ : „Im Bereich der landwirtschaftlichen Verarbeitungs- und Vermarktungsunternehmen, z.B. im Molkerei- und Schlachthofsektor, bestehen wettbewerbliche Benachteiligungen. Besonders vor dem Hintergrund einer wachsenden Konzentration auf der Nachfrageseite (Lebensmitteleinzelhandel) müssen auch auf den regional oft sehr begrenzten landwirtschaftlichen Erfassungsmärkten Zusammenschlüsse möglich sein,

\footnotetext{
266 Vgl. BURKHARDT 1995, S. 207, Rdnr. 581

267 KG, Die AG 1986, 294 (299) „Gruner und Jahr/Zeit; MESTMÄCKER, Pressekonzentration, S. 97 ff.

${ }^{268} \mathrm{Vgl}$. EMMERICH 1999, S. 323

${ }^{269}$ KG, WuW/E OLG 4379 = Die AG 1990, 163 (167) „Flensburger Zeitungsverlag/SH Landeszeitung"

270 BKartA, WuW/E 1571 (1581f.); Die AG 1977, 165 (168)

${ }^{271} \mathrm{Vgl.}$ BundeSKARTELLAMT 2002, Stellungnahme, S. $16 \mathrm{f}$.

272 Vgl. BOSCH/SCHÜTZ 2000, S. 18, Rdnr. 43
} 
um Wettbewerbsnachteile auf den Absatzmärkten auszugleichen. Bei der Beurteilung der wettbewerblichen Auswirkungen von Zusammenschlüssen sollte den Besonderheiten landwirtschaftlicher Märkte Rechnung getragen und die Veränderung der Wettbewerbsbedingungen gegenüber den Marktpartnern einbezogen werden (...) Dies kann insbesondere auch der Fall sein, wenn der Zusammenschluss zu Verbesserungen auf den Absatzmärkten führt, die die Nachteile in bezug auf die Erfassungsmärkte überwiegen. Dies dürfte insbesondere dann nahe liegen, wenn die betroffenen landwirtschaftlichen Verarbeitungsunternehmen eine nur relativ schwache Marktstellung als Anbieter haben, die entweder die Folge überlegener Marktmacht der nachfragenden Unternehmen des Lebensmitteleinzelhandels oder die Folge eines hohen Konzentrationsgrades konkurrierender Anbieter innerhalb und außerhalb des Geltungsbereiches dieses Gesetzes ist."

\subsubsection{Landwirtschaft als Ausnahmebereich}

In § 28 ff. GWB schränkt der Gesetzgeber die Anwendung des Kartellrechtes für bestimmte Wirtschaftsbereiche ein. Sonderegelungen gelten für die Landwirtschaft ( $\S$ 28 ), die Kredit- und Versicherungswirtschaft ( $\$ 29$ ), die Urheberrechtsverwertungsgesellschaften ( $\S 30$ ) und seit der sechsten GWB-Novelle auch für den Sport ( $\S 31$ ). Die genauen Reglungen für die Landwirtschaft und die Erzeuger- und Verarbeitungsbetriebe landwirtwirtschaftlich erzeugter Produkte (Agrar- und Ernährungswirtschaft im Sinne des EG-Gründungsvertrages Anhang $\mathrm{II}^{274}$ ) kann dem Paragraphen 28 GWB entnommen werden. Allerdings bezieht sich die Sonderregelung lediglich auf $\S 1$ GWB, also auf das Verbot von Vereinbarungen zwischen miteinander im Wettbewerb stehenden Unternehmen, Beschlüsse von Unternehmensvereinigungen und aufeinander abgestimmte Verhaltensweisen, die eine Verhinderung, Einschränkung oder Verfälschung des Wettbewerbs bezwecken oder bewirken. Eine explizite Ausnahmeregelung im Anwendungsbereich der Zusammenschlusskontrolle gibt es nicht. Unternehmen der Agrar- und Ernährungswirtschaft genießen somit keinen Sonderstatus, können aber im Zuge der Ministererlaubnis und der Abwägungsklausel eventuell Argumente vortragen, die auf ein übergeordnetes Interesse der Allgemeinheit (z.B. Sicherung von Arbeitskräften in ländlichen Regionen, Sicherung der Ernährung...) abstellen.

\footnotetext{
${ }^{273}$ Vgl. WuW-Sonderheft 1998, S. $139 \mathrm{f}$.

${ }^{274}$ In der Fassung des Vertrages von Amsterdam im Anhang zu entnehmen
} 


\subsection{Das europäische Wettbewerbsrecht}

Die Basis des europäischen Wettbewerbsrechts bilden die Artikel 81 und 82 des EGVertrages. Artikel 81 beschreibt das Verbot von Kartellen: "Mit dem gemeinsamen Markt unvereinbar und verboten sind alle Vereinbarungen zwischen Unternehmen, Beschlüsse von Unternehmensvereinigungen und aufeinander abgestimmte Verhaltensweisen, welche den Handel zwischen Mitgliedstaaten zu beeinträchtigen geeignet sind und eine Verhinderung, Einschränkung oder Verfälschung des Wettbewerbs innerhalb des Gemeinsamen Marktes bezwecken oder bewirken." In Artikel 82 wird das Verbot des Missbrauchs einer marktbeherrschenden Stellung konkretisiert: „Mit dem Gemeinsamen Markt unvereinbar und verboten ist die missbräuchliche Ausnutzung einer beherrschenden Stellung auf dem Gemeinsamen Markt oder auf einem wesentlichen Teil desselben durch ein oder mehrere Unternehmen, soweit dies dazu führen kann, den Handel zwischen Mitgliedstaaten zu beeinträchtigen"275. Eine Fusionskontrolle war im europäischen Recht ursprünglich nicht vorgesehen ${ }^{276}$. Der Europäische Gerichtshof erklärte jedoch in der Entscheidung "Continental Can"277, dass auch ein Zusammenschluss als Missbrauch einer marktbeherrschenden Stellung im Sinne von Art. 82 EGV angesehen werden könne. Dies wäre der Fall, wenn eine bereits vorhandene marktbeherrschende Stellung durch einen Zusammenschluss derart verstärkt wird, dass der Restwettbewerb für die betreffenden Waren in einem wesentlichen Teil des gemeinsamen Marktes praktisch ausgeschaltet wird ${ }^{278}$. Dieses Urteil stellte den Anknüpfungspunkt für die Schaffung einer Fusionskontrolle dar, die die Kommission bereits 1973 erarbeitete. Allerdings wurde der Rechtsakt zur Fusionskontrolle erst am 29.12.1989 vom Europäischen Ministerrat verabschiedet ${ }^{279}$.

Wie bereits beim deutschen Wettbewerbsrecht beschrieben, stand im Zuge der sechsten GWB-Novelle die Harmonisierung mit dem europäischem Recht im Vordergrund. Die Zusammenschlusskontrolle des GWB sowie die europäische Fusionskontrolle stellen daher keine konkurrierenden oder voneinander unabhängigen Gesetzesnormen dar, sondern sind als miteinander ergänzende Vorschriften zu betrachten, die je nach Ausprägung des jeweiligen Zusammenschlusses ihre Anwendung finden ${ }^{280}$. Ein wesentlicher Unterschied findet sich jedoch in der institutionellen Ausgestaltung beider

\footnotetext{
275 Sogenannte Zwischenstaatlichkeitsklausel

276 Vgl. SCHMIDT/SCHMIDT 1997, S. 63

277 JuS 1973, 508 Nr. 1 "Continental Can"

278 Vgl. SCHMIDT 1999, S. 232

${ }^{279}$ Verordnung Nr. 4046/89

280 In der ursprünglichen Fassung des EWG-Vertrages wurde von der Zweischrankentheorie ausgegangen, wonach ein Zusammenschluss nach europäischem und nationalem Recht geprüft wird und sich das jeweils strengere Recht durchsetzt. Heute dagegen überwiegt das sogenannte Subsidiaritätsprinzip, nach
} 
sentlicher Unterschied findet sich jedoch in der institutionellen Ausgestaltung beider Rechtssysteme ${ }^{281}$. So wird das Kartellrecht auf Bundesebene durch eine selbständige Behörde angewendet, während auf EU-Ebene eine Anwendung durch die EuropäischeKommission stattfindet, die als ein politisches Organ auch für andere politische und ökonomische Entscheidungen zuständig ist ${ }^{282}$.

\subsubsection{Anwendungsbereich der EU-Fusionskontrolle}

In die Zuständigkeit der EU-Kommission fallen alle Zusammenschlüsse von sogenannter "gemeinschaftsweiter Bedeutung". Gemeinschaftsweite Bedeutung hat ein Zusammenschluss, wenn folgende Umsatzkriterien erfüllt sind (Art. 1 Abs. 2 a und b EGFusionskontrollverordnung):

- Alle am Zusammenschluss beteiligten Unternehmen haben zusammen einen weltweiten Gesamtumsatz von mehr als 5 Milliarden Euro, und

- von mindestens zwei der beteiligten Unternehmen wird ein gemeinschaftsweiter (EU-)Umsatz von jeweils mehr als 250 Millionen Euro erzielt; oder

- alle am Zusammenschluss beteiligten Unternehmen erwirtschaften zusammen einen weltweiten Gesamtumsatz von mehr als 2,5 Milliarden Euro, und

- von mindestens zwei der beteiligten Unternehmen wird ein gemeinschaftsweiter Umsatz von jeweils mehr als 100 Millionen Euro erzielt, und

- alle am Zusammenschluss beteiligten Unternehmen haben zusammen in mindestens drei Mitgliedstaaten einen Gesamtumsatz von mehr als 100 Millionen Euro erzielt und

- von mindestens zwei der beteiligten Unternehmen wird in jedem dieser drei Mitgliedsstaaten ein Umsatz von jeweils mehr als 25 Millionen Euro erzielt.

Eingeschränkt wird die Zuständigkeit der EU-Kommission durch die sogenannte ZweiDrittel-Klausel (Art. 1 Abs. 2 und 3). Danach ist auch bei Überschreiten der oben ge-

dem die Normen und Kompetenzen der unterschiedlichen Entscheidungsebenen aufeinander abgestimmt sind (one-stop-shop-Prinzip).

${ }^{281} \mathrm{Vgl}$. BUNDESKARTELLAMT 2000, Auslegungsgrundsätze, S. 26

282 Kritisiert u.a. in SCHMIDT/SCHMIDT 1997, S. 121 
nannten Schwellenwerte keine Zuständigkeit der EU-Kommission gegeben, sobald jeweils mehr als 2/3 des gemeinschaftsweiten (nicht weltweiten!) Umsatzes in einem Mitgliedsstaat erwirtschaftet wird. Mit dieser Klausel wird das Ziel verfolgt, dass Zusammenschlüsse, die sich überwiegend in einem Mitgliedsstaat auswirken, in der Zuständigkeit der nationalen Behörde verbleiben ${ }^{283}$.

\subsubsection{Zusammenschlusstatbestand}

Eine weitere Differenz zwischen dem europäischen und dem deutschen Zusammenschlussbegriff besteht darin, dass die Anwendung bei Minderheitsbeteilungen in der EG-FKVO generell nicht geklärt ist ${ }^{284}$. Nach Art. 3 Abs. 1 und 2 EG-FKVO wird ein Zusammenschluss dadurch bewirkt, dass

- zwei oder mehr bisher unabhängige Unternehmen fusionieren oder dass

- eine oder mehrere Personen, die bereits mindestens ein Unternehmen kontrollieren, oder ein oder mehrere Unternehmen durch den Erwerb von Anteilsrechten oder Vermögenswerten, durch Vertrag oder in sonstiger Weise die unmittelbare oder mittelbare Kontrolle über die Gesamtheit oder über Teile eines oder mehrerer anderer Unternehmen erwerben oder dass

- ein Gemeinschaftsunternehmen gegründet wird, das dauerhaft alle Funktionen einer selbständigen Einheit erfüllt.

Maßgeblich ist also das Kriterium Kontrolle über andere Unternehmen mit der Option, einen bestimmten Einfluss auszuüben ${ }^{285}$.

\subsubsection{Eingriffskriterium}

Ebenso wie in der deutschen Zusammenschlusskontrolle kann die Kartellbehörde im EG-Recht einen Zusammenschluss untersagen bzw. mit Auflagen freigeben, sobald eine Marktbeherrschung festgestellt wird. In der Rechtssache "United Brands" definierte der Europäische Gerichtshof (EuGH) eine marktbeherrschende Stellung als "wirtschaftliche Machtstellung eines Unternehmens (...), die dieses in die Lage versetzt, die Aufrechterhaltung eines wirksamen Wettbewerbs auf dem relevanten Markt zu verhindern, indem sie inm die Möglichkeit verschafft, sich seinen Wettbewerbern, seinen

\footnotetext{
${ }^{283}$ Vgl. BUNDESKARTELLAMT 1998, EG-Fusionskontrolle, S. 2

${ }^{284}$ Vgl. BOSCH/SCHÜTZ 2000, Einführg, Rdnr. 55 ff.

${ }^{285} \mathrm{Vgl}$. BUNDESKARTELLAMT 1998, EG-Fusionskontrolle, S. 3
} 
Abnehmern und schließlich den Verbrauchern gegenüber in einem nennenswerten Umfang unabhängig zu verhalten" ${ }^{\text {286. }}$. Die Eingriffsschwelle des Art. 82 EGV $^{287}$ setzt dort an, wo Unternehmen in der Lage sind, sich unabhängig vom Wettbewerb zu verhalten oder die Wettbewerbsbedingungen zu kontrollieren. Wesentlicher Wettbewerb widerspricht ähnlich wie im deutschen Recht nicht zwingend dem Vorliegen einer marktbeherrschenden Stellung ${ }^{288}$. Prinzipiell werden von der Europäische-Kommission folgende Prüfungskriterien herangezogen, um das Vorliegen einer marktbeherrschenden Stellung zu beurteilen ${ }^{289}$ :

- Marktstellung der fusionierten Unternehmen (Marktanteile, Finanzstärke, Entwicklungen, Vertriebsnetz etc.),

- Marktstärke der verbleibenden Unternehmen (Marktanteile, Finanzstärke, Kapazitäten etc.),

- Struktur der Nachfrageseite (Stärke der Verhandlungsposition der Nachfrager),

- Höhe der Marktschranken (potentieller Wettbewerb).

Darüber hinaus berücksichtigt der Gesetzgeber explizit das Kriterium "technischer und wirtschaftlicher Fortschritt" (Art. 2 Abs. 1 Buchstabe b FKVO), welches jedoch von der Rechtsprechung generell in Frage gestellt wurde ${ }^{290}$.

\subsubsection{Der relevante Markt in der europäischen Fusionskontrolle}

Um eine Marktbeherrschung festzustellen, ist es notwendig, den relevanten Markt abzugrenzen. Dabei impliziert die Bestimmung des relevanten Marktes schon eine Aussage über den Wettbewerb auf diesem Markt, da die Größe der Marktanteile abhängig von der "Weite" der getroffenen Marktabgrenzung ist ${ }^{291}$. Die Europäische-Kommission bedient sich dabei ähnlich wie das Bundeskartellamt dem Bedarfsmarktkonzept ${ }^{292}$,

\footnotetext{
${ }^{286}$ EuGH 14.02.1978, Slg. 1978, 207, 286 Tz. 63 und 66 "United Brands"

${ }^{287}$ EGV = Vertrag zur Gründung der Europäischen Gemeinschaft - Konsolidierte Fassung mit den Änderungen durch den Vertrag von Amsterdam vom 2.10.1997

${ }^{288}$ EUROPÄISCHE-KOMMISSION, Entscheidung vom 22.09.1999 „Airtours/First Choice”, Abl. EG 2000, L 93/1, Rdnr. 128 ff.

${ }^{289}$ In Anlehnung an SCHMIDT/SCHMIDT 1997, S. 90

${ }^{290}$ EUROPÄISCHE-KOMMISSION, Entscheidung vom 19.07.1995 „Nordic Satellite Distribution”, Abl. 1996, L53/20

291 Vgl. KOTTMANN 2000, S. 15

292 Einen Überblick über verschiedene Konzepte vermittelt SCHMIDT 1996, S.46 ff.
} 
wobei aktueller, potentieller und Substitutionswettbewerb sowohl der Nachfrager als auch der Anbieter berücksichtigt wird. Die räumliche Abgrenzung des relevanten Marktes (Begriff: "räumlicher Referenzmarkt") wird nach Art. 9 Abs. 7 FKVO folgendermaBen definiert: "Der räumliche Referenzmarkt besteht aus einem Gebiet, auf dem die betroffenen Unternehmen als Anbieter oder Nachfrager von Waren oder Dienstleistungen auftreten, in dem die Wettbewerbsbedingungen hinreichend homogen sind und das sich von den benachbarten Gebieten unterscheidet. Bei der Beurteilung ist besonders auf das Vorhandensein von Zugangsschranken, Verbrauchergewohnheiten sowie auf das Bestehen erheblicher Unterschiede bei den Marktanteilen der Unternehmen oder nennenswerter Preisunterschiede zwischen dem betreffenden Gebiet und den benachbarten Gebieten abzustellen".

Der im Rahmen der FKVO geprägte Begriff des räumlichen Referenzmarktes beinhaltet keinen grundsätzlichen neuen Ansatz bei der Bestimmung relevanter Märkte. Es handelt sich vielmehr um eine Zusammenführung der im deutschen und USamerikanischen Kartellrecht entwickelten wettbewerbspolitischen Ansätze zur Marktabgrenzung ${ }^{293}$.

Die Abgrenzung des relevanten Marktes darf keinesfalls unterschätzt werden. Nach der Entscheidungspraxis der Europäischen-Kommission wird ein Zusammenschluss als bedenklich eingestuft, sobald der Marktanteil auf dem relevanten Markt die $25 \%$ Anteilsgrenze überschreitet. Wie bereits erwähnt, führt nicht allein dieses Kriterium zu einer Begründung einer marktbeherrschenden Stellung, dennoch werden solche Zusammenschlüsse besonders kritisch geprüft und erfordern eine detaillierte Rechtfertigung seitens der fusionswilligen Unternehmen ${ }^{294}$.

\subsubsection{Das Verfahren der europäischen Fusionskontrolle}

Unternehmenszusammenschlüsse, die in den Anwendungsbereich der europäischen Fusionskontrolle fallen, sind anmeldepflichtig. Das Verfahren gliedert sich in mehrere Schritte (vgl. Abbildung 22). Zunächst einmal erfolgt die Kontaktaufnahme mit zuständigen Mitarbeitern der "Task Force - Fusionskontrolle" in der Generaldirektion Wettbewerb der Europäischen Kommission. Bei dieser Voranmeldung kann zunächst geklärt werden, ob der geplante Zusammenschluss unter dem europäischen Wettbewerbsrecht erfasst wird, bzw. welche Schwierigkeiten ins Kalkül gezogen werden müs-

\footnotetext{
293 Vgl. KotTMANN 2000, S. 95

294 Eine detaillierte Analyse über die Auslegung einer marktbeherrschenden Stellung nach der VO Nr. 4064/89 verschafft KNITTEL 1999
} 
$\operatorname{sen}^{295}$. Erfolgt die endgültige Anmeldung, so tritt das Verfahren in die sogenannte erste Phase (Vorverfahren). Innerhalb von vier Wochen ${ }^{296}$ entscheidet die Kommission, ob das Zusammenschlussvorhaben in den Anwendungsbereich der Fusionskontrollverordnung fällt, das Vorhaben Anlass zu ernsthaften Bedenken hinsichtlich seiner Vereinbarkeit mit dem Gemeinsamen Markt gibt ${ }^{297}$ oder ob der Zusammenschluss ohne weitere Nachprüfungen und Auflagen freizugeben ist. Entscheidet die Kommission nicht innerhalb dieser Frist, gilt das Vorhaben als genehmigt. Bei ernsthaften Bedenken seitens der Kommission wird das Hauptverfahren (zweite Phase) eingeleitet. Innerhalb von vier Monaten erklärt die Kommission, ob der Zusammenschluss für vereinbar, für unvereinbar oder vereinbar mit bestimmten Bedingungen und Auflagen gilt $^{298}$, wobei innerhalb der ersten drei Monate Unternehmen Verpflichtungen ${ }^{299}$ anbieten können, um gegebenenfalls die Zulässigkeit des Vorhabens sicherzustellen. Vor der endgültigen Entscheidung ist den Beteiligten zusätzlich Akteneinsicht und eine mündliche Anhörung zu gewähren. Wird auch hier nicht innerhalb der Frist entschieden, gilt der Zusammenschluss als freigegeben. Eine Klage der Beteiligten oder auch Dritter (Konkurrentenklage) kann vor dem Europäischen Gerichtshof bzw. dem Gericht erster Instanz erhoben werden ${ }^{300}$.

\footnotetext{
295 Vgl. BUNDESKARTELLAMT 1999, Inlandsauswirkung, S. 13

${ }^{296}$ Frist kann auf sechs Wochen verlängert werden, falls Unternehmen Zugeständnisse anbieten oder ein Mitgliedsstaat eine Verweisung auf eine nationale Behörde beantragt.

297 In diesem Falle wird die zweite Phase (Hauptverfahren) eingeleitet.

${ }^{298}$ Vgl. FKVO Art. 10

${ }^{299}$ Einteilung in: a) Veräußerungszusagen: WuW/E EU 103 „Nestlé/Perrier", b) Marktöffnungszusagen: WuW/E EV 1616 "Alcatel/Telettra" oder c) Entflechtungszusagen: WuW/E EU-V 64 "Anglo American/Lanrho"

300 Vgl. EMMERICH 1999, S. 493
} 
Abbildung 22: Übersicht über das EG- Fusionskontrollverfahren

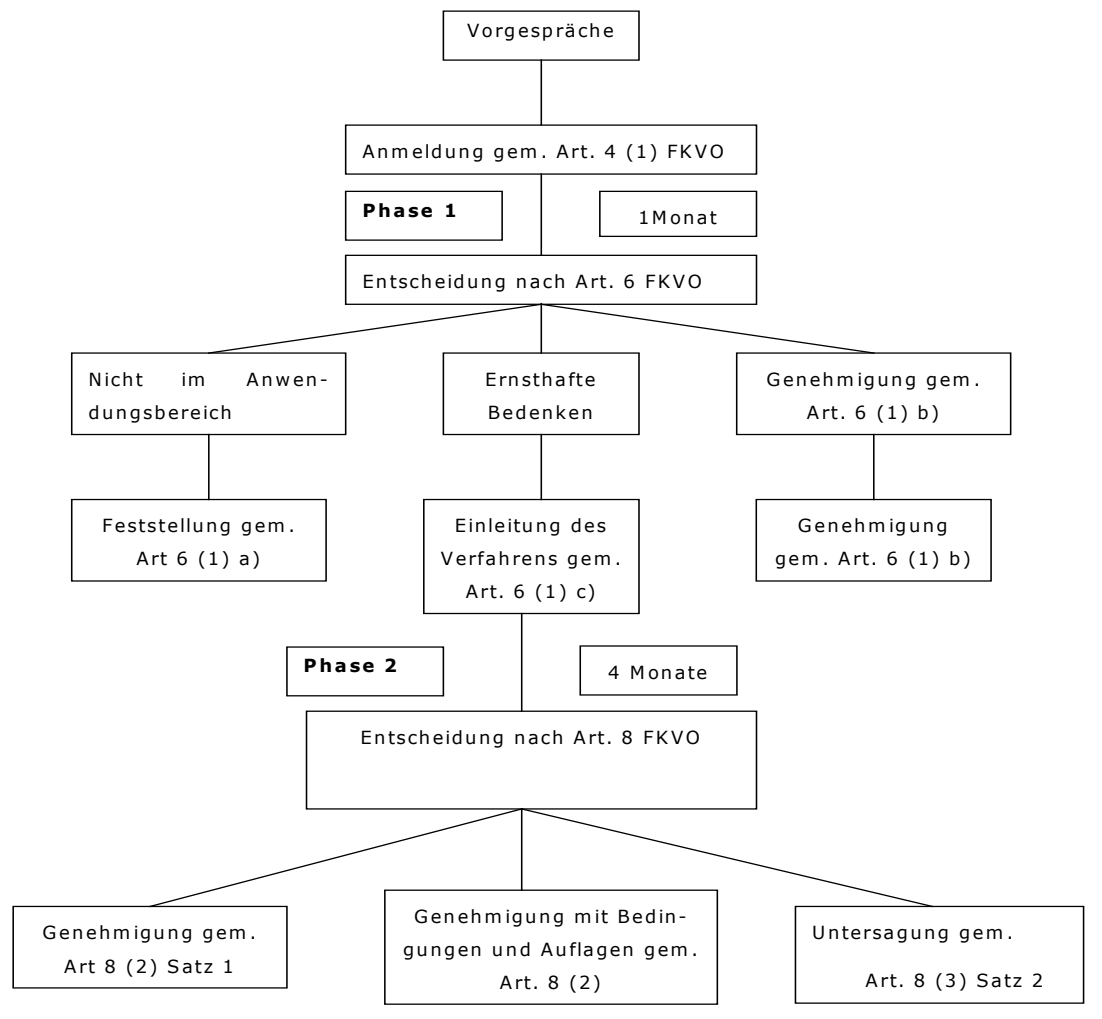

Quelle: DRAUZ/SCHRöDER 1992, S. 114

\subsubsection{Sonstige Anmerkungen zum europäischen Wettbewerbsrecht}

Landwirtschaftliche Betriebe sowie ihr vor- und nachgelagerter Bereich gelten im EGWettbewerbsrecht als Ausnahmebereich ${ }^{301}$. Art. 42 EGV besagt, dass die Wettbewerbsregeln des EU-Rechtes auf die Produktion und Vermarktung landwirtschaftlicher Erzeugnisse $^{302}$ nur insoweit anwendbar sind, als dass die Ziele zur Verwirklichung der gemeinsamen Agrarpolitik (Art. 39 EGV) nicht behindert werden. Wettbewerbsbeschränkungen, die, wie z.B. nationale Absatzsyndikate für Milch oder Liefervorrechte für nationale Erzeugerbetriebe, den Rahmenverträgen mit den Verarbeiterbetrieben den Zwecken der gemeinsamen Agrarpolitik direkt zuwiderlaufen, bleiben daher (Ver-

\footnotetext{
$301 \mathrm{Vgl.} \mathrm{WINKLER} \mathrm{1997,} 2159 \mathrm{ff}$.

${ }^{302}$ Alle Erzeugnisse, die in der VO 26/62 Anhang II ausdrücklich erwähnt sind.
} 
stoß gegen Art. 81) verboten ${ }^{303}$. Die europäische Fusionskontrolle kennt ebenso wie die deutsche Zusammenschlusskontrolle keine Bereichsausnahme für die Agrar- und Ernährungswirtschaft.

Eine weitere Gemeinsamkeit zwischen dem deutschen und europäischen Recht besteht darin, dass beide Gesetzesnormen eine Abwägungsklausel enthalten ${ }^{304}$. Zwar ist im europäischen Recht die Abwägungsklausel weit unschärfer konkretisiert, dennoch findet nach Art. 2 Abs. 1 b eine gewisse Abwägung statt, und auch die Rechtsprechung berücksichtigt eine mögliche Abwägung der Vor- und Nachteile eines Zusammenschlusses $^{305}$.

Eine Ministererlaubnis (vgl. § 42 GWB) kennt das europäische Wettbewerbsrecht nicht. Dennoch steht die Beurteilung von Zusammenschlussvorhaben im Spannungsfeld zwischen Industrie- und Wettbewerbspolitik. So wurden in einigen Fällen Unternehmenszusammenschlüsse trotz erheblich wettbewerbspolitischer Bedenken aufgrund politischer Einflussnahme genehmigt ${ }^{306}$. Beispielsweise intervenierte Bundeswirtschaftsminister Rexrodt im Fall Mercedes-Benz/Kässbohrer ${ }^{307}$ aus arbeitsmarkt- und regionalpolitischen Gründen, da die Kommission ursprünglich drohte, den Zusammenschluss der Überlandbushersteller (75 \% Marktanteil in Deutschland) zu untersagen. Letztendlich genehmigte die Kommission die Fusion ohne weitere Auflagen mit dem Verweis auf die Relativierung der hohen Marktanteile durch potentiellen Wettbewerb ${ }^{308}$.

Abschließend sei die Möglichkeit einer Entflechtung von marktbeherrschenden Unternehmen erwähnt. Auch in diesem Punkt harmonisiert das europäische mit dem deutschen Recht. Eine Auflösung von Unternehmenszusammenschlüssen ist nur dann rechtmäßig, sobald ein Zusammenschluss ohne die Zustimmung der Kommission vollzogen wurde ${ }^{309}$ (Art. 8 Abs. 2 FKVO). Ein wesentlicher Unterschied zum USamerikanischem Wettbewerbsrecht besteht darin, dass in den USA darüber hinaus die Möglichkeit besteht, bereits in der Vergangenheit vollzogene Fusionen wieder rückgängig zu machen. Beispielsweise wurde im Jahr 1982 das Monopol der Telefongesell-

\footnotetext{
${ }^{303}$ Vgl. EUROPÄISCHE-KOMMISSION, Entscheidung v. 07.12.1984, Abl. 1985 Nr. L 35/35; v. 26.11.1986, Abl. Nr. L 348/50; v. 18.12.1989, Abl. Nr. L 31/32 "Zuckerrüben"; v. 30.07.1992, Abl. Nr. L 246/37

${ }^{304} \mathrm{Vgl.} \mathrm{BOSCH} / \mathrm{SCHÜTZ} \mathrm{2000,} \mathrm{Einführung,} \mathrm{Rdnr.} 43$

${ }^{305} \mathrm{Vgl.} \mathrm{FLEISCHER/KÖRBER} \mathrm{2001,} \mathrm{S.} 10 \mathrm{ff}$.

306 Vgl. SCHMIDT/SCHMIDT 1997, S. 115

307 Vgl. EUROPÄISCHE-KOMMISSION 1995, Abl. L 211, S. $1 \mathrm{ff}$

308 Vgl. SCHMIDT/SCHMIDT 1997, S. 116

309 Präventive Fusionskontrolle
} 
schaft AT\&T in den USA von den Wettbewerbsbehörden zerschlagen ${ }^{310}$. Eine solche Regelung existiert weder im europäischen noch im deutschen Wettbewerbsrecht.

\subsubsection{Vergleich, Entwicklung und Ausblick}

\subsubsection{Vergleich der Untersagungskriterien}

Die Voraussetzungen für die Untersagung von Unternehmenszusammenschlüssen sind nur in wenigen Rechtsordnungen vollkommen identisch. Grundsätzlich lassen sich jedoch zwei Gruppen von Untersagungskriterien unterscheiden: Die meisten Mitgliedsstaaten der Europäischen Union wie auch die Europäische Kommission selbst stellen bei der Beurteilung eines Zusammenschlusses darauf ab, ob dieser zur Begründung oder Verstärkung einer marktbeherrschenden Stellung (MB) führt. Demgegenüber sind gemäß den Vorschriften aus den USA, Kanada sowie Australien solche Fusionen zu untersagen, die zu einem "substantial lessening of competition" (SLC) - also zu einer wesentlichen Reduzierung des Wettbewerbs - führen ${ }^{311}$. Trotz formalrechtlicher Unterschiede in der Begrifflichkeit und der Vorgehensweise verschiedener Wettbewerbsordnungen kommen die Wettbewerbsbehörden in den meisten Fällen zu dem gleichen Urteil $^{312}$. Unterschiede in den Rechtsprechungen werden auf Differenzen wettbewerbspolitischer Leitbilder, unterschiedliche Informationen oder industriepolitische übergeordnete Interessen begründet ${ }^{313}$. In der folgenden Abbildung (Tabelle 16) soll dennoch ein grober Vergleich der deutschen, europäischen und US-amerikanischen Fusionskontrolle vorgenommen werden ${ }^{314}$.

\footnotetext{
310 Vgl. SCHMIDT 1999, S. 249

${ }^{311}$ Für einen detaillierten Vergleich s.: BUNDESKARTELLAMT 2001

312 Besondere Aufmerksamkeit erlangte der geplante Unternehmenszusammenschluss von General Electric und Honeywell (2001) in den USA, wobei die Europäische Kommission im Gegensatz zu den USamerikanischen Behörden für eine Untersagung des Zusammenschlusses plädierte.

${ }^{313} \mathrm{Vgl.}$ BUNDESKARTELLAMT 2001, S. $36 \mathrm{ff}$.
} 
Tabelle 16: Beurteilungskriterien in der Fusionskontrolle (nach Maßgabe der gesetzlichen Grundlagen)

\begin{tabular}{|c|c|c|c|}
\hline & Deutschland & Europäische Union & USA \\
\hline $\begin{array}{l}\text { Untersagungskri- } \\
\text { terium }\end{array}$ & $\begin{array}{l}\text { Zusammenschluss, von dem zu } \\
\text { erwarten ist, dass er eine markt- } \\
\text { beherrschende Stellung begrün- } \\
\text { det oder verstärkt }\end{array}$ & $\begin{array}{l}\text { Zusammenschlüsse, die eine } \\
\text { beherrschende Stellung begrün- } \\
\text { den oder verstärken, durch die } \\
\text { wirksamer Wettbewerb (...) er- } \\
\text { heblich behindert würde }\end{array}$ & $\begin{array}{l}\text { No person shall (...) where (...) } \\
\text { the effect of such acquisition may } \\
\text { be substantially to lessen compe- } \\
\text { tition, or to tend to create a mo- } \\
\text { nopoly }\end{array}$ \\
\hline $\begin{array}{l}\text { Marktanteil der } \\
\text { beteiligten Unter- } \\
\text { nehmen } \\
\text { /Quantitative } \\
\text { Vermutungs- } \\
\text { schwellen }\end{array}$ & $\begin{array}{l}\text { MB-Vermutung: } \geq 30 \% \\
\text { Oligopolvermutung: } \\
\mathrm{CR}_{3} \geq 50 \% \\
\mathrm{CR}_{5} \geq 67 \%\end{array}$ & $\begin{array}{l}\text { Marktstellung (...) der beteiligten } \\
\text { Unternehmen }\end{array}$ & $\begin{array}{l}\text { Vermutung nachteiliger Wirkung: } \\
\geq 35 \% \\
\text { Falls Alternativen für wesentli- } \\
\text { chen Teil der Verbraucher unzu- } \\
\text { reichend } \\
\text { SLC-Vermutung: } \\
\text { HHI }>1000(+>100) \text { oder } \mathrm{HHI}> \\
1800(+>50)\end{array}$ \\
\hline $\begin{array}{l}\text { Marktstruktur/ } \\
\text { Marktkonzentra- } \\
\text { tion }\end{array}$ & $\begin{array}{l}\text { Oligopolvermutung: } \\
\mathrm{CR}_{3} \geq 50 \% \\
\mathrm{CR}_{5} \geq 67 \% \\
\begin{array}{l}\text { Abstände und Verteilung der } \\
\text { Marktanteile }\end{array}\end{array}$ & $\begin{array}{l}\text { Struktur aller betroffenen } \\
\text { Märkte }\end{array}$ & $\begin{array}{l}\text { SLC-Vermutung } \\
\mathrm{HHI}>1000(+>100) \text { oder } \\
\mathrm{HHI}>1800(+>50)^{315}\end{array}$ \\
\hline $\begin{array}{l}\text { Tatsächlicher } \\
\text { oder potentieller } \\
\text { Wettbewerb }\end{array}$ & $\begin{array}{l}\text { Tatsächlicher oder potentieller } \\
\text { Wettbewerb durch innerhalb oder } \\
\text { außerhalb des Geltungsbereichs } \\
\text { (...) ansässige Unternehmen } \\
\text { Wettbewerb durch Randsubstitu- } \\
\text { tion }\end{array}$ & $\begin{array}{l}\text { Tatsächlicher oder potentieller } \\
\text { Wettbewerb durch innerhalb oder } \\
\text { außerhalb der Gemeinschaft } \\
\text { ansässige Unternehmen }\end{array}$ & $\begin{array}{l}\text { Verbindlicher Marktzutritt }(= \\
\text { Neuer Wettbewerb, der den } \\
\text { Aufwand bedeutsamer „sunk } \\
\text { costs“ des Marktzu- und -austritts } \\
\text { erfordert) }\end{array}$ \\
\hline $\begin{array}{l}\text { Marktzutritts- } \\
\text { schranken }\end{array}$ & $\begin{array}{l}\text { Rechtliche und tatsächliche } \\
\text { Schranken für den Marktzutritt } \\
\text { anderer Unternehmen }\end{array}$ & $\begin{array}{l}\text { Rechtliche oder tatsächliche } \\
\text { Marktzutrittsschranken }\end{array}$ & $\begin{array}{l}\text { Rechtzeitigkeit, Wahrscheinlich- } \\
\text { keit und Hinlänglichkeit des } \\
\text { Marktzutritts }\end{array}$ \\
\hline $\begin{array}{l}\text { Entwicklung des } \\
\text { Marktes }\end{array}$ & \begin{tabular}{|l|} 
Marktphase (Wachstum, Innova- \\
tionstempo, Veränderung der \\
Wettbewerbsbedingungen)
\end{tabular} & $\begin{array}{l}\text { Entwicklung des Angebots und } \\
\text { der Nachfrage }\end{array}$ & $\begin{array}{l}\text { Veränderung der Marktbedingun- } \\
\text { gen (neue Technologien, Ent- } \\
\text { wicklung der Marktanteile) }\end{array}$ \\
\hline $\begin{array}{l}\text { Ausweichmög- } \\
\text { lichkeiten und } \\
\text { Interessen der } \\
\text { Marktgegenseite }\end{array}$ & $\begin{array}{l}\text { Möglichkeit der Marktgegenseite, } \\
\text { auf andere Unternehmen auszu- } \\
\text { weichen }\end{array}$ & $\begin{array}{l}\text { Wahlmöglichkeiten der Lieferan- } \\
\text { ten und Abnehmer } \\
\text { Interessen der Zwischen- und } \\
\text { Endverbraucher }\end{array}$ & $\begin{array}{l}\text { Grad der Substituierbarkeit mit } \\
\text { Produkten/Gebieten außerhalb } \\
\text { des relevanten Marktes }\end{array}$ \\
\hline
\end{tabular}

${ }^{314}$ Entnommen aus BUNDESKARTELLAMT 2001

315 Die Richtlinien unterscheiden zwischen Märkten, die nach dem Zusammenschluss unkonzentriert sind $(\mathrm{HHI}<1000)$, mäßig konzentrierten (HHI 1000 - 1800) und hochgradig konzentrierten (HHI > 1800) Märkten. Betrifft das Vorhaben nach dem Zusammenschluss mäßig konzentrierte Märkte, so können den Richtlinien zufolge schwerwiegende Wettbewerbsbedenken auftreten, wenn der Zusammenschluss einen Anstieg des HHI um mehr als 100 Punkte bewirkt. Auf hochgradig konzentrierten Märkten genügt bereits ein Anstieg um 50 Punkte. 
Tabelle 16 (Fortsetzung)

\begin{tabular}{|c|c|c|c|}
\hline & Deutschland & Europäische Union & USA \\
\hline $\begin{array}{l}\text { Weitere wettbe- } \\
\text { werbliche Fakto- } \\
\text { ren }\end{array}$ & $\begin{array}{l}\text { Finanzkraft bzw. überlegene } \\
\text { Ressourcen } \\
\text { Zugang zu den Beschaffungs- } \\
\text { oder Absatzmärkten (vertikale } \\
\text { (Integration, Sortiment) } \\
\text { Gegengewichtige Marktmacht } \\
\text { Verflechtung mit anderen Unter- } \\
\text { nehmen } \\
\text { Umstellungsflexibilität } \\
\text { Abwägungsklausel }\end{array}$ & $\begin{array}{l}\text { Wirtschaftliche Macht und Fi- } \\
\text { nanzkraft } \\
\text { Zugang zu Beschaffungs- und } \\
\text { Absatzmärkten }\end{array}$ & $\begin{array}{l}\text { Wahrscheinlichkeit abgestimmten } \\
\text { Verhaltens (Verfügbarkeit von } \\
\text { Informationen, Produktheteroge- } \\
\text { nität, Preissetzungs- und Marke- } \\
\text { tingpraktiken, Transaktionsfre- } \\
\text { quenz) } \\
\text { Wahrscheinlichkeit einseitiger } \\
\text { Maßnahmen (insbesondere } \\
\text { Preiserhöhungsspielraum) } \\
\text {... }\end{array}$ \\
\hline $\begin{array}{l}\text { Sonstige Fakto- } \\
\text { ren }\end{array}$ & $\begin{array}{l}\text { Kausalität/ Sanierungsfunktion } \\
\text { Möglichkeit der Ministererlaubnis } \\
\text { bei überwiegenden gesamtwirt- } \\
\text { schaftlichen Vorteilen bzw. einem } \\
\text { überragenden Interesse der } \\
\text { Allgemeinheit }\end{array}$ & $\begin{array}{l}\text { Entwicklung des technischen und } \\
\text { wirtschaftlichen Fortschritts so- } \\
\text { fern diese dem Verbraucher dient } \\
\text { und den Wettbewerb nicht behin- } \\
\text { dert } \\
\text { Kausalität/ Sanierungsfunktion }\end{array}$ & $\begin{array}{l}\text { Erkennbare Effizienzen, die die } \\
\text { Wettbewerbsbeschränkung des } \\
\text { Zusammenschlusses aufwiegen } \\
\text { Failing firm defense }\end{array}$ \\
\hline
\end{tabular}

Anm.: D: Vgl. §§ 19, 36 GWB, Auslegungsgrundsätze Bundeskartellamt; EU: Vgl. Artikel 2 FKVO und Erwägungsgrund 15 FKVO; USA: Vgl. Section 7 Clayton Act sowie Horizontal Merger Guidelines (US), Abschnitte 1-3

Quelle: Bundeskartellamt 2001

\subsubsection{Entwicklung und Ausblick}

Die Bemühungen um Rechtsangleichung außerhalb der EU/EWR oder Schaffung vergleichbarer regionaler Organisationen bis hin zum Weltkartellrecht mit einer Weltfusionskontrolle befinden sich noch im Anfangsstadium ${ }^{316}$. Dabei steht ein einheitliches materielles Recht noch gar nicht zur Diskussion, vielmehr geht es um verfahrensrechtliche oder institutionelle Regelungen ${ }^{317}$, wie beispielsweise den Austausch von Informationen. Aufgrund der zunehmenden Zahl globaler Fusionen ist daher besonders erfreulich, dass bereits seit 1991 ein intensiver Kontakt zwischen der EuropäischenKommission und den USA (Federal Trade Commission) besteht, so dass im Bereich der transatlantischen Zusammenarbeit Abstimmung erreicht werden kann ${ }^{318}$. Ähnliche Verhandlungen werden seit 1999 mit Kanada geführt. Weitere Arbeitsgruppen bzw. intensive Kooperationen bestehen mit der WTO (World Trade Organization), der OECD (Organization of Economic Cooperation and Development) sowie der UNCTAD (United

\footnotetext{
${ }^{316}$ Vgl. BosCH/SCHÜTZ 2000, S. 48

317 Vgl. EU-Kommission 1999, Wettbewerbspolitik, S.101 ff.

${ }^{318}$ Vgl. EU-KOMMISSION 1999, Wettbewerbspolitik, S. 105; zum Einfluss des US-Rechtes auf das EU-Recht vgl.: FLEISCHER/KÖRBER 2001 in WuW 2001, S. 6 ff.
} 
Nations Conference on Trade and Development). Dabei steht allerdings zunächst die allgemeine Wettbewerbspolitik im Vordergrund. Der Schwerpunkt der WTOÜbereinkommen liegt im Abbau bzw. in der Angleichung staatlicher Beihilfen ${ }^{319}$. Die OECD beschäftigt sich neben der Ausrichtung einer zukünftigen Wettbewerbspolitik ${ }^{320}$ mit der Etablierung von Wettbewerbsnormen, die zum Teil in Entwicklungsländern erst erschaffen werden müssen ${ }^{321}$. Die UNCTAD, als Vertretung der Vereinten Nationen, übt dagegen eine wettbewerbsüberwachende Funktion aus. So beobachtet und kontrolliert die UNCTAD beispielsweise das faire Funktionieren von Märkten, den Zugang zu Ressourcen oder die Entwicklung von Korruption ${ }^{322}$.

Innerhalb der Europäischen Union steht die Harmonisierung mit den nationalen Rechten weiterhin im Vordergrund. Aktuell wird in dem Grünbuch ${ }^{323}$ der Kommission über die Anrechnung positiver Effekte (Abwägungsklausel), eine Änderung der Schwellenwerte bzw. die Zuweisung an nationale Wettbewerbsbehörden diskutiert, um die Kommission aufgrund der zunehmenden Anzahl der zu beurteilenden Fälle arbeitstechnisch zu entlasten ${ }^{324}$. Einen weiteren Schwerpunkt stellt die anstehende Integration der EU-Beitrittskandidaten dar. Überdies arbeitet die Kommission intensiv daran, die Wettbewerbspolitik transparenter und anwendungsfreundlicher zu gestalten, damit ein Entscheidungsprozess „bürgernäher" vermittelt werden kann ${ }^{325}$.

\subsection{Zusammenfassung der Rechtsmaterie}

Grundsätzlich sind Unternehmenszusammenschlüsse in Deutschland erlaubt. Erst beim Überschreiten bestimmter Umsatzgrenzen greift die Fusionskontrolle bzw. bei Erreichen eines kritischen Ausmaßes der Wettbewerbsbeschränkungen (Marktbeherrschung) das Verbot des Bundeskartellamts ein. Unterschieden wird zwischen horizontalen, vertikalen und konglomeraten Unternehmenszusammenschlüssen. Die Prüfung der Zusammenschlüsse findet Anwendung, sofern weltweit ein Umsatzerlös von mehr

\footnotetext{
${ }^{319}$ EU-KoмmISSION 1999, Wettbewerbspolitik, S. 108

320 OECD 1999, Trade and Competition Policies; Policies for Tomorrow

321 OECD 1999, A Framework for the Design and Implementation of Competition Law and Policy

${ }^{322} \mathrm{Vgl}$. dazu internet: www.unctad.org/competition

${ }^{323}$ Grünbuch $=$ Zur Diskussion stehender Vorschlag

${ }^{324}$ Vgl. EU-Kommission 1999, Wettbewerbspolitik, S. 5

${ }^{325}$ Vgl. EU-Kommission 1999, Wettbewerbspolitik, S. 5
} 
als 500 Mio. Euro vorliegt und mindestens ein beteiligtes Unternehmen im Inland Jahresumsatzerlöse von mehr als 25 Mio. Euro erzielt. Sobald die Umsatzerlösschwelle von fünf Mrd. Euro überschritten wird oder gemeinschaftsweite Bedeutung vorliegt, ist die Europäische Kommission exklusiv für die Prüfung des Zusammenschlussvorhabens zuständig. Hierdurch soll im wesentlichen sichergestellt werden, dass Zusammenschlüsse von Unternehmen mit hohen Umsätzen von der europäischen Fusionskontrolle erfasst werden. Zusammenschlüsse von Unternehmen, die insgesamt weniger als 500 Mio. Euro Umsatz erzielen, unterliegen in Deutschland keiner Fusionskontrolle.

Das Bundeskartellamt untersagt einen Zusammenschluss, von dem zu erwarten ist, dass er eine marktbeherrschende Stellung begründet oder verstärkt, es sei denn, die beteiligten Unternehmen weisen nach, dass durch den Zusammenschluss auch Verbesserungen der Wettbewerbsbedingungen eintreten und dass diese Verbesserungen die Nachteile der Marktbeherrschung überkompensieren. Voraussetzung für die Feststellung von Marktbeherrschung ist die Abgrenzung des für die Beurteilung des Zusammenschlusses relevanten Marktes in sachlicher und räumlicher, mitunter auch zeitlicher Hinsicht.

Die deutsche Fusionskontrolle basiert nicht auf einem bestimmten Theorieansatz. Dies ergibt sich schon aus dem Kriterienkatalog, den der Gesetzgeber bei der Definition der überragenden Marktstellung des $\S 19$ Abs. 2 Nr. 2 GWB zur Prüfung einer marktbeherrschenden Stellung eingefügt hat. Maßgebend hierfür war der Gedanke, dass sich die Marktstellung eines Unternehmens in der Regel nicht allein aufgrund der Prüfung eines Strukturkriteriums (z.B. Marktanteil, aktueller oder potentieller Wettbewerb), sondern nur anhand einer Gesamtbetrachtung aller relevanten Umstände des Einzelfalles beurteilen lässt. In einzelnen Fällen kann dies dazu führen, dass selbst Zusammenschlüsse, bei denen hohe Marktanteile erreicht werden, nicht auf Untersagung stossen, da andere Strukturfaktoren, wie z.B. ein leistungsfähiger, potentieller Wettbewerb aus dem Ausland oder eine nachfragestarke Marktgegenseite, allen Erwartungen nach keine unkontrollierten wettbewerblichen Verhaltensspielräume der Zusammenschlussbeteiligten zulassen.

Auf europäischer Ebene definierte der Europäische Gerichtshof in der Rechtssache "United Brands" eine marktbeherrschende Stellung als "wirtschaftliche Machtstellung eines Unternehmens (...), die dieses in die Lage versetzt, die Aufrechterhaltung eines wirksamen Wettbewerbs auf dem relevanten Markt zu verhindern, indem sie ihm die Möglichkeit verschafft, sich seinen Wettbewerbern, seinen Abnehmern und schließlich den Verbrauchern gegenüber in einem nennenswerten Umfang unabhängig zu verhalten". Die Eingriffsschwelle des Art. 82 (EG-Vertrag) setzt dort an, wo ein Unternehmen in der Lage ist, sich unabhängig vom Wettbewerb zu verhalten oder die Wettbewerbs- 
bedingungen zu kontrollieren. Wie auch im deutschen Recht spricht auf der Ebene der EU wesentlicher Wettbewerb unter diesen Umständen nicht zwingend gegen das Vorliegen einer marktbeherrschenden Stellung. Die Prüfung der marktbeherrschenden Stellung orientiert sich, ähnlich wie im GWB, im wesentlichen an markt- und unternehmensbezogenen Strukturkriterien.

Nachdem die vorigen Ausführungen den rechtlichen Rahmen schilderten und dazugehörige Rechtssprechungen aus der "allgemeinen Wirtschaft” herangezogen wurden, wird im anschließenden Kapitel die Anwendung in den Branchen der Milch- und Zuckerindustrie näher analysiert. 


\section{Beurteilung ausgewählter Fusionen in der Er- nährungsindustrie}

In diesem Kapitel werden Zielsetzung, Vorgehensweise und die praktische Beurteilung der Kartellbehörden anhand ausgewählter Unternehmenszusammenschlüsse in der Milch- und Zuckerindustrie vorgestellt und gewertet. Die Erkenntnisse wurden aus Befragungen/Akteneinsicht bei den zuständigen Mitarbeitern der Kartellbehörden, Interviews bei den betroffenen Unternehmen und Auswertungen der Fragebögen sowie sonstigen Veröffentlichungen wie Beschlüsse, Geschäftsberichte und Presseartikel, gewonnen. Zunächst werden die Ergebnisse der Unternehmensbefragungen vorgestellt. In einem weiteren Schritt erfolgt eine fallweise Darstellung von ausgewählten Unternehmenszusammenschlüssen und deren Beurteilung durch die Kartellbehörden.

\subsection{Auswertung der Befragung}

Einen Bestandteil dieser Untersuchung bildet die Auswertung eines Fragebogens, durch den die Motivation von Unternehmenszusammenschlüssen, die Quantifizierung und Qualifizierung der Erfolge/Misserfolge, aber auch Kritik und mögliche Verbesserungsvorschläge an der Kartellrechtspraxis sowie die Besonderheiten der Agrarbranche erfasst werden sollten. Jedoch stellte sich nach Versand der Fragebögen heraus, dass einige Unternehmen ein persönliches Gespräch präferierten. Daher bestand der Fortgang der Untersuchung einerseits in der Auswertung der eingesandten Bögen und andererseits in der Interpretation und Einordnung der geführten Interviews. Die Unternehmen wurden nach der Fusionsaktivität innerhalb der letzten sieben Jahre selektiert. Folgende Unternehmen wurden näher untersucht bzw. haben nähere Auskünfte abgelehnt (vgl. Tabelle 17). 
Tabelle 17: Befragung ausgewähIter Unternehmen

\begin{tabular}{|l|c|c|c|}
\hline & Fragebogen & Gespräch & Keine Teilnahme \\
\hline Zuckerindustrie: & $\mathrm{X}$ & & \\
\hline Jülich (Westzucker) & $\mathrm{X}$ & & \\
\hline Nordzucker & $\mathrm{X}$ & $\mathrm{X}$ & \\
\hline Südzucker & & $\mathrm{X}$ & $\mathrm{X}$ \\
\hline Pfeifer \& Langen & & & \\
\hline Union-Zucker & & & \\
\hline
\end{tabular}

Milchindustrie:

\begin{tabular}{|l|c|c|c|}
\hline Nordmilch & & $\mathrm{X}$ & \\
\hline Humana & $\mathrm{X}$ & $\mathrm{X}$ & \\
\hline Hochwald/Eifelperle & & & $\mathrm{X}$ \\
\hline Tuffi Campina/emzett & & \\
\hline
\end{tabular}

Beratungsgesellschaft:

AFC-International

$X$

Quelle: eigene Darstellung

Die Ergebnisse einer Befragung sollten nicht überbewertet werden, da sie z.T. vom Zeitpunkt der Befragung, subjektiven Faktoren und individuellen Einschätzungen abhängig sind (vgl. Kapitel 4.2). Dennoch konnten durchaus prinzipielle Probleme und Entwicklungen erfasst werden, die der Fragestellung dieser Untersuchung "Nährboden" und wichtige Hinweise verschafften. Ein Auszug der schriftlichen Befragung wird in der folgenden Darstellung (Tabelle 18) wiedergegeben, wobei $1=$ trifft nicht zu und $7=$ trifft voll zu. Auf die Auflistung der mündlichen Ergebnisse wird an dieser Stelle verzichtet; soweit diese von besonderer Erkenntnis waren werden sie an entsprechender Stelle im Verlauf der Arbeit erläutert.

Tabelle 18: Ergebnisse der Befragung

\begin{tabular}{|c|c|c|c|c|c|c|c|}
\hline \multirow{3}{*}{\begin{tabular}{|l} 
A. Kartellrechtspraxis \\
$\begin{array}{l}\text { Der geplante Zusammenschluss wurde dem Kar- } \\
\text { tellamt rechtzeitig gemeldet }\end{array}$
\end{tabular}} & \multirow{2}{*}{\multicolumn{2}{|c|}{$\begin{array}{c}\begin{array}{c}X=\text { Südzu- } \\
\text { cker }(1)\end{array} \\
\text { trifft nicht zu }\end{array}$}} & $\begin{array}{l}X=\text { Nordzu- } \\
\text { cker }(2)\end{array}$ & & \multicolumn{2}{|c|}{$X=\underset{(4)}{\operatorname{Humana}}$} & \multirow[b]{2}{*}{ t voll zu } \\
\hline & & & & & & & \\
\hline & 1 & 2 & 3 & 4 & \rangle & & $X X$ \\
\hline $\begin{array}{l}\text { Die Verhandlungsgespräche mit der Kartellbehör- } \\
\text { de verliefen schwierig }\end{array}$ & $X X$ & 2 & 3 & 4 & \rangle & 6 & $x$ \\
\hline $\begin{array}{l}\text { Der Zusammenschluss wurde nur mit Zugeständ- } \\
\text { nissen/Auflagen genehmigt }\end{array}$ & $X x$ & 2 & 3 & 4 & 5 & & $X X$ \\
\hline Die zuständigen Mitarbeiter erschienen kompe- & 1 & $x$ & 3 & $X$ & 5 & X & $x$ \\
\hline
\end{tabular}




\begin{tabular}{|c|c|c|c|c|c|c|c|}
\hline tent, informiert und aufgeschlossen & & & & & & & \\
\hline $\begin{array}{l}\text { Das "deutsche" Verfahren erweist sich als akzep- } \\
\text { tabel }\end{array}$ & 1 & $x$ & 3 & & 5 & 6 & $x$ \\
\hline $\begin{array}{l}\text { Wir fühlen uns gegenüber ausländischen Mitbe- } \\
\text { werbern durch die restriktive Zusammenschluss- } \\
\text { kontrolle benachteiligt }\end{array}$ & $x x$ & 2 & 3 & 4 & $x$ & 6 & $x$ \\
\hline $\begin{array}{l}\text { Die Kartellamtspraxis berücksichtigt nicht in aus- } \\
\text { reichendem Maße die besondere Stellung des } \\
\text { Agrarsektors (Einfluss der Agrarpolitik) }\end{array}$ & $x x$ & 2 & 3 & 4 & 5 & 6 & $x x$ \\
\hline $\begin{array}{l}\text { Mit der Änderung der Agrarpolitik (z.B. Abschaf- } \\
\text { fung von Quoten...) ist auch eine Umorientierung } \\
\text { der Kartellrechtspraxis notwendig }\end{array}$ & $x$ & 2 & 3 & & 5 & 6 & $X X$ \\
\hline $\begin{array}{l}\text { Die Machtposition des Handels (insb. LEH) wurde } \\
\text { nur unzureichend berücksichtigt }\end{array}$ & $X$ & 2 & 3 & 4 & 5 & $X$ & $x$ \\
\hline
\end{tabular}

\section{B. Sonderstellung der Zucker- o- der Milchbranche}

\begin{tabular}{|c|c|c|c|c|c|c|c|}
\hline $\begin{array}{l}\text { Die } \\
\text { lung/Exporterstattung) verzerrt die Wettbewerbs- } \\
\text { fähigkeit meines Unternehmens in erheblichem } \\
\text { Maße }\end{array}$ & $x$ & 2 & X & $X$ & 5 & 6 & 7 \\
\hline $\begin{array}{l}\text { Expansion kann nur über externes Wachstum } \\
\text { erfolgen, da insbesondere durch die Quotenrege- } \\
\text { lung der Zugriff auf den Beschaffungsmarkt (Roh- } \\
\text { stoff Milch/Zucker) stark eingeschränkt ist }\end{array}$ & 1 & 2 & 3 & 4 & 5 & 6 & $X X X$ \\
\hline $\begin{array}{l}\text { Hieraus ergibt sich meines Erachtens ein Konflikt } \\
\text { (Verzerrung durch Agrarpolitik versus strenge } \\
\text { Kartellrechtspraxis) }\end{array}$ & $x$ & 2 & 3 & X & 5 & 6 & $x$ \\
\hline $\begin{array}{l}\text { Landwirte bzw. Vertreter wurden von Beginn an } \\
\text { (Überlegung } \\
\text { on/Unternehmenspartnerauswahl) informiert }\end{array}$ & 1 & 2 & 3 & 4 & 5 & 6 & $X X X$ \\
\hline $\begin{array}{l}\text { Landwirte erweisen sich als schwierige Verhand- } \\
\text { lungspartner }\end{array}$ & & 2 & 3 & 4 & $X X$ & 6 & 7 \\
\hline $\begin{array}{l}\text { Widerstand/Skepsis ist eher bei Betriebsleitern } \\
\text { kleinerer landwirtschaftlicher Betriebe zu erwarten }\end{array}$ & $X$ & 2 & 3 & 4 & & 6 & 7 \\
\hline $\begin{array}{l}\text { Die größere Unternehmung schafft gegenüber } \\
\text { Landwirten eine verbesserte Verhandlungsposition }\end{array}$ & $x$ & 2 & 3 & & 5 & 6 & 7 \\
\hline $\begin{array}{l}\text { Mit dem Zusammenschluss fühlen wir uns gestärkt } \\
\text { gegenüber einer Änderung der Agrarpolitik }\end{array}$ & $x$ & 2 & 3 & 4 & & 6 & $X X$ \\
\hline
\end{tabular}

\section{Beschreibung der Ziele des Zu- sammenschlusses}

C1. Folgende Ziele sind mit der Akquisition vor der Übernahme verbunden gewesen

\begin{tabular}{|l|c|c|c|c|c|c|c|}
\hline Vergrößerung der Marktmacht & 1 & 2 & 3 & $X$ & 5 & 6 & $X \times$ \\
\hline $\begin{array}{l}\text { Zugang zu neuen Technologien, Mitarbeitern, } \\
\text { Kunden }\end{array}$ & 1 & 2 & 3 & 4 & $X$ & $X$ & 7 \\
\hline $\begin{array}{l}\text { Realisierung finanzieller Synergiepotentiale (incl. } \\
\text { steuerl. Vorteile) }\end{array}$ & 1 & 2 & 3 & 4 & $X$ & 6 & $X$ \\
\hline
\end{tabular}




\begin{tabular}{|c|c|c|c|c|c|c|c|}
\hline Ausschöpfung funktionaler Synergiepotentiale & 1 & 2 & 3 & 4 & 5 & $\mathrm{XX}$ & $\mathrm{x}$ \\
\hline Ausweichen in schnell wachsende Marktsegmente & & $x$ & 3 & 4 & $\mathrm{x}$ & 6 & 7 \\
\hline $\begin{array}{l}\text { Erreichen einer "kritischen Mindestgröße" für den } \\
\text { eigenen Geschäftsbereich }\end{array}$ & & 2 & $\mathrm{x}$ & 4 & 5 & 6 & $\mathrm{x}$ \\
\hline Ausweichen in profitable Marktsegmente & $x \times$ & 2 & 3 & $\mathrm{x}$ & 5 & 6 & 7 \\
\hline Erwerb eines Markennamens & $\mathrm{x}$ & $\mathrm{x}$ & 3 & 4 & 5 & 6 & 7 \\
\hline Reaktion auf "Globalisierung" & 1 & 2 & 3 & $x$ & & $x$ & 7 \\
\hline $\begin{array}{l}\text { Notwendige Reaktion auf Verhalten der Konkur- } \\
\text { renz }\end{array}$ & 1 & $x$ & 3 & 4 & & 6 & $\mathrm{x}$ \\
\hline Streben nach Macht, Einfluss, Prestige & $\mathrm{XX}$ & 2 & 3 & 4 & 5 & $x$ & 7 \\
\hline Beeindrucken des Wettbewerbs & $\mathrm{X} \times$ & 2 & 3 & 4 & $x$ & 6 & 7 \\
\hline
\end{tabular}

\section{C2. Spezifizierung der synergetischen Ziele}

\subsection{Finanzielle Synergie}

\begin{tabular}{|c|c|c|c|c|c|c|c|}
\hline Risikostreuung & 1 & 2 & 3 & 4 & $\mathrm{XX}$ & 6 & 7 \\
\hline $\begin{array}{l}\text { Verminderung des Liquiditätsrisikos (Pooling von } \\
\text { Liquidität) }\end{array}$ & 1 & $x$ & 3 & 4 & $x$ & 6 & 7 \\
\hline Nutzung von Verlustvorträgen (steuerlicher Vorteil) & $x$ & $\mathrm{x}$ & 3 & 4 & 5 & 6 & 7 \\
\hline $\begin{array}{l}\text { Abzugsfähigkeit von FK-Zinsen (steuerlicher Vor- } \\
\text { teil) }\end{array}$ & $x$ & $x$ & 3 & 4 & 5 & 6 & 7 \\
\hline $\begin{array}{l}\text { Erleichterte Aufnahme von Fremdkapital (Zunah- } \\
\text { me der Kreditwürdigkeit) }\end{array}$ & & $x$ & 3 & $\mathrm{X}$ & 5 & 6 & 7 \\
\hline $\begin{array}{l}\text { Aufnahme von Fremdkapital zu verbesserten } \\
\text { Konditionen }\end{array}$ & & 2 & $X$ & $\mathrm{X}$ & 5 & 6 & 7 \\
\hline $\begin{array}{l}\text { Verkürzung der durchschnittlichen Bindungsdauer } \\
\text { des Gesamtkapitals }\end{array}$ & 1 & $X$ & 3 & & 5 & 6 & 7 \\
\hline
\end{tabular}

\subsection{Funktionale Synergien}

\subsubsection{Vertrieb/ Marketing}

\begin{tabular}{|c|c|c|c|c|c|c|c|}
\hline Gemeinsamer Markenname & & $x$ & 3 & $\mathrm{x}$ & 5 & 6 & 7 \\
\hline Kombinierter Produktabsatz & 1 & $x$ & 3 & 4 & $x$ & 6 & \\
\hline $\begin{array}{l}\text { Gemeinsame Nutzung der in- und ausländischen } \\
\text { Niederlassungen (incl. Läger, Fuhrpark) }\end{array}$ & & $x$ & 3 & $\mathrm{x}$ & 5 & 6 & 7 \\
\hline Gemeinsamer Außendienst & 1 & $x$ & 3 & 4 & 5 & $x$ & \\
\hline $\begin{array}{l}\text { Gemeinsamer Kundendienst/ Anwendungstechni- } \\
\text { ken/ Service }\end{array}$ & 1 & 2 & 3 & 4 & $X X$ & 6 & \\
\hline Gemeinsame Auftragsabwicklungssystem & 1 & 2 & 3 & $x$ & $x$ & 6 & \\
\hline $\begin{array}{l}\text { Zusammenlegung der Marketingabteilung / Markt- } \\
\text { forschung (incl. Werbeabteilung) }\end{array}$ & 1 & 2 & 3 & $x$ & 5 & 6 & $x x$ \\
\hline Gemeinsames physisches Distributionssystem & 1 & 2 & 3 & 4 & 5 & 6 & $x x$ \\
\hline Einführung vorteilhafterer Vertriebskonditionen & 1 & 2 & 3 & 4 & 5 & 6 & $x$ \\
\hline $\begin{array}{l}\text { Nutzung/ Schaffung einer gemeinsamen Finanzie- } \\
\text { rung (-sorganisation) für Abnehmer oder Distribu- } \\
\text { toren }\end{array}$ & 1 & $\mathrm{x}$ & 3 & 4 & 5 & 6 & 7 \\
\hline
\end{tabular}




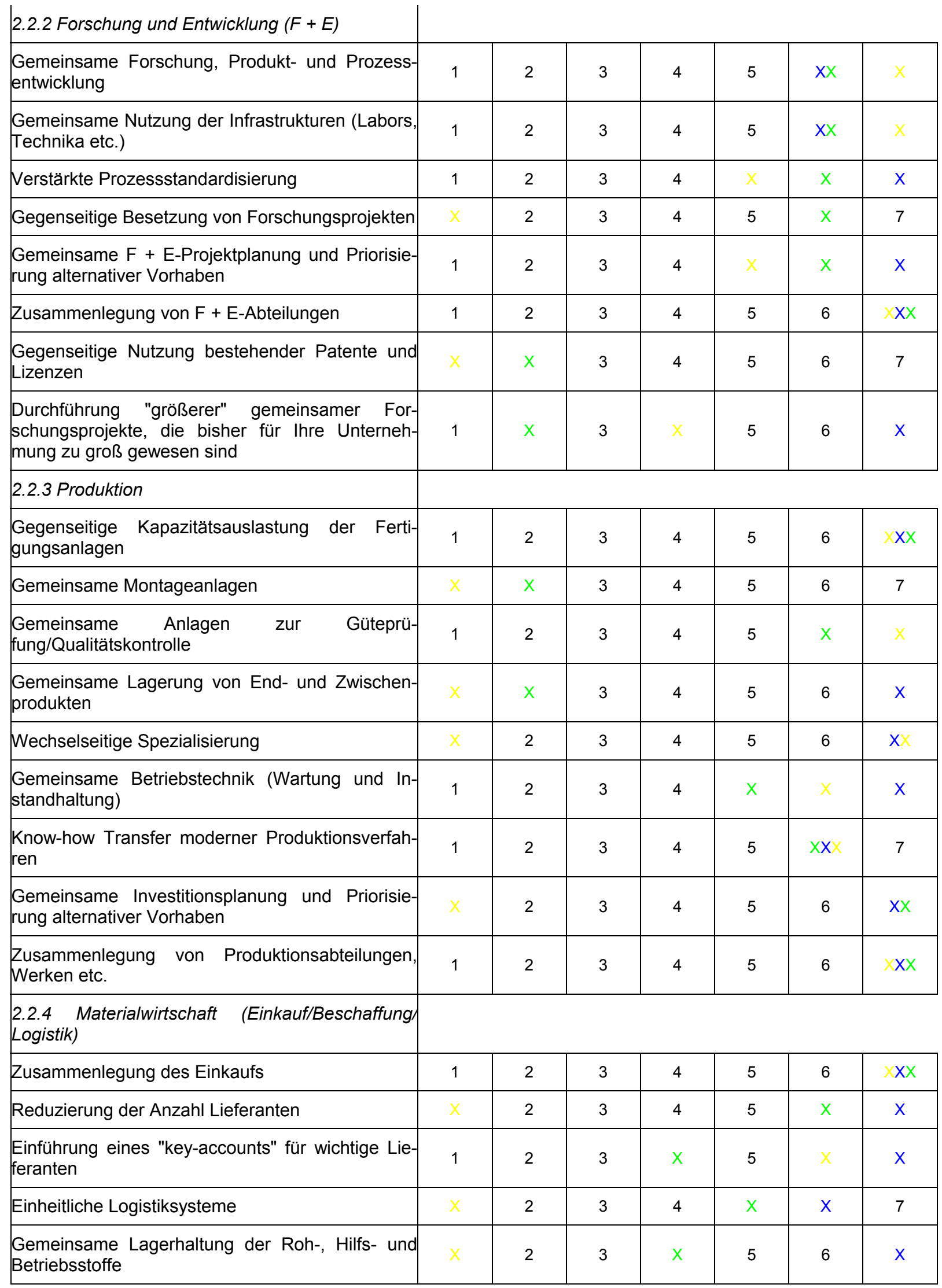




\begin{tabular}{|c|c|c|c|c|c|c|c|}
\hline Zusammenlegung der Logistikabteilungen & 1 & 2 & 3 & 4 & & 6 & $\mathrm{XX}$ \\
\hline \multicolumn{8}{|l|}{ 2.2.5 Verwaltung } \\
\hline Zentralisierung des Rechnungswesens & 1 & 2 & 3 & 4 & 5 & 6 & $x x$ \\
\hline Gemeinsame Nutzung des Rechenzentrums & 1 & 2 & 3 & 4 & 5 & 6 & $x$ \\
\hline Gemeinsame Softwareentwicklung/-anpassung & 1 & 2 & 3 & 4 & 5 & 6 & $x x$ \\
\hline Zentralisierung der Organisationsabteilungen & 1 & 2 & 3 & 4 & 5 & 6 & $x x$ \\
\hline Zusammenfassung der Personalabteilungen & 1 & 2 & 3 & 4 & 5 & 6 & $x x$ \\
\hline Zusammenlegung der Zentralen Dienste & 1 & 2 & 3 & 4 & 5 & 6 & $x x$ \\
\hline Zusammenfassung der Öffentlichkeitsarbeit & 1 & & 3 & 4 & 5 & 6 & $X X$ \\
\hline Zusammenlegung der Rechtsabteilung & & 2 & 3 & 4 & 5 & 6 & $x$ \\
\hline $\begin{array}{l}\text { Zusammenlegung der Planungs- und Controlling- } \\
\text { abteilungen }\end{array}$ & 1 & 2 & 3 & 4 & 5 & 6 & $x x$ \\
\hline Zentralisierung der Beteiligungsführung & & 2 & 3 & 4 & 5 & 6 & $X X$ \\
\hline Zusammenfassung der Revisionsabteilungen & & 2 & 3 & 4 & 5 & 6 & $x$ \\
\hline Zentralisierung an einem Verwaltungsstandort & 1 & 2 & 3 & 4 & & 6 & $x X$ \\
\hline
\end{tabular}

\subsection{Folgende potentielle Wettbewerbsvorteile} wurden durch Ausschöpfung der funktionalen Synergien vor der Übernahme erwartet/erhofft

\subsubsection{Vertrieb/Marketing}

\begin{tabular}{|c|c|c|c|c|c|c|c|}
\hline Niedrigere Werbungskosten & & $\mathrm{X}$ & 3 & $x$ & 5 & 6 & 7 \\
\hline Verstärkung durch Produktimage/Ruf & & $\mathrm{X}$ & $\mathrm{X}$ & 4 & 5 & 6 & 7 \\
\hline $\begin{array}{l}\text { Niedrigere Kosten für das Auffinden neuer Ab- } \\
\text { nehmer }\end{array}$ & & $\mathrm{X}$ & $\mathrm{X}$ & 4 & 5 & 6 & 7 \\
\hline Niedrigere Verkaufskosten & 1 & $\mathrm{X}$ & & $x$ & 5 & 6 & 7 \\
\hline Niedrigere Vertriebsgemeinkosten & 1 & $\mathrm{x}$ & 3 & 4 & 5 & $x x$ & 7 \\
\hline Geringere Außendienstkosten & 1 & $\mathrm{X}$ & 3 & 4 & $x$ & & 7 \\
\hline Umsatzerweiterung durch Produktergänzung & & 2 & 3 & 4 & $x$ & $x$ & 7 \\
\hline Niedrigere Kosten für Marktforschung & 1 & & 3 & 4 & $\mathrm{x}$ & 6 & 7 \\
\hline $\begin{array}{l}\text { Kosteneinsparung im Bereich der Verkaufsförde- } \\
\text { rung }\end{array}$ & & 2 & 3 & 4 & $\mathrm{X}$ & 6 & 7 \\
\hline $\begin{array}{l}\text { Größere Verhandlungsstärke gegenüber den } \\
\text { Vertriebskanälen (verbesserter Service, Anwen- } \\
\text { dungstechnik, Regalplazierung etc.) }\end{array}$ & & 2 & 3 & 4 & 5 & $x$ & $x$ \\
\hline $\begin{array}{l}\begin{array}{l}\text { Kostenreduzierung für Hilfseinrichtungen der Ver- } \\
\text { triebsinfrastruktur }\end{array} \\
\end{array}$ & & 2 & 3 & $x$ & 5 & $x$ & 7 \\
\hline Qualifizierteres Verkaufspersonal & & 2 & 3 & 4 & $\mathrm{x}$ & $x$ & 7 \\
\hline $\begin{array}{l}\text { Durch Know-how Transfer technologisch verbes- } \\
\text { serter Kundendienst, Service, Anwendungstechnik }\end{array}$ & & 2 & 3 & 4 & $\mathrm{x}$ & $x$ & 7 \\
\hline
\end{tabular}




\begin{tabular}{|c|c|c|c|c|c|c|c|}
\hline $\begin{array}{l}\text { Umsatzerweiterung durch Schaffung einer Finan- } \\
\text { zierung(-sorganisation) }\end{array}$ & $x \mathrm{X}$ & 2 & 3 & $\mathrm{x}$ & 5 & 6 & 7 \\
\hline Niedrigere Auftragsabwicklungskosten & 1 & 2 & 3 & & $\mathrm{x}$ & $\mathrm{x}$ & 7 \\
\hline
\end{tabular}

2.3.2 Forschung und Entwicklung

\begin{tabular}{|l|c|c|c|c|c|c|c|}
\hline Senkung der Forschungs- und Entwicklungszeiten & $\times$ & $X$ & 3 & 4 & 5 & $X$ & 7 \\
\hline Senkung der direkten F+E-Kosten & $x$ & $X$ & 3 & 4 & 5 & $X$ & 7 \\
\hline Reduzierung der F+E-Gemeinkosten & $\times$ & 2 & 3 & 4 & 5 & $X \times$ & 7 \\
\hline $\begin{array}{l}\text { Erhöhung der F+E-Qualität (Output), z.B Zunahme } \\
\text { der angemeldeten Patente }\end{array}$ & $\times$ & 3 & 4 & 5 & 6 & $X$ \\
\hline $\begin{array}{l}\text { Erhöhte Innovationskraft durch Transfer von Neu- } \\
\text { entwicklungen }\end{array}$ & 2 & 3 & $\times$ & 5 & 6 & $\times$ \\
\hline
\end{tabular}

2.3.3 Produktion

\begin{tabular}{|c|c|c|c|c|c|c|c|}
\hline $\begin{array}{l}\text { Niedrigere Fertigungskosten durch verbesserte } \\
\text { Auslastung }\end{array}$ & 1 & 2 & 3 & 4 & 5 & $x$ & $x$ \\
\hline Reduzierung der Montagekosten & & 2 & 3 & 4 & $x$ & 6 & 7 \\
\hline Niedrigere Kosten der Qualitätskontrolle & 1 & 2 & 3 & 4 & $x$ & $x$ & 7 \\
\hline $\begin{array}{l}\text { Eine technologische Verbesserung führt zur bes- } \\
\text { serer Qualitätskontrolle }\end{array}$ & 1 & 2 & 3 & $X$ & 5 & $X$ & 7 \\
\hline $\begin{array}{l}\text { Verbesserte Qualität (Output) in der Produktion } \\
\text { (Senkung der Fehlerquote) durch verbesserte } \\
\text { Produktionsverfahren }\end{array}$ & $X$ & 2 & 3 & 4 & 5 & & $x$ \\
\hline $\begin{array}{l}\text { Senkung der Wartungs- und Instandhaltungskos- } \\
\text { ten }\end{array}$ & 1 & 2 & 3 & 4 & 5 & $x X$ & $x$ \\
\hline $\begin{array}{l}\text { Erhöhte Rendite bzw. schnellere Amortisation der } \\
\text { Investition innerhalb der Produktion }\end{array}$ & 1 & 2 & 3 & 4 & 5 & $x x$ & $\mathrm{X}$ \\
\hline Senkung der Produktionsgemeinkosten & 1 & 2 & 3 & 4 & 5 & & $x$ \\
\hline $\begin{array}{l}\text { Senkung der Lagerkosten von End- und Zwi- } \\
\text { schenprodukten }\end{array}$ & 1 & 2 & 3 & 4 & $x$ & 6 & $x$ \\
\hline Senkung der Rüstkosten durch Spezialisierung & & 2 & 3 & 4 & 5 & $x$ & $x$ \\
\hline
\end{tabular}

\subsubsection{Materialwirtschaft (Einkauf/Beschaffung/ Logistik)}

\begin{tabular}{|c|c|c|c|c|c|c|c|}
\hline $\begin{array}{l}\text { Bessere Einkaufskonditionen (Senkung der Input- } \\
\text { Kosten) }\end{array}$ & 1 & 2 & 3 & 4 & & $x$ & $\mathrm{x}$ \\
\hline Verbesserte Qualität der eingekauften Güter & & 2 & 3 & 4 & $x$ & 6 & $\mathrm{x}$ \\
\hline $\begin{array}{l}\text { Senkung der Lagerkosten durch flexiblere Liefer- } \\
\text { bedingungen im Einkauf (häufigere, kleinere Liefe- } \\
\text { rungen) }\end{array}$ & 1 & 2 & & 4 & $x$ & 6 & $x$ \\
\hline Reduzierung der Transportkosten & & 2 & 3 & 4 & 5 & $x$ & $\mathrm{x}$ \\
\hline $\begin{array}{l}\text { Aufspüren günstigerer Lieferanten durch Speziali- } \\
\text { sierung (z.B. nach Branchen) des Einkaufs }\end{array}$ & & 2 & $x$ & 4 & 5 & 6 & $\mathrm{x}$ \\
\hline $\begin{array}{l}\text { Reduzierung der Eingangsprüfkosten durch Verla- } \\
\text { gerung der Qualitätsprüfung auf Lieferanten }\end{array}$ & & 2 & $\mathrm{x}$ & 4 & 5 & 6 & $\mathrm{X}$ \\
\hline $\begin{array}{l}\text { Senkung der Lagerkosten durch Zentralisierung } \\
\text { der Lagerhaltung }\end{array}$ & 1 & 2 & $x X$ & 4 & 5 & 6 & $x$ \\
\hline
\end{tabular}




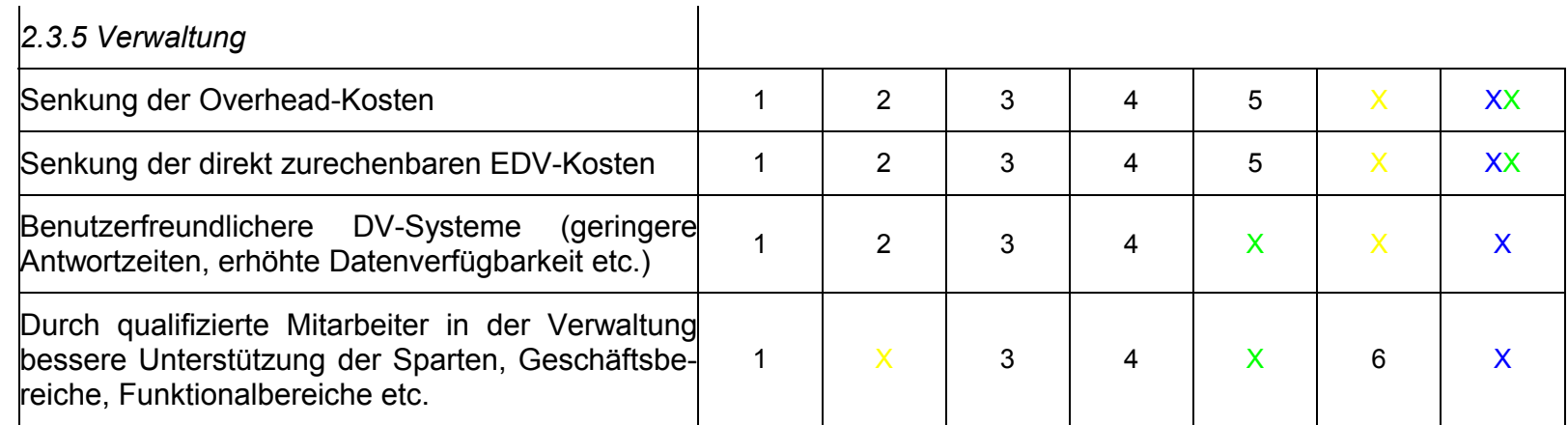

Anmerkung: (1) Südzucker/ Saint Louis Sucre mit Auflagen genehmigt (EU-Kommission); (2) ZVN/ ZAG/ Union Zucker mit Auflagen genehmigt;

(4) Westmilch/Milchwerke Westfalen:

Freigabe

\section{Quelle: eigene Darstellung}

Die Ergebnisse werden aufgrund der zuvor aufgeführten Anmerkungen nicht im einzelnen diskutiert. Festzuhalten bleibt, dass sich der Verdacht bestätigt, dass Zusammenschlüsse durch eine Vielzahl von Motiven begründet sind und eine klare Abgrenzung der Motive nicht immer möglich ist. Erwartungsgemäß versprechen sich die Unternehmen durch den Zusammenschluss eine Ausnutzung von Synergievorteilen, um optimale Betriebsgrößen zu erreichen, aber auch die Erzielung von Marktmacht (gegengewichtige Position gegenüber den Abnehmern) scheint ein wichtiger Beweggrund zu sein. Durch die Spezifika der agrarpolitischen Regelungen ist externes Wachstum im Zuge von Fusionen und Kooperationen oftmals der einzige Weg, die Größenvorteile zu erlangen. Die Beurteilung der Kartellrechtspraxis durch die betroffenen Unternehmen ergibt ein indifferentes Bild. Offensichtlich besteht ein Zusammenhang zwischen der Kritik an der Zusammenschlussbeurteilung der Kartellbehörden und den erteilten Unternehmensauflagen. Die konkrete Prüfung ausgewählter Zusammenschlüsse wird in den anschließenden Abschnitten näher aufgegriffen.

\subsection{Zusammenschlussbeurteilung ausgewählter Fälle durch die Wettbewerbsbehörden}

\subsubsection{Zuckerindustrie}

In den letzten zehn Jahren waren es im wesentlichen fünf Unternehmenszusammenschlüsse, die das Geschehen der deutschen Zuckerindustrie prägten: (1) 1992: Südzucker AG/Zuckerfabrik Soest, (2) 1995: Bedburg/Jülich, (3) 1997: Entstehung der Nordzucker AG, (4) 2001: Südzucker AG/Saint Louis Sucre, (5) 2002: Nordzucker AG/Union-Zucker.

In dieser Studie werden die drei jüngsten Zusammenschlüsse näher analysiert. Dabei lässt sich die Vorgehensweise der Kartellbehörde zur Beurteilung von Fusionen auf 
andere Unternehmenszusammenschlüsse problemlos übertragen. Der Zusammenschluss Südzucker/Saint Louis Sucre im Jahre 2001 erreichte Umsatzschwellen, die eine Prüfung durch die Europäischen-Kommission erforderlich machten. Somit wird in diesem Kapitel ein Überblick zunächst über die deutsche und anschließend über die europäische Beurteilungsweise gegeben.

\subsubsection{Nordzucker AG (1997)}

Ebenso wie bei der Milchindustrie nimmt die Zahl der Zuckerfabrikgesellschaften in der EU weiterhin $a b$. Die fünf größten Rübenzucker erzeugenden Unternehmen produzieren heute ca. 58 \% des EU-Rübenzuckers (vgl. Kapitel 2.5). In den Jahren 1996/97 verhandelten die vier norddeutschen Zuckerunternehmen Zuckerverband Nord AG $(Z V N)$, Zucker-AG Uelzen-Braunschweig (ZAG), Union-Zucker Südhannover GmbH und die Zuckerfabrik Harsum AG über eine mögliche Fusion, um dadurch zu der neuen Nordzucker AG zu verschmelzen ${ }^{326}$. Bis auf die der Harsum AG stimmten die Gesellschafter diesen Plänen zu, so dass das Vorhaben beim Bundeskartellamt ( $\S 39$ GWB) angezeigt werden konnte. Das Bundeskartellamt erklärte am 14. November 1997, dass es dem Fusionsvorhaben nicht zustimmen würde, da durch den Zusammenschluss eine marktbeherrschende Stellung erreicht werden würde. Einem Zusammenschluss unter den drei verbleibenden Unternehmen würde nur zugestimmt, wenn diese insgesamt 100.000 t Weißzuckerquote an Konkurrenzunternehmen abgäben. Von dieser Auflage abgeschreckt, trat die Union-Zucker von dem Fusionsvorhaben zurück. Eine Fusion zwischen der verbleibenden ZVN und der Zucker AG Uelzen-Braunschweig wurde durch das Bundeskartellamt ohne weitere Auflagen genehmigt ${ }^{327}$.

\subsection{Motive der Fusion}

Bevor Argumentation und Vorgehensweise des Bundeskartellamtes vorgestellt werden, sollen zunächst Motive von Seiten der Zusammenschlusspartner illustriert werden (in Ergänzung mit Kapitel 6.1). Folgende Kennzahlen prägten die unabhängigen Unternehmen vor der Fusion (Tabelle 19):

\footnotetext{
${ }^{326} \mathrm{Vgl.}$ ZUCKERINDUSTRIE 47 (1997) 8, S. 666

327 Vgl. ZUCKERINDUSTRIE 48 (1998) 1, S. 49
} 
Tabelle 19: Kennzahlen der Zusammenschlusspartner 1996/97

\begin{tabular}{|l|c|c|c|c|}
\hline & ZVN & ZAG & Union-Zucker & Harsum \\
\hline Bruttoumsatz (Mio. DM) & 1145 & 588 & 173 & 23 \\
\hline Werke & 7 & 4 & 1 & 1 \\
\hline Stamm-Mitarbeiter & 1148 & 677 & 213 & 39 \\
\hline Aktionäre/Gesellschafter & 12884 & 8111 & 2015 & 291 \\
\hline Anbaufläche (ha/Jahr) & 98600 & 68300 & 15200 & 2146 \\
\hline Rübenverarbeitung (1000 t) & 4655 & 3126 & 752 & 110 \\
\hline Tagesverarbeitung (t/Tag) & 56000 & 38000 & 9300 & 1800 \\
\hline Zuckererzeugung (t/Jahr) & 762000 & 486000 & 121000 & 17363 \\
\hline EU-Höchstquote (t/Jahr) & 639105 & 410543 & 101383 & 15245 \\
\hline
\end{tabular}

Quelle: Niederhut-Bollmann/Schrader 2001, S. 11

Die Argumente der Fusionsbefürworter bewegten sich in erster Linie dahingehend, den gestiegenen Anforderungen der Nachfrageseite gerecht zu werden. Die zunehmende Europäisierung im LEH erfordere ebenfalls eine Präsenz am europäischen Markt. Nur dann könnten Ergebnisverbesserungen durch eine Zusammenlegung des Einkaufs und die Erzielung höherer Rabatte realisiert werden ${ }^{328}$. Eine Liberalisierung des Welthandels und Öffnung der europäischen Märkte würde ferner das zukünftige Wettbewerbsgeschehen am Zuckermarkt bestimmen. Ziel sei es daher, bereits zuvor alle möglichen Einsparungspotentiale zu mobilisieren ${ }^{329}$. Möglich wäre dies durch ${ }^{330}$ :

- den Abbau von Überkapazitäten,

- die Konzentration der Zuckersortenproduktion auf geeignete marktnahe Standorte,

- die Steigerung der Produktivität in allen Werken,

- die Zusammenlegung der vier Verwaltungen unter Einbindung der Vertriebsgesellschaft,

- die Verbesserung der Rübenlogistik,

- die Zusammenfassung und den Ausbau der Forschungs- und Entwicklungsaktivitäten.

\footnotetext{
${ }^{328} \mathrm{Vgl}$. ZVN 1995/96, S. 61

${ }^{329} \mathrm{Vgl}$. ZVN 1995/96, S. $62 \mathrm{ff}$.

${ }^{330}$ Vgl. ZVN 1995/96, S. 66 f.
} 
Monetär wurden Synergieeffekte der beiden Zusammenschlusspartner von jährlich 25 Mio. $€$ pro Jahr geschätzt ${ }^{331}$.

\subsection{Prüfung durch das Bundeskartellamt}

Aus Geheimhaltungsgründen werden im folgenden keine detaillierten Daten oder Namen genannt. Dies scheint zur Verdeutlichung des Prinzips der Überprüfung auch nicht notwendig.

Im ersten Schritt reichte die Nordzucker AG, vertreten durch eine Kanzlei, alle notwendigen Anträge ein und ergänzte diese um detaillierte Angaben über die Funktion der Zuckermarktordnung, Verarbeitungsmengen und -kapazitäten und weitere Kennzahlen der Beteiligten wie auch der Konkurrenzunternehmen in Deutschland (und z.T. in Europa). Hingewiesen wurde ferner auf eine sinkende Verarbeitungsspanne und den zunehmenden Kostendruck durch höhere Inputpreise (Arbeit, Bau, Umwelt), was einen Rationalisierungszwang hervorrufe. Ebenso wurden die größten Abnehmer mit den entsprechenden Absatzmengen benannt. Neben den oben angeführten produktionstechnischen Vorteilen würde ein Zusammenschluss des weiteren die Herstellung von Produkten ermöglichen, die vorher nicht produziert wurden. Dies käme der zunehmenden Forderung des Handels nach dem „Bezug aller Produkte aus einer Hand" nach. Außerdem wurde auf die z.T. wettbewerbsfähigeren Konkurrenzunternehmen hingewiesen und das Argument des Erhalts einer norddeutschen Zuckerproduktion angeführt. Es wurde außerdem ein Vorschlag für die Abgrenzung des sachlich und geographisch relevanten Marktes unterbreitet sowie Position zu einer evtl. marktbeherrschenden Stellung bezogen.

Nach Erhalt der Informationen begannen die Recherchen des Bundeskartellamtes. Nachdem die Unterlagen auf ihre Richtigkeit geprüft wurden, erfolgte zunächst eine Unterrichtung der Konkurrenzunternehmen von dem Vorhaben. Diese bezogen nun Stellung zu einzelnen Punkten wie Abnehmerstruktur, Importe, Lieferantenentfernungen, Zuckersorten etc. und hatten die Möglichkeit, auch Einwände und mögliche Nachteilseinschätzungen zu äußern. Ebenso wurden Mengen- und Umsatzdaten der Konkurrenten erhoben und mit den Angaben der Nordzucker verglichen. Besondere Beachtung fanden ferner Zuckerabnehmer, die sich im "typischen" Nordzuckerabnahmeterritorium befinden. Anhand von Postleitzahlengebieten wurde letztendlich der

\footnotetext{
${ }^{331} \mathrm{Vgl.} \mathrm{SOMMER} 1998$, S. 38
} 
Marktanteil der fusionsbeteiligten Unternehmen für die Märkte Industrie-, Haushaltsund Flüssigzucker festgelegt.

Es herrschte im wesentlichen bei den darauf folgenden Gesprächen zwischen Kartellbehörde und Unternehmensvertretern Uneinigkeit über den relevanten Markt. Der vorgeschlagene Radius von 200 bis 300 km Umkreis vom Produktionsstandort wurde von Vertretern der Zuckerunternehmen abgelehnt, denn vor allem strategische Partnerschaften mit multinationalen Abnehmerunternehmen erforderten eine nationale oder gar europäische Präsenz. Die Kartellbehörde kam jedoch aufgrund eines nahezu $100 \%$ igen Marktanteils in Schleswig-Holstein oder Hamburg zu dem Schluss, dass dem Vorhaben nur unter Abgabe von ca. 10\% der Höchstquote oder Ausscheiden eines Partners zugestimmt werden könne. Durch die ZMO sei der Wettbewerb in der Zuckerindustrie besonders stark eingeschränkt. Deshalb sei der Erhalt eines Restwettbewerbes von außerordentlicher Wichtigkeit, um auch nach einer evtl. Beendigung der ZMO einem anderen Unternehmen Zugang zum norddeutschen Markt zu ermöglichen.

Nach Mitteilung der Bedenken wurden außer den Konkurrenten zusätzlich die größten Abnehmer über die Unternehmenspolitik der fusionierenden Konzerne und mögliche Auswirkungen eines Zusammenschlusses befragt, wobei bestätigt wurde, dass der Zuckermarkt sehr reglementiert war, Preise gar abgestimmt erschienen und sogar eine nationale Abgrenzung z.T. als unzureichend (zu groß) angesehen wurde.

Abschließend wurden weitere Argumente der Zuckerunternehmen geprüft, wie z.B. mögliche Synergievorteile (Abwägungsklausel), Besitzverhältnisse an den Zuckerrübenlieferrechte, und die Notwendigkeit einer Aufholfusion (Anschluss an Größenordnungen wie beispielsweise der Südzucker AG) analysiert und z.T. mit Hilfe Dritter (Bundeslandwirtschaftsministerium) überprüft.

Die Argumente der Unternehmensvertreter konnten jedoch die Beschlussabteilung nicht davon überzeugen, dass insbesondere der Restwettbewerb innerhalb der ZMO besonders schützenswert wäre, und somit die oben genannten Auflagen zu erfüllen seien. Die Kartellbehörde stimmte dem Unternehmenszusammenschluss nach Ausscheiden der Zuckerfabrik Harsum und der Union Zucker ohne weitere Auflagen zu.

\subsection{Zwischenergebnis}

Einige besonders interessante Punkte seien an dieser Stelle hervorgehoben: Der Beschaffungsmarkt (Landwirte) spielte bei der Untersuchung nur eine untergeordnete oder gar keine Rolle. Ebenso wurden Rechtsform oder die dahinterstehenden Besitzverhältnisse nicht näher analysiert. Auf der Abnehmerseite wurde das in den Fragebögen ausgewertete Hauptmotiv der Nachfragemacht des LEH überhaupt nicht berücksichtigt, da nach Aussage der Kartellbehördenmitarbeiter alle Zuckerunternehmen 
gleichermaßen von der Konzentration des LEH betroffen seien. Eine Abwägung zwischen Synergievorteilen und Marktmachteffekten wurde nur geringfügig, basierend auf Einschätzungen von Konkurrenzunternehmen, vorgenommen. Wie exakt die Höhe der abzugebenden Quote (entspricht gleichzeitig der Höchstquote der Union-Zucker) bzw. die Notwendigkeit des Ausscheidens eines Unternehmens ermittelt wurde, konnte nicht zufriedenstellend geklärt werden.

\subsubsection{Nordzucker AG und Union-Zucker (2002)}

\subsection{Hintergrund}

Die Einschätzung aus dem Jahre 1997 ergab, dass einem Zusammenschluss mit der Union-Zucker nicht ohne Auflagen zugestimmt worden wäre. Während der Recherchen wurden die Kartellbehörden darauf aufmerksam, das der Vertrieb des Zuckers von der Nordzucker AG wie auch der Union Zucker fast vollständig über die Nordzucker KG Vertriebsgesellschaft erfolgte und die Union-Zucker über keinen eigenen Vertrieb verfügte $^{332}$. Das Bundeskartellamt untersagte damit am 18. März 1999 wegen Verstoß gegen $\S 1$ GWB in Verbindung mit $\S 28$ GWB (= Ausnahmebereich Landwirtschaft) und Art. 81 Abs. 1 EG die gemeinsame Vertriebsgemeinschaft und forderte die unverzügliche Auflösung. Überraschenderweise schätzte das Kammergericht Berlin diese Konstellation anders ein und hob den Beschluss der Kartellbehörde nach Klage von den beteiligten Unternehmen auf ${ }^{333}$. Dabei kritisierte das Kammergericht die damalige Erlaubnis zur Fusion von ZVN und ZAG zur Nordzucker AG. Die Union Zucker sei in dieser Situation nicht mehr wettbewerbsfähig, für Großkunden als alleine agierendes Unternehmen uninteressant, und somit gäbe es keine wesentlichen Auswirkungen, wenn die Unternehmen zusammen oder getrennt agieren würden ${ }^{334}$. Dieses Urteil sah die Geschäftsführung im Jahr 2002 als Anlass, erneut den Versuch einer Fusion von Nordzucker und Union Zucker zu unternehmen ${ }^{335}$.

\subsection{Erneute Beurteilung}

Die sachliche Marktabgrenzung wurde in Industrie-, Haushalts- und Flüssigzucker vorgenommen. Zwar weist Industriezucker fast die gleichen Charakteristika wie Haushaltszucker auf, kann inn aber aufgrund der fehlenden endverbrauchergerechten Por-

\footnotetext{
332 Ausnahme: ca. 10.000 - 14.000 t des Zuckers werden über das Zuckerhandelshaus Schlüter\&Maack vertrieben (Folge der Übernahme der Zuckerfabrik Harsum von Pfeifer \& Langen und der Union-Zucker).

333 KAMMERGERICHT, Beschluss vom 10.10.2001 (Kart 30/99; B2-91/98)

${ }^{334}$ Vgl. BundeSKARTELLAMT 2002, B2 - 31/02, "Nordzucker/Union-Zucker",

${ }^{335} \mathrm{Vgl}$. NORDZUCKER Pressemitteilung 25.07.2002
} 
tionierung funktionell nicht ersetzen ${ }^{336}$. Als dritter Markt sei Flüssigzucker angeführt. Eine Marktuntersuchung der Kartellbehörden ergab, dass aus der Perspektive der Abnehmer ein Austausch von Flüssig- mit Weißzucker nicht in Betracht kommt, da damit ein erheblicher zeitlicher, technischer und personeller Aufwand verbunden sei ${ }^{337}$.

Der räumlich relevante Markt bei Zuckerprodukten wird im wesentlichen durch die Transportkosten bestimmt, da Innovations- und Preiswettbewerb zwischen den Herstellern kaum stattfindet ${ }^{338}$. Die Zuckerhersteller bieten ihre Waren mit Preisen "frei Haus" an. Da die Distanz von Fabrik zum Abnehmer somit den Preis bestimmt, bieten Konkurrenzunternehmen zu einem nicht konkurrenzfähigen Preis an oder geben überhaupt kein Angebot $a b^{339}$. Somit wurde ein Lieferradius von 200 km Luftlinie um die Produktionsstätte als räumlich relevanter Markt festgelegt.

Nach Ermittlung der Postleitzahlbezirke wurde der Anteil der abgesetzten Mengen von der Gesamtproduktion der Nordzucker von 90-100\% für die drei genannten sachlichen Märkte bestimmt.

Anschließend wurde auf die Marktstruktur unter Berücksichtigung von Importen und Konkurrenzunternehmen gefolgert (vgl. Tabelle 20). Dabei ergibt sich für den relevanten Markt folgender (mengenmäßiger) Anteil:

Tabelle 20: Marktstruktur des Zuckermarktes in Deutschland

\begin{tabular}{|l|c|c|c|}
\hline & Industriezucker & Haushaltszucker & Flüssigzucker \\
\hline Nordzucker KG & $\begin{array}{c}40-50 \% \\
(5-10 \%)\end{array}$ & $\begin{array}{c}40-50 \% \\
(10-20 \%)\end{array}$ & $30-40 \%$ \\
\hline $\begin{array}{l}\text { Union-Zucker (nicht an } \\
\text { KG gelierfert) }\end{array}$ & $<5 \%$ & - & $25-35 \%$ \\
\hline Pfeifer \& Langen & $20-30 \%$ & $30-40 \%$ & $5-10 \%$ \\
\hline Südzucker & $10-20 \%$ & $10-20 \%$ & $10-20 \%$ \\
\hline Danisco & $5-10 \%$ & - & - \\
\hline Jülich & $<5 \%$ & $<5 \%$ & - \\
\hline Importe & $7,96 \%$ & $9,41 \%$ & $7,5 \%$ \\
\hline
\end{tabular}

Quelle: BundeskARTELLAMt, Beschluss 2002, S. 16

Bei Betrachtung der Marktanteile kann festgestellt werden, dass die Nordzucker in allen drei Märkten bereits vor der Fusion eine marktbeherrschende Stellung nach $\S 19$

\footnotetext{
${ }^{336}$ Vgl. BUndeSKARTELLAMT 2002, B2 - 31/02, "Nordzucker/Union-Zucker", S. 8

337 Auch KG, Beschluss vom 10.10.2001 (Kart 30/99; B2-91/98), S. 19

${ }^{338}$ Vgl. BUndeSKARTELLAMT 2002, B2 - 31/02, "Nordzucker/Union-Zucker", S. 9

${ }^{339}$ Ergebnisse der Befragung von Zuckerabnehmern
} 
Abs. 3 Satz 1 GWB einnahm. Angesichts des zum Teil deutlichen Marktanteilsabstands zum nächstgroßen Wettbewerber (über 20\%) überwiegt weitgehend die Einzelmarktbeherrschungsvermutung. Zudem erfüllt die Nordzucker zusammen mit Pfeifer \& Langen die Oligopolvermutung nach $\S 19$ Abs. 3 Satz 2 Nr.1 GWB. Wichtig für die Beurteilung eines Zusammenschlusses ist nicht nur die Frage der aktuellen, sondern auch der zukünftigen Verstärkung einer marktbeherrschenden Stellung. Eine Verstärkung tritt bei der Fusion von Nordzucker und Union-Zucker nach Ansicht der Beschlussabteilung nicht ein, und auch eine Verschlechterung der Marktstruktur wird verneint ${ }^{340}$. Die Beschlussabeilung gibt dazu folgende (sinngemäße) Erläuterung: Marktanteile können nicht einfach addiert werden, da bereits vor dem Zusammenschluss der Zucker der Union mit der Nordzucker KG gemeinsam vertrieben wurde ${ }^{341}$ (seit 1987). Ein Aufbau eines eigenen Vertriebs und einer eigenen Marke wäre wirtschaftlich nicht rentabel und höchst unwahrscheinlich. Es scheidet also kein aktueller Wettbewerber aus dem Markt aus! Wichtig ist es dennoch, die Frage des potentiellen Wettbewerbs zu klären. Dabei werden folgende Gedankengänge zugrundegelegt: Es wird geprüft, ob von der Union Zucker a) alleine oder b) in Verbindung mit einem anderen Unternehmen Wettbewerbsimpulse auf dem räumlich relevanten Markt zu erwarten sind.

A) Festzuhalten ist zunächst, dass die Union aufgrund vertraglicher Bindung bis zum 30. Juni 2015 verpflichtet ist, den Zucker gemeinsam über die Nordzucker KG zu vermarkten (fällt unter die Sonderregelung nach § 28 GWB). Diese Gestaltung bedeutet, dass die Union Zucker nach mindestens 28 Jahren ohne eigene Vertriebstätigkeit einen komplett neuen Vertrieb aufbauen und Kunden akquirieren müsste ${ }^{342}$. Die UnionZucker alleine wäre darüber hinaus nicht in der Lage, Zuckerprodukte wie Flüssigzucker, Fructose oder Fondant herzustellen. Dies stellt einen gravierenden Wettbewerbsnachteil dar, da Großkunden ein vollständiges Sortenspektrum erwarten ${ }^{343}$. Die UnionZucker müsste unter diesen Bedingungen die fehlenden Produkte von Wettbewerbern beziehen. Für Haushaltszucker stellte sich das Problem in erster Linie in der fehlenden Marke (Handelsmarken sind nicht so lukrativ). Ein weiterer Aspekt betrifft das Produktionsrisiko. Mit nur einer Produktionsstelle ist das Risiko des Totalausfalls bzw. der fristgerechten Produktion wesentlich größer als bei den übrigen Mitbewerbern, die über mehrere Fabriken verfügen ${ }^{344}$. Dies wäre bei einer Kooperation mit der Nordzucker

\footnotetext{
340 Vgl. BundeSKARTELLAMT 2002, B2 - 31/02, "Nordzucker/Union-Zucker", S. 20

${ }^{341} \mathrm{Vgl}$. BundeSKARTELLAMT 2002, B2 - 31/02, "Nordzucker/Union-Zucker", S. 21

342 Vgl. BundeSKARTELLAMT 2002, B2 - 31/02, "Nordzucker/Union-Zucker", S. 23

343 Vgl. BundeSkARTELLAMT 2002, B2 - 31/02, "Nordzucker/Union-Zucker”, S. 23

${ }^{344}$ Vgl. BundeskARTELLAMT 2002, B2 - 31/02, "Nordzucker/Union-Zucker", S. 24
} 
nicht der Fall. Abschließend sei auf eine eingeschränkte Forschungs- und Entwicklungstätigkeit hingewiesen, die aufgrund der beschränkten Finanzkraft zumindest nicht in der heutigen Höhe betrieben werden könnte ${ }^{345}$. In der Summe bietet die Kooperation mit der Nordzucker der Union-Zucker so erhebliche Vorteile, dass eine Beendigung der Kooperation 2015 oder später ausgeschlossen werden kann ${ }^{346}$.

B) Ab 2015 oder danach wäre dagegen eine Kooperation mit einem anderen Unternehmen denkbar. Dies würde aus wettbewerbspolitischer Sicht zu Wettbewerbsimpulsen auf dem norddeutschen Zuckermarkt führen. § 36 Abs. 1 GWB verlangt hierzu konkrete „Erwartungen", die eine Marktbeherrschung begründen oder verstärken. Dazu wurden nationale und europäische Mitbewerber konkret befragt, wobei sich herausstellte, dass lediglich das Unternehmen Pfeifer \& Langen ernsthaft an einer Kooperation interessiert wäre ${ }^{347}$. "Aus ideologischen Gründen scheint allerdings eine solche Fusion als unwahrscheinlich, da die bislang agrargenossenschaftlich organisierte UnionZucker sich einer kapitalgesellschaftlich organisierten Unternehmensstruktur unterordnen müsste ${ }^{348 " .}$. Dennoch wird diese hypothetische Fusion zwischen Pfeifer \& Langen und der Union Zucker unterstellt und Marktanteile gemäß der obigen Vorgehensweise in den relevanten Märkten berechnet. Ergebnis ist, dass die Konzentration allenfalls zu einem symmetrischen Duopol führen würde und letztendlich ein solcher $\mathrm{Zu}$ sammenschluss kartellrechtlich unzulässig wäre ${ }^{349}$.

Besondere Beachtung findet ferner der Aspekt des Ressourcenzuwachses bei einer Fusion zwischen Union-Zucker und Nordzucker. Da die Fabrik der Union-Zucker zu einer der modernsten zählt, wird die Möglichkeit geschaffen, unrentable Produktionsstätten zu schließen, weitere Rationalisierungsvorteile auszuschöpfen und entsprechende Kostensenkungspotentiale zu realisieren. Erst wenn dadurch ein "Entmutigungs- oder Abschreckungseffekt" gegenüber den Wettbewerbern eintritt (kurzfristige Absenkung der Preise, Ausscheiden der Mitbewerber, alleiniger Anbieter), sind die Potentiale negativ zu bewerten ${ }^{350}$. Da die verbleibenden Mitbewerber ähnliche oder gröBere Produktionsstätten aufweisen und ein Preiswettbewerb durch die ZMO stark ein-

\footnotetext{
${ }^{345}$ Zusammen mit der Nordzucker AG betreibt die Union-Zucker ein Zuckerinstitut in Braunschweig, das sich insbesondere mit Sonder- und Spezialprodukten befasst.

346 Vgl. BundeSKARTELLAMT 2002, B2 - 31/02, "Nordzucker/Union-Zucker”, S. 25

347 Eine Expansion der Südzucker AG ist aufgrund kartellrechtlicher Gründe eingeschränkt.

348 Vgl. BundeskARTELLAMT 2002, B2 - 31/02, "Nordzucker/Union-Zucker", S. 27

349 Vgl. BundeSKARTELLAMT 2002, B2 - 31/02, "Nordzucker/Union-Zucker”, S. 33

350 Vgl. BundeskARTELLAMT 2002, B2 - 31/02, "Nordzucker/Union-Zucker", S. 33
} 
geschränkt ist, ist dieses Argument hier zu vernachlässigen ${ }^{351}$. Da die Unternehmen durch die ZMO über den Interventionspreis in ihrer Existenz weitgehend abgesichert sind, ist die Ausübung einer Verdrängungsstrategie (Kostensenkungspotentiale an Kunden weitergeben) zwischen den Unternehmen eher unrealistisch ${ }^{352}$. Mit diesen Begründungen hat das Bundeskartellamt entschieden, das angemeldete Zusammenschlussvorhaben nicht zu untersagen.

Hervorgehoben sei an dieser Stelle, dass die ZMO von entscheidender Bedeutung für die Beurteilung von Unternehmenszusammenschlüssen ist. Für Zukunftseinschätzungen ergibt sich dadurch ein hohes Unsicherheitspotential. Grundsätzlich können mögliche Veränderungen der Rechtslage nicht zum Gegenstand der vorausschauenden Betrachtung der Kartellbehörden und -gerichte gemacht werden ${ }^{353}$. Bei konkreten, mit hoher Wahrscheinlichkeit bevorstehenden Gesetzesänderungen können diese jedoch berücksichtigt werden ${ }^{354}$. Neben der Agrarpolitik wurden in diesem Fall auch Rechtsform und potentielle Neugestaltung des Unternehmens berücksichtigt. Der Beschaffungsmarkt und mögliche Synergieeffekte wurden jedoch nicht näher analysiert. Letztendlich hat eine Legalisierung des gemeinsamen Vertriebskartells durch das Gericht zu einer völlig neuen Beurteilungssituation geführt.

\subsubsection{Südzucker/Saint Louis Sucre (2001)}

Die zwei soeben genannten Unternehmenszusammenschlüsse aus der Zuckerindustrie wurden von der deutschen Kartellbehörde - dem Bundeskartellamt - überprüft. Der folgende Fall wurde von der Europäischen Kommission - Generaldirektion Wettbewerb - in Brüssel beurteilt. Es handelt sich um die geplante Fusion zwischen dem französischen Zuckerproduzenten Saint Louis Sucre und der deutschen Südzucker AG. Da die Umsatzschwellen bei diesem grenzübergreifenden Zusammenschluss überschritten wurden, ist die Kommission die zuständige Beurteilungsbehörde, wobei in der praktischen Vorgehensweise die betroffenen nationalen Kartellbehörden zur Informationsund Entscheidungsgrundlage beitragen. Am 20. Juli 2001 wurde das entsprechende Vorhaben angemeldet. Es handelt sich um folgende Parteien:

\footnotetext{
${ }^{351}$ Vgl. BundeSKARTELLAMT 2002, B2 - 31/02, "Nordzucker/Union-Zucker”, S. 34

352 Vgl. BundeSKARTELLAMT 2002, B2 - 31/02, "Nordzucker/Union-Zucker”, S. 35

353 BGH WuW/E DE-R 24, 28 "Stromversorgung Aggertal"

${ }^{354}$ Vgl. BundeSKARTELLAMT 2002, B2 - 31/02, "Nordzucker/Union-Zucker”, S. 31
} 
a) Die Südzucker AG gehört zu den weltweit größten Zuckerherstellern und produziert neben Zuckerprodukten Süßungsmittel, Lebensmittelzusatzstoffe, Stärke, Melasse, landwirtschaftliche Produkte, Speiseeis ${ }^{355}$ und Tiefkühlkost. Der Schwerpunkt der Aktivitäten des Konzerns liegt in Deutschland, Belgien, Österreich sowie Mittel- und Osteuropa. Die Konzernbeteiligungen erstrecken sich auch auf die belgische Raffinerie Tirlemontoise und die österreichische Agrana.

b) Saint Louis Sucre (SLS) ist der zweitgrößte französische und siebtgrößte europäische Hersteller von Zucker. Daneben produziert die SLS Alkohol und handelt mit Melasse. Frankreich, Mittel- und Osteuropa gehören zu den Produktionsgebieten. Über eine Holdinggesellschaft ist die SLS zudem mit 13,8\% am größten spanischen Zuckerproduzenten „Ebro Puleva" und mit 44,5\% an dem französischen Zuckerproduzenen "Sucrière Distellerie des Ouvré Fils" beteiligt.

Da bei einem Zusammenschluss weltweit die Umsatzgrenze von 5 Mrd. Euro und gemeinschaftsweit eine Umsatzgrenze von mehr als 250 Mio. $€$ überschritten wird, fällt das Vorhaben unter die europäische Fusionskontrollverordnung (vgl. dazu Kapitel 5.2).

Aus den Anmeldungsunterlagen geht hervor, dass sich das Zusammenschlussvorhaben geopraphisch auf Deutschland, Belgien und Frankreich auswirkt, wobei die Effekte auf die Zuckermärkte in Süddeutschland und Belgien zu wettbewerbsbedenklichen Konstellationen führen würden ${ }^{356}$. Der sachlich relevante Markt bezieht sich nach Angaben der Unternehmen auf Industrie- und Haushaltszucker sowie auf die Lieferung von Zucker für Handelsmarken. Die Unterteilung der zwei zuerst erwähnten sachlichen Märkte führt zu keinen weiteren Bedenken, da bereits ältere Entscheidungen eine ähnliche Unterteilung vornahmen ${ }^{357}$. Die Unterteilung in Handelsmarken ist dagegen unbekannt. Dennoch handelt es sich nach Ansicht der Behörden ${ }^{358}$ um einen getrennten Markt, da ein Handelsunternehmen Qualität, Marketing und andere Spezifika selbst bestimmen und somit unabhängig von den Interessen der Hersteller agieren kann. Kundenkriterien beziehen sich lediglich auf Preis, Qualität, freie Kapazitäten sowie Lieferzuverlässigkeit ${ }^{359}$. In diesem Fall findet also keine Betrachtung aus Sicht des Endkunden (Verbraucher), sondern des Bezugsunternehmens statt. Uneinigkeit dagegen

\footnotetext{
355 Seit 01.10.2001 nicht mehr im Konzernportfolio

${ }^{356}$ Vgl. EUROPÄISCHE-KOMMISSION, Fall Nr. COMP/M.2530 - Südzucker/Saint Louis Sucre, S. 4, Rdnr. 12

357 Vgl. EUROPÄISCHE-KOMMISSION, Entscheidung 97/624/EG vom 14. Mai 1997 in der Sache IV/F-3/M.34.621

- Irish Sugar u.a., ABI. L 258 vom 22.9.1997, S. 1 (Rn. 90) und EU-KommISSION, Entscheidung

1999/210/EG vom 14. Oktober 1998 in der Sache IV/F-3/33.708 - British Sugar u.a., ABI. L 76 vom

22.3.1999, S. 1 (Rn. 59)

358 Nach einer eigenen Marktuntersuchung

${ }^{359}$ Vgl. EUROPÄISCHE-KOMMISSION, Fall Nr. COMP/M.2530 - Südzucker/Saint Louis Sucre, S. 6
} 
herrscht in der räumlichen Abgrenzung des relevanten Marktes. So tragen die Vertreter der Unternehmen vor, dass die Märkte entweder national oder gar europaweit abzugrenzen wären. Die Kartellbehörde ist teilweise einer anderen Auffassung. Zwar treffe eine nationale Marktabgrenzung für Staaten wie z.B. Frankreich zu, da die Zuckerunternehmen innerhalb Frankreichs keine geographische Gebietsaufteilung aufweisen ${ }^{360}$, im Falle Deutschlands könne allerdings maximal der süddeutsche Raum als relevanter Markt angenommen werden, da Marktuntersuchungen von Kunden und Wettbewerbern die regionale Aufteilung bestätigten ${ }^{361}$. Nachdem zunächst auch der Handelsmarkenzucker regional abgegrenzt werden sollte, konnten die Unternehmen die Kartellbehörden davon überzeugen, dass dieser Markt weitläufiger abgegrenzt werden müsste. Da nur wenige europäische marktmächtige Großkunden Handelsmarken nachfragen und auch die Marktuntersuchung keine einheitlichen Ergebnisse lieferte, wird für dieses Marktsegment eine größere Marktabgrenzung offen gehalten ${ }^{362}$.

Eine Marktuntersuchung ergibt, dass insgesamt der Wettbewerb durch die ZMO äuBerst eingeschränkt ist. Ferner stellt sich heraus, dass die Marktpreise 10-20\% über den EU-Interventionspreisen liegen ${ }^{363}$. Europaweit würde die Südzucker ihren Anteil an der europäischen Höchstquote von 16,4 auf 21,5\% erhöhen können. Am Absatzmarkt würden folgende Marktanteile (Tabelle 21) in den jeweiligen Gebieten entstehen:

Tabelle 21: Marktstruktur des Zuckermarktes in Europa

\begin{tabular}{|c|c|c|c|c|c|c|}
\hline $\begin{array}{l}\text { Sachlich rele- } \\
\text { vanter Markt }\end{array}$ & $\begin{array}{l}\text { Räumlich rele- } \\
\text { vanter Markt }\end{array}$ & $\begin{array}{l}\text { Mengen } \\
\text { (in } 1000 t \text { ) }\end{array}$ & Südzucker & $S L S$ & Wettbewerber 1 & Wettbewerber 2 \\
\hline \multirow[t]{6}{*}{$\begin{array}{l}\text { Industrie- } \\
\text { zucker }\end{array}$} & Frankreich & 1660 & $0-10 \%$ & $10-20 \%$ & $\begin{array}{l}\text { 20-30\% } \\
\text { (Beghin-Say) }\end{array}$ & $\begin{array}{l}10-20 \% \\
\text { (Sucre Union) }\end{array}$ \\
\hline & Italien & 891 & $10-20 \%$ & $0-10 \%$ & $\begin{array}{l}35-45 \% \\
\text { (Beghin-Say) }\end{array}$ & $\begin{array}{l}10-20 \% \\
\text { (SFIR) }\end{array}$ \\
\hline & Belgien & 502 & $60-70 \%$ & $0-10 \%$ & $\begin{array}{l}15-25 \% \\
\text { (Sucrier) }\end{array}$ & $\begin{array}{l}0-10 \% \\
\text { (Cosucra) }\end{array}$ \\
\hline & Süddeutschland & 878 & $75-85 \%$ & $0-10 \%$ & $\begin{array}{l}0-10 \% \\
\text { (Pfeifer) }\end{array}$ & $\begin{array}{l}0-10 \% \\
\text { (Beghin Say) }\end{array}$ \\
\hline & Deutschland & 2045 & $30-40 \%$ & $0-10 \%$ & $\begin{array}{l}25-35 \% \\
\text { (Nordzucker) }\end{array}$ & $\begin{array}{l}15-25 \% \\
\text { (Pfeifer) }\end{array}$ \\
\hline & EU & 10055 & $10-20 \%$ & $0-10 \%$ & - & - \\
\hline
\end{tabular}

\footnotetext{
${ }^{360}$ Die Zuckerproduktionsstätten befinden sich im wesentlichen im Norden und Nordosten des Landes.

361 Offiziell wird das mit den steigenden Transportkosten bei zunehmender Entfernung begründet.

362 Vgl. EUROPÄISCHE-KOMMISSION, Fall Nr. COMP/M.2530 - Südzucker/Saint Louis Sucre, S. 10 f.

${ }^{363}$ Vgl. EUROPÄISCHE-KOMMISSION, Fall Nr. COMP/M.2530 - Südzucker/Saint Louis Sucre, S. 14
} 
Tabelle 21 (Fortsetzung)

\begin{tabular}{|l|l|l|l|l|l|l|}
\hline $\begin{array}{l}\text { Sachlich rele- } \\
\text { vanter Markt }\end{array}$ & $\begin{array}{l}\text { Räumlich rele- } \\
\text { vanter Markt }\end{array}$ & $\begin{array}{l}\text { Mengen } \\
\text { (in 1000 t) }\end{array}$ & Südzucker & SLS & Wettbewerber 1 & Wettbewerber 2 \\
\hline $\begin{array}{l}\text { Haushalts- } \\
\text { zucker }\end{array}$ & Frankreich & 510 & $0-10 \%$ & $20-30 \%$ & $\begin{array}{l}20-30 \% \\
\text { (Beghin-Say) }\end{array}$ & $\begin{array}{l}5-15 \% \\
\text { (Union SDA) }\end{array}$ \\
\hline & Belgien & 59 & $85-95 \%$ & $0-10 \%$ & - & - \\
\hline & Süddeutschland & 180 & $80-90 \%$ & $0-10 \%$ & $\begin{array}{l}10-20 \% \\
\text { (Pfeifer) }\end{array}$ & $\begin{array}{l}0-10 \% \\
\text { (Nordzucker) }\end{array}$ \\
\hline $\begin{array}{l}\text { Zucker für } \\
\text { Handelsmar- } \\
\text { ken }\end{array}$ & Deutschland & 152 & $15-25 \%$ & $5-15 \%$ & $\begin{array}{l}20-30 \% \\
\text { (Nordzucker) }\end{array}$ & $\begin{array}{l}19 \% \\
\text { (Pfeifer) }\end{array}$ \\
\hline
\end{tabular}

Quelle: EUROPÄISCHE-Kommission, Fall Nr. COMP/M.2530 - Südzucker/Saint Louis Sucre, S. 16

Danach kommt es hauptsächlich in Belgien und Süddeutschland für Industrie- und Haushaltszuckermärkte zu wettbewerbsrechtlich bedenklichen Konzentrationsgraden. Die Marktuntersuchung hat ergeben, dass kaum Anreize für andere Unternehmen bestehen, das jeweilige traditionelle Absatzgebiet zu verlassen, da sich die Konkurrenten unmittelbar der Gefahr von Vergeltungsmaßnahmen ausgesetzt sehen. Solche Vergeltungsmaßnahmen sind in der Zuckerindustrie besonders leicht möglich, weil es sich bei Zucker um ein homogenes Massenprodukt handelt und die Märkte angesichts der bestehenden Produktionsquoten und Interventionspreise transparent sind ${ }^{364}$. Daher ist die Kommission zu dem Ergebnis gelangt, dass sich die Südzucker bereits zuvor in Süddeutschland in einer Position befand, die es ihr erlaubte sich gegenüber Abnehmern, Wettbewerbern und letztendlich Verbrauchern unabhängig zu verhalten ${ }^{365}$. Ähnliches gilt für den belgischen Markt.

Im weiteren geht es um die Klärung der Frage, ob eine marktbeherrschende Stellung verstärkt wird. Dabei führen nach Auffassung der Kommission folgende drei Faktoren zu einer Verstärkung der marktbeherrschenden Stellung bei Südzucker ${ }^{366}$ :

- Erstens würde der Zusammenschluss zu einer entscheidenden Verringerung von potentiellem Wettbewerb für den süddeutschen und belgischen Markt führen;

\footnotetext{
${ }^{364}$ Vgl. EUROPÄISCHE-KOMMISSION, Fall Nr. COMP/M.2530 - Südzucker/Saint Louis Sucre, S. 18

${ }^{365}$ Vgl. EUROPÄISCHE-KOMMISSION, Fall Nr. COMP/M.2530 - Südzucker/Saint Louis Sucre, S. 18, Rdnr. 69

${ }^{366}$ Vgl. EUROPÄISCHE-KOMMISSION, Fall Nr. COMP/M.2530 - Südzucker/Saint Louis Sucre, S. 20, Rdnr. 79
} 
- zweitens würde ein bisher kaum vorhandenes Vergeltungspotenzial geschaffen, durch das sich die Südzucker insbesondere in Süddeutschland und Belgien besser gegen ausländischen Wettbewerb schützen könnte. Der Konzern hätte fortan die Möglichkeit, effektiv Vergeltungsmaßnahmen auf dem französischen Zuckermarkt durchzuführen und so etwaige französische Wettbewerber vom Vordringen auf den süddeutschen und belgischen Markt abzuschrecken;

- schließlich würde der Zusammenschluss Südzucker im Vergleich zu seinen Hauptwettbewerbern erlauben, industrielle Großkunden über nationale Grenzen hinweg im Wege „europäischer Deals" mit Zucker zu versorgen.

Um die Bedenken der Wettbewerbsbehörde im Hinblick auf die süddeutschen und belgischen Zuckermärkte auszuräumen, hat die Südzucker die folgenden Zugeständnisse gemacht $^{367}$ :

Südzucker verpflichtet sich, seine $68 \%$ Beteiligung an der belgischen Zuckerfabrik Veurne zu veräußern ${ }^{368}$. In Süddeutschland verpflichtet sich die Südzucker ferner, einem unabhängigen Zuckerhandelsunternehmen 90.000 t Quotenzucker pro Jahr aus ihren Zuckerfabriken zur Verfügung zu stellen, wobei bei den Lieferbedingungen vom Interventionspreis ausgegangen wird. Die Wettbewerbsbehörde begrüßt die Zusagen und wertet diese als ausreichend ${ }^{369}$. Ein durchgeführter Markttest ${ }^{370}$ bestätigte diese Einschätzung. Für den belgischen Markt wären demnach 10\% des gesamten Marktvolumens und mehr als $10 \%$ des Industriezuckermarktvolumens Belgiens für Wettbewerber verfügbar. Darin sieht die Behörde eine ausreichende Stärkung der belgischen Zuckerindustrie und einen hinreichenden Ausgleich für den Wegfall von SLS ${ }^{371}$. Für den süddeutschen Raum würde die Kommission eine ähnliche Zusage (Veräußerung einer Fabrik) gegenüber einer Mengenabgabe favorisieren. Unter den besonderen Umständen hätte allerdings in Süddeutschland - im Gegensatz zu Belgien - der Verkauf einer Zuckerfabrik keinen hinreichenden Erfolg gebracht. Speziell handelt es sich um Bedenken bei der Bereitschaft der betroffenen Landwirte, die zugleich als Anteilseigner der Südzucker fungieren. Darüber hinaus kündigte das deutsche Landwirtschaftsminis-

\footnotetext{
367 Vgl. EUROPÄISCHE-KOMMISSION, Fall Nr. COMP/M.2530 - Südzucker/Saint Louis Sucre, S. 36 Rdnr. 151 und 152. Die zuvor vorgeschlagene Zusage der Südzucker, sich von der spanischen Beteiligung trennen zu wollen, wurde zurückgezogen, nachdem die Kommission herausstellte, dass eine Genehmigung des Zusammenschlusses nicht von der Beteiligung an der Ebro Puleva abhänge.

${ }^{368}$ Veurne: Höchstquote ca. $60000 \mathrm{t}$

${ }^{369}$ Vgl. EUROPÄISCHE-KOMMISSION, Fall Nr. COMP/M.2530 - Südzucker/Saint Louis Sucre, S. 37

${ }^{370}$ Als Markttest wird in diesem Falle eine Kunden- und Konkurrenzbefragung im Hauptprüfverfahren bezeichnet. In anderen Fällen kann der Markttest auch ökonometrische Untersuchungen beinhalten.

${ }^{371}$ Vgl. EUROPÄISCHE-KOMmISSION, Fall Nr. COMP/M.2530 - Südzucker/Saint Louis Sucre, S. 37, Rdnr. 156
} 
terium an, dass es keinen Quotenübertragungen zustimmen werde, die nicht im Sinne der betroffenen Landwirte vollzogen würden ${ }^{372}$. Aus Sicht der Produktionsstruktur ergäben sich außerdem keine Verbesserungen, da im Gegensatz zu Belgien eine hohe Spezialisierung einzelner Fabriken in Süddeutschland vorhanden ist und eine Herauslösung einzelner Fabriken aus dem Strukturverbund gesamtwirtschaftlich eher nachteilig wäre. Aus diesen Gründen tritt die Kommission von der Veräußerungsauflage ab und akzeptiert die von der Südzucker vorgeschlagene Lösung. Die Abgabe von 90.000 t Zucker entspricht ca. 8\% des gesamten Marktvolumens in Süddeutschland und mehr als $10 \%$ des Volumens auf dem süddeutschen Markt für Industriezucker. Somit ist die Zusage im Verhältnis vergleichbar mit den „belgischen" Zusagen. Da eine erhebliche Differenz zwischen Bezugspreis und Marktpreis (vgl. dazu Kapitel 8.1.5.1) für den Zuckerhändler existiert ist, besteht die Möglichkeit, den derzeitigen Marktpreis in Süddeutschland zu unterbieten und somit für zusätzliche Wettbewerbsimpulse im süddeutschen Gebiet zu sorgen ${ }^{373}$. Die Auswirkungen dieser Auflage erbringt letztendlich nach Ansicht der Kommission den gleichen Effekt wie bei einer Veräußerung einer Fabrik $^{374}$. Eine derartige Zusage sei ein hinreichender Ausgleich für den Wegfall der SLS am süddeutschen Markt. Anhand der geschilderten Zusagen ist somit die Kommission der Ansicht, dass der geplante Zusammenschluss weder zu einer Verstärkung einer marktbeherrschenden Stellung auf dem süddeutschen noch dem belgischen Markt führen wird.

\subsection{Zwischenergebnis}

Es bleibt festzuhalten, dass die europäische Vorgehensweise hinsichtlich Wettbewerbsrecht mit der deutschen korrespondiert. Interessanterweise finden politische Argumente Berücksichtigung. Im allgemeinen kann herausgestellt werden, dass Rechtsvorschriften lediglich den groben Rahmen vorgeben. Die individuelle Entscheidung hängt offensichtlich von den Einschätzungen der jeweiligen Beschlussabteilung, aber auch vom Verhandlungsgeschick der Vertragspartner ab.

\footnotetext{
372 Vgl. EUROPÄISCHE-KOMMISSION, Fall Nr. COMP/M.2530 - Südzucker/Saint Louis Sucre, S. 39, Rdnr. 166

${ }^{373}$ Der Händler hat darüber hinaus das Recht unterschiedliche Qualitäten und von jedem süddeutschen Standort aus Zucker von der Südzucker zu beziehen.

${ }^{374}$ Vgl. EUROPÄISCHE-KOMMISSION, Fall Nr. COMP/M.2530 - Südzucker/Saint Louis Sucre, S. 41, Rdnr. 176
} 


\subsubsection{Milchindustrie}

Im Bereich der Milchwirtschaft regten die Entstehung der "neuen Nordmilch" mit den anschließenden Käufen, die kontinuierliche Vergrößerung der Humana, der Zusammenschluss von Tuffi-Campina-Emzett und seit 2001 der Zusammenschluss der Hochwald-Eiffelperle die Diskussion in Deutschland an. Gemessen am Gesamtumsatz waren nach Berechnungen der RABOBANK im Jahr 2000 schon 45\% der Akteure innerhalb der Branche an Unternehmenskäufen und -zusammenschlüssen beteiligt, während die Quote zwei Jahre zuvor erst $13 \%$ betrug $^{375}$. Insgesamt ist die Zahl der Milchverarbeiter in Deutschland auf 250 Unternehmen zurückgegangen (vgl. Kapitel 2.6). Die zunehmende Konzentration lässt sich auch an der verarbeiteten Milchmenge ablesen: Im Jahr 2000 erreichten die 5 Top Unternehmen einen Anteil von ca. 40\% an der verarbeiteten Milchmenge. Allein an dieser Kennziffer wird offensichtlich, welchen Umstrukturierungsprozess die Milchbranche hinter sich und auch weiterhin vor sich hat. Dabei stellt auch der Wissenschaftliche Beirat die Frage, inwieweit die Molkereistruktur mit der Handhabung des Kartellrechtes in Deutschland im Zusammenhang steht ${ }^{376}$.

\subsubsection{Nordmilch}

Bei dem Beispiel der Nordmilch handelt es sich um einen Zusammenschluss, der unter das deutsche Kartellrecht fällt. Besonders interessant im Gegensatz zu den vorigen Fallbeispielen ist, dass der Fokus der Untersuchungen nicht auf der Absatzseite, sondern auf dem Beschaffungsmarkt lag. Inwieweit landwirtschaftliche Besonderheiten berücksichtigt worden sind, wird im folgendem näher erläutert.

\subsection{Motivation}

Vier norddeutsche Molkereien, Bremerland-Nordheide, Hansano, MZO, und die Nordmilch, meldeten Anfang 1999 ihr Zusammenschlussvorhaben beim Bundeskartellamt an, um zu einem Unternehmen (neuer Name: Nordmilch) zu verschmelzen. In der folgenden Tabelle (Tabelle 22) sind wichtige Kennzahlen (1997) der Unternehmen zusammengefasst.

\footnotetext{
375 Vgl. RABOBANK 2000, S. 10

${ }^{376}$ Vgl. WissensChAFTLICHER BEIRAT 2000, S. 35
} 
Tabelle 22: Kennzahlen der Zusammenschlusspartner

\begin{tabular}{|l|c|c|c|c|}
\hline & $\begin{array}{c}\text { Bremerland/ } \\
\text { Nordheide }\end{array}$ & Hansano & MzO & Nordmilch \\
\hline Milcherfassung (Mio. kg) & 519 & 354 & 1369,2 & 1471 \\
\hline Umsatz (Mio. DM) & 465 & 352 & 1763,6 & 1511 \\
\hline Investitionen (Mio. DM) & 12 & 9,6 & 19,5 & 24 \\
\hline Abschreibungen (Mio. DM) & 14,5 & 10,8 & 31,7 & 28,8 \\
\hline Anlagevermögen (Mio. DM) & 77,6 & 25,6 & 208,9 & 119,3 \\
\hline Eigenkapital (Mio. DM) & 78,7 & 40 & 129,6 & 108,4 \\
\hline Bilanzsumme (Mio. DM) & 142 & 84,2 & 494,5 & 327,6 \\
\hline Mitarbeiter & 530 & 250 & 1757 & 1434 \\
\hline
\end{tabular}

Quelle: NORDMILCH 1999, S. 6

Die Standorte der vier Parteien können der folgenden Grafik (Abbildung 23) entnommen werden.

Abbildung 23: Standorte der „Nordmilch"-Molkereien

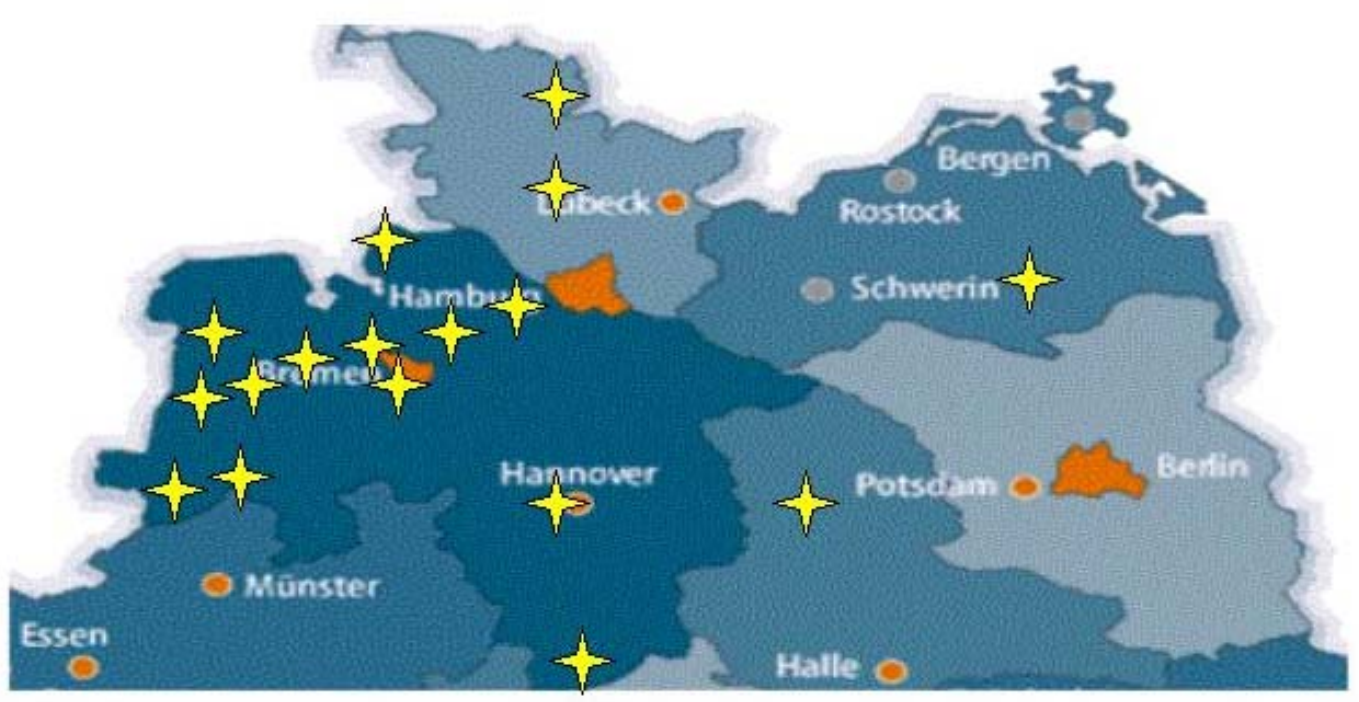

Quelle: NoRdmiLCH 1998b, S. 14

Offiziell erhofft sich die neue Nordmilch, folgende Ziele durch den Zusammenschluss verwirklichen zu können ${ }^{377}$ :

- $\quad$ Stärkung der Wettbewerbsposition gegenüber den Mitbewerbern

\footnotetext{
377 Vgl. NORDMILCH, laufende Geschäftsberichte; Nordmilch 1998a+b; Nordmilch 1999; siehe auch HOFFMANN/VENEMA 2001, S. 15
} 
- Stärkung der Position gegenüber dem Handel

- Konzentration und Verbesserung der Vermarktungsleistung als starker Partner gegenüber dem Handel

- Verbesserung der Wertschöpfung durch Produktinnovation und verstärkte Marketingaktivitäten

- Verbesserte Positionierung und Straffung des Markenportfolios

- Nutzung von Synergien und Kostenvorteilen

- Senkung von Produktions- und Erfassungskosten

- Begegnung der Herausforderung durch die Agrarpolitik ${ }^{378}$

- Eröffnung neuer Wachstumschancen

- $\quad$ Sicherstellung eines angemessenen Auszahlungspreises

\subsubsection{Beurteilung durch das Bundeskartellamt}

Zunächst einmal handelt es sich um einen Unternehmenszusammenschluss der unter den Anwendungsbereich des GWB fällt. Es liegt kein Zusammenschluss mit gemeinschaftsweiter Bedeutung vor, da alle beteiligten Unternehmen jeweils mehr als 2/3 ihres Umsatzes innerhalb Deutschlands erzielen und eine inländische Umsatzschwelle von 25 Mio. $€$ überschritten wird.

Auf den Absatzmärkten wird ohne weitere Prüfung festgestellt, dass die geographische Abgrenzung national erfolgen kann. Durch Auswertung von Unternehmensangaben wird ein Marktanteil von max. $15 \%$ ermittelt, was keine weiteren Bedenken auslöst ${ }^{379}$. Wesentlich aufwendiger gestaltet sich die Abgrenzung des Beschaffungsmarktes für Rohmilch. Dabei sind die Ausweichmöglichkeiten der Landwirte auf andere Milchabnehmer zu prüfen. Zu berücksichtigen sind neben den eigentlichen Milchverarbeitungsstätten kleinere Molkereien, die eine Vorbehandlung der Rohmilch durchführen, um diese dann an entfernt liegende Großmolkereien weiterzureichen. Letztendlich wird jedoch von der Position des Landwirtes ausgegangen ${ }^{380}$. Beim räumlich relevanten Markt sind zunächst Erfassungsgebiet (in dem die Molkereien tatsächlich die Rohmilch

\footnotetext{
378 Liberalisierung der Milchmarktpolitik

379 Vgl. BUNDESKARTELLAMT 1999, B2 - 15510 - ZU - 127/98, S. 14; Rdnr. 31

380 Vgl. BUNDESKARTELLAMT 1999, B2 - 15510 - ZU - 127/98, S. 5, Rdnr. 12
} 
beziehen) und Einzugsgebiet (theoretisch mögliches Rohmilchbezugsgebiet) zu unterscheiden. Hauptsächlich das Einzugsgebiet stellt eine dynamische Größe dar, die mit Verringerung der Haltepunkte, Anstieg der Milchdichte und Einfluss der Transportkosten durchaus unterschiedlich sein kann (vgl. Kapitel 8.2.1). Während in älteren Beschlüssen von einem Einzugsgebietsradius von $120 \mathrm{~km}$ um den Molkereistandort ausgegangen wurde, wird zunächst unter den norddeutschen Verhältnissen ein Radius von 150 km (Gebiet 1) bzw. 200 km (Gebiet 2) zugrundegelegt ${ }^{381}$. Diese Vorgehensweise wurde jedoch korrigiert, da Umfragen ergaben, dass zahlreiche andere Wettbewerber von weiter entfernten Standorten ebenfalls aus dem Kerngebiet Milch beziehen. Eine weiträumigere Erfassung ist auch für Unternehmen aus größerer Entfernung durchaus wirtschaftlich rentabel, da die relativ großen norddeutschen Betriebsstrukturen auch größere Transportentfernungen zulassen (weniger Haltepunkte, Infrastruktur etc.). Daher wird der räumlich relevante Markt in zwei Gebiete unterteilt: a) Schleswig-Holstein, Niedersachsen und der nördliche Teil Nordrhein-Westfalens bilden den Nordwestmarkt, während b) Mecklenburg-Vorpommern, Sachsen-Anhalt, Berlin und Brandenburg zum Nordostmarkt zusammengefasst werden. Ausländische Molkereien, z.B. in Dänemark, werden zunächst nicht einbezogen, da diese zwar Absatzalternativen für große, aber nicht unbedingt für kleinere, weiter entfernt liegende Milchviehbetriebe darstellen ${ }^{382}$. Mit Hilfe von Daten der ZMP wurde dann das Marktvolumen der jeweils abgegrenzten Märkte berechnet ${ }^{383}$. Im Nordwestmarkt kommen die beteiligten Unternehmen demnach auf einen Marktanteil von 40\%, während dieser im Nordostmarkt bei etwa $15 \%$ liegt $^{384}$. Problematisch wäre hiernach in wettbewerbsrechtlicher Hinsicht lediglich der Nordwestmarkt. Da sich aber der nordwestliche Milchproduktionsstandort wiederum für nordöstlich gelegene Molkereien als äußerst attraktiv gestaltet (aufgrund Betriebsgrößenstruktur), ist eine Verringerung des Wettbewerbes äuBerst unwahrscheinlich ${ }^{385}$. Auch die Ansiedlung kleinerer "Versandmolkereien", die in den letzten Jahren deutliche Marktanteile hinzugewinnen konnten, stützt diese Einschätzung. Für den nordwestdeutschen Raum sind außerdem die Wettbewerbsimpulse von ausländischen Großmolkereien nicht zu vernachlässigen, da auch kleinere Milchproduzenten zumindest über Liefergenossenschaften ${ }^{386}$ aufgrund der geringen Entfernung Absatzalternativen finden könnten. Die Rechtsform der Genossenschaft (Anteils-

\footnotetext{
${ }^{381}$ Dies entspräche auch dem Vorschlag der beteiligten Unternehmen.

382 Vgl. BundesKARTELLAMT 1999, B2 - 15510 - ZU - 127/98, S. 7, Rdnr. 18

${ }^{383}$ NRW wurde zur Hälfte angesetzt, weil keine genauere Aufteilung möglich war.

${ }^{384} \mathrm{Vgl}$. BUNDESKARTELLAMT 1999, B2 - 15510 - ZU - 127/98, S. 11 ff., Rdnr. 22 und 23

385 Vgl. BundeSKARTELLAMT 1999, B2 - 15510 - ZU - 127/98, S. 13, Rdnr. 26

${ }^{386}$ Sog. Milcherzeugergemeinschaft: vgl. dazu AgRARMARKT 1/2000, S. 51 ff.
} 
eigner sind Landwirte) ist bei der Beurteilung insofern von Relevanz, dass die Milchlieferer mit Genossenschaftsanteil über eine zweijährige Kündigungsfrist an das Mutterunternehmen gebunden sind. Dadurch sind die Ausweichmöglichkeiten der Landwirte zwar tendenziell eingeschränkt, aber laut Wettbewerbsbehörde noch hinnehmbar ${ }^{387}$. Der Gedanke, dass die Genossenschaft im Sinne der betroffenen Landwirte handelt, wird von den Kartellbehörden bei der Beurteilung einer möglichen Ausübung von Marktmacht nicht berücksichtigt. Aufgrund der zuvor ausgeführten Erwägungen wurde der Zusammenschluss somit am 29. Juli 1999 ohne Auflagen genehmigt.

\subsubsection{Europäische-Kommission: Friesland Coberco/Nutricia (2001)}

Abschließend wird in diesem Abschnitt ein weiterer Fall aus der Milchindustrie geschildert, der unter die europäische Fusionskontrollverordnung fällt. Es handelt sich um den Zusammenschluss der Friesland Coberco Dairy Foods Holding mit Nutricia Dairy \& Drinks Group. Während Coberco die Milchmenge aus den Niederlanden bezieht ${ }^{388}$, ist die Nutricia neben den Niederlanden auch in Belgien, Deutschland, Großbritannien, Ungarn, Rumänien, Tschechischen Republik und in der Slovakei tätig. Der Umsatz beider Unternehmen zusammengenommen liegt bei ca. 4,5 Mio. Euro. Da auch die weiteren Kriterien der Fusionskontrollverordnung erfüllt sind (vgl. Kapitel 5.2), fällt der Zusammenschluss unter die Beurteilung der Kommission. Überlappungen werden in den Produktmärkten für Kaffeeweißer und aromatisierte Milchgetränke festgestellt. Der geographische Markt wird auf die nationalen Grenzen definiert, was auch durch die durchgeführte Marktbefragung gestützt wird. Gemäß der mehrfach dargestellten Vorgehensweise wurden anschließend für den Handel wie auch für das "food service center" die entsprechenden Marktanteile ermittelt, wobei die engere Marktabgrenzung zunächst offen blieb (vgl. dazu Abbildung 24). Hier kam es zu unterschiedlichen Einschätzungen der beteiligten Parteien.

\footnotetext{
${ }^{387}$ EUROPÄISCHE-KOMMISSION, Beschluss Comp/M.2399, S. 13, Rdnr. 29
} 
Abbildung 24: Der sachlich relevante Markt Coberco/Nutricia

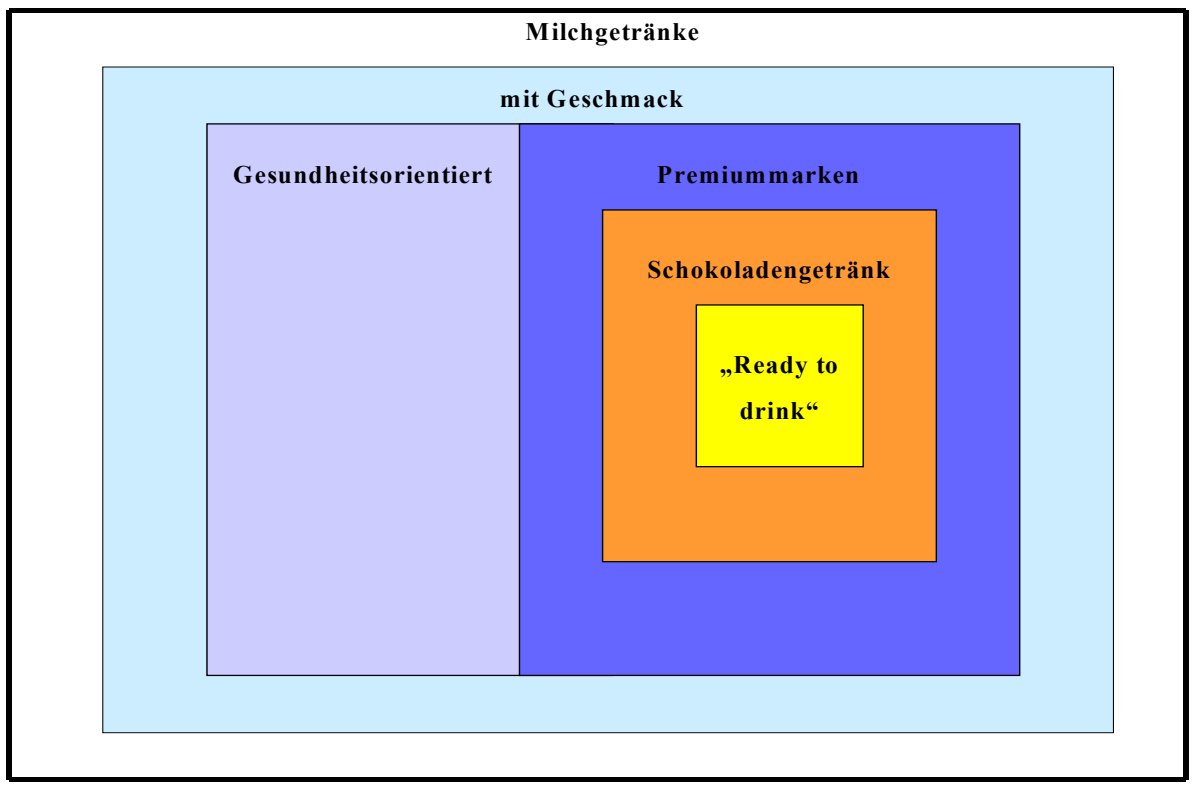

Quelle: EuRopäIsCHe-Kommission, Beschluss Comp/M.2399, S. 13

Beispielhaft sind in der folgenden Tabelle (Tabelle 23) die ermittelten Werte abgebildet (für aromatisierte Milchgetränke, LEH):

Tabelle 23: Marktanteile der Zusammenschlusspartner (v.H.)

\begin{tabular}{|l|c|c|c|}
\hline & Friesl. Coberco & Nutricia & Zusammen \\
\hline Milchgetränke & $10-15$ & $5-10$ & $15-20$ \\
\hline Mit Geschmacksrichtung & $5-10$ & $15-20$ & $20-30$ \\
\hline Gesundheitsorientiert & $15-20$ & $<1$ & $15-20$ \\
\hline Premiumsegment & $1-5$ & $20-30$ & $20-30$ \\
\hline Schokoladengeschmack & $5-10$ & $40-50$ & $60-70$ \\
\hline Schoko- "Ready to drink" & $5-10$ & $50-60$ & $60-70$ \\
\hline
\end{tabular}

Quelle: EU-Koмmission, Beschluss Comp/M.2399, S. 5

Lediglich der Markt für Schokoladenprodukte könnte hiernach zu bedenklichen Strukturen führen ${ }^{389}$. Nach genauerer Untersuchung des Marktes stellte die Kommission jedoch fest, dass der wesentliche Teil des Konkurrenzangebotes von noch größeren Unternehmen wie der Nestlé AG oder Campina bestritten wird, welche als finanzkräftige, marktstarke Unternehmen gelten. Auch eine Marktmachtausübung gegenüber den Verbrauchern wurde bezweifelt, da der Absatz weitgehend über den niederländischen

${ }^{388}$ Von der Dutch dairy co-operative Zuivelcoöperatie De Zeven Provicien U.A.

${ }^{389}$ Vgl. EU-Kommission, Beschluss Comp/M.2399, S. 5, Rdnr. 22 
LEH erfolgt. Aufgrund der hohen Konzentration des LEH und der damit verbundenen potentiellen Handelsmarkenproduktion ${ }^{390}$, der Struktur der Wettbewerber und der niedrigen Markteintrittsbarrieren ${ }^{391}$ kam die Behörde zu dem Schluss, dass auch nach dem Zusammenschluss die hohen Marktanteile keine wesentliche Ausübung von Marktmacht ermöglichten ${ }^{392}$. Entsprechend wurde der "Food Service Sector" für Kaffeeweißer und aromatisierte Milchprodukte untersucht. Da es in allen weiteren Bereichen aufgrund der Marktanteile zu keinen weiteren Bedenken kam, wurde die Fusion am 08.08.2001 freigegeben.

Interessanterweise spielen bei dieser Molkereifusion der Beschaffungsmarkt oder die Agrarpolitik keine Rolle. Was in dem deutschen Fall durchaus zur Untersagung bzw. Genehmigung mit Auflagen führen könnte, wurde auf europäischer Ebene gar nicht untersucht, obwohl der niederländische Milchmarkt als sehr konzentriert angesehen wird $^{393}$. Dagegen wurden Argumente wie gegengewichtige Marktmacht und Anpassungsdruck durch zunehmende Konzentration des LEHs im Gegensatz zur deutschen Beurteilung berücksichtigt.

Anhand der vorgestellten Fälle scheint sich der Verdacht zu bestätigen, dass trotz ähnlicher Sachlage, unterschiedliche Beschlüsse möglich sind. Dies kann darin begründet sein, dass Entscheidungen zum Großteil auf Erfahrungswissen von Mitarbeitern der Wettbewerbsbehörden beruhen. Kenntnisse über Wettbewerbsverhältnisse und zukünftige Verhaltensweisen werden weitgehend durch Befragungen der Hauptkonkurrenten und -abnehmer gewonnen. Ökonomische Instrumente kamen, zumindest bei den dargestellten Zusammenschlüssen, kaum zur Anwendung. In Kapitel 6 werden einige Aspekte thematisiert, die besonders kontrovers von Seiten der beteiligten Unternehmen und Behörden diskutiert werden.

\footnotetext{
390 Liegt für diese Märkte über $50 \%$ in den Niederlanden

${ }^{391}$ Da für Molkereien die Umrüstung einer Produktionslinie (z.B. Erdbeer zu Schokolade) mit geringen Kosten verbunden sind

392 Vgl. EUROPÄISCHE-KOMmission, Beschluss Comp/M.2399, S. 6 ff.

393 Vgl. Deutsche MilchwirTsChaft Spezial 2000, S. 88
} 


\section{Berücksichtigung und Wertung wichtiger Komponen- ten der Zusammenschlusskontrolle}

In den vorigen Ausführungen wurden ökonomische Wirkungszusammenhänge, juristische Rechtsvorgaben und die praktische Beurteilung von Unternehmenszusammenschlüssen dargestellt. In diesem Kapitel sollen die wichtigsten Kritikpunkte an der Wettbewerbspraxis zur Beurteilung von Zusammenschlüssen hervorgehoben und mögliche Verbesserungen vorgeschlagen werden. Insbesondere die Abgrenzung des relevanten Marktes, Berücksichtigung von Nachfragemacht, mangelnde Kenntnis agrarpolitischer Wirkungszusammenhänge, geringe Transparenz des Verfahrens und die Heranziehung der Abwägungsklausel stehen im Fokus der Kritik.

\subsection{Konzepte der Marktabgrenzung und ihre Problematik}

Für die partialanalytische Betrachtung tritt im Zusammenhang mit differenzierten Gütern das Problem der Marktabgrenzung auf. Differenziert wird in den Rechtsvorschriften nach sachlich, räumlich und zeitlich relevanten Märkten. Dabei geht es um die Frage, welche Güter demselben Markt zugeordnet werden. Diese Problematik wird beispielhaft am Produkt "Orangensaft" als sachlich relevanter Markt durch die folgende Grafik illustriert (Abbildung 25).

Abbildung 25: Abgrenzung des sachlich relevanten Produktmarktes
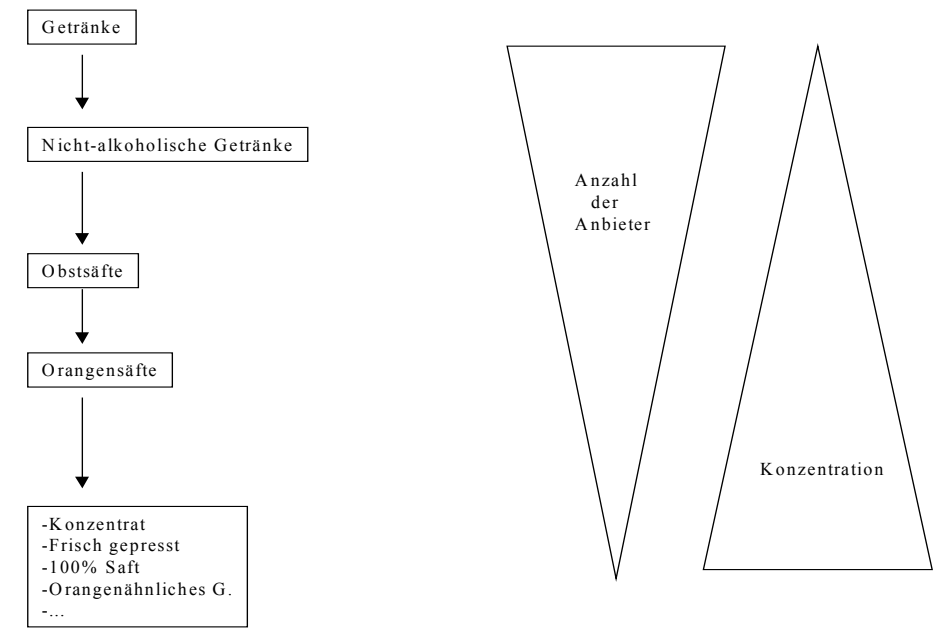
Ähnlich problematisch ist die Abgrenzung des räumlich relevanten Marktes. So wird in der ökonomischen Theorie der räumlich relevante Markt traditionell als "das geographische Gebiet, in dem sich durch ungehinderten Handel die Preise unter Berücksichtigung der Transportkosten tendenziell aneinander angleichen ${ }^{394 " ~ d e f i n i e r t . ~ I n ~ d e r ~ w e t t-~}$ bewerbspolitischen Praxis stellen Märkte ein komplexes intransparentes, z.T. von Unvollkommenheit geprägtes Konstrukt dar, und eine räumliche Abgrenzung ist somit mit erheblichen Schwierigkeiten verbunden.

Basiert die Beurteilung einer Marktsituation auf der Verteilung der Marktanteile, so hat die gewählte Marktabgrenzungsmethode großen Einfluss auf das Ergebnis. Im Prinzip sollten zum relevanten Markt all die Güter bzw. Produzenten zählen, die in wirksamer Konkurrenz zueinander stehen. Die Abgrenzung des relevanten Marktes spielt bei wettbewerbsrechtlichen Fragen eine entscheidende Rolle, da das Konzept der Marktbeherrschung von der Größe des betrachteten Marktes abhängt. Eine zu enge (weite) Marktabgrenzung führt in der Regel dazu, die Anzahl der Wettbewerber zu unterschätzen (überschätzen) und damit zu hohe (geringe) Marktmacht festzustellen. Im wesentlichen geht es also darum, die Gruppe von Anbietern und Nachfragern so zu bestimmen, dass von den nicht zur Gruppe gehörenden Marktteilnehmern kein Einfluss auf das Wettbewerbsgeschehen in der Gruppe ausgeht ${ }^{395}$.

Zur Ermittlung des relevanten Marktes erlangten mehrere theoretische Marktabgrenzungskonzepte wettbewerbspolitische Relevanz ${ }^{396}$. Den Ausgangspunkt der Überlegungen bildet das Industriekonzept. Ihm folgen das Produktflexibilitäts- und Lancaster- sowie dem eng verwandten Substitutionskonzept.

\subsubsection{Das Industriekonzept}

Nach MARSHALL ${ }^{397}$ lassen sich Branchen untereinander durch

- technisch-physischen Kriterien, z.B. technische Basis, verwendete Rohmaterialien, Produktionsprozess (technische Homogenität, Angebotshomogenität) und

- Nachfragekriterien, z.B. Verwendungsmöglichkeiten bzw. Bedarf (Kriterium: Substituierbarkeit, Nachfragehomogenität)

\footnotetext{
${ }^{394}$ Vgl. KaLLFASS 1996, S. 2

395 Vgl. SCHMIDT 1996, S. 46

${ }^{396}$ Für weitere Marktabgrenzungskonzepte siehe SCHENGBER 1996, 86 ff. sowie BAUER 1989

397 Vgl. MARSHALL 1961
} 
abgrenzen. Ein Hauptkritikpunkt an diesem Konzept ist, dass technische Homogenität und Nachfragehomogenität nicht übereinstimmen müssen (Beispiel: Fensterrahmen aus Holz oder Aluminium, Textilien für Kleidung oder Gardinen) und somit wettbewerbsrelevante Märkte zu weit abgegrenzt werden ${ }^{398}$.

\subsubsection{Das Produktionsflexibilitätskonzept}

Beim Produktionsflexibilitätskonzept wird versucht, potentielle Konkurrenten zu berücksichtigen. Demzufolge wären auch gleiche aktuelle Konzentrationsgrade unterschiedlich zu beurteilen, bzw. es müssen potentielle Konkurrenten zum relevanten Markt gerechnet werden. Dieser Gedanke wird in der Theorie des Angebotsraumes (supply space nach NARVER) vertieft ${ }^{399}$. Hiernach werden in erster Linie die großen diversifizierten Unternehmen als ein Pool von Ressourcen verstanden, der schnell in andere Märkte eindringen kann. Der supply space umfasst demgemäß alle Bereiche, in denen ein Unternehmen bereits tätig ist, sowie jene, in die es aufgrund seines Ressourcenpotentials schnell vorstoßen könnte. Das Produktflexibilitätskonzept ist demnach eng verwand mit der Theorie der "contestable markets" (BAUMOL/PANZAR/WiLLIG (1982)), wonach potentieller Wettbewerb (keine Restriktionen für Marktein- und austritte) einen ausreichenden Wettbewerb unabhängig von der Marktstruktur garantieren kann.

\subsubsection{Das Lancaster-Konzept}

Eine weitere Möglichkeit der Marktabgrenzung geht auf LANCASTER ${ }^{400}$ zurück. Die Konsumtheorie LANCASTERS basiert auf der Annahme, dass Konsumenten an bestimmten Gütern deshalb interessiert sind, weil sich mit ihnen bestimmte Eigenschaften verbinden. Die Nachfrage nach Produkten hängt somit nicht von der physischen Beschaffenheit, sondern von der Kombination ihrer Eigenschaften $a b^{401}$. Diese Eigenschaften müssen objektiv messbar sein und sich in einer Konsumtechnologiematrix darstellen lassen, deren Zeilenvektoren aus Eigenschaften und deren Spaltenvektoren aus Gütern bestehen. Wenn nur die Blockdiagonale der Konsumententechnologiematrix besetzt ist, lassen sich sog. "intrinsic groups”, also wesensgleiche Gruppen, unterschei-

\footnotetext{
398 Vgl. HERDZINA 1999, S. 74

${ }^{399} \mathrm{Vgl}$. SCHENGBER 1996, S. 113

400 Vgl. LANCASTER 1966, S. 1971

${ }^{401} \mathrm{Vgl.}$ Wied-NebBeLING 1997, S. 12
} 
den, denn dann sind die Eigenschaften, welche die Güter einer Gruppe aufweisen, völlig verschieden von denjenigen der anderen Gruppen ${ }^{402}$. Dieser Idealfall ist jedoch in der Realität eher unwahrscheinlich.

Somit ist auch das Konzept der Marktabgrenzung nach Gütereigenschaften nicht unproblematisch; nicht allein deshalb, weil die nur (block-)diagonal besetzte Matrix unwahrscheinlich ist, sondern weil es weiterer Annahmen hinsichtlich der Nutzenfunktion der Konsumenten bedarf, um Substitutionsmöglichkeiten zwischen den Gruppen auszuschließen $^{403}$.

\subsubsection{Das Substitutionskonzept}

Auf der Grundlage der Vorstellung, dass Produkte gemäß ihrer Eigenschaft der Befriedigung von spezifischen Bedürfnissen miteinander konkurrieren, wird beim Substitutionskonzept versucht, die Marktabgrenzung seitens der Nachfrage vorzunehmen. Dabei wird unterstellt, das gesamte Spektrum heterogener Güter lasse sich in verschiedene Bedarfsmärkte (ARNDT (1958); ABBOTT (1958)) aufteilen. Güter sind einem Bedarfsmarkt zuzuordnen, wenn sie die gleichen Grundbedürfnisse befriedigen. Gleiche Grundbedürfnisse zu definieren bzw. empirisch zu ermitteln, ist jedoch problematisch. Zur empirischen Beurteilung solcher Güter können

- Expertenbefragungen und demoskopische Umfragen (nach der sog. funktionalen Austauschbarkeit) oder

- Beobachtungen tatsächlichen Nachfrageverhaltens (sog. reaktive Austauschbarkeit)

zugrundegelegt werden. Während bei Befragungen von Experten deren subjektive Wertungen nicht auszuschließen sind, erfordern Nachfragebeobachtungen, dass bei einzelnen Angeboten Parameteränderungen (z.B. Preisänderungen) durchgeführt werden, auf welche die Nachfrager reagieren. Wettbewerbsrelevante Märkte wären dann durch hohe Kreuzpreiselastizitäten der Nachfrage gekennzeichnet, schwache Kreuzpreiselastizitäten hingegen zeigen Marktgrenzen $\mathrm{an}^{404}$. Im günstigsten Fall ergeben

\footnotetext{
402 Vgl. LANCASTER 1971, S. 126

403 Dazu ausführlich mit Beispiel in BAUER 1989, S. $61 \mathrm{ff}$.

${ }^{404}$ Vgl. HeRdZina 1999, S. 74
} 
sich bei diesem Verfahren in der Kette der Substitute deutliche Substitutionslücken ${ }^{405}$, so dass sich einzelne Gütergruppen klar voneinander trennen lassen.

Bezüglich der geläufigen Marktabgrenzungskonzepte kann festgestellt werden, dass sie zunächst begriffliche Raster (Bedarfsmarkt, Substitutionslücken, Produktionsflexibilität, Angebotsraum) sowie Messkonzepte (Kreuzpreiselastizitäten von Nachfrage und Angebot) zur Ermittlung der Enge der Substitutionsbeziehungen liefern. Offen bleibt allerdings, ob Kreuzpreiselastizitäten überhaupt empirisch zu ermitteln sind. Des weiteren ist die Frage, bei welchem Wert der Kreuzpreiselastizitäten die Marktgrenzen liegen, objektiv nicht zu beantworten ${ }^{406}$. Dennoch scheint aus ökonomischer Sicht das Konzept der Substitutionslücken einen brauchbaren Ansatz zu liefern. Daher wird im folgenden dieser Ansatz vertieft.

\subsubsection{Substitutionslücken als Basis für die räumliche Marktabgrenzung}

Innerhalb eines Marktes mit verteilten Angebots- und Nachfragestandorten können die Anbieter aufgrund unterschiedlich hoher Raumüberwindungskosten über unterschiedlich starke Wettbewerbspositionen verfügen. Der Markt endet an der Stelle, wo keine Substitutionsmöglichkeiten mehr vorliegen und umfasst dadurch alle Anbieter, die untereinander austauschbare Bezugsalternativen darstellen. Der auf diese Weise abgegrenzte Markt lässt sich von einem Monopolisten oder dem Kollektiv der vorhandenen Anbieter beherrschen, da für die Nachfrager keine Ausweichmöglichkeiten mehr bestehen ${ }^{407}$. Unter Zugrundelegung einer Ansiedlung einer großen Zahl von Nachfragern und zweier räumlich getrennter Anbieter entlang einer Strecke lässt sich der Fall einer räumlichen Substitutionslücke wie folgt illustrieren ${ }^{408}$ (Abbildung 26).

\footnotetext{
${ }^{405}$ Vgl. RoBinson 1961, S. 4 ff.

406 Vgl. HERDZina 1999, S. 76

407 Vgl. ROBINSON 1961, S. 4

${ }^{408}$ In Anlehnung an KotTmanN 2000, S. 34. Es wird ausschließlich der Preiswettbewerb berücksichtigt.
} 


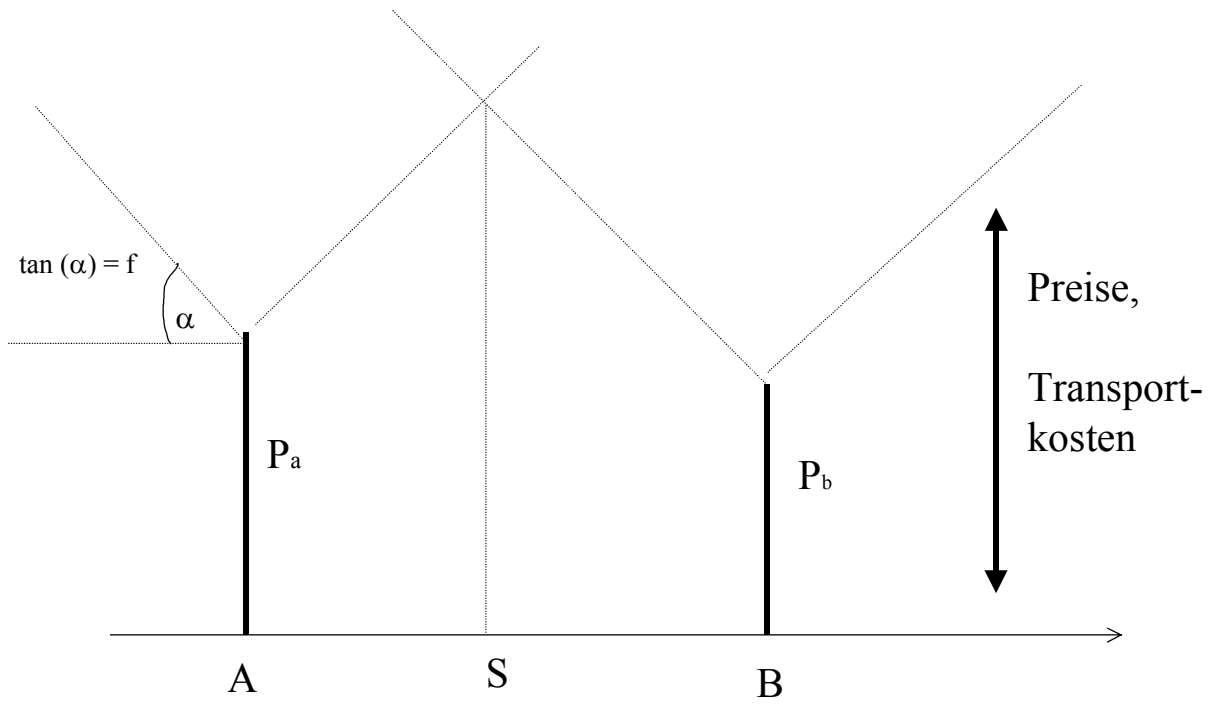

Quelle: in Anlehnung an Kottmann 2000, S. 34

Der Preis am Ort der Nachfrage (Ortspreis $\mathrm{P}_{\mathrm{e}}$ ) ist eine Funktion des Ab-Werk-Preises an den Produktionsstandorten $A$ oder $B\left(P_{a} b z w . P_{b}\right)$ und der anteiligen Transportkosten für eine normierte Mengeneinheit des betrachteten Gutes ( $f=$ Frachtsatz für eine normierte Mengeneinheit, $\mathrm{e}=$ geographische Entfernung zwischen Produktionsstandort und Ort der Nachfrage). Dabei wird die Abwesenheit sachlicher, zeitlicher und persönlicher Präferenzen unterstellt. Der Preis ist der entscheidende Wettbewerbsparameter.

$P_{e}=P+f e$

Unter der Voraussetzung, dass keine räumliche Preisdiskriminierung möglich ist, lassen sich hieraus die Überschneidungen zwischen den individuellen Absatzgebieten herleiten. Für den Ortspreis auf der Konkurrenzgrenze $S$ gilt:

$P_{a}+f_{a} e_{a}=P_{b}+f_{b} e_{b}$

Ist der Ortspreis in $S$ aus Sicht der Nachfrager prohibitiv hoch, gibt es an dieser Stelle eine räumliche Substitutionslücke. Die Nachfrager in $S$ fragen weder bei Anbieter $A$ noch bei Anbieter $B$ nach. Es bestehen keine unmittelbaren Wettbewerbsbeziehungen zwischen den beiden Anbietern; ihre wechselseitige Kreuzpreiselastizität ist Null. 
ChAMBerlin entwickelte durch die Erweiterung dieses Grundmodells das Konzept der "chain relationship" ${ }^{409}$. In diesem Konzept führen wettbewerbswirksame Überschneidungen zwischen räumlich benachbarten (Teil-)Märkten dazu, dass die Anbieter- und Nachfragerreaktionen zwischen jeweils benachbarten Märkten miteinander verkettet sind und Wettbewerbsbeziehungen nicht nur zwischen unmittelbaren Nachbarn, sondern auch zwischen weiter entfernt liegenden Anbietern bestehen können. Abbildung 27 verdeutlicht diese Verkettung.

Abbildung 27: „Chain relationship" bei räumlich verteilten Marktteilnehmern

\section{Anbieter-} sicht

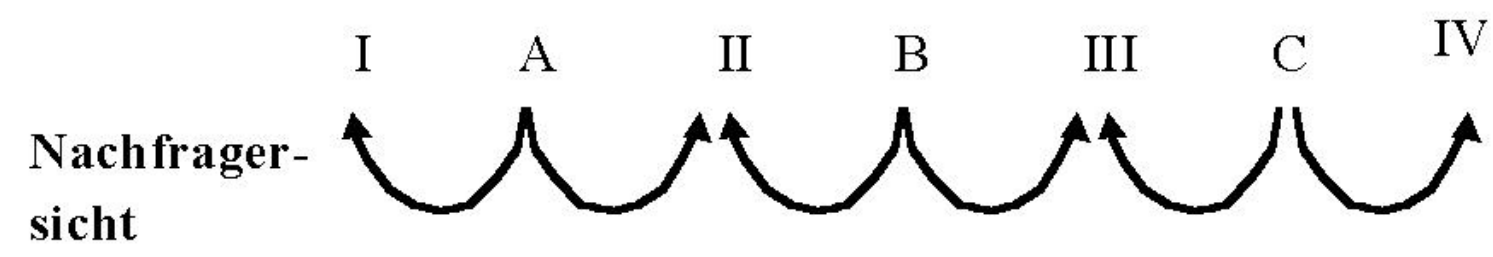

Quelle: eigene Darstellung in Anlehnung an Kottmann 2000, S. 35

Die Abbildung zeigt vier, in gleichen Distanzen angeordnete Anbieter (I, II, III, IV). Die gleichmäßig verteilten Nachfrager (zwischen den Anbietern) werden durch $A, B$ und $C$ repräsentiert. Senkt Anbieter IV zunächst den Preis, wird Anbieter III ebenfalls reagieren, da beide Anbieter um Nachfrage von $C$ konkurrieren. Durch die Verkettung der Absatzmärkte sind jedoch auch die Anbieter II und I von diesem Wettbewerbsvorstoß betroffen. Zwischen den vier Anbietern besteht eine Reaktionsinterdependenz.

Auch wenn es gelingt, die Substitutionsbeziehungen der (Teil-)Märkte zu bestimmen, muss festgelegt werden, bis zu welchem Grad der Substitution ein Gut bzw. ein Anbieter als noch zum Markt zugehörig angesehen werden soll ${ }^{410}$.

\footnotetext{
409 Vgl. ChAMBERLin 1950, S. $103 \mathrm{ff}$.

${ }^{410}$ Vgl. KANTZENBACH/KRÜGER 1990, S. 475
} 


\subsubsection{Elastizitäten als Maß für Substitutionslücken}

Um Abhängigkeitsbeziehungen und Verhaltensweisen verschiedener Akteure in der Wirtschaftswissenschaft demonstrieren zu können, werden häufig Elastizitätsmaße herangezogen. „Elastizitäten sind ein geeignetes Instrument zur Analyse wettbewerblicher Interdependenzen und bilden ein grundlegendes Konzept zur Abgrenzung relevanter Märkte" (KotTMAnn 2000, S. 38). Tabelle 24 fasst die zur Beurteilung herangezogenen Elastizitätsmaße zusammen.

Tabelle 24: Überblick verschiedener Elastizitätsmaße

\begin{tabular}{|c|c|c|}
\hline Nachfragerverhalten: & & Eigenschaften: \\
\hline $\begin{array}{l}\text { Direkte (Eigen-) Preise- } \\
\text { lastitizität der Nachfrager }\end{array}$ & $\varepsilon_{X i P i} \equiv($ & $\begin{array}{l}\text { Gibt an, um wieviel Prozent sich die bei An- } \\
\text { bieter } \mathrm{i} \text { nachgefragte Menge } X_{i} \text { bei einer pro- } \\
\text { zentualen Änderung des Preises } P_{i} \text { ändert. }\end{array}$ \\
\hline $\begin{array}{l}\text { Kreuzpreiselastizität } \\
\text { der Nachfrage }\end{array}$ & $\varepsilon_{X j P i}$ & $\begin{array}{l}\text { Gibt an, um wieviel Prozent sich die bei An- } \\
\text { bieter } \mathrm{j} \text { nachgefragte Menge } \mathrm{X}_{\mathrm{j}} \text { bei einer pro- } \\
\text { zentualen Preisänderung durch Anbieter } \mathrm{i} \\
\text { ändert. Je höher der Wert der Kreuzpreis- } \\
\text { elastizität ist, desto stärker reagiert die auf } \\
\text { Anbieter j gerichtete Nachfrage auf Preisver- } \\
\text { änderungen des Anbieters } \mathrm{i} \text {. }\end{array}$ \\
\hline $\begin{array}{l}\text { Beweglichkeit der Nach- } \\
\text { frage }\end{array}$ & $\varepsilon_{X j i P i} \equiv$ & $\begin{array}{l}\text { Gibt den Prozentsatz der auf Anbieter } \mathrm{j} \text { ge- } \\
\text { richteten Gesamtnachfrage an, die bei einer } \\
\text { prozentualen Preisänderung durch i von } \mathrm{j} \\
\text { nach i übergeht oder von i nach } \mathrm{j} \text { abwandert. }\end{array}$ \\
\hline Anbieterverhalten: & & Eigenschaften: \\
\hline $\begin{array}{l}\text { Kreuzpreiselastizität } \\
\text { des Angebots }\end{array}$ & $\varepsilon_{Y j P i} \equiv$ & $\begin{array}{l}\text { Gibt an, um wieviel Prozent sich die von An- } \\
\text { bieter } \mathrm{j} \text { angebotene Menge } \mathrm{Y}_{\mathrm{j}} \text { bei einer pro- } \\
\text { zentualen Preisänderung durch Anbieter i } \\
\text { ändert. Je höher der Wert der Kreuzpreis- } \\
\text { elastizität ist, desto stärker reagiert Anbieter } \\
\text { j auf Preisveränderungen des Anbieters i. }\end{array}$ \\
\hline $\begin{array}{l}\text { Reaktionsverbundenheit } \\
\text { der Anbieter in bezug auf } \\
\text { Einsatz verschiedener } \\
\text { Wettbewerbsparameter }\end{array}$ & $\begin{array}{l}\varepsilon_{W j W i} \equiv\left(d W_{i} / d W_{j}\right) *\left(W_{j} / W_{i}\right) \\
W_{j} \text { und } W_{i} \text { stehen für den Einsatz } \\
\text { eines bestimmten Wettbewerbs- } \\
\text { parameters } \mathrm{P} \text {. }\end{array}$ & $\begin{array}{l}\text { Gibt an, um wieviel Prozent sich der Einsatz } \\
\text { des Wettbewerbsparameters } P \text { bei Anbieter } \mathrm{j} \\
\text { bei einer Variation des Parametereinsatzes } \\
\text { durch Anbieter i verändert. Dabei wird unter- } \\
\text { stellt, dass der Einsatz aller übrigen Wettbe- } \\
\text { werbsparameter unverändert bleibt. }\end{array}$ \\
\hline $\begin{array}{l}\text { Reaktionskoeffizienten } \\
\text { (conjectural variation) }\end{array}$ & $a \equiv\left(^{d}\right.$ & $\begin{array}{l}\text { Gibt an, um wieviel Prozent } j \text { seinen Output } \\
\text { ändert, wenn } i \text { eine Mengenänderung vor- } \\
\text { nimmt (Reaktion) }\end{array}$ \\
\hline
\end{tabular}

Quelle: eigene Zusammenstellung auf Grundlage Kottmann 2000, S. 48 
Obwohl Elastizitätsmaße theoretisch gute Indikatoren zur Abbildung von Wettbewerbsbeziehungen darstellen, werden sie aufgrund zahlreicher konzeptioneller und empirischer Probleme bei der räumlichen Abgrenzung realer Märkte jedoch nur selten errechnet und sinnvoll interpretiert ${ }^{411}$. Außerdem steht bei partiellen Elastizitäten die ceteris-paribus-Annahme in der $\mathrm{Kritik}^{412}$. So beinhaltet ein Problem die Vernachlässigung des Nicht-Preis-Wettbewerbs. Instrumente der Unternehmenspolitik wie z.B. Werbung, Sortiments- und Markenpolitik können eine funktionale Verknüpfung der Nachfragemenge mit diesen Aktionsparametern - und somit eine Änderung der Präferenzen - hervorrufen. Theoretisch könnte dieser Mangel durch die Bildung von Kreuzwerbe-, Kreuzverkaufsförderungselastizitäten etc. gemildert werden, die Operationalisierung sämtlicher Instrumente der Unternehmenspolitik zur Berechnung von Kreuzelastizitäten scheint jedoch sehr aufwendig ${ }^{413}$. Ein weiterer Problembereich besteht darin, dass faktisch eine Vorab-Marktabgrenzung vorgenommen werden muss, da die in die Untersuchung einbezogenen Produkte aus informationsökonomischen Gründen vorher bestimmt werden müssen. Bedingung dafür ist, dass die Produkte zuvor am Markt vorhanden sind (ex-post-Maß). In einigen Fällen kann die Substituierbarkeit zweier Güter aus Nachfragersicht von der Anbieterperspektive abweichen (extreme Beispiele: links- und rechtsgesteuerte Autos sind für Anbieter Substitute, für Nachfrager keinesfalls, bei Wein und Bier ist dies umgekehrt ${ }^{414}$. Ungeklärt ist außerdem die Bestimmung eines numerischen Grenzwertes, ab dem Güter dem gleichen (unterschiedlichen) Untersuchungsmarkt (-märkten) zugeordnet werden können.

Trotz der angeführten Kritik ist das Elastizitätskonzept besonders geeignet, wenn sich der Wettbewerb vorwiegend durch Preisvariation ausdrückt und weitere absatzpolitisch orientierte Maßnahmen vernachlässigt werden können.

\subsubsection{Empirische Tests zur räumlichen Marktabgrenzung relevanter Märkte}

\subsubsection{Preisdaten}

Anhand von Preisdaten wird versucht, einen räumlich relevanten Markt abzugrenzen. Dabei werden verschiedene Testkonzepte genutzt. HorowITzs Test (Test auf uniforme Preisentwicklung) beruht auf der Vorstellung, dass sich verschiedene Regionen eines

\footnotetext{
${ }^{411}$ Vgl. KotTMANN 2000, S. 46

412 Vgl. Needham 1978, S. 113 ff.; Stegemann 1974, S. 151 ff.

${ }^{413} \mathrm{Vgl.} \mathrm{SCHENGBER} 1996$, S. 117

${ }^{414}$ Vgl. BAUER 1989, S. 53
} 
Marktes langfristig durch eine stabile Preisrelation zueinander auszeichnen, die mit Hilfe eines statistischen Regressionsverfahrens identifiziert werden kann ${ }^{415}$. HoROwITZ geht davon aus, dass sich durch exogene Schocks verursachte, schwankende Preisdifferenzen zwischen Teilgebieten eines Marktes im Zeitablauf immer wieder auf eine "natürliche" Preisdifferenz bewegen. Unter den Bedingungen einer sich dynamisch entwickelnden Umwelt ist der Test von HoROwITz zur räumlichen Marktabgrenzung daher wenig geeignet. Der Test zeigt zum Beispiel fälschlicherweise getrennte Märkte an, wenn sich die Preise innerhalb eines räumlichen Marktes langfristig infolge steigender Markttransparenz und sinkender Transportkosten annähern. Es bleibt unklar, welche Zeitperiode zu betrachten ist. Das Schätzverfahren ist sehr aufwendig, weil alle Faktoren, von denen die Preise an den Standorten gemeinsam beeinflusst werden ${ }^{416}$, einzubeziehen sind. Trotz der angeführten Schwachpunkte konnten erhebliche Fortschritte bei der Anwendung dieser Modelle erreicht werden. So können mittlerweile bei ausreichender Datengrundlage über sogenannte Marktintegrationsmodelle (besonders Cointegrationsmodelle) statistisch signifikante Preisbeziehungen zwischen Märkten definiert werden ${ }^{417}$

Eine Alternative zu dem unmittelbaren Preisvergleich zwischen verschiedenen Regionen bildet der von STIGLER und SHERWIN vorgeschlagene Test auf die Ähnlichkeit von Preisschwankungen in verschiedenen Gebieten ${ }^{418}$ (Test auf parallele Preisentwicklung). Zwei Regionen bilden dann einen gemeinsamen Markt, wenn sich die regionalen Preise im Zeitablauf parallel entwickelt haben, d.h., wenn der Test eine signifikante Korrelation zwischen den an verschiedenen Orten gemessenen Preisserien ergibt. Lassen sich über einen gewissen Zeitraum Unterschiede in der regionalen Preisentwicklung beobachten, die nicht auf eine Veränderung der Transportkosten zurückzuführen sind, gilt dies als ein Indiz dafür, dass die betroffenden Gebiete verschiedenen Märkten zuzuordnen $\sin d^{419}$.

Ohne die jeweiligen Vor- und Nachteile der verschiedenen Preistests detailliert zu beschreiben, werden im folgenden prinzipielle Eigenschaften der Preistests zusammengefasst $^{420}$ :

\footnotetext{
${ }^{415} \mathrm{Vgl.} \mathrm{HOROWITZ} 1981$

${ }^{416}$ Kritisch auch KALLFASS 1996, S. 6

417 Vgl. Cramon-Taubadel/Loy/Musfeldt 1995, S. 119 ff.

${ }^{418}$ Vgl. StigLeR/SHERWin 1985, S. 557 ff.

${ }^{419}$ Vgl. KotTMANN 2000, S. 53

${ }^{420}$ In Anlehnung an KOTTMANN 2000, S. 55
} 
- Sie unterstellen eine umkehrbar eindeutige Beziehung zwischen Preisen und den verursachenden Marktstrukturen.

- Sie erfassen Zusammenhänge zu bestimmten Zeitpunkten und sind nur für komparativ-statische Analysen geeignet. Eine dynamische Entwicklung der Umweltbedingungen kann nur schwer berücksichtigt werden. Eine hohe Volatilität der empirisch ermittelten Daten erschwert ihre Interpretation im Rahmen der Tests.

- Empirisch ermittelte Preisdaten unvollkommener Märkte müssen auf einheitliche Produktvarianten normiert werden. Ausgeprägte sachliche und persönliche Präferenzen erschweren die Interpretation der Daten.

- Da exogene Einflüsse zu Scheinkorrelationen führen oder bestehende Korrelationen überdecken können, müssen sie in den Tests vollständig und richtig berücksichtigt werden.

- Tests auf der Basis von Preisdaten können nicht zwischen dem Einfluss aktueller und potentieller Konkurrenten differenzieren.

\subsubsection{Handelsströme und Marktanteile}

Auch Daten über Lieferstrukturen können als geeignete Indikatoren für die räumliche Ausdehnung eines relevanten Marktes angesehen werden ${ }^{421}$. Vorausgesetzt für die Integration regionaler Teilmärkte zu einem zusammenhängenden relevanten Markt sei, dass Anreize für einen überregionalen Handel bestehen und die erforderliche Mobilität der Marktteilnehmer nicht durch hohe Transportkosten oder andere Handelshemmnisse behindert wird. ELzINGA (1981) wies darauf hin, dass eine explizite Prüfung dieser Faktoren nicht erforderlich sei, wenn sich ihre Wirkung aus Daten über Im- und Exporte oder aus der regionalen Verteilung von Marktanteilen ableiten lässt ${ }^{422}$. Auch bei diesen Ansätzen zur räumlichen Marktabgrenzung wird unterstellt, dass Arbitrageprozesse verschiedene Regionen zu einem einheitlichen räumlichen Markt integrieren. Im Unterschied zu den oben beschriebenen Tests werden aber nicht Preisbewegungen, sondern Mengenbewegungen bzw. Marktanteile als Resultat der Mengenbewegung betrachtet $^{423}$. Die Ansätze legen zugrunde, dass Arbitrageprozesse Handelsströme auf der Angebots- und Nachfrageseite des betrachteten Marktes erzeugen. Alternativ zu

\footnotetext{
${ }^{421}$ Solche Daten sind oft nur bei der Abgrenzung nationaler Märkte verfügbar, weil die Daten in der Regel nur an Ländergrenzen erhoben werden.

422 Vgl. ELZINGER 1981, S. 742

${ }^{423}$ Vgl. KotTMANn 2000, S. 57
} 
den Verfahren, die an den Preisdaten als Ergebnis der Arbitrageprozesse ansetzen, kann die räumliche Marktabgrenzung daher auf einer Analyse der beobachtbaren Lieferstruktur aufbauen. Auf eine detaillierte Darstellung einzelner Modelle wird verzichtet $^{424}$. Für eine speziellere Darstellung sei auf die Literatur von ELZINGA und HogARTY (1973), SHRIEVES (1978) sowie LANDES und POSNER (1981) verwiesen. Zusammenfassend werden Eigenschaften der auf Mengen basierenden Tests hervorgehoben ${ }^{425}$ :

- Sie unterstellen die parallele Entwicklung von Mengen- und Preisbewegungen.

- Sie erfassen Zusammenhänge zu bestimmten Zeitpunkten und sind nur für komparative Statistiken geeignet. Eine dynamische Entwicklung der Umweltbedingungen kann nur schwer berücksichtigt werden. Eine hohe Volatilität der empirisch ermittelten Daten erschwert ihre Interpretation im Rahmen der Tests.

- Mengenbewegungen enthalten keine Informationen über die Lieferkonditionen. Fälle, in denen ein Anbieter mehrere gesonderte Märkte beliefert, können nicht unterschieden werden von Fällen, in denen das gesamte Absatzgebiet zu einheitlichen Konditionen beliefert wird.

- Das Ausmaß, in dem Mengenbewegungen Einfluss auf die Verhaltensspielräume der Anbieter ausüben, ist abhängig von den regionalen Angebots- und Nachfrageelastizitäten.

- Daten über Mengenbewegungen können oft auch dann noch sinnvoll interpretiert werden, wenn sie sich nicht auf eine einheitliche Produktvariante beziehen bzw. Ausdruck verschiedener Marktunvollkommenheiten sind.

\subsubsection{Preiserhöhungstest}

Der Preiserhöhungstest basiert nicht auf der Analyse von einzelnen Wettbewerbsbeziehungen zwischen regionalen (Teil-)Märkten, sondern auf der Erfassung der Gesamtwirkung aller Wettbewerbsbeziehungen, unabhängig von welchen "Quellen" sie im einzelnen ausgehen. Durch die Unterstellung eines hypothetischen Monopols bildet der Zusammenhang zwischen der "Weite" eines Marktes, der Preiselastizität der Marktnachfrage und dem Ausmaß an Marktmacht die Grundlage dieses Verfahrens. Ziel ist es zu prüfen, über welche Regionen sich der Einfluss eines hypothetischen Monopolis-

\footnotetext{
${ }^{424}$ Beschreibung mit Beispiel in KaLLFASS 1996, S. $10 \mathrm{ff}$.

${ }^{425}$ In Anlehnung an KotTMAnN 2000, S. 62
} 
ten insgesamt erstrecken müsste, damit dieser dort dauerhaft einen signifikanten Preisanstieg durchsetzen könnte ${ }^{426}$. Der Preissetzungsspielraum bzw. die Marktmacht, über die der regionale Monopolist verfügen kann, ist abhängig von den Substitutionsmöglichkeiten der Nachfrager in den betrachteten Regionen. Eine geringe Preiselastizität ist somit Indiz für Substitutionslücken und markiert die Grenze eines Marktes. Zur räumlichen Abgrenzung eines Marktes im Rahmen des Preiserhöhungstests werden Schätzungen über die Preiselastizität der Nachfrage bei räumlich unterschiedlich weit abgegrenzten Märkten vorgenommen. Ein Markt wird dabei durch die schrittweise Integration weiterer Substitute solange erweitert, bis die auf diesen Markt gerichtete Nachfrage relativ preisunelastisch geworden ist ${ }^{427}$. Angewendet wird der Preiserhöhungstest sowohl von den US-amerikanischen als auch den europäischen Wettbewerbsbehörden. In den USA wird im Rahmen des SSNIP-Tests (small but significant and nontransitory increase in price) eine fünfprozentige hypothetische Preiserhöhung analysiert ${ }^{428}$. Der Schwellenwert ist theoretisch nicht begründbar und auch die Betrachtung von Fixkosten wird vernachlässigt, da ausschließlich Grenzkosten berücksichtigt werden. Dennoch wird diese Methodik als wettbewerbspolitisch akzeptabel interpretiert, weil aufgrund fehlender Information ein für alle denkbaren Fälle optimaler Schwellenwert nicht angegeben werden kann. Die Festlegung eines "offiziellen" Schwellenwertes hat jedoch den Vorteil, dass sich Unternehmen aufgrund ihrer eigenen Informationen auf die Rahmenbedingungen einstellen können ${ }^{429}$.

An dieser Stelle sollen die Eigenschaften solcher auf Preis-Mengen-Reaktionen basierenden Tests stichpunktartig zusammengefasst werden ${ }^{430}$ :

- Die bei der Definition partieller Elastizitäten unterstellten c.p.-Bedingungen können nicht eingehalten werden. Auf realen Märkten wird die Interpretation der gemessenen Zusammenhänge zwischen Preisen und Mengenbewegungen durch Angebotsrestriktionen, regionale Einkommensschwankungen usw. erschwert.

\footnotetext{
${ }^{426}$ Vgl. Kottmann 2000, S. 66

427 Vgl. KotTMAnN 2000, S. 67

${ }^{428}$ Die europäische Wettbewerbsbehörde führt Preiskorrelationsanalysen mit Stationaritätstests durch. Dazu ausführlich LEXECON 2001.

${ }^{429}$ Ausführlich zu den Vor- und Nachteilen von Preiserhöhungstests in CAMESASCA 2000, S. 84 ff. und der dort zitierten Literatur

${ }^{430}$ In Anlehnung an KotTmann 2000, S. 69; kritisch KalLFASS 1996, S. 17 ff.
} 
- Sie erfassen Zusammenhänge zu bestimmten Zeitpunkten und sind nur für komparativ-statische Analysen geeignet. Die Aussagen beruhen auf Daten, die in der Vergangenheit erhoben wurden. Eine dynamische Entwicklung der Umweltbedingungen kann nur schwer berücksichtigt werden. Eine hohe Volatilität der empirisch ermittelten Daten erschwert eine Interpretation im Rahmen der Tests.

- Der Preiserhöhungstest erfordert regional begrenzte und voneinander unabhängige exogene Schocks.

- Die Weite der Marktabgrenzung wird beeinflusst von der zum Betrachtungszeitpunkt herrschenden Wettbewerbssituation bzw. den Preissetzungsspielräumen regionaler Anbieter.

- Kreuzpreiselastizitäten werden durch große Differenzen im Mengenvolumen der regionalen Teilmärkte beeinflusst.

\subsubsection{Praktische Implementierung im deutschen und europäischen Recht}

\subsubsection{Der deutsche Rechtsrahmen}

In der deutschen Kartellrechtspraxis bezieht sich die Abgrenzung des relevanten Marktes ausschließlich auf die Sicht der Nachfrager ${ }^{431}$. Die Marktabgrenzung orientiert sich am Bedarfsmarktkonzept, das auf Arbeiten von ARNDT und ABBOT zurückgeht und auf die im Urteil der Nachfrager bestehende Austauschbarkeit verschiedener Angebote abstellt. Zu einem Bedarfsmarkt sind nach ARNDT Güter zusammenzufassen, deren heterogene Eigenschaften lediglich differenzierte Nachfragepräferenzen reflektieren, wobei die Güter grundsätzlich alle in gleicher Weise zur Deckung eines bestimmten gesellschaftlichen Bedarfs geeignet sind und von den Nachfragern daher als prinzipiell gegeneinander austauschbar angesehen werden ${ }^{432}$. Ähnlich definiert Аввот einen Markt als Zusammenfassung von Gütern, die alle zur Befriedigung eines bestimmten Bedürfnisses geeignet sind ${ }^{433}$.

\footnotetext{
${ }^{431}$ Vgl. Frankfurter Kommentar 1998, § 22 Textziffer $63 \mathrm{ff}$.

$432 \mathrm{Vgl}$. ARNDT 1958, S. $223 \mathrm{ff}$.

${ }^{433} \mathrm{Vgl}$. Аввотт 1958, S. 94 ff., in Anlehnung an KotTmann 2000, S. 80
} 
Der räumlich relevante Markt wird formalrechtlich auf das deutsche Staatsgebiet beschränkt $\mathrm{t}^{434}$. Allerdings wird zwischen der wirtschaftlichen Betrachtung und der Feststellung der Voraussetzungen für die formale und materielle Anwendung des GWB unterschieden. Für das Bundeskartellamt kann ein Markt in wirtschaftlicher Betrachtung größer sein als das Gebiet der Bundesrepublik Deutschland ${ }^{435}$. Die Marktanteile ausländischer Anbieter werden anhand deren tatsächlichen Lieferungen in das Bundesgebiet ermittelt. Dabei wird zwischen Unternehmen aus anderen Ländern der Europäischen Union und Unternehmen aus Drittländern unterschieden. Bei Unternehmen aus Drittländern wird angenommen, dass ihre wettbewerbliche Bedeutung geringer ist, als es ihren Marktanteilen im Inland entspräche ${ }^{436}$. Der potentielle Wettbewerb aus dem Ausland und die Höhe der Marktzutrittsschranken gegenüber ausländischen Anbietern haben dagegen bei den Entscheidungen des Bundeskartellamtes und der Rechtssprechung bisher eine untergeordnete Bedeutung eingenommen. Dies hat sich durch die sechste Novellierung des GWB ${ }^{437}$ geändert, wobei ausdrücklich betont wird, der Internationalisierung des Wettbewerbs größere Bedeutung beizumessen, indem der potentielle ausländische Wettbewerb berücksichtigt wird ${ }^{438}$.

\subsubsection{Abgrenzung des relevanten Marktes in der EU}

Grundsätzlich orientiert sich das in der europäischen Zusammenschlusskontrolle verfolgte Prinzip ebenfalls an dem Bedarfsmarktkonzept. Die Bestimmungen des räumlichen Referenzmarktes weisen jedoch Parallelen zu der im US-amerikanischen Kartellrecht entwickelten Abgrenzungsmethode auf. So bildet auch hier die Frage nach der mutmaßlichen Reaktion der Marktteilnehmer auf eine hypothetische Erhöhung der aktuellen Marktpreise die gedankliche Grundlage für eine Analyse der Wettbewerbsbeziehungen. Dabei stützt man sich in der Entscheidungspraxis auf eine Reihe von Faktoren, die als geeignete Indikatoren für eine Begrenzung des relevanten Marktes angesehen werden. Hierzu zählen sowohl Marktergebniskriterien wie Preise und Mengenbewegungen als auch strukturelle Faktoren, die als mögliche Ursache für eine räumliche Begrenzung relevanter Märkte in Betracht kommen ${ }^{439}$.

\footnotetext{
${ }^{434}$ Siehe Rechtsprechung „Backofenurteil", BGH, 24.10.1995

${ }^{435} \mathrm{Vgl}$. MONOPOLKOMMISSION 2000, S. 28; vgl. BUNDESKARTELLAMT 2001: Tätigkeitsbericht, S. 20

${ }^{436} \mathrm{Vgl.}$ BundeSKARTELLAMT 1990, S. 18

437 Zum 01. Januar 1999 in Kraft getreten

${ }^{438}$ Vgl. KoOPMANn 2000, S. 18

${ }^{439}$ Ausführliche Vorgehensweise und Kriterien in: EUROPÄISCHE KOMMISSION 1997, Official Journal 372, 9.12.1997
} 
Die Europäische Kommission definiert den sachlich relevanten Markt folgendermaßen: "Der sachlich relevante Markt umfasst all jene Erzeugnisse und/oder Dienstleistungen, die vom Verbraucher hinsichtlich ihrer Eigenschaften, Preise und ihres vorgesehenen Verwendungszwecks als austauschbar oder substituierbar angesehen werden"440. Unter dem räumlich relevanten Markt ist folgendes zu verstehen: „Der geographisch relevante Markt umfasst das Gebiet, in dem die beteiligten Unternehmen Produkte oder Dienstleistungen anbieten, in dem die Wettbewerbsbedingungen hinreichend homogen sind und das sich von benachbarten Gebieten, insbesondere aufgrund merklich unterschiedlicher Wettbewerbsbedingungen, die in diesen Gebieten herrschen, abgrenzt." ${ }^{441}$.

Die für die Wettbewerbspolitik der Europäischen Union zuständige Europäische Kommission muss bei der Beurteilung von Unternehmenszusammenschlüssen "den tatsächlichen oder potentiellen Wettbewerb durch innerhalb oder außerhalb der Gemeinschaft ansässige Unternehmen" berücksichtigen ${ }^{442}$. Anders als in der deutschen Fusionskontrolle ist bei der europäischen Fusionskontrolle deshalb kein exogen vorgegebenes, fiktives nationales oder europäisches Marktgebiet zu unterstellen ${ }^{443}$. Dies spiegelt sich in der Entscheidungspraxis der Kommission wider: Die räumliche Marktabgrenzung reicht in Fusionsfällen vom lokalen Markt bis hin zum Weltmarkt. Hohe Marktanteile fusionswilliger Unternehmen können zudem durch niedrigere Marktzutrittsschranken relativiert werden ${ }^{444}$. Nach Möglichkeit wird bei der räumlichen Marktabgrenzung die vorhersehbare Öffnung nationaler Märkte und die damit einhergehende Verschiebung der Marktgrenzen antizipiert. Die wirtschaftliche Bedeutung politischer Grenzen ist zu prüfen, und die Entwicklung der wirtschaftlichen Verbindungen zwischen einzelnen nationalen Märkten ist zu prognostizieren ${ }^{445}$.

\subsection{Kritische Anmerkungen über die Wettbewerbspraxis}

Das Bedarfsmarktprinzip basiert auf der Annahme, dass der Gesamtmarkt in eine endliche Zahl von Teilmärkten zerlegbar ist. Da alle Teilmärkte jedoch über Kosten und Preise in mehr oder weniger ausgeprägter Interdependenz stehen, besteht die Aufga-

\footnotetext{
${ }^{440}$ Siehe Formblatt A/B zur VO 17/62 sowie Formblatt CO zur Verordnung EWG 4064/89 in EU-COMmISSION (1997): http://europa.eu.int/comm/competition/antitrust/relevma_en.html

${ }^{441}$ Siehe Formblatt A/B zur VO 17/62 sowie Formblatt CO zur Verordnung EWG 4064/89 in EU-COMmISSION (1997): http://europa.eu.int/comm/competition/antitrust/relevma_en.html

${ }^{442}$ FKVO Art. 2, Abs. 1a

${ }^{443}$ Vgl. Kallfass 1997, S. 112 zitiert in KoOPMANN 2000, S. 18

${ }^{444}$ Vgl. KoOpmann 2000, S. 18

445 Vgl. Kottmann 2000, S. 23
} 
be der Partialmarktabgrenzung darin, einen sachlich begründeten Marktausschnitt aus der Vielzahl weiterer Substitutionsbeziehungen zu bestimmen. Der Ausschluss der irrelevanten Marktbeziehungen setzt erfahrungswissenschaftlich daher voraus, dass die Intensität wettbewerblicher Intendepenzen sachgerecht identifiziert wird ${ }^{446}$. Folgende Probleme treten dabei auf ${ }^{447}$ :

1) Die Marktabgrenzung erfordert vom Rechtsanwender ein ausgeprägtes Erfahrungswissen über die faktische Intensität von Wettbewerbs- und Substitutionsbeziehungen, weil es ihm ansonsten nicht möglich ist, den Abgrenzungsvorgang auf die tatsächlichen Machtbereiche der Unternehmen zu vollziehen.

2) Die Vernachlässigung der „irrelevanten Substitutionsbeziehungen" lässt sich gleichwohl nicht ausschließlich empirisch nachvollziehen. Der relevante Markt lässt sich daher nicht objektiv bestimmen, eine Objektivierung ist jedoch sehr wohl möglich.

LADEMANN empfiehlt den Einsatz von erfahrungswissenschaftlichen Instrumenten, wobei von einem Kriterienbündel zur Messung von Substitutionsbeziehungen ausgegangen wird. Er legt somit einen mehrdimensionalen, multivariaten Ansatz zugrunde, um die Substituierbarkeit zwischen Waren nicht nur an einem ggf. willkürlich bestimmten Kriterium, wie etwa dem Preis, zu messen, sondern um ein ganzes Bündel von Merkmalen berücksichtigen zu können ${ }^{448}$. Mit Hilfe erfahrungswissenschaftlicher Untersuchungen ist es ebenfalls möglich, eine empirisch fundierte Bewertung von Markteintrittsbarrieren zu bestimmen ${ }^{449}$.

Die Europäische-Kommission schränkt den relevanten Markt eines Produktes auf die Anbieter ein, für die ein gedankliches Experiment ab einer dauerhaften 5 bis $10 \%$ igen Preisänderung ein Überwechseln der Nachfrager auf einen anderen Marktpartner erwarten lässt ${ }^{450}$ (analog bei Beschaffungsmärkten). Es werden in der Praxis meist Expertengespräche mit ausgewählten Mitbewerbern und Abnehmern geführt und markt-

\footnotetext{
${ }^{446}$ Vgl. LADEMANN 2000, S. 70

447 Vgl. LADEMANN 1988, S. 578

448 Vgl. LADEMANN 2000, S. 70, 82 ff., 91: Insbesondere repräsentative Verbraucherbefragungen und die entsprechenden Auswertungen ermöglichen somit ein realitätsnahes Abbild von den Beziehungen verschiedener Güter zueinander und sollten daher bei der Abgrenzungspraxis der relevanten Märkte als Primärquellen herangezogen werden. Instrumente können demnach Techniken der Marktforschung wie Cluster-, Comjointanalysen usw. sein.

${ }^{449}$ Vgl. LADEMANN 2000, S. 70

450 Vgl. IVALDI/VERBOVEN 2001, S. 3; dieser Test stammt aus der amerikanischen Wettbewerbspraxis: SSNIPTest; dazu NUSSKERN 1999, S. $125 \mathrm{ff}$.
} 
bezogene Sekundärquellen ausgewertet ${ }^{451}$. LADEMANN kritisiert vor allem die begrenzte Anzahl dieser Gespräche, so dass eine solche Vorgehensweise nur auf Märkten mit wenigen Teilnehmern zu brauchbaren Ergebnissen führen könne ${ }^{452}$. Ferner handele es sich lediglich um ein gedankliches Experiment, bei dem von einer fiktiven dauerhaften Preis-änderung ausgegangen wird und somit die Praxisrelevanz bestritten werden könne. Zudem macht LADEMANN auf die mangelnde Transparenz und erschwerte Nachprüfbarkeit solcher Untersuchungen aufmerksam ${ }^{453}$.

Die 10 \%-Schwelle stellt einen weiteren Kritikpunkt dar. So gibt es Märkte, in denen geringere Preisveränderungen zu einem Überwechseln der Nachfrager führen (Konsumgüterindustrie), während bei anderen Produkten oder Dienstleistungen wesentlich stärkere Preisdifferenzen erforderlich sind (Benzin) ${ }^{454}$. Auch hätte eine tatsächliche $10 \%$ Preiserhöhung eine weitreichende Verschiebung der Substitutionsbeziehungen zur Folge, die bei der Betrachtung der aktuellen Marktpartner kaum berücksichtigt wird ${ }^{455}$. CAMESASCA (2000) wies ebenfalls auf die Problematik des Preiserhöhungstests hin, da dynamischen Effekten nicht Rechnung getragen wird ${ }^{456}$. Auch KaLLFASS kam zu dem Schluss, dass ein Preiserhöhungstest allein nicht von den Wettbewerbsbehörden zur Abgrenzung des relevanten Marktes eingesetzt werden sollte ${ }^{457}$. Außerdem ist zweifelhaft, ob die ausschließliche Ausrichtung auf den Wettbewerbsparameter Preis, die Ganzheitlichkeit des Wettbewerbs wiedergeben kann. So berichten Marktforscher aus dem Konsumgüterbereich, dass Marktanteilserhöhungen eher von einer Erhöhung des Werbemitteleinsatzes als infolge von Preissenkungen ausgelöst werden ${ }^{458}$. In den geführten Gesprächen dieser Studie mit Mitarbeitern der europäischen Kartellbehörde wurde jedoch betont, dass der SSNIP-Test lediglich als eine Komponente bei der Beurteilung von Zusammenschlüssen zu werten und die Bedeutung bei der Gesamtabwägung aller Faktoren nur geringeren Ausmaßes sei. Der Kritik einiger Autoren, dass der SSNIP-Test zu Fehleinschätzungen führe, scheint demnach nicht besonders schwerwiegend zu sein.

\footnotetext{
${ }^{451}$ Vgl. LADEMANN 2000, S. 74, sowie eigene Erhebung

452 Vgl. LADEMANN 2000, S. 75

${ }^{453}$ Vgl. LADEMANN 2000, S.75

${ }^{454} \mathrm{Vgl}$. LADEMANN 2000, S. 76

${ }^{455} \mathrm{Vgl}$. LADEMANN 2000, S.76

${ }^{456} \mathrm{Vgl.}$ CAMESASCA 2000, S. 87

${ }^{457} \mathrm{Vgl}$. KALLFASS 1996, S. $18 \mathrm{ff}$.

${ }^{458}$ Vgl. LADEMANN 2000, S. 77
} 


\subsubsection{Zwischenergebnis}

Zur Ermittlung des relevanten Marktes sind verschiedene Marktabgrenzungskonzepte entwickelt worden, wobei die beschriebenen Konzepte einen erheblichen Ermessungsspielraum zulassen. Aus empirischer Sicht wird durch die unterschiedlichen Einschätzungen der Gerichte und Kartellbehörden dieses Problem belegt (vgl. dazu Kapitel 5.1.3, Kapitel 5.2.4 sowie Kapitel 6.2). Die deutsche und europäische Wettbewerbspraxis orientiert sich am Bedarfsmarktkonzept nach ARNDT und ABOTT. Danach bilden all diejenigen Produkte einen gemeinsamen Markt, die aus Sicht eines "verständigen" Nachfragers gleichwertige Alternativen darstellen. Hierbei ist nicht die Homogenität der Güter entscheidend sondern vielmehr die funktionelle (Produkteigenschaft, Verwendungszweck etc.), räumliche und zeitliche Austauschbarkeit. Wird der relevante Markt auf diese Weise abgegrenzt, werden nur die kurzfristigen Austauschbeziehungen der Nachfrage berücksichtigt (aktuelle Konkurrenz), und langfristige Substitutionsprozesse, die von Unternehmen auf angrenzenden Märkten ausgehen, sowie Wettbewerbseinflüsse, die noch nicht am Markt befindliche (potentielle) Konkurrenten ausüben, vernachlässigt. Während die sachliche und zeitliche Marktabgrenzung bei der Berücksichtigung grenzüberschreitender Wettbewerbsbeziehungen weniger Probleme bereitet, können bei Bestimmung der räumlichen Marktgrenzen mitunter Schwierigkeiten auftreten. Denn stehen ausländische Konkurrenten in unmittelbarem Wettbewerb zu heimischen Unternehmen, so ist es ökonomisch wenig überzeugend, das nationale Staatsgebiet als räumlich relevante Marktobergrenze anzusehen. Üblicherweise werden als Indikatoren zur räumlichen Marktabgrenzung die Testverfahren auf uniforme und parallele Preisentwicklung, der Test der Handelsströme sowie verschiedene Methoden zur Bestimmung der Preis-Mengen-Reaktionen verwendet. Da Elastizitätsmaße die Grundlage vieler Testverfahren bilden, wird nochmals auf mögliche Schwachpunkte bei der Abgrenzung des relevanten Marktes - insbesondere mit Hilfe der Kreuzpreiselastizitäten - hingewiesen: Erstens erweist sich die empirische Bestimmung zuverlässiger Werte für diese Elastizitäten als schwierig und aufwendig, und zweitens ist diejenige kritische Höhe der Kreuzpreiselastizität, oberhalb der ein Gut dem relevanten Markt zugeordnet werden sollte, theoretisch umstritten. Ein drittes entscheidendes Problem besteht in der Abhängigkeit der Kreuzpreiselastizität vom Preissetzungsverhalten der Anbieter. Sie stellt also keine exogene Größe dar, sondern wird endogen durch den Wettbewerb im Markt bestimmt. Wenn also Kreuzpreiselastizitäten verwendet werden, um den Bereich der wirksamen Konkurrenz zu definieren, so wird implizit 
ein $M a ß$ benutzt, welches selbst vom Konkurrenzverhalten der Anbieter beeinflusst wird $^{459}$.

Oftmals lassen die einzelnen Testresultate keine eindeutigen Rückschlüsse auf räumliche Marktgrenzen zu. Sie werden dann überwiegend zur Absicherung der mit Hilfe von Plausibilitätsüberlegungen ermittelten geographischen Marktgrenzen verwendet ${ }^{460}$.

Ein weiterer kritischer Bereich der Wettbewerbsprüfung betrifft die Einschätzung des substitutiven und potentiellen Wettbewerbs als Ergänzung zum aktuellen Wettbewerb. Besonders die Gewichtung der einzelnen Faktoren bildet eines der Hauptprobleme.

\subsection{Beurteilung von Nachfragemacht}

In den Gesprächen bzw. schriftlichen Befragungen dieser Untersuchung gaben nahezu alle Unternehmer als Hauptfusionsmotiv die Erreichung einer gegengewichtigen Marktstellung gegenüber dem Handel an. Obwohl die Konzentration im Handel bereits weit vorangeschritten ist, ist eine Stagnation dieser Entwicklung noch nicht voraussehbar. Die Ernährungsindustrie befürchtet bei voranschreitender Konzentration des Lebensmitteleinzelhandels auch eine zunehmende Ausübung von Marktmacht ${ }^{461}$. Selbst prominente Vertreter aus der Politik unterstützen diese Marktmachtthese. So eröffnete der Bundeskanzler die Anuga 2001 mit dem Zitat: „Der Handel bestimmt, was in die Regale kommt und somit auch, was gekauft wird ${ }^{462 " .}$

Auch hat sich in letzter Zeit die Auffassung über die Funktionsfähigkeit im Lebensmitteleinzelhandel unter vielen Wissenschaftlern und Wettbewerbshütern geändert. So entsteht zunehmend die Meinung, dass die gewachsenen oligopolistischen Strukturen des Handels mitunter auch regulierende Eingriffe erfordern ${ }^{463}$. Beispielsweise fordern die OECD und die britischen Wettbewerbsbehörden einen kritischeren Blick auf die

\footnotetext{
${ }^{459}$ Vgl. BeSter 2000, S. 21

${ }^{460} \mathrm{Vgl}$. MONOPOLKOMMISSION 1984, S. 447

461 Stellungnahme des BUNDESVERBANDES FÜR ERNÄHRUNGSINDUSTRIE vom 17.10.2001: www.bveonline.de/aktuell/texte/221001/industry.html

462 Zitat Gerhard Schröder zur Eröffnung der Anuga 2001, zitiert in LZ Nr. 42 vom 18. Oktober 2001, S. 1

${ }^{463} \mathrm{Vgl.} \mathrm{Aiginger/WieSER/Wüger} \mathrm{1999,} \mathrm{S.} 52$
} 
Wettbewerbsverhältnisse im Handel ${ }^{464}$. Auch die Europäische-Kommission zeigt in den letzten Jahren gegenüber Handelsfusionen eine geänderte Einstellung ${ }^{465}$.

Ziel dieses Kapitels ist es, das Nachfragemachtargument bei der Begründung von Fusionen näher zu untersuchen. Dabei werden primär die Relationen der Hersteller zum Handel erläutert. Darauf aufbauend wird beschrieben, inwieweit die Marktmachthypothese in der Beurteilungspraxis Berücksichtigung findet und welche Verbesserungsvorschläge zukünftig aufgegriffen werden sollten.

\subsubsection{Marktmacht im LEH: Problemstellung und Stand der Literatur}

\subsubsection{Problemstellung}

Die Lebensmittelindustrie betrachtet die zunehmende Konzentration des LEH als ernsthafte Gefahr. Die überwiegend mittelständisch strukturierten Verarbeitungsbetriebe sind der Nachfragemacht der großen Handelsunternehmen zunehmend ausgesetzt. Dies führt soweit, dass zum Teil kleinere Herstellerunternehmen in den Ausweichmöglichkeiten akut eingeschränkt sind und sich somit der Verhandlungsstärke der Abnehmerseite erlegen fühlen ${ }^{466}$. In 2000 repräsentierten allein die Top 10Handelsunternehmen einen Marktanteil von nahezu 84 Prozent. „Prognosen gehen für 2010 von einer weiteren Umsatzverdichtung aus, so dass der zuvor genannte Wert dann bereits von lediglich fünf maßgeblichen „Playern" erzielt wird", prognostiziert die Beratungsgesellschaft KPMG ${ }^{467}$. Laut einer Umfrage der KPMG International bei führenden Unternehmen der Ernährungsindustrie sehen 80 \% der Befragten die größte Bedrohung für ihr Unternehmen in der zunehmenden Konzentration des Handels.

Der Handel dagegen verweist auf einen intensiven Wettbewerb, der durch niedrige Umsatzrenditen geprägt ist, und damit nur geringe Möglichkeiten zur Erzielung abnormaler Renditen zulässt. Diesem Argument sollte jedoch nur eingeschränkt Aufmerksamkeit geschenkt werden, da die Umsatzrentabilität keinen geeigneten Indikator zur Messung der Wettbewerbsfähigkeit darstellt ${ }^{468}$. Abbildung 28 verdeutlicht das Verhältnis der Konzentration zwischen dem LEH und der Ernährungsindustrie in Europa:

\footnotetext{
${ }^{464}$ Vgl. HEWITT 1998

${ }^{465}$ Im Fall Kesko/Toko in Finnland bestand die Kommission sogar auf eine Entflechtung bereits fusionierter Unternehmensteile. Vgl. EU-Kommission, Competition Letters 1996 (2), S. 35; 1996 (3), S. 34; 1997 (1), S. 17; vgl. Untersuchung in Auftrag der EU-Kommission: DOBSON CONSULTING (1999)

${ }^{466}$ Vgl. dazu DRESCHER 1999, S. 231 verweist auf Artikel, die diese These stützen.

${ }^{467}$ Vgl. KPMG/EHI 2001, S. 18

${ }^{468} \mathrm{Vgl}$. DRESCHER 1999, S. 231 und die dort zitierte Literatur
} 
Abbildung 28: Unternehmenskonzentration - Ernährungsindustrie und Einzelhandel im Vergleich 1998

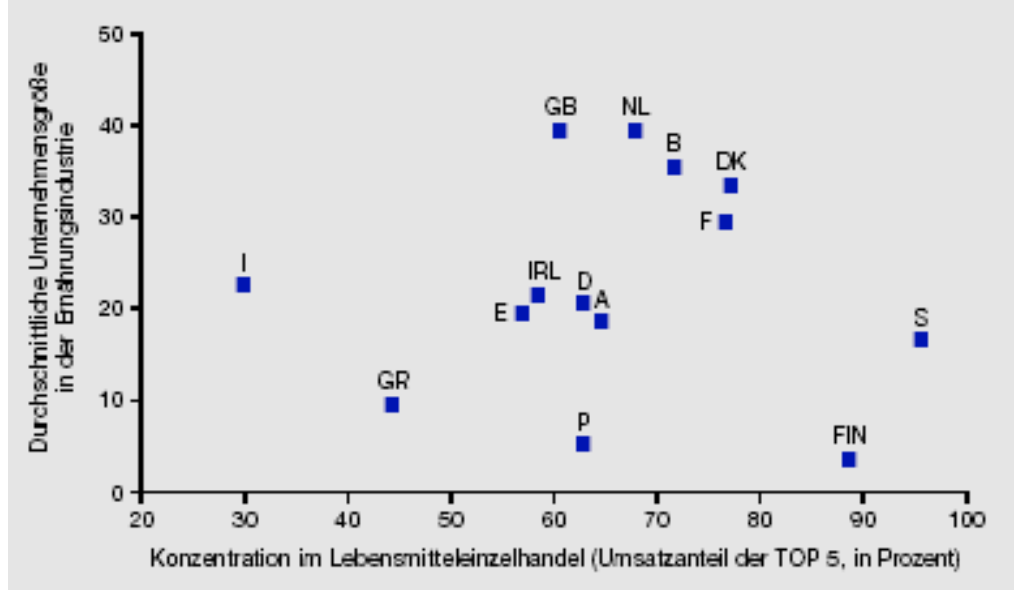

Anm.: Durchschnittliche Unternehmensgröße: Produktionswert pro Unternehmen in Mio. Euro

Quelle: Deutsche Genossenschaftsbank 2000, S. 59

\subsubsection{Stand der Forschung}

Die Preisgestaltung im LEH ist schon länger Gegenstand der wissenschaftlichen Forschung, sowohl vom positiven als auch normativen Standpunkt aus. Normative Studien untersuchen in erster Linie die Preisgestaltung von einzelnen Produkten oder Produktgruppen. Die meisten Untersuchungen greifen auf Modelle der Preisdiskriminierung zurück (BLATTBERG und NESLIN 1990; KIM et al. 1995) und unterstellen die Marktform der monopolistischen Konkurrenz. Demnach kann der LEH bis zu einem gewissen Grad Marktmacht ausüben. Marktmacht begründet sich aufgrund steigender Suchkosten seitens der Verbraucher oder infolge einer gewissen Bequemlichkeit (Beziehen aller Einkäufe aus einem Geschäft) ${ }^{469}$. Die positive Ökonomie befasst sich dagegen in erster Linie mit der Preisgestaltung bei unterschiedlichen Wettbewerbsverhältnissen und kann somit dem klassischen Gebiet der Industrieökonomik zugeordnet werden. Umstritten ist dabei, ob es sich beim Lebensmitteleinzelhandel um ein Polypol, ein Oligopol oder gar um die Marktform der monopolistischen Konkurrenz handelt ${ }^{470}$.

In Europa sind bisher nur sehr wenige empirische Studien zum Thema Angebotsmacht bzw. Nachfragemacht im Handel erschienen. Der überwiegende Teil der Arbeiten stammt aus den USA, wo aufgrund der langen Antitrust Tradition schon seit längerem

\footnotetext{
${ }^{469}$ Vgl. DRESCHER 1999, s. 233 und die dort zitierte Literatur

${ }^{470}$ Vgl. DRESCHER 1999, S. 233
} 
anspruchsvolle Untersuchungen vorliegen und auch die entsprechende Datenbasis Rückschlüsse auf regionale Konzentrationsentwicklungen erlaubt ${ }^{471}$.

Die Arbeiten von MARIon et al. (1979), LAMm (1981), CotTERILl (1986), MARION (1989), BRESNAHAN/REISS (1991) sowie BINKLEY/CONNOR (1995) zeigten durchweg einen (statistisch signifikant) positiven Zusammenhang zwischen Unternehmens- bzw. Verkaufsstättenkonzentration und dem Preisniveau. Die Preisniveaudifferenzen waren dabei nicht nur auf Kostenunterschiede sondern eindeutig auch auf die aus steigender Konzentration resultierende höhere Marktmacht der Unternehmen zurückzuführen ${ }^{472}$. BLINKLEY und CONNOR (1998) zeigten, dass im Bereich von haltbaren Markenprodukten mit intensivem Verarbeitungsgrad eine hohe Konzentration der Hersteller mit erhöhten Preisen für die Endverbraucher einherging. Die Beobachtungen einiger Autoren über die Zusammenhänge von Konzentration und Profitabilität im europäischen Handel ergeben kein geschlossenes Bild. DRESCHER (1999) kam mit Hilfe eines Cournot-Ansatzes zu dem Schluss, dass bei Unterstellung einer quadratischen Konzentrationsfunktion des LEHs ab einem bestimmten Konzentrationsgrad das Preisniveau stieg ${ }^{473}$. In der jüngsten Studie untersuchten HerRmann/Möser/Werner (2002) Preissetzungs- und Verbraucherverhalten am Point-of-Sale und stellten insgesamt fest, dass das Verbraucherverhalten stark auf die Preispolitik reagierte. So konnte die allgemeine Aussage der agrarökonomischen Literatur, dass "die Preiselastizität der Nachfrage nach Nahrungsmitteln im Absolutbeitrag niedrig ist" ${ }^{144}$, angezweifelt werden.

Aus den genannten Untersuchungen kann jedoch noch kein direkter Schluss auf die Aufteilung der Marktspannen und somit die Relation der Machtverhältnisse gezogen werden. Während auf Verbraucherebene die Einführung von Scannerdatensystemen erhebliche Transparenz erbrachte ${ }^{475}$, führt die mangelnde Verfügbarkeit von Herstellerabgabepreisen zu Unsicherheit über die unterschiedliche Aufteilung der Gewinnmargen und die strukturbedingten Verteilungen innerhalb des Absatzkanals. So kann die Aussage, es bestehe ein direkter Zusammenhang zwischen Größe und Macht, bislang nicht verallgemeinert werden ${ }^{476}$.

Die Studien zur Nachfragemacht, in denen die Gegenmachthypothese überprüft wird und wonach Gegenmacht (countervailing power) die unerwünschten Wirkungen von

\footnotetext{
${ }^{471}$ Vgl. Aiginger/Wieser/Wüger 1999, S. 187; DRESCHER 1999, S. 232

$472 \mathrm{Vgl.} \mathrm{Aiginger/WieSER/WÜGER} \mathrm{1999,} \mathrm{S.} 161 \mathrm{ff}$.

473 Vgl. DRESCHER 1999

474 Vgl. HeRrMANn/MÖSER/WERnER 2002, S. 99

475 Vgl. Herrmann/Möser/Werner 2002, S. 103

476 Vgl. OLBRICH 1998 , S. 480
} 
Marktmacht weitgehend neutralisiert, stammen ebenfalls weitgehend aus den USA. Während ältere Arbeiten von LUSTGARTEN (1975), MARTIN $(1982,1983)$ oder WATERSON (1980) die These bejahten, dass Konzentration der Nachfrager bzw. deren relative Größe die Gewinne der vorgelagerten Sektoren reduzierte, negiert die jüngere Literatur überwiegend diese Hypothese. FARRIS/AILAWADI (1992) kamen in ihrer Untersuchung der Lebensmittelmärkte in den USA über den Zeitraum 1972 - 1990 zu dem Ergebnis, dass sowohl die Renditen der Händler als auch jene der größeren Hersteller tendenziell gestiegen sind, während die kleineren Hersteller Gewinneinbußen erlitten. MESSINGER/NARASIMHAN (1995) wiederum stellten für die siebziger und achtziger Jahre keine Gewinnverschiebungen zwischen Händlern und Herstellern fest. Zuletzt verwarfen CONNER et al. (1996) die These von der wohlfahrtserhöhenden Wirkung von Gegenmacht für die Lebensmittelmärkte in den USA.

In den neueren europäischen Untersuchungen werden die Erkenntnisse der amerikanischen Forschung, wonach starke Markenhersteller von der zunehmenden Konzentration im Lebensmitteleinzelhandel weniger betroffen sind - und damit die Gegenmachthypothese nicht zutrifft -, weitgehend bestätigt ${ }^{477}$. KAAS/GEGENMANTEL kommen anhand der Untersuchung von Auslistungsverlusten zu dem Resultat, dass der Handel aufgrund seiner systematischen Sortimentsstruktur einen Vorteil hat. Da die Verkaufsfläche des Handels eine weniger spezifische Ressource ist als die Produktionsanlage und das immaterielle Markenkapital des Herstellers, kann daher insgesamt der Handel Auslistungsverluste besser kompensieren als die Hersteller ${ }^{478}$ (vgl. Kapitel 7.2.4.2.1). LADEMANN, HERMES, ROMEISER weisen jedoch darauf hin, dass tendenziell umsatzschwächere Hersteller der Marktmacht des Handels unterlegen sind, sich aber in individuellen Situationen durchaus andere Hersteller-Handels-Relationen ergeben können. In den anschließenden Ausführungen werden Indikatoren und Auswirkungen von Nachfragemacht näher beschrieben.

\subsubsection{Struktur und Entwicklung im LEH}

Der Lebensmitteleinzelhandel wird seit Mitte der 80er Jahren von einer zunehmenden Konzentration geprägt. Dieses Phänomen lässt sich sowohl auf deutscher wie auch europäischer Ebene beobachten (vgl. Abbildung 29 und Abbildung 30):

\footnotetext{
477 Vgl. hierzu die Studien von LADEMANn 1996, 2001; BendL 2000, AIGINGER/WieSER/WüGER 1999; SCHULZE 1998; ZENTES/HURTH 1996; CULLEN/ WHELAN 1997; ROMEISER 1998

478 In Anlehnung an AIGINGER/WIESER/WÜGER 1999 Kurzfassung: Internet: www.bmwa.gv.at/service/weservice/marktmachtkurz.html, S. $8 \mathrm{f}$
} 


\section{Abbildung 29: Konzentration im deutschen Lebensmittelhandel}

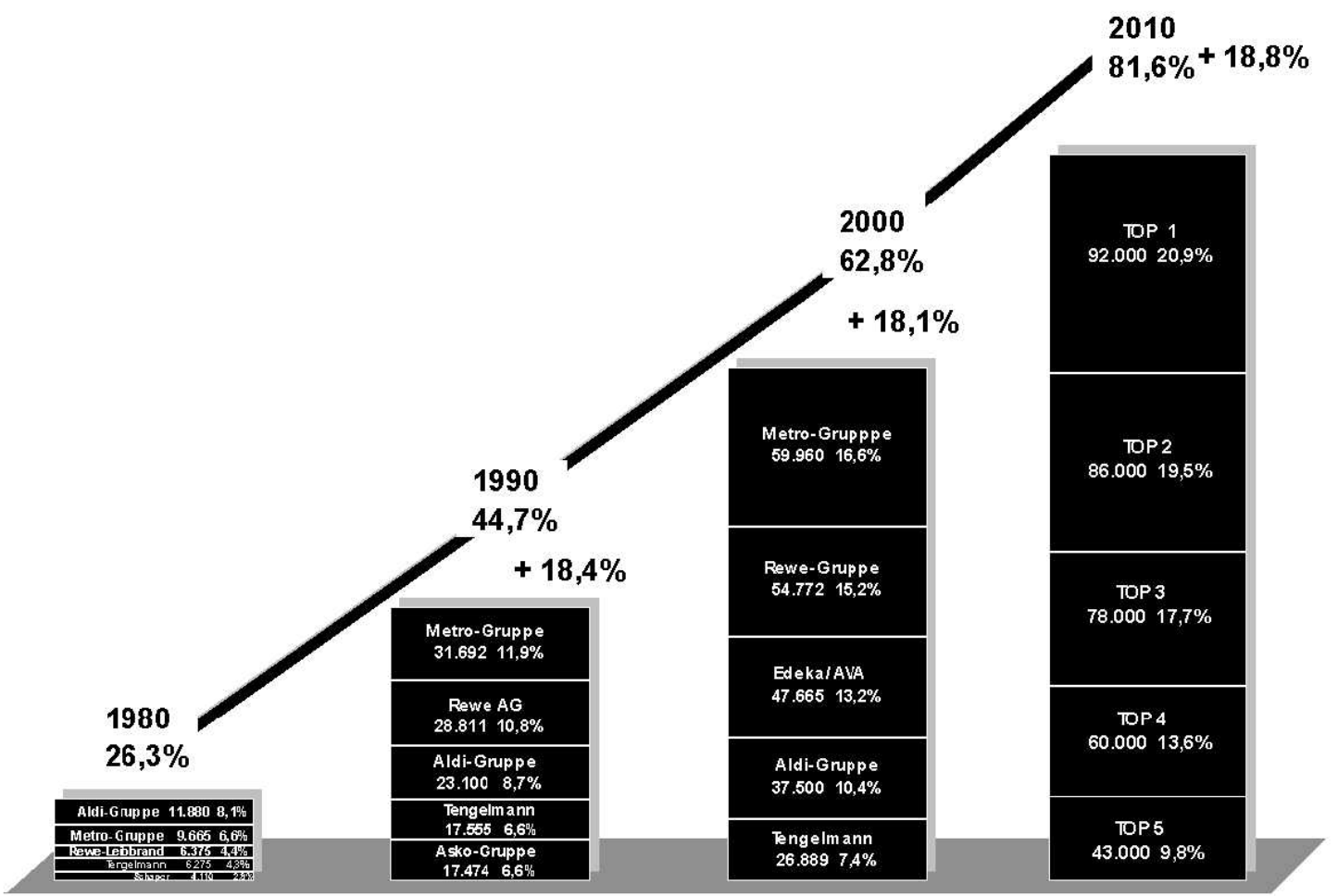

Anm: Umsatzangaben: 2001-2010: durchschnittliche Umsatzsteigerungen 1980-1990-2000 sowie eine angenommene Durchschnittsinflationsrate von 2,0 \% unterstellt; 2000: Gesamtumsätze Mio. DM brutto, Prognose; 1990: Gesamtumsätze Mio. DM brutto, Gesamtdeutschland; 1980: Gesamtumsätze Mio. DM brutto, nur BRD

Quelle: M+M Eurodata 2002

Abbildung 30: Konzentration im europäischen LEH (Top 5)

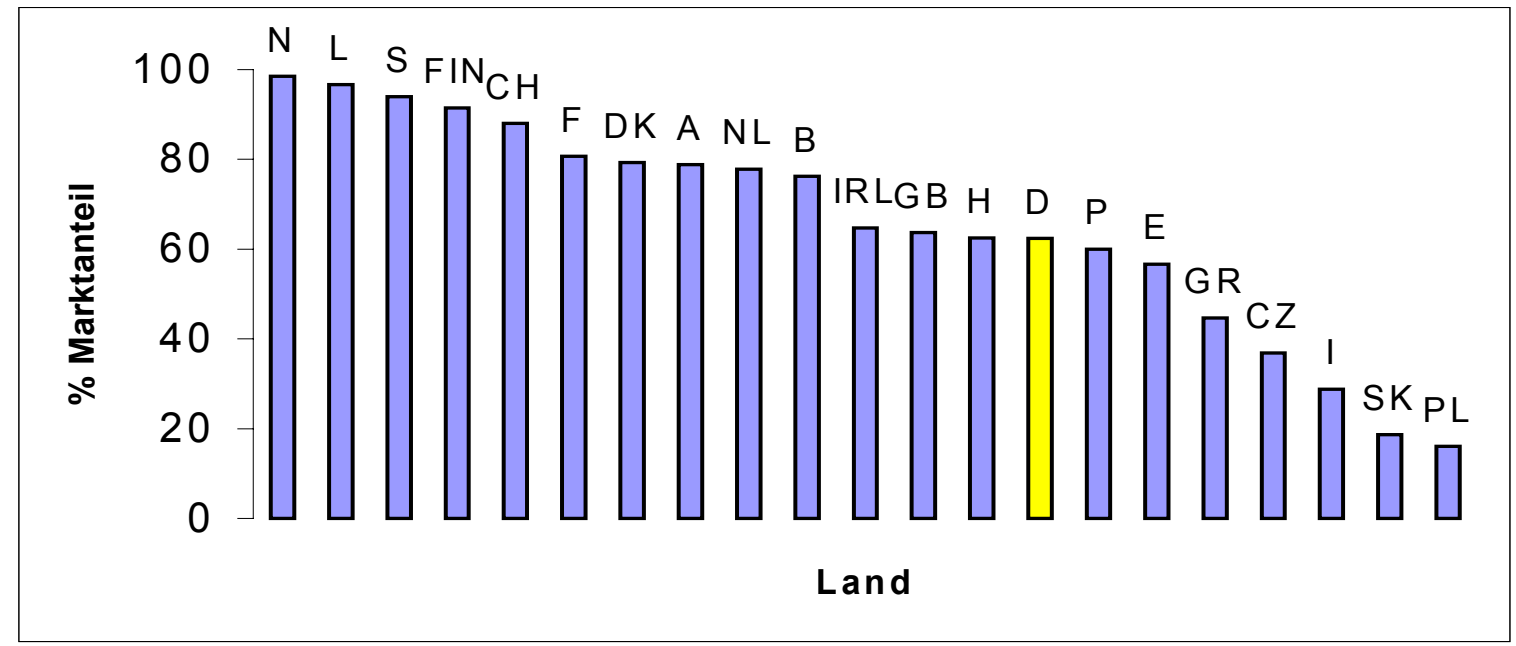

Quelle : M+M EuROdATA 2002 
DRESCHER ${ }^{479}$ führte diese Entwicklung im wesentlichen darauf zurück, dass sich eine Reihe von Handelsunternehmen wegen wirtschaftlicher Probleme oder sonstigen Gründen vom Markt zurückgezogen hat oder in Teilen an die Konkurrenz veräußert wurde. Einige bedeutende Handelsübernahmen der letzen Jahre werden in der folgenden Tabelle (Tabelle 25) zusammengefasst:

Tabelle 25: Bedeutende Fusionen des Handels

\begin{tabular}{|ll|ll|}
\hline Jahr & beteiligte Unternehmen & Jahr & beteiligte Unternehmen \\
\hline $\mathbf{1 9 9 6}$ & Auchan/Docks de France & $\mathbf{1 9 9 9}$ & Karstadt/Quelle \\
$\mathbf{1 9 9 7}$ & Metro/Makro & Sainsbury/Star Markets \\
& Migros/Globus & & Rewe/Meinl \\
& Carrefour/Promodes/Com.Mod. \\
& Wal Mart/Wertkauf & & Wal Mart/Asda \\
$\mathbf{1 9 9 8}$ & Wntermarché/Spar & Wal Mart/Interspar & Carrefour/GIB \\
& Sommerfield/Kwik Save & Ahold/ CA \\
& Kingfisher/Castorama & Ahold/Standa \\
& Carrefour/Comptoires Modernes & & BML/Standa \\
& De Boer/ Vendex Food & $\mathbf{2 0 0 1}$ & Edeka/AÖAG \\
& Metro/Allkauf & $\mathbf{2 0 0 2}$ & Casino/Laurus \\
& Ahold/Giant Food & & \\
\hline
\end{tabular}

Quelle: eigene Zusammenstellung (Stand: Sept. 2002)

Als Gründe für ein Streben nach zunehmender Größe wurden aktuellen Umfragen von Beratungsunternehmen zufolge in erster Linie die Erzielung von besseren Einkaufskonditionen (durch Einkaufsbündelung) sowie die Erhöhung der eigenen "Masse" (um nicht selber Opfer von Übernahmeversuchen zu werden) genannt ${ }^{480}$.

Um auch zukünftig als Handelunternehmen überlebensfähig zu bleiben, ist beim Umsatzvolumen eine Größenordnung von ca. 20 Mrd. DM anzustreben ${ }^{481}$.

Neben der steigenden Konzentration des Gesamtlebensmitteleinzelhandels lässt sich darüber hinaus eine deutliche Verschiebung innerhalb des Sektors erkennen ${ }^{482}$. So stieg der Marktanteil von Discountern beispielsweise von 6,2\% im Jahr 1983 auf fast $30 \%$ im Jahr 2000 an $^{483}$ (vgl. Abbildung 31).

\footnotetext{
${ }^{479}$ Vgl. DRESCHER 1999, S. 6

${ }^{480}$ Vgl. KPMG/EHI 2001, S. 44

${ }^{481}$ Vgl. KPMG/EHI 2001, S. 46; MONOPOLKOMMISSION 1994; M+M EURODATA, 31. Oktober 2000

482 Die Charakteristika der Formen des Lebensmitteleinzelhandels sind näher in Anhang $D$ beschrieben

${ }^{483}$ Vgl. M+M EURODATA 2001
} 
Abbildung 31: Entwicklung verschiedener Vertriebsformen in Deutschland

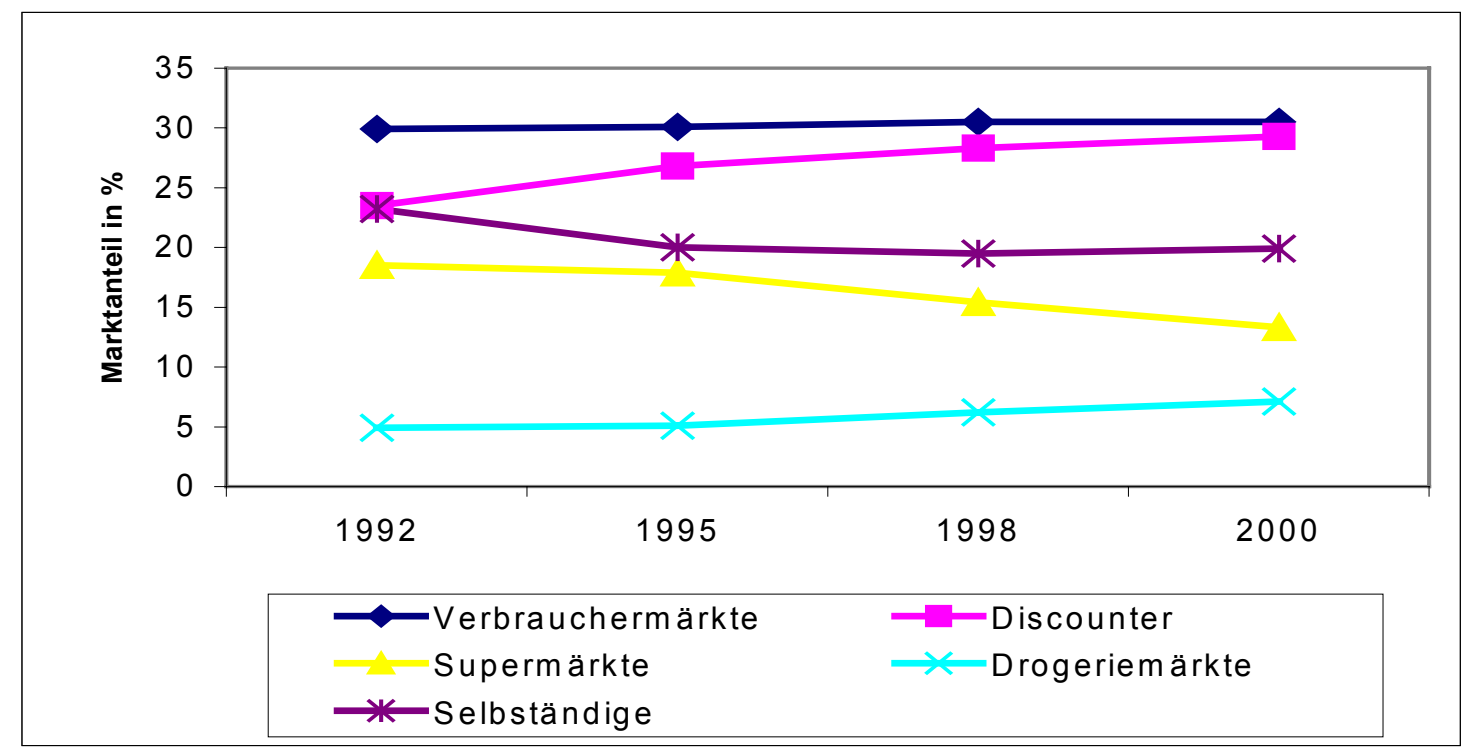

Quelle: M+M EUROdATA 2001

Die unterschiedlichen Vertriebsformen können unterschiedliche Beschaffungspolitiken zur Folge haben. So steht beispielsweise bei der Vertriebsform des Discountmarktes in erster Linie der Preis und weniger Attribute wie Kundenservice oder Regalpflege im Vordergrund der Verhandlungen. Somit führt eine Änderung der Vertriebsform gleichzeitig zu unterschiedlichen Relationen zwischen Industrie und Handel.

Die beschriebene Entwicklung beim Lebensmitteleinzelhandel indiziert die Situation in der sich die meisten Konsumgüterhersteller derzeit befinden. Der fortlaufende Konzentrationsprozess im LEH hat zumindest laut Aussage der Hersteller zu einem Umschwung der Marktmachtverhältnisse vom Angebots- zum Nachfragemarkt geführt (vgl. dazu Kapitel 7.2.4.2.3). Den Herstellern stehen immer weniger Handelsorganisationen mit permanent zunehmendem Beschaffungsvolumen gegenüber ${ }^{484}$. Kaum ein Hersteller kann somit darauf verzichten, eine der Top 10 Handelsorganisationen nicht zu beliefern ${ }^{485}$.

\subsubsection{Definition von Nachfragemacht}

Der Begriff der Nachfragemacht ist nicht eindeutig definierbar. So kann das Phänomen der Nachfragemacht als „Abweichung von einem Gleichgewichtszustand", als „wettbe-

\footnotetext{
${ }^{484}$ Vgl. u.a. MATSCHUK/VIETH 1989, S. 135 ff.

${ }^{485}$ Vgl. BENDL 2000, S. 26
} 
werbsbeschränkter Verhaltensspielraum", als "bilaterales Beherrschungsabhängigkeitsverhältnis" oder als „Überwindung von Widerstand der Marktgegenseite" betrachtet werden ${ }^{486}$. Werden diese Ansätze theoretischer Natur mit empirischen Konzepten in Verbindung gebracht, ergibt sich eine Vielzahl von Definitionen für Nachfragemacht. Die OECD definierte Nachfragemacht folgendermaßen: "a situation which exists when a firm or a group of firms, either because it has a dominant position as a purchaser of a product or service or because it has strategic or leverage advantages as a result of its size or other characteristics, is able to obtain from a supplier more favourable terms than those available to other buyers" ${ }^{\prime 487}$.

Im weiteren wird die Definition LADEMANNs herangezogen. Danach ist unter Nachfragemacht "die Fähigkeit von Personen in nachfragenden Wirtschaftsorganisationen gegenüber Personen aus Anbieterorganisationen, die Bewertung der Tauschbedingungen im Sinne der eigenen Unternehmensziele durchsetzen zu können" zu verstehen ${ }^{488}$. Einigkeit besteht weitestgehend darin, dass Nachfragemacht stets zeitpunktbezogen ist ${ }^{489}$; so kann sie zu einem gegebenen Zeitpunkt auf materieller Verfügungsgewalt beruhen (z.B. Regalflächen im Einzelhandel). Veränderungen dieser Situation, möglicherweise aufgrund der Mobilisierung von Ressourcen seitens eines Herstellers, zeugen davon, dass Nachfragemacht keineswegs von dauerhafter Natur ist und somit als dynamischer Prozess ${ }^{490}$ interpretiert werden muss. Wie bereits im vorigen Abschnitt angedeutet, sind grundsätzlich Marktmachtbeziehungen zwischen Hersteller und Handel als interdependente Verflechtung zu verstehen. Eine strukturelle Änderung auf der Handelsseite hat unmittelbar Auswirkungen auf die Herstellerebene (vgl. dazu Abbildung 32). Ändert sich beispielsweise durch die erfolgreiche Einführung neuer Betriebsformen die Qualität des Marktzugangs für die Herstellerseite, wird dieses auch entsprechende Anpassungen im Konditionsgefüge nach sich ziehen. Konditionsspreizungen sind somit auch als Spiegelbild von unterschiedlichen Gratifikationspotentialen im Handel und daher als solche nur durch das Zusammenwirken beider Marktseiten zu verstehen ${ }^{491}$.

\footnotetext{
${ }^{486}$ Hierzu ausführlich: Lademann 1986, S. 9 ff.; SCHUlze 1998, S. 11; ApPel 1992, S. 125 ff.; BendL 2000 S. 191; Aiginger/Wieser/Wüger 1999, S. 30 ff.

${ }^{487} \mathrm{Vgl.}$ OECD 1981

${ }^{488}$ Vgl. LADEMANN 1986, S. 44

489 Vgl. LADEMANN 1986, S. 33

${ }^{490}$ Vgl. OLSON 1970, S. 2; WRONG 1968, S. 673 ff. - zitiert nach LADEMANN 1986, S. 33

${ }^{491}$ Vgl. LADEMANN 1986, S. 245
} 
Abbildung 32: Wettbewerb im Handel: Produktspezifische Beurteilung

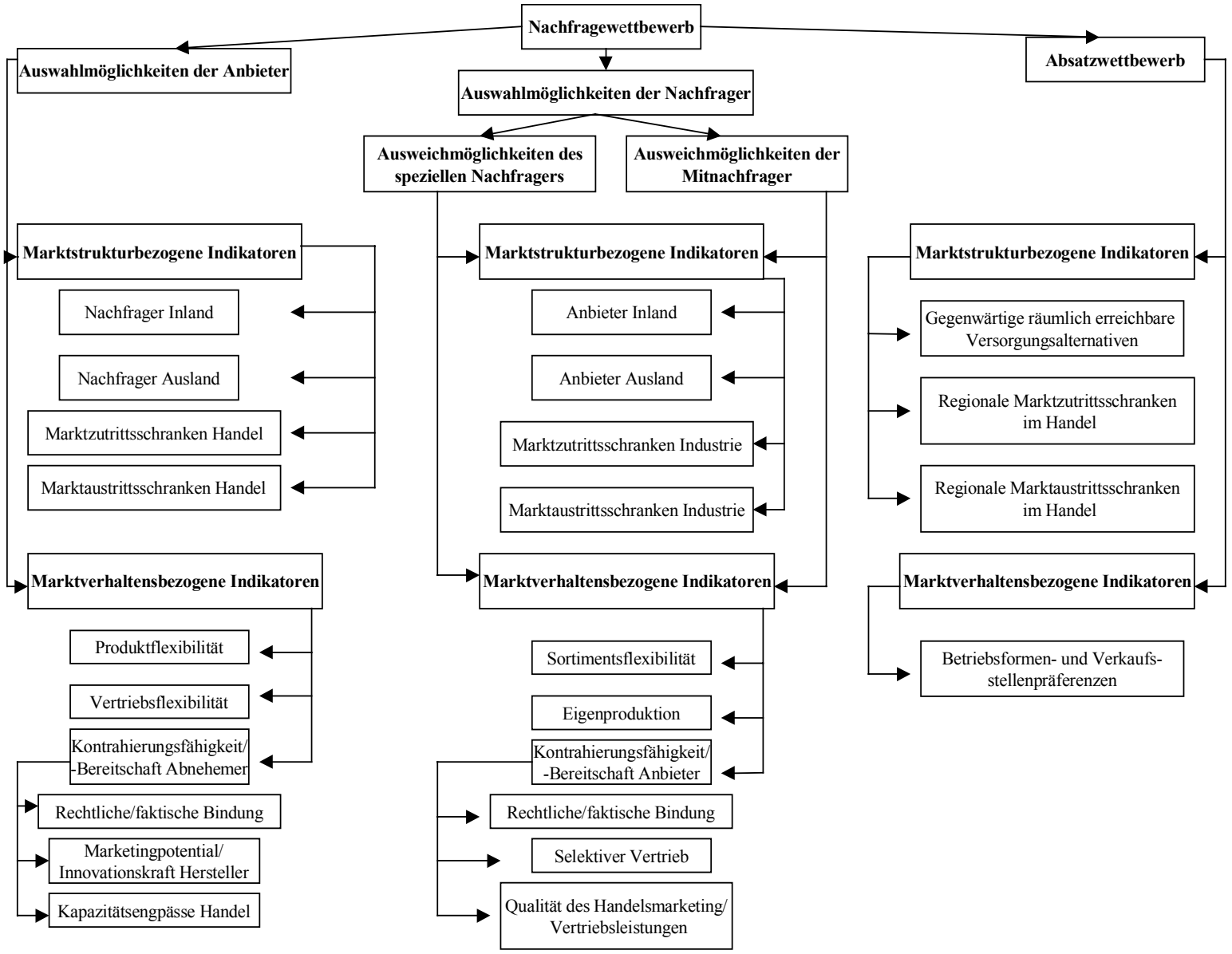

Quelle: Hermes 1988, S. 145

In der Abbildung 32 werden Indikatoren der Marktstruktur und des Marktverhaltens schematisch dargestellt, die den Verhandlungsspielraum zwischen Hersteller- und Handelsunternehmen beeinflussen. Einige Aspekte werden in den nächsten Abschnitten näher aufgegriffen.

\subsubsection{Entstehung von Nachfragemacht zwischen Hersteller und Handel}

Gemäß der Ausführungen in den vorigen Abschnitten ist Nachfragemacht als interaktiver Prozess zu verstehen. Ein eingeschränkter Wettbewerb auf der Handelsseite führt unter anderem dazu, dass dem Hersteller weniger Abnehmer gegenüberstehen. Daher beschreiben die nächsten beiden Abschnitte mögliche Ursachen von Macht des Handels auf der horizontalen Ebene und anschließend im vertikalen Prozess. 


\subsubsection{Begrenzter Wettbewerb auf der horizontalen Ebene des Handels}

Die Entstehung von Angebots- und Nachfragemacht auf den Handelsmärkten ist zum Teil strukturbedingt, kann aber auch im strategischen Marktverhalten der Unternehmen begründet sein. $\mathrm{Zu}$ den strukturellen Ursachen zählen institutionelle Markteintrittsbarrieren, wie rechtliche und regulatorische Vorschriften (Raumordnungsgesetze, Bauordnungen, Gewerbeordnungen und Ladenschlussregelungen), überwiegend aber das Einkaufs- und Konsumverhalten der Endverbraucher ${ }^{492}$. Da Konsumenten ihren Nutzen optimieren, indem sie versuchen, Zeit-, Transport- und Suchkosten zu reduzieren ${ }^{493}$, reagieren die Unternehmen mit einer entsprechenden Standortpolitik, wie einer Vergrößerung von Verkaufsflächen und Sortimenten und dem Versuch, die Konsumenten verstärkt an ihre Verkaufsläden zu binden, indem durch Aktionen (kostenlose Zugaben, Treuekarten und -marken, Gewinnspiele) sowie Preis- und Differenzierungsstrategien das Einkaufen in den Geschäften der Konkurrenten unattraktiver werden soll, so dass sich die Wechselkosten der Konsumenten erhöhen ${ }^{494}$.

Der Erfolg für ein neu eintretendes Handelsunternehmen (potentielle Konkurrenz) wird zunächst dadurch begrenzt, dass etablierte Unternehmen beispielsweise über mehr Erfahrung (Lernkurveneffekte), Reputation und Konsumentenloyalität, die der neue Wettbewerber erst erarbeiten müsste, verfügen. Zusätzlich müssen Newcomer für einen Markteintritt spezifische Investitionen, etwa in Distributions- oder Marketingsysteme tätigen, die zumindest im großflächigen Lebensmitteleinzelhandel mit hohen versunkenen Kosten (sunk costs) verbunden sind und oftmals nur von großen, finanzkräftigen Unternehmen zu bewältigen sind ${ }^{495}$.

Weitere Begründungen für die Entstehung von Marktmacht im Einzelhandel liegen in den zunehmenden Skalenerträgen und Verbundvorteilen großflächiger Formate, die Kostenvorteile gegenüber kleineren Mitbewerbern eröffnen.

Erschwerend kommt hinzu, dass strategische Maßnahmen den Markteintritt neuer Wettbewerber behindern können. Verschiedene Preisstrategien (Absprachen, Kampfpreise, Preisdiskriminierung und Lockvogelpreise) sowie Formen vertikaler Bindungen

\footnotetext{
492 Vgl. Aiginger/Wieser/Wüger 1999, S. 35

493 Dadurch erklären RoMEISER 1998; LADEMANN 1996, S. 215 u.a. die Zunahme sog. One-Stop-Shopping Handelsplätze (alle Produkte an einem Ort $=$ Malls).

${ }^{494} \mathrm{Vgl.}$ Aiginger/Wieser/Wüger 1999, S. $39 \mathrm{f}$.

495 Dazu auch Deutsche Genossenschaftsbank-ReSEARCH 1999, S. 12 ff.
} 
(Alleinvertrieb, Markenzwang, Preisbindungen und Marktaufteilung) wirken potentiell wettbewerbsbeschränkend ${ }^{496}$.

\subsubsection{Vertikale Ebene: Thesen zur Marktmacht des Handels gegenüber den Lieferanten}

Es können verschiedene Begründungen für unterschiedliche Marktmachtstellungen des Handels gegenüber der Industrie angeführt werden. Einige weit verbreitete Thesen werden nachfolgend dargestellt.

\subsection{Flexibilitätsthese}

Nach der Flexibilitätsthese hat es der Handel leichter, seine Sortimente umzustellen, als die Hersteller in der Lage sind, deren Produktion auf andere Märkte zu verlagern. Durch diese unterschiedliche Flexibilität bei der Leistungserstellung in beiden Wirtschaftsstufen kommt es zu einer asymmetrischen Konstellation bei den gegenseitigen Ausweichmöglichkeiten auf andere Marktpartner ${ }^{497}$.

Schließlich kann der Hersteller versuchen, die Ausweichmöglichkeiten des Handels einzuschränken, indem er durch konsumentenbezogenes Marketing beim Endverbraucher den Wunsch stimuliert, sein Produkt kaufen und speziell dieses auch in den Regalen des Handels vorfinden zu wollen. Eine solche Markenartikelstrategie ${ }^{498}$ führt allerdings nicht nur zu einer einseitigen Abhängigkeit des Handels vom Hersteller, sondern zu einer verstärkten wechselseitigen Abhängigkeit von Hersteller und Handel. Indem ein Kennzeichen eines Markenartikels das Versprechen ist, diesen „überall" erhalten zu können (Ubiquitätsanspruch), ist der Hersteller auf einen hohen Distributionsgrad angewiesen und somit vor allem auf eine Listung durch die nahezu flächendeckend agierenden Filialunternehmen des Handels ${ }^{499}$.

\subsection{Sortimentsthese}

Die Sortimentsthese gründet auf der typischerweise unterschiedlichen Sortimentsbreite von Handels- und Industrieunternehmen. Da Handelsunternehmen ein sehr viel

\footnotetext{
${ }^{496}$ Vgl. dazu Beschlüsse Verkauf unter Einstandspreis: BUNDESKARTELLAMT: B-09-74/00; B-09-84/00; B09$85 / 00$

497 Vgl. APPEL 1992, S.145 und die dort zitierte Literatur

$498 \mathrm{Vgl.} \mathrm{MefFERT} \mathrm{2000,} \mathrm{S.} 872 \mathrm{f}$.

${ }^{499}$ Vgl. APPEL 1992, S. 147
} 
breiteres Sortiment führen als Unternehmen der Industrie, ist der Ertrag eines Handelsunternehmens in geringerem Maße von einem einzelnen Produkt abhängig als der Ertrag eines produzierenden Unternehmens. Daher sind beide Marktstufen nicht in gleicher Weise aufeinander angewiesen ${ }^{500}$.

Es kann ebenso wie bei der Flexibilitätsthese kritisiert werden, dass bestimmten Produkten eine besondere Funktion zugeordnet wird. So kann ein Verzicht auf bestimmte Markenprodukte (USP-Position ${ }^{501}$ ) kaum durch Produktquantität kompensiert werden. Hinzukommend wies APPEL u.a. darauf hin, dass der Bezugsmarktanteil allein noch keine Aussagen über die "Wichtigkeit" oder Verdienstspanne eines Produktes zulässt ${ }^{502}$.

\subsection{Käufermarkthypothese}

Einzelne Handelsunternehmen verfügen gegenüber Herstellern über vertikale Nachfragemacht. Die Ursache liegt laut MONOPOLKOMMISSION ${ }^{503}$ in der Existenz einer anhaltenden Käufermarktsituation. Käufermärkte zeichnen sich dadurch aus, dass zu bestehenden Preisen die Angebotsmengen größer sind als die nachgefragten Mengen ${ }^{504}$. Angebotsüberkapazitäten bestehen regelmäßig dann für längere Zeit, wenn auf den betroffenen Märkten Preisinflexibilität herrscht. Das Preissystem verliert seine Funktion, Knappheiten anzuzeigen und für eine Räumung der Märkte zu sorgen. Solange die Preise auf den Beschaffungsmärkten oberhalb des Gleichgewichtspreises ${ }^{505}$ liegen, erwachsen den Handelsunternehmen allein daraus erhebliche Verhaltensspielräume gegenüber den Anbietern ${ }^{506}$.

Fordert ein Handelsunternehmen in einer Käufermarktlage von einem Lieferanten Preiszugeständnisse, indem es droht, auf einen anderen ebenfalls nicht ausgelasteten Anbieter auszuweichen, kann der Lieferant diese Forderung nur selten ablehnen. In dieser Situation wird er kaum einen alternativen Abnehmer finden, der für eine Übernahme der ausgefallenen Liefermenge nicht gleiche oder ähnliche Preiszugeständnisse fordert. Um einen allgemeinen Preisverfall zu verhindern, setzt dabei ein "Geheim-

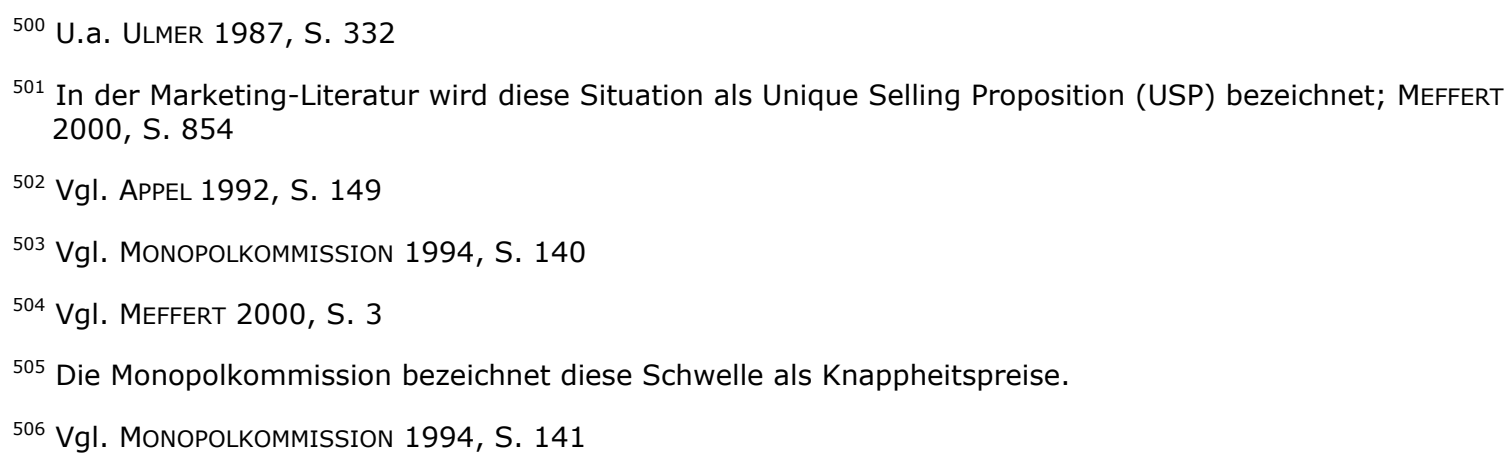


wettbewerb" zwischen den Lieferanten ein, der typischerweise mit den Aktionsparametern Rabatte, Serviceleistungen und sonstige Konditionsvorteile für die Nachfrager ausgetragen wird ${ }^{507}$.

\subsubsection{Gefahren}

\subsubsection{Wohlfahrtsökonomische Gefahren}

Die wohlfahrtsökonomischen Implikationen der Nachfragemacht unterscheiden sich in Abhängigkeit vom Vorhandensein von Marktmacht auf der Lieferstufe ${ }^{508}$. Nachfragemacht kann als Gegenmacht dann wohlfahrtserhöhend wirken, wenn seitens der Lieferantenseite eine hohe Konzentration vorliegt und gleichzeitig die nachfragemächtigen Käufer auf ihren Absatzmärkten einem wirksamen Wettbewerb ausgesetzt und dadurch gezwungen sind, die erreichten Vorteile an ihre Konsumenten weiterzugeben (Gegenmachthypothese) $^{509}$.

Nachfragemacht kann dagegen negative Wohlfahrtswirkungen haben, wenn die Lieferantenseite kleinstrukturiert ist und in scharfem Wettbewerb steht. Eine solche Situation erlaubt es mächtigen Nachfragern, die Produzentenrente der Lieferstufe abzuschöpfen. Im Zuge der Rentenumverteilung von den Herstellern zum Handel ergeben sich Wohlfahrtsverluste, die auf eine Unterbeschäftigung von Ressourcen, eine Aushöhlung der Innovationskraft und eine Abschreckung potentieller Newcomer auf Lieferantenseite zurückzuführen sind. Soweit der Handel nicht gleichzeitig auf den Absatzmärkten mit intensivem Wettbewerb konfrontiert ist, werden zusätzlich die Konsumentenrenten reduziert ${ }^{510}$.

\subsubsection{Gefahr einer Kartellbildung}

Eine zusätzliche Gefahr bei Verringerung der Anzahl möglicher Abnehmer besteht im wesentlichen darin, dass bei Koordination der Abnehmer ein sogenanntes Nachfragekartell gebildet werden kann ${ }^{511}$. Dieses Nachfragekartell zielt zunächst durch Verringerung der nachgefragten Menge auf die Senkung der Einkaufspreise. Die durch das

\footnotetext{
${ }^{507}$ Vgl. dazu auch SCHULZE, S. $16 \mathrm{ff}$.

508 Vgl. dazu ausführlich Marktstrukturmodelle (Bilaterales Monopol, Monemporist, Monopsonist etc.) z.B. in: DOBSON CONSULTING 1999, S. 10 ff.; MARTIN 2001, S. 23 ff.

${ }^{509}$ Vgl. dazu Ausführungen Aiginger/Wieser/Wüger 1999, S. 33 ff.

${ }^{510} \mathrm{Vgl.}$ AigingeR/Wieser/WüGER 1999, S. 33 ff.

511 Vgl. OLBRICH 1998, S. 477
} 
Nachfragekartell initiierte Reduktion der nachgefragten Menge wird auf dem Absatzmarkt zu Preiserhöhungen gemäß den gegebenen Elastizitäten führen. Durch oligopolistisches Parallelverhalten kann es somit dem Handel gelingen, Konsumentenrenten abzuschöpfen, die nicht an die Hersteller weitergegeben werden. Allerdings wird die Stabilität eines solchen Kartells angezweifelt ${ }^{512}$. Für Außenseiter oder Kartellmitglieder kann es in dieser Situationen vorteilhaft sein, offen oder heimlich zusätzliche Mengen zu geringfügig höheren Preisen zu kaufen. Ein solches Vorgehen wird den Marktanteil dieses Unternehmens auf der Bezugs- und Abnehmerseite erhöhen und ist zumindest kurzfristig auch profitabel ${ }^{513}$. Die Wahrscheinlichkeit eines abgestimmten Kartellverhaltens nimmt mit Abnahme der unabhängigen Handelsunternehmen zu. Festzuhalten bleibt, dass eine präventive Fusionskontrolle somit nur mit einer begleitenden Missbrauchsaufsicht einen fairen Wettbewerb garantieren kann ${ }^{514}$.

\subsubsection{Praktische Auswirkungen und Indikatoren von Nachfragemacht}

WEINDELMAIER und FLORIOT stellten bei ihrem Vergleich des deutschen mit dem französischen LEH fest, dass niedrigere Einkaufspreise eine entscheidende Wettbewerbsstrategie darstellen, so dass der LEH seine Machtposition zur Durchsetzung immer niedrigerer Einstandspreise bzw. zur ständigen Forderung nach Konditionenverbesserung ausnutzt ${ }^{515}$. Andere Autoren beschreiben die Entwicklung als Bestreben, im Nachfragewettbewerb den Einkauf auf weniger Lieferanten mit möglichst umfassendem Produktprogramm oder auf eine geringere Artikelzahl zu konzentrieren. "Dies lässt den Konditionendruck auf die wenigen innovativen, von Auslistung bedrohten Herstellern grundsätzlich in dem Maße anwachsen, wie der Handel ansonsten Rationalisierungspotentiale erschließen könnte. Sie dürften durch Konditionenzugeständnisse zwar i.d.R. ihre Listung sichern, aber auch in erheblichem Maß den im horizontalen Wettbewerb der Lieferstruktur erzeugten Innovations- und Rationalisierungsdruck bezahlen" ${ }^{\text {516. }}$.

In der Praxis treten Marktmachtphänomene nur in den seltensten Fällen direkt in Form niedrigerer Preisforderungen gegenüber den Herstellern auf ${ }^{517}$. Vielmehr sind es indirekte Maßnahmen, die sich letztendlich auch auf die Produktpreise auswirken, jedoch nicht unmittelbar einem Produkt zugeordnet werden können. Vor allem sind es Kondi-

\footnotetext{
512 Dazu APPel 1992, S. 143

${ }^{513} \mathrm{Vgl}$. dazu auch Kapitel 4.1.3

${ }^{514} \mathrm{Vgl}$. Aiginger/Wieser/WügeR 1999, S. 52 und S. 187

515 Vgl. WEINDLMAIER/FLORIOT 2001, S. 143

516 Vgl. LADEMANN 1996, S. 26

517 Vgl. HeRMES 1988, S. 9
} 
tionen, die zu einem Spiegelbild der Wettbewerbsverhältnisse zwischen einem Anbieter und seinem Abnehmer führen ${ }^{518}$.

Dabei können bei einzelnen Produkten beachtliche absolute Beträge beobachtet werden (vgl. Abbildung 33):

Abbildung 33: Bedeutung von Rabatten und Konditionen

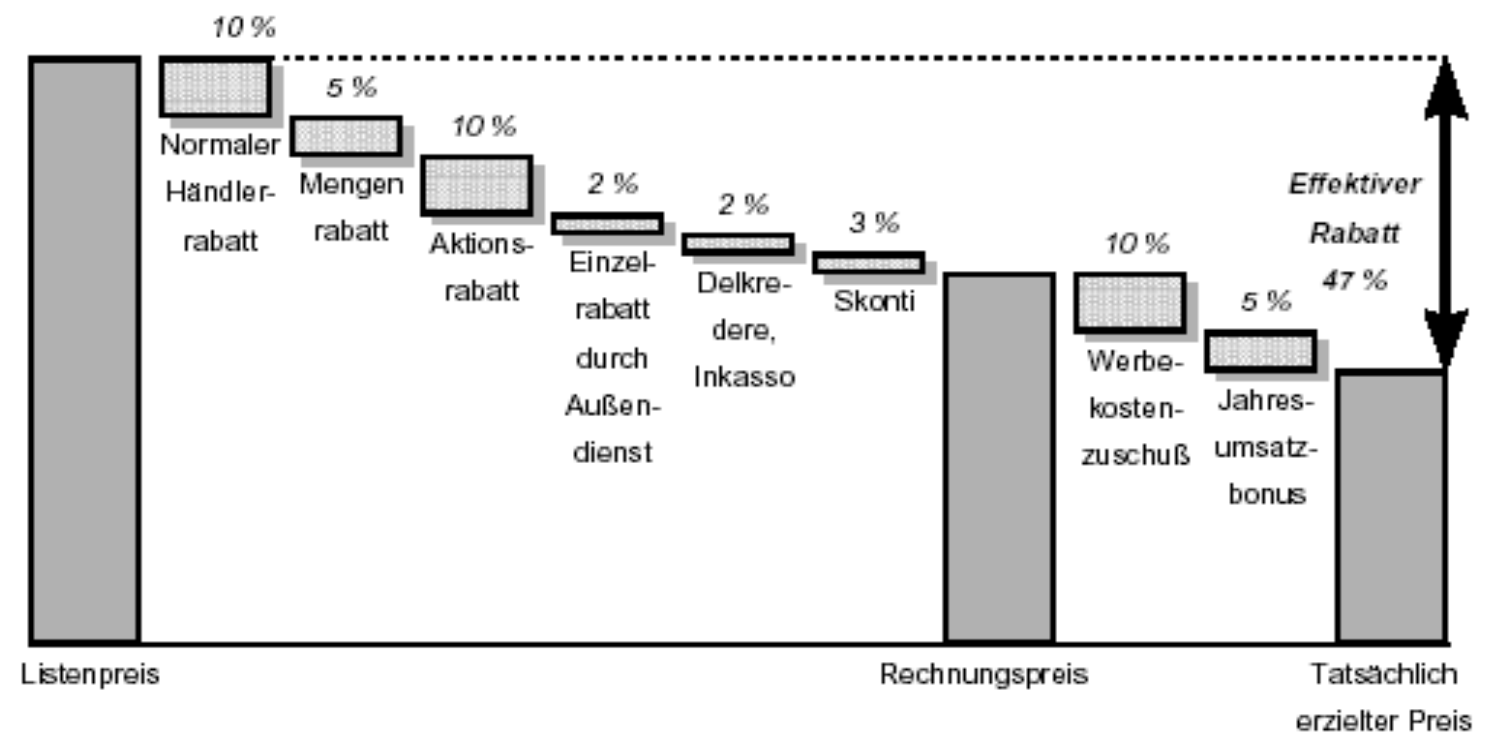

Anm.: Listen-, Rechnungs- und tatsächlich erzielter Preis entspricht dem Endverbraucherpreis

Quelle: BendL 2000, S. 218

BENDL führte in seiner Studie eine Umfrage zur Höhe und praktischen Gestaltung von Konditionsvereinbarungen durch. Unter anderem prüfte er die Hypothese, ob die Höhe der Konditionenvergütung durch den Grad der Abhängigkeit eines Herstellers von seinen Handelskunden beeinflusst wird. Stark abhängige Hersteller müssten somit höhere Konditionen gewähren ${ }^{519}$. Er kommt in seiner Untersuchung zu folgenden Ergebnissen (vgl. Tabelle 26 und Tabelle 27).

518 Vgl. BENDL 2000, S. 25

${ }^{519}$ Vgl. BENDL 2000, S. 50 
Tabelle 26: Verteilung nach Branchen

\begin{tabular}{|l|c|c|c|c|c|}
\hline Branche & $\begin{array}{c}\text { Anzahl der } \\
\text { Nennungen }\end{array}$ & $\begin{array}{c}\text { Durchschnitt- } \\
\text { liche Kondi- } \\
\text { tionenhöhe }\end{array}$ & $\begin{array}{c}\text { Häufigste } \\
\text { Bandbreite }\end{array}$ & $\begin{array}{c}\text { Niedrigste } \\
\text { Bandbreite }\end{array}$ & $\begin{array}{c}\text { Höchste } \\
\text { Bandbreite }\end{array}$ \\
\hline Fleischverarbeitung & 11 & $7,05 \%$ & $5-10 \%$ & $0-5 \%$ & $10-15 \%$ \\
\hline $\begin{array}{l}\text { Herstellung und Verarbei- } \\
\text { tung von Ölen und Fetten }\end{array}$ & 3 & $22,5 \%$ & $20-30 \%$ & $15-20 \%$ & $20-30 \%$ \\
\hline Verarbeitung von Milch & 3 & $5,83 \%$ & $5-10 \%$ & $0-5 \%$ & $5-10 \%$ \\
\hline Sonstige Ernährung & 25 & $16,9 \%$ & $5-10 \%$ & $0-5 \%$ & $>40 \%$ \\
\hline $\begin{array}{l}\text { Herstellung von Geträn- } \\
\text { ken }\end{array}$ & 29 & $12,59 \%$ & $5-10 \%$ & $0-5 \%$ & $30-40 \%$ \\
\hline
\end{tabular}

Quelle: BENDL 2000, S. 50

Tabelle 27: Verteilung nach Umsatzkonzentration und horizontaler Marktposition

\begin{tabular}{|l|c|c|c|}
\hline $\begin{array}{l}\text { 80\% der Herstellerumsätze } \\
\text { werden erzielt mit... }\end{array}$ & $\begin{array}{l}\text { Anzahl der Nenn- } \\
\text { ungen }\end{array}$ & $\begin{array}{l}\text { Durchschnittliche } \\
\text { Konditionenhöhe }\end{array}$ & $\begin{array}{l}\text { Häufigste Bandbrei- } \\
\text { te }\end{array}$ \\
\hline 10 oder weniger Kunden & 35 & $18,5 \%$ & $20-30 \%$ \\
\hline 20 oder weniger Kunden & 26 & $13,85 \%$ & $5-10 \%$ \\
\hline mehr als 20 Kunden & 28 & $10,18 \%$ & $5-10 \%$ \\
\hline Marktposition der Hersteller & \multicolumn{2}{|c|}{} \\
\hline Marktführend (Nr. 1 oder 2) & 22 & $20,11 \%$ & $20-30 \%$ \\
\hline Marktbestimmend & 28 & $15,09 \%$ & $5-10 \%$ \\
\hline Nicht marktbestimmend & 38 & $11,05 \%$ & $5-10 \%$ \\
\hline
\end{tabular}

Quelle: BendL 2000, S. 50

BENDL zog aus seinen Untersuchungen folgenden Schluss: Obwohl von Industrie und Handel der Wunsch nach fairen und leistungsbezogenen Konditionssystemen vorgetragen wird, sind die Konditionsverhandlungen in der Praxis nach wie vor durch eine opportunistische Verhaltensweise der Verhandlungsparteien geprägt, die vorrangig an der Höhe der gewährten Konditionen ausgerichtet ist. Die Hersteller versuchen, für eine Handlungsleistung möglichst geringe Konditionen zu vergüten bzw. für eine Kondition eine maximale Gegenleistung zu erhalten, wohingegen die Handelsunternehmen bestrebt sind, immer mehr Konditionen bei möglichst geringer Gegenleistung zu vereinnahmen. Als Bestimmungsfaktor der Konditionenhöhe wird vor allem die Machtposition des Handels gegenüber den Herstellern angeführt und nicht die betriebswirtschaftlich begründete und an der optimalen Gestaltung der Wertschöpfung ausgerichtete Festlegung der Vergütungshöhen und -staffeln ${ }^{520}$.

Die verschiedenen Konditionsforderungen führen generell bei einem Vergleich verschiedener Leistungen zu einer mangelnden Transparenz. Hinzu kommt, dass es sich

${ }^{520}$ Vgl. BENDL 2000, S. 149 
um sehr "sensible" Daten handelt und somit der Zugriff erschwert ist ${ }^{521}$. Trotz Bestehen eines sogenannten "Geheimwettbewerbs" kam HERMES zu dem Fazit, dass Konditionen des Handels zumindest mittelfristig innerhalb der jeweiligen Branche bekannt werden $^{522}$. HERMES vertrat die These, dass somit von Verkaufspreisen auf Einkaufspreise in der Regel rückgeschlossen werden kann ${ }^{523}$.

Marktanteile als alleiniger Indikator zur Beurteilung der Machtverhältnisse scheinen wenig aussagefähig. Als eines der Hauptprobleme beim Vergleich der Marktanteile ist die Abgrenzung des relevanten Marktes zu nennen. Der Marktanteil sagt keineswegs etwas über das Abhängigkeitsverhältnis zwischen Hersteller und Handel aus, denn die Ausweichmöglichkeiten beider Seiten bleiben unberücksichtigt ${ }^{524}$. Ebenso bleiben dynamische Effekte, räumlich unterschiedliche Zugänglichkeiten sowie potentieller Wettbewerb unberücksichtigt, und es kann daher bei isolierter Betrachtung des aktuellen Marktanteils zu Fehlschlüssen kommen.

Nicht der Marktanteil, sondern vielmehr der Bezugsanteil kann dagegen als ein geeigneter Indikator zur Beschreibung der geschilderten Relationen dienen ${ }^{525}$. Ein hoher Bezugsanteil von einem Hersteller ermöglicht einer Handelsorganisation die Ausübung von Druck auf Preise und Konditionen. Je höher der Bezugsanteil eines Abnehmers ist, desto schwieriger und kostenintensiver wird es für einen Hersteller, Absatzmengen, die bei einem Abbruch der Geschäftsbeziehungen verloren gehen, bei anderen Abnehmern auszugleichen ${ }^{526}$. Der in der Regel steigende Bezugsanteil der Abnehmer von Handelsmarken und die mit der Produktion von Handelsmarken verbundenen spezifischen Transaktionskosten erhöhen somit die Abhängigkeit der Hersteller von einzelnen Abnehmern ${ }^{527}$.

GRACIA und ALBISu ${ }^{\mathbf{5 2 8}}$ versuchten anhand des Anteils von Handelsmarken (vgl. zur Bedeutung in Europa Abbildung 34) bzw. Herstellermarken am Gesamtsortiment des Handelsunternehmens, die Marktmachtbeziehungen abzuleiten.

\footnotetext{
${ }^{521}$ Vgl. BENDL 2000, S. 194 erklärt damit die niedrige Rücklaufquote seiner Befragung.

522 Vgl. HeRMES 1988, S. 11

${ }^{523} \mathrm{Vgl}$. HERMES 1988, S. 12

524 Vgl. LADEMANN 1986, S. 80

${ }^{525}$ Vgl. BendL 2000, S. 192

${ }^{526}$ Vgl. BÖGER 1990, S. 130

527 Vgl. SCHULZE 1998, S. 56

${ }^{528}$ Gracia/Albisu 2001, S. 467 ff.
} 
Abbildung 34: Bedeutung von Handelsmarken in Europa 1999

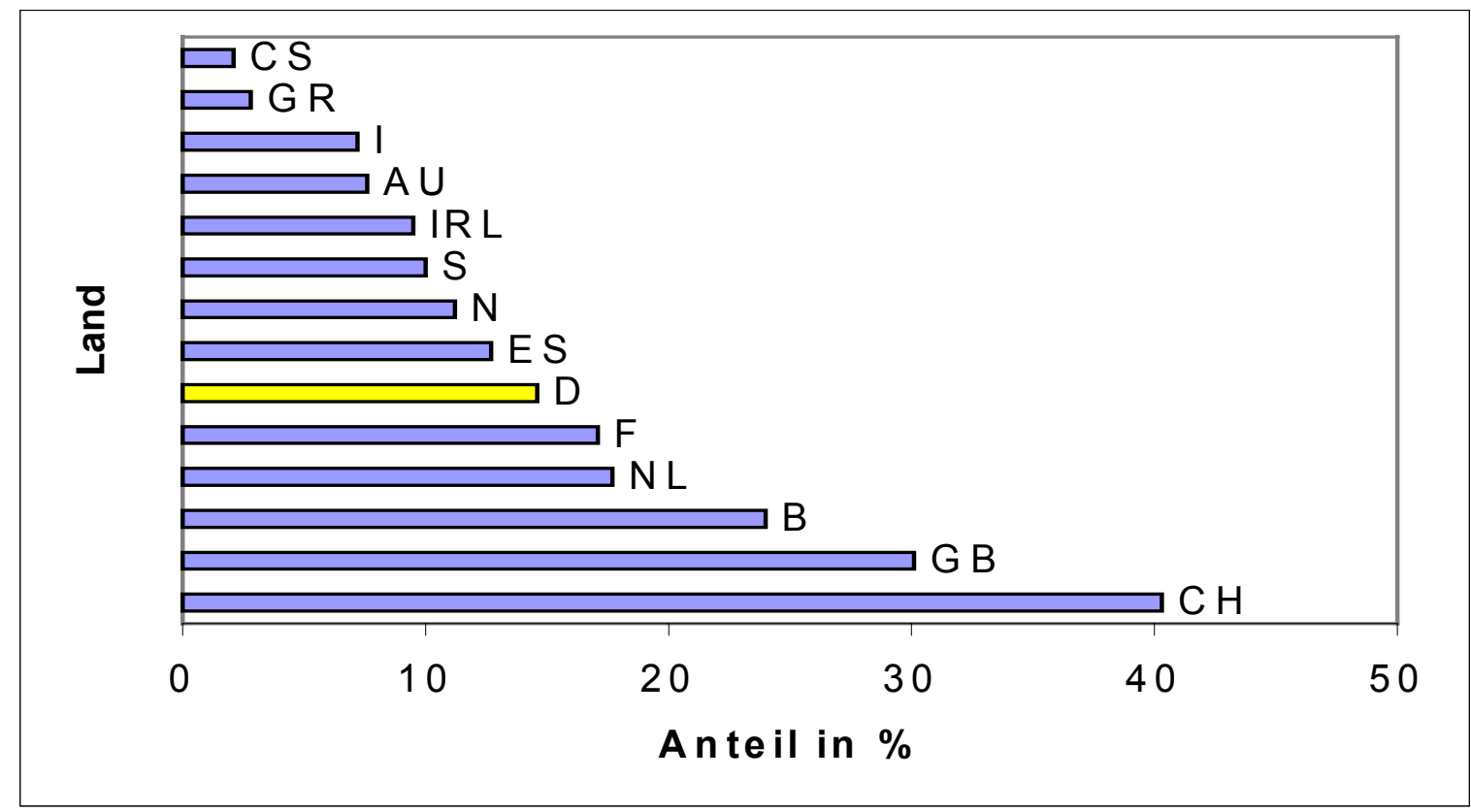

Quelle: AC NIELSEN 2001

Da sich auch im Zeitverlauf eine Verschiebung zugunsten der Handelsmarken abzeichnet, folgern die Autoren, dass sich Marktmacht tendenziell von den Herstellern zu den Handelsunternehmen verlagert ${ }^{529}$. Die Kritik an diesem Ansatz ergibt sich zum Teil bereits aus den vorigen Ausführungen dieses Kapitels (vgl. Kapitel 7.2.4.2). Ein wesentlicher Aspekt besteht darin, dass der Handel ein hohes Maß an Beschaffungssicherheit sowie kontinuierliche Sortimentsstrukturen zu gewährleisten versucht, um allein aus Gründen der Imagepflege und der ausgeprägten Markenbindung der Konsumenten, eine Einkaufsstättentreue zu erreichen. Somit sind Produkte bestimmter Hersteller nicht ohne weiteres durch Produkte anderer Hersteller bzw. andere Produktgruppen zu ersetzen. Außerdem wird die Produktion von Handelsmarken von vielen Herstellern als eine Chance zur Auslastung ihrer Produktionskapazitäten und zur Schaffung neuer Absatzpotentiale genutzt.

Vielversprechender erscheint der Ansatz LADEMANNs zu sein, in welchem er die Fluktuation von Herstellern näher untersucht ${ }^{530}$. Leider fehlt es hier noch an detaillierten Kenntnissen, so dass der durchschnittliche Wert von ca. 2-3\% beim Austausch der Lieferbeziehungen für spezifische Produkte nur geringe Aussagekraft besitzt.

Weiterer Betrachtung bedarf es bei der Gestaltung der Absatzkanäle. Zwar nimmt unbestritten der klassische Lebensmitteleinzelhandel eine dominierende Form beim Dist-

\footnotetext{
${ }^{529}$ Vgl. Gracia/Albisu 2001, S. 482; Aiginger/Wieser/Wüger 1999, S. 81 ff.
}

${ }^{530}$ Vgl. LADEMANN 1996, S. 32; LADEMANN 2001, S. 44 
ributionsweg ein, dennoch gibt es für spezifische Produkte alternative Absatzmöglichkeiten. Für jedes einzelne Produkt müssen also individuelle Ausweichmöglichkeiten und deren Wechselkosten und Potentiale ermittelt werden, damit sich ein Abbild des möglichen Machtspielraums eines Abnehmers machen lässt. Für Lebensmittel können mehrere Vertriebsformen miteinander konkurrieren (Abbildung 35).

\section{Abbildung 35: Vertriebsform von Lebensmitteln}

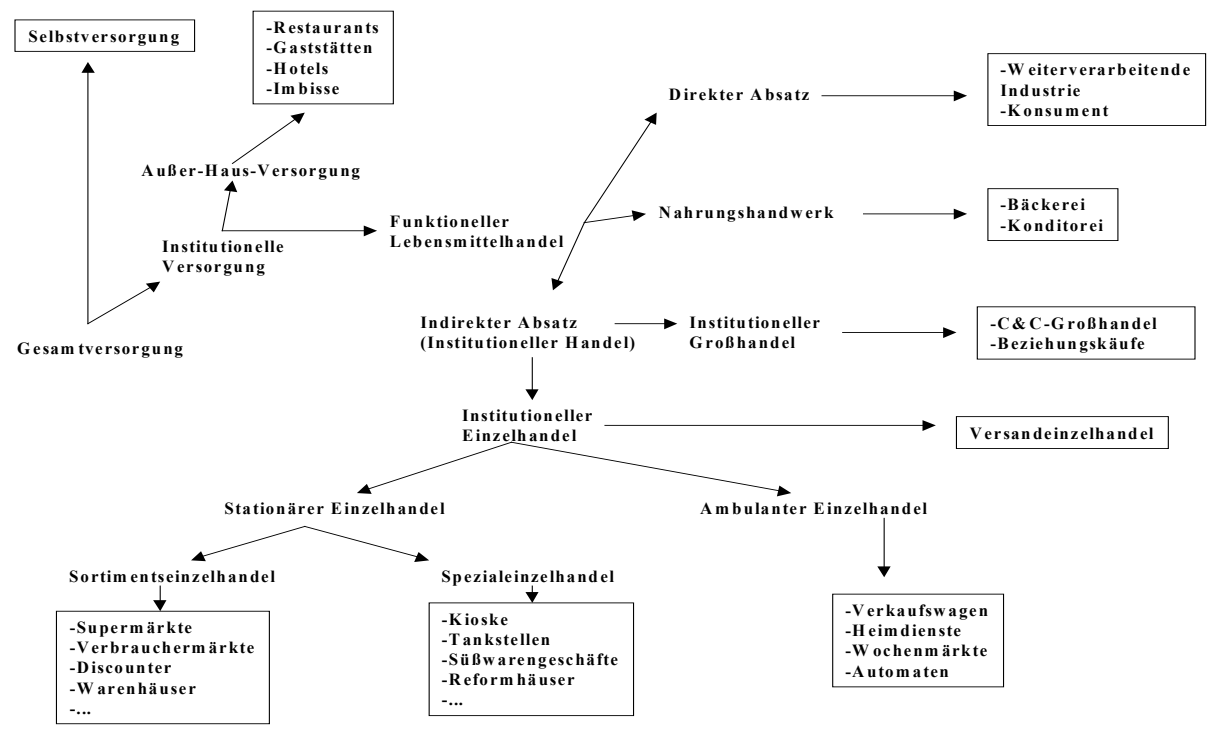

Quelle: LAdemann 1996, S. 194

Das Machtpotential der nachgelagerten Stufe wird demnach nicht nur von den Markteintritts- und -austrittskosten innerhalb einer Vertriebsform sondern auch durch die (potentiellen) Wechselkosten unterschiedlicher Absatzkanäle bestimmt.

\subsubsection{Wettbewerbspolitische Praxis}

Auf Nachfragemacht kann nicht allein aufgrund der horizontalen Konzentration eines Wirtschaftszweiges geschlossen werden, vielmehr sollte neben der horizontalen auch die vertikale Verflechtung bei der Beurteilung zugrundegelegt werden. Machtausübung von Handelsunternehmen kann einerseits zu einer allgemeinen und dauerhaften Renditeminderung des jeweils betroffenen Herstellers führen. Zum anderen führt Handelsmacht dazu, das weniger nachfragemächtige Handelsunternehmen gegenüber ihren Wettbewerbern im Handel Nachteile, welche sie mittel- bis langfristig zum Ausscheiden bzw. zur Aufgabe der Selbständigkeit zwingen, erleiden. Dies führt zu einer 
beschleunigten Konzentration auf der Handelsstufe ${ }^{531}$. Daher schlagen einige Autoren ein Konzept der wechselseitigen Austauschbeziehungen vor ${ }^{532}$. Inwieweit die Argumente und Auswirkungen in der Beurteilungspraxis berücksichtigt werden, wird in den nächsten Abschnitten vertieft (ergänzend zu Kapitel 6.2).

\subsubsection{Die Regelung im GWB}

Marktbeherrschung kann gemäß GWB § 19 Abs. 2 Satz 1 Nr. 2 durch gegengewichtige Marktmacht relativiert werden. Voraussetzung ist allerdings, dass ein marktstarker Nachfrager seine Aufträge nach marktstrategischen Überlegungen verteilt, um Abhängigkeiten von einem marktbeherrschenden Zulieferer zu reduzieren ${ }^{533}$. Wettbewerbspolitischer Maßstab ist der strenge Marktmachtbezug. Angebots- bzw. Nachfragemacht muss stets für einen ganz bestimmten Markt nachgewiesen werden. Die dazu erforderliche Abgrenzung des relevanten Marktes erfolgt dabei auf Grundlage des Bedarfsmarktkonzepts (vgl. Kapitel 7.1.6). Es ist jeweils auf die Ausweichmöglichkeit der Marktgegenseite abzustellen. Danach verfügt ein Marktpartner über Nachfragemacht, wenn die Möglichkeiten der Anbieterseite, auf andere Nachfrager auszuweichen, beschränkt sind.

Dem Argument, dass besonders kleinere und mittlere Unternehmen ${ }^{534}$ der fortschreitenden Konzentration des Handels unterlegen sind, versucht der Gesetzgeber mit einer besonderen Regelung Folge zu leisten. So wird marktmächtigen Unternehmen und Unternehmenskooperationen nach § 20 Abs. 2 und 3 GWB untersagt, gegenüber ihren abhängigen Lieferanten, denen auf einem bestimmten Markt ausreichende und zumutbare Abnehmer fehlen, Vorzugsbedingungen zu erhandel ${ }^{535}$. Zumindest theoretisch versucht der Gesetzgeber, mit dieser Regelung das Nachfragemachtpotential gegenüber kleineren Herstellern zu begrenzen. In der Unternehmerbefragung dieser Untersuchung wird allerdings die praktische Wirksamkeit der Regelung bestritten.

\subsubsection{Die Berücksichtigung von Nachfragemacht auf europäischer Ebene}

Bzgl. der Berücksichtigung von Nachfragemacht gibt der europäische Gesetzgeber folgenden Rechtsrahmen vor (Art. 2 Abs. 1 b FKVO): Bei der Prüfung berücksichtigt die Kommmission die Marktstellung, wirtschaftliche Macht und die Finanzkraft der beteilig-

\footnotetext{
${ }^{531} \mathrm{Vgl.}$ APPEL 1992, S. 152

532 Vgl. HeRmes 1988, S. 145; Romeiser 1998, S. 275 ff., LADEMANn 2000 S. 71 ff.

${ }^{533} \mathrm{Vgl.}$ BUNDESKARTELLAMT 2001, S. 22

${ }^{534}$ definiert in HGB § 267 (in D: ca. 25 Mio. €; EU ca. 40 Mio €)

${ }^{535} \mathrm{Vgl}$. dazu auch LADEMANN 2001, S. $44 \mathrm{f}$
} 
ten Unternehmen, die Wahlmöglichkeiten der Lieferanten und Abnehmer, samt ihrem Zugang zu den Absatz- und Beschaffungsmärkten, rechtliche oder tatsächliche Marktzutrittsschranken, die Entwicklung des Angebotes und der Nachfrage bei den jeweiligen Erzeugnissen und Dienstleistungen, die Interessen der Zwischen- und Endverbraucher sowie die Entwicklung des technischen und wirtschaftlichen Fortschritts, sofern diese dem Verbraucher dient und den Wettbewerb nicht behindert.

Es wird somit explizit auf die Interessen der Zwischen- und Endverbraucher eingegangen. Für den Nachweis einer beherrschenden Stellung eines Nachfragers nutzt die Kommission bzw. der EuGH eine Vielzahl von Instrumenten. Im Fall "United Brands"536 wurde beispielsweise ein Preis-Kosten-Vergleich angewandt, um daraus die Gewinnmargen zu ermitteln. Ein anderes Instrument stellt das Vergleichsmarktkonzept dar, wonach der reale Preis einem fiktiven Preis gegenübergestellt wird und entsprechende "Spielräume" ermittelt werden können ${ }^{537}$. Bei der sogenannten Sockeltheorie (beforeafter-Ansatz) wird der Preis des beherrschenden Unternehmens mit einem früheren, von demselben Unternehmen auf demselben Markt geforderten Preis verglichen ${ }^{538}$. Auch ökonometrische Verfahren wurden bei einzelnen Fällen zur Ermittlung von Marktmacht angewendet ${ }^{539}$. Bei dem in dieser Studie vorgestellten Fall Friesland/Coberco wurde die Nachfragemacht explizit berücksichtigt und führte u.a. dazu, die wettbewerblichen Bedenken auszuräumen.

\subsubsection{Vorgehensweise des Bundeskartellamtes}

Die Mehrzahl der interviewten Unternehmensvertreter kritisierten an der Vorgehensweise des Bundeskartellamtes, dass das Hauptmotiv der Fusion, eine Gegenmacht gegenüber dem marktmächtigen Handelsunternehmen zu erschaffen, nur unzureichend berücksichtigt wurde.

Der Kritik aus Wirtschaft und von den zuvor angeführten Autoren kann aufgrund der Recherchen über die praktische Vorgehensweise des Bundeskartellamtes zur Beurteilung von Unternehmenszusammenschlüssen nur zum Teil zugestimmt werden. Zwar wurde die Frage nach der Berücksichtung des Nachfragemachtargumentes von den zuständigen Mitarbeitern der Beschlussabteilung eindeutig verneint, allerdings stellte sich jedoch aufgrund der Akteneinsichten heraus, dass durchaus die Relationen zwi-

\footnotetext{
${ }^{536}$ Vgl. EuGH vom 14.02.1978, Slg. 1978, S. 305

537 Vgl. SCHULZE 1998, S. 234 und die dort zitierte Literatur

538 Vgl. SCHWARz 1987, S. 98; Anwendung z.B. im Fall „British Leyland": EuGH vom 11.11.1986, Slg. 1986, S. 3303

539 Vgl. Aiginger/WieSER/WüGER 1999, S. 187
} 
schen Hersteller und Handel näher untersucht wurden. Zumindest wurden die größten Abnehmer der fusionswilligen Unternehmen befragt, wie sie die Strukturveränderungen beurteilen würden, welche Konsequenzen sich daraus für deren Unternehmen ergäben und welche Alternativen nach einer Fusion der Hersteller in Betracht kämen. Es wurden keineswegs nur Gesamtmarktanteile ermittelt und gegenübergestellt, sondern durchaus nach Abnehmern und räumlicher Nähe differenziert, um möglichen Folgewirkungen Rechnung zu tragen. Ebenso fanden die Meinungen der jeweils größten Mitbewerber Berücksichtigung. Zugestimmt werden kann der Kritik insofern, dass nur die unmittelbaren und vor allem aktuellen Marktpartner einbezogen worden sind. Dynamische Effekte wurden somit in der Nachfragemachtdiskussion nur zum Teil erfasst. AuBerdem fanden die Verschiebungen innerhalb des Absatzkanals keine Beachtung. Da das Bundeskartellamt personell wie auch zeitlich gewissen Restriktionen unterliegt, soll bereits hier die Empfehlung ausgesprochen werden, auf Expertenwissen zurückzugreifen, um zumindest sehr spezifischen und komplexen Konstellationen gerecht zu werden. Wohlfahrtsökonomisch ist zudem nicht nur die Wirkung innerhalb der Handels-Hersteller-Relationen zu untersuchen, sondern es ist ergänzend die Verbraucherebene heranzuziehen, damit eine gesamtwirtschaftliche Folgeabschätzung durchgeführt werden kann.

In der Gesamtbeurteilung kamen die Entscheidungsträger der Kartellbehörden in den untersuchten Fällen zu dem Ergebnis, dass keine besondere Gefahr aus der zunehmenden Konzentration des Lebensmitteleinzelhandels hervorgeht ${ }^{540}$ und daher der Gegenmachtargumentation der Unternehmen nicht Folge geleistet wird.

\subsubsection{Beurteilung der Lebensmitteleinzelhandelkonzentration durch die Monopol- kommission}

Die MonOPOLKOMmission schließt aufgrund ihres 23. Sondergutachtens von 1994 darauf, dass die Marktstrukturentwicklungen trotz der erkennbaren Verlagerung des Wettbewerbsgeschehens auf die großen Filialunternehmen und Kooperationen des Einzelhandels nicht auf ein Nachlassen der Wettbewerbsintensität hindeuten würden ${ }^{541}$. Versuche, die Position auf den Handelsmärkten durch Unternehmensübernahmen zu stärken, um dadurch verbesserte Einkaufskonditionen zu erzielen, würden regelmäßig und vergleichsweise schnell durch Reaktionen der Wettbewerber ausgeglichen. Durch die seit Mitte der 80er Jahre erfolgten Unternehmenszusammenschlüsse, die zunehmende Konzernierung der Verbundgruppen und die Konzentrationsvorgänge bei den

\footnotetext{
$540 \mathrm{Vgl}$. auch hierzu Ausführungen: BundeSKARTELLAMT 2001, S. IV und $143 \mathrm{f}$.

${ }^{541} \mathrm{Vgl.} \mathrm{MONOPOLKOMMISSION} \mathrm{1994,} \mathrm{S.} 19$
} 
Einkaufskooperationen sei die Zahl der unabhängig voneinander einkaufenden Abnehmer aus Sicht der Industrie zwar zurückgegangen, marktbeherrschende Nachfragestellungen seien dabei jedoch noch nicht entstanden ${ }^{542}$. Eine dauerhafte Gewinnverschiebung von der Industrie auf die Handelsunternehmen wäre ein Anhaltspunkt für Veränderungen in der Machtbalance zugunsten der Nachfrager. Dies konnte empirisch nicht nachgewiesen werden ${ }^{543}$. Da allerdings die Machtbalance zwischen Industrie und Handel stärker als zuvor von der jeweiligen Leistungsstärke der Marktgegenseite bestimmt wird, spricht vieles dafür, dass die Konzentrationsentwicklung im Handel auch die Konzentration in der Industrie vorantreiben würde ${ }^{544}$.

Auch die Vorgehensweise des Kartellamtes wird von der Monopolkommission gestützt. So könne zwar der Verzicht auf eine detailliertere Analyse der Nachfrageseite Bedenken auslösen, dennoch könnten Fortentwicklungsmöglichkeiten des Nachfragekonzeptes mehr "Schaden als Nutzen" hervorbringen ${ }^{545}$.

\subsubsection{Zwischenergebnis}

Die Ursachen für Machtgefälle zwischen Industrie und Handel verbergen sich in groBem Maße hinter Wettbewerbsvorteilen einzelner Marktteilnehmer im Horizontalprozess, die zu unterschiedlichen Gratifikationspotentialen im Vertikalprozess führen. Ob ein Handelsunternehmen über Nachfragemacht verfügt, lässt sich deshalb nicht bereits aus der Unternehmensgröße ermitteln. Vielmehr spielen wechselseitige Relationen der auf beiden Seiten eingesetzten Wettbewerbsparameter eine entscheidende Rolle. Diese sind weder aus Marktanteilen eines Nachfragers noch mit Hilfe von Umsatzrenditen feststellbar. In diesem Zusammenhang konnte gezeigt werden, dass Handelsunternehmen sowohl Nachfragemacht besitzen, als auch von Angebotsmacht betroffen sein können. Ohne eine umfassende bilaterale Analyse kann also keine generelle Aussage zur Handel-Hersteller-Relation getroffen werden. In diese Richtung deutet auch die Empfehlung bei der Beurteilung von Nachfragemacht bei Unternehmenszusammenschlüssen: Nachfragemacht sollte grundsätzlich in die Beurteilungspraxis mit einbezogen werden. Für die jeweils betroffenen Unternehmen und Produkte müssen individuelle Relationen auf der Absatzseite ermittelt werden, um die Machtverhältnisse beurteilen zu können. Eine Auflistung der allgemeinen Marktanteile des Handels ist somit

\footnotetext{
542 Vgl. MONOPOLKOMMISSION 1994, S. 21

${ }^{543} \mathrm{Vgl}$. MONOPOLKOMMISSION 1994, S. 21; S. $74 \mathrm{f}$.

${ }^{544} \mathrm{Vgl.} \mathrm{MONOPOLKOMMISSION} \mathrm{1994,} \mathrm{S.} 21$
} 
nur begrenzt aussagefähig. Mögliche Indikatoren wurden diskutiert (z.B. Bezugsanteile, Wechselkosten, Absatzalternativen). Unter Zuhilfenahme moderner Marktforschungs- und Preisbeobachtungstechniken kann die Preispolitik einzelner Unternehmen sowohl auf Handels- als auch auf Herstellerebene ermittelt werden. Daraus können ergänzend Schlussfolgerungen über potentiellen Wettbewerb und dynamische Veränderungen hergeleitet werden. Angemerkt sei an dieser Stelle auch, dass der Kritik angeführter Autoren nicht ausnahmslos zugesprochen wird. So kann beispielsweise der wiederkehrenden Behauptung, die Beurteilungspraxis orientiere sich lediglich an allgemeinen Strukturkriterien, nicht zugestimmt werden.

\subsection{Agrarpolitische Rahmenbedingungen}

Der Fragebogen bzw. die Interviews bei den betroffenen Unternehmen enthüllten z.T. die Kritik, dass agrarpolitische Rahmenbedingungen kaum bei der Beurteilungspraxis des Ernährungssektors berücksichtigt worden sind. Die Praxisfälle im Bereich der Zuckerfusionen können diese Einschätzung jedoch nicht bestätigen. Dennoch entsteht vereinzelt der Eindruck, dass nur eine geringe Kenntnis über die agrarpolitischen Reglungen und deren ökonomischen Wirkungen bei Entscheidungsträgern vorhanden ist. Um dieses Defizit bei einer Beurteilung zu vermeiden, wird ein Überblick über die Regelungen im Milch- und Zuckersektor vermittelt.

\subsubsection{Milch und Milchprodukte}

Milch und Milcherzeugnisse sind mit einem Anteil von ca. $18 \%$ an den gesamten Verkaufserlösen landwirtschaftlicher Produkte ein wichtiger Markt für die europäische Agrarwirtschaft ${ }^{546}$. Auch aus Sicht der Konsumenten sind Milch und Molkereiprodukte ein bedeutendes Sortiment, denn ca. 20\% der Konsumausgaben für Nahrungsmittel werden im Bereich dieser Produktgruppe aufgewendet ${ }^{547}$. Der Milchmarkt stellt damit einen Markt von agrarpolitischer Bedeutung dar. Die europäische Milchmarktpolitik stützt sich auf zwei gesetzliche Regelungen (Milchmarktordnung und Garantiemengenverordnung), die im folgenden näher erläutert werden.

\footnotetext{
${ }^{545} \mathrm{Vgl.} \mathrm{MONOPOLKOMMISSION} \mathrm{1994,} \mathrm{S.} 22$

${ }^{546}$ Vgl. EU-Kommission 1999: Fact Sheet, S. 1

547 Vgl. OSMERS 2001, S. 632
} 


\subsubsection{EG-Marktordnung für Milch und Milcherzeugnisse}

Die Markt- und Preispolitik für Milch- und Molkereiprodukte im Gemeinsamen Markt ist durch die EG-Marktorganisation für Milch und Milcherzeugnisse geregelt ${ }^{548}$. Um eine Einkommenssicherung für die Milcherzeuger zu gewährleisten, wurden staatliche Richtpreise eingeführt. Daraus resultierten seit Anfang der 70er Jahre ständig steigende Überschüsse, die sich in einem zunehmenden Finanzbedarf äußerten und in den 80 er Jahren zur Reform der Milchmarktordnung ${ }^{549}$ führten. Die Reform umfasst im wesentlichen zwei Eckpfeiler: die Binnenmarktregelung sowie die Außenhandelsregelung. Im Zuge der gemeinsamen Agrarpolitik wurde die Verordnung erneut reformiert und an die Beschlüsse der AGENDA 2000 angepasst ${ }^{550}$. National regelt seit dem 12.01.2000 die sogenannte Zusatzabgabenverordnung das Garantiesystem und hat somit die vorangegangene Michgarantiemengenverordnung (MGV) abgelöst.

\subsection{Binnenmarktregelung}

Die Binnenmarktregelung zur Stützung des inländischen Preises besteht aus einem staatlichen Ankauf sowie einem differenzierten Beihilfesystem für den Absatz von Butter, Magermilch und einigen Käsesorten ${ }^{551}$. Zusätzlich werden Beihilfen für Lagerkosten sowie bestimmte Sonderprogramme ${ }^{552}$ geleistet, die saisonale Schwankungen ${ }^{553}$ der Produktion und des Verkaufs glätten sollen.

Die gemeinsame Marktorganisation für Milch und Milcherzeugnisse sieht auch nach der Einführung der Agenda 2000 Reform die Festlegung eines Richtpreises ${ }^{554}$ für Milch und die Beibehaltung von Interventionspreisen für Butter und Magermilchpulver vor. Die jährlich vom Rat der EU vor dem 1. August festgesetzten Preise für Milch und Milcherzeugnisse besitzen für ein Milchwirtschaftsjahr Geltung ${ }^{555}$.

\footnotetext{
548 Ursprünglich in der EG-Verordnung 804/68 vom 27. Juni 1968

549 Einführung der sogenannten Milchgarantiemengenregelung

${ }^{550}$ EG-Verordnung 1255/1999 sowie EG-Verordnung 1256/1999

${ }^{551}$ Speziell Grano Padano, Parmigiano Regiano, Provolone (geregelt in EU-VO 1255/ 1999 Artikel 8)

552 Verbilligte Preise für z.B. gemeinnützige Einrichtungen, Schulen, ... vgl. EU-VO 1255/ 1999 Artikel 13

${ }^{553}$ Im Frühjahr meist Produktionsüberhang, im Herbst/Winter dagegen tendenziell Nachfrageüberhang

${ }^{554}$ Der Richtpreis ist der Milchpreis, der für die im Milchwirtschaftsjahr von den Erzeugern insgesamt verkaufte Milch angestrebt wird. Bei seiner Festsetzung sollen die Absatzmöglichkeiten auf dem Binnenmarkt sowie auf Märkten außerhalb der Gemeinschaft berücksichtigt werden. Der Richtpreis bezieht sich auf Milch mit einem Fettgehalt von 3,7 \%, frei Molkerei. Vgl. EU-VO 1255/ 1999 Artikel 3

555 Das Milchwirtschaftsjahr beginnt am 1. Juli und endet am 30. Juni des Folgejahres; das Garantiemengenjahr (Zwölfmonatszeitraum) dagegen beginnt am 1. April und endet am 31. März des Folgejahres.
} 
Neben dem Richtpreis setzt der Ministerrat jährlich Interventionspreise für Butter und Magermilchpulver (MMP) fest. Die Interventionspreise für Butter und MMP sind Preise, zu denen die staatlichen Interventionsstellen die o.a. Erzeugnisse ankaufen. Seit 1987 erfolgen die Ankäufe der Interventionsstelle zum Ankaufspreis. Dieser wird von der EU-Kommission im Ausschreibeverfahren festgelegt ${ }^{556}$.

Der Richtpreis für Milch und der Interventionspreis für Butter sind seit dem Milchwirtschaftsjahr 1995/96 unverändert geblieben. Zur Verbesserung der Absatzmöglichkeiten werden die Preise ab dem Wirtschaftsjahr 2005/06 bis zum Jahr 2007/08 in drei Schritten von je $5 \%$ um insgesamt $15 \%$ abgesenkt ${ }^{557}$ (vgl. dazu Tabelle 28 ).

Tabelle 28: Entwicklung wichtiger Milchpreise

\begin{tabular}{|l|c|c|c|c|}
\hline Milch- und Milcherzeugnisse: EU Interventions- und Richtpreis je 100 kg in C \\
\hline Wirtschaftsjahr & $\mathbf{2 0 0 0 - 2 0 0 5}$ & $\mathbf{2 0 0 5 - 2 0 0 6}$ & $\mathbf{2 0 0 6 - 2 0 0 7}$ & $\mathbf{2 0 0 7 - 2 0 0 8}$ \\
\hline Butter: Interventionspreis & 328,2 & 311,79 & 295,38 & 278,97 \\
\hline $\begin{array}{l}\text { Magermilchpulver: Interven- } \\
\text { tionspreis }\end{array}$ & 205,52 & 195,24 & 184,97 & 174,69 \\
\hline $\begin{array}{l}\text { Milch (Fettgehalt 3,7\%, frei } \\
\text { Molkerei): Richtpreis }\end{array}$ & 30,98 & 29,23 & 27,47 & 25,72 \\
\hline
\end{tabular}

Quelle: EUROPÄISCHE-KommisSION, Fact Sheet 1999, S. 2

Im Zuge der Agenda 2000 wird die Preisstützung zwar abgebaut, jedoch wird die Intervention auch weiterhin beibehalten. Der Ankauf erfolgt unter der Bedingung, dass die Erzeugnisse in einem EU-Land hergestellt worden sind und die festgelegten Qualitätsanforderungen erfüllen.

Bei Butter erfüllt die Intervention künftig nur noch die Funktion eines Sicherheitsnet$z^{2} s^{558}$. Fallen die Buttermarktpreise in einem oder mehreren Mitgliedsstaaten während eines repräsentativen Zeitraums unter $92 \%$ des Interventionspreises, so nehmen die Interventionsstellen der betroffenen Mitgliedstaaten Interventionskäufe vor. Der von der Kommission festgesetzte Ankaufspreis darf jedoch nicht unter 90\% des Interventionspreises liegen. Die Intervention soll so angewendet werden, dass Wettbewerbsfähigkeit und ursprüngliche Qualität der Butter erhalten bleiben und eine möglichst rationelle Lagerhaltung gewährleistet ist.

Bei Magermilchpulver wird nur in der Periode vom 1. März bis 31. August jeden Jahres interveniert $^{559}$. Der Ankaufspreis entspricht bis zu einer Menge von 109.000 t pro Jahr dem Interventionspreis frei Interventionslagerhaus. Das Überschreiten der Mengen-

\footnotetext{
${ }^{556}$ Vgl. EU-VO 1255/1999 Artikel 6

557 Vgl. EU-VO 1255/1999 Artikel 3

${ }^{558}$ Vgl. EU-VO 1255/1999 Artikel 6

${ }^{559}$ Vgl. EG-VO 1255/1999 Artikel 4 b
} 
schwelle bei der Andienung von MMP führt zu Preissenkungen (Ausschreibungsverfahren) bzw. zum Aussetzen der Intervention ${ }^{560}$.

Der Absatz der intervenierten Erzeugnisse soll ohne Störungen des Marktgleichgewichts erfolgen. Dabei ist allen Käufern gleicher Zugang zu den zu verkaufenden Erzeugnissen sowie gleiche Behandlung zu garantieren. Der Absatz der Interventionsbutter erfolgt zu einem Mindestpreis. Für Interventionserzeugnisse, die innerhalb eines Milchwirtschaftsjahres keinen Absatz finden, können besondere Maßnahmen ergriffen werden, z.B. die verbilligte Abgabe an bestimmte Käufer (Sozialhilfeempfänger, Streitkräfte) sowie der Export zu verbilligten Preisen ${ }^{561}$.

\section{Beihilfen}

Seit 1987 können also die Interventionskäufe bei Vorliegen bestimmter Bedingungen zeitweise ausgesetzt bzw. bei Butter durch ein Ausschreibungsverfahren ersetzt werden. Bei Aussetzung der Intervention ist eine Marktentlastung durch die Zahlung von Beihilfen für die private Lagerhaltung vorgesehen. Die über die Interventionsstellen auf Lager genommene Butter wird (über Exporterstattung) auf den Weltmärkten verkauft oder im Binnenmarkt verbilligt abgesetzt. Um saisonale Marktschwankungen auszugleichen, kann die EG Beihilfen anstelle der Intervention für die private Lagerung von Butter, Magermilchpulver und bestimmte Käsesorten leisten. Die Beihilfe kommt privaten Lagerhaltern zu und ihre Höhe ist von den Lagerhaltungskosten und der voraussichtlichen Marktentwicklung abhängig. Außerdem werden zur Steigerung des Verbrauchs und zur Entlastung der Interventionen verschiedene Beihilfen für die Verfütterung von Magermilch und MMP gewährt. Auch der Bezug von Rahm, Butter oder Butterfett durch gemeinnützige Organisationen, Backwaren- oder Speiseeishersteller werden ebenso wie die Abgabe von Milch und Milcherzeugnissen an Schulen durch Gemeinschaftsbeihilfen gefördert.

${ }^{560}$ Vgl. EG-VO 1255/1999 Artikel 7 Abs. 2

${ }^{561}$ Vgl. EG-VO 1255/1999 Artikel 7 Abs. 4 


\subsection{Außenhandel}

\section{Bisherige Regelung}

Um ein Unterlaufen des Interventionspreises durch Einfuhren von Drittländern zu unterbinden, wurde bislang ein sogenannter Schwellenpreis festgelegt zu dem Drittländer ihre Produkte einführen konnten ${ }^{562}$. Dieser lag ca. $13 \%$ über dem Interventionspreis. Diese Spanne sollte die Transportkosten (Hafen zu Verbrauchsgebiet) und die Handelsspanne abdecken.

Bei der Ausfuhr von Butter und MMP konnten Exporteuren Erstattungen aus Haushaltsmitteln der EG gewährt werden, damit eine Konkurrenz der EG-Ware am Weltmarkt sichergestellt war. Die Erstattung deckte die Preisdifferenz zwischen dem EGMarktpreis und dem Weltmarktpreis (fob) ab.

\section{Aktuelle Regelung}

1995 traten die GATT-/WTO-I-Vereinbarungen in Kraft. Für die EU-Milchwirtschaft hatte die Umsetzung bis zum Jahr 2000 folgende Konsequenzen ${ }^{563}$ :

- Die Umwandlung der variablen Importabschöpfung in feste Zolläquivalente um $36 \%$ bei Butter und um $20 \%$ bei MMP,

- Durch den Mindestmarktzugang mussten 5\% des inländischen Verbrauchs für Importe zur Verfügung stehen. Dies bedeutete für die EU zusätzliche zollfreie Importquoten von 10.000 t Butter, 69.000 t MMP und 104.000 t Käse, entsprechend ca. 1,2 Mio. t Milch.

- Die subventionierten Ausfuhren waren um $21 \%$ zu reduzieren, die Ausfuhrerstattungen gleichzeitig um 36\% einzuschränken. Dies erhöhte das Milchangebot in der EU um rund 0,6 Mio. $t$.

\section{Einfuhr - und Ausfuhrlizenzen}

Für die Einfuhr von Milch und Milcherzeugnissen in die EU sind Einfuhrlizenzen erforderlich. Ausfuhren mit Erstattungen erfordern grundsätzlich eine Ausfuhrlizenz ${ }^{564}$. Die Lizenzen werden von den Mitgliedstaaten vergeben und sind jedem Antragsteller aus der EU zu erteilen. Für die Erteilung von Lizenzen ist die Stellung einer Kaution erforderlich.

\footnotetext{
562 Vgl. GLOY 1995, S. 20 ff.

${ }^{563}$ Vgl. LLM SCHWÄBISCH GMÜND 2002a, S. 108

${ }^{564}$ Vgl. EG-VO 1255/1999 Art. 26
} 


\section{Zölle/Zollkontingente/Mindestmarktzugang}

Grundsätzlich finden die Zölle aus dem gemeinsamen Zolltarif auf Milch und Milchprodukte Anwendung ${ }^{565}$. Zusätzlich können Zölle zwecks Vermeidung von Nachteilen für den Gemeinschaftsmarkt erhoben werden, wenn - wie im Rahmen der Uruguay-Runde festgelegt - Einfuhrpreise unterschritten oder Importmengen überschritten werden.

Zollkontingente, die sich aus den Vereinbarungen des GATT ergeben, werden jährlich gestaffelt eröffnet. Um sicherzustellen, dass die Märkte in allen Sektoren um ein bestimmtes Maß geöffnet werden, sieht das GATT für jedes Land einen bestimmten Mindestmarktzugang vor. Diese Importe können zum ermäßigten Zollsatz eingeführt werden.

\section{Erstattungen}

Für exportierte Milch und Milcherzeugnisse in Länder außerhalb der EU können auf Antrag Erstattungen gewährt werden. Sie werden aus der Differenz zwischen den Preisen innerhalb der Gemeinschaft und den Preisen am Weltmarkt errechnet. Die Einhaltung von mengenmäßigen Exportbegrenzungen wird anhand der Ausfuhrlizenzen gewährleistet ${ }^{566}$.

\subsubsection{Garantiemengenregelung für Milch}

\subsection{Milchquotenregelung}

Das Quotensystem wurde zum 1.4.1984 eingeführt. Es war zunächst auf fünf Jahre begrenzt und wurde seither mehrmals - zunächst bis zum 31.3.2000 - verlängert. Mit der Verordnung (EG) Nr. 1256/1999 über die Erhebung einer Zusatzabgabe ist die Quotenregelung bis März 2008 verlängert worden. Gleichzeitig wurde festgehalten, dass die Regelung im Jahr 2003 mit dem Ziel, die derzeitige Quotenregelung zu beenden ${ }^{567}$, überprüft werden solle.

\subsection{Garantiemenge}

Für die gesamte EU ist eine Garantiemilchmenge festgelegt ${ }^{568}$, die abgabefrei geliefert werden kann. Für jeden Mitgliedstaat wurde daraus eine Gesamtgarantiemenge bestimmt, welche sich auf Lieferungen an Molkereien und Direktverkäufe durch die

\footnotetext{
${ }^{565}$ Vgl. EG-VO 1255/1999 Art. 29 ff.

566 Vgl. LLM SCHWÄBISCH GMÜND 2002, S. 108

567 Vgl. EU-Kommission 1999, Fact Sheet S. 4

${ }^{568}$ Vgl. EG-VO 1256/1999
} 
Landwirte aufteilt. Sollte die Menge der an die Molkereien gelieferten Milch oder der direkt abgesetzten Milch die Gesamtgarantiemenge überschreiten, so wird der Überschuss mit einer Abgabe belegt. Dadurch soll ein Anwachsen der Erzeugung verhindert und die Finanzierung des Überschussabsatzes ermöglicht werden ${ }^{569}$.

Die Fortführung der Milchquotenregelung war bei den Verhandlungen zur AGENDA 2000 nur durch umfangreiche Kompromisse in Form von Quotenerhöhungen für Italien, Spanien, Irland, Griechenland und Nordirland um insgesamt fast 1,4 Mio. $t$ in den Jahren 2000 und 2001 („Spezifische Erhöhung") erreichbar. Die Garantiemengen der übrigen EU-Staaten werden in drei gleichen Schritten ab 2005 um insgesamt 1,5\% (ebenfalls rund 1,4 Mio. t) erhöht (Lineare Erhöhung). Insgesamt erhöht sich dadurch die europäische Gesamtgarantiemenge um 2,4\%.

\subsection{Referenzmenge}

Bei der internen Weiterverteilung ihrer Garantiemenge auf die Milcherzeuger konnten die Mitgliedstaaten ursprünglich zwischen der einzelbetrieblichen Festlegung der Referenzmenge für jeden Milcherzeuger (Formel A: Deutschland, Niederlande und Belgien) und der Festlegung einer Referenzmenge für die Molkereien als Milchkäufer oder Milcherfasser (Formel B: Frankreich, Italien, Großbritannien, Irland, Dänemark, Luxemburg und Griechenland) wählen ${ }^{570}$. Seit 1992 gibt es nur noch einzelbetriebliche Referenzmengen. Direktverkäufe von Erzeugern an Verbraucher sind in das Referenzmengensystem einbezogen, und entsprechende Direktverkaufsreferenzmengen wurden jedem Mitgliedstaat zugeteilt (vgl. Tabelle 29).

Da eine rein mengenmäßige Begrenzung der Milcherzeugung nicht verhindern konnte, dass durch steigende Fettgehalte in der Milch die Milchfetterzeugung zunahm, wurde festgelegt, dass je Anstieg des Fettgehalts der gelieferten Milch um 0,1\%, die gelieferte Milchmenge rechnerisch um 1,8\% erhöht wird. Diese berichtigte Milchmenge ist Grundlage für die Erhebung der Übermengen-Abgabe.

\footnotetext{
${ }^{569}$ Vgl. LLM SCHWÄBISCH GMÜND 2001, S. 3
} 
Tabelle 29: Gesamtreferenzmenge gemäß EG-VO 1256/1999 Artikel 3 Absatz 2 in Tonnen

\begin{tabular}{|c|c|c|c|c|c|}
\hline Mitgliedsstaat & $\begin{array}{c}2002-2005 \\
\text { Molkerei }\end{array}$ & $\begin{array}{c}2005 \text { - } 2006 \\
\text { Molkerei }\end{array}$ & $\begin{array}{c}2006 \text { - } 2007 \\
\text { Molkerei }\end{array}$ & $\begin{array}{c}2007 \text { - } 2008 \\
\text { Molkerei }\end{array}$ & $\begin{array}{l}2002-2008 \\
\text { Direktverkäufe }\end{array}$ \\
\hline Belgien & 3140696 & 3157248 & 3173800 & 3190352 & 169735 \\
\hline Dänemark & 4454640 & 4476917 & 4499193 & 4521470 & 708 \\
\hline Deutschland & 27767036 & 27906360 & 28045684 & 28185008 & 97780 \\
\hline Griechenland & 699817 & 699817 & 699817 & 699817 & 696 \\
\hline Spanien & 6007564 & 6007564 & 6007564 & 6007564 & 109386 \\
\hline Frankreich & 23793932 & 23915111 & 24036290 & 24157469 & 441866 \\
\hline Irland & 5386575 & 5386575 & 5386575 & 5386575 & 9189 \\
\hline Italien & 10298399 & 10298399 & 10298399 & 10298399 & 231661 \\
\hline Luxemburg & 268098 & 269443 & 270788 & 272134 & 951 \\
\hline Niederlande & 10991900 & 11047273 & 11102647 & 11158020 & 82792 \\
\hline Österreich & 2543979 & 2557726 & 2571473 & 2585220 & 205422 \\
\hline Portugal & 1835461 & 1844823 & 1854186 & 1863548 & 37000 \\
\hline Finnland & 2394528 & 2406551 & 2418573 & 2430596 & 10000 \\
\hline Schweden & 3300000 & 3316515 & 3333030 & 3349545 & 3000 \\
\hline Großbritannien & 14393669 & 14466619 & 14539569 & 14612520 & 216078 \\
\hline
\end{tabular}

Anm.: Gesamtreferenzmenge $=$ Molkerei + Direktverkäufe; Molkerei $=$ Lieferung an Molkerei

Quelle: eigene Darstellung, Daten EG-VO 1256/1999

\subsubsection{4 Übermengenabgabe}

Wird die nationale Milchgarantiemenge überschritten, muss für die überlieferte Menge eine Strafabgabe geleistet werden. Sie beträgt für alle Milchanlieferungen oder Direktverkäufe $115 \%$ des Milchrichtpreises ${ }^{571}$. Die Abgabe wird auf alle Milcherzeuger verteilt, die zur Mengenüberschreitung beigetragen haben. Das Geldaufkommen aus der Übermengenabgabe wird zur Finanzierung der Ausgaben im Milchsektor eingesetzt.

\subsection{Saldierung}

Die Unter- und Überlieferungen bei den Referenzmengen der einzelnen Milcherzeuger werden zur Ermittlung der Strafabgabe in zwei Stufen miteinander verrechnet (saldiert). Die erste Stufe ist die Saldierung auf Ebene der Molkereien. Dazu werden die ungenutzten Referenzmengen von Unterlieferern den Überlieferern im Verhältnis derer Referenzmengen zugeteilt.

570 Vgl. LLM SCHWÄBISCH GMÜND 2001, S. 4; FRANCOISE 1997, S. 69 ff.

${ }^{571}$ Vgl. BRAUn 2000, S. 8 
In der zweiten Stufen werden alle aus den Molkereisaldierungen noch verbleibenden einzelbetrieblichen Unterlieferungen den verbleibenden einzelbetrieblichen Überlieferungen gegenübergestellt. Hier sind die Referenzmengen der Überlieferer bedeutungsIos; bei der Verteilung wird nur das Verhältnis von Unter- zu Überlieferungen zugrundegelegt.

\subsection{Quotenübertragung}

Die Quotenübertragungsmodalitäten sind mit der AGENDA 2000 und dem neuen Garantiemengensystem seit dem 1.4.2000 geändert worden. Die EG hat hierzu folgende Regelungen erlassen ${ }^{572}$ :

- Die Mitgliedstaaten bilden einzelstaatliche Reserven zur Verteilung an aktive Milcherzeuger. Diese werden durch teilweise Abzüge bei zeitweiliger oder endgültiger Quotenübertragung aufgefüllt. Dieser Reserve fallen auch Quoten von Milcherzeugern zu, die ein Jahr keine Milch oder Milcherzeugnisse vermarktet haben, die Milcherzeugung nicht wieder aufnehmen wollen und ihre Referenzmenge nicht an andere Milcherzeuger übertragen haben.

- Zur Umstrukturierung der Milcherzeugung und zur Verbesserung der Umweltbedingungen können die Mitgliedstaaten Quoten von Erzeugern, die sich verpflichten, ganz oder teilweise die Milcherzeugung aufzugeben, gegen Entschädigung in die nationale Reserve übernehmen (Milchrente). Sie können die flächenlose Quotenübertragung zulassen, hierzu Übertragungsregionen festlegen und die Bedingungen der endgültigen Quotenübertragung insgesamt selbst festlegen.

- Die Mitgliedstaaten können die Quotenübertragung anders als durch individuelle Übertragungen zwischen Erzeugern ermöglichen.

Deutschland hat von den neuen Regelungen Gebrauch gemacht und die Quotenübertragung zum 1.4.2000 völlig neu geregelt. Die Kernpunkte der Neuregelung sind ${ }^{573}$ :

1. Ein Neuverpachtungs- und Leasingverbot,

2. die Quotenübertragung erfolgt nur noch durch Verkauf über regionale Verkaufsstellen (Börsen),

3. es erfolgt kein Eingriff in laufende Pachtverträge (Pächterschutz),

\footnotetext{
572 Vgl. EG-VO 1256/1999 Art. 8

${ }^{573}$ Vgl. DUMSTORF 1999, S. 558 ff.
} 
4. die Regelung ist mit kostendämpfenden Elementen (Abzugs- und Verteilregelungen) zur Begrenzung der Quotenpreise ausgestattet,

5. es erfolgt ein entschädigungsloser Einzug der Quote in die Landesreserve, wenn im Garantiemengenjahr keine Milch vermarktet wurde.

\subsection{AGENDA 2000}

Im Rahmen der AGENDA 2000 wurden für den Milchbereich im wesentlichen folgende Beschlüsse gefasst (danach wird die Milch-Garantiemengenregelung bis zum Jahr 2007/2008 fortgeführt ${ }^{574}$ ):

- Ab dem Garantiemengenjahr 2005/06 wird die Garantiemenge in drei gleichen Schritten für alle Mitgliedstaaten mit Ausnahme von Griechenland, Spanien, Irland, Italien und Nordirland linear um insgesamt 1,5 \% bzw. 1,44 Mio. Tonnen erhöht. Deutschland erhält davon einen Anteil von 418.000 Tonnen $^{575}$,

- ab dem Milchwirtschaftsjahr 2005/06 werden die Interventionspreise für Butter und Magermilchpulver in drei gleichen Schritten um insgesamt 15\% gesenkt. Gleichzeitig erfolgt eine Senkung des Milchrichtpreises um $17 \%{ }^{576}$,

- die Milcherzeuger erhalten dafür einen Ausgleich je Kilogramm Milchreferenzmenge. Die Ausgleichsbeträge ergeben sich demnach wie folgt ${ }^{577}$ :

- $5,75 € /$ t im Kalenderjahr 2005

- $11,49 € /$ t im Kalenderjahr 2006

- $17,24 € /$ t im Kalenderjahr 2007

Als weiteren Ausgleich für die Preissenkung erhält Deutschland ab dem Jahr 2005 jährlich einen nationalen Zusatzbetrag ${ }^{578}$. Die EG weist den Mitgliedstaaten hierzu Mittel zu, die bis zu festgelegten Höchstprämien durch nationale Zahlungen ergänzt werden können (vgl. Tabelle 30). Die Mitgliedstaaten können damit flexibel auf Bedürfnis-

\footnotetext{
${ }^{574}$ Vgl. EG-VO 1256/1999 sowie $1255 / 1999$

575 Vgl. EG-VO 1256/1999 Anhang II

576 Vgl. EG-VO 1255/1999 Art. 3 und 4

577 Vgl. EG-VO 1255/1999 Art. 16

${ }^{578}$ Vgl. EG-VO 1255/1999 Art. 17 ff.
} 
se ihrer Milcherzeuger reagieren. Dieser Zusatzbetrag wird ebenso wie die Grundprämie in drei Schritten erhöht und beträgt in der Endstufe 216 Mio. Euro. Dabei sind die Mitgliedstaaten ermächtigt, die Zusatzbeträge als Ergänzung des Ausgleichs je kg Referenzmenge oder als Flächenprämie zu gewährleisten. Ferner können sie mit der Gewährung des Zusatzbetrages bestimmte Umweltauflagen verbinden ${ }^{579}$.

Tabelle 30: Entwicklung der Direktzahlungen

\begin{tabular}{|l|c|c|c|}
\hline Kalendarjahr & $\mathbf{2 0 0 5}$ & $\mathbf{2 0 0 6}$ & $\mathbf{2 0 0 7}$ \\
\hline Prämie für Milcherzeuger: $€ / \mathrm{t}$ & 5,75 & 11,49 & 17,24 \\
\hline $\begin{array}{l}\text { Gesamtprämie: Höchstprämie }+ \\
\text { Prämienzuschlag: } € / \mathrm{t}\end{array}$ & 13,9 & 27,8 & 41,7 \\
\hline $\begin{array}{l}\text { Flächenzahlung je ha Dauer- } \\
\text { grünland }\end{array}$ & Max. 350€ & Max. 350€ & Max 350€ \\
\hline
\end{tabular}

Quelle: EU-Koмmission 1999, Fact Sheet, S. 3

Der Agrarat hat sich verpflichtet, auf Grundlage eines Berichtes der Kommission im Jahr 2003 eine Halbzeitbewertung mit dem Ziel vorzunehmen, das gegenwärtige Quotenregime nach dem Jahr 2006 auslaufen zu lassen. Ziele der Umgestaltung sind ${ }^{580}$ :

- Milch und Milcherzeugnisse wettbewerbsfähiger zu machen,

- eine umweltverträglichere Landwirtschaft,

- eine bessere Basis für WTO-Verhandlungen,

- die Vorbereitung auf die EU-Osterweiterung und

- die Schaffung größerer Ermessensspielräume für die Mitgliedstaaten.

\subsubsection{Bewertung einer Kontingentierung}

\subsection{Kosten der Regelung}

Seit der Einführung der Quotenregelung sind die Haushaltsausgaben für Zuschüsse im Sektor Milch und Milcherzeugnisse relativ betrachtet stark zurückgegangen ${ }^{581}$. Die Ausgaben sanken von 27 \% der EAGFL-Garantieausgaben 1983 auf 6,5 \% in 1999. Die Gesamtsumme - in Höhe von 2,5 Mrd. $€$ jährlich - ist nach wie vor relativ hoch (vgl. Abbildung 36). Hinzukommen die Belastungen für den Verbraucher innerhalb der

\footnotetext{
${ }^{579}$ Vgl. EG-VO 1255/1999 Art. 19

${ }^{580}$ Vgl. Eu-RECHNUNGSHOF 2001, S. 16 u. S. 34

${ }^{581} \mathrm{Vgl.}$ EU-RECHNUNGSHOF 2001, S. 7
} 
EU, der die höheren Preise für die Milcherzeugnisse "tragen muss". Laut einer Schätzung der OECD betragen die zusätzlichen Kosten für die EU-Verbraucher bei diesen Erzeugnissen mehr als 20 Mrd. $€$ pro Jahr ${ }^{582}$. Die Europäische-Kommission dagegen ist der Meinung, dass eine Liberalisierung des Milchmarktes insgesamt keine niedrigeren Kosten für den europäischen Verbraucher zur Folge hat. Ihrer Meinung nach zeigten Erfahrungen, dass sich eine Senkung der Erzeugerpreise nicht automatisch auf die Verbraucherpreise auswirke ${ }^{583}$.

Abbildung 36: Kosten der Marktorganisation (nach Kategorie aufgelistete Ausgaben)

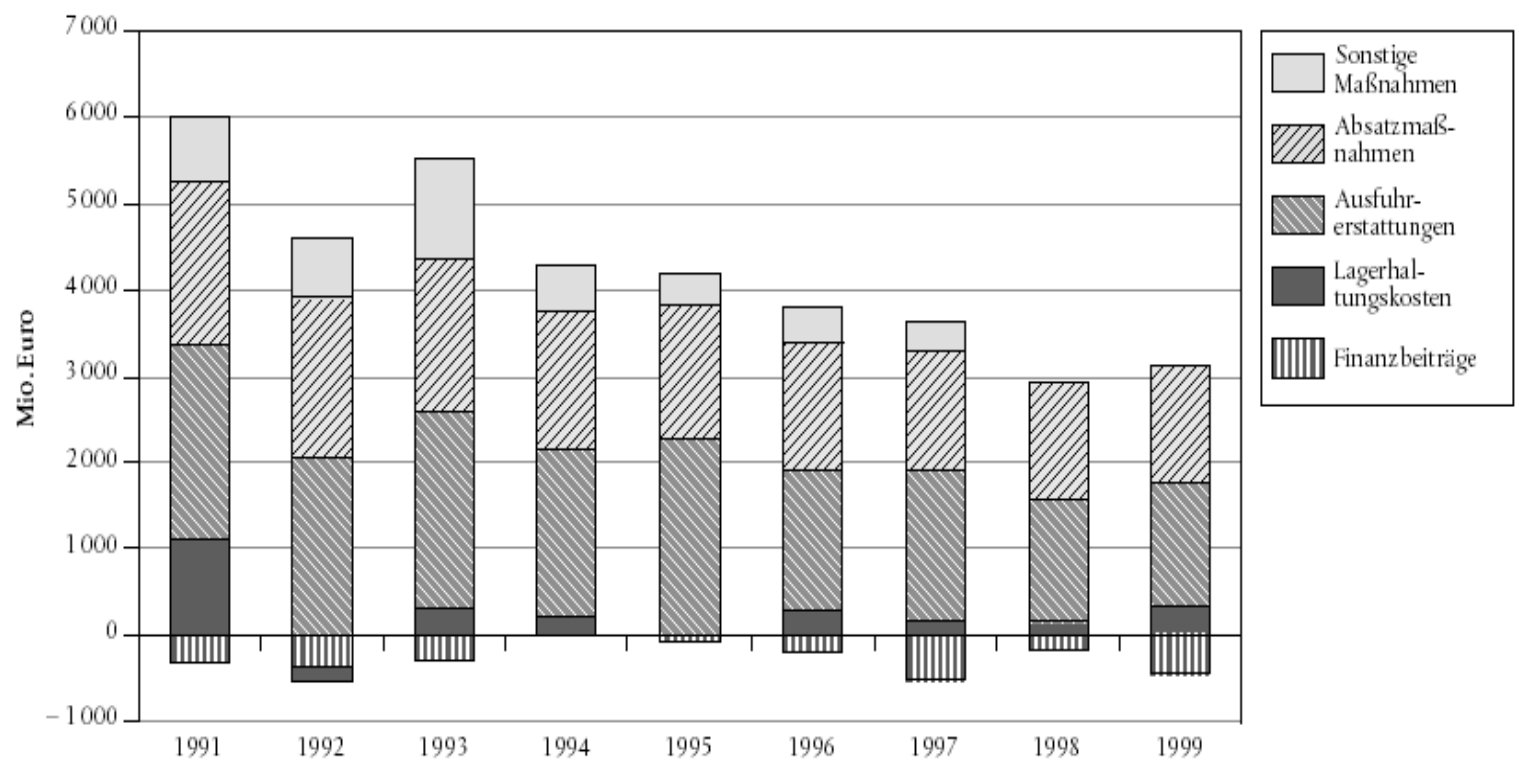

QUelle: EUROPÄISCHER-RECHNUNGSHOF 2001, S. 7

\subsection{Zukunft und Wirkung der Quotenregelung bzgl. des Wettbewerbs}

Die Zukunft der Milchquotenregelung ist bislang ungewiss. Zur Zeit sind Großbritannien, Schweden, Dänemark und Italien (sog. London Club) eindeutige Befürworter der Abschaffung einer Mengenregelung, während Frankreich, Spanien, Belgien, Luxemburg, Griechenland, Portugal, Österreich, Irland und Finnland für die Beibehaltung einer Quotenregelung plädieren. Unentschlossen sind derzeit die Länder Deutschland und die Niederlande ${ }^{584}$. Befürworter der derzeitigen Quotenregelung fassen die Vorteile (nach deren Einschätzung) folgendermaßen zusammenfassen ${ }^{585}$ :

\footnotetext{
582 OECD 2001: Agricultural policies in OECD countries, monitoring and evaluation 2000, zitiert in: EUROPÄISCHER-RECHNUNGSHOF 2001, S. 7 und 9

583 Vgl. EUROPÄISCHER-RECHNUNGSHOF 2001, S. 27 und S. 33

${ }^{584}$ Vgl. IDF 2001, S. 532

${ }^{585}$ Vgl. Wille 2001, S. 597
} 
- Planungssicherheit für Erzeuger, Verarbeiter und Abnehmer (Handelsstufe),

- große Preis- und Einkommensstabilität,

- stabile regionale Verteilung der Produktion,

- kontinuierliche Rohstoffversorgung der Molkereien,

- Quoten können ein effizientes und kostengünstiges Instrument der Marktsteuerung und der regionalen Planung von Milch- und Verarbeitungsstandorten $\operatorname{sein}^{586}$.

Argumente gegen die Quotenregelung sind:

- Kontingentierungsmaßnahmen stellen eine Absage an das Prinzip der marktwirtschaftlichen Ordnung dar. Sie führen mehr als andere Instrumente der Markt- und Preispolitik dazu, weitere staatliche Eingriffe nach sich zu ziehen ${ }^{587}$;

- eine Mengenbegrenzung könnte dazu führen, dass Wachstumspotentiale auf dem Weltmarkt nicht voll genutzt werden;

- zwischen Inlands- und Weltmarktpreisen entstehen zu große Preisunterschiede, so dass die europäische Milchproduktion international nicht wettbewerbsfähig ist $^{588}$;

- Quoten bewahren unwirtschaftliche Strukturen ${ }^{589}$ und haben auch zu Ineffizienzen in der verarbeitenden Industrie geführt ${ }^{590}$;

- Quoten sind in den Beitrittsländern Mittel- und Osteuropas nicht zu administrieren $^{591}$;

- einzelbetriebliches Wachstum ist zwar möglich, jedoch oftmals nur unter Inkaufnahme hoher Quotenkosten;

- für Neueinsteiger ist die Milchquotenregelung eine Markteintrittsbarriere, die sich in der Praxis als quasi unüberwindbar erweist ${ }^{592}$;

\footnotetext{
586 Vgl. IDF 2001, S. 532

587 Bereits 1983 machte der WISSENSCHAFTLICHE BEIRAT (Milchmarktpolitik) auf diese Gefahr aufmerksam

${ }^{588}$ Vgl. IDF 2001 , S. 532

${ }^{589} \mathrm{Vgl}$. IDF 2001, S. 532

$590 \mathrm{Vgl}$. BeRTELSMEIER/JÄGERSBERG 2002, S. 11

${ }^{591}$ Vgl. IDF 2001, S. 532

592 Vgl. BeRTELSMEIER/JÄGERSBERG 2002, S. 10
} 
- im Zuge des Strukturwandels hat die Zahl der Inhaber von Milchquoten, die nicht mehr selber melken, sondern ihre Quote aktiven Milcherzeugern gegen Entgelt zur Nutzung überlassen oder übertragen, stark zugenommen. Damit findet ein erheblicher Einkommenstransfer aus der Milchwirtschaft heraus statt;

- das Argument der Preis- und Einkommensstabilität hat an Schlagkraft verloren: Da die Europäische-Kommission bei der Intervention von Butter nur noch $90 \%$ des Interventionspreises zahlt, und die Intervention von Magermilchpulver grundsätzlich nur noch in den Sommermonaten bis zu einer Höchstmenge möglich ist, können die Milchpreise auch deutlich unter das Interventionspreisniveau sinken;

- erhebliche wohlfahrtsökonomische Kosten (Kosten der Marktordnung sowie für die Verbraucher).

Neben der Marktordnung und der Quotenregelung für Milch wird die Zukunft auch von steigendem Wettbewerbsdruck geprägt sein, der u.a. durch die WTO-Verhandlungen und die damit verbundene Öffnung der Märkte begründet ist ${ }^{593}$. Derzeit wird zunehmend die Einführung eines A/C-Modelles nach Vorbild der Zuckermarktordnung diskutiert (vgl. 7.3.2) ${ }^{594}$. Danach erhält der Landwirt eine Grundquote, für die ein erhöhter Binnenpreis gezahlt wird. Der Landwirt kann darüber hinaus Milch zum Weltmarktpreis in beliebiger Menge produzieren ${ }^{595}$. Allerdings muss die WTO-Kompatibilität dieser Regelung geprüft werden und der Kompromiss zwischen Freihandel und Regulierung hinterfragt werden ${ }^{596}$.

\subsubsection{Zucker}

\subsubsection{Gesetzliche Grundlagen}

Die Grundlage der gemeinsamen Marktorganisation für Zucker bildet die EGVerordnung 1260/2001. Sie enthält einheitliche Regelungen für die Sektoren Zucker, Isoglukose und seit dem Wirtschaftsjahr 1994/95 Inulinsirup ${ }^{597}$ und besteht in einer

\footnotetext{
${ }^{593}$ Vgl. WiLLE 2001, S. 600

${ }^{594}$ Vgl. AgRA-EUROPE 2002, 25. März, Länderbericht 44 sowie AGA-EUROPE 2002, 18. März, EuropaNachrichten Nr. 5

595 Diese Marktregulierung besteht bereits in Kanada

${ }^{596}$ Vgl. AgRA-EUROPE 2002, 25. März, Länderbericht 44

597 Vgl. LLM 2002b, S. 1
} 
Mengenbeschränkung mit einem komplexen Außenschutz. Die Besonderheit der Zuckermarktordnung gegenüber anderen Marktordnungen liegt in der Quotenfestlegung und der Möglichkeit zur Erhebung von Produktionsabgaben, die eine Selbstfinanzierung der Marktorganisation sicherstellen sollen. Die wesentlichen Elemente der Zuckermarktordnung (ZMO) - insbesondere die Quotenregelung - bestehen bereits seit $1967^{598}$. Trotz zunehmender Kritik wurde die Zuckermarktordnung im Herbst 2000 überarbeitet und zunächst bis 2006 verlängert, wobei im Jahr 2003 im Rahmen des „mid-term review"599 die Zukunft der Marktorganisation für Zucker nach 2006 geregelt werden soll.

\subsubsection{Administrative Preise}

Der Europäische Rat legt für jedes Wirtschaftsjahr ${ }^{600}$ einen Richtpreis für Weißzucker der Standardqualität (unverpackt, ab Werk verladen) fest. Gleichzeitig werden Interventionspreise fixiert. Für Mitgliedstaaten ohne Zuschussbedarf ${ }^{601}$ an Zucker wird ein einheitlicher Interventionspreis für Weißzucker ${ }^{602}$ vereinbart $^{603}$. Für Mitgliedstaaten mit Zuschussbedarf ${ }^{604}$ wird von einem einheitlichen Interventionspreis jeweils ein Interventionspreis für Weißzucker abgeleitet, der um die Transportkosten vom Hauptüberschussgebiet in die jeweilige Region erhöht ist. Der Interventionspreis für Rohzucker ergibt sich aus dem Interventionspreis für Weißzucker unter Berücksichtigung von Pauschalbeträgen für die Verarbeitung und den Transport.

Ebenfalls jährlich setzt der Rat einen Grundpreis für Zuckerrüben für eine bestimmte Standardqualität und Anlieferungsstufe fest. Der Grundpreis wird abgeleitet aus dem Interventionspreis für Weißzucker unter Berücksichtigung einer festgelegten Verarbeitungsspanne, den Transport- und Abnahmekosten sowie den Erlösen aus dem Melasseverkauf 605 .

\footnotetext{
598 Vgl. EWG-VO 206/68 und EWG-VO 431/68; vgl. Ausführungen NEI 2000, S. 5

${ }^{599}$ Im Zuge der AGENDA 2000 beschlossene Bewertung der Agrarpolitik. Darin sollen konkrete Entscheidungen über die Zukunft europäischer Marktordnungen nach 2006 bzw. 2008 geregelt werden.

${ }^{600}$ Das Zuckerwirtschaftsjahr beginnt am 1. Juli und endet am 30. Juni des folgenden Jahres.

${ }^{601}$ Zuschussbedarf gilt für traditionelle Zuckernettoexporteure (Spanien, Finnland, Irland, Portugal, Vereinigtes Königreich)

602 Standardqualität, unverpackt, ab Werk, verladen

${ }^{603}$ Vgl. EG-VO 1260/2001 Art. 2

${ }^{604}$ Kein Zuschussbedarf gilt für traditionelle Zuckernettoimporteure (Belgien, Deutschland, Dänemark, Frankreich, Griechenland, Italien, Luxemburg, Niederlande, Österreich, Schweden)

${ }^{605}$ Vgl. EG-VO 1260/2001 Art. 2
} 
In Abhängigkeit vom Grundpreis wird jährlich je ein Mindestpreis für A-Zuckerrüben und B-Zuckerrüben bestimmt. Die Mindestpreise betragen für A-Zucker gegenwärtig $98 \%$ und für B-Zucker $60,5 \%$ des Grundpreises ${ }^{606}$. Für Gebiete mit Zuschussbedarf sind die Mindestpreise analog der abgeleiteten Interventionspreise höher ${ }^{607}$ ( $\mathrm{vgl}$. Tabelle 31).

Tabelle 31: Marktordnungspreise für Zuckerrüben in ECU bzw. $\mathbf{C / d t}$

\begin{tabular}{|l|l|l|l|l|l|l|l|l|l|l|}
\hline & $\mathbf{1 9 9 3}$ & $\mathbf{1 9 9 4}$ & $\mathbf{1 9 9 5}$ & $\mathbf{1 9 9 6}$ & $\mathbf{1 9 9 7}$ & $\mathbf{1 9 9 8}$ & $\mathbf{1 9 9 9}$ & $\mathbf{2 0 0 0}$ & $\mathbf{2 0 0 1}$ & $\mathbf{2 0 0 2}$ \\
\hline A-Mindestpreis & 3,869 & 3,869 & 4,672 & 4,672 & 4,672 & 4,672 & 4,672 & 4,672 & 4,672 & 4,672 \\
\hline B-Mindestpreis & 2,389 & 2,389 & 2,884 & 2,884 & 2,884 & 2,884 & 2,884 & 2,844 & 2,844 & 2,844 \\
\hline $\begin{array}{l}\text { Interventionspr. } \\
\text { Weißzucker }\end{array}$ & 52,33 & 52,33 & 63,19 & 63,19 & 63,19 & 63,19 & 63,19 & 63,19 & 63,19 & 63,19 \\
\hline
\end{tabular}

Quelle: BARTENS, Ifd. Jgg.

\subsubsection{Quotenregelung}

Die Mengenzuordnung der Quote ist historisch, sie wurde aufgrund der damaligen europäischen Produktion vorgenommen. Diese wurde nach demselben Verfahren für Beitrittskandidaten der EU bzw. EG erweitert (vgl. Tabelle 32).

Neben reinem Rübenzucker erstreckt sich die Marktordnung ebenso auf Rohrzuckeranbau, Isoglukose ${ }^{608}$ (seit 1981) und Inulinsirup ${ }^{609}$ (seit 1994). Ziel der Quotenregelung ist die Sicherstellung der Zuckerbinnenversorgung. Über die Quotenregelung hinaus produzierte Mengen müssen als sogenannter C-Zucker ohne Exporterstattungen auf dem Weltmarkt abgesetzt werden ${ }^{610}$.

\footnotetext{
${ }^{606}$ Vgl. LLM SCHWÄBISCH GMÜND 2002b, S. 1

${ }^{607}$ Vgl. EG-VO 1260/2001 Art. 4

${ }^{608}$ Aus Mais oder Weizenstärke hergestellter Fructosesirup (High Fructose Corn Sirup = HFCS)

${ }^{609}$ Aus Zichorien gewonnener Polyfructansirup

${ }^{610}$ Vgl. EG-VO 1260/2001 Art. 10 ff.
} 
Tabelle 32: Aktuelle Aufteilung der Quoten

\begin{tabular}{|c|c|c|c|c|c|c|}
\hline Region & $\begin{array}{l}\text { Grundmenge } \\
\text { A Zucker (in } \\
\text { Tonnen Weiß- } \\
\text { zucker) }\end{array}$ & $\begin{array}{l}\text { Grundmenge } \\
\text { A Isoglu- } \\
\text { kose (in } \\
\text { Tonnen Tro- } \\
\text { ckenstoff) }\end{array}$ & $\begin{array}{l}\text { Grundmenge } \\
\text { A für Inulin- } \\
\text { sirup (in } \\
\text { Tonnen Tro- } \\
\text { ckenstoff, } \\
\text { ausgedrückt } \\
\text { in Weißzu- } \\
\text { cker-/Iso- } \\
\text { glukose- } \\
\text { Äquivalent) }\end{array}$ & $\begin{array}{l}\text { Grundmenge } \\
\text { B Zucker (in } \\
\text { Tonnen Weiß- } \\
\text { zucker) }\end{array}$ & $\begin{array}{l}\text { Grundmenge } \\
\text { B Isoglu- } \\
\text { kose (in } \\
\text { Tonnen Tro- } \\
\text { ckenstoff) }\end{array}$ & $\begin{array}{l}\text { Grundmenge } \\
\text { B für Inulin- } \\
\text { sirup (in } \\
\text { Tonnen Tro- } \\
\text { ckenstoff, } \\
\text { ausgedrückt } \\
\text { in Weißzu- } \\
\text { cker-/Iso- } \\
\text { glukose- } \\
\text { Äquivalent) }\end{array}$ \\
\hline DK & 325000 & - & - & 95745,5 & - & - \\
\hline D & 2612913,3 & 28643,3 & - & 803982,2 & 6745,8 & - \\
\hline GR & 288638 & 10435 & - & 28863,8 & 2457,5 & - \\
\hline ES & 957082,4 & 74619,6 & - & 39878,5 & 7959,4 & - \\
\hline $\mathrm{F}$ & 2506487,4 & 15747,1 & 19847,1 & 752259,5 & 4098,6 & 4674,2 \\
\hline F-Übersee & 463872 & - & - & 46372,5 & - & - \\
\hline IRL & 181145,2 & - & - & 18114,5 & - & - \\
\hline I & 1310903,9 & 16432,1 & - & 246539,3 & 3869,8 & - \\
\hline NL & 684112,4 & 7364,6 & 65519,4 & 180447,1 & 1734,5 & 15430,5 \\
\hline A & 314028,9 & - & - & 73297,5 & - & - \\
\hline$P$ & 63380 & 8027 & - & 6338 & 1890,3 & - \\
\hline Azoren & 9048,2 & - & - & 904,8 & - & - \\
\hline FIN & 132806,3 & 10792 & - & 13280,4 & - & - \\
\hline $\mathrm{S}$ & 334784,2 & - & - & 33478 & - & - \\
\hline B/LUX & 674905,5 & 56150,6 & 174218,6 & 144906,1 & 15441 & 41028,2 \\
\hline GB & 1035115,4 & 21502 & - & 103511,5 & 5735,3 & - \\
\hline
\end{tabular}

Quelle: EU-VO 1260/2001 Art. 11

Ursprünglich entsprach die Menge der A-Quote in etwa dem Zuckerverbrauch innerhalb der Gemeinschaft, für den eine volle Abnahme- und Preisgarantie gewährt wird. Die B-Quote sollte den steigenden Verbrauch absichern ${ }^{611}$. Für die B-Quote gilt die volle Abnahme-, aber eine verminderte Preisgarantie. A- und B-Quote zusammen entsprechen der Höchstquote. Die Mitgliedstaaten können die den einzelnen Unternehmen zustehenden A- und B-Quoten um bis zu 10\% kürzen und die freiwerdende Menge neu verteilen. Zucker, der über die Summe von A- und B-Zucker hinaus erzeugt wird (C-Zucker) und nicht unter Anrechnung der A-Quote auf das folgende Wirtschaftsjahr übertragen wird, darf nicht innerhalb der Gemeinschaft abgesetzt werden, sondern muss in unverarbeiteter Form vor dem auf das betreffende Wirtschaftsjahr folgenden 1. Januar exportiert werden ${ }^{612}$. Der Export erfolgt zum Weltmarktpreis, also

\footnotetext{
${ }^{611}$ Vgl. NEI 2000, S. 6 ff.

612 Vgl. EG-VO 1260/2001 Art. 13
} 
ohne jegliche Erstattung der EG (vgl. Abbildung 37). Diese Bestimmung kann aufgehoben werden, wenn der Zucker zur Versorgung innerhalb der EU benötigt wird.

\section{Abbildung 37: Prinzip der europäischen Quotenregelung}

\section{EU-Quotensystem}
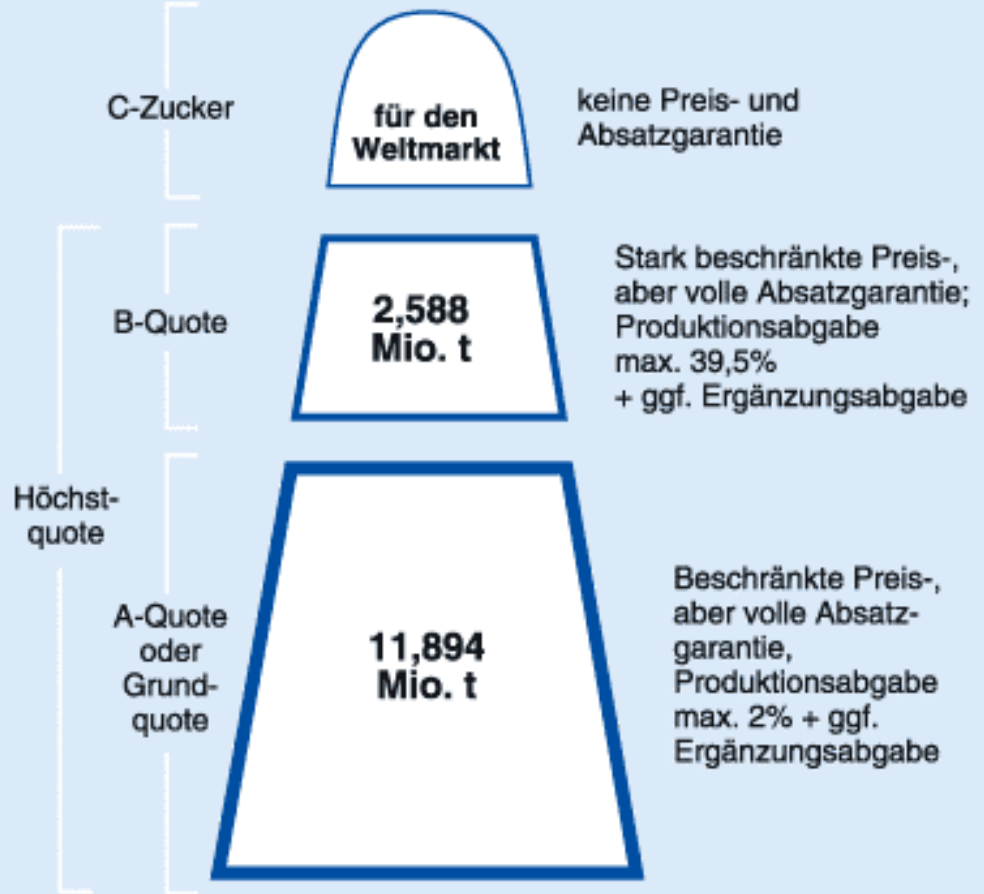

\section{Quelle: Wirtschaftliche Vereinigung ZUCKeR 2001}

Durch den GATT-Vertrag sind die subventionierten Zuckerausfuhren mengenmäßig von 1995 bis 2001 sukzessive um 21 \% reduziert worden (ausgabenmäßig um 36\%), und zudem wurde eine Einfuhrzollsenkung um $20 \%$ vorgenommen ${ }^{613}$. Aus diesem Grund sieht die Zuckermarktordnung vor, dass die Quoten jährlich flexibel angepasst werden, wenn die Absatzsituation dies erfordert. Eine notwendige Reduzierung der Quote soll im Bedarfsfall nicht bereits vor der Aussaat, sondern zum 2. Oktober eines jeden Wirtschaftsjahres durchgeführt werden ${ }^{614}$. Dies hat zur Folge, dass aus Quotenzucker C-Zucker würde, der entweder ohne Erstattungen in Drittländer ausgeführt oder aber auf die folgende Kampagne übertragen werden könnte.

\subsubsection{Regelungen des EU-Binnenmarktes}

Die Interventionsstellen der einzelnen Mitgliedstaaten sind während des gesamten Wirtschaftsjahres verpflichtet, den ihnen angebotenen und aus der Gemeinschaftsern-

\footnotetext{
${ }^{613}$ Vgl. EUROPÄISCHE-KOMMISSION 4.10.2000, S. 8; NEI 2000, S. 14

${ }^{614}$ Vgl. EG-VO 1260/2001 Art. 15
} 
te stammenden Weiß- und Rohzucker zum entsprechenden Interventionspreis anzukaufen. Für Abweichungen von der Standardqualität gibt es Zu- oder Abschläge. Die Quotenregelung schränkt die Ankaufspflicht jedoch ein und begrenzt die Preis- und Absatzgarantie auf bestimmte Erzeugungsmengen ${ }^{615}$.

Die Abgabe (Verkauf) des intervenierten Zuckers ist nur zu einem über dem Interventionspreis liegenden Preis möglich. Ausnahmen sind jedoch bei Abgabe zum Zwecke der Tierfütterung, der Herstellung bestimmter Waren für den Export sowie der Abgabe an Wohltätigkeitseinrichtungen möglich ${ }^{616}$.

\subsubsection{Regelung des Außenhandels}

\subsection{Außenhandelsverträge}

Außenhandelsverträge beziehen sich auf Abkommen mit Drittländern oder internationalen Handelsorganisationen. Für Zucker betrifft das die AKP-Staatenregelung, die EBA-Initiative sowie die GATT/WTO-Abkommen.

Mit den GATT-Verträgen der Uruguay-Runde sind 1994 auch auf dem Zuckermarkt erste Einschnitte in die bestehende Marktordnung eingeleitet worden. Ziel der GATT/WTO-Vereinbarungen, die den Abbau von Handelshemmnissen und verzerrungen vorsehen, ist ein liberalisierter Weltmarkt. Aufgrund der in der Uruguay Runde eingegangenen Verpflichtungen wurden die variablen Importabschöpfungen durch die festen Zollsätze des GZT (Gemeinsamer Zolltarif) ${ }^{617}$ ersetzt und Kontingente für vergünstigte Importe und subventionierte Exporte eröffnet. Alle Ein- und Ausfuhren werden mittels umfassender Lizenzpflichten kontrolliert. Für die Zuckermarktordnung bedeutet dies den Abbau von Exporterstattungen ${ }^{618}$.

Bei der Ein- oder Ausfuhr ist die Vorlage einer Lizenz erforderlich, die gegen Kaution erteilt wird ${ }^{619}$. Die Anwendung der Zollsätze kann für Rohzucker und Melasse ausgesetzt werden, wenn der Weltmarktpreis zuzüglich Zoll den Interventionspreis übersteigt. Zusatzzölle können GATT-konform erhoben werden, wenn von Einfuhren Störungen des Gemeinschaftsmarktes ausgehen. Geprägt ist das Einfuhrsystem für Zucker durch die vereinbarten Zollkontingente für Präferenzzucker v.a. aus AKP-Staaten

\footnotetext{
${ }^{615}$ Vgl. EG-VO 1260/2001 Art. 7

${ }^{616}$ Vgl. EG-VO 1260/2001 Art. 9

${ }^{617}$ Vgl. EG-VO 1260/2001 Art. 23

${ }^{618} \mathrm{Vgl.} \mathrm{TRUMM} \mathrm{2001,} \mathrm{S.} 301$

${ }^{619}$ Vgl. EG-VO 1260/2001 Art. 22
} 
und Indien ${ }^{620}$; dieser Zucker wird zollfrei eingeführt ${ }^{621}$. Durch den Vertrag von Lomé hat sich die EU zur Abnahme beträchtlicher Zuckermengen (1,3 Mio. t Weißzuckerwert) aus 68 Staaten Afrikas, der Karibik und des Pazifiks (AKP) zu Festpreisen verpflichtet, welche wieder zu Weltmarktpreisen reexportiert werden müssen ${ }^{622}$.

Bei der Ausfuhr kann eine Erstattung gewährt werden, die den Unterschied zwischen den Preisen in der Gemeinschaft und den (normalerweise niedrigeren) Weltmarktpreisen ausgleicht ${ }^{623}$. Besteht die Gefahr, dass die im GATT vereinbarten Exportmengen bzw. Haushaltsausgaben im Zuckersektor überschritten werden, kann die Kommission die beantragten Lizenzen nach einer einheitlichen Erteilungsquote kürzen. Daneben räumt sich die Kommission die Möglichkeit ein, Lizenzanträge abzulehnen, denen noch nicht stattgegeben wurde. Wenn der Weltmarktpreis über den Interventionspreis ansteigt, kann bei der Zuckerausfuhr eine Abschöpfung erhoben werden; sie hat das Ziel, die innergemeinschaftliche Marktversorgung zu sichern. Kann die Versorgung der Gemeinschaft nicht durch eigene Kapazitäten gedeckt werden, können Einfuhrsubventionen gewährt werden ${ }^{624}$.

\subsection{LDC-Regelung (EBA-Abkommen)}

Die Europäische Gemeinschaft gewährt seit 1971 allgemeine Zollpräferenzen für bestimmte landwirtschaftliche Erzeugnisse und gewerbliche Waren aus Entwicklungsländern. Im "Cotonou-Abkommen" zwischen den AKP-Staaten und der EU aus dem Jahr 2000 wurde zudem vereinbart, dass spätestens in 2005 der zollfreie Zugang zum EUMarkt für im wesentlichen alle Waren aus den am wenigsten entwickelten Staaten ermöglicht wird ${ }^{625}$. Zusätzlich hat die EU beschlossen für alle Waren (ausgenommen Waffen und Munition = everything but arms = EBA-Abkommen) mit Ursprung aus den 48 am geringsten entwickelten Ländern (Least Developed Countries = LDC), zollfreien Zugang ohne Mengenbeschränkung zum EU-Markt zu gewähren ${ }^{626}$. Für Zucker wurde jedoch eine Sonderegelung eingeführt. So werden die Zollsätze zunächst in drei Stufen

\footnotetext{
${ }^{620}$ Vor dem EG-Beitritt importierte Großbritannien einen Großteil von Rohzucker aus den ehemaligen Kolonien, um diesen in Großbritannien zu verarbeiten - mit Aufnahme Großbritanniens (1975) zur EG wurden die entsprechenden Lieferverträge mit übernommen.

${ }^{621}$ Vgl. EG-VO 1260/2001 Art. 35 ff.

622 Vgl. LLM Schwäbisch Gmünd 2000, S. 63

${ }^{623}$ Vgl. EG-VO 1260/2001 Art. 27

${ }^{624}$ Vgl. EG-VO 1260/2001 Art. 33

${ }^{625} \mathrm{Vgl}$. SOMmer 2002, S. 41

${ }^{626}$ Vgl. EG-VO Nr. 416/2001
} 
abgebaut $^{627}$ und parallel zollfreie Einfuhrquoten für Rohrzucker (der in den LDCLändern erzeugt wird und zur Raffination bestimmt ist) eingeführt. Die Mengen werden beginnend von 2001 bis 2008 von ca. 75.000 t auf etwa. 200.000 t erhöht ${ }^{628}$. Erst ab dem Jahr 2009 gilt der freie Marktzugang. Gleichzeitig enthält die Verordnung allerdings auch eine Sicherungsklausel, so dass die Präferenzen ausgesetzt werden können, sobald die Einführung dieser Waren ernsthafte Störungen auf den Märkten der Gemeinschaft und bei den Regulationsmechanismen verursachen ${ }^{629}$.

\subsubsection{Finanzierung der Kosten}

Bei der EG-Zuckermarktordnung wird die Zielsetzung verfolgt, durch eine entsprechende Erzeugerbeteiligung den Zuckermarkt für die EG-Kasse kostenneutral zu halten. Daher wird für jedes Zuckerwirtschaftsjahr festgestellt, um welchen Betrag die hergestellte Menge an A- und B-Zucker (einschl. Isoglukose, Inulinsirup) den Verbrauch übertrifft (Überschuss). Es wird weiterhin vorausgeschätzt, in welcher Höhe die Gemeinschaft Exporterstattungen für Zucker gewähren und Einnahmen aus der Abschöpfung für Zucker erzielen wird. Die Differenz der beiden Beträge, also die Nettoaufwendungen der Gemeinschaft für den Außenhandel mit Zucker, wird durch die exportierte Menge dividiert. Daraus ergibt sich der durchschnittliche Verlust (Gewinn) der Gemeinschaft je Tonne exportierten Zuckers. Mit diesem Betrag wird der in der Gemeinschaft erzeugte Zuckerüberschuss bewertet und führt so zum Gesamtverlust (-gewinn) des Zuckerwirtschaftsjahres ${ }^{630}$.

\subsection{Produktionsabgabe}

Der Gesamtverlust wird zunächst auf die erzeugten A- und B-Zucker-, Isoglukose- und Inulinsirupmengen umgelegt und von den Herstellern erhoben. Diese Grundproduktionsabgabe darf jedoch beispielsweise für Weißzucker $2 \%$ des Interventionspreises nicht überschreiten ${ }^{631}$.

Kann damit der Gesamtverlust nicht gedeckt werden, so wird der Restbetrag auf die erzeugte Menge B-Zucker, B-Isoglukose und B-Inulinsirup umgelegt, jedoch nur bis zu maximal 30\% des Interventionspreises für Weißzucker (B-Abgabe). Ist auch mit dieser Umlage eine vollständige Deckung des Verlustes nicht möglich, so kann der Ministerrat

\footnotetext{
$6272006=20 \%, 2007=50 \%, 2008=80 \%$

${ }^{628} \mathrm{Vgl.}$ ZUCKERINDUSTRIE 2001, S. 232

${ }^{629} \mathrm{Vgl}$. SOMMER 2002, S. 41

${ }^{630}$ Vgl. EG-VO 1260/2001 Art. $10 \mathrm{ff}$.

${ }^{631}$ Vgl. NEI 2000, S. 7
} 
für das nachfolgende Wirtschaftsjahr eine Erhöhung des Umlagesatzes bei der B-Quote bis auf $37,5 \%$ beschließen. Gleichzeitig wird der Mindestpreis für B-Rüben entsprechend angepasst.

Liegt der Betrag der Grundproduktionsabgabe unter 2\% oder der Betrag der B-Abgabe unter $37,5 \%$, sind die Zuckerhersteller verpflichtet, den Rübenerzeugern $60 \%$ des Unterschieds zwischen dem Höchstbetrag der Abgabe und der tatsächlichen Abgabe zu zahlen $^{632}$.

\subsection{Ergänzungsabgabe}

Da auch durch eine Erhöhung des Umlagesatzes bei der B-Quote auf 37,5\% der Gesamtverlust nicht immer gedeckt werden kann, ist seit 1988 eine sogenannte Ergänzungsabgabe vorgesehen, die für die zuckererzeugenden Unternehmen anteilig zu ihrer zu zahlenden Grundproduktions- und B-Produktionsabgaben erhoben wird ${ }^{633}$. Diese Ergänzungsabgabe wird so berechnet, dass die Gesamtverluste der Gemeinschaft für das betreffende Wirtschaftsjahr in jedem Fall gedeckt werden. Die Unternehmen können eine Rückerstattung von den Zuckerrübenerzeugern in Höhe von bis zu $60 \%$ verlangen.

\subsubsection{Exkurs: Garantierte Verarbeitungsspanne}

Durch Festlegung eines Interventions- und Rübenpreises ist dem Verarbeiter eine feste Spanne vorgegeben. Die Kalkulationen beruhen seit 1995 auf folgenden Daten ${ }^{634}$ :

Interventionspreis:

Verarbeitungskosten:

Melasseverkaufserlös:

Transportkosten (Rüben)

Weißzuckerpreis

Grundpreis (16\% Zucker, Faktor 0,013) $=47,67 € /$ t Weißzucker
$+631,9 € /$ t Weißzucker

$-243,6 € /$ t Weißzucker

$+22,5 € /$ t Weißzucker

$-44,1 € /$ t Weißzucker

$366,7 € /$ t Weißzucker

Landwirte erhalten somit $58 \%$ des Interventionspreises $(366,7 € / t / 631,9 € / t)$, während die Verarbeiter einen Anteil von $42 \%$ erzielen. In der Realität erwirtschaften die Verarbeiter jedoch noch einen größeren Anteil, da der Marktpreis in den meisten Fällen

\footnotetext{
632 Vgl. EG-VO 1260/2001 Art. 18

${ }^{633}$ Vgl. EG-VO 1260/2001 Art. 16

${ }^{634} \mathrm{Vgl}$. ZUCKERINDUSTRIE, Ifd. Jgg. der Monate September und Oktober
} 
über dem Interventionspreis liegt ${ }^{635}$. Im wesentlichen wird an der Verarbeitungsspanne kritisiert, dass sie bereits seit vielen Jahren unverändert geblieben ist und somit keine technischen Fortschritte sowie Rationalisierungsvorteile Berücksichtigung fanden und die Europäische-Kommission überdies lediglich Datenmaterial seitens der Zuckerindustrievertreter zur Rechtfertigung heranzieht ${ }^{636}$. Die Kommission dagegen ist der Auffassung, dass aufgrund der unveränderten Annahme der Marktspannen real eine Senkung der Verarbeitungsspannen stattfindet ${ }^{637}$. "Auch wenn der Anschein eines nur geringen Wettbewerbs innerhalb der Zuckerbranche erzeugt wird," so weisen immer wieder Verstöße gegen das Kartellrecht (British Sugar 1997; Irish Sugar 1999; Nordzucker 2000) sowie das Streben nach Fusionen auf einen funktionierenden Wettbewerb $\operatorname{hin}^{638}$.

\subsubsection{Kosten der Zuckermarktordnung}

1999 beliefen sich die im Haushalt aus der GMO entstehenden Kosten auf brutto 2,1 Milliarden $€$, wovon 1,2 Mrd. $€$ über die Produktions- und Lagerkostenabgaben ${ }^{639}$ einbezogen wurden (vgl. Tabelle 33). Der Verbraucher kommt über den Zuckerpreis für einen beträchtlichen Teil dieser Kosten auf ${ }^{640}$.

Die volkswirtschaftlichen Kosten der hohen Zuckerpreise in der EU können nur durch detaillierte Untersuchungen ermitteln werden ${ }^{641}$. Relativ einfach lassen sich die Kosten an der Differenz zwischem gestützten EG-Preis und dem Weltmarktpreis messen, woraus sich jährliche Maximalkosten in Höhe von rund 6,5 Mrd. Euro für den Verbraucher ergäben ${ }^{642}$. Der derzeit niedrige Weltmarktpreis kann aber nicht als wirklich objektiver Faktor betrachtet werden. Dennoch vermittelt diese Kalkulation eine grobe Vorstellung, in welcher Größenordnung sich die Kosten bewegen ${ }^{643}$.

Die Erfahrungen in anderen Industrieländern lassen darauf schliessen, dass alternative Süßstoffe - ohne entsprechende Quotenregelung - zu Lasten von Zucker einen we-

\footnotetext{
${ }^{635}$ Vgl. NEI 2000, S. 9

${ }^{636} \mathrm{Vgl}$. EUROPÄISCHER-RECHNUNGSHOF 2000, S. 10

${ }^{637}$ Vgl. EUROPÄISCHE-KOMMISSION 2000 (Amtsblatt), S. 27

${ }^{638} \mathrm{Vgl.} \mathrm{EUROPÄISCHE-KOMMISSION} 2000$ (Amtsblatt), S. 25

639 Die Lagerkostenabgabe wurde in der Reform von 2000 abgeschafft, vgl. Neuregelung

${ }^{640} \mathrm{Vgl.}$ EUROPÄISCHER-RECHNUNGSHOF 2000, S. 3

${ }^{641} \mathrm{Vgl}$. EUROPÄISCHER-RECHNUNGSHOF 2000, S. 14

642 Revidierte Ansätze der Kommission für den Haushaltsplan 2000

${ }^{643} \mathrm{Vgl.} \mathrm{EUROPÄISCHER-RECHNUNGSHOF} \mathrm{2000,} \mathrm{S.} 14$
} 
sentlich höheren Anteil am gesamten Süßmittelmarkt einnehmen würden, da sie preiswerter und für einige Lebensmittel besser geeignet sind ${ }^{644}$.

Auch die geschätzten Kontroll- und Verwaltungskosten der EG für Zucker können nur einen Hinweis geben. Der Betrag wird vom Rechnungshof auf ca. 290 Millionen $€$ jährlich geschätzt ${ }^{645}$.

Die Kommission dagegen argumentiert, dass die Nettokosten der GMO für die Zuckerausfuhr (1,6 Mio. t), die aus AKP-Ländern und Indien gemäß Protokoll des AKP-EWGAbkommens und dem Abkommen mit Indien eingeführt wurde, 90 \% der Nettokosten entspricht. Es handelt sich also um eine Art Entwicklungshilfe, die lediglich im Agrarhaushalt verrechnet wird ${ }^{646}$. Die Kommission beurteilt den Weltmarkpreis als nicht objektiv, da er zum Großteil aus quersubventionierten Exportmengen zustande kom$m e^{647}$. Die Kommission zweifelt außerdem an, ob eine Senkung des Zuckerpreises sich auch auf den Endverbraucher auswirkt ${ }^{648}$. In anderen Industrieländern unterscheiden sich beispielsweise die Zuckerpreise kaum von denen innerhalb der EU:

- in den USA, Kanada oder Australien seien die Preise ca. 2,9 mal so hoch wie auf dem Weltmarkt

- Norwegen: trotz Einkauf zum Weltmarktpreis seien die Verbraucherpreise für Weißzucker im Inland etwa genauso hoch wie in der Gemeinschaft und für Verarbeitungszucker sogar höher ${ }^{649}$.

\footnotetext{
${ }^{644}$ Vgl. RABOBANK 1999, S. 35; zitiert aus EUROPÄISCHER-RECHNUNGSHOF 2000, S. 14

${ }^{645} \mathrm{Vgl}$. EUROPÄISCHER-RECHNUNGSHOF 2000, S. 14

${ }^{646} \mathrm{Vgl.} \mathrm{EUROPÄISCHE-KOMMISSION} \mathrm{15.} \mathrm{Feb.} \mathrm{2001,} \mathrm{S.} 23$ u. 24

${ }^{647} \mathrm{Vgl.}$ EUROPÄISCHE-KOMMISSION 15. Feb. 2001, S. 27

${ }^{648}$ Vgl. EUROPÄISCHE-KOMMISSION 15. Feb. 2001, S. 28; EU-KOMMISSION 2000 (Amtsblatt), S. 28; EUROPÄISCHE-KOMMISSION 2000, S. 4
} 
Tabelle 33: EU-Kosten der Marktorganisation für Zucker (in Mio. Euro)

\begin{tabular}{|c|c|c|c|c|c|c|}
\hline & 1995 & 1996 & 1997 & 1998 & 1999 & 2000 \\
\hline \multicolumn{7}{|l|}{ Ausgaben } \\
\hline \multicolumn{7}{|l|}{ Maßnahme } \\
\hline $\begin{array}{l}\text { Ausfuhrerstattung bei Zucker und Isoglu- } \\
\text { kose }\end{array}$ & 1312,1 & 1230 & 1115,7 & 1265,5 & 1591,1 & 1493 \\
\hline Vergütung der Lagerkosten & 398,8 & 361,1 & 362,4 & 349,5 & 342,8 & 316 \\
\hline $\begin{array}{l}\text { Erstattung für die Verwendung in der che- } \\
\text { mischen Industrie }\end{array}$ & 70,4 & 80,9 & 81,8 & 104,8 & 128,3 & 135 \\
\hline $\begin{array}{l}\text { Maßnahmen zur Förderung des Absatzes } \\
\text { von Rohzucker }\end{array}$ & 15 & 15,4 & 14,2 & 12,8 & 11,2 & 12 \\
\hline Sonstige Intervention für Zucker & 34,7 & 23,9 & 33,8 & 44,1 & 39,4 & 40 \\
\hline Ausgaben insgesamt & 1831 & 1711,3 & 1607,8 & 1776,7 & 2112,8 & 1996 \\
\hline \multicolumn{7}{|l|}{ Abgaben (Einnahmen) } \\
\hline Produktionsabgabe für Zucker & 726 & 711,6 & 746,5 & 780,5 & 817,5 & 788,3 \\
\hline Produktionsabgabe für Isoglukose & 5,9 & 6,8 & 6,6 & 6,4 & 7,1 & 6,8 \\
\hline Produktionsabgabe für Inulin & 1,1 & 1,6 & 2,2 & 2,8 & 2,0 & 3,0 \\
\hline Lagerkostenabgabe für Zucker & 542 & 490,3 & 349 & 280,1 & 288,5 & 286,9 \\
\hline $\begin{array}{l}\text { Auf nicht ausgeführten C-Zucker und } \\
\text { C-Isoglukose Mengen erhobene Beträge }\end{array}$ & 0,3 & 0,3 & 9,9 & 0,3 & 0,4 & - \\
\hline $\begin{array}{l}\text { Für Substitutions-C-Zucker und } \\
\text { C-Isoglukose erhobene Beträge }\end{array}$ & 0,8 & 0 & - & 0 & 0 & - \\
\hline Ergänzungsabgabe & 40,3 & 2,1 & 0 & 0 & 88,1 & 77,7 \\
\hline Abgaben insgesamt (Einnahmen) & 1316,4 & 1213,7 & 1114,1 & 1070,1 & 1203,6 & 1162,7 \\
\hline Haushaltsnettoausgaben & 514,6 & 497,6 & 493,7 & 706,6 & 909,2 & 833,3 \\
\hline
\end{tabular}

Quelle: EUROPÄISCHER-RECHNUNGSHOF 2000, S. 18

\subsubsection{Kritik an der ZMO}

Die Kritik an der geltenden Zuckermarktordnung ist vielfältig. Einen Kritikpunkt stellt die Verarbeitungsspanne dar. Die Verarbeitungsspanne, die EU-weit gilt, einheitlich festgesetzt wird und ursprünglich (1968) eine Vollkostendeckung im Bereich der AQuotenproduktion ermöglichen sollte, stellt für effizient wirtschaftende Unternehmer trotz gestiegener Faktorpreise ein erhebliches Gewinnpotential dar ${ }^{650}$. Allein aufgrund der vollzogenen Fusionen in der Zuckerindustrie und der damit einhergehenden Rationalisierungsmaßnahmen konnten die Tagesverarbeitungen erheblich gesteigert werden. Render zeigte beispielsweise in seiner Studie ${ }^{651}$, dass bei einer Ausdehnung der

\footnotetext{
${ }^{649} \mathrm{Vgl.}$ EUROPÄISCHE-KOMMISSION 15. Feb. 2001, S. 27

650 Vgl. SCHMIDT 1991, S. 344

${ }^{651}$ Vgl. RENDER 1989
} 
Tagesverarbeitung von 3000 auf 10.000 t Produktionskostenunterschiede von $30 \%$ realistisch sind.

Aus ökonomischer Sicht hat die Quotenregelung dazu beigetragen, dass der Wettbewerb zwischen Verarbeitern nicht effizient funktioniert und es keinen echten "gemeinsamen Markt" für Zucker gibt. Aus Gesprächen mit den verschiedenen Interessensgruppen wurde deutlich, dass nationale Verarbeitungsbetriebe kaum daran interessiert sind, mit Verarbeitungsbetrieben außerhalb der Landesgrenzen zu konkurrieren. Sie beschränken ihre Tätigkeit weitgehend auf ihre angestammten Märkte. Quoten und Preisregelungen in Verbindung mit dem Ausfuhrprogramm sichern den Verarbeitern somit Stabilität und ein garantiertes Einkommen ${ }^{652}$.

Zusätzlich wird die Sonderstellung von Zucker gegenüber anderen Ackerfrüchten kritisiert $^{653}$. Da im allgemeinen Preise für andere Agrarprodukte gesunken sind, ist die relative Vorzüglichkeit von Zucker überdimensional gestiegen ${ }^{654}$

Auch führt die derzeitige ZMO zu verzerrten Einkommensverteilungen: Hohe Markteintrittsbarrieren ${ }^{655}$ machen es kaum oder nur zu hohen Kosten möglich, in den Zuckerrübenanbau einzusteigen. Durch regional zugeteilte Lieferrechte mit geringen Übertragungsmöglichkeiten wird außerdem eine Verschiebung der Produktion zu den effizientesten Betrieben oder Regionen ${ }^{656}$ behindert. Unterschiede zwischen den innerhalb verschiedener Regionen gezahlten Preisen für Rübenkontingente von mehr als hundert Prozent belegen nachdrücklich, wie stark die Rentabilität und damit die Wettbewerbsfähigkeit des Rübenanbaus zwischen verschiedenen Territorien variiert. Die Wanderung der Zuckerproduktion an die bestmöglich geeigneten Standorte bezieht sich außerdem nicht nur auf den Rübenanbau, auch die Allokation der Verarbeitungsstandorte wird durch die ZMO behindert ${ }^{657}$.

Auf vertikaler Ebene existiert wegen der Vorgabe von Mindestpreisen kaum Wettbewerb zwischen Landwirt und Zuckerfabrik. Da Lieferrechte in der Praxis auf Unterneh-

\footnotetext{
652 Vgl. EUROPÄISCHER-RECHNUNGSHOF 2000, S. 15

${ }^{653}$ Vgl. WisSENSCHAFTLICHER BEIRAT 1993, S. 3

${ }^{654}$ Vgl. dazu Berechnungen NEI 2000, S. $146 \mathrm{ff}$.

${ }^{655} \mathrm{Vgl}$. EUROPÄISCHER-RECHNUNGSHOF 2000, S. 9

${ }^{656}$ Vgl. WISSENSCHAFTLICHER BEIRAT 1993, S. 5

${ }^{657}$ Vgl. MAHLER 1992, S. 118
} 
mensebene nur sehr begrenzt übertragbar sind ${ }^{658}$, gibt es zwischen den Unternehmen faktisch kein Wettbewerb um den Rohstoff Zuckerrübe ${ }^{659}$.

Eine Quotierung führt zu einer mangelnden Anpassung der Produktion an die Nachfrage. Hauptursache für die Kostenbelastung ist eine zu hoch angesetzte Kontingentmenge, so dass ständig ein künstlicher Überschuss erzeugt wird, welcher auf dem Weltmarkt über Exporterstattungen abgesetzt werden muss ${ }^{660}$.

Die Belastung der Verbraucherhaushalte, auf die letztendlich die Finanzierung überwälzt wird, wurde bereits unter dem Kostenaspekt diskutiert.

Durch den relativ hohen Preis für Rübenzucker wurde die Produktion von Zucker aus anderen Pflanzen oder Verfahren (Zuckersubstitute) lukrativer (z.B. Isoglukose, Inulinsirup). Daraufhin hat der Gesetzgeber auch für diese Produkte Quoten eingeführt und somit eine volkswirtschaftlich ineffiziente Lösung herbeigeführt ${ }^{661}$.

Auch international treten durch die EG-Zuckermarktordnung Störungen des internationalen Handels ein: Quersubventionierung von C-Zucker kann zu verzerrten Weltmarktpreisen ${ }^{662}$ führen und somit andere Volkswirtschaften beeinflussen.

Abschließend sei auf die Ineffizienz der AKP-Regelung hingewiesen, die als teure Entwicklungshilfe durch unnötige Transporte und Handelsströme gekennzeichnet ist (Transaktionskosten) $^{663}$.

Befürworter der Regelung argumentieren, dass die ZMO die Ziele der Gemeinsamen Agrarpolitik erfülle. So werde der Binnenmarkt stetig und regelmäßig mit Weißzucker versorgt und eine Ausgabenbelastung aufgrund der Eigenfinanzierung in Grenzen gehalten. Überdies sichere die ZMO in einem von starken Schwankungen der Weltmarktpreise betroffenen Sekor ein stabiles Einkommen der Zuckerrübenerzeuger.

Interventionsmaßnahmen kämen schon seit längerem nicht mehr zur Anwendung, weil die Zuckermarktpreise innerhalb der EU seit 1986 oberhalb des Interventionspreises lägen ${ }^{664}$.

\footnotetext{
${ }^{658} \mathrm{Vgl}$. DOHMS 2002

${ }^{659}$ Vgl. WiSSENSCHAFTLICHER BEIRAT 1993, S. 6

660 Vgl. WiSSENSCHAFTLICHER BEIRAT 1993, S. 7

${ }^{661} \mathrm{Vgl}$. SCHMIDT 1991, S. 340 ff.; NEI 2000, S. 88 ff.

662 Vgl. WiSSENSCHAFTLICHER BEIRAT 1993, S. 13; EUROPÄISCHER-RECHNUNGSHOF 2000, S. 9

${ }^{663}$ Vgl. WisSENSCHAFTLICHER BEIRAT 1993, S. 15

${ }^{664}$ Vgl. NEI 2000, S. 8
} 


\subsubsection{Neuregelung}

Für den Zuckerbereich waren die letzten Wirtschaftsjahre von den GATTVerpflichtungen zur Reduzierung der von der EG gestützten Exporte gekennzeichnet. Das dafür eingeführte Instrument der Deklassierung, also der Herabsetzung der maximalen Ausnutzung der Höchstquote, kam erstmals im Wirtschaftsjahr 2000/2001 zur Geltung, so dass die Quoten entsprechend des zur Verfügung stehenden Exporterstattungsbudgets um ca. 115.000 t reduziert wurden ${ }^{665}$. Als weitere Maßnahmen wurden die Abschaffung des Lagerkostenausgleichs ${ }^{666}$ eingeleitet sowie die Mindestlagermengenreglung ${ }^{667}$ gestrichen. Des weiteren wurde die vollständige Finanzierung der Produktionserstattungen für sog. Chemiezucker durch Produktionsabgaben der Zuckerwirtschaft anstelle des bislang dafür aufkommenden EG-Haushaltes eingeführt ${ }^{668}$ (ausführliche Marktdaten im Anhang $\mathrm{C}$ ).

\subsubsection{Zwischenergebnis}

Die Berücksichtigung der Ziele anderer Politikbereiche tragen nicht zu einer Vereinfachung und Transparenz einer Entscheidung bei. So stellte der ehemalige Direktor der Generaldirektion Wettbewerb der Europäischen Kommission Van Miert heraus: „Die Wettbewerbspolitik der Kommission findet nicht in einem Vakuum statt. Sie hat stets auch ihre Auswirkungen in andere Politikbereiche der Kommission mit in Betracht zu ziehen, wie etwa Industrie-, Regional-, Sozial- und Umweltpolitik" ${ }^{\prime \prime 69}$. Umgekehrt zeigt sich aber auch im Bereich der in dieser Studie vorgestellten Zusammenschlüsse, dass beispielsweise die Zuckermarktordnung für erhebliche wettbewerbliche Verzerrungen sorgt. So ist ein fast ausgeschalteter Wettbewerb in der Zuckerindustrie nicht auf eine unrestriktive Wettbewerbsrechtspraxis zurückzuführen, sondern vielmehr auf agrarpolitische Rahmenbedingungen. Neben Preisverzerrungen und Einschränkungen der Handelbarkeit von Zuckerrechten wurde zusätzlich jeglicher potentieller Substitutionswettbewerb, der einen belebenden Effekt auf den Zuckermarkt ausüben könnte, aus-

\footnotetext{
${ }^{665}$ Vgl. SOMMER 2002, S. 40; F.O. LICHT 2000, 24.10.2000, S. 576

666 Um einen gleichbleibenden Preis für Zucker über das komplette Jahr zu garantieren, wurden sogenannte Lagerkostenzuschüsse gezahlt, die die Kosten für Lagerung und Versicherung des Zuckers übers Jahr hinweg konstant halten sollten.

667 Um immer eine gewisse "Notreserve" für Krisenzeiten zur Verfügung stellen zu können, waren die Unternehmen bislang verpflichtet, eine Mindestreserve einzulagern.

${ }^{668}$ Bislang gab es für Chemiezucker (beschränkte Menge) eine Beihilfe damit die europäische Chemieindustrie gegenüber außereuropäischen Produzenten, die ihren Zucker auf dem Weltmarkt beziehen, wettbewerbsfähig sind.

669 Zitat: VAN MIERT 1995, S. 554
} 
geschaltet ${ }^{670}$. Der gering verbleibende Wettbewerb findet innerhalb der Zuckerbranche somit im wesentlichen über Fusionen und Übernahmen statt. Mangelnder Wettbewerb ist also weniger auf den geltenden wettbewerbsrechtlichen Gesetzesrahmen, sondern vielmehr auf politische Verzerrungen (Marktunvollkommenheiten) aus anderen Bereichen zurückzuführen. Eine Berücksichtigung dieses Rahmens ist zwingend notwendig, eine Beseitigung von marktverzerrenden Fehlentwicklungen jedoch kaum möglich.

\subsection{Positive und negative Effekte von Fusionen}

\subsubsection{Abwägung}

Ziel jeder Wirtschaftspolitik sollte es u.a. sein, eine effiziente Nutzung der zur Verfügung stehenden Ressourcen zu gewährleisten. Die theoretischen wie empirischen Untersuchungen ergeben bislang ein differenziertes Bild. Trotz Unterstellung einer preiserhöhenden Wirkung als wesentliches Merkmal von Zusammenschlüssen deutet dies noch nicht unbedingt darauf hin, dass die damit verbundenen Marktmachteffekte gegenüber den potentiellen Effizienzgewinnen überwiegen (vgl. dazu Kapitel 4.1). Wird demnach Effizienz als Ziel angestrebt, so müsste eine entsprechende Abwägung wesentlicher Bestandteil der Zusammenschlusskontrolle sein ${ }^{671}$. Eine solche Vorgehensweise ist durch den vorgegebenen Rechtsrahmen zwar möglich (vgl. Abwägungsklausel), wurde aber bislang in der Rechtspraxis nur eingeschränkt angewendet ${ }^{672}$. Aus der Fallrechtsprechung ergibt sich, dass die Kommission Effizienzvorteile nur dann anerkennt, wenn ihre gesamtwirtschaftliche Wirkung mit Vorteilen für den Verbraucher und ohne Nachteile für den Wettbewerb verbunden ist ${ }^{673}$. In der bisherigen Fallpraxis konnte bislang kein Fall diese Bedingungen erfüllen. Ebenso erweist sich eine transparente Ermittlung von möglichen Effizienzwirkungen als äußerst schwierig ${ }^{674}$. Vielmehr indiziert die Rechtspraxis, dass, sobald die Marktanalyse auf die Existenz einer marktbeherrschenden Stellung hindeutet, Effizienzgewinne in ihrem Bestehen verneint werden ${ }^{675}$. Es findet somit nur eine eingeschränkte Ausbalancierung zwischen Effizienzvor-

\footnotetext{
${ }^{670}$ Ernstzunehmende Zuckerersatzstoffe unterliegen ebenfalls einer mengenmäßigen Einschränkung.

671 Z.B. USA, Kanada; vgl. dazu BOFINGER 2001, S. 21

672 Vgl. KeRBER 2000, S. 88

${ }^{673}$ Vgl. KINNE 2000, S. 127; auch Grünbuch FKVO 2001, Rdnr. 169/ 170

${ }^{674} \mathrm{Vgl.}$ RÖLLER/STENEK/VERBOVEN 2000 , S. 118 ff. diskutieren mögliche Vorgehensweisen

${ }^{675} \mathrm{Vgl.} \mathrm{KINNE} \mathrm{2000,} \mathrm{S.} 130$
} 
teilen und Marktmachteffekten statt, obwohl gerade unter dieser Konstellation für eine Urteilsfindung die Mitberücksichtigung positiver Effekte entscheidend wäre.

Im Gegensatz zu der in einigen anderen Ländern herrschenden Verwaltungspraxis ${ }^{676}$ wird der Schwerpunkt der europäischen Kartellbehörden auf die Ermittlung des Ausmaßes der gestiegenen Marktmacht gesetzt ${ }^{677}$. Es wird implizit oder explizit versucht, durch einen Zusammenschluss entstehende Preiserhöhungsspielräume auszuloten. Bei der Beurteilung werden u.a. Marktanteil, Wettbewerbsaktivität der Konkurrenzunternehmen, Eintrittsbarrieren und potentieller Wettbewerb berücksichtigt, um daraus das Marktmachtpotential des Zusammenschlusses abzuleiten.

\subsubsection{Negative Effekte}

Mit Unternehmenszusammenschlüssen kann ein effizienzminderndes Potential einhergehen. Dies äußert sich in der erhöhten Wahrscheinlichkeit einer Kollusion, einer Verringerung der Vielfalt von Produkten, einer Errichtung von strategischen Markteintrittsbarrieren und schließlich in der zuvor diskutierten Ausübung von Marktmacht. Für Unternehmen positiv besetzte Motive für einen Zusammenschluss können unter Effizienzgesichtspunkten negativ beurteilt werden. Die vorgestellten industrieökonomischen Modelle (vgl. Kapitel 1) deuten darauf hin, dass Preise nach einem Zusammenschluss steigen, wenn Synergiepotentiale nicht in erheblichem Maße ausgenutzt werden können. Die Ausnutzung von Rationalisierungsvorteilen allein ist dabei oftmals nicht ausreichend. Zwar disziplinieren die verbleibenden Wettbewerber die Preiserhöhung zu einem gewissen Grad, ähnlich wie in der Situation eines partiellen Kartells profitieren aber auch die verbleibenden Konkurrenten von dem verminderten Wettbewerbsdruck und haben die Möglichkeit zu Preiserhöhungen. Bei der Beurteilung von Zusammenschlüssen sollte somit die Wettbewerbsbehörde folgenden potentiellen Gefahren besondere Aufmerksamkeit schenken (Abbildung 38).

\footnotetext{
${ }^{676}$ Vgl. RÖLleR/Stenek/VERBoven 2000, S. $91 \mathrm{ff}$.

677 Vgl. BOFINGER 2001, S. 21
} 
Abbildung 38: Mögliche negative Effekte einer Fusion

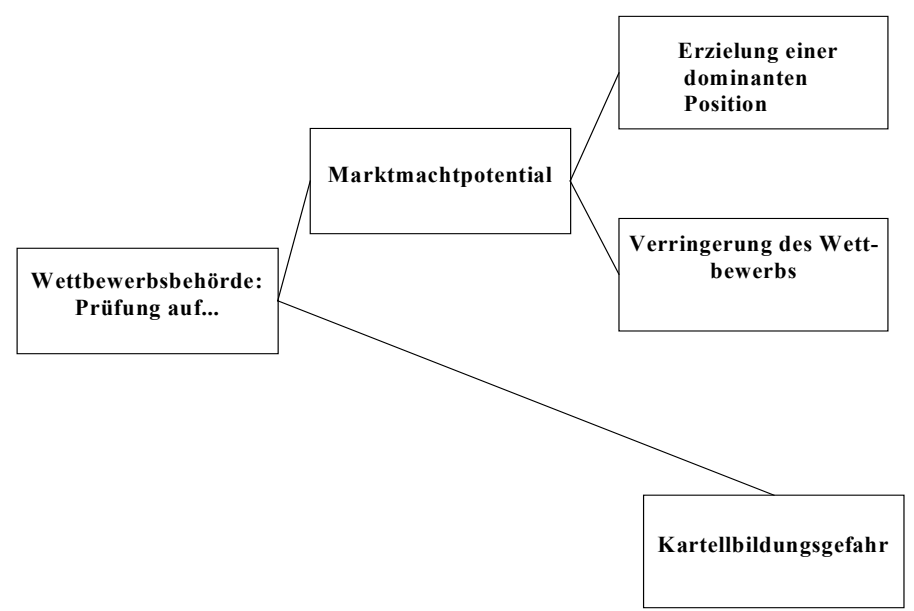

Quelle: eigene Darstellung

Die Praxis deutet darauf hin, das lediglich das Kriterium der Erzielung einer dominanten Position berücksichtigt wird ${ }^{678}$. Aus ökonomischer Sicht sind jedoch auch weitere Effekte zur Erfassung des gesamten Wohlfahrtsverlustes von extremer Wichtigkeit. Danach ist nicht nur das Niveau der Marktmacht (Prüfung einer dominanten Position), sondern auch eine mögliche Steigerung dieser Macht (Potential) von Belang ${ }^{679}$. Im Gegensatz zu den USA ${ }^{680}$ wird eine Fusion unter den europäischen Rechtsvorschriften nur bei Erlangung einer dominanten Stellung untersagt. Besondere Berücksichtigung sollten zusätzlich Faktoren finden, die eine Kollusion erleichtern ${ }^{681}$ :

1. Produktdifferenzierung,

2. Kapazitätsbeschränkungen der Outsider,

3. Substitutionsmöglichkeiten der Produkte,

4. Marktverhalten anderer (ausländischer) Anbieter,

5. Markteintrittsbarrieren,

6. absolutes Niveau der Preise,

\footnotetext{
678 Vgl. STENNEK 2002, S. 31

${ }^{679}$ Vgl. STENNEK 2002, S. 31

${ }^{680}$ dort werden beide Effekte (Niveau und Veränderung) berücksichtigt

${ }^{681} \mathrm{Vgl.}$ STENNEK 2002, S. 34; vgl. Motta 2000, S. 7 ff.
} 
7. Symmetrie (Marktanteile, Produktportfolio, Anzahl der Produkte, Kosten, technisches Wissen, Kapazitäten $)^{682}$.

\subsubsection{1 Ökonomische Instrumente zur Ermittlung negativer Effekte}

Die Ermittlung von Preiselastizitäten scheint ein brauchbares Instrument zur Feststellung von Marktmacht zu sein ${ }^{683}$. Die Kommission nutzt vermehrt den Einsatz von Preiselastizitäten in der Fallpraxis und verspricht sich gegenüber den Deutungen von Konzentrationsraten einen höheren Informationsnutzen ${ }^{684}$. Die zunehmende Preistransparenz (Scannerdaten) und die Ermittlung von Kosten auf Betriebs- und Branchenebene können zur Identifikation der Angebots- und Nachfragefunktionen beitragen, so dass mit Hilfe des Lerner-Indexes ${ }^{685}$ auf das Machtpotential einzelner Unternehmen rückgeschlossen werden kann ${ }^{686}$. „With full knowledge of the demand system (own and cross-elasticities), the nature of an oligopoly behaviour (including the behaviour of prospective entrants), and cost of all firms in an industry; with knowledge of these functions not just locally but in a significant range; and with knowledge of how costs and behaviour would change with a proposed merger; merger analysis would become merely a matter of computation"687.

Preistests, die auf ökonometrischen Beziehungen basieren, können im Gegensatz zu dem aktuell angewandten SSNIP Test nicht nur Aussagen über den relevanten Markt treffen, sondern überdies Informationen über Preiserhöhungsspielräume und alternative Absatzmöglichkeiten und -verschiebungen liefern ${ }^{688}$. IVALDI und VERBOVEN (2001) zeigten in ihrer Analyse beispielhaft anhand der Fusion Volvo/Scania, wie solche Untersuchungen ergänzend zu der bisherigen Vorgehensweise der Kartellbehörden eingesetzt werden könnten und welche nützlichen praxisnahen Informationen geliefert wür$\operatorname{den}^{689}$.

\footnotetext{
682 Vgl. MOTTA 2000, S. 20

${ }^{683}$ NeVen/NutTel/SEABright 1993, S. 20 ff.; S. 46 f. schlagen bereits 1993 den Vorzug des Einsatzes von Preiselastizitäten gegenüber der Ermittlung von Grenzkosten vor.

${ }^{684}$ Vgl. CAMESASCA 2000, S. 111 ; S. $131 ;$ S. 232

${ }^{685}$ Vgl. dazu ausführlich Anhang B

${ }^{686}$ zum Einsatz von Elastizitäten bei der EU-Kommission: CAMESASCA 2000, S. 71

687 Vgl. BAKER 1997, S. 347

${ }^{688} \mathrm{Vgl}$. IVALDi/VERBOVEN 2001, S. 16

${ }^{689}$ Für angewandte ökonometrische Untersuchungen vgl. Literaturübersicht IVALDI/VERBOVEN 2001, S. 2 SOWie RöLLER/STENNEK/VERBOVEN 2000, S. 109
} 
Auch wenn es noch einen gewissen Zeitrahmen in Anspruch nehmen wird, bis tatsächlich alle Kosten und Beziehungen von verschiedenen Gütern ermittelt werden können, besteht eine sinnvolle zukünftige Entwicklung in dem Einsatz von ökonometrischen Verfahren. In Kombination mit den herkömmlichen Parametern kann somit ein detaillierteres Abbild des Wettbewerbsgeschehens geschaffen werden. In diesem Punkt kann die US-amerikanische Rechtspraxis als vorbildhaft angesehen werden, denn ökonometrische Untersuchungen und das Zurückgreifen auf Expertengutachten gehören schon seit längerem zu dem Beurteilungsstandard der USA ${ }^{690}$. Denkbar und empfehlenswert wäre bei zunehmend leichterer Verfügbarkeit von Preisdaten ein Wettbewerbsmonitoring auf ökonometrischer Basis, dass bei der Beurteilung von Zusammenschlüssen - vor allem für die Missbrauchsaufsicht - als ein relativ preiswertes Instrumentarium genutzt werden könnte ${ }^{691}$. Die EU-Kommission ist dieser Entwicklung gegenüber offen und wird auch in Zukunft Anwendungsmöglichkeiten prüfen ${ }^{692}$.

Das Bundeskartellamt wendet ökonometrische Analysemethoden bislang nicht an, beschäftigt sich aber intensiv mit zukünftigen Anwendungsmöglichkeiten. „Problematisch sind vor allem die hierfür erforderlichen Daten über Preise, Nachfragemengen usw., die bestimmten Voraussetzungen genügen müssen, um beispielsweise Elastizitäten oder ähnliches berechnen zu können. Der damit verbundene personelle, finanzielle und zeitliche Aufwand ist in der Regel nur bei wirklich kritischen Fällen gerechtfertigt" 693 .

Besonders vielversprechend scheint der Einsatz von AIDS ${ }^{694}$-Modellen. Gegenüber den konventionellen $\mathrm{CDSM}^{695}$ (logit)-Modellen würden wesentlich weniger Informationen über Kreuzpreiselastizitäten benötigt. Lediglich Kenntnisse über Marktanteile, Branchen- und Markenelastizität genügten, um die Effekte einer Fusion vollständig zu simulieren. In einem Beispiel für die Analyse eines Zusammenschlusses in der Cerealienindustrie zeigen EPSTEIN und RUBINFELD (2001), dass für eine ökonometrische Analyse anstelle der bisher 40.000 benötigen Daten lediglich die Schätzung von 199 Elastizitäten notwendig ist ${ }^{696}$. Damit wäre der Einsatz auch für Fälle denkbar - und vom Aufwand zumutbar -, für die bisher keine ausreichenden Preisdaten (z.B. LEH

\footnotetext{
690 Vgl. CameSACA 2000, S. 404; auch RölleR/Stennek/Verboven 2000 S. 110 u. 113

${ }^{691}$ Vgl. Neven/Nuttel/Seabright 1993, S. 56; Aiginger/Wieser/Wüger 2001, S. 10

692 Grünbuch FKVO 2001, S. 43, Rdnr. 150 ff.

${ }^{693}$ Auskunft Bundeskartellamt vom 05.09.2002

${ }^{694}$ AIDS = Almost Ideal Demand System

${ }^{695}$ CDSM $=$ calibrated demand simulation models (logit model)

${ }^{696}$ Vgl. EPSTEIN/RUBINFELD 2001, S. 14
} 
wand zumutbar -, für die bisher keine ausreichenden Preisdaten (z.B. LEH Scannerdatensätze) vorliegen ${ }^{697}$.

\subsubsection{Positive Effekte}

Ebenso sind bei der Kontrolle positive Effekte von Fusionen zu beachten. Effizienzen können z.B. durch Rationalisierung, economies of scale, technischen Fortschritt, Forschung \& Entwicklung, Einkaufsvorteile sowie die Vermeidung von Managermüdigkeit ${ }^{698}$ begründet sein und spiegeln sich in niedrigeren Produktionskosten wider. Die fusionsbetroffenen Parteien haben dann generell zwei Möglichkeiten, hiervon zu profitieren: a) Preis steigern und Menge reduzieren oder b) Preis senken und Menge ausdehnen. A priori kann keine allgemeine Aussage getroffen werden. Tendenziell überwiegt jedoch die zweite Strategie - je größer die Synergievorteile sind ${ }^{699}$. Insider und Verbraucher profitieren in diesem Fall, während die Outsider das Nachsehen haben. Ökonomisch sind Fusionen mit Synergievorteilen zu begrüßen, da beispielsweise eine Vermeidung von Fixkosten erreicht wird und somit Ressourcen eingespart werden. Dennoch muss aus Sicht einer verbrauchernahen Wettbewerbspolitik garantiert sein, dass diese Vorteile auch an die Konsumenten in Form von niedrigeren Preisen (bessere Qualität, Produktvielfalt etc.) weitergegeben werden (sonst profitieren lediglich die Produzenten $)^{700}$.

Das GWB trägt zusammenschlussbedingten Effizienzvorteilen im Rahmen eines gesonderten Ausnahmeverfahrens (Ministererlaubnis) Rechnung. Dabei wird die Prüfung und Gegenüberstellung der Vor- und Nachteile eines Zusammenschlusses einer politischen Instanz, dem Bundesministerium für Wirtschaft, übertragen. Sobald gesamtwirtschaftliche Vorteile auch unter Berücksichtigung wettbewerbsfremder Gründe mit einem Zusammenschluss resultieren (Gesamtwohlfahrtsansatz), kann das Ministerium einen Zusammenschluss trotz Bedenken des Kartellamtes erlauben ${ }^{701}$ (vgl. Kapitel 5.1.7). Der wesentliche Unterschied zwischen deutscher und europäischer Zusammenschlusskontrolle besteht also darin, dass mit der Ministererlaubnis im deutschen Recht zusätzlich gesellschaftspolitische Gemeinwohlvorteile berücksichtigt werden können ${ }^{702}$. Der europäische Ansatz stellt ein nicht zu vernachlässigendes Problem in der Fusions-

\footnotetext{
${ }^{697}$ Vgl. EPSTEIN/RUBINFELD 2001, S. 12

${ }^{698}$ Gefahr besteht, von besseren ersetzt zu werden, daher optimaler Einsatz

${ }^{699} \mathrm{Vgl}$. MOTTA 2000, S. 11

700 Unter Umständen kann sogar die Fusion eines Duopols zu einem Monopol aus Sicht der Verbraucher positiv sein, wenn die Kostenvorteile die Marktmachtnachteile übersteigen (Williamson Modell).

${ }^{701}$ Vgl. KinNe 2000, S. 94
} 
kontrolle dar ${ }^{703}$. Allein funktionell handelt es sich im Gegensatz zum Bundeskartellamt bei der Kommission um eine politische Institution. In der Literatur wird von daher aufgrund gewisser Entscheidungen die wettbewerbspolitische Unabhängigkeit angezweifelt $^{704}$ (Industriepolitik). Die deutsche Regelung hat in diesem Punkt den Vorteil, dass eine politische Einflussnahme nur durch eine Ministererlaubnis denkbar ist und somit transparent wird ${ }^{705}$. Dennoch muss zur Entlastung der Kommission darauf aufmerksam gemacht werden, dass der Rechtsprechung ein relativ enger Rahmen ${ }^{706}$ vorgegeben ist und somit willkürliche Entscheidungen nur schwer durchsetzbar sind. Durch die Möglichkeit einer Drittklage ist außerdem ein weiteres Instrumentarium geschaffen worden, welches die Gefahr einer Beeinflussbarkeit weiter mindert ${ }^{707}$.

Ein Problem ergibt sich bei der Bewertung der Synergievorteile. Durch eine gewisse Informationsasymmetrie sind die Entscheidungsträger auf externe Informationen angewiesen. Dabei haben die Fusionspartner den Anreiz, Synergievorteile möglichst hoch anzugeben, während die Konkurrenz genau den gegenteiligen Effekt fördern wird ${ }^{708}$. Expertenbefragungen und empirische Studien, die den Zusammenhang von Produktionskosten und Preisen erforschen, aber auch Marktbeobachtungen können somit wesentlich zur Informationsgewinnung beitragen ${ }^{709}$.

\footnotetext{
702 Vgl. dazu KinNe 2000, S. 170

${ }^{703} \mathrm{Vgl}$. KeRBER 2000, S. 89

${ }^{704} \mathrm{Vgl.} \mathrm{SCHMIDT/SCHMIDT} \mathrm{1997,} \mathrm{S.} 103 \mathrm{ff}$.

705 Vgl. MARSCHALL 2002, S. 26

${ }^{706}$ Vgl. KeRBER 2000, S. 90

707 Vgl. dazu im deutschen Recht $\S 63$ Abs. 2 und $\S 54$ Abs. 2 Nr. 3 sowie Einflussnahme über $\S 25$ GWB. Auf europäischer Ebene Art 18 FKVO; für die praktische Anwendung und Bedeutung: vgl. BöGE 2001

708 Vgl. MOTTA 2000, S. 12

709 Zu begrüßen ist der aktuelle Versuch der Europäischen-Kommission einen „Expertenrat" aufzubauen, zu dem sachkundige Institutionen aus Wissenschaft und Wirtschaft als Sachverständige bei Fusionsfällen herangezogen werden (http://europa.eu.int/comm/dgs/competition/call_for_experts/).
} 


\section{Praktische Anwendung ökonomischer Instrumente zur Ermittlung von Marktmachteffekten und Effizienzvor- teilen}

In den vorigen Abschnitten wurde darauf hingewiesen, dass bei der wettbewerbspolitischen Beurteilung von Fusionen positive Effekte (Synergieeffekte) berücksichtigt werden sollten. Dabei stehen die Behörden vor einem Informationsproblem, denn nur Branchenkenner verfügen über die relevanten Informationen. Daher werden in diesem Abschnitt mögliche Größenvorteile für Unternehmen der Zucker- und Milchindustrie im Detail beschrieben (Studien und Expertengespräche). Ebenfalls wird versucht zu anaIysieren, inwieweit die Vorteile einer Fusion tatsächlich in die Realität umgesetzt werden konnten (ex post Analyse anhand einer Bilanzanalyse), um daraus Einschätzungen für zukünftige Beurteilungen zu gewinnen. Außerdem wird der Versuch unternommen, Erkenntnisse über das Wettbewerbsverhalten anhand eines Datensatzes zu überprüfen. Hingewiesen sei an dieser Stelle darauf, dass es sich bei den Ausführungen um einen Auszug möglicher ökonomischer Instrumente (weitere wurden bereits in den vorigen Ausführungen aufgegriffen) handelt, die jeweils mit Vor- und Nachteilen behaftet sind. Anhand ausgewählter Zusammenschlüsse aus der Zucker- und Milchindustrie wird konkret gezeigt, welche Erkenntnisse aus den Methoden gewonnen werden könnten. Dadurch würden zukünftige Entscheidungsfindungen der Kartellbehörden weiter gestützt.

\subsection{Kosten der Zuckerproduktion und mögliche Einsparungspo- tentiale: Erkenntnisse aus Expertengutachten}

Die Kosten der Zuckerproduktion hängen von vielen Faktoren ab. Sowohl natürliche wie auch technische Einflussfaktoren bestimmen den Erfolg und die Wettbewerbsfähigkeit verschiedener Unternehmen. In Abbildung 39 sind die wesentlichen Wirkungszusammenhänge kostenrelevanter Faktoren dargestellt. 
Abbildung 39: Wirkungszusammenhänge kostenrelevanter Faktoren bei der Zuckerproduktion

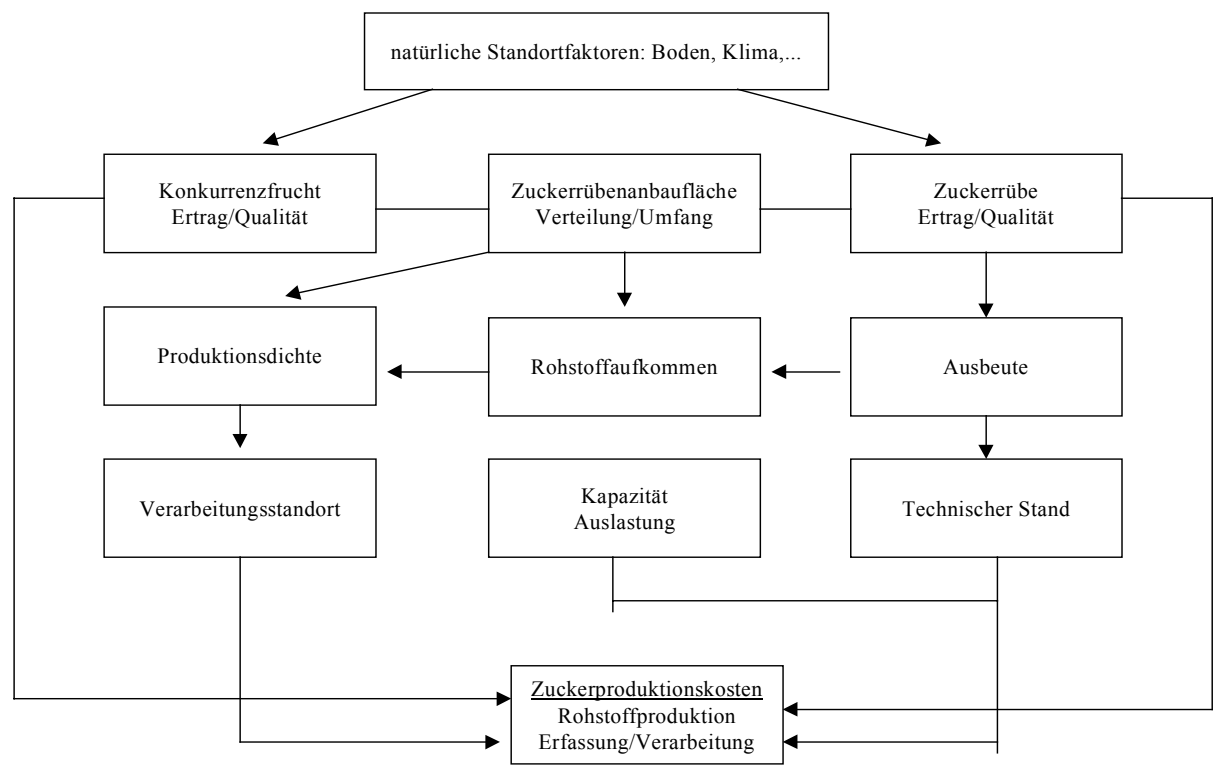

Quelle: RENDER 1989, S. 18

\subsubsection{Vorüberlegungen zur Standortbestimmung der Zuckerrübenver- arbeitung}

Die Raumwirtschaftstheorie legt zugrunde, dass sich unter den Bedingungen des vollkommenen Wettbewerbs eine räumliche Verteilung der Wirtschaftsstrukturen ergibt, die sich im Gleichgewicht befindet. Der Gleichgewichtsbegriff beinhaltetet das Aufeinanderwirken der verschiedenen Standortfaktoren, die zu einer regionalen Konzentration bzw. Dekonzentration ökonomischer Systeme führen.

Von der Lage der Fabrik hin zu den Rohstoffeinzugsgebieten geht ein dominierender Einfluss auf die Kosten der Verarbeitungsstufe aus. Bei Betrachtung der Standorte der Zuckerindustrie ist eine eindeutige Ausrichtung der Verarbeitung an den Produktionsstandorten feststellbar ${ }^{710}$. Die Rohstofforientierung der Verarbeitung begründet sich durch das Input-Outputverhältnis des Verarbeitungsprozesses von ca. sechs bis sieben

710 So auch RENDER 1989, S. 41 
Gewichtsteilen Rüben zu einem Gewichtsteil Zucker ${ }^{711}$. Die Einflussfaktoren der Standortwahl werden in Abbildung 40 skizziert.

Abbildung 40: Interdependenzen bei der Standort- und Betriebsgrößenbestimmung in der Zuckerindustrie

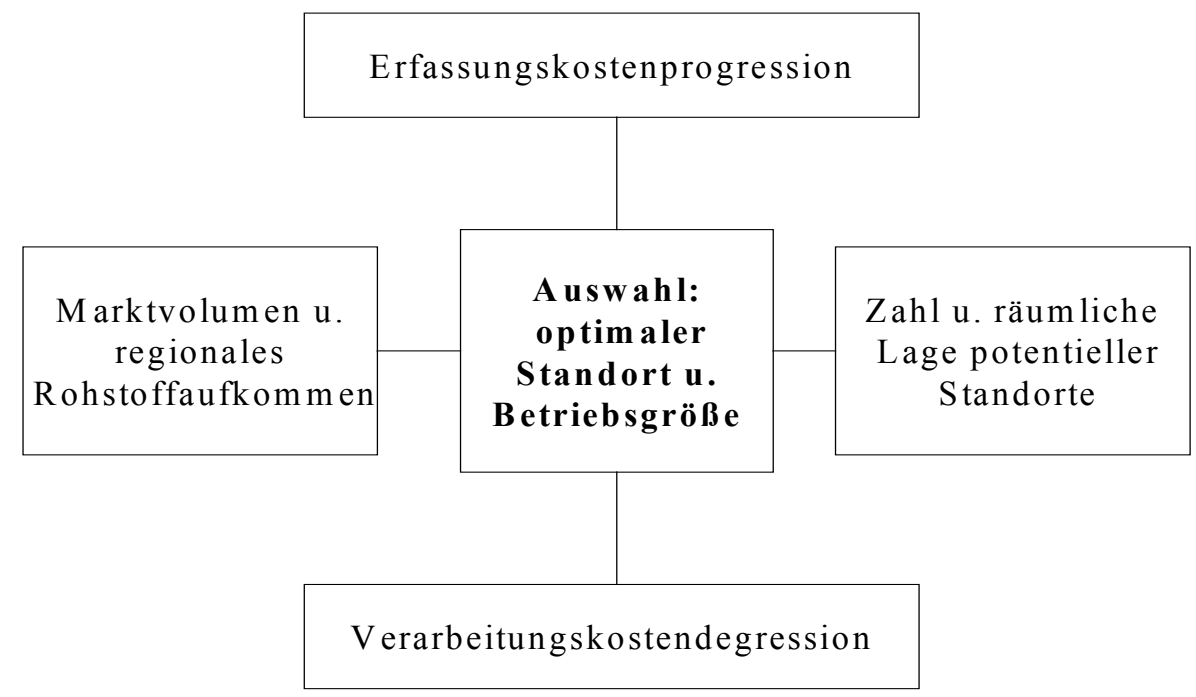

Quelle: RENDER 1989, S. 43

Prinzipiell wird das Optimum der Zuckerproduktion durch die Verarbeitungskostendegression und die Erfassungskostendegression bestimmt. Einige Faktoren, die Entscheidungsvariablen der zuckerproduzierenden Unternehmen darstellen, werden nachfolgend näher erläutert (Faktoren wie Rübenertrag pro ha oder Zuckergehalt pro Rübe haben zwar erheblichen Einfluss auf die Rentabilität der Zuckerproduktion, sind aber weitestgehend von den Entscheidungen der Manager unbeeinflussbar und betreffen alle Teilnehmer innerhalb einer Branche gleichermaßen).

\subsubsection{Erfassungskosten versus Fixkostendegression}

\subsubsection{Erfassungskosten}

Bei gegebenen Frachtsätzen hängen die durchschnittlichen Erfassungskosten je Tonne Weißzucker einer Fabrik ab von ${ }^{712}$ :

- der regionalen Produktionsdichte an Rüben,

- (dem regionalen Marktanteil der Fabrik),

\footnotetext{
${ }^{711} \mathrm{Vgl.} \mathrm{ABN-AMRO} 1996$, S. 6 sowie eigene Berechnungen

712 Vgl. RENDER 1989, S. 54; BLUMENCRON 2000, S. 47
} 
- der jährlichen Produktionsmenge,

- dem ausbeutbaren Zuckergehalt,

- dem Schmutzanteil der Rüben,

- den Verkehrs- und topographischen Verhältnissen,

- dem Erfassungssystem.

Beispielhaft sind in der folgenden Tabelle (Tabelle 34) Faktoren und deren Einfluss dargestellt:

Tabelle 34: Erfassungskosten einer Zuckerfabrik in DM/t Weißzucker

\begin{tabular}{|c|c|c|c|c|c|c|c|c|c|c|c|c|}
\hline \multirow{4}{*}{$\begin{array}{l}\text { Pro- } \\
\text { dukti- } \\
\text { ons- } \\
\text { dichte } \\
\text { t/qkm }\end{array}$} & \multirow{4}{*}{$\begin{array}{l}\text { Markt- } \\
\text { anteil } \\
\%\end{array}$} & \multirow{4}{*}{$\begin{array}{c}\text { Schmutzan- } \\
\text { hang } \\
\\
\%\end{array}$} & \multicolumn{10}{|c|}{ tägliche Verarbeitungsmenge in Tonnen/Tag } \\
\hline & & & \multicolumn{2}{|c|}{3000} & \multicolumn{2}{|c|}{6000} & \multicolumn{2}{|c|}{10000} & \multicolumn{2}{|c|}{15000} & \multicolumn{2}{|c|}{20000} \\
\hline & & & \multirow[b]{2}{*}{12} & \multirow[b]{2}{*}{14} & \multicolumn{4}{|c|}{ Zuckerausbeute in \% } & \multirow[b]{2}{*}{12} & \multirow[b]{2}{*}{14} & \multirow[b]{2}{*}{12} & \multirow[b]{2}{*}{14} \\
\hline & & & & & 12 & 14 & 12 & 14 & & & & \\
\hline 50 & 50 & 10 & 107 & 92 & 127 & 109 & 160 & 138 & 197 & 169 & 226 & 193 \\
\hline 50 & 50 & 20 & 117 & 100 & 139 & 119 & 175 & 150 & 215 & 184 & 246 & 211 \\
\hline 50 & 100 & 10 & 84 & 72 & 107 & 92 & 122 & 105 & 139 & 119 & 160 & 138 \\
\hline 50 & 100 & 20 & 92 & 79 & 117 & 100 & 133 & 114 & 152 & 130 & 175 & 150 \\
\hline 200 & 50 & 10 & 72 & 61 & 84 & 72 & 99 & 85 & 114 & 97 & 122 & 105 \\
\hline 200 & 50 & 20 & 78 & 67 & 92 & 79 & 108 & 93 & 124 & 106 & 133 & 114 \\
\hline 200 & 100 & 10 & 62 & 53 & 72 & 61 & 79 & 68 & 89 & 76 & 98 & 84 \\
\hline 200 & 100 & 20 & 68 & 58 & 78 & 67 & 86 & 74 & 97 & 83 & 107 & 92 \\
\hline 500 & 50 & 10 & 59 & 50 & 69 & 59 & 74 & 64 & 84 & 72 & 94 & 80 \\
\hline 500 & 50 & 20 & 64 & 55 & 75 & 64 & 81 & 69 & 92 & 79 & 102 & 87 \\
\hline 500 & 100 & 10 & 49 & 42 & 58 & 50 & 68 & 58 & 72 & 61 & 75 & 64 \\
\hline 500 & 100 & 20 & 53 & 45 & 63 & 54 & 74 & 63 & 78 & 67 & 82 & 70 \\
\hline
\end{tabular}

Quelle: Render 1989, S. 56; BLUMENCRON 2000, S. 47

\subsubsection{Kosten der Verarbeitung}

Den tendenziell steigenden Erfassungskosten stehen die sinkenden Kosten der Zuckerrübenverarbeitung gegenüber. Diese sind ebenfalls von verschiedenen Faktoren abhängig. Neben der Entwicklung der variablen Kosten wie Energie und Arbeitskosten ist im wesentlichen der Anteil der Fixkosten von besonderer Bedeutung, der potentiell durch Mehrauslastung oder Kapazitätsvergrößerung einer Fabrik gesenkt werden kann. Dazu einige nähere Erläuterungen. 


\subsection{Kampagnelänge}

Naturgemäß fällt der Rohstoff in den Herbst-/Wintermonaten an (Saisonalität des Rohstoffangebots). Da Zuckerrüben einer Verderblichkeit (Verschlechterung der Qualitätseigenschaften) unterliegen und eine Lagerung somit nur begrenzt möglich ist (Funktion von Kosten und Qualität), ist die Eingrenzung des Verarbeitungszeitraumes naturgemäß vorgegeben. Zwar könnte eine Verlängerung der Kampagne erhebliche Einsparungspotentiale bergen (vgl. Tabelle 35), ist aber aufgrund der Verderblichkeit des Rohstoffes stark eingeschränkt ${ }^{713}$.

Tabelle 35: Kostendegression der Zuckerproduktion in Abhängigkeit von der Kapazitätsauslastung in DM/t Weißzucker

\begin{tabular}{|c|c|c|}
\hline $\begin{array}{c}\text { Zeitliche Auslastung } \\
\text { (Tage) }\end{array}$ & $\begin{array}{c}\text { Kapazität 6000 t/Tag } \\
\text { DM/t Weißzucker }\end{array}$ & $\begin{array}{c}\text { Kapazität 10.000 t/Tag } \\
\text { DM/t Weißzucker }\end{array}$ \\
\hline 60 & 720 & 601 \\
\hline 70 & 651 & 547 \\
\hline 80 & 601 & 509 \\
\hline 90 & 566 & 482 \\
\hline 100 & 543 & 466 \\
\hline
\end{tabular}

Quelle: SCHMIDT 1991, S. 345 in Anlehnung an ReNDER 1989, S. 53

Die empirischen Kampagnelängen der Zuckerfabriken in Deutschland und in Europa sind Tabelle 36 zu entnehmen.

Tabelle 36: Durchschnittliche Kampagnelänge

Kampagnelänge (in Tagen)

$\begin{array}{llllllllll}\text { Wirtschaftsjahr } 90 / 91 & 91 / 92 & 92 / 93 & 93 / 94 & 94 / 95 & 95 / 96 & 96 / 97 & 97 / 98 & 98 / 99 & 99 / 00\end{array}$

$\begin{array}{lllllllllll}\text { Deutschland } & 89 & 81 & 85 & 92 & 75 & 80 & 84 & 82 & 85 & 90\end{array}$

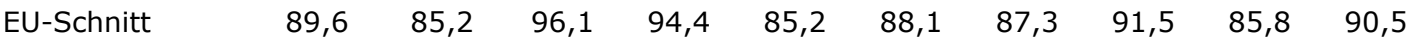

Quelle: CEFS 2000, S. 2

\subsection{Investitionskosten}

Nach RENDER, der anhand von Investitionsdaten der Beratungsfirma IPRO auf Größenvorteile in Abhängigkeit von der Betriebsgröße schloss, kann die Erkenntnis gewonnen werden, dass sich ebenfalls beim Investitionsvolumen mit Anstieg der Zuckerrübenverarbeitungsmengen erhebliche Einsparungspotentiale vermuten lassen. Dazu folgende Auflistung (Tabelle 37):

${ }^{713}$ Vgl. auch ABN-AMRO 1996, S. 6 
Tabelle 37: Kosten der Zuckerverarbeitung (DM je Tonne Weißzucker)

\begin{tabular}{|c|c|c|c|c|c|}
\hline Kapazität in t Rüben/Tag & 3000 & 6000 & 10000 & 15000 & 20000 \\
\hline Verarbeitungskosten & 679,2 & 569,5 & 485,6 & 453,3 & 437,4 \\
\hline Fixkosten & 465,4 & 373,3 & 295,4 & 266,3 & 252 \\
\hline Abschreibung (1) & 156,2 & 115,7 & 86,8 & 82 & 79,6 \\
\hline davon Gebäude & 23 & 17 & 12,8 & 12,1 & 11,7 \\
\hline davon Anlage & 133,3 & 98,7 & 74 & 69,9 & 67,9 \\
\hline Zinsen (2) & 58,1 & 43,1 & 32,3 & 30,5 & 29,6 \\
\hline Reparaturen (3) & 72,2 & 53,5 & 40,1 & 37,9 & 36,8 \\
\hline Personalkosten & 135,2 & 128,6 & 111,9 & 93 & 83,8 \\
\hline sonst. Kosten (4) & 43,7 & 32,4 & 24,3 & 23 & 22,3 \\
\hline variable Kosten & 213,8 & 196,3 & 190,2 & 187 & 185,4 \\
\hline Energie (5) & 109,3 & 109,3 & 109,3 & 109,3 & 109,3 \\
\hline Personalkosten & 39,7 & 22,2 & 16,1 & 12,9 & 11,3 \\
\hline Sonst. Betriebsmittel (6) & 23,9 & 23,9 & 23,9 & 23,9 & 23,9 \\
\hline Zinsen, Umlaufkapital (7) & 30,7 & 30,7 & 30,7 & 30,7 & 30,7 \\
\hline Ausbeuteverlust (8) & 10,2 & 10,2 & 10,2 & 10,2 & 10,2 \\
\hline
\end{tabular}

Anm.: (1) zu Wiederbeschaffungskosten des Jahres 1986, bei einer Nutzungsdauer des Gebäudes von 50 Jahren und der Anlagen von 16 Jahren; (2) kalkulatorischer Zinssatz von 3 \%, ohne Grund und Boden; (3) Instandhaltung $2 \%$ des Anlageneuwertes bzw. $1 \%$ des Gebäudeneuwertes; (4) Steuern, Versicherungen, sonstige Kosten $1 \%$ vom Anschaffungswert; (5) 3 \% schweres Heizöl (500 DM/t); (6) 3,5\% Kalkstein (25 $\mathrm{DM} / \mathrm{t}$ ), 0,35 \% (400 DM/t); (7) $5 \%$ Zinssatz; (8) 0,28\% bewertet mit $500 \mathrm{DM} / \mathrm{t}$ Weißzucker

\section{Quelle: RENDER 1989, S. 49}

Aus den Berechnungen kann geschlossen werden, dass

1. die Verarbeitungskosten je t Weißzucker aufgrund der großen Fixkostenunterschiede eine beachtliche Spannweite (680 bis 437 DM) aufweisen,

2. die variablen Kosten einen Anteil von ca. 30-40\% an den gesamten Verarbeitungskosten ausmachen und betriebsgrößenabhängig nur geringe Unterschiede vorliegen,

3. der Kostensenkungseffekt bei einer Vergrößerung der Zuckerfabrik von $\mathbf{3 0 0 0}$ t/Tag auf 10.000 t/Tag rund 33\% beträgt, während eine Kapazitätserhöhung auf 20.000 t/Tag nur noch Kosteneinsparungen von $10 \%$ ermöglicht. 


\subsubsection{Abwägung}

Die Ausführungen zeigen, dass Zuckerfabriken kapitalintensive Investitionen sind. Aus den vorgestellten Berechnungen und Ergänzungen von WALKENHORST ${ }^{714}$ ergeben sich Investitionskosten von 150 bis 200 Millionen Euro für eine moderne Zuckerfabrik (bei Zugrundelegung einer Nutzungsdauer von 30 bis 40 Jahren). Aufgrund der hohen Fixkostenbelastung ist es daher nicht verwunderlich, dass eine Erhöhung der täglichen Verarbeitung immense economies of scale freisetzen kann ${ }^{715}$. Auch können erhebliche Teile der Arbeitskosten durch Automatisierungsprozesse in großen Fabriken eingespart werden. Demgegenüber stehen die steigenden Erfassungskosten. Werden beide Komponenten berücksichtigt, ergibt sich, dass der hohe Fixkostenanteil und die starke Kostendegression bei der Zuckerrübenverarbeitung zunächst das Wirksamwerden der Erfassungsprogression überdecken. Der Einfluss stark ansteigender Transportkosten von Rüben aus Randbereichen auf die durchschnittlichen Erfassungs- und Verarbeitungskosten der gesamten Rübenmenge ist vergleichsweise gering (vgl. Abbildung 41).

Abbildung 41: Die durchschnittlichen Zuckerrübenverarbeitungs- und erfassungskosten in den kalkulierten Weißzuckerfabriken in Abhängigkeit von der Kapazität und Produktionsdichte

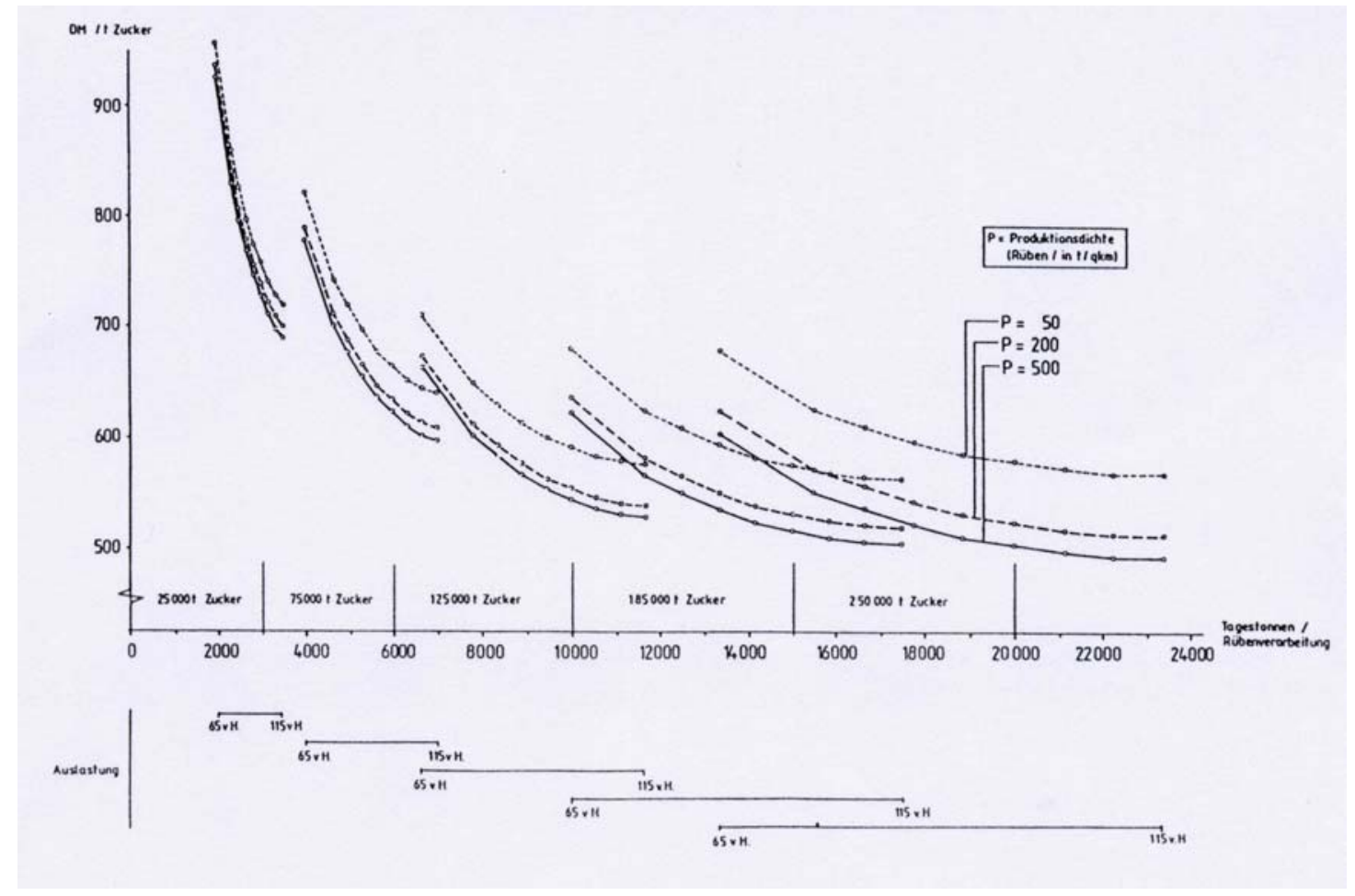

Quelle: RENDER 1989, S. 59

\footnotetext{
714 Zitiert in NEI 2000, S. 179

715 Dazu auch ABN-AMRO 1996, S. 6
} 
Insgesamt herrscht jedoch Uneingigkeit über die optimale Betriebsgröße einer Fabrik. So kam WALKENHORST zu dem Schluss, dass diese bei etwa 10.000 t/Tag erreicht ist, während MAHLER durchaus $20.000 \mathrm{t} /$ Tag als realistisch ansah ${ }^{716}$. Abhängig ist dies von der jeweiligen Produktionsdichte und den damit verbundenen Transportkosten. Tendenziell führt eine niedrigere Produktionsdichte zu niedrigeren optimalen Auslastungsgraden der Fabriken, da Transportkosten die Kostenvorteile einer größeren Fabrik überkompensieren würden ${ }^{717}$.

Bei Betrachtung der durchschnittlichen europäischen Fabrikgröße ${ }^{718}$ kann ein Optimum bei etwa 10.000 bis 15.000 t Tagesverarbeitung vermutet werden. Dazu folgende Übersicht (Tabelle 38):

Tabelle 38: Entwicklung der tatsächlichen Betriebsgrößenstrukturen

Anzahl und Kapazitäten der Zuckerfabriken

\begin{tabular}{|c|c|c|c|c|c|c|c|c|c|c|}
\hline Jahr & $90 / 91$ & $91 / 92$ & $92 / 93$ & $93 / 94$ & $94 / 95$ & $95 / 96$ & $96 / 97$ & $97 / 98$ & $98 / 99$ & $99 / 0 \mathrm{C}$ \\
\hline \multicolumn{11}{|l|}{ Deutschland } \\
\hline$<5000 t$ & 8 & 30 & 21 & 10 & 8 & 6 & 6 & 4 & 3 & 4 \\
\hline $5000-8000 \mathrm{t}$ & 18 & 19 & 16 & 18 & 17 & 13 & 9 & 7 & 8 & 6 \\
\hline $8000-12000 t$ & 6 & 6 & 10 & 11 & 11 & 13 & 20 & 20 & 21 & 19 \\
\hline$>12000 t$ & 5 & 5 & 5 & 4 & 6 & 7 & 1 & 3 & 2 & 3 \\
\hline total & 37 & 60 & 52 & 43 & 42 & 39 & 36 & 34 & 34 & 32 \\
\hline
\end{tabular}

\section{Europa}

$\begin{array}{lcccccccccc}<5000 \mathrm{t} & 53 & 72 & 51 & 29 & 25 & 26 & 29 & 20 & 12 & 12 \\ 5000-8000 \mathrm{t} & 69 & 67 & 59 & 59 & 53 & 53 & 42 & 39 & 43 & 37 \\ 8000-12000 \mathrm{t} & 44 & 42 & 46 & 46 & 53 & 61 & 78 & 81 & 83 & 78 \\ >12000 \mathrm{t} & 21 & 23 & 27 & 26 & 31 & 32 & 16 & 19 & 16 & 20 \\ \text { total } & 187 & 204 & 183 & 160 & 162 & 172 & 165 & 159 & 154 & 147 \\ \text { CEFS countries } & 202 & 218 & 196 & 172 & 174 & 184 & 167 & 161 & 156 & 149\end{array}$

Quelle: CEFS 2000, S. 24

\footnotetext{
${ }^{716} \mathrm{Vgl}$. MAHLER 1992, S. 124 (wie in den vorigen Abschnitten verdeutlicht, gibt es durchaus einzelbetriebliche Abweichungen, die beispielsweise von der Produktionsdichte oder Infrastruktur abhängig sind.)

717 Vgl. NEI 2000, S. 179

${ }^{718}$ Es ist zu bedenken, dass durch die eingeschränkte Übertragbarkeit der Lieferrechte keine optimale Allokation zu den geeignetsten Standorten erfolgen kann.
} 


\subsubsection{Veränderung der Kosten der Zuckerverarbeitung}

Die Untersuchungen von RENDER sind mittlerweile etwa 15 Jahre alt und tragen somit den in der Zwischenzeit aufgetretenen Änderungen der Kostenzusammensetzung insbesondere im Bereich der variablen Kosten, nicht mehr Rechnung. Eine genauere Untersuchung der Veränderung der variablen Kostenzusammensetzung scheint jedoch auch bei Diskussion der Wettbewerbsfähigkeit lohnenswert, da die Rechtfertigung einer angemessenen Verarbeitungsspanne, die der Rat jährlich festlegt, auf eine solide Basis gestellt werden würde und somit der Kritik von Vertretern der zuckerabnehmenden Industrie entgegen käme ${ }^{719}$. Eine konstante Verarbeitungsspanne bei sinkenden variablen Kosten würde somit das Gewinnpotential der Zuckerproduktion erhöhen und einen Fusionsanreiz (externes Wachstum, da Quote nur bedingt handelbar ist) darstellen.

Nach der NEI Studie entwickelten sich die Zuckerproduktionskosten pro Tonne zwischen 1993 und 1998 folgendermaßen (vgl. Tabelle 39 - basierend auf Daten der Rabobank):

Tabelle 39: Entwicklung der Verarbeitungskosten in $€$ pro Tonne

\begin{tabular}{|l|c|c|}
\hline & $\mathbf{1 9 9 3}$ & $\mathbf{1 9 9 8}$ (geschätzt) \\
\hline Rüben & 400 & 388 \\
\hline Personal & 100 & 80 \\
\hline Unterhaltungsmaterial & 52 & 37 \\
\hline Energie & 18 & 15 \\
\hline Kalk & 14 & 9 \\
\hline Gemeinkosten & 20 & 15 \\
\hline Total & $\mathbf{6 2 4}$ & $\mathbf{5 5 9}$ \\
\hline
\end{tabular}

Quelle: RABOBANK 1995, S. 39

\subsubsection{Versuch der Ermittlung einzelner Komponenten}

Einzelbetrieblich können Ausmaß und Zusammensetzung der Kosten durchaus unterschiedliche Größen annehmen. Exemplarisch wird im folgenden versucht, die Entwicklung zweier wichtiger Positionen näher zu analysieren.

719 Z.B. CAOBISCO; IZZ; Deutscher Süßwarenverband 


\subsection{Zur Diskussion des Energieeinsatzes}

Der Energieeinsatz macht laut der Erhebung der europäischen Zuckerhersteller etwa $30 \%$ der Verarbeitungskosten aus ${ }^{720}$.

Anhand der verschiedenen Inputgüter ist die Tendenz feststellbar, das aufgrund von technischem Fortschritt fortlaufend geringere Mengen an Energie und Wasser zur Erzeugung einer Einheit Zucker notwendig sind ${ }^{721}$.

Für den Energiebedarf wird dies auch durch die Untersuchungen des RWI-Essen ${ }^{722}$ bestätigt (siehe Abbildung 42).

Abbildung 42: Spezifischer Energiebedarf in der Zuckerindustrie 1953/54 bis 2005 in kWh/100 kg Rüben

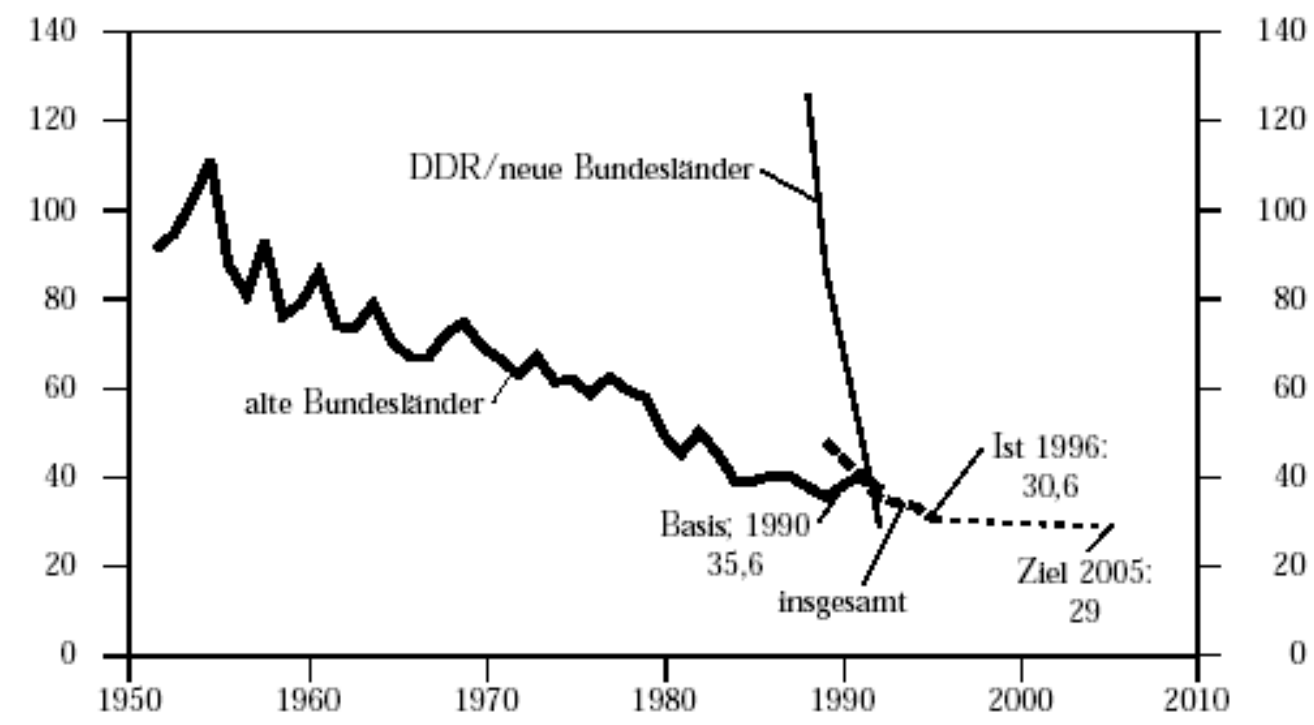

Quelle: RWI-ESSEN 1998, S. 191

Für die gesamte deutsche Zuckerindustrie kann diese Entwicklung nur für die Stromversorgung nachgewiesen werden, da keine ausreichende Datengrundlage vorliegt. Eigene Berechnungen, die auf der Datenbasis des statistischen Bundesamtes beruhen, bestätigen die Ergebnisse. Danach hat sich der Energiebedarf folgendermaßen entwikkelt (vgl. Abbildung 43).

\footnotetext{
${ }^{720}$ Vgl. CIUS, laufende Jahrgänge, vgl. dazu Anhang C

${ }^{721}$ Vgl. NORDZUCKER Umweltberichte, laufende Jahrgänge; wird auch durch Angaben der WVZ 2001, S. 54 bestätigt
} 
Abbildung 43: Entwicklung des Stromverbrauchs pro kg Zuckererzeugung

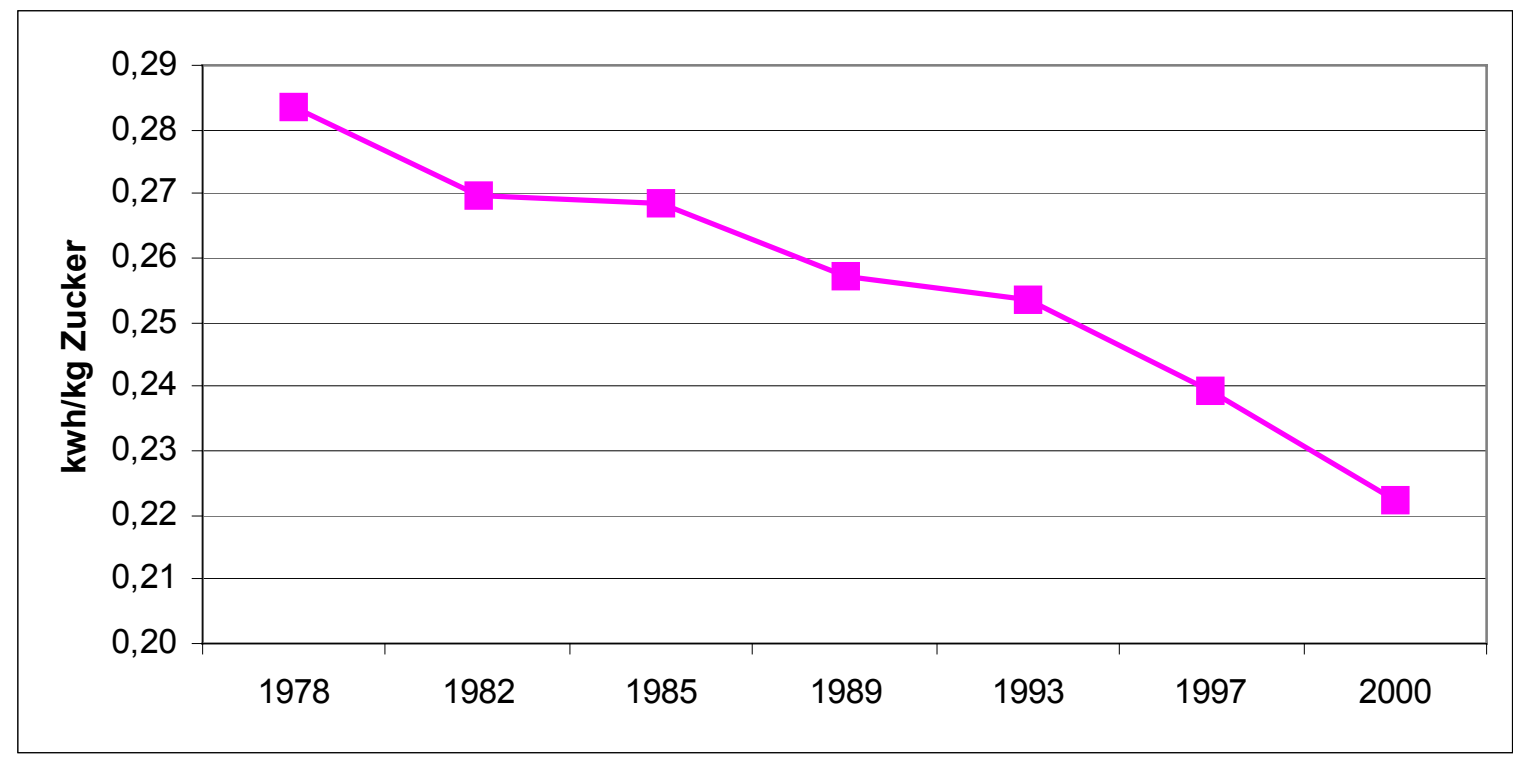

Quelle: eigene Berechnungen; Statistitisches Bundesamt; WVZ

Die Ausgaben für Energie sind bekanntermaßen eine Funktion aus Menge und Preis. Der spezifische Energiebedarf hat sich, wie zuvor dargestellt, stark verringert. Es ist also der Frage nach der Energiepreisentwicklung nachzugehen (vgl. Abbildung 44). Dabei lässt sich feststellen, dass die verschiedenen Energieträger ganz unterschiedlichen Entwicklungen unterlagen.

Abbildung 44: Entwicklung der Preisindices verschiedener Inputfaktoren (Basis 1995)

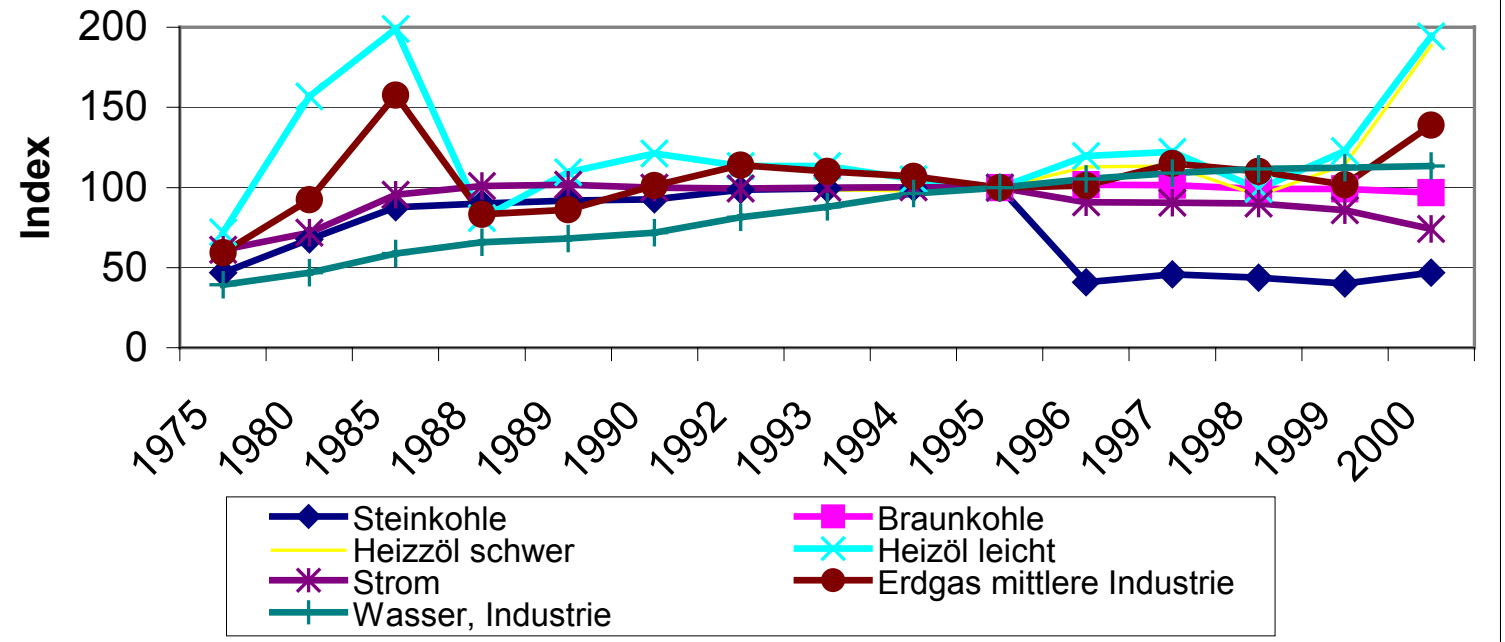

Quelle: VIK 2000, S. 24

722 Rheinisch Westfälisches Institut für Wirtschaftsforschung 
Alles in allem dürfte jedoch die Mengeneinsparung die Preissteigerung (und dadurch z.T. die induzierte Veränderung des Energieträgerportfolios) überkompensiert haben, so dass insgesamt ein geringerer Energiekostenblock pro kg verarbeiteten Zuckers zu Buche schlägt als noch vor 15 Jahren.

\subsection{Der Faktor Arbeit}

Einen weiteren größeren Kostenbestandteil stellt der Faktor Arbeit (Personal) dar, der etwa $25-29 \%$ der Verarbeitungskosten ausmacht ${ }^{723}$. In Gesamtdeutschland betrug die Zuckerproduktion pro Arbeitsstunde 1978165 kg. Durch Produktivitätserhöhung und technischen Fortschritt erhöhte sich die Produktion um den Faktor 3,5 auf $582 \mathrm{~kg}$ im Jahr 2000 (vgl. Abbildung 45).

Abbildung 45: Zuckerproduktion pro Arbeitsstunde

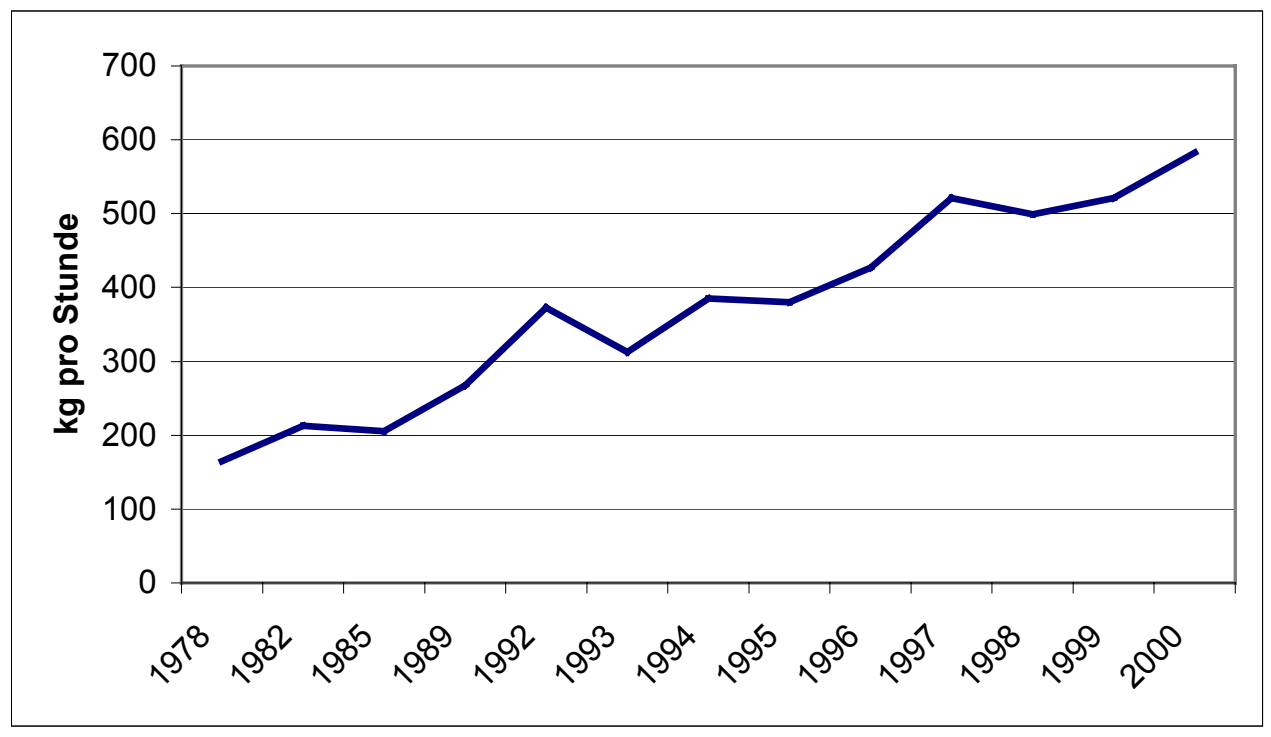

Quelle: STATISTISChes BUndesamT; WVZ; eigene Berechnungen

Obwohl der Stundenlohn in den vergangenen Jahren ständig angestiegen ist (vgl. Abbildung 46), konnten durch Arbeitsproduktivitätszuwächse die Gesamtkosten für Personal reduziert werden (Abbildung 47).

${ }^{723}$ Nach CIUS, vgl. dazu Anhang C 
Abbildung 46: Personalkosten in der Zuckerindustrie

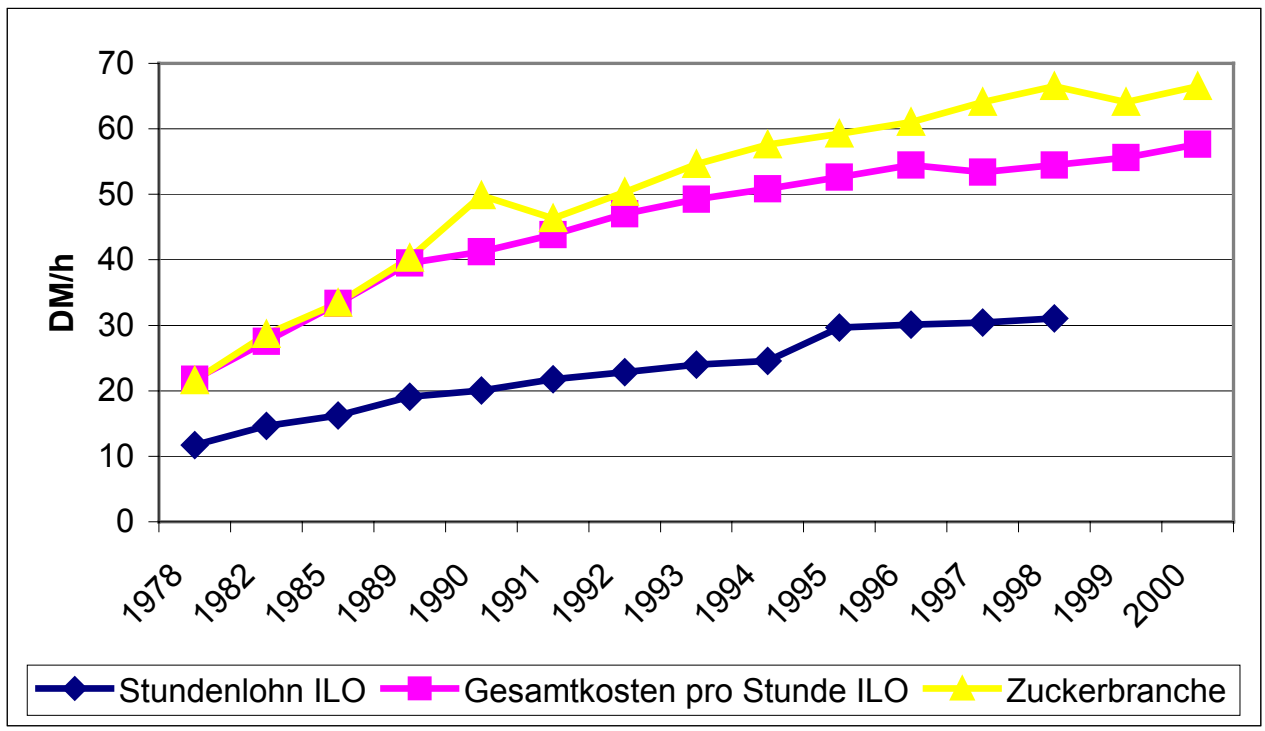

Quelle: ILO Yearbook; StATISTISChes BundesAMT; WVZ; eigene Berechnungen

Abbildung 47: Personalkosten pro kg Zuckererzeugung

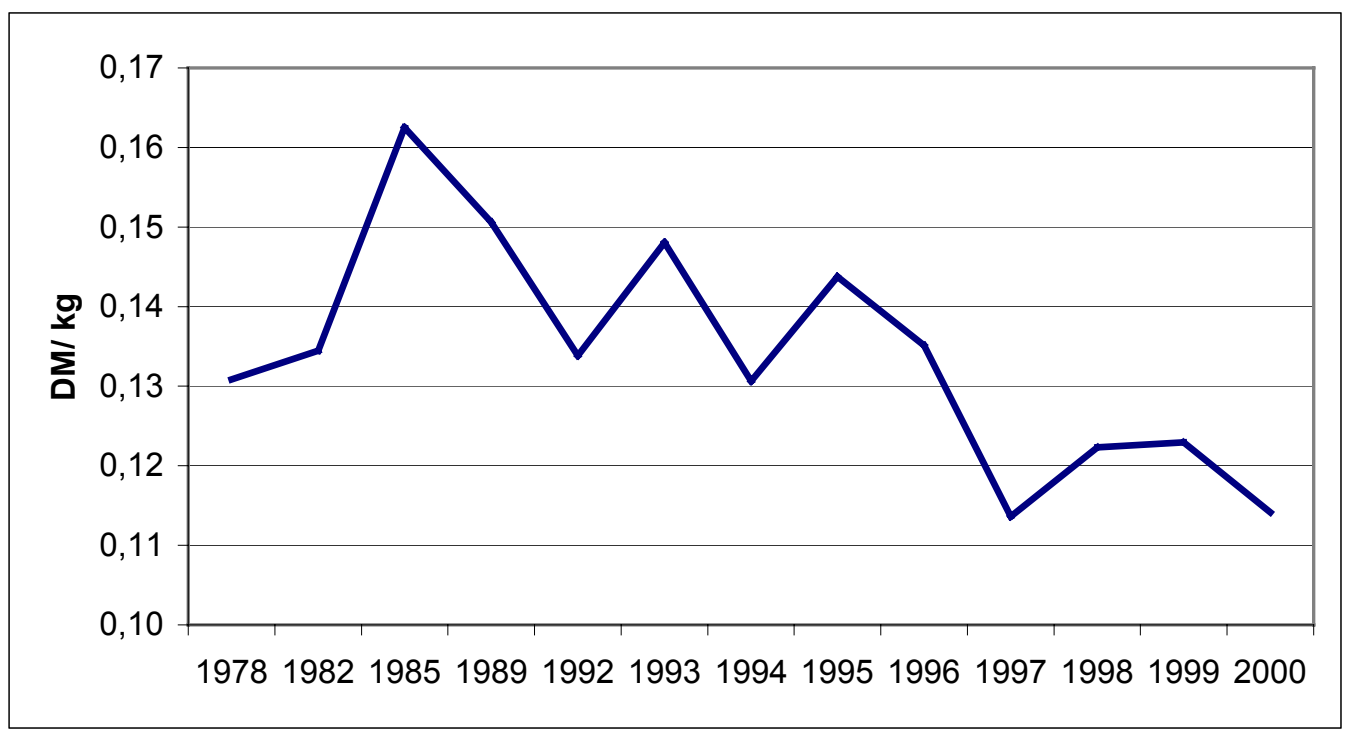

Quelle: STATISTISChes BUndesamt, eigene Berechnungen

Auch beim Kostenfaktor Arbeit zeigt sich, dass zwar die Löhne und Abgaben pro Stunde gestiegen sind, aber die Mehrkosten aufgrund der Produktivitätssteigerung mehr als aufgefangen wurden, so dass insgesamt eine Abnahme der Arbeitskosten zu verzeichnen ist. 


\subsection{Wertung}

Zwei wesentliche Komponenten der Kostenstruktur weisen nach den Untersuchungen sinkende Werte auf. Da die Faktorkosten absolut nicht gesunken sind, müssen die Vorteile auf technischen Fortschritt oder Ausschöpfung von Rationalisierungsvorteilen (Synergievorteile) zurückzuführen sein. Die Kritik an einer sich in der Vergangenheit kaum geänderten Verarbeitungsspanne scheint damit gerechtfertigt zu sein. Dazu jedoch einige nähere Ausführungen.

\subsubsection{Kritik Verarbeitungsspanne}

Wie in dem Kapitel über die Grundregelung der ZMO dargelegt worden ist, setzt der Ministerrat jährlich den Rübenmindest- sowie Interventionspreis für Zucker fest. Die Differenz wird als sogenannte Verarbeitungsspanne bezeichnet und soll die Kosten der Verarbeitung decken. Der europäische Zusammenschluss der Zuckerverwender CIUS hat die Bruttofabrikationsspanne für die Herstellung von einer Tonne Zucker nach einzelnen Kostenarten aufgegliedert. CIUS kommt zu dem Schluss, dass wesentlich niedrigere Kosten angesetzt werden müssten als von der Zuckerwirtschaft veranschlagt. Dabei sind vor allem ein höherer Zuckerertrag pro Tonne Rüben, eine steigende Arbeitsproduktivität und damit verbundene Rationalisierungsgewinne, gesunkene Zinsen, geringere Energiekosten und ein abnehmender Energieverbrauch in Ansatz gebracht worden $^{724}$. Mit Hilfe offizieller Informationsquellen wurden folgende Berechnungen erstellt (Tabelle 40). 
Tabelle 40: Gewinnmarge der Zuckerindustrie in ECU bzw. $\mathrm{C} /$ Tonne

\begin{tabular}{|c|c|c|c|c|c|c|c|c|c|c|c|}
\hline Jahr 19.. & $88 / 89$ & $89 / 90$ & $90 / 91$ & $91 / 92$ & $92 / 93$ & $93 / 94$ & $94 / 95$ & $95 / 96$ & $96 / 97$ & $97 / 98$ & 98/99 \\
\hline Basic Beet Price & 41 & 40 & 40 & 40 & 40 & 39 & 39 & 47,67 & 47,67 & 47,67 & 47,67 \\
\hline $\begin{array}{l}\text { Beet Price adj. } \\
\text { For "Switchover" }\end{array}$ & 49,38 & 48,38 & 48,3 & 48,3 & 48,3 & 47,67 & 47,67 & 48 & 48 & 48 & 48 \\
\hline A+B Levy \% & 10,9 & 6,1 & 9 & 7,4 & 8,3 & 10,1 & 9,2 & 7,7 & 7,7 & 7,7 & 7,7 \\
\hline Levy ECU/Mt & 5,38 & 2,95 & 4,35 & 3,57 & 4,01 & 4,81 & 4,39 & 3,67 & 3,67 & 3,67 & \\
\hline $\begin{array}{l}\text { Beet Price Net of } \\
\text { Levy ECU/Mt }\end{array}$ & 43,99 & 45,43 & 43,95 & 44,73 & 44,29 & 42,86 & 43,29 & 44 & 44 & 44 & 44 \\
\hline $\begin{array}{l}\text { Cost of Beet @ } \\
13 \% \text { extraction }\end{array}$ & 338,41 & 349,49 & 338,1 & 344,05 & 340,7 & 329,67 & 332,97 & 338,46 & 338,46 & 338,46 & 338,46 \\
\hline $\begin{array}{l}\text { Actual extraction } \\
\text { yield \% }\end{array}$ & 87,9 & 87,6 & 87 & 87,8 & 88 & 89,9 & 89,9 & 88,7 & 90 & 90 & 90 \\
\hline $\begin{array}{l}\text { Actual extraction } \\
\text { from Beet @ } \\
16 \%\end{array}$ & 14,1 & 14 & 13,9 & 14 & 14,1 & 14,4 & 14,4 & 14,2 & 14,4 & 14,4 & 14,4 \\
\hline $\begin{array}{l}\text { Cost of Beet @ } \\
\text { actual extraction }\end{array}$ & 312,76 & 324,03 & 315,92 & 318,54 & 314,4 & 298,08 & 301,15 & 310,18 & 305,58 & 305,58 & 305,58 \\
\hline $\begin{array}{l}\text { Transport Charge } \\
\text { ECU/mt }\end{array}$ & 37,3 & 37 & 37 & 37 & 37 & 39,8 & 39,89 & 40,42 & 39,82 & 39,82 & 39,82 \\
\hline $\begin{array}{l}\text { Sugar Interven- } \\
\text { tion Price (IVP) } \\
\text { ECU/Mt }\end{array}$ & 542 & 542 & 530 & 530 & 530 & 523 & 523 & 632 & 632 & 632 & 632 \\
\hline $\begin{array}{l}\text { Sugar IVP adj. } \\
\text { For "switchover" }\end{array}$ & 654,23 & 654,23 & 640,1 & 640,1 & 640,1 & 631,89 & 631,89 & 632 & 632 & 632 & 632 \\
\hline Levy ECU/Mt & 71,31 & 39,91 & 57,61 & 47,37 & 53,13 & 63,82 & 58,13 & 48,66 & 48,66 & 48,66 & 48,66 \\
\hline $\begin{array}{l}\text { Sugar IVP net of } \\
\text { Levy ECU/ Mt }\end{array}$ & 582,92 & 614,32 & 582,49 & 592,73 & 586,97 & 568,07 & 573,76 & 583,24 & 583,24 & 583,24 & 583,24 \\
\hline $\begin{array}{l}\text { Theoretical Gross } \\
\text { Margin }\end{array}$ & 207,21 & 227,83 & 207,39 & 211,69 & 209,27 & 194,3 & 200,9 & 204,37 & 204,97 & 204,97 & 204,97 \\
\hline $\begin{array}{l}\text { Theoretical Gross } \\
\text { Margin \% }\end{array}$ & 35,5 & 37,1 & 35,6 & 35,7 & 35,7 & 34,2 & 35 & 35 & 35,1 & 35,1 & 35,1 \\
\hline $\begin{array}{l}\text { Gross Margin @ } \\
\text { actual extraction }\end{array}$ & 232,86 & 253,29 & 229,58 & 237,2 & 235,57 & 225,88 & 232,72 & 232,64 & 237,84 & 237,84 & 237,84 \\
\hline $\begin{array}{l}\text { Gross Margin @ } \\
\text { actual extraction } \\
\text { as \% }\end{array}$ & 39,9 & 41,2 & 39,4 & 40 & 40,1 & 39,8 & 40,6 & 39,9 & 40,8 & 40,8 & 40,8 \\
\hline $\begin{array}{l}\text { Income from By } \\
\text { Products }\end{array}$ & 32,62 & 28,35 & 29,33 & 27,79 & 30,65 & 29,6 & 26,92 & 28,1 & 30,98 & 30,98 & 31,69 \\
\hline $\begin{array}{l}\text { Sales Price Pre- } \\
\text { mium/IVP \% }\end{array}$ & 10 & 10 & 10 & 11 & 11 & 11 & 12 & 13 & 13 & 13 & 13 \\
\hline $\begin{array}{l}\text { Price Premium } \\
\text { ECU/Mt }\end{array}$ & 65,42 & 65,42 & 64,01 & 67,23 & 68,61 & 69,98 & 74,04 & 81,26 & 81,26 & 81,26 & 81,26 \\
\hline $\begin{array}{l}\text { Average Sales } \\
\text { Price }\end{array}$ & 648,34 & 679,74 & 646,5 & 659,97 & 655,58 & 638,05 & 647,8 & 664,51 & 664,51 & 664,51 & 664,51 \\
\hline $\begin{array}{l}\text { Total Real Gross } \\
\text { Margin ecu }\end{array}$ & 330,9 & 347,06 & 322,92 & 332,22 & 334,83 & 325,47 & 333,68 & 342 & 350,08 & 350,08 & 350,79 \\
\hline
\end{tabular}

${ }^{724}$ Vgl. CIUS 1999 


\begin{tabular}{|c|c|c|c|c|c|c|c|c|c|c|c|}
\hline $\begin{array}{l}\text { Tabelle } 40 \\
\text { (Fortsetzung) }\end{array}$ & & & & & & & & & & & \\
\hline Jahr $19 .$. & $88 / 89$ & $89 / 90$ & $90 / 91$ & $91 / 92$ & $92 / 93$ & 93/94 & 94/95 & $95 / 96$ & $96 / 97$ & $97 / 98$ & 98/99 \\
\hline $\begin{array}{l}\text { Total Real Gross } \\
\text { Margin \% }\end{array}$ & 46 & 48,2 & 45,9 & 47 & 47,2 & 46,4 & 47,3 & 48 & 49,1 & 49,1 & 49,2 \\
\hline \multicolumn{12}{|l|}{$\begin{array}{l}\text { Processing Cost } \\
\text { Estimates }\end{array}$} \\
\hline Labour & 69,74 & 68,78 & 65,01 & 63,86 & 61,23 & 58,7 & 56,45 & 54,19 & 52,14 & 50,15 & 48,23 \\
\hline Energy & 80,07 & 65,08 & 69,46 & 68,62 & 72,28 & 59,34 & 56,07 & 64,12 & 72,72 & 55,71 & 38,04 \\
\hline Depreciation & 33,1 & 33,1 & 32,39 & 32,54 & 32,6 & 29,35 & 31,06 & 29,95 & 28,53 & 27,62 & 27,62 \\
\hline Other & 11,02 & 11,56 & 10,99 & 11,22 & 11,14 & 10,85 & 11,01 & 11,3 & 11,3 & 11,3 & 11,3 \\
\hline $\begin{array}{l}\text { Distribution \& } \\
\text { Administration }\end{array}$ & 54,46 & 57,1 & 54,31 & 55,44 & 55,07 & 53,6 & 54,42 & 55,82 & 55,82 & 55,82 & 55,82 \\
\hline Total Cost & 248,39 & 235,62 & 232,15 & 231,67 & 232,32 & 211,83 & 209,01 & 215,38 & 220,5 & 200,59 & 181,01 \\
\hline $\begin{array}{l}\text { Total Cost as } \\
\text { percentage }\end{array}$ & 38,3 & 34,7 & 35,9 & 35,1 & 35,4 & 33,2 & 32,3 & 32,4 & 33,2 & 30,2 & 27,2 \\
\hline $\begin{array}{l}\text { Operating Margin } \\
\text { ECU/Mt }\end{array}$ & 82,51 & 11,45 & 90,76 & 100,54 & 102,51 & 113,64 & 124,67 & 126,63 & 129,58 & 149,49 & 169,76 \\
\hline $\begin{array}{l}\text { Operating Margin } \\
\%\end{array}$ & 12,7 & 16,4 & 14 & 15,2 & 15,6 & 17,8 & 19,2 & 19,1 & 19,5 & 22,5 & 25,6 \\
\hline $\begin{array}{l}\text { Interest Receiv- } \\
\text { able }\end{array}$ & 67,02 & 78,72 & 78,41 & 71,34 & 47,95 & 41,19 & 22,16 & 26,37 & 30,5 & 28,93 & 28,93 \\
\hline $\begin{array}{l}\text { Interest Rates } \\
\text { Euro \% }\end{array}$ & 8,3 & 9,3 & 9,8 & 8,7 & 5,9 & 5,2 & 2,8 & 3,2 & 3,7 & 3,5 & 3,5 \\
\hline Net Profit ECU/Mt & 149,53 & 190,17 & 169,18 & 171,89 & 150,46 & 154,83 & 146,83 & 153 & 160,08 & 176,43 & 198,7 \\
\hline Net Profit \% & 23,1 & 26 & 26,2 & 26 & 23 & 24,3 & 22,7 & 23 & 24,1 & 26,9 & 29,9 \\
\hline $\begin{array}{l}\text { Cash Flow } \\
\mathrm{ECU} / \mathrm{Mt}\end{array}$ & 806,51 & 845,57 & 804,22 & 820,97 & 815,51 & 793,71 & 805,83 & 826,62 & 826,62 & 826,62 & 826,62 \\
\hline
\end{tabular}

Quelle: CIUS 1999, S. 21 ff.

Aus den Berechnungen ergibt sich ein erhebliches Preisreduktionspotential, welches anhand der Auflistung in Tabelle 41 verdeutlicht wird.

Tabelle 41: Differenz zwischen theoretischer und praktischer Bewertung

\begin{tabular}{|l|l|l|l|}
\hline$€ /$ Tonne & $\begin{array}{l}1998 / 99 \\
\text { Offizielle } \\
\text { Daten }\end{array}$ & $\begin{array}{l}1999 / 2000 \quad \text { Neu- } \\
\text { berechnete Daten }\end{array}$ & Preisreduktionspotential \\
\hline Zuckerrübenmindestpreis & 47,67 & 47,67 & 0 \\
\hline Zuckerertrag & $13 \%$ & $14,4 \%$ & $1,4 \%$ \\
\hline Kosten des Rübenanteils & $\mathbf{3 6 6 , 6 9}$ & $\mathbf{3 3 1 , 0 4}$ & $\mathbf{3 5 , 6 5}$ \\
\hline Transportkosten & 44,1 & 39,79 & 4,31 \\
\hline Produktionskosten & & & \\
\hline Arbeitskosten & - & 48,23 & \\
\hline Energiekosten & - & 38,04 & \\
\hline
\end{tabular}


Tabelle 41 (Fortsetzung)

\begin{tabular}{|l|l|l|l|}
\hline$€ /$ Tonne & $\begin{array}{l}1998 / 99 \\
\text { Offizielle } \\
\text { Daten }\end{array}$ & $\begin{array}{l}1999 / 2000 \quad \text { Neu- } \\
\text { berechnete Daten }\end{array}$ & Preisreduktionspotential \\
\hline Abschreibung & - & 27,62 & \\
\hline Sonstige & - & 11,3 & \\
\hline Distribution/Verwaltung & - & 55,82 & \\
\hline Summe Produktionskosten & $\mathbf{2 4 3 , 6}$ & $\mathbf{1 8 1 , 0 1}$ & $\mathbf{6 2 , 5 9}$ \\
\hline Verkauf Nebenprodukte & 22,5 & 22,5 & \\
\hline Nettointerventionspreis & $\mathbf{6 3 1 , 8 9}$ & $\mathbf{5 2 9 , 3 4}$ & $\mathbf{1 0 2 , 5 5}$ \\
\hline Lagerkostenabgabe & $\mathbf{2 0}$ & 14,74 & 5,26 \\
\hline Bruttointerventionspreis & $\mathbf{6 5 1 , 8 9}$ & $\mathbf{5 4 4 , 0 8}$ & $\mathbf{1 0 7 , 8 1}$ \\
\hline
\end{tabular}

Quelle: IZZ 2000, S. 4 (auf Grundlage ABN-AMRO 1996)

Bei der theoretischen Gewinnmarge der Berechnung der ABN-AMRO Bank wurde von der Marge als Differenz zwischen den Kosten und dem EU-Interventionspreis ausgegangen. In der Realität liegen jedoch die tatsächlich erzielten Preise weitaus über dem Interventionspreis, so dass sich eine weitaus größere Gewinnmarge ergibt. Dieser Aspekt wird im nächsten Abschnitt näher ergründet.

\subsubsection{Erlöse: Rückschlüsse mit Hilfe von Preisbeobachtungen}

Nicht nur die Kostenseite entscheidet über den Erfolg eines Unternehmens, sondern ebenso die Erlösseite. Entscheidend ist in erster Linie der Anteil bzw. das Verhältnis zwischen A-, B- und C-Quote, da durch garantierte A-Mengen-Preise und relativ lukrative B-Mengen-Preise gewisse Erlöse sichergestellt sind. Das Verhältnis in den jeweiligen EU-Ländern wurde historisch festgelegt ${ }^{725}$.

Mittlerweile sind einige Zuckerkonzerne europa- oder gar weltweit tätig, so dass insgesamt unterschiedliche A/B-Quotenverteilungen erkennbar sind (vgl. Tabelle 42) und somit unterschiedliche Erlöspotentiale erwartet werden können.

${ }^{725}$ Vgl. zur Begründung NEI 2000, S. 167 
Tabelle 42: Unternehmenskennzahlen (Angaben in Tausend Tonnen)

\begin{tabular}{|l|l|l|l|l|l|l|l|}
\hline Unternehmen & $\begin{array}{l}\text { A- } \\
\text { Quote }\end{array}$ & $\begin{array}{l}\text { B- } \\
\text { Quote }\end{array}$ & A+B & $\begin{array}{l}\text { A/B- } \\
\text { Verhältnis }\end{array}$ & $\begin{array}{l}\text { Tatsächl. } \\
\text { Produk- } \\
\text { tion }\end{array}$ & $\begin{array}{l}\text { EU- } \\
\text { Marktanteil }\end{array}$ & $\begin{array}{l}\text { Kampagne- } \\
\text { länge (Tage) }\end{array}$ \\
\hline Südzucker & 1,865 & 521 & 2,386 & 3,6 & 2,800 & 16,4 & 90 \\
\hline $\begin{array}{l}\text { Eridania Béghin- } \\
\text { Say }\end{array}$ & 1,585 & 365 & 1,95 & 4,3 & 2,110 & 13,4 & 80 \\
\hline ABF (British S.) & 1,040 & 104 & 1,144 & 10 & 1,353 & 7,8 & 120 \\
\hline Danisco & 759 & 162 & 921 & 4,7 & 885 & 6,3 & 95 \\
\hline Saint Louis & 524 & 141 & 665 & 3,7 & 700 & 4,6 & 90 \\
\hline Ebro Agricolas & 518 & 22 & 540 & 23,5 & 669 & 3,7 & 75 \\
\hline CSM & 260 & 68 & 328 & 3,8 & 362 & 2,2 & 100 \\
\hline Greencore & 182 & 18 & 200 & 10,1 & 177 & 1,4 & 100 \\
\hline Cultor & 133 & 13 & 146 & 10,2 & 142 & 1 & 75 \\
\hline Top 9 & 6,866 & 1,414 & 8,280 & 4,9 & 9,004 & 56,8 & 91 \\
\hline Andere & 5,108 & 1,194 & 6,302 & 4,3 & 8,209 & 43,2 & 94 \\
\hline Total EU-ZR & 11,974 & 2,608 & 14,582 & 4,6 & 17,213 & 100 & 92 \\
\hline
\end{tabular}

(Anm: Fusion zwischen Südzucker und Saint Louis Sucre noch nicht berücksichtigt)

Quelle: ABM-AMRO 1996, S. 7

\subsubsection{Preise}

Durch die Zuckermarktordnung ist der Preisrahmen für Zucker weitgehend vorgegeben. In der Realität werden Abweichungen zum Interventionspreis vorgefunden. Dies liegt einerseits in unterschiedlichen Qualitäten und sogenannten Serviceleistungen (Verpackungsgrößen, Just-in-Time Belieferung, Regalpflege, kontinuierliche Belieferung etc.) und ist andererseits in Transportkosten begründet. Dennoch weisen zahlreiche Zuckerverwender darauf hin ${ }^{726}$, dass es sich z.T. um kartellartige Absprachen innerhalb bestimmter Regionen handele und jeglicher Wettbewerb der Zuckerunternehmen untereinander ausgeschaltet würde. Auch die europäische Kartellrechtspraxis bestätigt eine auffallend hohe Anzahl von Preisabsprachen innerhalb der europäischen Zuckerbranche. Besonders schwierig erweist sich der Vergleich von Zuckerproduktpreisen innerhalb Europas, da kaum Veröffentlichungen vorhanden sind. CAOBISCO (Verband der europäischen Zuckerverwender) führt einen regelmäßigen Preisvergleich von Industriezucker in Europa durch. Dabei zahlten die Verwender folgende Preise frei Fabrik (vgl. Abbildung 48).

\footnotetext{
${ }^{726} \mathrm{Vgl}$. Frankfurter Allgemeine Zeitung (online) vom 22.01.2002 sowie 21.02.2001, S.19; FinANCIAL Times DEUTSCHLAND (online: Sven Clausen) vom 29.11.2000; HANDELSBLATT (online: Ingo Reich) vom 20.11.2000
} 
Abbildung 48: Entwicklung der Industriezuckerpreise in Deutschland und Europa

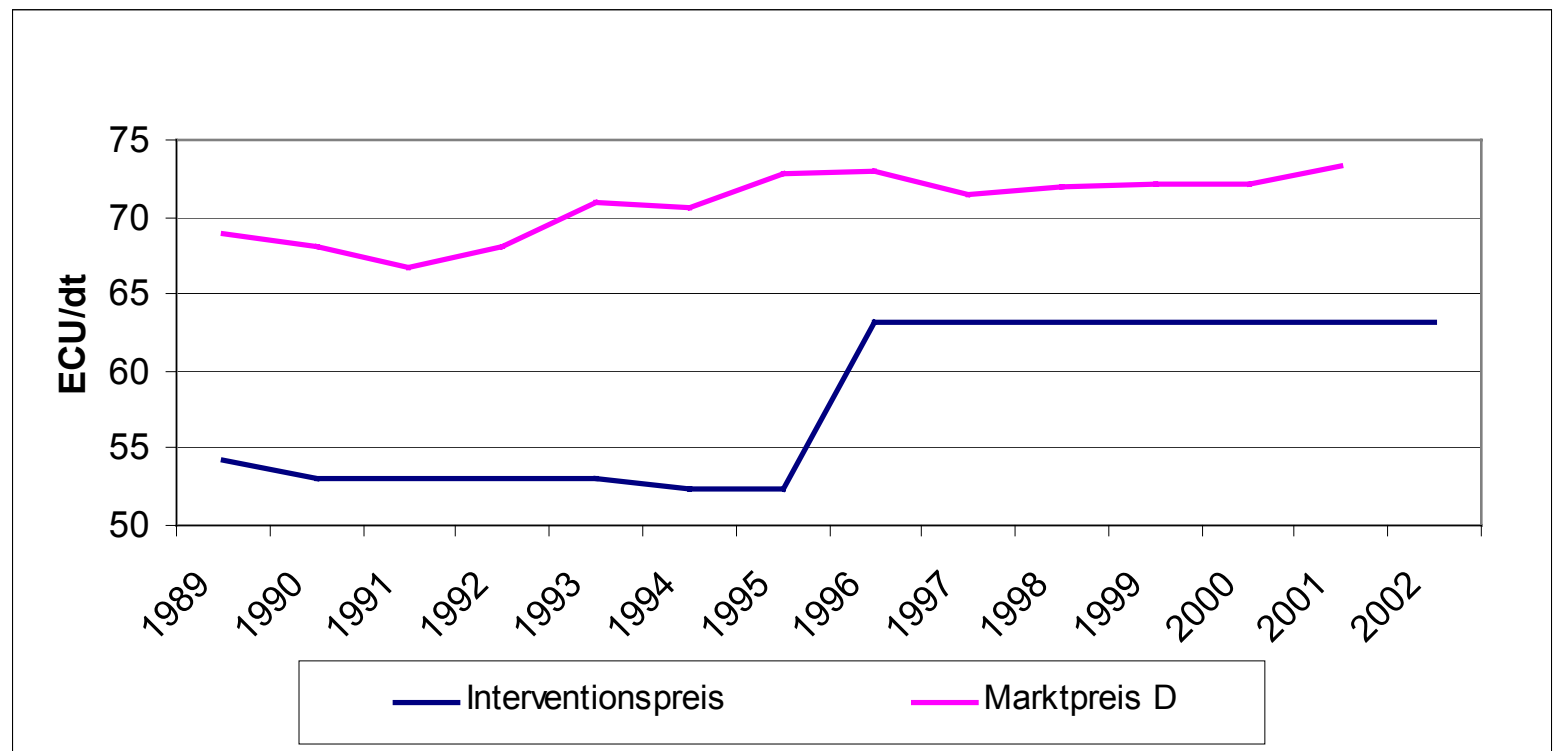

Quelle: WVZ laufende Jahrgänge; CIUS; CAOBISCO

\section{Industriezuckerpreise in der EU}

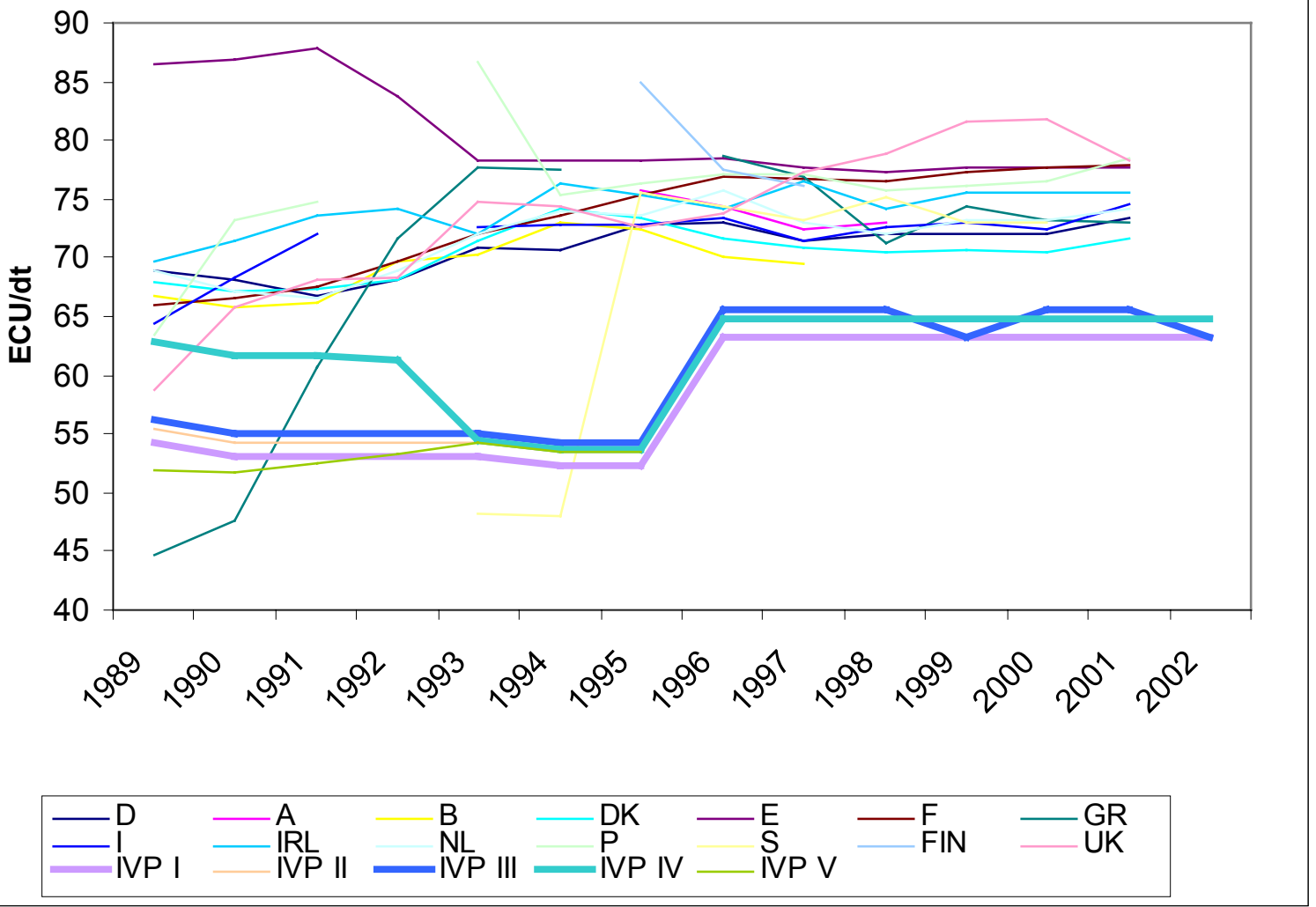

Anm.: IVP = Interventionspreis; IVP I: B, D, DK,. F, GR, L, NL; IVP II: GB, IRL; IVP III: I; IVP IV: E; IVP V: $\mathrm{P}$; Datengrundlage siehe Anhang $\mathrm{C}$

Quelle: Cius; Caobisco 
Auffallend ist, dass die Preise über dem Interventionspreis liegen und in den einzelnen Ländern unterschiedliche Margen vorliegen. Ob die Differenz in den Transportkosten begründet ist, konnte nicht zufriedenstellend geklärt werden, da die Zuckertransportspeditionen Aussagen über Transportkosten verweigerten. Ohne die besonderen Anforderungen von Zuckertransporten zu berücksichtigen, lagen die Transportkosten nach einer Untersuchung von HeRRY (2001) bei einer Zuladung von $16 \mathrm{t}$ bei ca. 1,02 $€$ pro gefahrenem km (LKW inkl. Fahrer) bzw. zwischen 37 und $38 €$ pro Stunde bei einer unterstellten Tagestour von $600 \mathrm{~km}^{727}$.

Anzumerken sei jedoch an dieser Stelle, dass prinzipiell der Machtspielraum durch den Interventionspreis in Addition mit den Transportkosten vom Handelsplatz zum Abnehmer begrenzt ist, denn spätestens, wenn die binnenländischen Zuckerpreise die Interventionspreise inklusive Transportkosten langfristig überschreiten, wird ein Bezug von Drittlandsware für Abnehmer lukrativ (vorausgesetzte gleiche Qualitätseigenschaften).

Auch für Haushaltszucker gibt es nur sporadische Veröffentlichungen in Deutschland und Europa. Daher wurde anhand eines LEH-Scannerdatensatzes der Firma MADAKOM versucht, Rückschlüsse auf das Wettbewerbsverhalten der Zuckerunternehmen zu ziehen. Es handelt sich dabei um gesammelte Preisdaten von sieben Handelsorganisationen und insgesamt 207 Geschäften innerhalb Deutschlands im Zeitraum von 2000 bis 2001.

Zunächst wurde die Frage nach der örtlichen Verfügbarkeit von Zuckerprodukten unterschiedlicher Hersteller überprüft. Folgendes Ergebnis konnte ermittelt werden (Abbildung 49).

${ }^{727}$ Vgl. HERRY 2001, S. 38 ff. 
Abbildung 49: Verfügbarkeit verschiedener Hersteller

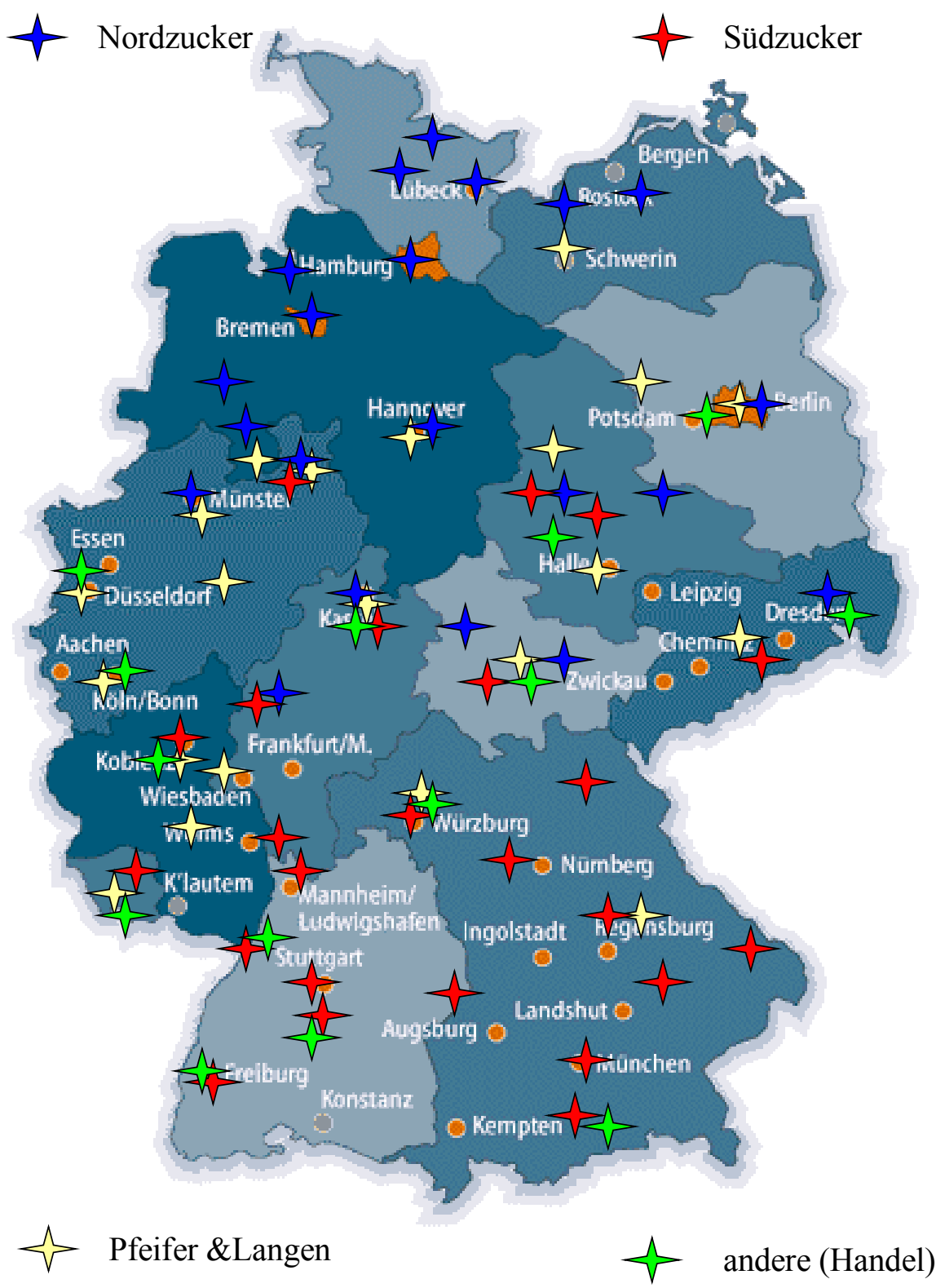

Quelle: eigene Darstellung

Die Produktionsstandorte der Zuckerhersteller in Deutschland sind der Abbildung 50 zu entnehmen. 


\section{Abbildung 50: Standorte der Zuckerindustrie}

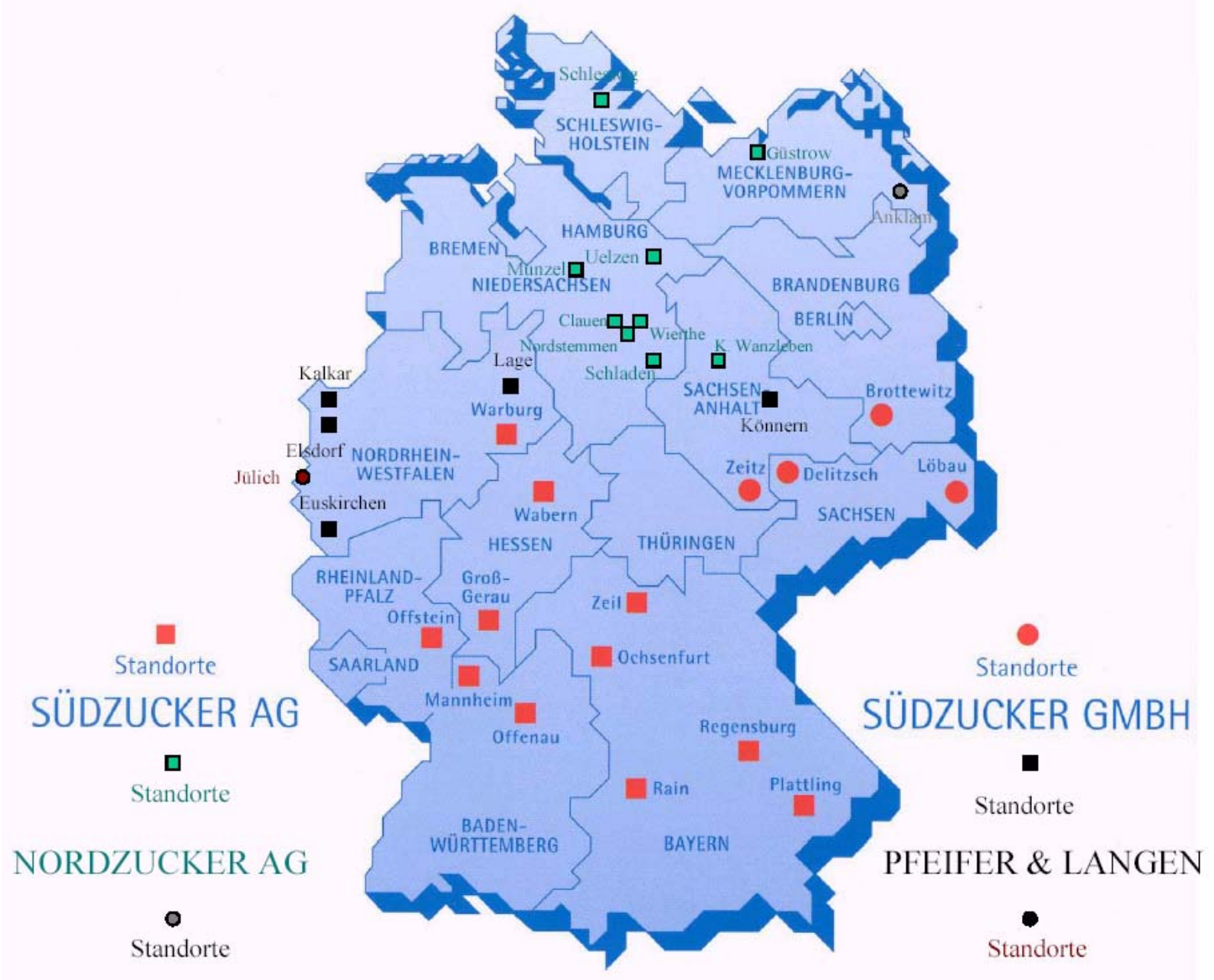

DANISCO

JÜLICH

\section{Quelle: SÜDZUCKER 2001}

Die These, dass in den Produktionsgebieten bestimmter Hersteller lediglich Produkte dieser Hersteller zur Verfügung stehen, kann weitgehend bestätigt werden ${ }^{728}$. Dies könnte jedoch auf Transportkosten (Serviceleistungen) oder - wenngleich relativ unwahrscheinlich - auf regionale Präferenzen zurückzuführen sein. Interessanter scheint es deshalb, dort einen Preisvergleich vorzunehmen, wo Überschneidungsgebiete verschiedener Zuckerhersteller vorliegen, und somit zusätzliche Wettbewerbsimpulse zu erwarten sind. Diese Situation war gemäß des MADAKOM Datensatzes besonders in den Regionen Kassel, Thüringen und Detmold vorzufinden, wo jeweils die Hersteller Nordzucker, Pfeifer \& Langen und Südzucker vertreten sind.

\footnotetext{
${ }^{728}$ In der Entscheidung der EU-Kommission zu Kartellabsprachen in der britischen Zuckerbranche (Amtsblatt Nr. L 076 vom 22.03.1999, Rdnr. 16) bestätigen Tate \& Lyle, dass aus Furcht vor Vergeltungsmaßnahmen anderer Unternehmer eine Überschreitung der traditionellen Absatzgebiete gemieden wird.
} 
Abbildung 51a: Preise innerhalb Deutschlands für Südzucker Raffinade (1000g)

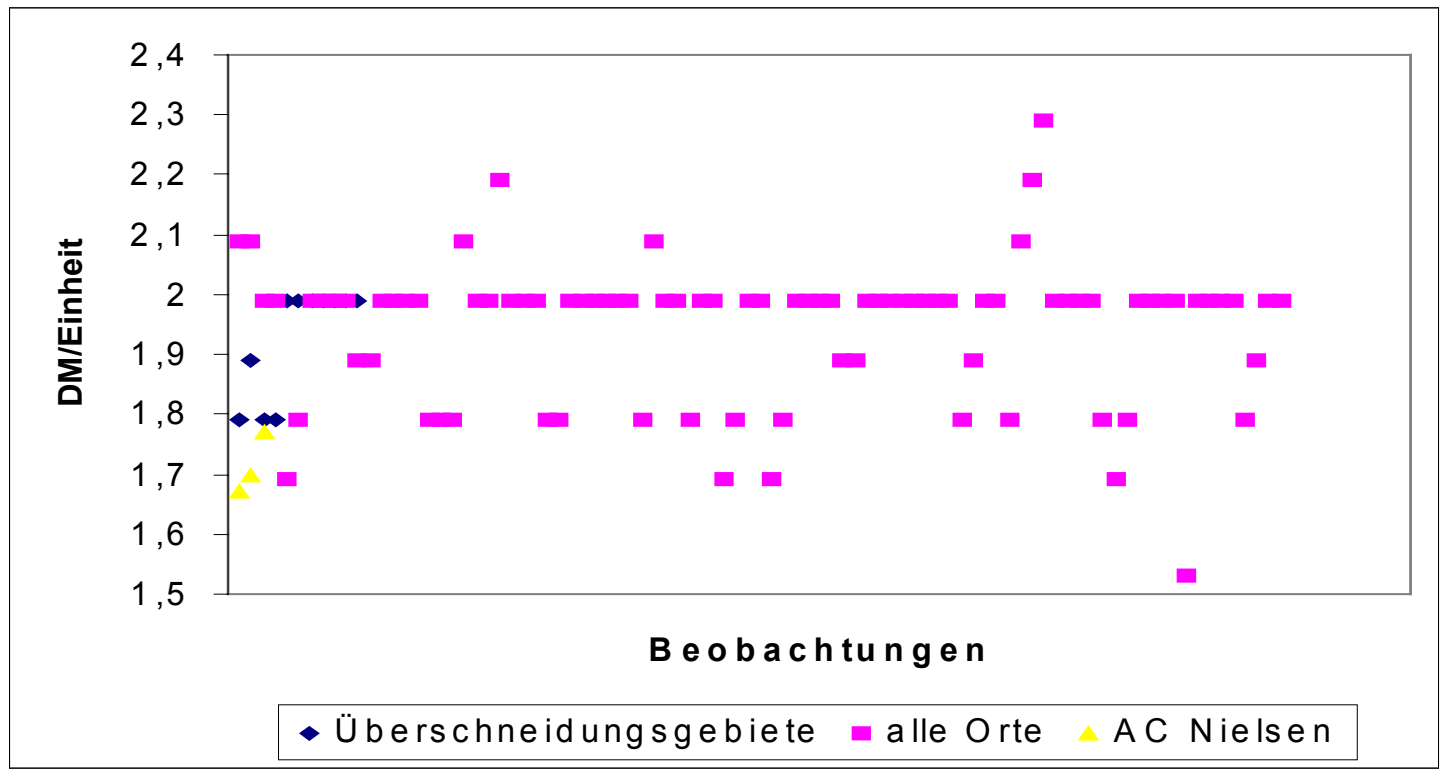

Quelle: eigene Berechnungen

Abbildung 51b: Statistische Auswertung

\begin{tabular}{|lrr|}
\hline \multicolumn{1}{|c}{ SZ-Raffinade 1000g } & Variable 1 & Variable 2 \\
\hline Mittelwert & 1,944382022 & 1,92636364 \\
Varianz & 0,014390807 & 0,00854545 \\
Beobachtungen & 89 & 11 \\
Hypothetische Differenz der Mittelwerte & 0 & \\
Freiheitsgrade (df) & 15 & \\
t-Statistik & 0,588147513 & \\
P(T<=t) einseitig & 0,282592099 & \\
Kritischer t-Wert bei einseitigem t-Test & 1,753051038 & \\
P(T<=t) zweiseitig & 0,565184198 & \\
Kritischer t-Wert bei zweiseitigem t-Test & 2,131450856 & \\
\hline
\end{tabular}

Anm.: Signifikanzniveau $\alpha=0,05$

Quelle: eigene Berechnungen

Exemplarisch sei dies in Abbildung 51a und Abbildung 51b anhand eines Südzucker Produktes dargestellt, wobei die violetten Quadrate die Preise in den Überschneidungsorten, die blauen Rauten die Preise in allen übrigen Gebieten Deutschlands und die gelben Dreiecke die Durchschnittspreiserhebung laut der Marktforschungsgesellschaft AC-Nielsen wiedergeben. Alle weiteren Produkte (aller untersuchten Hersteller) sind im Anhang $C$ aufgeführt. 
Tabelle 43: Gesamtauswertung untersuchter Zuckerprodukte anhand des MADAKOM Datensatzes

\begin{tabular}{|l|c|c|c|}
\hline & $\begin{array}{l}\text { Nullhypothese nicht } \\
\text { verworfen }\end{array}$ & $\begin{array}{l}\text { Nullhypothes verwor- } \\
\text { fen }\end{array}$ & gesamt \\
\hline Nordzucker & 6 & 2 & 9 \\
\hline Südzucker & 6 & 5 & 9 \\
\hline Pfeifer \& Langen & 4 & 10 & 26 \\
\hline Gesamt & 16 & 3 & 8 \\
\hline
\end{tabular}

Anm.: Nullhypothese: „Es gibt keine signifikante Preisabweichung in den Überschneidungsgebieten gegenüber dem Gesamtgebiet"; Signifikanzniveau $\alpha=0,05$

\section{Quelle: eigene Berechnungen}

Festzuhalten bleibt, dass von insgesamt 26 untersuchten Zuckerprodukten ${ }^{729}$ bei 16 Erzeugnissen keine signifikante Preisabweichung in den wettbewerbsintensiven Gebieten festzustellen war (vgl. dazu Tabelle 43). Damit scheint sich die These geringen Preiswettbewerbs zwischen Zuckerunternehmen zu bestätigen.

Bei den Untersuchungen wurde vollkommener Wettbewerb auf Handelsebene unterstellt, d.h., eine Preiserhöhung der Zuckerproduzenten wird direkt über den Handel auf die Verbraucherpreise überwälzt. Diese Annahme kann kritisch hinterfragt werden, denn unterschiedliche Preisschwankungen könnten vielmehr auf differenzierte Preisstrategien der nachgelagerten Handelsketten zurückzuführen $\operatorname{sein}^{730}$. Idealerweise sind für die Analyse des Preissetzungsverhaltens Zuckerabgabe- (Hersteller) bzw. Zuckerbezugspreise (Handel) heranzuziehen. Diese Auskünfte wurden sowohl von den Herstellern als auch dem Handel abgelehnt. Somit ist bedauerlicherweise keine detaillierte Analyse der Transportkosten oder der Handelsspanne möglich ${ }^{731}$. Dieser Weg wäre allerdings den Kartellbehörden zugänglich, da das Statistische Bundesamt, aber auch die betroffenen Parteien im Falle eines Verfahrens der Auskunftspflicht unterliegen ${ }^{732}$. Insbesondere könnten dabei Handelsströme wie auch Preisdaten im Zeitverlauf (vor und nach einer Fusion) Aufschluss über Preissetzungsstrategien und Marktmachtpotentiale geben. Die Auskünfte aus den Preisbeobachtungen ließen außerdem Aussagen über die Wettbewerbsintensität der übrigen Mitbewerber zu und könnten Rückschlüsse auf eine Kollusionswahrscheinlichkeit ermöglichen (was der

\footnotetext{
${ }^{729}$ Bedingung für die Auswahl der Produkte war das Vorhandensein von mindestens 3 Beobachtungen in den Überschneidungsgebieten.

730 Vgl. dazu auch HeRRMANN/MÖSER/WERNER 2002

${ }^{731}$ Anhand der Beschlüsse des Bundeskartellamts zum Verkauf unter Einstandspreisen (B9-74/00; B984/00; B9-85/00) wird der Zuckerabgabepreis frei Haus auf 1,48 bzw. 1,53 DM pro kg Raffinade und 1,76 bzw. 1,85 DM pro kg Würfelzucker beziffert. Die Differenz zwischen Zuckerabgabepreis und Verbraucherpreis entspräche der Marge des LEHs.

${ }^{732}$ Vgl. für Ermittlung durch Bundeskartellamt: § 57 - 59 GWB; für Ermittlung der EU-Kommission: Art. 11 FKVO
} 
auf eine Kollusionswahrscheinlichkeit ermöglichen (was der Forderung der industrieökonomischen Erkenntnisse - vgl. Kapitel 4.1.5 - entspräche).

\subsubsection{Bilanzanalyse: Rückschlüsse von vergangenheitsbezogenen Beobachtungen auf zukünftiges Verhalten}

\subsubsection{Vorüberlegungen zur Bilanzanalyse}

In diesem Abschnitt soll der Frage nachgegangen werden, welche Veränderungen sich in den Jahresabschlüssen fusionierter Unternehmen erkennen lassen, um daraus Schlussfolgerungen für eine wettbewerbliche Beurteilung gewinnen zu können. Ziel ist es, anhand von Kennziffern abzuleiten, inwieweit ein Unternehmen Synergievorteile durch den Zusammenschluss erzielen konnte. Der Gedankengang dieser Untersuchung beruht auf der Annahme, dass soweit keine Änderungen in der Marktsituation (Preisoder Erlössituation) vorliegen, eine Erhöhung (Verminderung) des Jahresüberschusses auf die Verbesserung (Verschlechterung) der Kostenstruktur zurückzuführen ist. Dass heißt es, könnten im Zuge einer Fusionen Synergievorteile realisiert werden, die unter Fortbestehen der einzelnen Unternehmen nicht möglich wären ${ }^{733}$. Des weiteren wird der Versuch unternommen, anhand der Jahresabschlüsse auf den Marktmachtspielraum der Unternehmen rückzuschliessen. Zumindest theoretisch müsste sich dieser aus der Differenz der Erlöse und Kosten (im modelltypischen Idealfall: $\mathrm{p}-\mathrm{GK}=$ Marktmachtpotential) ableiten lassen.

Eine Jahresabschlussanalyse kann für ein Unternehmen sowohl über mehrere Geschäftsjahre hinweg (Längsschnittvergleich oder vertikaler Vergleich), als auch im Vergleich zu anderen Unternehmen der Branche erfolgen (Querschnittsvergleich oder horizontaler Vergleich). Neben den allgemeinen Einsatzbereichen der Jahresabschlussanalyse ${ }^{734}$ kommt ihr im Zusammenhang mit Fusionen eine besondere Bedeutung zugute. So dient sie oftmals der Vorauswahl potentieller Zusammenschlusspartner, da der Jahresabschluss oft die einzige offizielle Quelle zur Einschätzung von Kooperationen, Akquisitions- oder Fusionskandidaten ist ${ }^{735}$ (vgl. auch Kapitel 4.2.3). In den weiteren Ausführungen soll aber auch verdeutlicht werden, dass die Jahresabschlussanalyse einen Beitrag zu wohlfahrtsökonomischen Erkenntnissen liefern kann. Darüber

\footnotetext{
${ }^{733}$ Es wird davon ausgegangen, dass die Betriebe vor der Fusion optimal für die entsprechende Unternehmensgröße geführt worden sind.

734 Vgl. dazu ausführlich GRÄFER 1997, S. 19 ff.; SCHULT 1999, S. 8 ff.

735 Bestätigt in den Unternehmensbefragungen dieser Untersuchung. Dabei wurde insbesondere darauf hingewiesen, dass oftmals Überraschungen im positiven wie auch negativen Sinn die Unternehmensleitung trafen, die nicht aus den Jahresabschlüssen erkenntlich waren, und somit einer Korrektur des Sollplanes bedurften.
} 
hinaus wird versucht, die Entwicklung nicht nur vor dem Zusammenschluss (premerger), sondern auch nach der Fusion (postmerger) zu analysieren, um somit Aussagen über den Erfolg oder Misserfolg eines Zusammenschlusses treffen zu können.

Kritisch für die Analyse der ausgewählten Unternehmen ist die Tatsache, dass zwar monetäre Daten ausreichend ausgewiesen sind, naturale Daten hingegen, wie z.B. die absolute Anzahl der produzierten Einheiten einzelner Produkte und entsprechende Umsätze, zum Teil gar nicht oder nur mangelhaft zur Verfügung stehen (vgl. dazu Publikationspflicht in Anhang F). Dieser Umstand kommt besonders bei der Untersuchung von Konzernen zur Geltung ${ }^{736}$, wo sich im Konsolidierungskreis Unternehmen befinden, die in völlig anderen Geschäftsfeldern agieren. Eine Folgerung auf Kennziffern eines bestimmten Geschäftsbereiches ist somit nur unter Annahmen (evtl. mit Hilfe anderer Quellen) möglich. Die unterschiedliche Ausübung von Wahlrechten ${ }^{737}$ führt dazu, dass lediglich Längsschnittvergleiche aufgrund des Stetigkeitsgrundsatzes einigermaßen auswertbar sind; bei Querschnittsanalysen kann eine Vergleichbarkeit auf Grenzen stoßen ${ }^{738}$. In diesem Zusammenhang wird auch auf die mangelnde Vergleichbarkeit internationaler Unternehmen bzw. Unternehmen mit unterschiedlichen Rechnungslegungsstandards (IAS, HGB, US GAAP) hingewiesen.

Bei einem Vergleich zwischen genossenschaftlichen ${ }^{739}$ und privatwirtschaftlichen Unternehmen ist zu beachten, dass beispielsweise genossenschaftliche Molkereien geringere Jahresüberschüsse aufweisen, weil sie ihre Lieferanten (Genossen) bereits mit höheren Milchauszahlungspreisen vergüten (Erhöhung des Materialaufwandes gegenüber einem privaten Unternehmen). BRAUN beispielsweise, der die Ergebnisse zwecks Vergleichbarkeit mit anderen Unternehmen korrigierte, errechnete im Durchschnitt allerdings lediglich Abweichungen der Kennziffern von max. 0,5\% (siehe dazu ausführlich im Anhang E). In der Zuckerindustrie können sogenannte Zusatzleistungen, die additiv den Zuckerrübengrundpreisen zugerechnet werden, die direkte Vergleichbarkeit zwischen verschiedenen Unternehmen erschweren ${ }^{70}$. Auch auf die Einbeziehung von sogenannten "Soft-Faktoren" 741 wird bei der Bilanzanalyse verzichtet, da (wenn überhaupt vorhanden) zumindest langfristig eine unterschiedliche Unternehmenspolitik entweder beim Vergleich der Preise (Bezugs- oder Absatzpreise) oder bei den Bilanz-

\footnotetext{
${ }^{736}$ Andere Probleme bei der Untersuchung von Konzernen erläutert u.a. SCHULT 1999, S. 229 ff.

${ }^{737}$ Ansatz-, Bewertungs- und sonstige Wahlrechte, vgl. dazu ausführlich SCHULT 1999, S. 10 ff.

738 Näheres im Detail vgl. BRAUn 2000 b, S. 7 f.

${ }^{739}$ Die Anteilseigner sind die beliefernden Landwirte. Oftmals Vergütung über höheren Preis.

${ }^{740} \mathrm{Vgl}$. BICKERT 2002, S. 23

${ }^{741}$ Z.B. Betriebsklima, Marketing, Umsetzungsgeschwindigkeit
} 
kennziffern erkennbar sein müsste und somit in der Gesamtanalyse berücksichtigt wird. Erkenntnisse und Entscheidungen aufgrund reiner Jahresabschlusskennziffern und Marktpreisdaten sind stets mit ergänzenden Erwägungen durchzuführen, damit realistische, aussagefähige Prognosen erstellt werden können ${ }^{742}$.

Als Kennziffern werden Maßstäbe der Rentabilität, Liquidität und Stabilität herangezogen. Die genaue Definition der Kennziffern ist Anhang G zu entnehmen.

Um die premerger mit der postmerger Situation vergleichbar zu gestalten, werden die Posten der einzelnen Bilanzen der beteiligten Unternehmen vor der Fusion addiert (es wird also eine fiktive Fusion unterstellt) und mit den Werten nach der Fusion verglichen. Die detaillierte Auswertung aller Kennziffern ist dem Anhang $\mathrm{H}$ dargestellt.

\subsubsection{Bilanzanalyse in der Zuckerindustrie - Beurteilung der Nordzucker AG}

Rein theoretisch ist die Gewinnmarge durch die Quotierung und die festgelegten Preise für Zucker vorgegeben. Zudem schützen fast unüberwindbare Eintrittsbarrieren den Markt vor neuen Wettbewerbern. Abweichungen in den Betriebsergebnissen einzelner Unternehmen können dennoch durch eine unterschiedliche Unternehmenspolitik, beispielsweise durch den Verkauf von C-Zucker (Weltmarkt) oder Zuckernebenprodukten (Melasse) und anderen Aktivitäten der Zuckerindustrie ${ }^{743}$, wie das Engagement in anderen Geschäftsbereichen, begründet sein. Deshalb erweist sich das Problem der Vergleichbarkeit einzelner Zuckerunternehmen als größer als zunächst vermutet ${ }^{744}$.

Aufgrund mangelnder Informationen ist es schwierig, die Wirtschaftlichkeit verschiedener Zuckerunternehmen zu vergleichen ${ }^{745}$.

Im Zuckerindustriesektor steht der Zusammenschluss zur Nordzucker AG aus dem Jahr 1997 im Mittelpunkt. Als Vergleichsunternehmen wurden die Zuckerfabrik Jülich sowie die Union Zucker herangezogen. Gemessen an der Höchstquote handelt es sich bei diesen Vergleichsunternehmen um eher kleinere Einheiten (vgl. Kapitel 2.5). Sie weisen jedoch den Vorteil auf, dass sie ihre Unternehmung ausschließlich mit Zucker tätigen, und daher unverzerrte Kennziffern ablesbar sind. Die zugrundeliegenden Daten der Auswertung sind in detaillierter Form dem Anhang $\mathrm{H}$ zu entnehmen. Wichtige erkenntnisreiche Kennziffern werden im folgenden vorgestellt.

\footnotetext{
742 Auch SCHULT 1999, S. 17

${ }^{743}$ Vgl. ABN-AMRO 1996, S. 4

${ }^{744} \mathrm{Vgl}$. ABN-AMRO 1996, S. 8

${ }^{745}$ Vor allem bei Unternehmen, die in verschiedenen Geschäftsbereichen operieren, sind kaum Rückschlüsse auf Kennzahlen der reinen Zuckerproduktion möglich.
} 
Als zentrales Problem ergibt sich, dass Konzern- und Betriebszahlen z.T. erheblich voneinander abweichen. Dies ist auf den Konsolidierungskreis zurückzuführen. So engagieren sich die Nordzucker und ihre Vorgänger beispielsweise in Bereichen der Pharmazie. Auch eine Teilung der Aktivitäten in Ost- und Westdeutschland ist anhand der Konzernzahlen ersichtlich. Seit dem Zusammenschluss (1997) werden die Zahlen der Nordzucker AG und nicht des Konzerns herangezogen. Mittel- und osteuropäische Zuckerfabrikbeteiligungen sowie ein Engagement außerhalb der Zuckerbranche werden also nicht berücksichtigt. In den vorangegangenen Jahren kann dies z.T. anders gewesen sein, da eine eindeutige Trennung der Geschäftsbereiche nicht immer möglich war. Daher sollten die Abweichungen z.B. beim Umsatz nicht überbewertet werden. Trotz der Abgrenzungsproblematik lassen sich kaum Umsatzunterschiede (Vermarktungsseite) vor und nach dem Zusammenschluss identifizieren (vgl. Abbildung $52)$.

Abbildung 52: Entwicklung von Jahresüberschuss und Umsatz der Nordzucker AG

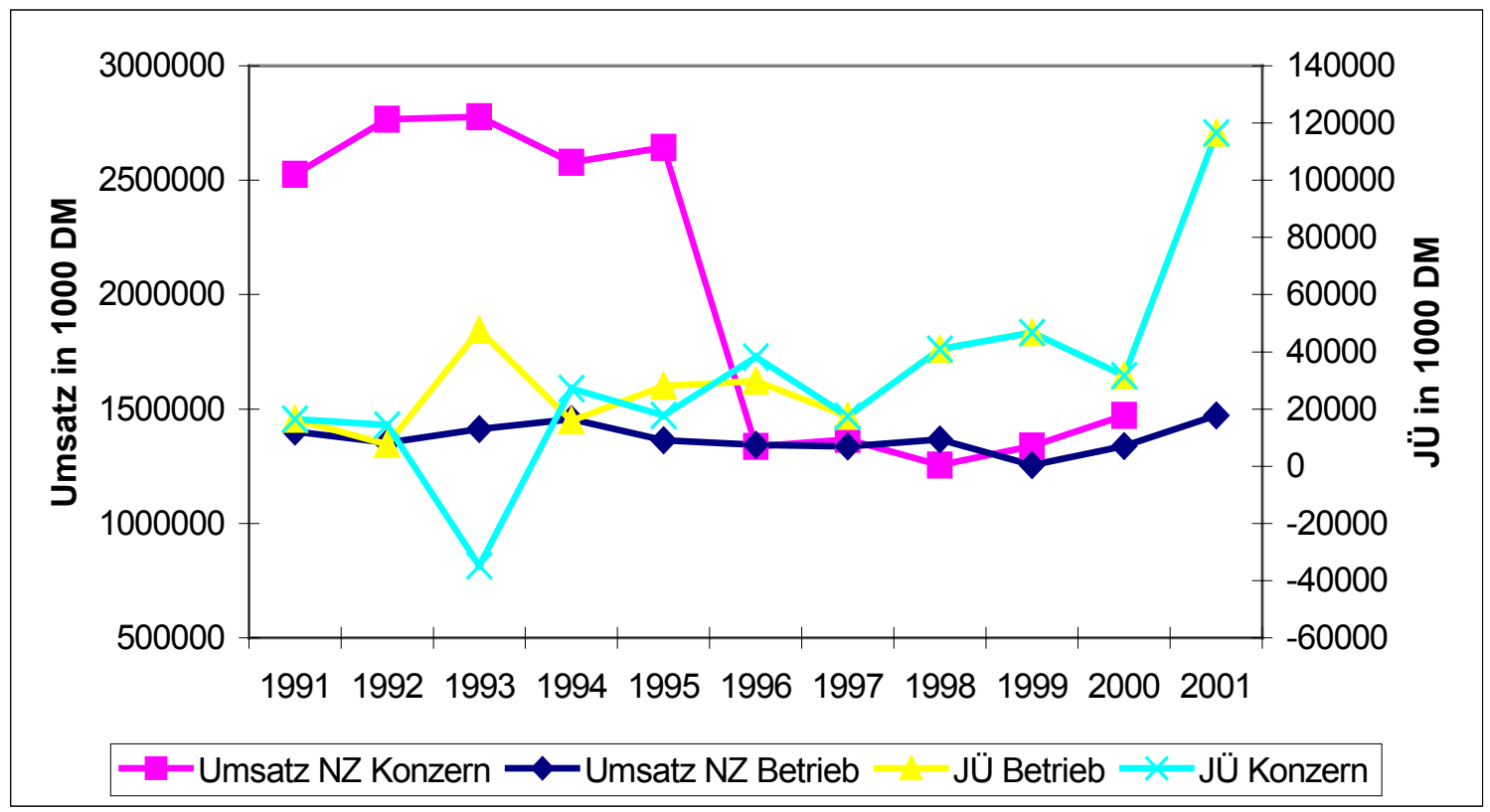

Quelle : eigene Berechnungen auf Grundlage Ifd. Jahresberichte

Ein anderes Bild ergibt sich dagegen bei Betrachtung des Jahresüberschusses (Abbildung 52). Dieser konnte nach 1997 deutlich gesteigert werden, was den Erfolg einer Fusion indizieren könnte. Da bei konstanten Erträgen steigende Gewinne erzielt wurden, deutet dies auf eine Erreichung der im Zuge der Fusion umgesetzten Kosteneinsparungen hin.

Anhand des Anlagevermögens oder der Abschreibungen sind keine eindeutigen Ergebnisse ersichtlich (Abbildung 53). In den letzten zwei Jahren ist sogar eine gegenläufige Entwicklung erkennbar: trotz steigendem Anlagevermögen haben sich die Abschrei- 
bungen reduziert. Die Veräußerung oder Stillegung von Werken spiegelt sich demnach nicht eindeutig in den Zahlen wider.

Abbildung 53: Entwicklung von Anlagevermögen und Abschreibungen der Nordzucker

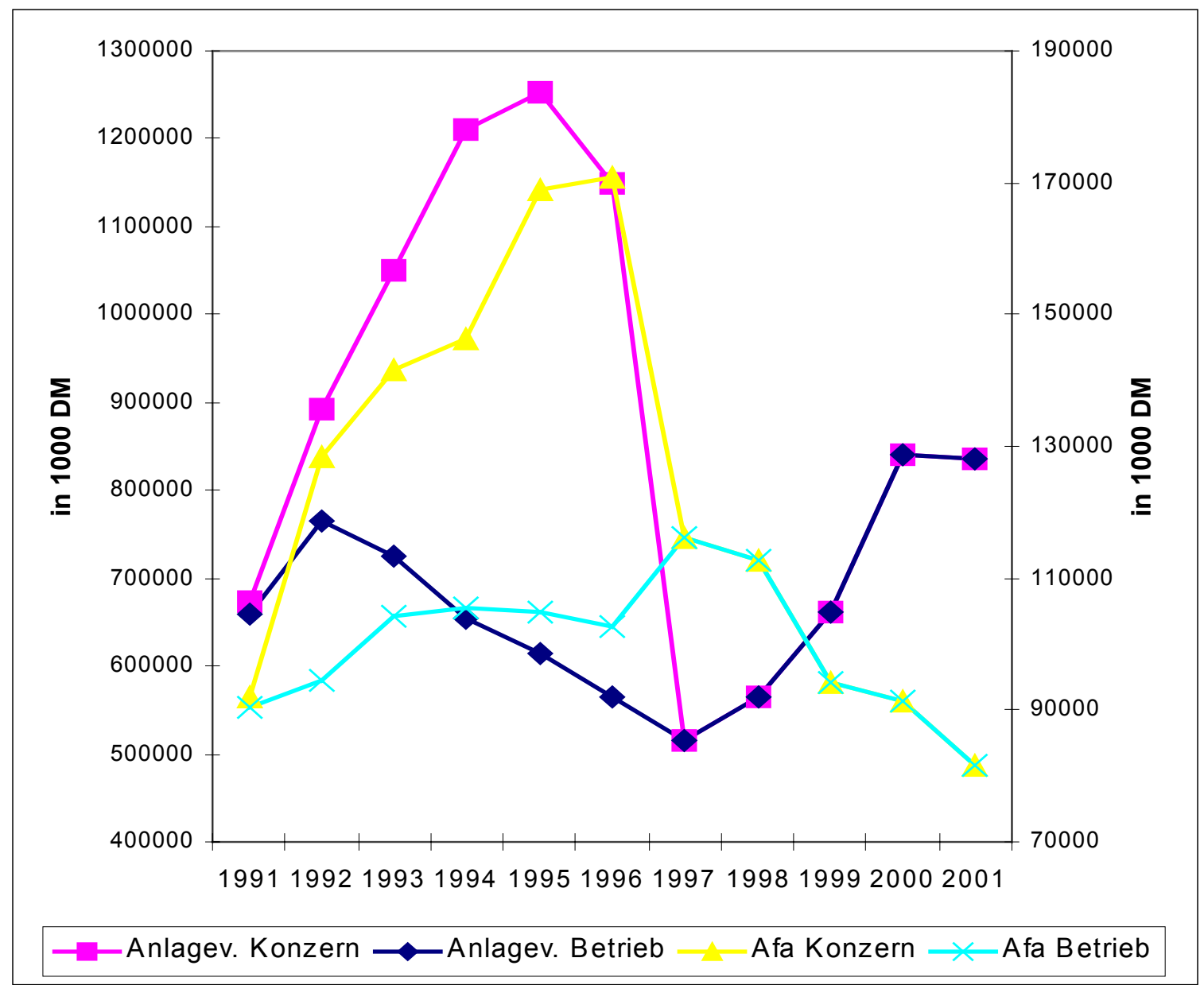

Quelle : eigene Berechnungen auf Grundlage Ifd. Jahresberichte

Eindeutige Ergebnisse zeigen die Grafiken hinsichtlich des Personalbestandes (Abbildung 54). Hier konnte ein erheblicher Abbau mit Implikationen auf die Aufwendungen realisiert werden. 
Abbildung 54: Entwicklung der Mitarbeiterzahl und des Personlaufwandes (pro Mitarbeiter) der Nordzucker

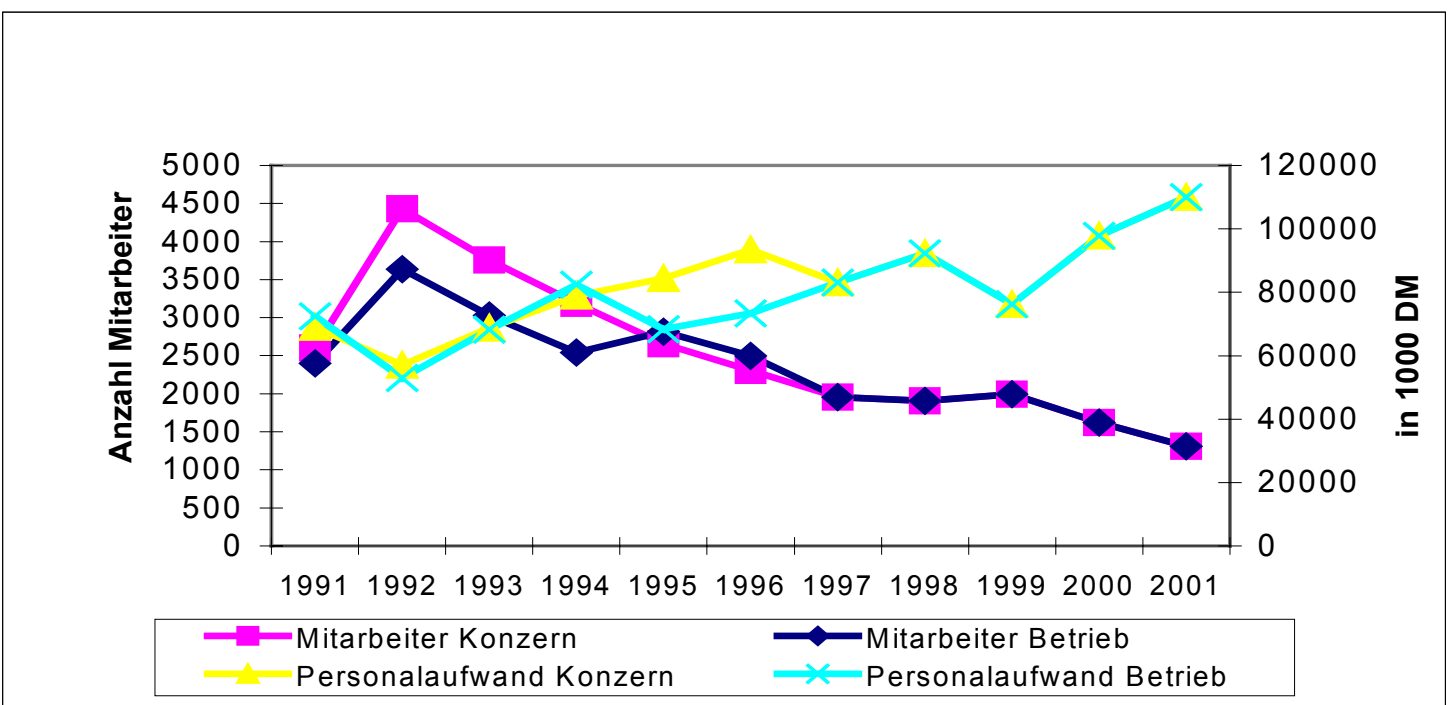

Quelle : eigene Darstellung auf Grundlage Ifd. Jahresberichte

Die Rübenverarbeitung pro Mitarbeiter (Abbildung 55) konnte gegenüber der vorigen Position ausgebaut werden. Allerdings konnten die Mitbewerber ebenfalls diese Position verbessern. Dies deutet darauf hin, dass die Produktivitätssteigerungen nicht nur auf Zusammenschlusseffekte zurückzuführen sind, sondern zum Großteil auf technischen Fortschritt, wovon die gesamte Branche profitiert.

Abbildung 55: Rübenverarbeitung pro Mitarbeiter

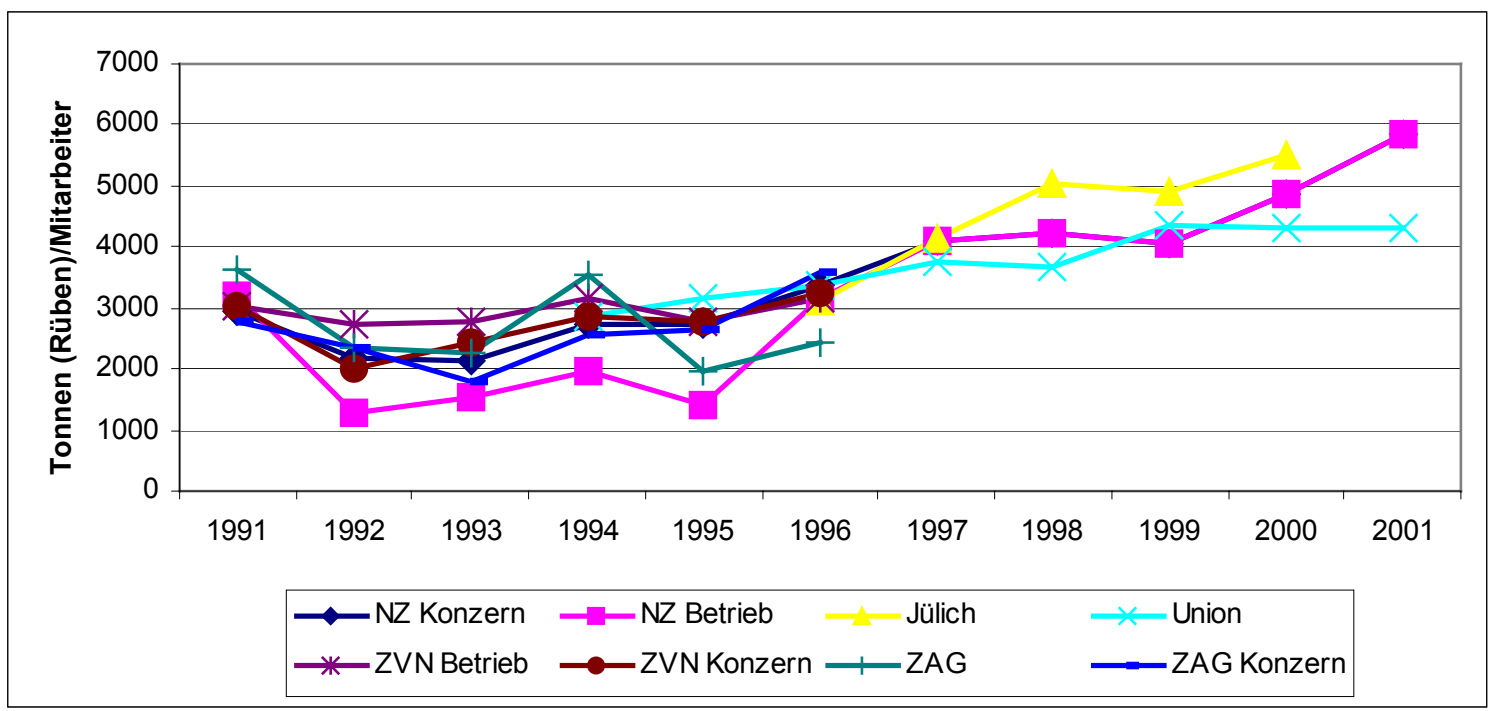

Quelle : eigene Darstellung auf Grundlage Ifd. Jahresberichte

Der vermehrte Umsatz pro Mitarbeiter (Abbildung 56) dürfte die gestiegenen Arbeitskosten überkompensieren. 
Abbildung 56: Entwicklung des Umsatzes pro Mitarbeiter

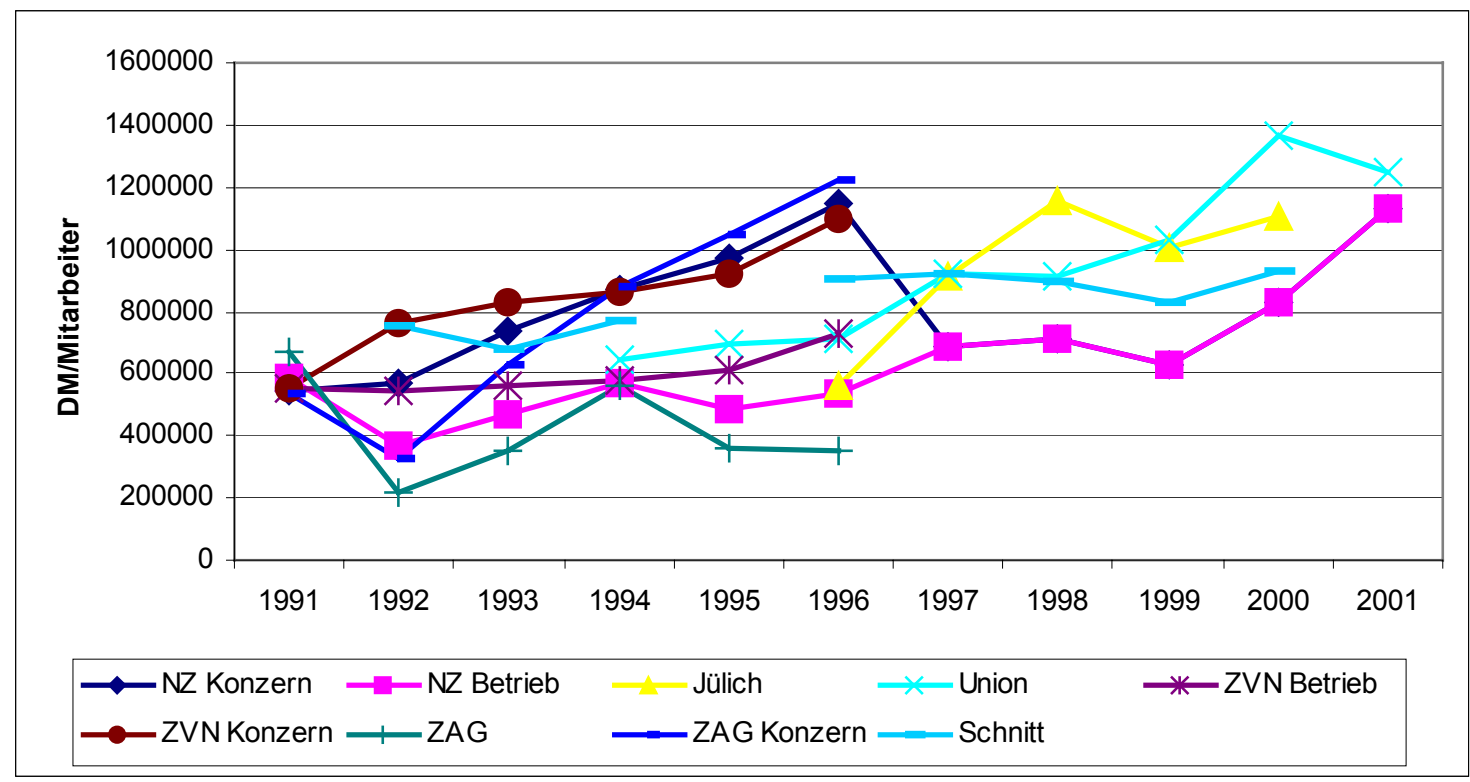

Quelle: eigene Darstellung auf Grundlage Ifd. Jahresberichte

Abbildung 57: Aufwand pro Mitarbeiter im Vergleich mit anderen Unternehmen

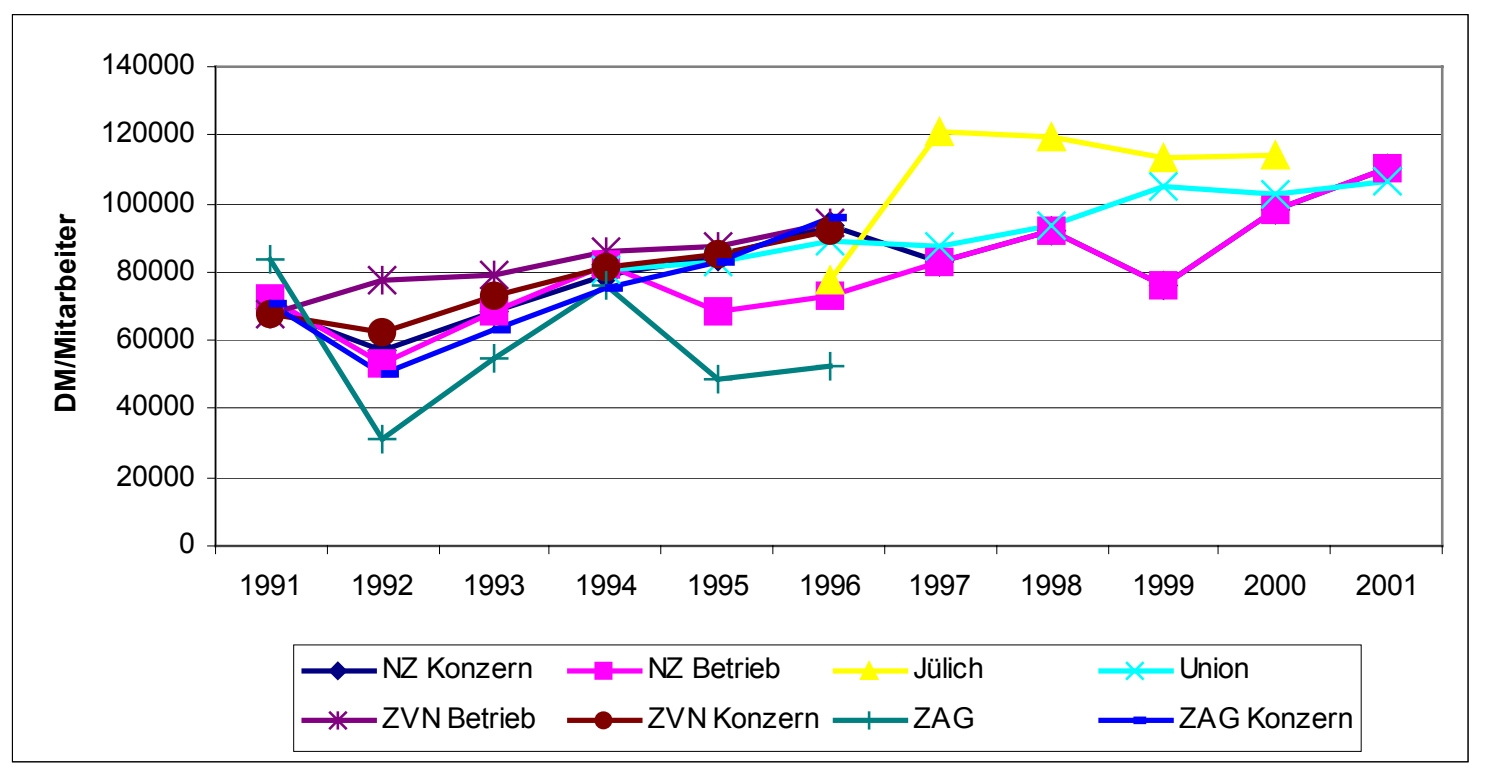

Quelle : eigene Darstellung auf Grundlage Ifd. Jahresberichte

Beim Aufwand pro Mitarbeiter (Abbildung 57) schneidet die Nordzucker besonders gut ab. Zwar ist dieser gegenüber den vergangenen Jahren erheblich gestiegen, liegt aber in den letzten Jahren unter den Werten der kleineren Mitbewerber. Hier könnte sich ein Größenvorteil verbergen. 
Abbildung 58: Umsatz pro kg Zucker

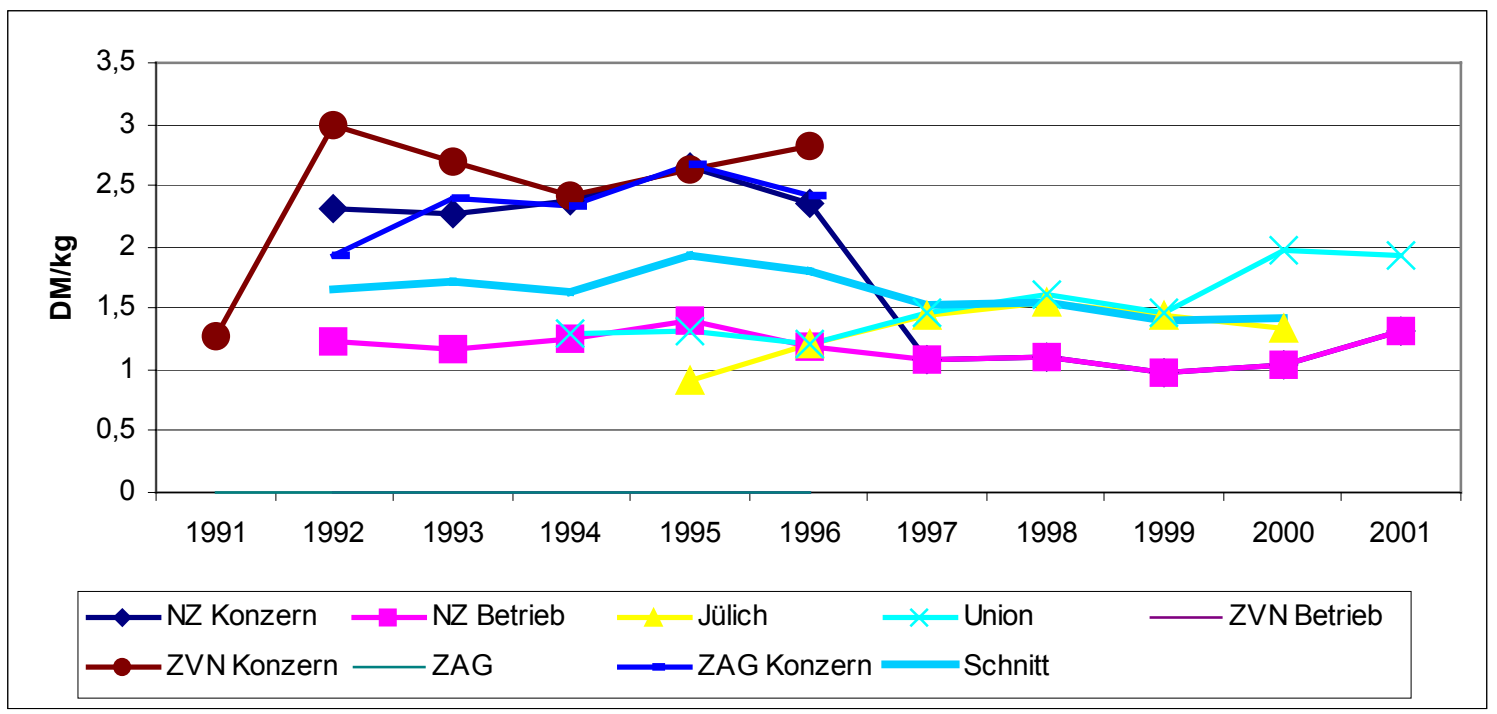

Quelle: eigene Darstellung auf Grundlage Ifd. Jahresberichte

Auf der Vermarktungsseite scheint der Zusammenschluss nicht unbedingt Effekte auf den erzielten Umsatz pro $\mathrm{kg}$ Zucker zu haben (Abbildung 58). So konnten vor dem Zusammenschluss z.T. deutlich bessere Ergebnisse erzielt werden. Auch im bundesdeutschen Vergleich scheint die Nordzucker keine Spitzenposition einzunehmen. Dennoch kann dadurch nicht unbedingt auf ein Marktmachtpotential gegenüber einzelnen Abnehmern geschlossen werden. Beispielsweise würde eine vermehrte Produktion von C-Zucker den durchschnittlich erzielten Preis pro kg Zucker vermindern.

\section{Abbildung 59: Anlagevermögen pro Tonne Rübenverarbeitung}

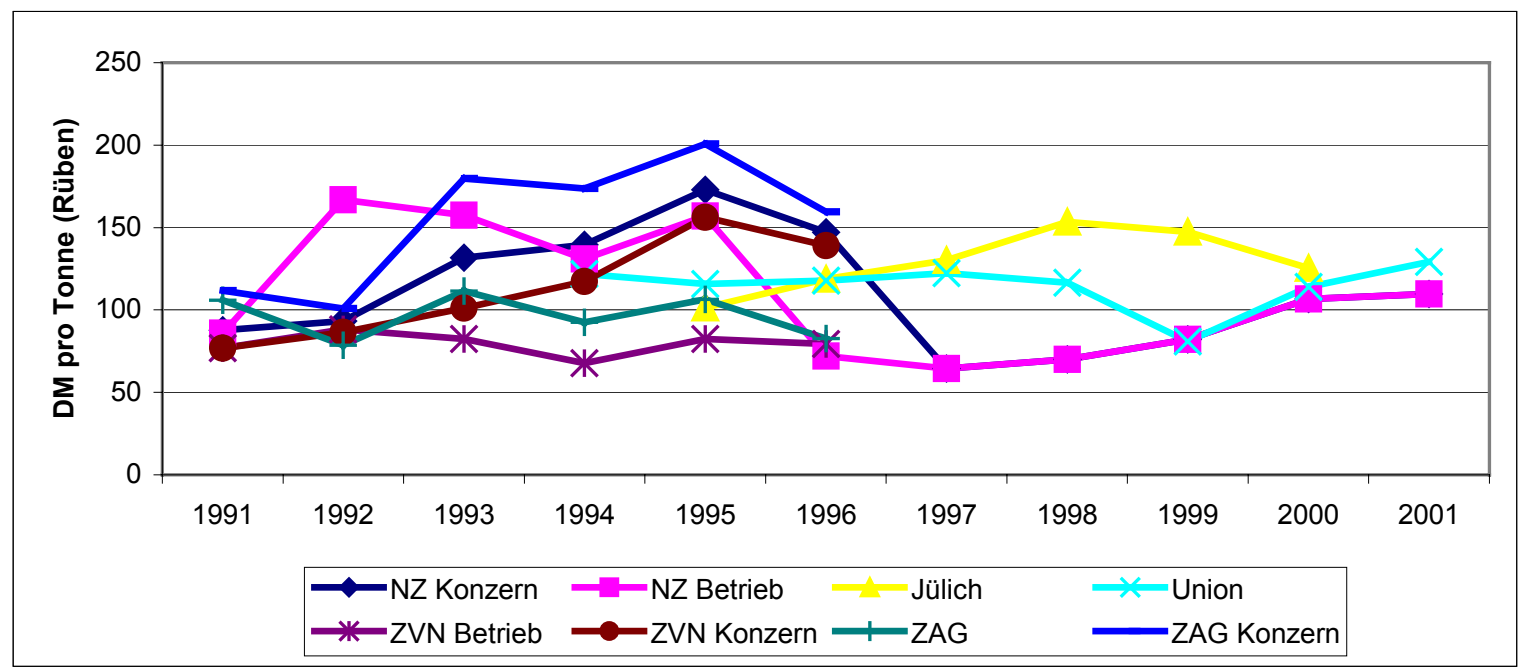

Quelle : eigene Darstellung auf Grundlage Ifd. Jahresberichte

Einen deutlichen Impuls auf das benötigte Anlagevermögen pro verarbeitete Tonne Rüben scheint die Unternehmensgröße auszuüben (Abbildung 59). Hier ist der Grö- 
Benvorteil sowohl innerhalb der Branche, als auch vor und nach dem Zusammenschluss offensichtlich.

Abbildung 60: Entwicklung des Umlaufvermögens pro Tonne Rübenverarbeitung

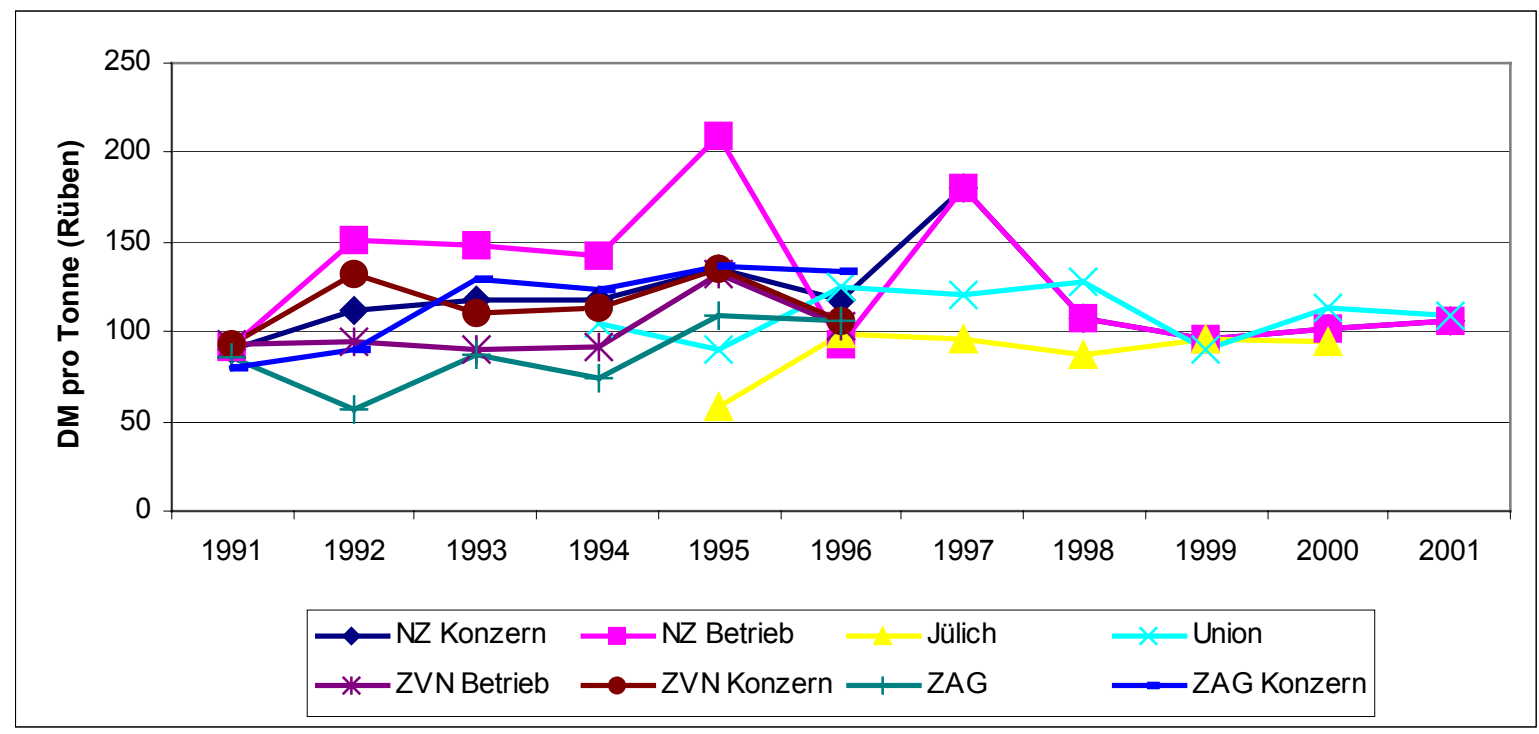

Quelle : eigene Darstellung auf Grundlage Ifd. Jahresberichte

Die Betrachtung des Umlaufsvermögens dagegen scheint keine eindeutigen Schlussfolgerungen zu ermöglichen (Abbildung 60). Innerhalb der letzten Jahre findet offenbar eine Angleichung der Werte bei großen und kleineren Unternehmen statt.

Abbildung 61: Entwicklung des theoretischen Gewinns pro kg Zucker

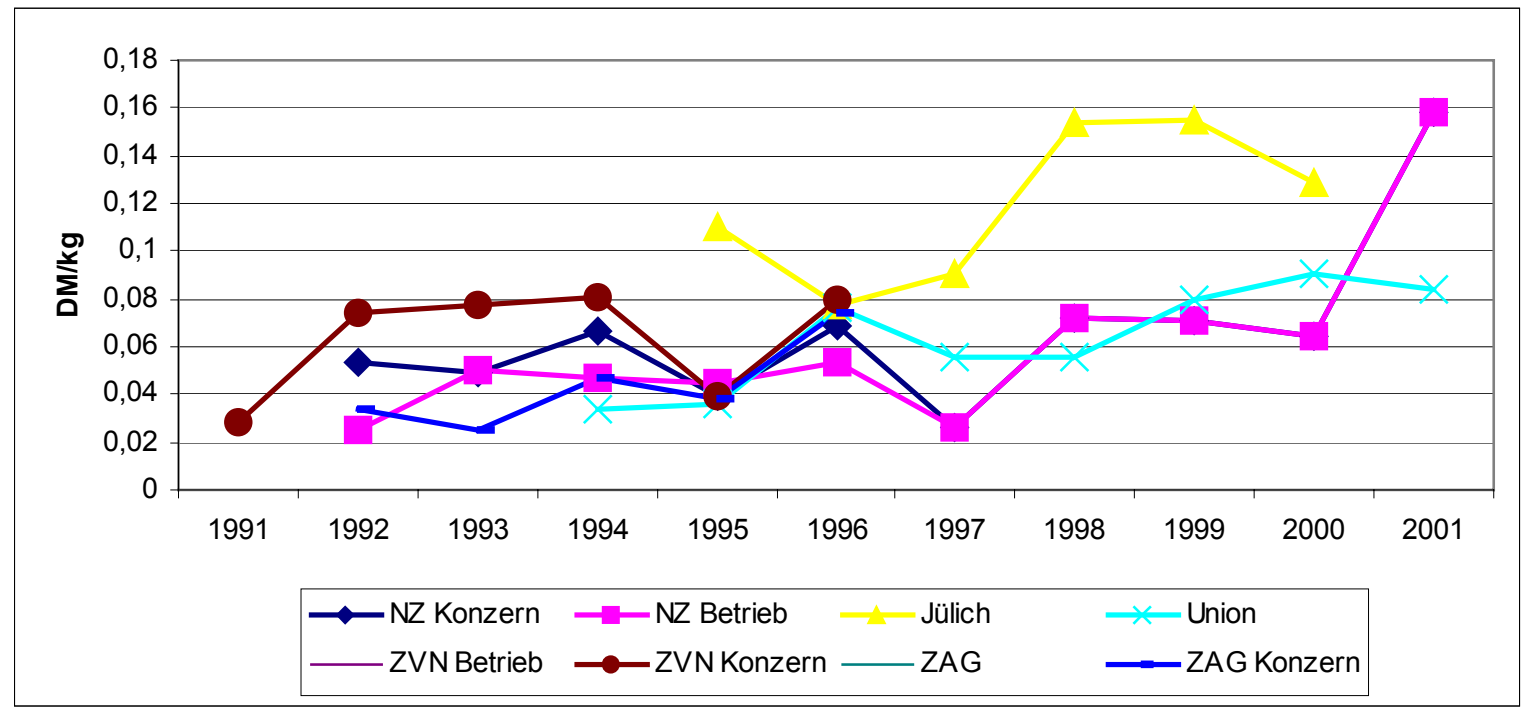

Quelle : eigene Darstellung auf Grundlage Ifd. Jahresberichte

Nach dem vereinfachten Verfahren (nach betriebswirtschaftlicher Definition des Gewinns ist die folgende Vorgehensweise nicht korrekt!) wurde der kalkulatorische Gewinn anhand einer simplifizierten Überlegung ermittelt. In den Oligopolmodellen ergibt sich: Erlös - Kosten = Gewinn. Der Erlös (Umsatz) ist bekannt. Als Gewinn wird das 
Betriebsergebnis herangezogen ${ }^{746}$. Im Gegensatz zur Milchindustrie (vgl. Kapitel 8.2.6) handelt es sich bei der Zuckerindustrie um eine lukrative Branche mit erheblich gestiegenen Gewinnen innerhalb der letzten Jahre. Da eine Gewinnsteigerung nicht nur als Resultat von Fusionsaktivität, sondern auch bei nicht fusionierten Unternehmen zu erkennen ist (Abbildung 61), liegt die Vermutung nahe, dass alle Unternehmen der Branche von einer evtl. Preiserhöhung bzw. Kostensenkung profitiert haben. Dies stützt deutlich die Argumentation der Verarbeitungsspannengegner, deren Kritik sich auf die mangelnde Anpassung der festgelegten Verarbeitungsspannen bezieht.

Abbildung 62: Eigenkapitalrentabilität in der Zuckerbranche (vor Steuern)

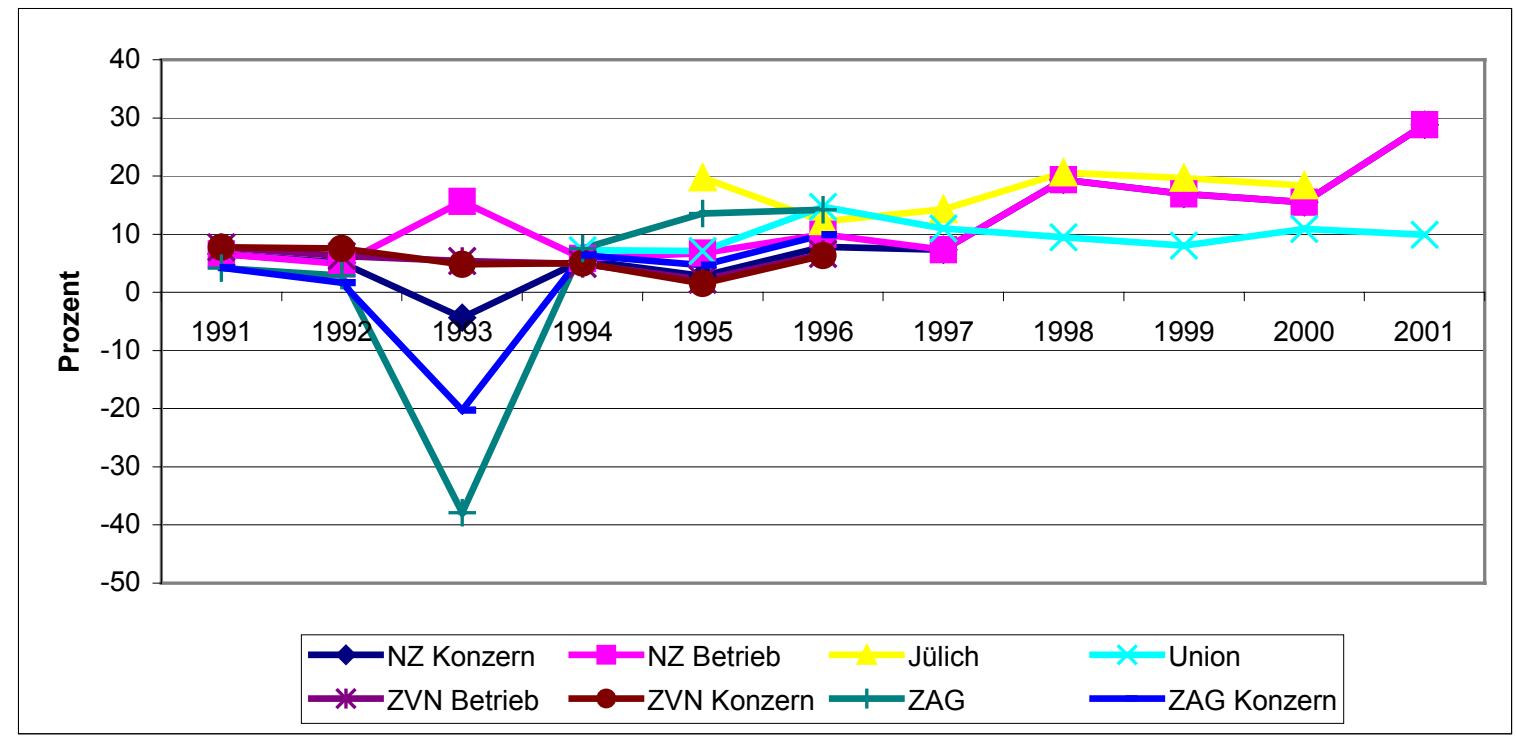

Quelle : eigene Darstellung auf Grundlage Ifd. Jahresberichte

Abschließend sei ein Maßstab der Rentabilität herausgegriffen. An der Eigenkapitalrentabilität kann abgelesen werden, wie sich das eingesetzte Kapital (Eigenkapital) verzinst $^{747}$. Es wird deutlich, dass sich beachtliche Renditen erzielen lassen (Abbildung 62). Eine solch lukrative Marge ließe erwarten, dass Marktneulinge in den Markt eintreten und sich die Gewinne langfristig denen der anderen Branchen angleichen müssten. Verhindert wird dieser Vorgang durch die Zuckermarktordnung, durch die eine fast unüberwindbare Markteintrittsbarriere geschaffen wurde.

\footnotetext{
${ }^{746}$ Außerordentliche Ergebnisse sowie Ertragssteuern finden demnach keine Berücksichtigung.

${ }^{747}$ Definiert als Betriebsergebnis * 100/Eigenkapital
} 


\subsubsection{Ausblick Zuckerindustrie}

Anfänglich stellte die unterschiedliche Eigentümerstruktur ein Hindernis zur Rationalisierung, Konzentration und Ausnutzung von Skalenerträgen dar. Mittlerweile sind diese Barrieren weitgehend überwunden, so dass ein zunehmendes Kostenbewusstsein die Kooperations- und Fusionsvorhaben bestimmt. Besonders in Frankreich und Italien, aber auch in Deutschland gibt es im Vergleich zu anderen europäischen Ländern durchaus noch Rationalisierungspotentiale (Konzentrationsanreize) und somit eine Annährung an die optimale Betriebs- bzw. Unternehmensgröße.

Festzuhalten bleibt, dass in Europa das Wachstumspotential im wesentlichen auf Rationalisierungsvorteile innerhalb der Branche zurückzuführen ist, da die meisten Faktoren durch politische Regulierung und klimatische Abhängigkeiten weitgehend unbeeinflussbar sind. Sobald eine Liberalisierung der Zuckermarktordnung umgesetzt wird, werden sich Unternehmen an neue Marktstrukturen des globalen Wettbewerbs gewöhnen müssen. Dabei ist vorauszusehen, dass sich finanzkräftige Unternehmen vermehrt kostenorientiert verhalten werden, so dass in Zukunft Unternehmensbeteiligungen und -kooperationen in erster Linie über die Grenzen Europas hinaus stattfinden werden (vgl. Tabelle 44).

Tabelle 44: Wettbewerbsfähigkeit der internationalen Zuckerproduktion: Durchschnittskosten der Zuckererzeugung in US \$/Tonne

\begin{tabular}{|l|l|c|c|}
\hline Produkt & Ländergruppe & $\begin{array}{c}\text { Kostenperiode } \\
\mathbf{1 9 8 9 - 1 9 9 4}\end{array}$ & $\begin{array}{c}\text { Kostenperiode } \\
\mathbf{1 9 9 4 - 1 9 9 8}\end{array}$ \\
\hline $\begin{array}{l}\text { Rübenzucker (Bil- } \\
\text { ligproduzenten) }\end{array}$ & $\begin{array}{l}\text { Belgien, Niederlande, Türkei, Chile, USA, Verei- } \\
\text { nigtes Königreich }\end{array}$ & $456(434-479)$ & 450 \\
\hline $\begin{array}{l}\text { Rübenzucker } \\
\text { (Hauptexporteure) }\end{array}$ & EU, Türkei, Ukraine & $656(566-713)$ & 710 \\
\hline $\begin{array}{l}\text { Rübenzucker (teu- } \\
\text { re Produzenten) }\end{array}$ & $\begin{array}{l}\text { Bulgarien, Kasachstan, Moldawien, Rumänien, } \\
\text { Russland, Ukraine, Japan }\end{array}$ & 989 (791-1221) & - \\
\hline $\begin{array}{l}\text { (Roh-) Rohrzucker } \\
\text { (Billigproduzenten) }\end{array}$ & $\begin{array}{l}\text { Brasilien, Kolumbien, Malawi, Guatumala, Sam- } \\
\text { bia }\end{array}$ & $198(177-219)$ & 197 \\
\hline $\begin{array}{l}\text { (Raff.) Rohrzucker } \\
\text { (Billigproduzenten) }\end{array}$ & $\begin{array}{l}\text { Brasilien, Kolumbien, Malawi, Guatemala, Sam- } \\
\text { bia }\end{array}$ & $280(258-303)$ & - \\
\hline $\begin{array}{l}\text { (Roh-) Rohrzucker } \\
\text { (Hauptexporteure) }\end{array}$ & $\begin{array}{l}\text { Australien, Brasilien, Kuba, Kolumbien, Guate- } \\
\text { mala, Thailand, Mauritius, Südafrika }\end{array}$ & $277(246-329)$ & 335 \\
\hline $\begin{array}{l}\text { (Raff.) Rohzucker } \\
\text { (Hauptexporteure) }\end{array}$ & $\begin{array}{l}\text { Australien, Brasilien, Kuba, Kolumbien, Guate- } \\
\text { mala, Thailand, Mauritius, Südafrika }\end{array}$ & $366(332-429)$ & \\
\hline
\end{tabular}

Quelle: NEI 2000, S. 113 (auf Grundlage HALEY 1998; HANNAH 1999) 


\subsection{Effizienzvorteile in der Milchindustrie}

Nachdem mögliche Synergievorteile und Erlöse der Zuckerbranche analysiert worden sind, soll dies im folgenden auch für die Milchwirtschaft geschehen. Auch in der Milchindustrie wird letztendlich das Optimum der Betriebsgröße durch economies of scale versus Transport- und Koordinationskosten bestimmt. Die Frage nach der optimalen Betriebsgrösse mit dem ihr zugrunde liegenden Motivationsansatz zur Erzielung von Effizienzvorteilen wird systematisch ergründet.

\subsubsection{Erfassungskosten}

Ein wesentliches Element der Kostenstruktur bei der Milchverarbeitung stellen die Milcherfassungskosten dar. So betrugen die Stückkosten der Milcherfassung 1998 im Durchschnitt 2,10 Pf pro kg Milch ${ }^{748}$. Sie sind daher ein bedeutender Kostenfaktor in der Wertschöpfungskette der Milchprodukte ${ }^{749}$. Die Milcherfassungskosten bestehen im wesentlichen aus den An- und Rückfahrtkosten vom Milchproduktionsstandort zur Milchverarbeitungsstätte. Einzelne Komponenten sind Kosten für Maschinen (LKW), Personal und sonstige Kosten für Reinigung, Rüsten usw. Im Zuge des Strukturwandels landwirtschaftlicher Milchviehbetriebe bewirkt die abnehmende Zahl der Milcherzeuger zwangsläufig eine Verringerung der Haltestellenzahl. Dies hat zur Folge, dass mit jedem geringeren Haltestopp auch dessen haltestellenspezifischer Fixkostenverbrauch entfällt. Zusätzlich steigt mit zunehmender Anlieferungsmenge pro Milcherzeuger die effektive Absaugleistung durch den Tanksammelwagen ${ }^{750}$. Die Entwicklung zu größeren Milchmengen pro Lieferant führt somit zu einer effizienteren Milchübernahme.

Geht dagegen die regionale Milchdichte zurück, dann muss entsprechend das Sammelfahrzeug eine weitere Entfernung zurücklegen, um das gleiche Volumen zu generieren. Dies gilt vor allem für Anhängertouren mit mehrmaliger Rückkehr zum Anhängerabstellplatz. Folglich steigen in diesem Fall die Sammelstreckenkosten einer Erfassungstour an. Steigt indessen die regionale Milchdichte, so ist von einer Reduzierung der Sammelstreckenkosten auszugehen.

Bei gleichbleibender Milchdichte und damit weitgehend unverändertem Fahrstreckenaufwand durch eine effizientere und zugleich effektivere Milchübernahme an den Haltestellen kann die Leistung der Fahrzeuge gesteigert, der Zeitverbrauch pro Mengen-

\footnotetext{
${ }^{748}$ Vgl. WeINDLMAIER u.a. 1999, S. 882

${ }^{749}$ Vgl. BRAUn 2000, S. 32

750 Vgl. HAISCH/BETZ 1983
} 
einheit gesenkt und schließlich der Fuhrpark (plus Personal) verkleinert werden. Nimmt in einer Region die Milchdichte zu, so dass zusätzlich auch noch ein Sammelstreckeneffekt entsteht, wird sich diese positive Entwicklung verstärken ${ }^{751}$.

Die Erfassungskosten werden zusätzlich durch die Organisation der Logistik stark beeinflusst. So führt eine „zweitägige Abholung” gegenüber der täglichen Erfassung zu einer Halbierung der Haltestellenkontakte und zu einer Verdoppelung der Milchmenge pro Haltestelle und Abholung. Dadurch lässt sich der notwendige Fuhrpark sowie der Fahrstrecken- und Arbeitszeitaufwand für die Milcherfassung um bis zu $30 \%$ reduzieren $^{752}$. Je geringer die regionale Milchdichte ist, desto größer sind die aus dieser Maßnahme zu erwartenden Einsparungen. BRAUN hob hervor, dass auch bei den sonstigen Erfassungskosten mit Einsparungen bei Ausdehnung der Erfassungsmenge zu rechnen ist, da sich Rüst- und Reinigungskosten entsprechend der Fuhrparkentwicklung anpassen, Aufwendungen für Güteuntersuchungen und Milchgeldabrechnung proportional zur Zahl der Lieferanten sinken und die Kosten für die Erzeugerberatung tendenziell zurückgehen, da sowohl die absolute Zahl der Milchlieferanten als auch der relative Anteil der überdurchschnittlich beratungsintensiven kleinen Milcherzeuger abnehmen $^{753}$.

Bei der Kalkulation der Erfassungskosten besteht nach den vorigen Ausführungen eine Beziehung zwischen den Anfahrtskosten ${ }^{754}$ und der Erfassungsmenge pro Betriebsstätte. Dieser Zusammenhang basiert auf der Erfordernis, mit steigender Milchmenge das Erfassungsgebiet auszudehnen und die Milch auch von entfernteren Sammelgebieten abzuholen. Hierdurch steigt die mittlere Distanz zwischen der Molkerei und ihren einzelnen Sammelgebieten. BRAUN errechnete Anfahrtskosten, die pro Entfernungskilometer zwischen 2,57 DM bei einem Sammelfahrzeug mit 9 t Kapazität und 2,93 DM bei zusätzlichem Einsatz eines Anhängers mit 16 t Kapazität varierten ${ }^{755}$.

Bei voller Kapazitätsauslastung liegen die Anfahrtsstückkosten pro 100 Entfernungskilometer folglich zwischen 1,17 und 2,86 Pf/kg erfasster Milch. Es wird also von zur Entfernung zwischen Molkerei und Sammelgebiet proportionalen Anfahrtsstückkosten ausgegangen. Dies bedeutet bei einer konzentrischen Ausdehnung des Erfassungsge-

\footnotetext{
${ }^{751}$ Vgl. dazu ausführlich BRAUn 2000, S. 33

752 Vgl. WeindLMAier/Betz 1997, S. 22

${ }^{753}$ Vgl. BRAUn 2000, S. 56

$754=$ An- und Rückfahrt

${ }^{755}$ Vgl. BRAUn 2000, S. 58. Folgende Annahmen liegen der Kalkulation zugrunde: a) Kosten für den Tanksammelwagen je gefahrenen Kilometer: $0,70 \mathrm{DM} / \mathrm{km}$; b) Kosten für den Anhänger je gefahrenen Kilometer: 0,18 DM/km; Personalkosten je Stunde Fahrzeit: $35 \mathrm{DM} / \mathrm{h}$; durchschnittliche Fahrgeschwindigkeit: $60 \mathrm{~km} / \mathrm{h}$ (hoch bemessen).
} 
bietes um die Betriebsstätte, dass die Anfahrtsstückkosten unterproportional zur Erfassungsmenge steigen. Der Grund für dieses Kostenverhalten ist die mit dem Kreisradius quadratisch wachsende Kreisfläche, d.h., bei einer Verdoppelung des Erfassungsradius vervierfacht sich die Erfassungsmenge usw. Ausgehend von diesen Überlegungen kann z.B. analysiert werden, wie sich eine regionale Reduktion der Anfahrtskosten auswirken würde ${ }^{756}$.

Zu vermerken ist, dass die Nähe zum Rohstoff und die damit verbundenen Milcherfassungskosten von entscheidender Bedeutung für die Dimensionierung einer Betriebsstätte bzw. eines Unternehmens sind. Dies stützte auch die Erhebung von WELLERT, der 38 Molkereibetriebe nach der Bedeutung von Standortfaktoren befragte (vgl. Abbildung 63).

Abbildung 63: Unterschiedliche Bedeutung einzelner Standortfaktoren für die befragten Molkereien

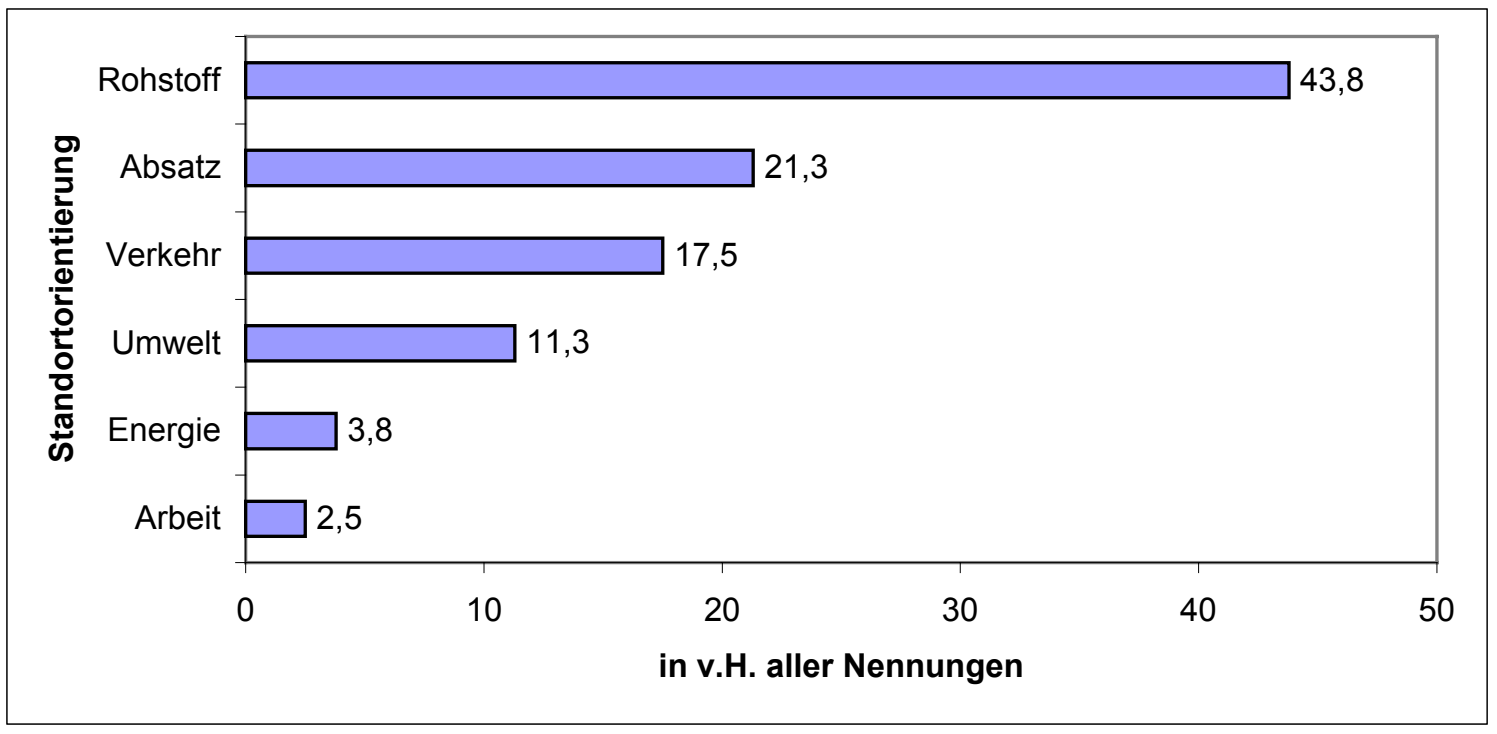

Quelle: WeLLert 1994, S. 150

\subsubsection{Produktion und Vermarktung}

Im vorigen Abschnitt wurde verdeutlicht, dass eine Vergrößerung des Milcheinzugsgebietes zu höheren Milcherfassungskosten führen kann, insbesondere wenn im Zuge von Fusionen Betriebsstätten geschlossen werden und größere Distanzen zwischen

\footnotetext{
756 Dazu ausführlich BRAUn 2000, S. 58: Es handelt sich bei der Annahme der konzentrischen Ausdehnung in der Praxis allerdings eher um eine Ausnahme. „Vielmehr liegen die Betriebsstätten oft am Rand ihres Erfassungsgebietes bzw. sind sie durch die Erfassungsgebiete anderer Molkereien oder Gebiete ohne Milcherzeugung von diesen getrennt. Die Erfassungsgebiete selber lassen sich z.T. auch weniger als kreisförmig oder quadratisch beschreiben, sondern eher als unregelmäßige bzw. zerklüftetet Gebilde."
} 
Erzeuger und Verarbeitungsstätte in Kauf genommen werden müssen. Demgegenüber stehen die Einsparungen durch Schließung von Werken und die dadurch bessere Auslastung der verbleibenden Betriebsstätten.

Ein bedeutender Vorteil größerer Unternehmen liegt in der Möglichkeit, sta-tische economies of scale verwirklichen zu können. Hinzu kommen eine bessere Möglichkeit der Nutzung des technischen Fortschritts bzw. der Automatisierung, eine Überlegenheit im Marketing und eine bessere Verhandlungsposition zum hochkonzentrierten $\mathrm{LEH}^{757}$. Einige Faktoren werden im weiteren näher untersucht.

\subsubsection{Kostenstruktur}

Eine Vergrößerung der Betriebsgröße, die in mutative und multiple Betriebsgrößenvariationen unterschieden wird, kann bezogen auf die Kostenstruktur verschiedene Effekte auslösen. Eine mutative Betriebsgrößenvariation ist dadurch gekennzeichnet, dass bei einer Betriebsvergrößerung die Verfahrenstechnik und die damit implizierte Art der eingesetzten Betriebsmittel verändert wird ${ }^{758}$. Dadurch ändert sich die Relation von fixen zu variablen Kosten. Zur Erläuterung sei die Tabelle 45 herangezogen.

Tabelle 45: Kostenstruktur in Molkereien (ohne Rohstoff)

\begin{tabular}{|l|c|c|c|}
\hline & Minimum & Maximum & $\begin{array}{c}\text { Durchschnitt } \\
\text { (gewichtet) }\end{array}$ \\
\hline Personal & $19 \%$ & $38 \%$ & $24 \%$ \\
\hline Verpackungsmaterial & $4 \%$ & $34 \%$ & $15 \%$ \\
\hline Abschreibungen & $4 \%$ & $13 \%$ & $8 \%$ \\
\hline Hilfs- und Zusatzstoffe & $2 \%$ & $15 \%$ & $7 \%$ \\
\hline $\begin{array}{l}\text { Energie-, Wasser-, } \\
\text { Betriebsstoffe }\end{array}$ & $2 \%$ & $10 \%$ & $6 \%$ \\
\hline Externe Instandhaltung & $2 \%$ & $5 \%$ & $3 \%$ \\
\hline Sonstige Kosten & $21 \%$ & $49 \%$ & $36 \%$ \\
\hline
\end{tabular}

Quelle: Institut für BWL des FML Weihenstephan, Unternehmensvergleich 1996, S. 40 (Basis: acht Unternehmen, die in ihrer Gesamtheit alle bedeutenden Molkerieprodukte herstellen)

Wird dagegen die Anzahl der Gebrauchsfaktoren (Betriebsmittel) ohne Einfluss auf die Verfahrenstechnik verändert, wird von multiplen Betriebsgrößenvariationen gesprochen $^{759}$.

\footnotetext{
757 Vgl. WeindLMAIER 1998, S. 242

758 Vgl. WÖHE 1996, S. 531

759 Vgl. WÖHE 1996, S. 531
} 
Anhand verschiedener Verarbeitungsprozesse wird im folgenden demonstriert, welche Potentiale vorhanden sind und welche damit verbundene Effekte die Kostenstruktur beeinflussen.

\subsubsection{Milcheingang}

Sobald die Milch erfasst und an der Betriebsstätte angeliefert wird, wird sie der allgemeinen Milchbehandlung unterzogen ${ }^{760}$. Durch Konzentration der Produktion auf einen bzw. wenige Standort(e) wird oftmals erst die Voraussetzung geschaffen, multiple und mutative Skaleneffekte in der Betriebsinfrastruktur zu realisieren ${ }^{761}$. Tabelle 46 zeigt dies exemplarisch für den Bereich der allgemeinen Milchbehandlung.

Tabelle 46: Herstellkosten ohne Rohstoff und statische Skaleneffekte in der Modellabteilung allgemeine Milchbehandlung

\begin{tabular}{|c|c|c|c|c|c|c|c|}
\hline \multirow[t]{2}{*}{ Auslastung } & \multicolumn{6}{|c|}{ Kapazität bzw. Stückkosten } & \multirow{2}{*}{$\begin{array}{l}\text { Skalen- } \\
\text { effekt (1) }\end{array}$} \\
\hline & Modell 1 & Modell 2 & Modell 3 & Modell 4 & Modell 5 & Modell 6 & \\
\hline $\begin{array}{l}\text { Kapazität in } \\
\text { Mio. } \mathrm{kg} / \mathrm{a}\end{array}$ & 118 & 196 & 275 & 549 & 1099 & 1648 & \\
\hline $100 \%$ in $\mathrm{Pf} / \mathrm{kg}$ & 1,27 & 0,91 & 0,75 & 0,60 & 0,53 & 0,51 & $149,8 \%$ \\
\hline $65 \%$ in Pf/kg & 1,62 & 1,14 & 0,92 & 0,72 & 0,64 & 0,61 & $165,6 \%$ \\
\hline
\end{tabular}

(1) Mehrkosten des kleinsten im Verhältnis zum größten Modell bei gleichem Kapazitätsauslastungsgrad

Quelle: BRAUn 2000, S. 64

\subsubsection{Be- und Verarbeitung}

Nachdem die allgemeine Milchbehandlung durchlaufen ist, erfolgt die Zuordnung des Rohstoffs Milch an die jeweilige Abteilung mit dem entsprechenden Verwendungszweck. Die entstehenden Kosten sowie mögliche Einsparungspotentiale werden in der nachfolgenden Tabelle (Tabelle 47) aufgeführt.

\footnotetext{
760 Dieser Verfahrensschritt umfasst die Reinigung, Kühlung und Vorstapelung der Milch

761 Vgl. Braun 2000, S. 64
} 
Tabelle 47: Herstellkosten einschließlich Rohstoff und statische Skaleneffekte in ausgewählten Modellabteilungen

\begin{tabular}{|c|c|c|c|c|c|c|}
\hline \multirow{2}{*}{$\begin{array}{l}\text { Modellabtei- } \\
\text { lung }\end{array}$} & \multirow[t]{2}{*}{ Auslastung } & \multicolumn{4}{|c|}{ Kapazität bzw. Stückkosten } & \multirow[t]{2}{*}{ Skaleneffekt (1) } \\
\hline & & Modell 1 & Modell 2 & Modell 3 & Modell 4 & \\
\hline H-Milch & $\begin{array}{l}\text { Kapazität in Pckg./a } \\
-100 \% \text { in Pf/Pckg. } \\
-63 \% \text { in Pf/Pckg. }\end{array}$ & $\begin{array}{l}30560 \\
78,4 \\
80,2\end{array}$ & $\begin{array}{l}61119 \\
77,0 \\
78,4\end{array}$ & $\begin{array}{l}122239 \\
76,0 \\
77,2\end{array}$ & $\begin{array}{l}183358 \\
75,7 \\
76,8\end{array}$ & $\begin{array}{l}3,6 \% \\
4,4 \%\end{array}$ \\
\hline Joghurt & $\begin{array}{l}\text { Kapazität in Pckg./a } \\
-100 \% \text { in Pf/Be. } \\
-60 \% \text { in Pf/Be. }\end{array}$ & $\begin{array}{l}138 \\
27,6 \\
28,5\end{array}$ & $\begin{array}{l}415 \\
25,7 \\
26,3\end{array}$ & $\begin{array}{l}829 \\
24,8 \\
25,4\end{array}$ & & $\begin{array}{l}11,1 \% \\
12,2 \%\end{array}$ \\
\hline $\begin{array}{l}\text { Pasteurisierte } \\
\text { Milch }\end{array}$ & $\begin{array}{l}\text { Kapazität in Pckg./a } \\
-100 \% \text { in Pf/Pckg. } \\
-64 \% \text { in Pf/Pckg. }\end{array}$ & $\begin{array}{l}30327 \\
75,5 \\
76,0\end{array}$ & $\begin{array}{l}64024 \\
74,1 \\
74,4\end{array}$ & $\begin{array}{l}128048 \\
73,8 \\
74,2\end{array}$ & & $\begin{array}{l}2,3 \% \\
2,4 \%\end{array}$ \\
\hline $\begin{array}{l}\text { Schnittkäse } \\
\text { (Gouda) }\end{array}$ & $\begin{array}{l}\text { Kapazität in t/a } \\
-100 \% \text { in } \mathrm{Pf} / \mathrm{kg} \\
-65 \% \text { in } \mathrm{Pf} / \mathrm{kg}\end{array}$ & $\begin{array}{l}5120 \\
555,7 \\
574,6\end{array}$ & $\begin{array}{l}11540 \\
542,2 \\
555,2\end{array}$ & $\begin{array}{l}19220 \\
535,6 \\
547,6\end{array}$ & $\begin{array}{l}30770 \\
530,9 \\
541,9\end{array}$ & $\begin{array}{l}4,7 \% \\
6,0 \%\end{array}$ \\
\hline $\begin{array}{l}\text { Speisequark } \\
\text { mager } \\
500 \mathrm{~g} \mathrm{Be}- \\
\text { cher }\end{array}$ & $\begin{array}{l}\text { Kapazität in t/a } \\
-100 \% \text { in } \mathrm{Pf} / \mathrm{kg} \\
-67 \% \text { in } \mathrm{Pf} / \mathrm{kg}\end{array}$ & $\begin{array}{l}5550 \\
181,1 \\
187,8\end{array}$ & $\begin{array}{l}11110 \\
166,5 \\
170,9\end{array}$ & $\begin{array}{l}22250 \\
158,9 \\
161,7\end{array}$ & & $\begin{array}{l}14,0 \% \\
16,1 \%\end{array}$ \\
\hline $\begin{array}{l}\text { Sprühmager- } \\
\text { milchpulver }\end{array}$ & $\begin{array}{l}\text { Kapazität in t/a } \\
-100 \% \text { in } \mathrm{Pf} / \mathrm{kg} \\
-60 \%(2) \text { in } \mathrm{Pf} / \mathrm{kg}\end{array}$ & $\begin{array}{l}6921 \\
371,3 \\
382,4\end{array}$ & $\begin{array}{l}13790 \\
358,8 \\
366,6\end{array}$ & $\begin{array}{l}35303 \\
351,1 \\
356,6\end{array}$ & & $\begin{array}{l}5,8 \% \\
7,2 \%\end{array}$ \\
\hline Weichkäse & $\begin{array}{l}\text { Kapazität in } \mathrm{t} / \mathrm{a} \\
-100 \% \text { in } \mathrm{Pf} / \mathrm{kg} \\
-65 \%(3) \text { in } \mathrm{Pf} / \mathrm{kg}\end{array}$ & $\begin{array}{l}4900 \\
594,5 \\
645,2\end{array}$ & $\begin{array}{l}7300 \\
561,3 \\
602,0\end{array}$ & $\begin{array}{l}12800 \\
531,4 \\
565,8\end{array}$ & $\begin{array}{l}17000 \\
522,1 \\
553,5\end{array}$ & $\begin{array}{l}13,9 \% \\
16,6 \%\end{array}$ \\
\hline
\end{tabular}

Anm.: (1) Mehrkosten des kleinsten im Verhältnis zum größten Modell bei gleichem Kapazitätsauslastungsgrad; (2) bei 280 Produktionstagen; (3) ohne Anpassung der vor- und nachgelagerten Anlagen

Quelle: BRAUN 2000, S. 63

Die statischen economies of scale sind vor allem bei Joghurt, Speisequark und Weichkäse von großer Bedeutung. Aber auch bei Commodities, wie z.B. H-Milch, beeinflussen Kostendifferenzen von wenigen Prozentpunkten oft bereits die Wettbewerbsposition des Anbieters entscheidend ${ }^{762}$.

Die Analyse der langfristigen Stückkostenkurve zeigt allerdings, dass das Ausmaß der Kostendegression ab einer bestimmten Abteilungsgröße erheblich abnimmt. "Zwar liegt das mathematische Minimum eindeutig bei voller Kapazitätsauslastung der größten verfügbaren Anlage. Der kostenmäßige Vorteil gegenüber einer etwas kleineren 
Anlage ist jedoch nur noch gering. " 763 Unter strategischen Gesichtspunkten kann deshalb eine Abteilungsgröße, welche die Degressionseffekte zwar weitgehend, aber nicht unbedingt vollständig ausschöpft, durchaus akzeptabel sein ${ }^{764}$.

Beispielhaft sei dies anhand einer Schnittkäserei verdeutlicht (Tabelle 48 u. Abbildung $64)$.

Tabelle 48: Gesamte und entscheidungsrelevante Herstellkosten für Schnittkäse (ohne Rohstoff) bei verschiedenen Abteilungsgrößen und Kapazitätsauslastungsgeraden

\begin{tabular}{|c|c|c|c|c|}
\hline & Modell 1 & Modell 2 & Modell 3 & Modell 4 \\
\hline \multicolumn{5}{|l|}{$\begin{array}{l}\text { Produktionsmenge in t/a } \\
\text { bei... }\end{array}$} \\
\hline ...100 \% Auslastung & 5120 & 11540 & 19220 & 30770 \\
\hline ...65 \% Auslastung & 3328 & 7501 & 12493 & 20001 \\
\hline ...50 \% Auslastung & 2560 & 5770 & 9610 & 15385 \\
\hline ...33 \% Auslastung & 1690 & 3808 & 6343 & 10154 \\
\hline \multicolumn{5}{|l|}{$\begin{array}{l}\text { Herstellungskosten ohne } \\
\text { Rohstoff in Pf/kg Käse bei... }\end{array}$} \\
\hline ...100 \% Auslastung & 77,1 & 64,1 & 57,5 & 52,8 \\
\hline ...65 \% Auslastung & 96,0 & 77,1 & 69,5 & 63,8 \\
\hline ...50 \% Auslastung & 110,8 & 90,5 & 81,2 & 73,8 \\
\hline ...33 \% Auslastung & 145,2 & 120,3 & 107 & 97,1 \\
\hline $\begin{array}{l}\text { Abschreibung und Zinsen in } \\
\text { TDM }\end{array}$ & 1435 & 2621 & 3850 & 5540 \\
\hline \multicolumn{5}{|l|}{$\begin{array}{l}\text { Herstellungskosten ohne } \\
\text { Rohstoff, Abschreibungen } \\
\text { und Zinsen in Pf/kg Käse } \\
\text { bei... }\end{array}$} \\
\hline$\ldots 100 \%$ Auslastung & 49,1 & 41,4 & 37,5 & 34,8 \\
\hline ...65 \% Auslastung & 52,9 & 42,2 & 38,7 & 36,1 \\
\hline ...50 \% Auslastung & 54,8 & 45,1 & 41,1 & 37,8 \\
\hline ...33 \% Auslastung & 60,3 & 51,5 & 46,3 & 42,5 \\
\hline
\end{tabular}

Quelle: Braun 2000, S. 178; basiert auf Krell/Wietbrauk 1993, S. 150, 253 u. 256

${ }^{763} \mathrm{Vgl.}$ WeINDLMAIER 1998c, S. 245

${ }^{764}$ Vgl. Braun 2000, S. 63 
Abbildung 64: Schnittkäseproduktion

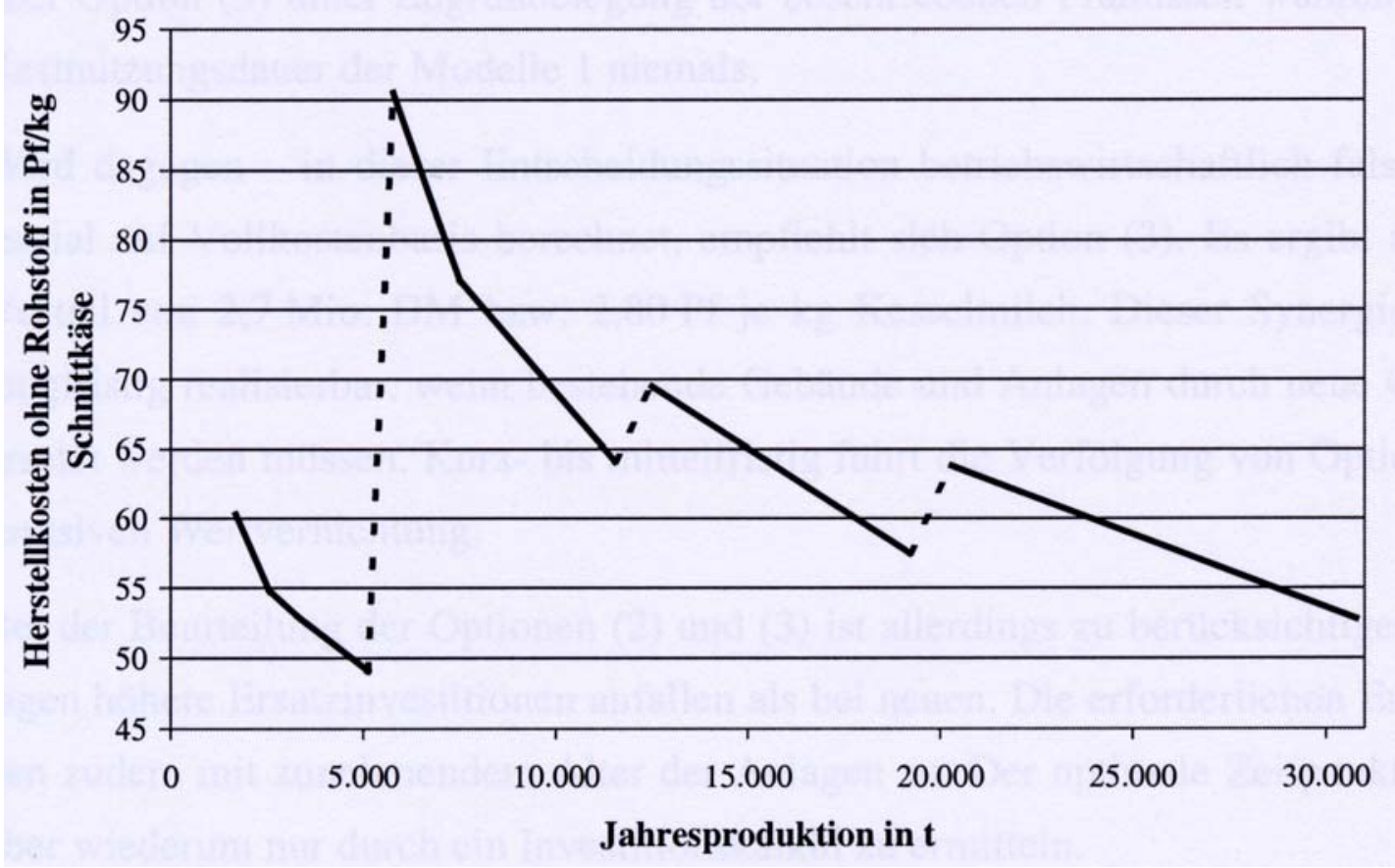

Quelle: BRAUn 2000, S. 179

Die vorgestellten Modellkalkulationen sind insofern nicht realitätsnah, da ein ganz wesentlicher Aspekt in Zusammenhang mit multiplen und mutativen Skaleneffekten noch nicht berücksichtigt ist: Molkereien können an einem Standort auch mehr als eine Produktionsanlage unterhalten. Der Vorteil einer solchen Konzentration liegt neben der einfacheren Rohstoffsteuerung und der transportkostengünstigeren Verwertung von Kuppelprodukten vor allem in einer Verteilung der Betriebsstätteninfrastruktur (Betriebsraum, Dampf-, Eiswassererzeugung, Kläranlage usw.) auf ein größeres Mengengerüst ${ }^{765}$.

Auch Weindlmaier zeigte am Beispiel einer Modellkalkulation für die Abfüllung von Konsummilch in Flaschen, dass bei 100\%iger Auslastungskapazität von etwa 1000 Einheiten/h - das entspricht etwa 5200 t Konsummilch pro Jahr - Kosten anfallen, die um etwa $10 \mathrm{Pf} / \mathrm{kg}$ höher liegen, als bei einer Anlage mit einer Maximalkapazität von 10000 Einheiten/h (entsprechend 52000 t Konsummilch pro Jahr). „Selbst Premiumanbieter werden kaum in der Lage sein, diesen Kostennachteil auf Dauer durch entsprechend höhere Erlöse zu kompensieren"766.

Ähnliches kann auch bei der Produktion von Schnittkäse festgestellt werden. Bei einer Käserei, in der in zwei Schichten ca. 3000 t Käse pro Jahr hergestellt werden, resultie-

\footnotetext{
${ }^{765}$ Vgl. BRAUN 2000, S. 64

766 Vgl. WeindLMAIER 1998, S. 246
} 
ren daraus Kosten von etwa 5,75 DM/kg inkl. Rohstoff. Eine wesentlich größere Käserei, in der in zwei Schichten ca. 31000 t Käse pro Jahr produziert werden, würde demgegenüber auf Kosten von 5,30 DM/kg kommen ${ }^{767}$. KRELL kalkulierte ähnliche Größen (vgl. Tabelle 49).

Tabelle 49: Modellkosten für die Herstellung von Goudakäse, 2-Schicht-Betrieb, 300 Produktionstage/Jahr, 1997

\begin{tabular}{|l|l|l|l|l|l|l|l|l|}
\hline Kostenarten/-gruppen & \multicolumn{1}{|l|}{$\begin{array}{l}\text { Modell 1 } \\
3300 \text { t Käse/a }\end{array}$} & $\begin{array}{l}\text { Modell 2 } \\
7500 \text { t Käse/a }\end{array}$ & $\begin{array}{l}\text { Modell 3 } \\
12500 \text { t Käse/a }\end{array}$ & $\begin{array}{l}\text { Modell } 4 \\
20000 \text { t Käse/a }\end{array}$ \\
& Pf/kg & $\%$ & Pf/kg & $\%$ & Pf/kg & $\%$ & Pf/kg & $\%$ \\
\hline Personal & 20,5 & 4 & 13,3 & 2 & 10,1 & 2 & 7,5 & 1 \\
Hilfs- u. Zusatzstoffe & 11,4 & 2 & 11,4 & 2 & 11,4 & 2 & 11,4 & 2 \\
Energie, Betriebsstoffe & 15,2 & 3 & 12,9 & 2 & 12,6 & 2 & 12,3 & 2 \\
Verpackung & 1,7 & 0 & 1,7 & 0 & 1,7 & 0 & 1,7 & 0 \\
Anlagekosten & 52,2 & 9 & 42,2 & 8 & 37,2 & 7 & 33,5 & 6 \\
Herstellungskosten & 101,0 & 17 & 81,5 & 15 & 73,0 & 13 & 66,4 & 12 \\
Rohstoffkosten & 484,5 & 83 & 479,1 & 85 & 479,1 & 87 & 479,1 & 88 \\
\hline Gesamtkosten & 585,5 & 100 & 560,6 & 100 & 552,1 & 100 & 545,5 & 100 \\
\hline
\end{tabular}

Quelle: KRELL et al. 1997, S. 88

\subsubsection{Lagerhaltung}

Ein weiterer Kostenfaktor ist die Lagerhaltung der fertigen Produkte. Skaleneffekte sind auch bei der Lagerhaltung realisierbar. Zum einen begründet ZIEGLER die Einsparung dadurch, dass mit steigender Lagerkapazität die Einstellung von qualifiziertem Personal für Planung, Steuerung und Kontrolle der Lagerprozesse rentabler wird ${ }^{768}$. Zum anderen rechnet sich aufgrund des hohen Investitionsvolumens ein automatisiertes Lagersystem erst ab einem bestimmten Lagervolumen ${ }^{769}$. Der Einsatz von spezialisierten Mitarbeitern sowie die Automatisierung ermöglichen eine bessere Flächen-/ Raumnutzung und verringern die Lagerverluste sowie den Personalbedarf. Dadurch sinken tendenziell die Lagerstückkosten ${ }^{770}$.

\footnotetext{
767 Vgl. WeINDLMAIER 1998, S. 246

768 Vgl. ZiEGLER 1997, S. 105

769 Vgl. BICHLER 1990, S. 188

770 Nach WeindLmaIER 1998, S. 246
} 


\subsubsection{Transport/Marketing/Absatz}

Im Bereich des Absatzes fallen im wesentlichen Distributions- und Marketingkosten an. Mit steigender Milchverarbeitungsmenge muss zwangsläufig auch das Absatzgebiet zumindest dann, wenn der Marktanteil im bisherigen Absatzgebiet nicht weiter erhöht werden kann - vergrößert werden. In Analogie zu den bei der Dimensionierung der Milcherfassung beschriebenen Zusammenhängen steigen folglich die Anfahrtskosten in die Absatzgebiete unterproportional zur Produktionsmenge. Verschiedene Faktoren beeinflussen diese Entwicklung ${ }^{771}$.

- Durch die nationale und internationale Konzentration des Lebensmitteleinzelhandels ist die durchschnittliche Nachfrage eines Handelsunternehmens stark angestiegen. Aus Kostengründen versuchen die Lebensmittelhändler inzwischen, die Zahl ihrer Lieferanten zu reduzieren. Ziel ist es, in jedem Produktsegment nur noch mit wenigen, leistungsfähigen Herstellern zusammenzuarbeiten. Dadurch entsteht für viele Molkereien u.a. der Zwang zu einer wenigstens national flächendeckenden Distribution ${ }^{772}$. Daher kann ein regional begrenzter Absatzradius zumindest bei haltbaren Produkten immer weniger aufrechterhalten werden. Eine weitere wichtige Entwicklung ist in diesem Kontext, dass der LEH immer mehr Logistikfunktionen an sich zieht. Dadurch nimmt aus Sicht der Molkereiwirtschaft die Bedeutung des Streckengeschäftes, d.h. die Direktbelieferung von Einzelhandeloutlets, zugunsten von Zentrallagerlieferungen stetig $a b^{773}$. Steht nun ein Molkereiunternehmen mit einem auf eine Region begrenzten Rohmilcherfassungsgebiet vor der Entscheidung, die Produktion in einer bzw. wenigen Betriebsstätte(n) zu konzentrieren, dann verändert sich durch diese Maßnahme die durchschnittliche Entfernung zwischen der konzentrierten Produktion und den Zentrallagern des Lebensmittelhandels nur geringfügig. Die Anfahrtsstückkosten steigen also kaum und können deshalb in der beschriebenen Entscheidungssituation bei der Dimensionierung von Betriebsstätten i.d.R. vernachlässigt werden ${ }^{774}$.

- Dies ist auch vor dem Hintergrund zu sehen, dass das Gewicht vieler Molkereiprodukte nur einen Bruchteil von dem des eingesetzten Rohstoffes Mich ausmacht (vgl. Tabelle 50). Die mengenabhängigen Anfahrtsstückkosten der Milcherfassung dominieren oft diejenigen der Distribution. Auch aus diesem

\footnotetext{
771 In Anlehnung an BRAUn 2000, S. 73

772 Vgl. auch ANNAS 1994, S. 52

${ }^{773}$ Vgl. KIEFER 1990, S. 159 f.; BAUTZ 1997, S. 87-89
} 
Grund haben die mengenabhängigen Anfahrtsstückkosten der Distribution keinen bzw. kaum einen Einfluss auf den Verlauf der Gesamtstückkostenkurve ${ }^{775}$.

Tabelle 50: Materialindizes verschiedener Molkereiprodukte

\begin{tabular}{|l|c|}
\hline Produktsegment & Materialindex (1) \\
\hline Erzeugnisse zum Frischverzehr & $1: 0,985$ \\
Trinkmilch zum Export & $1: 1,000$ \\
Frischkäse & $1: 4,061$ \\
Weichkäse & $1: 7,780$ \\
Schnittkäse & $1: 10,460$ \\
Magermilchpulver & $1: 10,870$ \\
Hartkäse & $1: 11,120$ \\
Molkepulver & $1: 16,000$ \\
Butter & $1: 21,020$ \\
\hline
\end{tabular}

(1) Gewicht des Fertigerzeugnisses / Gewicht der eingesetzten Milch bzw. Molke

Quelle: BRAUn 2000, S. 74

\subsubsection{Marketing}

Die Bedeutung der Verarbeitungsmenge soll auch anhand der Marketingkosten verdeutlicht werden. Ein erhebliches Defizit kleinerer Unternehmen liegt beim Angebot etablierter Herstellermarken sowie bei der für Markenartikel unverzichtbaren Werbung vor $^{776}$. Eine nationale Werbekampagne beansprucht heutzutage zweistellige Millionenbeträge ${ }^{777}$. Eine 10 Mio. $€$ Marketingkampagne, umgelegt auf eine Verarbeitungsmenge von 200 Mio. kg, würde eine Belastung von 5 Cent/kg Rohstoffeinsatz (RES) bedeuten. Bei einer Verarbeitungsmenge von 1 Mrd. kg Milch läge die entsprechende Belastung demgegenüber nur noch bei 1 Cent/kg RES!

Ein weiterer Vorteil ergibt sich aus der Möglichkeit der Belieferung des LEHs mit einem kompletten Sortiment anstelle einzelner Artikel. Neben der Lieferung von Markenartikeln fordert der Handel eine zusätzliche Belieferung mit Handelsmarken, was bei klei-

\footnotetext{
774 Vgl. auch DREWS 1982, S. 560

775 Vgl. HAISCH u.a. 1986, S. 294; KEANE 1998, S. 1

776 Vgl. WeIndLMAIER 1998, S. 247
} 
neren Unternehmen zu zusätzlichen Chargen mit hohen Stückkosten führt, während größere Molkereien durch den Einsatz verschiedener Abteilungen diesbzgl. eindeutige Vorteile besitzen ${ }^{778}$.

WEINDLMAIER verdeutlichte, dass u.a. durch den Einsatz moderner Instrumente wie $\mathrm{ECR}^{779}$ und Category Management beim LEH, dieser allein aus Kostengründen gezwungen sein wird, die Zahl seiner Lieferanten drastisch zu reduzieren. Dies erfordert seitens der Molkereien die Lieferung einheitlicher, garantierter Qualität zu konkurrenzfähigen Preisen ${ }^{780}$.

\subsubsection{Investition}

Mit Unternehmenszusammenschlüssen in der Molkereibranche sind oftmals Umstrukturierungen verbunden, die einen entsprechenden Investitionsbedarf beanspruchen. Evident ist zunächst, dass milchverarbeitende Unternehmen mit zunehmender Größe der Produktionsabteilungen einen zwar absolut betrachtet höheren, aber relativ gesehen geringeren Finanzbedarf haben ${ }^{781}$ (vgl. Tabelle 51).

\footnotetext{
777 Vgl. WeINDLMAIER 1998, S. 247; eigene Ergebnisse der Befragung

778 keine Umrüstkosten usw., vgl. WeINDLMAIER 1998, S. 246

779 Efficiency Consumer Response

780 Vgl. WeindLMAieR 1998, S. 248

781 Vgl. Braun 2000, S. $111 \mathrm{f}$
} 
Tabelle 51: Relativer Finanzbedarf in Abhängigkeit von der investierten Abteilungsgröße

\begin{tabular}{|c|c|c|c|c|c|c|c|c|}
\hline \multirow[b]{2}{*}{ Modellabteilung } & & \multicolumn{6}{|c|}{$\begin{array}{l}\text { Kapazität in Mio. kg RES bzw. Investition in Pf/kg RES (bei } 100 \% \\
\text { Kapazitätsauslastung) }\end{array}$} & \multirow[b]{2}{*}{$\begin{array}{l}\text { Skalen- } \\
\text { effekt (1) }\end{array}$} \\
\hline & & Modell 1 & Modell 2 & Modell 3 & Modell 4 & Modell 5 & Modell 6 & \\
\hline $\begin{array}{l}\text { Allgemeine } \\
\text { Milchbehand- } \\
\text { lung }\end{array}$ & $\begin{array}{l}\text { Kapazität } \\
\text { Investition }\end{array}$ & $\begin{array}{l}118 \\
4,0\end{array}$ & $\begin{array}{l}196 \\
2,6\end{array}$ & $\begin{array}{l}275 \\
2,0\end{array}$ & $\begin{array}{l}549 \\
1,4\end{array}$ & $\begin{array}{l}1099 \\
1,2\end{array}$ & $\begin{array}{l}1648 \\
1,1\end{array}$ & 3,63 \\
\hline H-Milch (2) & $\begin{array}{l}\text { Kapazität } \\
\text { Investition }\end{array}$ & $\begin{array}{l}32 \\
16,3\end{array}$ & $\begin{array}{l}63 \\
12,9\end{array}$ & $\begin{array}{l}126 \\
10,1\end{array}$ & $\begin{array}{l}189 \\
9,5\end{array}$ & & & 1,72 \\
\hline Joghurt & $\begin{array}{l}\text { Kapazität } \\
\text { Investition }\end{array}$ & $\begin{array}{l}16 \\
75,9\end{array}$ & $\begin{array}{l}47 \\
50,9\end{array}$ & $\begin{array}{l}95 \\
45,8\end{array}$ & & & & 1,66 \\
\hline $\begin{array}{l}\text { Pasteurisierte } \\
\text { Konsummilch } \\
\text { (2) }\end{array}$ & $\begin{array}{l}\text { Kapazität } \\
\text { Investition }\end{array}$ & $\begin{array}{l}31 \\
5,14\end{array}$ & $\begin{array}{l}66 \\
3,8\end{array}$ & $\begin{array}{l}132 \\
3,6\end{array}$ & & & & 1,5 \\
\hline $\begin{array}{l}\text { Schnittkäse } \\
\text { (Gouda) }\end{array}$ & $\begin{array}{l}\text { Kapazität } \\
\text { Investition }\end{array}$ & $\begin{array}{l}50 \\
21,6\end{array}$ & $\begin{array}{l}111 \\
17,2\end{array}$ & $\begin{array}{l}186 \\
15,1\end{array}$ & $\begin{array}{l}297 \\
13,5\end{array}$ & & & 1,6 \\
\hline $\begin{array}{l}\text { Speisequark } \\
\text { mager } 500 \mathrm{~g} \\
\text { Becher (4) }\end{array}$ & $\begin{array}{l}\text { Kapazität } \\
\text { Investition }\end{array}$ & $\begin{array}{l}25 \\
19,1\end{array}$ & $\begin{array}{l}50 \\
11,9\end{array}$ & $\begin{array}{l}100 \\
7,9\end{array}$ & & & & 2,42 \\
\hline $\begin{array}{l}\text { Sprühmager- } \\
\text { milchpulver (5) }\end{array}$ & $\begin{array}{l}\text { Kapazität } \\
\text { Investition }\end{array}$ & $\begin{array}{l}73 \\
11,1\end{array}$ & $\begin{array}{l}146 \\
7,9\end{array}$ & $\begin{array}{l}374 \\
5,4\end{array}$ & & & & 2,06 \\
\hline Weichkäse (2) & $\begin{array}{l}\text { Kapazität } \\
\text { Investition }\end{array}$ & $\begin{array}{l}41 \\
60,7\end{array}$ & $\begin{array}{l}62 \\
50,3\end{array}$ & $\begin{array}{l}108 \\
42,4\end{array}$ & $\begin{array}{l}144 \\
37,9\end{array}$ & & & 1,6 \\
\hline
\end{tabular}

Anm.: (1) Relative Investitionssumme des kleinsten im Verhältnis zum größten Modell ; (2) Annahme: RES $=1,03 \mathrm{~kg}$ pro Packung H-Milch bzw. pasteurisierte Konsummilch bzw. pro Kesselmilch in allen Modellen; (3) Annahme: RES $=4,11 \mathrm{~kg}$ pro $\mathrm{kg}$ Quark in allen Modellen; (4) Annahme: RES $=4,11 \mathrm{~kg}$ pro kg Quark in allen Modellen ; (5) Annahme: RES = 10,6 kg pro kg Magermilchpulver in allen Modellen

Quelle: BRAUn 2000, S. 112

Bspw. beträgt die zu finanzierende Investitionssumme in einer kleinen Schnittkäserei (Modell 1) mit 21,6 Pf pro kg Kesselmilch das 1,6-fache des Finanzbedarfes von Modell 4. Die Vergleichsfaktoren für die anderen Modellabteilungen liegen zwischen 1,5 und 3,63 .

Hervorzuheben ist, dass die relativ betrachtet geringere Investitionssumme von groBen Produktionsabteilungen nicht nur über niedrigere kalkulatorische Abschreibungen und Zinsen zu günstigeren Herstellungskosten führt. Vielmehr ermöglicht der relativ gesehen niedrigere Finanzbedarf erst viele Investitionen, bzw. es steht der Differenzbetrag für Zukunftsinvestitionen wie z.B. Markenstrategien, F+E-Projekte oder Anlagen zur Erhöhung des Veredlungsgrades zur Verfügung. Dies gilt zumindest für dieje- 
nigen genossenschaftlichen Molkereien, die sich hauptsächlich über von der Anlieferungsmilch abhängige Geschäftsanteile finanzieren ${ }^{782}$.

\subsubsection{Technische Fortschritte}

Neben statischen Gesichtspunkten sind ebenfalls dynamische Effekte u.a. bedingt durch die Möglichkeit zur Nutzung von technischem Fortschritt bzw. von Automatisierung, die oftmals im Zuge von Fusionen realisiert werden, nicht zu vernachlässigen. Solche Erneuerungen sind mit erheblichen Investitionen verbunden, die Kleinunternehmen oftmals nicht alleine aufbringen können. Tabelle 52 demonstriert, welchen Einfluss eine Automatisierung auf die Kosten und den Personalbedarf (teuerster Produktionsfaktor) ausübt.

Tabelle 52: Beispielhafte Auswirkungen der Automatisierung auf den Personalbedarf und die Kosten

\begin{tabular}{|l|c|c|}
\hline Vorgang & $\begin{array}{c}\text { Situation vor der Auto- } \\
\text { matisierung }\end{array}$ & $\begin{array}{c}\text { Situation nach der Auto- } \\
\text { matisierung }\end{array}$ \\
\hline Herstellung von Schnittkäse & 1886 & 2545 \\
Herstellungsmenge (t) & 60330 & 35420 \\
Personaleinsatz (h) & 31,99 & 13,92 \\
Personaleinsatz in (h/t) & 123682 & 129656 \\
Herstellung von Trinkmilch, Milch- \\
frischprodukten und Käse & 240 & 200 \\
Rohstoffeinsatz (1000 DM) & 16806 & 13550 \\
Personaleinsatz (Anzahl) & 7977 & 5185 \\
Personalkosten (1000 DM) & & \\
Energie- und Betriebsstoffkosten & & \\
(1000 DM) & & \\
\hline
\end{tabular}

Quelle: WeindLMAIER 1998, S. 247

Die geschilderten Beispiele haben einen Eindruck vermittelt, welche Faktoren zur Kostenminderung aber auch -steigerung beitragen können. Aus Sicht des strategischen Planers sind diese Faktoren gegeneinander abzuwägen und mit Hilfe ergänzender Betrachtungen zur Gestaltung des Optimums einer Betriebsstätte bzw. eines Unternehmes heranzuziehen. Diesem Kontext wird in dem nächsten Abschnitt nachgegangen.

782 Vgl. dazu Braun 2000; Weindlmaier 1998; WisSEnSCHAFtLiCher Beirat 2000 


\subsubsection{Optimale Betriebsgröße insgesamt: eine Abwägung}

In einer neueren Untersuchung versuchten HöPER et al. in ihrer Kalkulation, die optimale Betriebsgröße einer Molkerei zu ermitteln. Dabei werden die verschiedenen in den vorigen Abschnitten behandelten Aspekte in ihrem Zusammenwirken betrachtet und die positiven Effekte einer Betriebsvergrößerung den negativen gegenübergestellt. Dieses Modell und die zugrundeliegenden Annahmen werden im folgenden näher erläutert.

Für einzelne Funktionsbereiche wie Rohstofferfassung, Verarbeitung und Distribution wird unterstellt, dass die Unternehmen jeweils nur eine Betriebsstätte besitzen ${ }^{783}$. Zwischenbetriebliche Transporte von Halb- und Fertigprodukten finden also keine Berücksichtigung. Jede Betriebsstätte weist eine individuelle Produktionsstruktur auf, die sich u.a. im Einsatz des Rohstoffes in unterschiedliche Produktionsrichtungen niederschlägt. Um eine Vergleichbarkeit zu erzielen, wird von der Annahme ausgegangen, dass in allen modellierten Betriebsstätten das Produktionsprogramm identisch ist ( $\mathrm{vgl}$. Tabelle 53).

\subsubsection{Erfassung der Rohmilch}

Als Erfassungskosten im Rahmen der Rohmilch gelten diejenigen Kosten, die beim Betanken des Sammelwagens auf den landwirtschaftlichen Betrieben mit Rohmilch sowie dem Transport der Milch bis zur Rampe der Molkerei anfallen. Die Kosten setzen sich also aus den in den Erfassungsgebieten entstehenden Kosten und den damit verbundenen An- und Abfahrten zusammen. Folgende Rahmendaten wurden herangezogen $^{784}$ (Tabelle 53).

\footnotetext{
783 Vgl. HÖPER et al. 2000, S. 116; 1998 gab es im Bundesgebiet 165 Unternehmen und 294 Betriebsstättten (laut Milchindustrieverband (MIV) 1999).
} 
Tabelle 53: Struktur der Milcherzeugung in Deutschland (1997) sowie unterstellte Modellannahmen

\begin{tabular}{|l|l|}
\hline Strukturdaten: & $27,170 \mathrm{Mrd} . \mathrm{kg}$ \\
\hline Milchanlieferung p.a. & $357014 \mathrm{qkm}$ \\
\hline Fläche Deutschlands & $76,103 \mathrm{t} / \mathrm{qkm} / \mathrm{Jahr}$ \\
\hline Milchdichte & 163000 \\
\hline Milchkuhhalter & \multicolumn{2}{|l|}{} \\
\hline Annahmen: & LKW u. Anhänger: 23 t; Personal \\
\hline Transportkosten & 6230 \\
\hline Erfassungsgebiete (theoretisch) & $48 \%$ im zweitägigen Rhythmus \\
\hline Milcherfassung &
\end{tabular}

Quelle: Höper et al. 2000, S. 117 auf Basis von ZMP, STATISTISCHES BUNDESAMT

\subsubsection{Be- und Verarbeitung}

Bei den Berechnungen wird zwischen drei Stufen der Verarbeitung differenziert: erstens die allgemeine Milchbehandlung ${ }^{785}$. Dieser Verfahrensschritt umfasst die Reinigung, Kühlung und Vorstapelung der Milch. Anschließend wird die bereitgestellte Milch zu Konsummilch (pasteurisierte UHT-Milch), Butter, Schnittkäse, Speisequark und Joghurt verarbeitet, wobei hierbei als Koppelprodukte Buttermilch, Molke und Magermilch anfallen. In einem letzten Schritt werden diese Produkte zu Buttermilch-, Molken- und Magermilchpulver verarbeitet. Für die Betriebsstätten wird ein einheitliches Produktionsprogramm festgelegt, d.h., der prozentuale Anteil des Rohstoffeinsatzes für die Herstellung einzelner Produkte ist bei allen Betriebsstätten gleich ${ }^{786}$.

Die Modellrechnungen basieren auf einer stufenweisen Verrechnung der Kosten. Die für die Produktherstellung benötigten Faktorverbräuche werden als jahresfixe, tagesfi$x e$, chargenfixe und mengenproportionale Faktorverbräuche ausgewiesen und mit aktuellen Preisen bewertet. Berücksichtigt werden sämtliche Kostenarten, die bei der Produktion in den einzelnen Abteilungen anfallen.

\subsubsection{Distribution}

Die Berechnungen zur Distribution von Milch und Milchprodukten umfassen den Transport der Fertigprodukte von der Betriebsstätte zum Zentrallager des Lebensmitteleinzelhandels. Die Kommissionierung und Verteilung auf die einzelnen Outlets des LEH

\footnotetext{
784 Vgl. HöPER et al. 2000, S. 117

$785 \mathrm{Vgl}$. HÖPER et al. 2000, S. 118

${ }^{786} \mathrm{Vgl}$. HÖPER et al. 2000, S. 118
} 
(Streckenlieferung) wird nicht berücksichtigt, da diese Funktion zunehmend von den Handelsunternehmen selbst wahrgenommen wird $^{787}$.

Es wird davon ausgegangen, dass etwa 300 Läger, die mit Milchprodukten beliefert werden, existieren ${ }^{788}$. Je größer die Betriebsstätten sind, desto mehr Läger werden bedient. Hinsichtlich der Transportentfernung kann angenommen werden, dass kleinere Molkereien eher regional vermarkten, d.h. Läger in einem näher gelegenen Umfeld anfahren, und größere Betriebsstätten ihren Distributionsbereich ausdehnen (und nur einen Teil der Produkte an umliegende Läger liefern). Bei der Transportkostenkalkulation wird der Einsatz von Sattelaufliegern (20 t) mit Kühlvorrichtung unterstellt. Kostenarten sind LKW-Kosten sowie Personalkosten (jeweils abhängig von der Transportstrecke).

\subsubsection{Ergebnisse}

In Tabelle 54 sind die Ergebnisse zusammengefasst.

Tabelle 54: Modellspezifische Kosten der Erfassung, Be- und Verarbeitung und Distribution in Abhängigkeit unterschiedlicher Betriebsstättengrößen

\begin{tabular}{|l|l|l|l|l|l|l|l|}
\hline \multirow{2}{*}{$\begin{array}{l}\text { Größe der Be- } \\
\text { triebsstätte }\end{array}$} & \multicolumn{7}{|c|}{ Kosten } \\
in 1000 t RES & Gesamt & Rohstofferfassung & Be- und Verarbeitung & \multicolumn{2}{l|}{ Distribution } \\
\cline { 2 - 8 } & Pf/kg RES & Pf/kg RES & V.H. & Pf/kg RES & v.H. & Pf/kg RES & v.H. \\
\hline 50 & 20,8 & 1,36 & 6,52 & 19,3 & 92,75 & 0,15 & 0,73 \\
100 & 18,32 & 1,44 & 7,88 & 16,7 & 91,15 & 0,18 & 0,97 \\
200 & 17,21 & 1,55 & 8,98 & 15,4 & 89,44 & 0,27 & 1,59 \\
300 & 16,95 & 1,63 & 9,61 & 14,96 & 88,29 & 0,36 & 2,10 \\
400 & 16,85 & 1,67 & 9,92 & 14,74 & 87,53 & 0,43 & 2,55 \\
500 & 16,84 & 1,73 & 10,26 & 14,61 & 86,78 & 0,50 & 2,96 \\
600 & 16,85 & 1,76 & 10,46 & 14,53 & 86,2 & 0,56 & 3,34 \\
700 & 16,90 & 1,81 & 10,7 & 14,47 & 85,61 & 0,62 & 3,69 \\
800 & 16,93 & 1,83 & 10,81 & 14,42 & 85,16 & 0,68 & 4,03 \\
900 & 16,99 & 1,88 & 11,04 & 14,38 & 84,63 & 0,74 & 4,34 \\
1000 & 17,05 & 1,91 & 11,19 & 14,35 & 84,17 & 0,79 & 4,63 \\
\hline
\end{tabular}

Quelle: HöPER et al. 2000, S. 120

Bemerkenswert ist im Gegensatz zu den Ausführungen Brauns die Tatsache, dass die Kosten für Rohstofferfassung und Distribution zusammen nur ca. 9 bis $15 \%$ an den Gesamtkosten ausmachen. Zusammenfassend kann festgehalten werden, dass nach

\footnotetext{
787 Vgl. HÖPER et al. 2000, S. 119 nach WILD 1999, S. 58

788 Vgl. DREXLER 1999
} 
dieser Kalkulation die Gesamtkosten bis zu einer Betriebsstättengröße von etwa $500000 \mathrm{t}$ jährlicher Verarbeitungsmenge abnehmen. Besonders große Einsparungen ergeben sich bei einer Ausdehnung von 50000 t auf 300000 t mit einer Kostenersparnis von fast $4 \mathrm{Pf} / \mathrm{kg}$ RES. Bei Betriebsstätten, deren jährliche Verarbeitungsmenge 500.000 t überschreitet, erhöhen sich die Kosten je eingesetztem kg RES leicht (von 16,84 auf $17,05 \mathrm{Pf} / \mathrm{kg}$ ). Ab diesem Volumen werden die Kosteneinsparungen in der Be- und Verarbeitung von den Rohstofferfassungs- sowie Distributionskosten übertroffen.

Auch HülsEMEYER und HETZNER schätzten, dass die optimale Betriebsgröße bei mindestens 500 Mio. kg Milchverarbeitung pro Jahr liegt ${ }^{789}$. Solche pauschalen Feststellungen sind aber mit Vorsicht zu interpretieren, denn wie am Modell deutlich wurde, liegen den Berechnungen zahlreiche Annahmen zugrunde.

Unter strategischen Gesichtspunkten können schon (Mindest-)Abteilungsgrößen und Betriebsgrößen ausreichend sein, bei denen die Degressionseffekte zwar weitgehend, aber nicht vollständig ausgeschöpft werden. In diesem Kontext sind zwei weitere Aspekte von wesentlicher Bedeutung:

1) Welcher Preis muss für zusätzlich verarbeitete Milch bezahlt werden?

2) Kann die Mehrproduktion auch zu zufriedenstellenden Preisen abgesetzt werden?

Sowohl zu hohe Einkaufspreise für zusätzlich zu verarbeitende Milch als auch die Notwendigkeit von Preisnachlässen zum Absatz der Mehrproduktion haben einen wesentlich durchschlagenderen negativen Einfluss auf den Unternehmenserfolg als die nicht ganz vollständige Realisierung der Stückkostendegression ${ }^{790}$.

Niedrige Stückkosten sind zwar bei Verfolgung der Kostenführerschaftsstrategie der zentrale Wettbewerbsfaktor. Im Rahmen einer auch für Unternehmen der Molkereiwirtschaft zunehmend wichtiger werdenden kundenorientierten Differenzierungsstrategie verlieren jedoch die Kostensituation und der Preis gegenüber der Individualität und Qualität des Angebots, der Innovationskraft und dem angebotenen Service relativ an Bedeutung ${ }^{791}$. Jedoch müssen entsprechende Finanzmittel für Forschung und Entwicklung bzw. Marketingmaßnahmen zur Verfügung stehen, damit eine Differenzie-

\footnotetext{
789 Vgl. HÜLSEMEYER zit. in O.V. 1992c, S. 339; HetZneR zit. in FATHMANN 1998, S. 6

790 Vgl. BRAUn 2000, S. 56

791 Vgl. Weindlmaier 1998, S. 245 nach MäNNEL 1996, S. 63-95
} 
rungsstrategie konsequent angewendet werden kann. Wie auch durch die Unternehmensbefragung gestützt, ist ein wesentliches Fusionsmotiv das Streben nach Kostenführerschaft in der Produktion, um so Finanzmittel für andere Maßnahmen zur Verfügung zu haben.

\subsubsection{Empirie}

\subsubsection{Marktstruktur}

Auch empirisch lässt sich das Streben nach zunehmender Betriebsgröße beobachten (vgl. Tabelle 55). So ist der Anteil der Molkereien, die mehr als 300000 t Milch pro Jahr verarbeiten, sowohl absolut als auch relativ gegenüber den vorigen Jahren gestiegen (vgl. dazu auch Kapitel 2.6).

Tabelle 55: Zahl der Molkereiunternehmen

\begin{tabular}{|c|c|c|c|c|c|c|c|c|}
\hline \multirow{2}{*}{$\begin{array}{l}\text { Milchverarbeitung (1) im } \\
\text { Jahr von...bis unter... in } \\
1000 \mathrm{t}\end{array}$} & 1991 & 1994 & 1997 & 2000 & 1991 & 1994 & 1997 & 2000 \\
\hline & \multicolumn{4}{|c|}{ Unternehmen } & \multicolumn{4}{|c|}{ Anteil in \% } \\
\hline Weniger als 5 & 46 & 53 & 45 & 50 & 12,1 & 16,9 & 16,7 & 19,3 \\
\hline 5 bis unter 20 & 60 & 47 & 35 & 32 & 15,8 & 15,0 & 13,0 & 12,3 \\
\hline 20 bis unter 50 & 102 & 64 & 41 & 44 & 26,9 & 20,4 & 15,2 & 16,9 \\
\hline 50 bis unter 75 & 45 & 36 & 36 & 25 & 11,9 & 11,5 & 13,4 & 9,7 \\
\hline 75 bis unter 150 & 59 & 49 & 47 & 50 & 15,6 & 15,6 & 17,5 & 19,3 \\
\hline 150 bis unter 200 & 23 & 22 & 20 & 13 & 6,1 & 7,0 & 7,4 & 5,0 \\
\hline 200 bis unter 300 & 21 & 20 & 17 & 16 & 5,5 & 6,4 & 6,3 & 6,2 \\
\hline 300 und mehr & 23 & 23 & 28 & 29 & 6,1 & 7,3 & 10,4 & 11,2 \\
\hline Insgesamt & 379 & 314 & 269 & 259 & 100 & 100 & 100 & 100 \\
\hline
\end{tabular}

Anm.: (1) Milchverarbeitung = Milchanlieferung und Zukauf von Milch und Rahm sowie Einfuhr von Milch und Rahm der Unternehmen

Quelle: BMVEL 2001, S. 245

Auf der Verwendungsseite lässt sich die in Tabelle 56 ausgewiesene Struktur erkennen, wobei das Portfolio einzelner Unternehmen deutlich von den Durchschnittswerten abweichen kann. Der Einfluss agrarpolitischer Maßnahmen wie Interventionspreisfestlegung und Außenschutz dürfte große Auswirkungen auf die Veredlungsform von Milchprodukten haben. 
Tabelle 56: Molkereiunternehmen und durchschnittliche Herstellung von Milchprodukten

\begin{tabular}{|l|l|l|l|l|l|l|}
\hline \multirow{2}{*}{ Produktionsrichtung } & \multicolumn{3}{l}{ Zahl der Molkereiunternehmen } & \multicolumn{3}{l}{$\begin{array}{l}\text { Durchschnittliche Herstellung je } \\
\text { Unternehmen in Tonnen }\end{array}$} \\
\cline { 2 - 7 } & 1994 & 1997 & 2000 & 1994 & 1997 & 2000 \\
\hline Frischmilcherzeugnisse & 245 & 220 & 198 & 32119 & 37750 & 42686 \\
Darunter Konsummilch & 228 & 203 & 177 & 23845 & 28188 & 31854 \\
Kondensmilch & 17 & 16 & 16 & 31053 & 35388 & 35438 \\
Milchpulver & 51 & 48 & 43 & 10394 & 10256 & 11081 \\
Butter & 177 & 157 & 135 & 2606 & 2818 & 3147 \\
Hartkäse & 48 & 48 & 51 & 3744 & 3817 & 3682 \\
Schnittkäse & 90 & 84 & 84 & 4314 & 5958 & 6107 \\
Weichkäse & 60 & 48 & 37 & 1632 & 2540 & 3063 \\
Sauermilch-Kochkäse & 37 & 30 & 25 & 922 & 1130 & 1261 \\
Frischkäse & 125 & 112 & 94 & 5594 & 6703 & 8078 \\
Käse insgesamt & 248 & 217 & 193 & 5642 & 7328 & 8736 \\
Schmelzkäse & 22 & 23 & 20 & 7459 & 6526 & 8534 \\
Kasein/Kasinate & 5 & 3 & 4 & 1680 & 3533 & 3350 \\
\hline
\end{tabular}

Quelle: BMVEL 2001, S. 245

\subsubsection{Tatsächliches Synergiepotential}

Anhand der geführten Gespräche und publizierten Daten soll an dieser Stelle das geplante Synergiepotential nach Schätzungen der Unternehmensleitung dargestellt werden.

Beim Zusammenschluss der Tuffi/Campina/emzett im Jahre 2001 wurden neben Einsparungen beim gemeinsamen Einkauf von Roh-, Hilfs- und Betriebsstoffen in den Bereichen Geschäftsführung, Verwaltung, Logistik, Marketing, Vertrieb und Entwicklung Synergien erwartet ${ }^{792}$. Nachdem es zur unmittelbaren Schließung zweier Standorte bei der Fusion kam, wurde etwas später ein weiterer Standort geschlossen ${ }^{793}$, die Produktpalette von 1300 gelisteten Artikeln auf 700 gekürzt und die Verwaltung auf Köln konzentriert (vorher drei Verwaltungsstandorte) ${ }^{794}$. Durch die Umstrukturierung konnten 200 Arbeitsplätze eingespart werden ${ }^{795}$.

\footnotetext{
792 Vgl. CAMPINA Pressemitteilung vom 29.06.2001

${ }^{793} \mathrm{Vgl}$. Lebensmittelzeitung 23. Feb 2001: Standort Nr. 3 geschlossen

794 Vgl. TOP AGRAR (G. Veauthier) 10/2000, S. 109:

$795 \mathrm{Vgl}$. TOP AGRAR $11 / 2000$, S. R.31
} 
Die Hochwald/Eifelperle gibt zu den Motiven und Synergievorteilen bei ihrem Zusammenschluss im Jahre 2001 mehrere Begründungen ${ }^{796}$ :

- Verwaltung, Vertrieb und Koordination an einem Standort, ebenso Buchhaltung, Controlling, Betriebsorganisation;

- durch Fusion Komplettanbieter; Forderung des LEH wird nachgekommen;

- $\quad$ keine Werksschließung, da geringe Milchdichte hohe Transportkosten bedeutet. Zukünftig Transportkosten noch höher, deswegen Beibehaltung der Standorte präferiert.

- Kostenersparnis bei Milcherfassung, da vorher Überschneidungen im Milcheinzugsgebiet.

Die Humana (Zusammenschluss der Milchwerke Westfalen und der Westmilch im Jahr 1998) beziffert jährliche Einsparungspotentiale auf 29 Mio. DM ${ }^{797}$, was auf die Konzentration der Verwaltung, Verlagerung der Produktion von Frischmilchprodukten, Konzentration der Butterproduktion von drei auf einen Standort, gemeinsame Koordination im Marketing und Verkauf und Schließung eines Standortes zurückzuführen sei. Dabei wurden ca. 450 Stellen gestrichen ${ }^{798}$ und die Schließung von vier Standorten eingeleitet ${ }^{799}$. Allein durch eine Minderbelastung von Abschreibungen in Höhe von 20 Mio. DM/Jahr stehen ca. $1 \mathrm{Pf} / \mathrm{kg}$ Milch mehr an Finanzmitteln für das Unternehmen zur Verfügung. Durch den Zusammenschluss konnten auch höhere Preise gegenüber dem LEH durchgesetzt werden, da Humana durch die neue Größenordnung nicht einfach zu ersetzen ist ${ }^{800}$.

Die "neue" Nordmilch benannte Einsparungspotentiale im ersten Jahr auf ca. 17 Mio. DM, während die Summe im zweiten Jahr bereits auf 48 Mio. DM und im dritten Jahr nach der Fusion auf 52 Mio. DM angestiegen sei. Zurückzuführen sei dies u.a. auf Werksschließungen sowie eine gestärkte Position gegenüber dem Handel ${ }^{801}$.

Inwieweit sich die erwarteten Synergiepotentiale (auch) in den Erfolgskennziffern der Unternehmen niederschlagen, wird in den anschließenden Kapiteln thematisiert. Zuvor

\footnotetext{
796 Vgl. Agrarzeitung 15. Sept. 2001, S. 2 (Ernährungsdienstbeilage), sowie telefonische Auskunft

797 Vgl. LZ-News vom 20.04.1998

798 Vgl. LZ-News vom 29.06.2000

${ }^{799} \mathrm{Vgl}$. TOP AGRAR 6/99, S. 92

${ }^{800}$ Gespräch mit Humana-Direktionsassistent Sönke Voss vom 11.12.2001

${ }^{801} \mathrm{Vgl}$. LAND UND FORST 31.05.2001, S. 29, sowie eigene Erhebung
} 
wird jedoch das Argument der Marktmacht sowohl auf Anbieter- als auch Nachfrageseite überprüft.

\subsubsection{Rückschlüsse auf Marktmacht mit Hilfe von Preisbeobachtungen}

Im fallpraktischen Teil dieser Arbeit wurde erläutert, dass die Kartellbehörden beim Zusammenschluss der Nordmilch zunächst Bedenken gegen die Ausübung von Marktmacht auf Seiten des Beschaffungsmarktes äußerten. Der Rohstoff Milch nimmt als Kostenkomponente der Verbraucherpreise eine große Bedeutung ein (vgl. Tabelle 57 sowie Kostenstruktur im Anhang E).

Tabelle 57: Rohstoffkostenanteile an den Verbraucherpreisen bei Milch und Milcherzeugnissen

\begin{tabular}{|l|c|}
\hline Produkt & Anteil (\%) \\
\hline Konsummilch & 41,41 \\
Sahne & 41,48 \\
Joghurt & 12,77 \\
Kondensmilch & 36,12 \\
Butter & 73,02 \\
Hartkäse & 37,73 \\
Schnittkäse & 37,81 \\
Weichkäse & 27,65 \\
Frischkäse einschl. Speisequark & 26,40 \\
\hline
\end{tabular}

Quelle: LAHMANN 2001, S. 38

Eine Ausübung von Marktmacht auf der Beschaffungsseite würde sich in den an die Landwirte geleisteten Milchgeldauszahlungspreisen niederschlagen. Um diese Vermutung zu überprüfen, wurde mit Hilfe der jährlich erscheinenden ZMP-Milchdaten ein Milchpreisvergleich vorgenommen (vgl. Abbildung 65). Die Daten der ZMP sind aufgrund der Einbeziehung von Nebenkosten, wie z.B. Stoppkosten oder der Erwerb von Genossenschaftsanteilen, besonders geeignet ${ }^{802}$.

${ }^{802}$ Ausführliche Beschreibung der Methodik des Preisvergleiches ZMP: Milchpreisauswertung, verschiedene Jahrgänge 
Abbildung 65: ZMP-Vergleichspreise

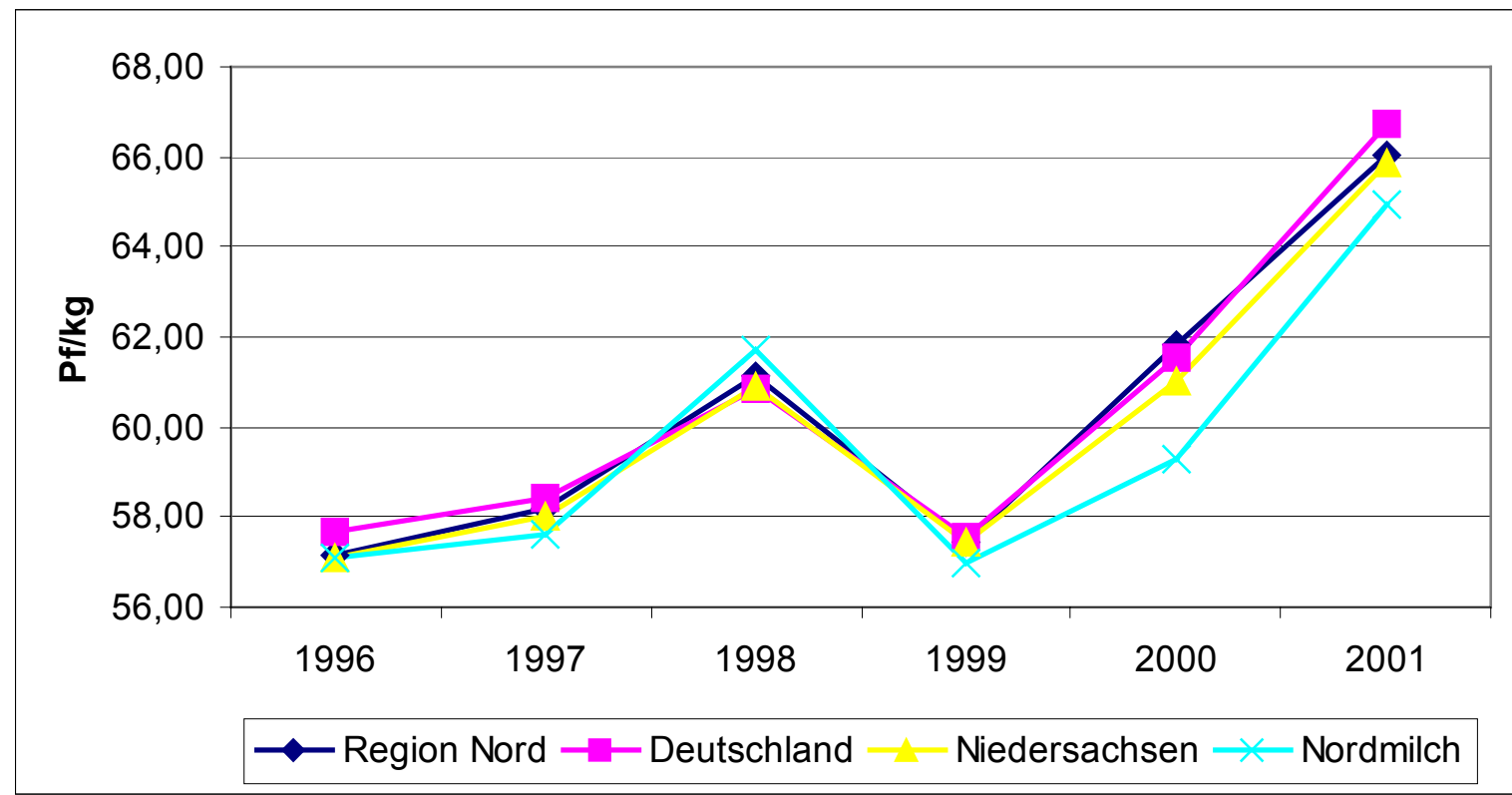

Quelle: eigene Darstellung auf Grundlage ZMP (Betrieb 500000kg Milchquote)

Interessanterweise zahlte die Nordmilch nach ihrem Zusammenschluss geringere Preise an die Landwirte aus als der Durchschnitt aller Molkereien, und zwar sowohl für Deutschland, Niedersachsen als auch die Nordregion (Mecklenburg-Vorpommern, Niedersachsen, Schleswig-Holstein)! Vor dem Zusammenschluss wurden durchschnittliche oder durchaus überdurchschnittliche Zahlungen getätigt (Detailangaben im Anhang E). Damit wäre ein Hinweis auf die Ausübung von Marktmacht auf dem Beschaffungsmarkt gegeben ${ }^{803}$. Inwieweit die verminderten Auszahlungspreise zur Vermögensbildung der Genossenschaft beitrugen, wird in der Bilanzanalyse näher aufgegriffen. Der Grund für die geringere Auszahlung könnte jedoch auch in einer Verminderung der Erlöse liegen. Die Entwicklung einiger wichtiger Preise wird in der nächsten Übersicht dargestellt (Abbildung 66) und soll die Marktentwicklung wiedergeben, von der die Unternehmen betroffen waren.

${ }^{803}$ Vgl. dazu Kapitel 6.2.2.1.2: Zunächst bekundete das Bundeskartellamt Bedenken auf dem Beschaffungsmarkt, da die Ausübung von Marktmacht ermöglicht worden wäre, konnte aber im Zuge der Beurteilung von der Unbedenklichkeit überzeugt werden. 


\section{Abbildung 66: Milchmarktpreisentwicklungen}

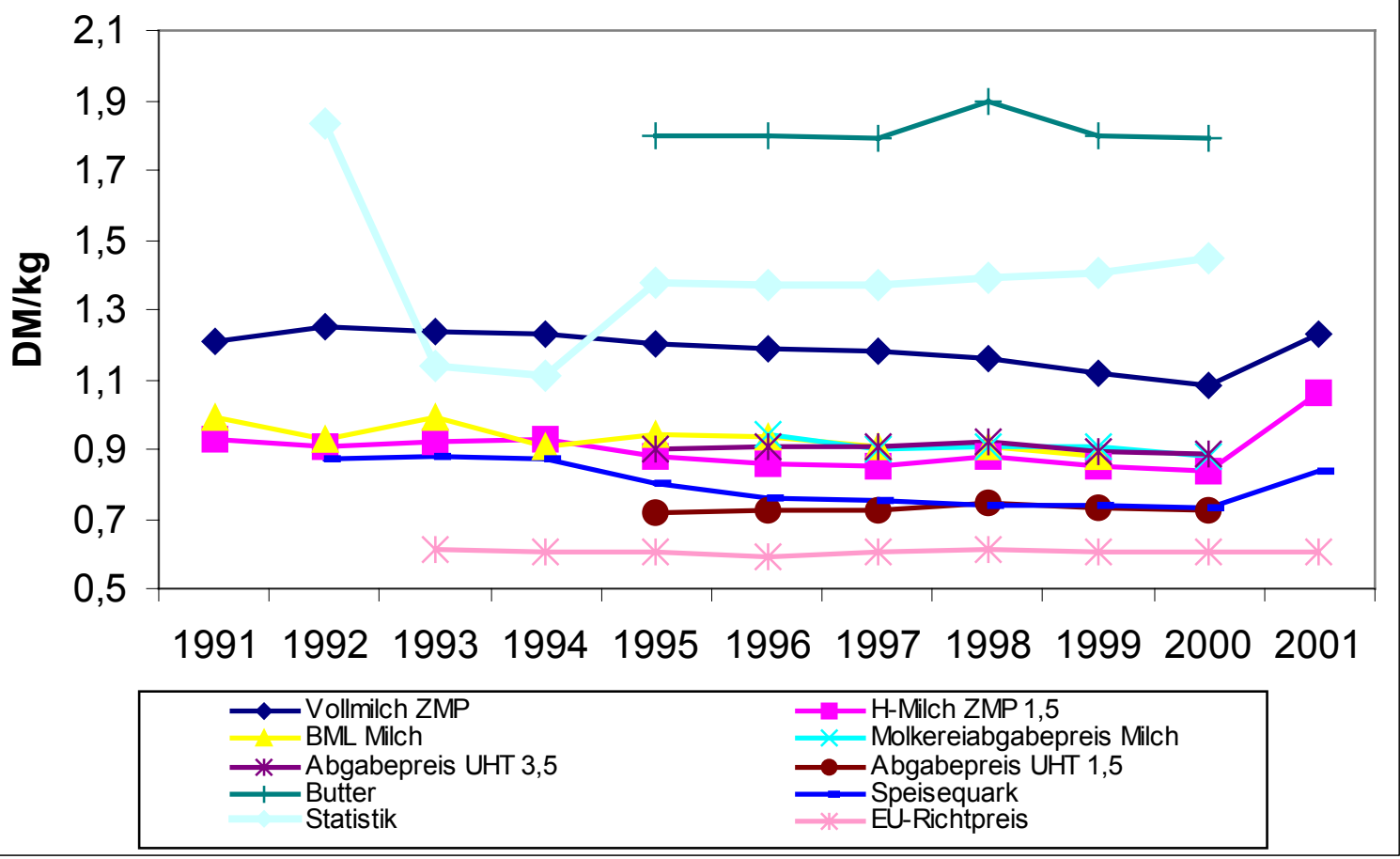

Anm.: Vollmilch ZMP = Einmalpackung $1 \mathrm{l}$; BML Milch = Milchabgabepreis (Molkerei) pro I laut Meldung des BML; Abgabepreis UHT 3,5 bzw. 1,5 = Abgabepreis Ultrahocherhitze Milch (1,5\% bzw. 3,5\% Fett) nach Abzug von Rabatten (BML); Butter = durchschnittlicher Verbraucherpreis (ZMP); Statistik: aus Daten des Statistisches Bundesamtes - Produzierendes Gewerbe - Durchschnittserlöspreis errechnet; H-Milch ZMP 1,5 $=$ Durchschnittsverbraucherpreis pro I (ZMP); Molkereiabgabepreis Milch = pasteurisierte Milch 3,5\% (BML); Speisequark = Magerstufe, $250 \mathrm{~g}$ Becher (ZMP)

Quelle: Berechnungen auf Grundlage von BML-/ZMP-Daten

Anhand eines LEH-Scannerdatensatzes der Firma MADAKOM wurde der Versuch unternommen, Rückschlüsse auf das Wettbewerbsverhalten der im Jahre 1998 fusionierten "Nordmilch" zu ziehen. Der MADAKOM Datensatz ${ }^{804}$ konnte durch Daten vom 30. September 1996 bis zum 28. Juni 1999 um Angaben von 38 Geschäften erweitert werden $^{805}$. Ein Preisvergleich wurde für Frisch- und Kondensmilch sowie für weitere Milramprodukte (Nordmilch), die in mindestens zwei Geschäftsfilialen über einen längeren Zeitraum geführt wurden, durchgeführt. Die Ergebnisse für Milram- und Frischmilchprodukte sind im folgenden illustriert (vgl. Abbildung 67).

${ }^{804}$ In Kapitel 8.1.5.1 näher beschrieben

${ }^{805} \mathrm{Vgl.} \mathrm{HeRRMANN/MÖSER/WERNER} 2002$ für genauere Selektion der Geschäfte 
Abbildung 67: Entwicklung von ausgewählten Frisch- und Kondensmilcherzeugnissen und Markenprodukten
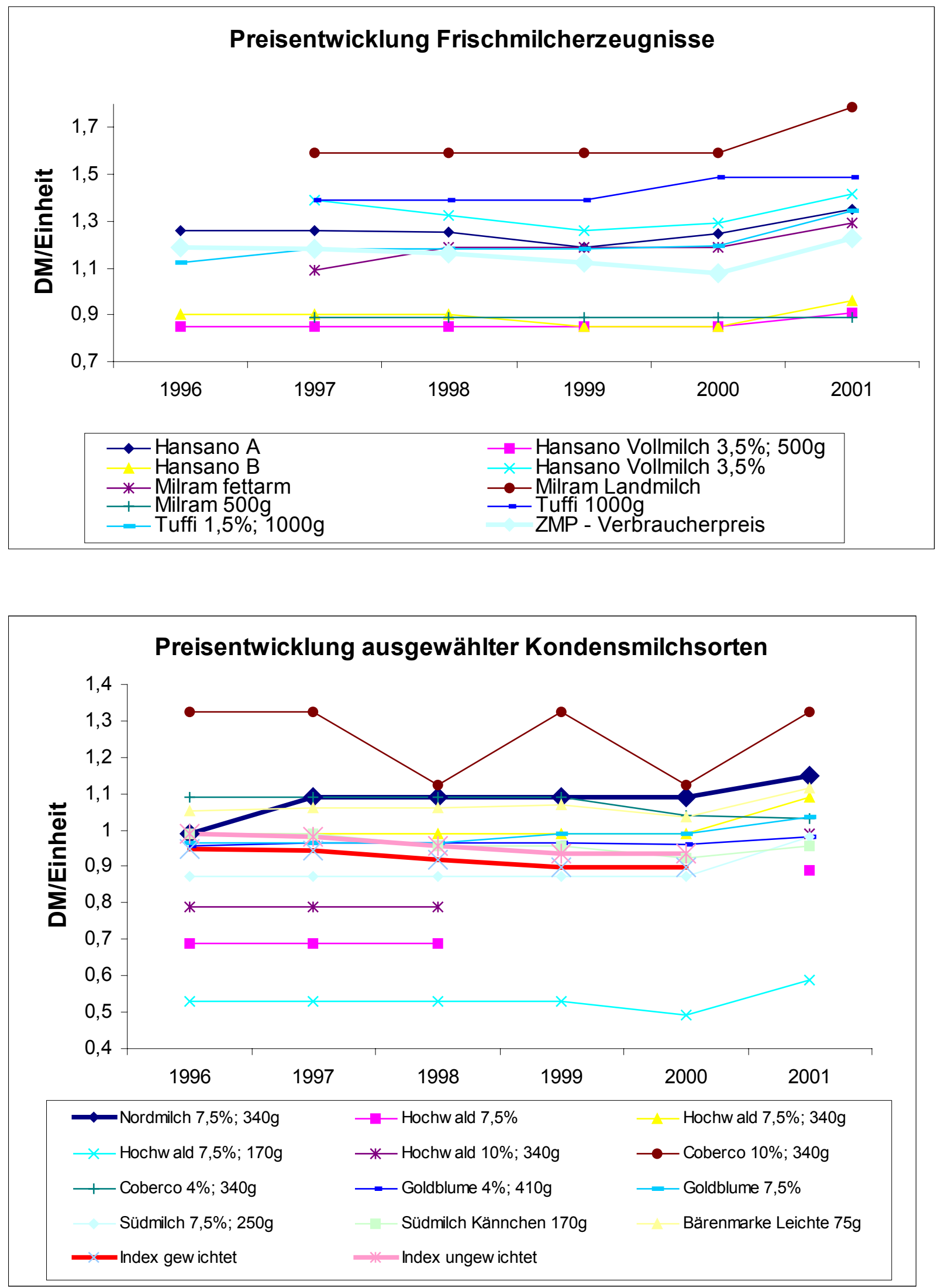

Anm.: erste Zahlenabgabe gibt den Fettgehalt an; zweite Zahlenangabe (soweit vorhanden) gibt die Packungsgröße an 


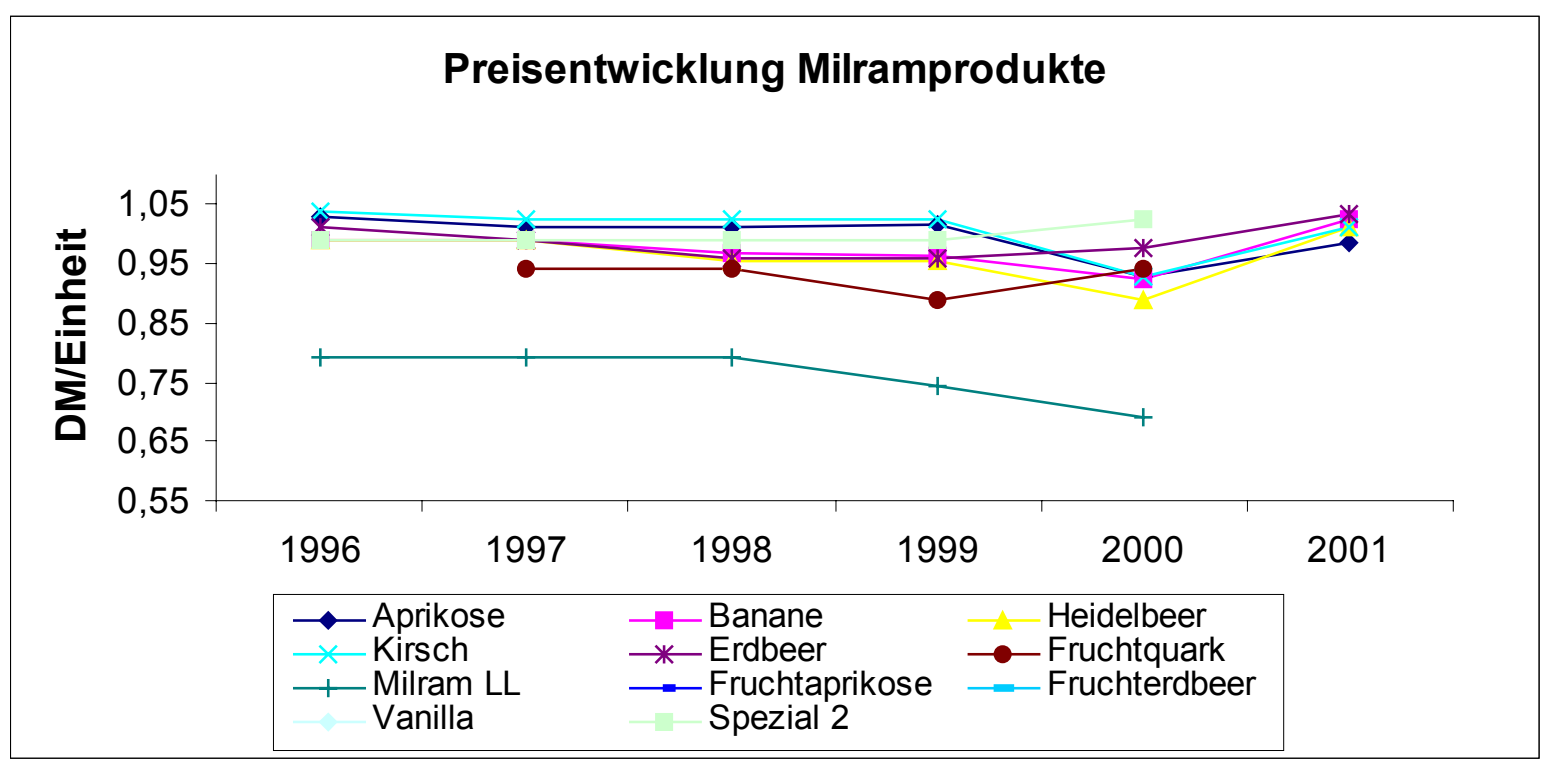

Quelle: eigene Berechnungen auf Grundlage von MADAKOM

Als besonders schwierig erweist sich die Vergleichbarkeit von verschiedenen Herstellern. So spiegeln unterschiedliche Verbraucherpreise auch den Markenwert verschiedener Produkte wider. Der Direktvergleich wird u.a. auch durch die Existenz verschiedener Packungsgrößen erschwert. Allerdings wird aus den Ergebnissen evident, dass der überwiegende Anteil der Produkte von ähnlichen Preisschwankungen gekennzeichnet ist (Parallelentwicklung). Eine überdimensionale Preiserhöhung herstellerspezifischer Produkte, die eine Ausübung von Marktmacht enthüllen würde, kann auf Verbraucherebene nicht festgestellt werden ${ }^{806}$. Damit wird die Einschätzung der Kartellbehörden, dass im Zuge der Fusion keine marktmächtige Position auf der Absatzseite realisiert werden könne, gestützt.

Die Ergebnisse zeigen (unter den zugrunde gelegten Annahmen) auch, dass Nordmilchprodukte nicht unterdurchschnittlich erfolgreich (gemessen am Marktpreis) waren. Dies würde bedeuten, dass bei einer konstanten Erlösseite und niedrigeren Milchauszahlungspreisen eine Verbesserung der Erfolgskennziffern in der Bilanz eintreten müsste. Diese und weitere Fragestellungen sind Gegenstand des nächsten Abschnittes.

806 Es wird davon ausgegangen, dass Preiserhöhungen der Hersteller über den Handel an den Verbraucher weitergegeben werden. Diese Vorgehensweise wurde gewählt, da keine unternehmensspezifischen Daten über Molkereiabgabepreise (veröffentlicht) verfügbar sind. Den Kartellbehörden wären detaillierte Hersteller- und Handelspreise zugänglich (vgl. Kapitel 8.1.5.1). 


\subsubsection{Bilanzanalyse ausgewählter Unternehmen der Milchindustrie: Nordmilch e.G.}

Bei der Bilanzanalyse der Milchindustrie wird auf die gewählte Methodik entsprechend der Zuckerindustrie zurückgegriffen (vgl. Kapitel 8.1.6.1). Im Fokus bei der Milchbranche steht der Zusammenschluss zur Nordmilch im Jahre 1998, wobei als Referenzunternehmen die Humana herangezogen wird, die 1997 aus der Westmilch und den Milchwerken Herford hervorgegangen ist.

Zunächst sei die Größe des Gesamtumsatzes betrachtet: Es konnte in den letzten Jahren eine Umsatzerhöhung, sowohl auf Betriebs- wie auch auf Konzernebene erreicht werden (Abbildung 68). Auch die Humana konnte Steigerungen gegenüber den vorigen Jahren verzeichnen (vgl. dazu Anhang $\mathrm{H}$ ). Ob dies auf eine evtl. Ausübung von Marktmacht (höhere Preise) zurückzuführen ist, kann allerdings nicht geklärt werden. Sicher jedoch ist, dass sich 2001 aufgrund gestiegener Verkaufserlöse als ein äußerst lukratives Jahr für die gesamte Molkereibranche erwies ${ }^{807}$.

Abbildung 68: Entwicklung der Umsatzerlöse

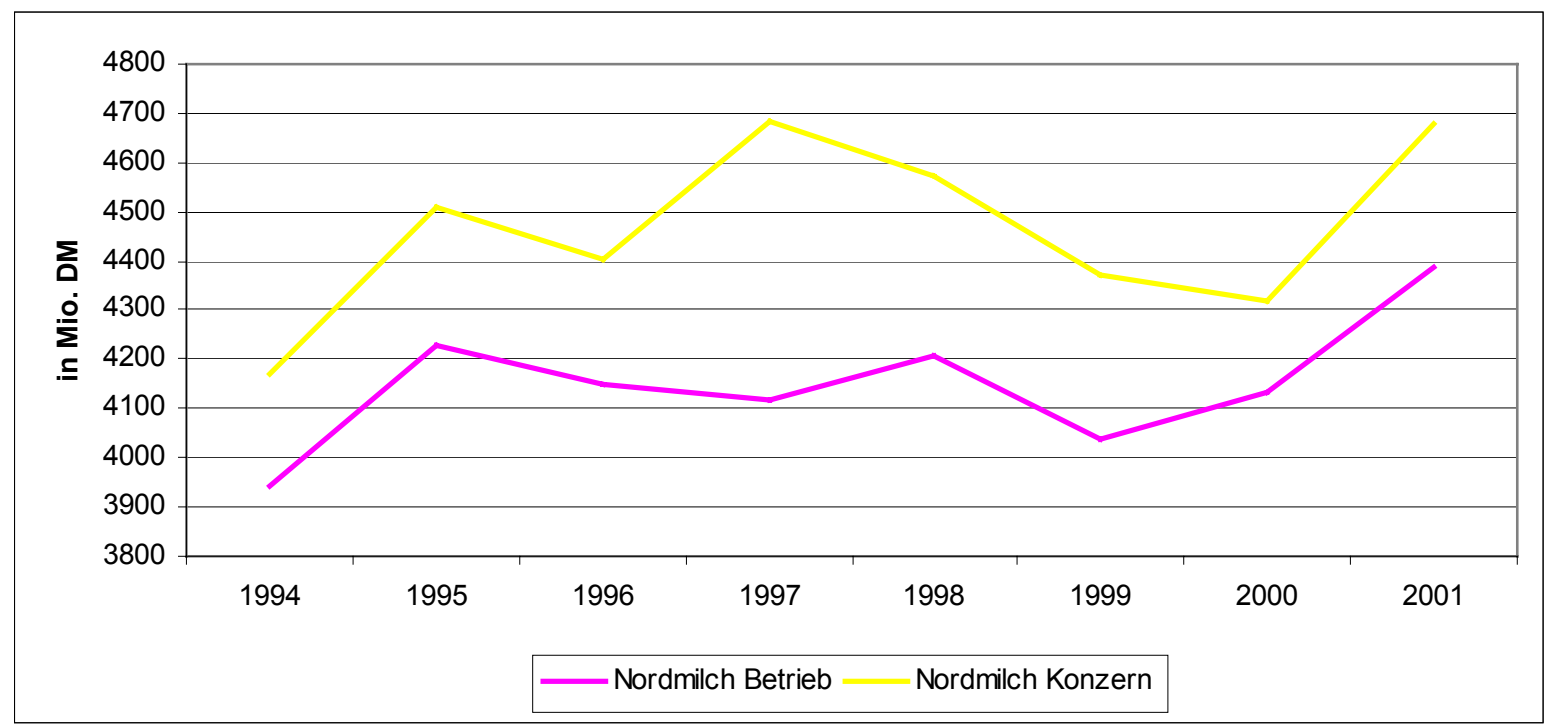

Quelle: eigene Darstellung auf Grundlage Ifd. Jahresberichte

Der Jahresüberschuss lässt diese Entwicklung nicht erkennen (Abbildung 69). Drei Jahre nach der Fusion war bei Nordmilch ein negatives Rekordergebnis zu verbuchen, während die Humana mit einer Spitzenleistung gegenüber den vergangenen Jahren glänzte. Es stellt sich demnach die Frage, ob die Kosten dermaßen angestiegen sind, dass trotz eines höheren Umsatzes die Jahresunterschüsse derartige Größenordnungen annehmen.

${ }^{807}$ Vgl. OSMERS 2001 
Abbildung 69: Entwicklung der Jahresüberschüsse/-unterschüsse

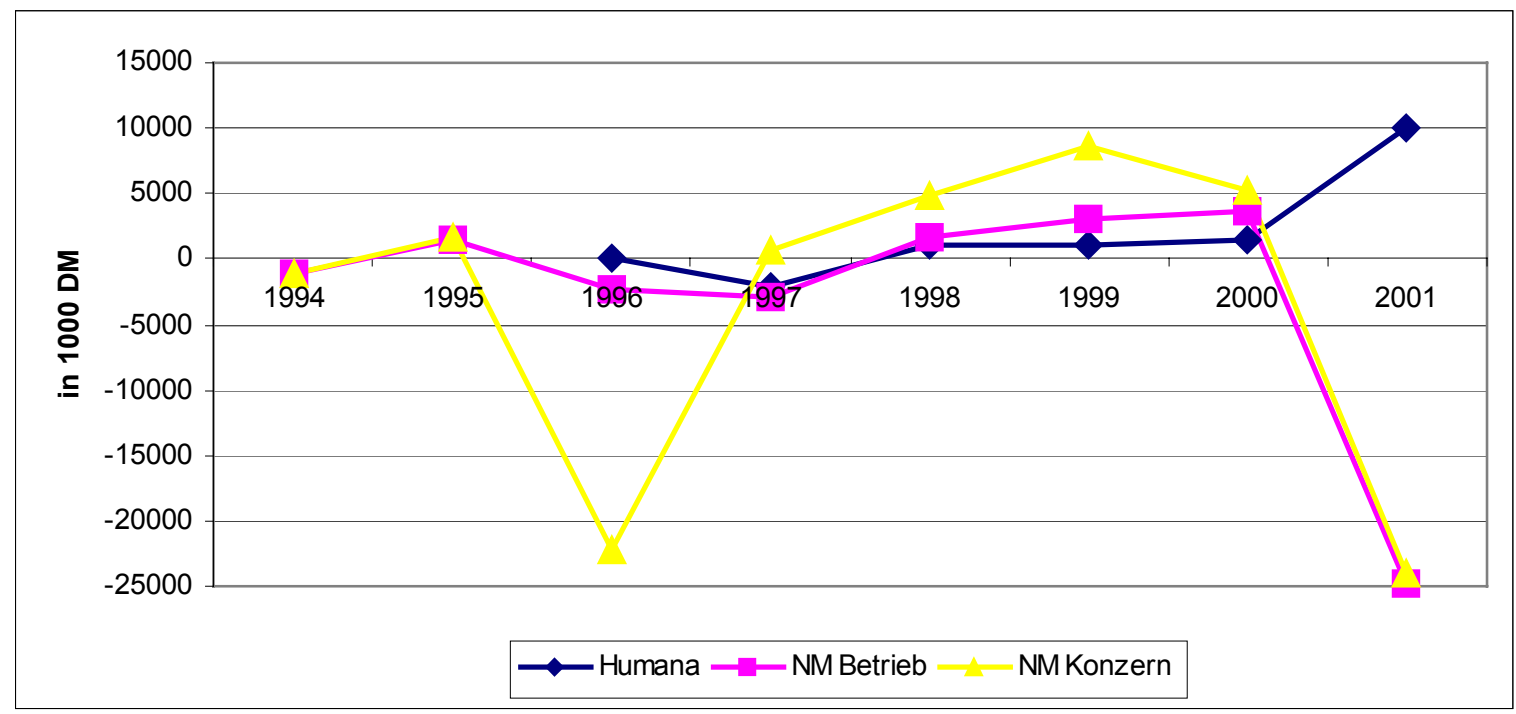

Quelle: eigene Darstellung auf Grundlage Ifd. Jahresberichte

Vielleicht lässt sich der immense Rückgang beim Jahresüberschuss durch eine vermehrte Investitionstätigkeit erklären. Sobald dies anhand von bilanzwirksamen Gütern geschehen ist (z.B. Maschinen oder Gebäude), müssten diese Aktivitäten durch das Anlagevermögen reflektiert werden. Aber auch eine Veräußerung von Standorten sollte sich beim Anlagevermögen bzw. bei den Abschreibungen wiedererkennen lassen (vgl. dazu Abbildung 70):

Abbildung 70: Entwicklung des Anlagevermögens und der Abschreibungen

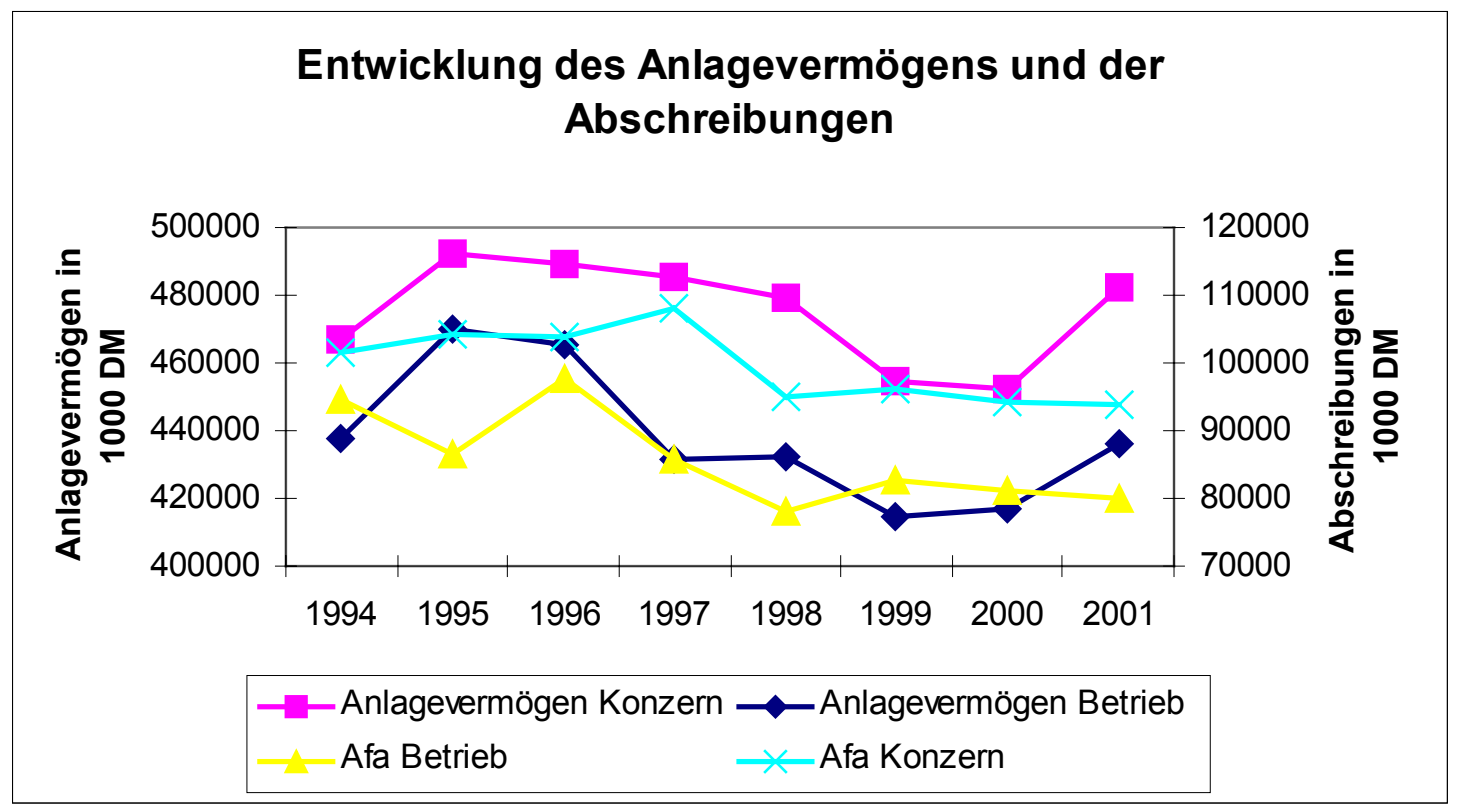

Quelle: eigene Darstellung auf Grundlage Ifd. Jahresberichte 
Zunächst einmal scheint tatsächlich der Effekt einer Veräußerung (Abnahme von Anlagevermögen und Abschreibungen) ersichtlich. Ein durch Investitionen bedingter Anstieg der zuvor genannten Kennziffern hebt jedoch die Belastungsverminderung auf. Die Humana weist als Pendant die gleiche Entwicklung auf. Verminderte Abschreibungsbelastungen scheinen nur kurzfristiger Natur nach Fusionen zu sein.

\section{Abbildung 71: Entwicklung des Personalaufwandes und der Mitarbeiterzahl}

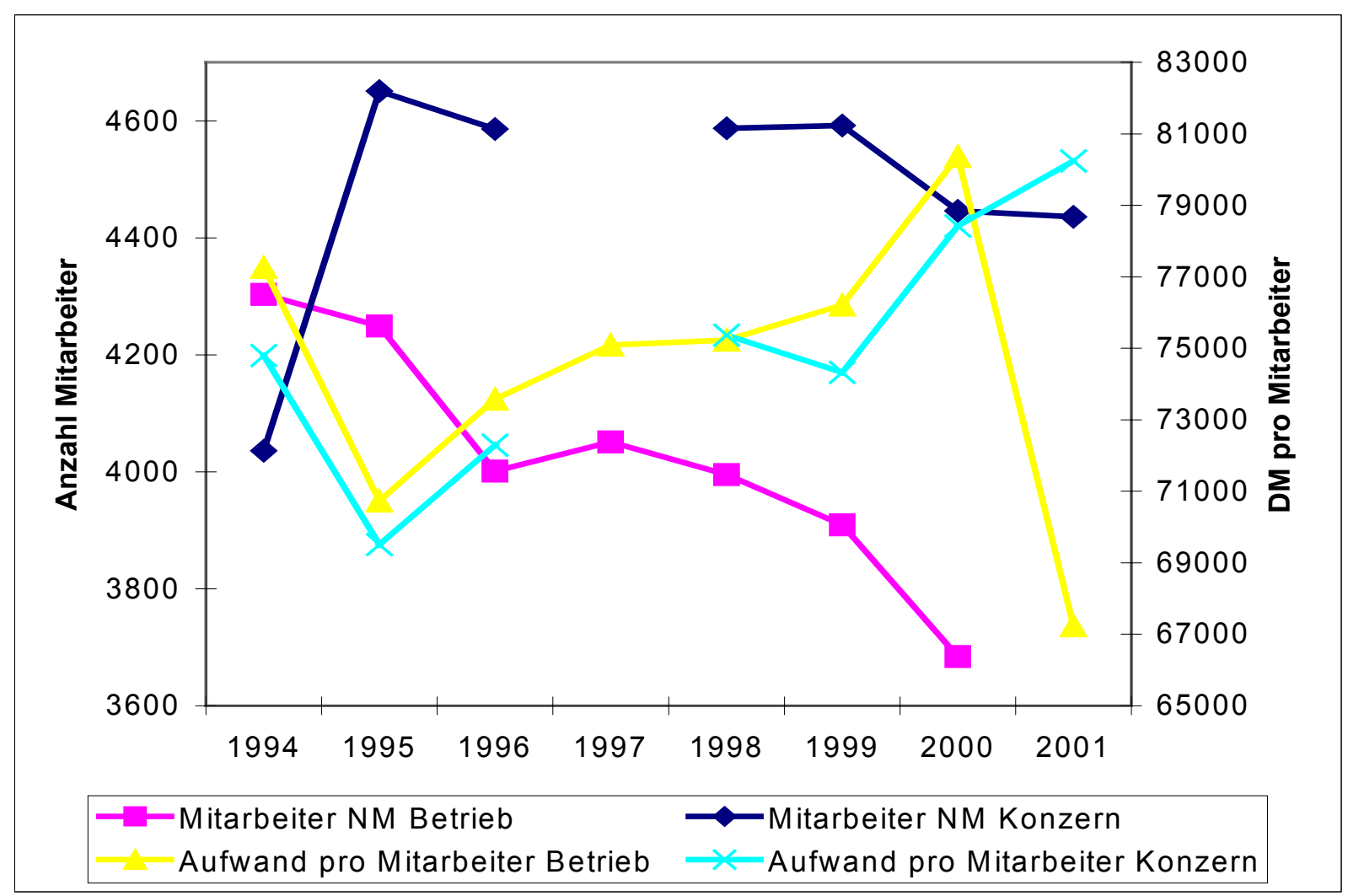

Quelle: eigene Darstellung auf Grundlage Ifd. Jahresberichte

Im Zeitraum nach der Fusion konnte bei beiden Zusammenschlüssen erwartungsgemäß Personal abgebaut werden, wobei interessanterweise die Nordmilch trotz weniger Beschäftigten höhere Personalaufwendungen zu bewältigen hat (vgl. Abbildung 71 und Abbildung 72). Ob dies allein auf eine Mehrbelastung durch Sozialpläne ${ }^{808}$ oder steigende Managervergütungen zurückzuführen ist, bleibt ungewiss.

\footnotetext{
${ }^{808}$ Bei größeren Unternehmen müssen bei Werksschließungen im Zuge von Fusionen Entschädigungszahlungen an die betroffenen Mitarbeiter gezahlt werden (= Sozialplan). Geregelt in Betriebsverfassungsgesetz $\S 112 \mathrm{ff}$.
} 
Abbildung 72: Personalaufwendungen verschiedener Unternehmen pro Mitarbeiter

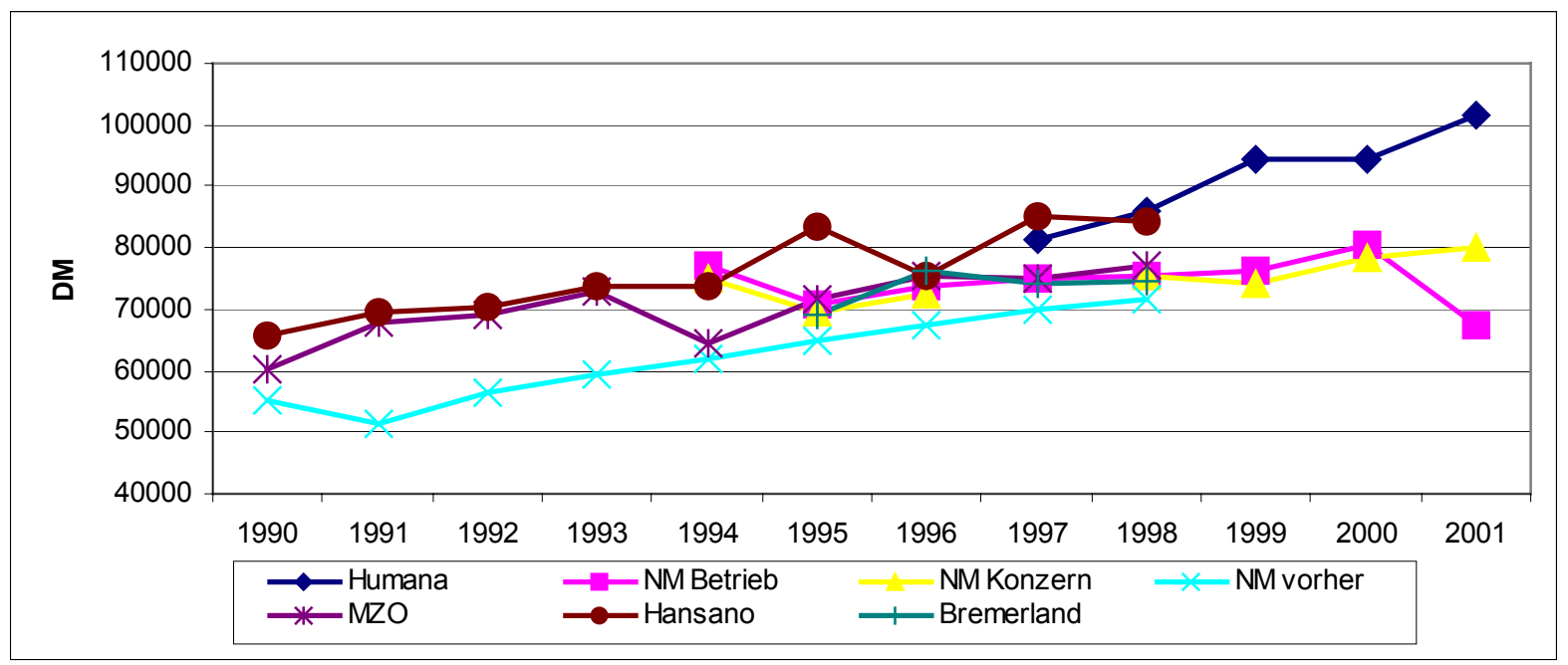

Quelle: eigene Darstellung auf Grundlage Ifd. Jahresberichte

Im Gegensatz zur Humana scheint die Arbeitsproduktivität (gemessen am Umsatz) bei der Nordmilch im Zuge der Fusion nicht besonders gestiegen zu sein (vgl. Abbildung 73).

Abbildung 73a: Entwicklung der Produktivität der Mitarbeiter (Umsatz pro Mitarbeiter)

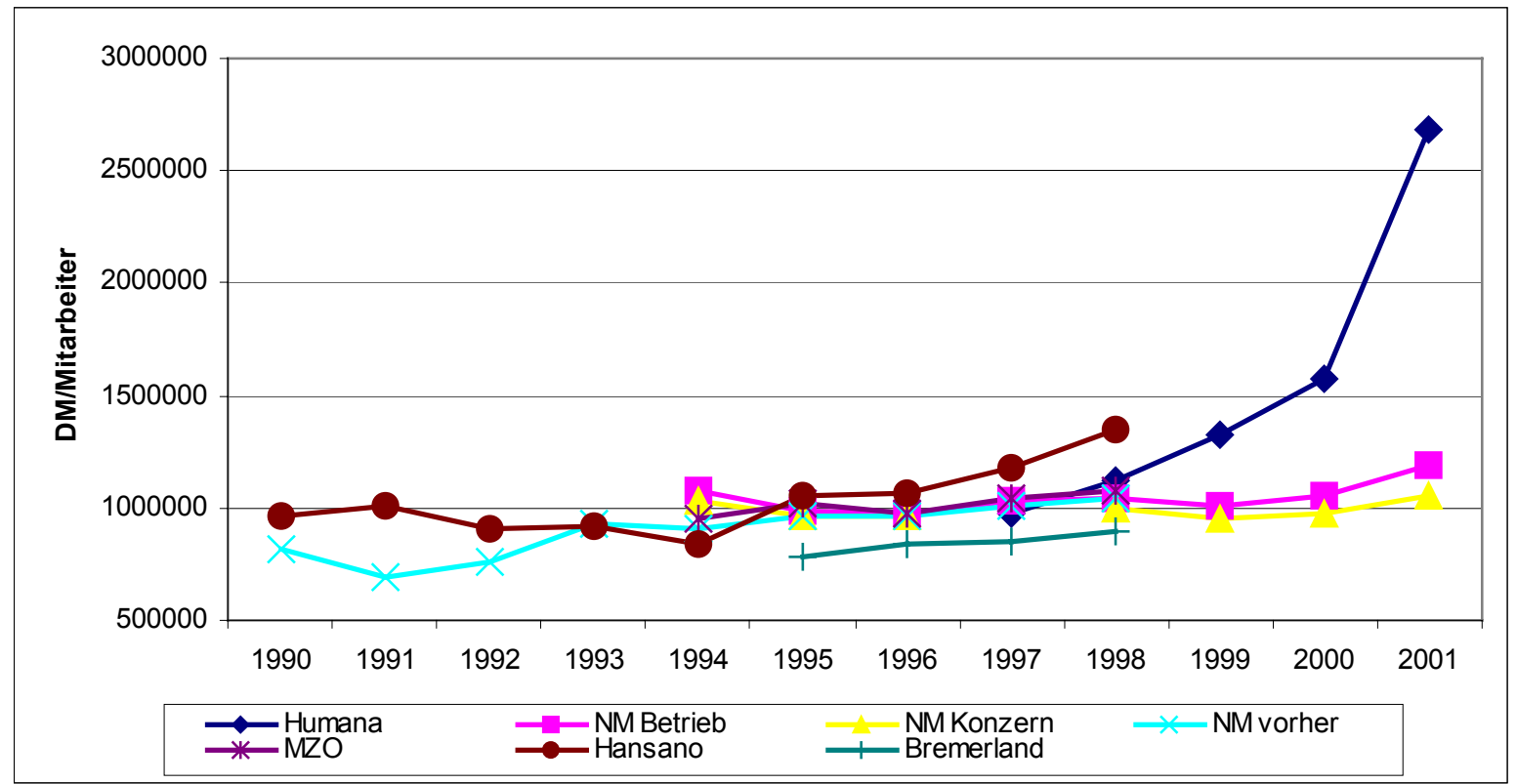

Quelle: eigene Darstellung auf Grundlage Ifd. Jahresberichte 
Abbildung 73b: Entwicklung der Produktivität der Mitarbeiter (Milchverarbeitung pro Mitarbeiter)

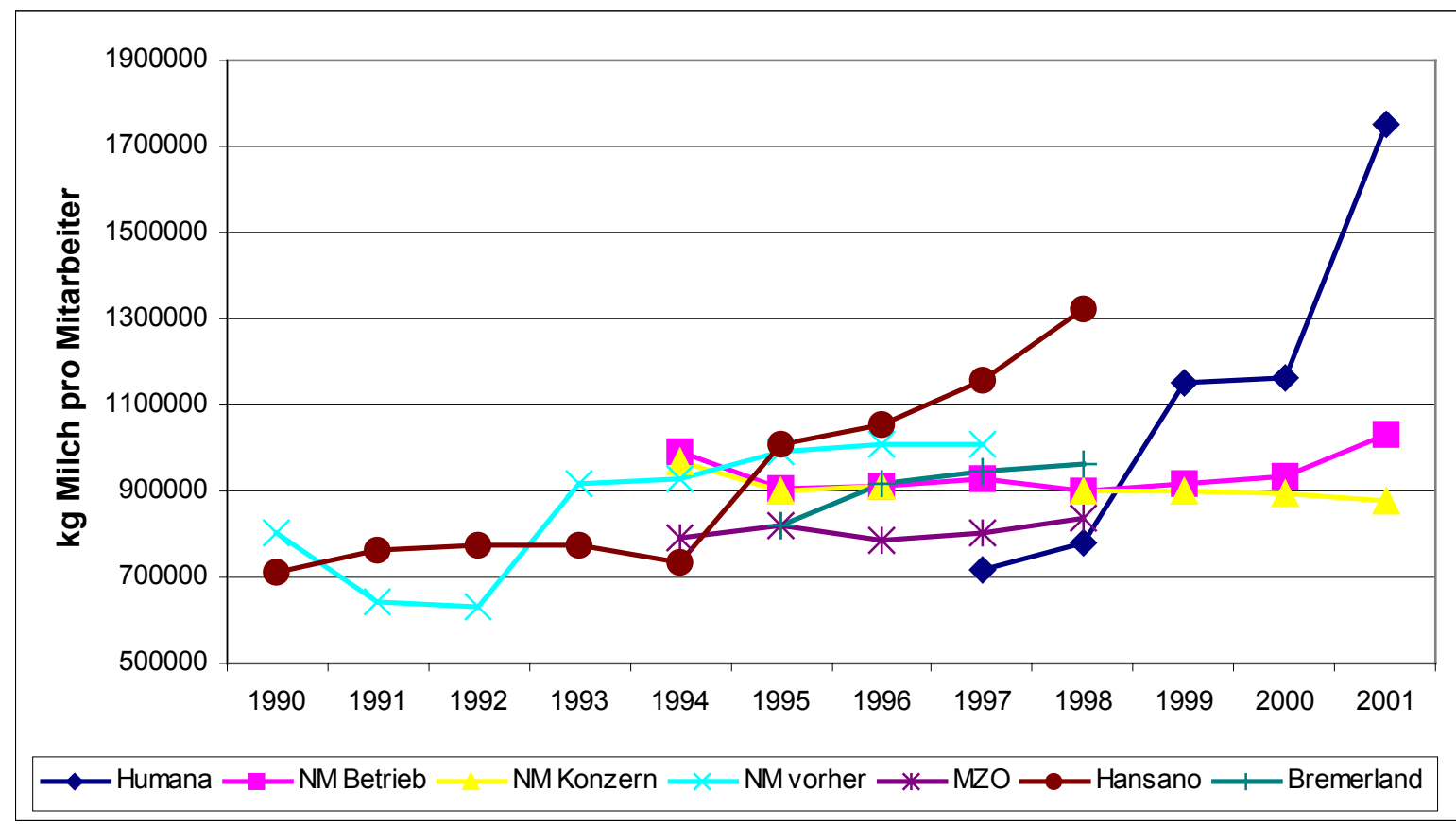

Quelle: eigene Darstellung auf Grundlage Ifd. Jahresberichte

Aufgrund steigender Löhne und höherer Nebenbelastungen scheinen alle Unternehmen von einem wachsenden Personalaufwand betroffen zu sein. Allerdings konnte die Humana eine wesentlich größere Produktivitätssteigerung erreichen. Dies kann z.B. in technischem Fortschritt begründet liegen, so dass ein wesentlich größerer Output bezogen auf ein Zeitintervall erreicht werden konnte, der die Lohnsteigerungen auffing.

Die Bandbreite der molkereispezifischen Bruttoverwertung (Umsatz/Rohstoffeingang) bewegte sich bspw. 1997 gemäß einer Analyse von 22 Jahresabschlüssen deutscher Molkereiunternehmen zwischen 64,3 und $174,3 \mathrm{Pf} / \mathrm{kg}^{809}$. Bei Betrachtung der Nordmilch zeigt sich, dass keine überdurchschnittliche Bruttoverwertung festgestellt werden kann (vgl. Abbildung 74). Vor dem Zusammenschluss erzielten die einzelnen an der Fusion beteiligten Partner höhere Werte. Anders dagegen verläuft die Entwicklung bei Humana, die nach ihrem Zusammenschluss 1998 wieder überdurchschnittliche Erlöse pro kg eingesetzter Milch erzielen konnte.

${ }^{809}$ Vgl. BraUn 2000c, S. 56 


\section{Abbildung 74: Durchschnittlicher Erlös pro kg Milch}

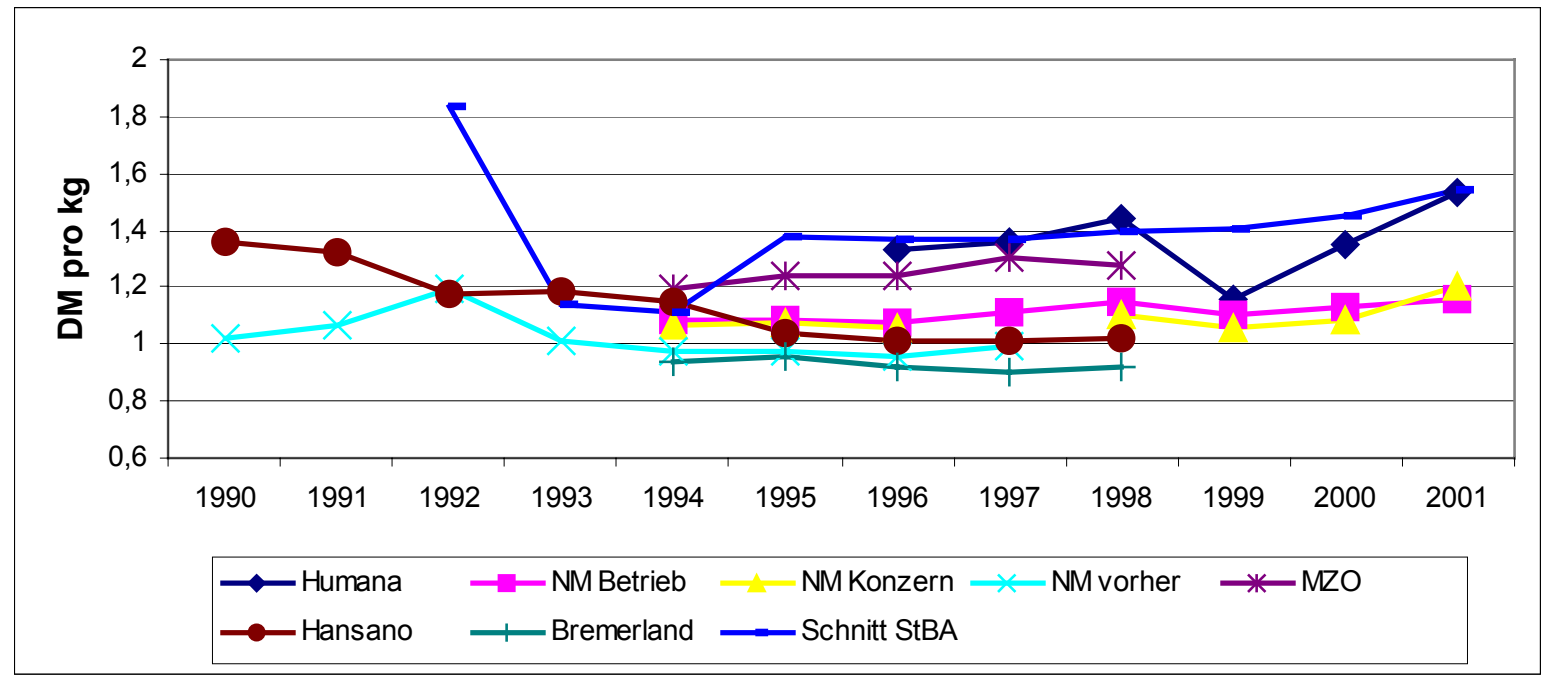

Quelle: eigene Darstellung auf Grundlage Ifd. Jahresberichte

Bei erneuter Betrachtung des Anlagevermögens stellt sich diesmal allerdings heraus, dass die Nordmilch wesentlich geringer belastet ist (vgl. Abbildung 75). Dies kann aber durch Unterschiede im Produktportfolio hervorgerufen sein, die kapitalintensive Produktionsmaschinen erfordern oder auch in der Struktur der einzelnen Standorte (der Betriebsstätten) begründet liegen.

\section{Abbildung 75: Entwicklung des Anlagevermögens pro t Milchverarbeitung}

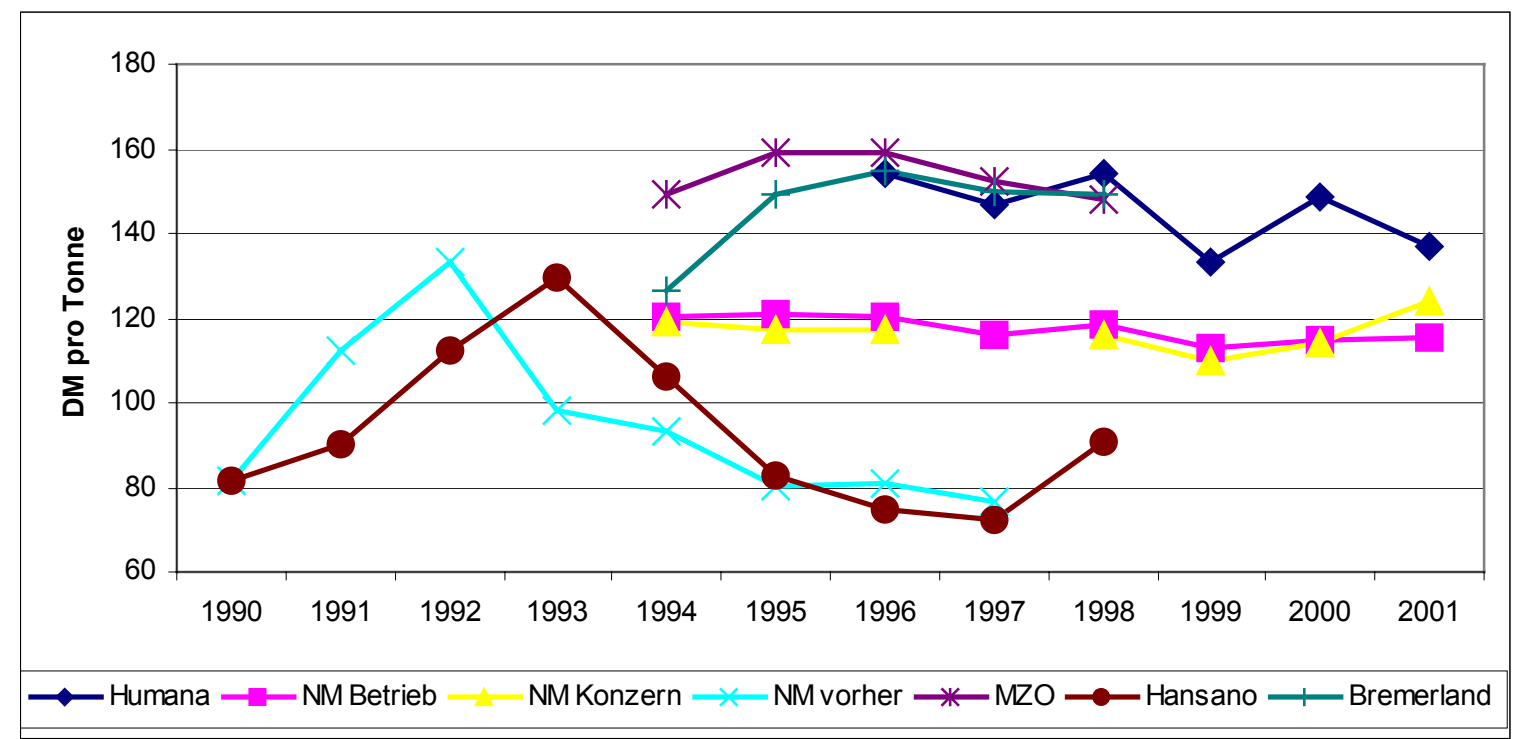

Quelle: eigene Darstellung auf Grundlage Ifd. Jahresberichte

Nicht zufriedenstellend konnte die Frage geklärt werden, warum trotz steigender Erlöse die Jahresüberschüsse bei der Nordmilch zurückgegangen sind. Die Antwort ist im Umlaufvermögen zu suchen. So ist die Belastung beim Umlaufvermögen (pro kg Milch) 
enorm angestiegen. Diese Beobachtung lässt sich ebenfalls bei der Humana machen (vgl. Abbildung 76).

\section{Abbildung 76: Entwicklung des Umlaufvermögens pro kg Milchverarbeitung}

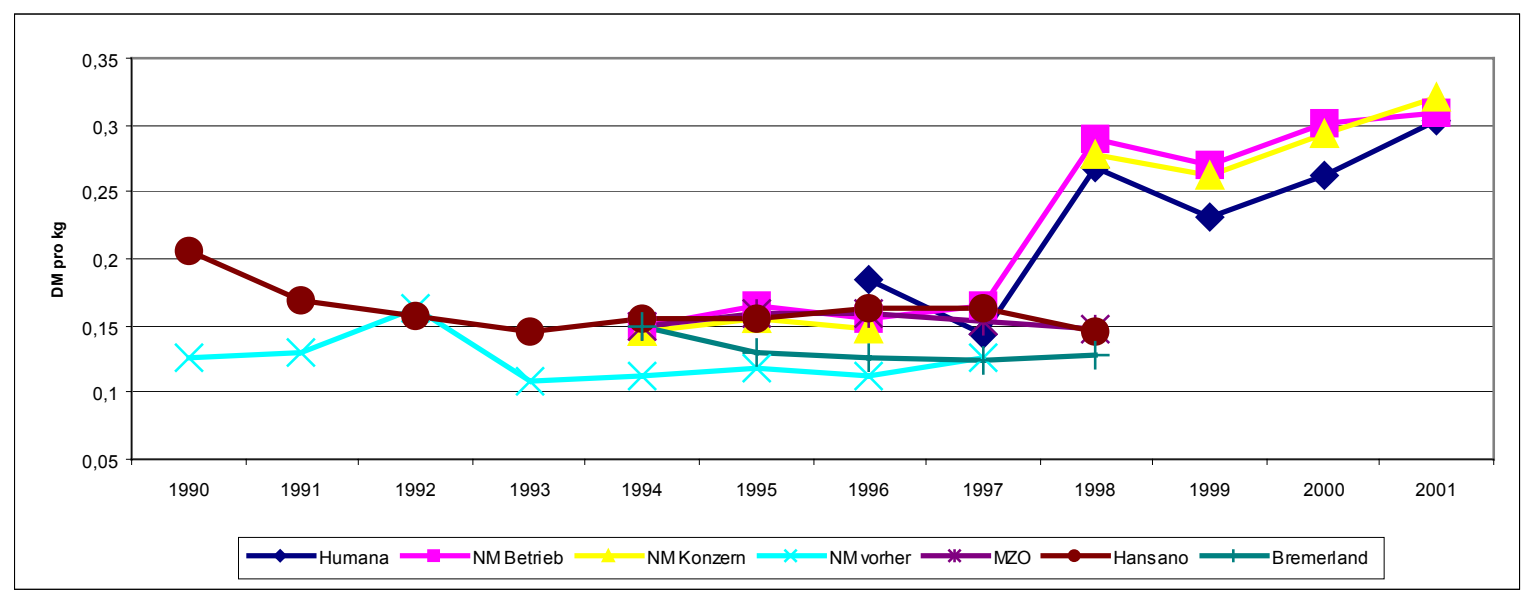

Quelle: eigene Darstellung auf Grundlage Ifd. Jahresberichte

Abschließend wird der theoretische Gewinn betrachtet. Es gelten auch hier die gleichen Prämissen wie bei der Jahresabschlussanlyse der Zuckerindustrie (vgl. Kapitel 8.1.6.2). Als Gewinn wird das Betriebsergebnis (bereinigt um geschäftstätigkeitsfremde Effekte; keine Verzerrung durch Ertragssteuern) herangezogen. Umgerechnet auf den Milcheingang ergibt sich folgende Gewinnentwicklung.

\section{Abbildung 77: Entwicklung des theoretischen Gewinns pro kg Milch (vor Steuer)}

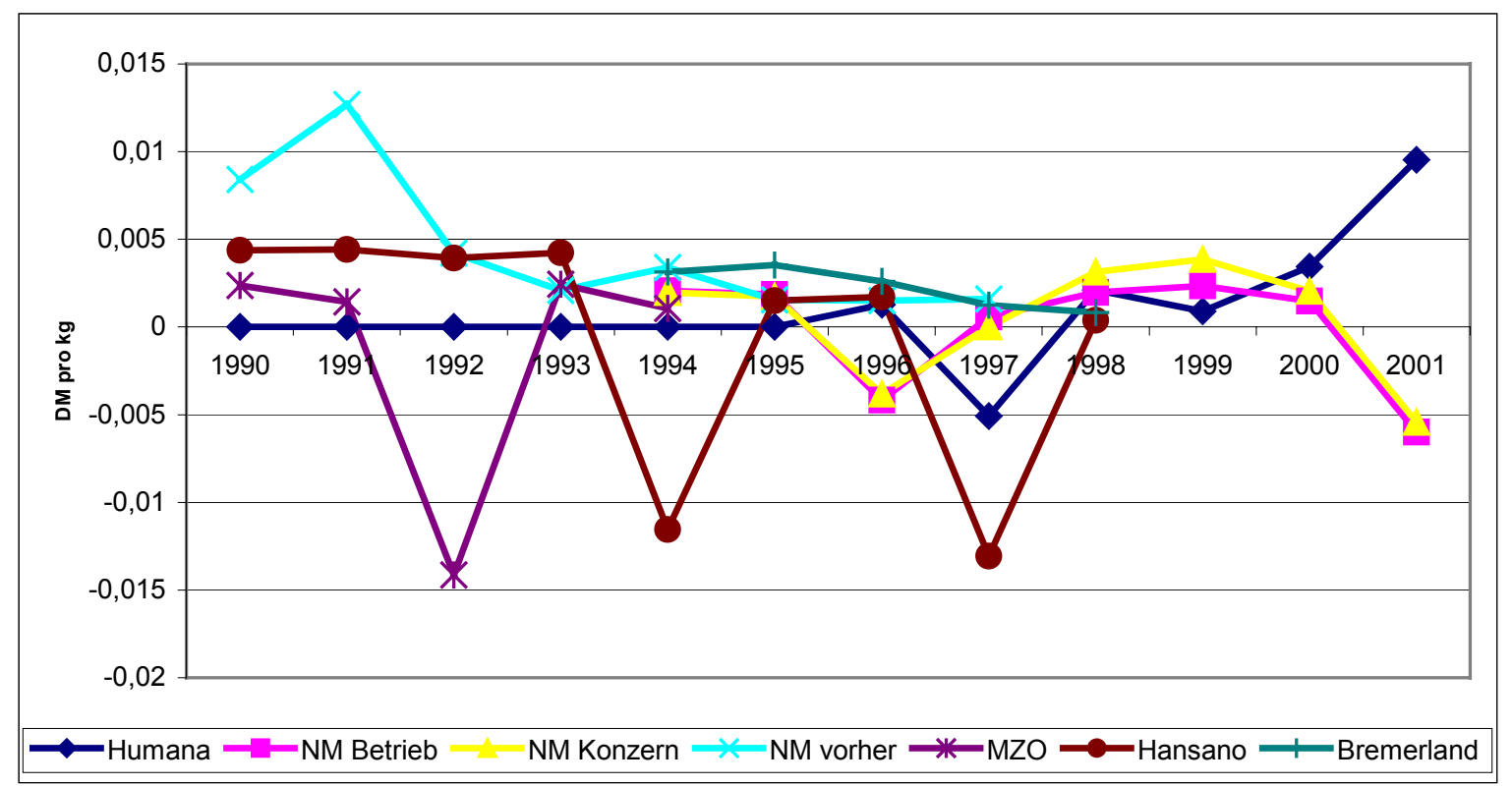

Quelle: eigene Darstellung auf Grundlage Ifd. Jahresberichte

Während die Humana steigende Gewinne verbuchen kann, ist eine negative Gewinnentwicklung bei der Nordmilch ersichtlich. Vor dem Zusammenschluss war zumindest die "alte" Nordmilch alleine erfolgreicher (vgl. Abbildung 77). 
Weitere Kennzahlen der Analyse sind im Anhang enthalten. Die wesentliche Entwicklung konnte den dargestellten Erfolgskennziffern entnommen werden. Alles in allem wird deutlich, dass Humana gestärkt aus dem Zusammenschluss hervorgeht und Synergiepotentiale realisieren konnte. Die Nordmilch dagegen schneidet sowohl bei den Milchauszahlungspreisen als auch der Bilanzanalyse nicht besonders gut ab. Es kann zumindest zum jetzigen Zeitpunkt nicht von Erfolg gesprochen werden (eine Frage der Definition von Erfolg ${ }^{810}$ ). Bei der Vorher-nachher-Analyse ist auch zu bedenken, dass die einzelnen Zusammenschlusspartner vor der Fusion bei einzelnen Kennzahlen z.T. erfolgreicher waren - allerdings kann dies in einer "Verzerrung der Substanz" begründet sein (geringer Erhaltungs- und Investitionsaufwand). Verwunderlich scheint abschließend auch, dass trotz höherer Umsätze und Synergievorteile keine Verbesserung des Ergebnisses, sondern sogar eine Erhöhung der Kosten eingetreten ist.

Im Jahr 1997 betrug die durchschnittliche, gewichtete Eigenkapitalrendite von 22 analysierten Jahresabschlüssen deutscher Molkereien unter Berücksichtigung eines genossenschaftlichen Korrekturbetrages ${ }^{811}$ 4,8 \% (die Umsatzrendite 0,7 \%). Die Auswertung der Bilanzanalyse zeigt, dass die Nordmilch wie auch die Humana niedrigere Werte vorweisen (z.T. sogar negativ). Diese im Vergleich zu anderen Wirtschaftsbereichen unattraktiven Renditen vermindern zumindest die Attraktivität des Markteintritts branchenfremder Investoren erheblich.

Anders als bei der Zuckerindustrie konnten durch die Jahresabschlussanalyse keine typischen Größenvorteile, bedingt durch eine Fusion, festgestellt werden. Vielmehr lässt sich der Erfolg oder Mißerfolg einzelner Unternehmen auf individuelle Strategien (Produktportfolio, Marketingstrategie etc.) zurückführen, die mit der in dieser Studie verwendeten Datengrundlage nicht ohne ergänzende Erwägungen erkennbar sind.

\footnotetext{
${ }^{810}$ Es kann nicht geklärt werden, ob die Unternehmen ohne Fusion überhaupt am heutigen Markt existent wären. Wird die Existenz allein als Erfolgsmaßstab betrachtet, konnte die Zielsetzung durch den Zusammenschluss erreicht werden.

${ }^{811}$ Zur Herleitung s. BRAUN 1999
} 


\subsection{Grenzen und Chancen der ex-post-Analyse}

In diesem Kapitel wurden drei ökonomische Instrumente vorgestellt, die die Beurteilung von Unternehmenszusammenschlüssen - unter Berücksichtigung der Zeit- und Personenrestrikton der Kartellbehörden - weiter "verfeinern" können. Durch Einbeziehung von Branchenstudien und Expertengespräche können neutrale Erkenntnisse über optimale Betriebsgrößen gewonnen werden, die den Kartellbehörden einen detaillierten Überblick über Größenvorteile verschaffen ${ }^{812}$.

Die Bilanzanalyse der ausgewählten Branchen zeigt deutlich, dass in der Zuckerproduktion gegenüber der Milchproduktion wesentlich höhere Renditen erwirtschaftet werden konnten. Die Begründung hierfür findet sich in erster Linie in der Gestaltung der ZMO, die die agierenden Unternehmen weitgehend vor einem intensiveren Wettbewerb schützt. Um im Zuge der Realisierung von Größenvorteilen Kosten einzusparen, scheint der Weg einer Übernahme oder Fusion die einzig gangbare Lösung zu $\operatorname{sein}^{813}$. Die Potentiale scheinen vor allem im Milchsektor erheblich zu sein. Kosteneinsparungen lassen sich anhand der Bilanzanalyse allerdings nur bedingt erkennen. Nur mit Hilfe zusätzlicher Informationen (Investitionsplan, Anlagespiegel etc.) können folglich Aussagen über die tatsächlichen Einsparungen getroffen werden. Auch die Erkenntnisse der fusionstheoretischen Untersuchungen (Insider verringern die angebotene Menge und erhöhen den Produktpreis) konnten nicht eindeutig anhand der Bilanzanalyse gezeigt werden. Die durchgeführten Preisuntersuchungen dagegen enthüllen durchaus Hinweise auf eine Ausübung von Marktmacht. Gerade in der Zuckerbranche liegt der Verdacht nahe, dass aufgrund der ZMO nicht an der Grenzkostenschwelle produziert wird und somit Modelle mit Marktmacht vor und nach dem Zusammenschluss heranzuziehen sind. Für eine Wettbewerbsbeurteilung sollte festgehalten werden, dass die Jahresabschlussanalyse allein kein Instrument der Abwägung sein kann. Dennoch verhilft sie, einen Überblick über die Kosten- und Erlösstruktur zu vermitteln.

Vergangenheitsanalysen können demnach wichtige Hinweise für Einschätzungen zukünftigen Wettbewerbsverhaltens geben. Einzelne Instrumente sind mit Vor- und Nachteilen behaftet und müssen auf die jeweilige Anwendungsmöglichkeit auf einen Zusammenschluss geprüft werden. So fehlt für ökonometrische Analysen z.T. die Da-

\footnotetext{
812 Durch das 3-5jährige Rotationsverfahren der Mitarbeiter in den Beschlussabteilungen (nach Branchen) ist es verständlicherweise für die Entscheidungsträger kaum möglich, ständig über neueste Erkenntnisse und Zusammenhänge Kenntnis zu erlangen. Durch Expertenstudien können Informationen mit relativ geringem Aufwand gewonnen werden.

${ }^{813}$ Vgl. Braun 2000c, S. 46
} 
tenbasis $^{814}$. Bei Jahresabschlussanalysen darf die Problematik der Vergleichbarkeit zwischen einzelnen Unternehmen und der Übereinstimmung von externen (veröffentlichten) Daten mit unternehmensinternen (unbereinigten) Daten nicht unterschätzt werden. Die nachfolgende Tabelle fasst schematisch eine mögliche Anwendbarkeit verschiedener ökonomischer Analyseverfahren für die näher untersuchten Fusionen zusammen.

Tabelle 58: Mögliche Anwendbarkeit ökonomischer Analysverfahren

\begin{tabular}{|c|c|c|c|}
\hline & Nordzucker & Südzucker & Nordmilch \\
\hline \multicolumn{4}{|l|}{$\begin{array}{l}\text { Preisbeobachtungen/ } \\
\text { Marktanalyse: }\end{array}$} \\
\hline Marktmachteffekte & \multicolumn{2}{|c|}{$\begin{array}{l}\text { Absatzmarkt: gut geeignet } \\
\text { Beschaffungsmarkt: nicht notwendig aufgrund politi- } \\
\text { scher Preisvorgabe }\end{array}$} & $\begin{array}{l}\text { Absatz-/Beschaffungs- } \\
\text { markt: gut geeignet }\end{array}$ \\
\hline Effizienzvorteile & \multicolumn{3}{|c|}{$\begin{array}{l}\text { nur indirekt geeignet (wenn Effizienzvorteile (langfristige) Preissenkungen hervor- } \\
\text { rufen) }\end{array}$} \\
\hline \multicolumn{4}{|l|}{ Bilanzanalyse: } \\
\hline Marktmachteffekte & $\begin{array}{l}\text { gut geeignet: deutlich } \\
\text { erkennbarer Effekt unter } \\
\text { Heranziehung naturaler } \\
\text { Daten }\end{array}$ & $\begin{array}{l}\text { nicht geeignet: mangelnde } \\
\text { Transparenz }\end{array}$ & $\begin{array}{l}\text { bedingt geeignet: detail- } \\
\text { liertere Daten erforderlich }\end{array}$ \\
\hline Effizienzvorteile & $\begin{array}{l}\text { gut geeignet (horizontaler } \\
\text { Vergleich) }\end{array}$ & $\begin{array}{l}\text { nicht geeignet: mangelnde } \\
\text { Transparenz }\end{array}$ & $\begin{array}{l}\text { gut geeignet (allerdings } \\
\text { z.T. widersprüchliche } \\
\text { Ergebnisse) }\end{array}$ \\
\hline Expertenbefragung & \multicolumn{3}{|c|}{$\begin{array}{l}\text { gut geeignet (besonders für die Analyse einzelner Komponenten wie z.B. die Wir- } \\
\text { kung einer agrarpolitischen Maßnahme oder Lieferkonditionen für Abnehmer). } \\
\text { Problem: Expertenwissen beruht ebenfalls auf Preisbeobachtungen, Bilanzanalysen } \\
\text { etc. bzw. z.T. auf subjektivem Erfahrungswissen }\end{array}$} \\
\hline
\end{tabular}

Quelle: eigene Darstellung

Deutlich wird, dass je nach Zusammenschluss unterschiedliche ökonomische Instrumente besonders geeignete Anwendung finden können. Die Beschränkung auf eine Analysemethode allein ist selten ausreichend. Ein weiteres Problem besteht darin, dass die verfügbare veröffentlichte Datengrundlage oftmals nicht ausreichend ist.

Allerdings haben die Kartellbehörden im Zuge des Verfahrens die Möglichkeit, Auskunft von den beteiligten Unternehmen und Dritten zu erlangen ${ }^{815}$, so dass bei Heranziehung präziserer Daten ein Abbild der Wettbewerbsverhältnisse, aber auch potentieller Effizienzvorteile ermittelbar wäre. In Kombination mit klassischen juristischen Ver-

\footnotetext{
${ }^{814}$ In den letzten Jahren konnten allerdings in diesem Bereich erhebliche Fortschritte erreicht werden (vgl. Kap. 7.4.2.1).

${ }^{815}$ Vgl. § 57-59 GWB sowie Art. 11 FKVO
} 
fahren (Anhörung der betroffen Parteien, Beweislast etc.) kann somit eine Art Mosaik, welches mit Hilfe vieler Einzelbausteine ein Gesamtwerk ergibt, erstellt werden.

Somit kann die notwendige ex-ante Einschätzung der Kartellbehörden über mögliches Wettbewerbsverhalten und potentielle Effizienzvorteile eines Unternehmens oder einer Branche im Zuge einer Fusion mit Hilfe von ex-post Analysen erleichtert werden. 


\section{Zusammenfassung und Empfehlung}

Beginnend wurde die Bedeutung von Unterternehmenszusammenschlüssen und -beteiligungen verdeutlicht. Die Anzahl von Transaktionen scheint global in Wellenform zu verlaufen, wobei nach dem deutlichen Anstieg der Beteiligungen in den letzten Jahren ein Rückgang erkennbar ist. Auch die europäische Ernährungsindustrie und speziell die Milch- und Zuckerbranche unterliegen dieser Entwicklung. Die europäische Zuckerindustrie hat im Vergleich zu anderen Industriezweigen bereits hohe Konzentrationsraten erreicht, so dass hier in den nächsten Jahren aufgrund der Kartellrechtsrestriktionen nur wenig Zusammenschlüsse zu erwarten sind. Anders verläuft die Entwicklung im Bereich der Milchindustrie, wo die noch relativ unkonzentrierte und kleinstrukturierte Marktstruktur weitere Kooperationsformen erwarten lässt.

Die Motivation für Fusionen ist äußerst vielfältig. Die Ausnutzung von Synergievorteilen sowie die Erzielung einer marktmächtigeren Stellung sind wichtige Beweggründe einer Fusion, aber auch branchenspezifische Motive wie beispielsweise die Sicherung eines Rohstoffmarktes können für eine Entscheidung ausschlaggebend sein. Bei Großunternehmen, die global agieren, können steuerliche und bewertungsrechtliche Aspekte von großer Relevanz sein. Ferner sind in diesem Zusammenhang manageralistische Interessen zu nennen, die einen Teil der gescheiterten Fusionen durch den PrinzipalAgent-Konflikt erklären können. Die Kenntnis von Motiven kann einerseits dazu dienen, die Entwicklung der Transaktionenanzahl im Zeitablauf zu erklären, andererseits können wichtige Rückschlüsse auf potentielle Effizienzvorteile oder Marktmachtspielräume gezogen werden.

Durch industrieökonomische und empirische Untersuchungen konnte gezeigt werden, dass mit Fusionen sowohl positive wie auch negative Wohlfahrtseffekte einhergehen können. Um zu einem bestimmten Beurteilungszeitpunkt Aussagen über das zukünftige Wettbewerbsverhalten der fusionierenden Unternehmen treffen zu können, sollten die Wettbewerbsbehörden neben Prüfung der Erzielung einer dominanten Stellung auch die Auswirkungen auf den Restwettbewerb untersuchen. Auch eine Analyse der Wahrscheinlichkeit einer möglichen Kollusion ist zwingend erforderlich. Die Orientierung an Strukturkriterien allein vermag das zukünftige Geschehen nicht ausreichend zu erklären. Ferner sollte von einem wohlfahrtsökonomischen Gesichtspunkt die Berücksichtigung von Effizienzvorteilen vorgenommen werden. Erst nach Abwägung der voraussichtlichen negativen Marktmachteffekte kann eine endgültige Entscheidung getroffen werden. 
Der deutsche und europäische Rechtsrahmen erfüllt weitgehend diese Forderungen. Der Schwerpunkt liegt insbesondere in der Prüfung der Erzielung einer marktbeherrschenden Stellung. Dabei betont der Gesetzgeber ausdrücklich, dass neben klassischen Strukturkriterien (Marktanteil, Konzentration etc.) weitere Faktoren wie beispielsweise technischer Fortschritt oder potentieller Wettbewerb heranzuziehen sind. Offen bleibt die Frage nach der Berücksichtigung positiver Effekte von Fusionen. Prinzipiell ist die Einbeziehung nach deutschem und europäischem Recht möglich. Die Rechtspraxis zeigt allerdings, dass dies nur vereinzelt (z.T. intransparent) und meist nur indirekt bei bedenkenlosen Zusammenschlüssen geschieht. Generell zeigt sich, dass das Gesetz recht dehnbare Begriffe vorgibt, die zu unterschiedlichen Auslegungen in den verschiedenen Beschlüssen führen. Die Entscheidungspraxis der Kartellbehörden beruht weitgehend auf Erfahrungswissen der Mitarbeiter und wird durch zusätzliche Befragungen der Branchenbeteiligten gestützt. Ökonomische Instrumente werden (zumindest bei den Zusammenschlüssen der Branchen dieser Studie) kaum genutzt. Auf europäischer Ebene ist dies zum Teil anders - insbesondere innerhalb der letzten Jahre werden zunehmend moderne Techniken herangezogen.

Einzelne Komponenten, die besonders kontrovers von den Vertretern der beteiligten Unternehmen und den Wettbewerbsbehörden diskutiert werden, wurden näher analysiert. Bei der Abgrenzung des relevanten Marktes konnte verdeutlicht werden, dass durch Zuhilfenahme von ökonometrischen Analysemethoden und Heranziehung von Expertengutachten Einschätzungen gestützt werden können. Nachfragemacht, die als ein Hauptfusionsmotiv in der Ernährungsindustrie benannt wird, sollte grundsätzlich Berücksichtigung finden. Auch hier könnten Preisbeobachtungen und Expertengespräche nützliche Hinweise auf den tatsächlichen Machtspielraum liefern. Besonders wichtig ist für die Beurteilung von Zusammenschlüssen in der Ernährungsindustrie die Kenntnis über agrarpolitische Zusammenhänge. Zwar können durch wettbewerbsrechtliche Einschränkungen keine Defizite eines marktwirtschaftlichen Ordnungsrahmens beseitigt werden, dennoch kann der Schutz eines Restwettbewerbs außerordentlich wichtig sein. Besonders im Bereich der Zuckerbranche zeigt sich, dass eine klare zukünftige Ausrichtung der Agrarpolitik wünschenswert wäre, um Argumente der fusionsinteressierten Unternehmen gewichten zu können.

Wohlfahrtsökonomisch sei die Heranziehung positiver Fusionseffekte empfohlen. Die Behörde steht allerdings bei der Berücksichtigung dieser Effekte vor einem Informationsproblem. Mit Hilfe von Expertengutachten, Preisbeobachtungen und der Jahresabschlussanalyse wurde verdeutlicht, wie mit relativ einfachen ökonomischen Techniken die Ausführungen der Unternehmensvertreter auf ihre Plausibilität überprüft werden 
könnten. Darüber hinaus lassen sich aus diesen Instrumenten Rückschlüsse auf zukünftiges Wettbewerbsverhalten oder den Marktmachtspielraum ziehen.

Zusammenfassend war es Ziel dieser Studie einen Überblick über das Forschungsfeld von Fusionen zu verschaffen. Der Schwerpunkt wurde auf wohlfahrtsökonomische Wirkungszusammenhänge und die praktische Beurteilung von Unternehmenszusammenschlüssen in der Zucker- und Milchindustrie gelegt. Sowohl juristische wie auch ökonomische Aspekte wurden dabei herangezogen. Um den zeitlich und personell vorgegebenen Restriktionen der Wettbewerbsbehörden gerecht zu werden, sei als Fazit dieser Untersuchung folgende Empfehlung ausgesprochen. Die in der Kritik stehende intuitive Entscheidungspraxis der Kartellbehörde könnte wesentlich entschärft werden, wenn neben den bewährten juristischen Verfahren die Heranziehung von ökonomischen Instrumenten verstärkt genutzt werden würde. Zumindest ex-post liessen sich Effizienzvorteile und Marktmachtspielräume ermitteln. Daraus könnte mit ergänzenden Erwägungen eine Abschätzung über zukünftiges Wettbewerbsverhalten vorgenommen werden. Ein weiterer Vorteil wäre, dass eine transparente Rechtfertigungsbasis geschaffen würde. Auf dieser Grundlage könnten Argumente der Unternehmensvertreter auf ihre Plausibilität geprüft werden. Spätestens dann, wenn die Forderung nach vermehrter Einbeziehung positiver Fusionseffekte in die Realität umgesetzt wird, sollten die vorgestellten Instrumente zwingender Bestandteil einer Fusionskontrolle sein. 


\section{Anhang}

A. Literaturverzeichnis

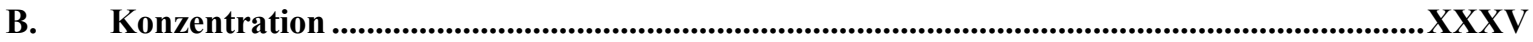

1. Begriffe und Formen der Unternehmenskonzentrationen....................................................XXXV

2. Messung von Konzentration ..................................................................................................XXVI

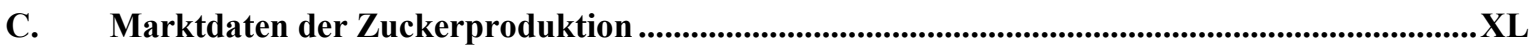

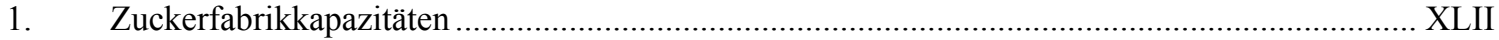

2. Veränderung der Kosten der Zuckerindustrie ................................................................... XLII

3. Daten Stromverbrauch $(\mathrm{kwh} / \mathrm{kg}$ Zucker) …................................................................. XLII

4. Energieeinsatz in der Zuckerindustrie ................................................................................

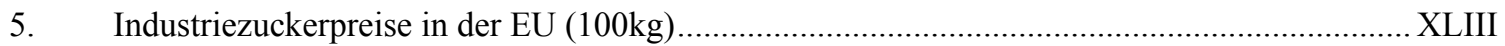

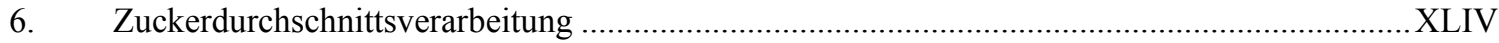

7. T-Test-Ergebnisse der MADAKOM-Datensatz Preisuntersuchung Preisuntersuchungen.......... XLV

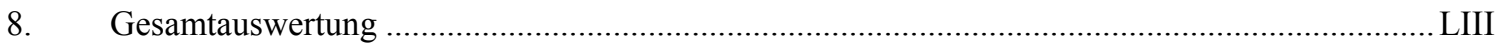

D. Lebensmitteleinzelhandel (LEH) Charakteristika........................................................................ LIV

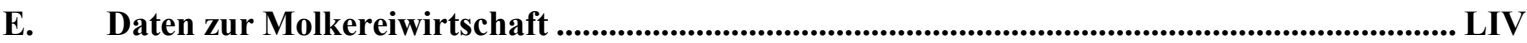

1. Entwicklung der Molkereistruktur in Deutschland und Europa ...............................................V

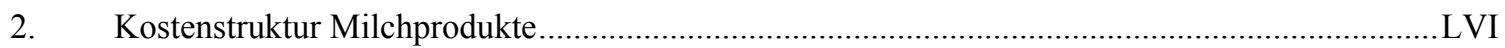

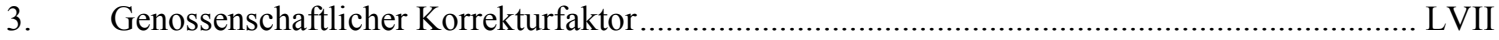

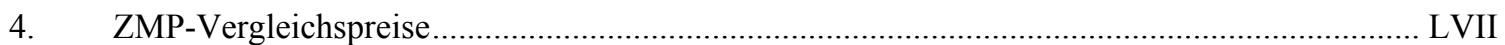

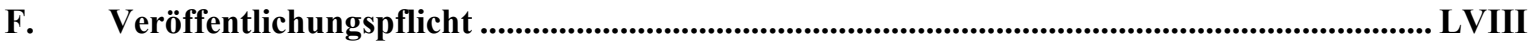

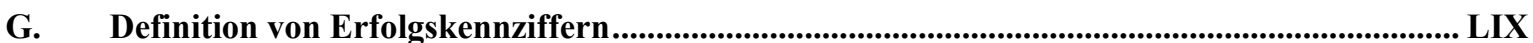

H. Ergebnisse der Bilanzanalyse ausgewählter Zusammenschlüsse und Vergleichsuntern............LX 


\subsection{Literaturverzeichnis}

- $\quad$ Аввот, L. (1958): Qualität und Wettbewerb, Beck-Verlag, München

- ABN-AMRO (1996): The Reinvestment Dilemma - A comparison of the top 10 European Sugar Manufactures, Hoare Govett, London

- AC Nielsen (2001): Daten zum Handel in Deutschland - Universen 2001, AC Nielsen, Frankfurt am Main

- AgRA-EURoPe (deutsche Fassung): unabhängiger europäischer Presse- und Informationsdienst für Agrarpolitik und Agrarwirtschaft, Bonn u.a.

- AgRARZeItUng: Beilage der Zeitung Ernährungsdienst - unabhängige Wirtschafts- und Börsenzeitung, Deutscher Fachverlag, Frankfurt am Main

- AHRNS, J.; Feser, H.-D. (1997): Wirtschaftspolitik: Problemorientierte Einführung- 7. Auflage, Verlag Oldenbourg, München, Wien

- Aiginger, K. (1999): The Use of Game Theoretical Models for Empirical Industrial Organization, Austrian Insitute of Economic Research, Wien

- Aiginger, K.; Wieser, R.; WüGeR, M. (1999): "Marktmacht im Lebensmitteleinzelhandel” (Zusammenfassung), in WIFO -Monatsbericht 12/1999, Wien, S. 797-818

- $\quad$ Aiginger, K.; Wieser, R.; WüGer, M. (1999): Marktmacht im Lebensmitteleinzelhandel, Studie des Österreichischen Instituts für Wirtschaftsforschung (WIFO) im Auftrag des Bundesministeriums für wirtschaftliche Angelegenheiten, Wien

- $\quad$ ALBACH, H. (1993): Betriebswirtschaftslehre als Wissenschaft, ZfB-Ergänzungsbeitrag, 3/93, S. 925

- $\quad$ ALBACH, H.; BOCK, K.; WARNKE, Th. (1985): Kritische Wachstumsschwellen der Unternehmensentwicklung, Schriften zur Mittelstandsforschung Nr.7, Stuttgart

- $\quad$ Alberts, W. (1989): "Assessing The Profitability of Growth by Acquisition", in: International Journal of Industrial Organization 7, S. 133-149

- AlbreCht, S. (1994): Erfolgreiche Zusammenschlussstrategien: eine empirische Untersuchung deutscher Unternehmen, Gabler-Verlag, Wiesbaden

- Alchian, A.; Demsetz, H. (1972): "Production, Information Costs and Economic Performance", in: American Economic Review, S. 777 ff.

- $\quad$ AMIT, R.; LIVNAT, J. (1988): "Diversification strategies, business cycles and economic performance", in: Strategic Management Journal, Vol 9, S. 99-101

- $\quad$ ANNAS, F. (1994): Endverbrauchersegmentierung auf dem deutschen Markt für Hart-, Schnitt- und Weichkäse und Konsequenzen für das Marketing der Anbieter, Dissertation Uni Kiel

- Ansoff, H.I.; Brandenburg, R.g.; Portner, F.E.; Radosevich, R. (1971): Acquisition Behavior of U.S. Manufacturing Firms, Nashville

- APPEL, V. (1992): Wettbewerbsprozesse in der deutschen Ernährungswirtschaft, Agrarwirtschaft Sonderheft 135, Agrimedia, Hamburg

- ARNDT, H. (1952): Schöpferischer Wettbewerb und klassenlose Gesellschaft, Duncker \& Humblot, Berlin

- $\quad$ ARNDT, H. (1958): Anpassung und Gleichgewicht am Markt, Bd. 170, Stuttgart

- $\quad$ ARNDT, H. (1976): Kapitalismus, Sozialismus, Konzentration und Konkurrenz, Mohr-Verlag, Tübingen

- ArRow, K.J. (1985): "The Economies of Behavior", in: Pratt, J.W.; Zeckhauser, R.J.: Principals and Agents: The structure of business, Boston (Mass.), S. $37 \mathrm{ff}$.

- $\quad$ Asch, P. (1972): Economic Theory and the Antitrust Dilemma, New York

- AVkiRAn, N.K. (1999): "The evidence of efficiency gain: The role of merger and the benefit to the public", in: Journal of Banking and Finance, Vol. 35, S. 245-256

- BAIK, K. H. (1995): "Horizontal Mergers of Price-Setting Firms with the Sunk Capacity Costs", in: Quarterly Review of Economics and Finance, 35, S. 245-256 
- BAIN, J.S. (1951): "Relation of Profit Rate to Industry Concentration in American Manifacturing 1936-1940", in: Quarterly Journal of economics 65, S. 293-324

- $\quad$ BAIN, J.S. (1956): Barriers to New Competition. Their Charakter and Consequences in Manifacturing Industries, Cambridge, Mass.

- BAKer, J.B. (1997): "Contemporary empirical merger analysis", in: Geo. Mason L. Rev., S. 347-356

- Baldwin, J.; GoReCKI, P. (1990): "Mergers Placed in the Context of Firm Turnover", in: Bureau of Consensus, Annual Research Conference, Proceedings, Washington D.C.: US Departement of Commerce, S. 53-73

- BarRos, P. (1998): "Endogenous Mergers and Size asymmetry of merger participants", in: Economic Letters, Vol. 60, S. 113-119

- $\quad$ BARTENS (laufende Jahrgänge): Taschenbuch Zuckerwirtschaft Europa, Albert Bartens, Berlin

- Barton, D. M.; Sherma, R. (1984): "The Price and Profit Effects of Horizontal Merger: A Case Study", in: Journal of Industrial Economics, Vol. 33, No. 2, S. 165-177

- $\quad$ BAUER, H.H. (1989): Marktabgrenzung, Duncker \& Humblot, Berlin

- Baulig, C.; Prellberg, M. (2001): „Beteiligungsverkäufe: Vorsicht Bauarbeiten”, in Financial Times Deutschland vom 27.12.2001

- Baumol W.J.; Panzar J.C.; Willig, R.C. (1982): Contestable Markets and the Theory of Industry Structure, Harcourt Brace Janovich, New York

- $\quad$ Baumol, W.J. (1959): Business Behavior, Value and Growth, Macmillan, New York

- $\quad$ BAUMOL, W. J. (1967): Business Behaviour, Value and Growth, Brace of World, New York

- BAUTZ, D. (1997): „Handel oder Systemanbieter? Molkereistreckendienst bleibt auf der Strecke”, in: Milch-Marketing Nr. 11, S. 86-89

- BECHTHOLD, R. (1999): Kartellgesetz/ Gesetz gegen Wettbewerbsbeschränkungen - Kommentar, 2. Aufl. Beck-Verlag, München

- Bend, H. (2000): Planung, Steuerung und Kontrolle leistungsbezogener Konditionen - eine empirische Untersuchung zwischen Industrie und Lebensmittelhandel, Dissertation, Uni- Stuttgart

- Berg, H. u.a. (1999). Vahlens Kompendium der Wirtschaftstheorie und Wirtschaftspolitik- Wettbewerbspolitik-, Bd. 2, 7.Aufl., Vahlen, München

- $\quad$ Berle, A. A.; Means, G. C. (1932): The Modern Corporation and Private Property, New York

- Bertelsmeier, M.; Jägersberg, P. (2002): „Einstieg in den Ausstieg aus der Milchquotenregelung? Auswirkungen für Betriebe", in: Welt der Mich, S. $9-14$

- $\quad$ BeSTER, H. (2000): Theorie der Industrieökonomik, Springer-Verlag, Berlin

- Bhaskar, K. (1972): "Three Case Studies - Guiness, Spillers and Nestles", in: Samuels, J.M.: Readings on Mergers and Takeovers, London, p. 97-122

- BICHLER, K. (1990): Beschaffungs- und Lagerwirtschaft, 5. Aufl., Gabler-Verlag, Wiesbaden

- $\quad$ BICKERT, C. (2002): „Abrechnungen im Vergleich", in: DLG-Mitteilungen 117 12, S. 22-24

- Biener, H. (1986): Die gesellschafts- und bilanzrechtlichen Gesetze nach Änderung durch das Bilanzrichtlinien Gesetz, Verlag Franz Rehm, München u.a.

- BinkLeY, J.K.; Connor, M. (1995): Concentration, Demographics, and Conundrums: MetropolitanArea Food Prices. Staff Paper, Department of Agricultural Economics Purdue University, West Lafayette, Indiana

- BinkLeY, J.K.; ConnoR, M. (1998): "Grocery Market Pricing and the New Competitive Environment", in Journal of Retailing, Vol. 74 (2), S. 273-294

- $\quad$ Blattberg, R.; Neslin, S. (1990): Sales Promotion, Englewood Cliffs

- BLEYMÜlLER, J.; GeHLERT, G. (1989): Konzentrationsmessung, in: WiSt, Wirtschaftswissenschaftliches Studium, 18

- $\quad$ BLUMENCRON MÜLleR VON, U. (2000): Anpassungserfordernisse der rheinischen Zuckerwirtschaft an verschärfte marktwirtschaftliche Rahmenbedingungen, Diplomarbeit Universität Bonn 
- BMVEL (BUNDESMINISTERIUM FÜR VERBRAUCHERSCHUTZ, LANDWIRTSCHAFT UND FORSTEN) (laufende Jahrgänge): Statistisches Jahrbuch über Ernährung, Landwirtschaft und Forsten, Landwirtschaftsverlag Münster Hiltrup

- BÖBEL, I. (1984): Wettbewerb und Industriestruktur - Industrial Organisationsforschung im Überblick, Berlin

- BOFINGER, P. (2001): Script zur Vorlesung „Wettbewerbspolitik", Universität Würzburg

- BÖGE, U. (2001): „Bonus- und Kronzeugenregelungen in Deutschland und in der EU", Vortrag zum 8. St. Gallener Kartellrechtsforum, St. Gallen, internet: www.bundeskartellamt.de

- BÖGER, R. (1990): Konditionenspreizung der Hersteller gegenüber dem Lebensmittelhandel, Göttingen 1990

- Bosch, W.; SCHÜTZ, J. (2000) in Gesetz gegen Wettbewerbsbeschränkungen und Europäisches Kartellrecht: Gemeinschaftskommentar/Begr. von Hans Müller-Henneberg u. Gustav Schwartz. Hrsg. von Christian Hootz, Carl Heymann Verlag, Köln, Berlin u.a.

- BRAUN, H. G. (1999): "Ermittelung eines genossenschaftlichen Korrekturbedarfes für die Analyse von Jahresabschlüssen in der Molkereiwirtschaft", in: Zeitschrift für Genossenschaftswesen, Nr. 4, S. $278-281$

- $\quad$ Braun, H. G. (2000): Unternehmenszusammenschlüsse in milchverarbeitenden Unternehmen, Dissertation Technische Universität München

- $\quad$ BRAUN, H. G. (2000b): Jahresabschlussanalyse unter Berücksichtigung molkereispezifischer Besonderheiten, Arbeitsbericht 00/1 Technische Universität München (FML Weihenstephan)

- $\quad$ Breshnahan, T.F. (1989): "Emperical Studies of Industries with Market Power", in: Schmalensee, R.; WILLIG, R.D.: Handbook on Industrial Organization, S. 1011-1057

- BResnahan, R.F.; ReISS, P.C. (1991): "Entry and competition in concentrated markets", in: Journal of Political Economy

- BROZEN, Y. (1970): „The Antitrust Taskforce Deconcentration Recommendation”, in: Journal of Law and Economics, 13, S. 279-292

- BROZEN, Y. (1971): "Bain's Concentration and Rates of Return Revisited”, in Journal of Law and Economics 13, S. 351-369

- Buccirossi, P.; MaretTe, S.; SchiavinA, A. (2002): "Competition Policy and the Agribusiness Sector in the European Union", in: Economic Review of Agricultural Economics, Vol. 29 (3), S. 373-397

- BÜHNER, R.; SPINDLER, H. (1986): "Synergieerwartungen bei Unternehmenszusammenschlüssen”, in: Der Betrieb 12, S. 601-606

- BÜHNER, R. (1990): Unternehmenszusammenschlüsse: Ergebnisse empirischer Analysen, PoeschelVerlag, Stuttgart

- BÜHNER, R. (1990): Erfolg von Unternehmenszusammenschlüssen in der Bundesrepublik Deutschland, Poeschel-Verlag, Stuttgart

- $\quad$ BundeskARTELLAMT (1990): Marktbeherrschung in der Fusionskontrolle, Köln

- $\quad$ BundeskARTELLAMT (1997): Tätigkeitsbericht 1995/96, Bundestagsdrucksache 13/7900

- BundeskARTELLAMT (1998): Merkblatt zum Anwendungsbereich der EG-Fusionskontrolle, internet: www.bundeskartellamt.de/EG-Fusionskontrolle/Merkblatt.pdf

- $\quad$ Bundeskartellamt (1999): 1999 B2 - 15510 - ZU - 127/98 - „Nordmilch", Bonn

- BUNDESKARTELLAMT (1999): Inlandsauswirkung net: www.bundeskartellamt.de/Inlandsauswirkung.pdf

- $\quad$ BundeSKARTELLAMT (1999): Tätigkeitsbericht 1997/98, Bundestagsdrucksache 14/1139

- BUndeSKARTELLAMT (2000): Auslegungsgrundsätze zur Beurteilung einer marktbeherrschenden Stellung, internet: www.bundeskartellamt.de/FusionskontrollChecklisteOkt2000.pdf

- BundeskARTELLAMT (2000): Das Bundeskartellamt und seine Arbeit, internet: www.bundeskartellamt.de

BundeSKARTELLAMT (2000): Merkblatt zur deutschen Fusionskontrolle, internet: www.bundeskartellamt.de/Merkblatt Fusionskontrolle D August 2000.pdf

BUNDESKARTELLAMT (2001): Das Untersagungskriterium in der Fusionskontrolle - Marktbeherrschende Stellung versus Substancial Lessening of Competition, Diskussionspapier vom 08.10.2001, Bonn 
- $\quad$ BundeSKARTElLAMt (2001): Tätigkeitsbericht 1999/ 2000, Bundestagsdrucksache 14/ 1139

- Bundeskartellamt (2002), B2 - 31/02, "Nordzucker/Union-Zucker", Berlin

- Bundeskartellamt (2002): Stellungsnahme zum Grünbuch der Kommission zur Revision der Verordnung Nr. 4064/89 des Rates über die Kontrolle von Unternehmenszusammenschlüssen, Bonn

- Bundeskartellamt (laufende Jahrgänge): Tätigkeitsbericht, Bonn

- BUNDESMINISTERIUM FÜR ERNÄHRUNG, LANDWIRTSCHAFT UND FORSTEN (laufende Jahrgänge): Strukturen der Molkereiwirtschaft - Daten Analyse, Referat 216, Bonn

- BUNDESMINISTERIUM FÜR VERBRAUCHERSCHUTZ, ERNÄHRUNG UND LANDWIRTSCHAFt (laufende Jahrgänge): Statistisches Jahrbuch über Ernährung, Landwirtschaft und Forsten, Landwirtschaftsverlag, Münster-Hiltrup

- $\quad$ BundesVerband DER ERnÄHRUngSindustrie (BVE) (laufende Jahrgänge): Jahresbericht, Bonn

- Bundesverband der ERnÄHrungsindustrie, Price Waterhouse CoOpers (1998): Fusionen, Akquisitionen und Unternehmensverkäufe in der deutschen Ernährungsindustrie, Fachverlag Moderne Wirtschaft, Frankfurt am Main

- BUNDESVERBANDES FüR ERNÄHRUNGSINDUSTRIE: (17.10.2001): Zukünftige Herausforderungen der Ernährungsindustrie, internet: www.bve-online.de/aktuell/texte/221001/industry.html

- $\quad$ BURKHARDT, J. (1995): Kartellrecht, Beck-Verlag, München

- Cable, J.R.; Palfrey, J.; Runge, J. (1980): "Economic Determinants and Effects of Mergers in West Germany", in: Zeitschrift für die gesamte Staatswissenschaft, Bd. 136, S. 226-248

- CamesascA, P. D. (1999): "The explicit efficiency defence in merger control: does it make the difference?", in: European Competition Law Review 20, S. 14

- CamesascA, P. D. (2000): European Merger Control: Getting the Efficiencies Right, Intersentia-Hart, Antwerpen u.a.

- Caplin, A.; Leahy, J. (1994): "Business as Usual, Market Crashs and Wisdom after the Fact", in: American Economic Review, Vol. 84, No. 3, S. 548-565

CAVES, R. (1989): "Mergers, Takeovers and Economic Efficiency", in: International Journal of Industrial Organization 7: S. 151-174

- CEFS (Comité Européen des Fabricants de Sucre) (2000): Sugar Statistics 2000, Brüssel

- Chakravaty, S. R. (1998): "Efficient Horizontal Mergers", in: Journal of Economic Theory 82, S. 277-289

- Chamberlin, E.H. (1950): The Theory of Monopolistic Competition, Havard University Press, Cambridge

ChATterJeE, S. (1986): "Types of Synergie and Economic Value: The Impact of Acquisitions on Merging and Rival Firms", in: Strategic Business Journal 7, S. 119-139

- CIUS (Committee of Industrial Users of Sugar) (1999): The Reduction of the EU-Sugar Price Through A Reassessment of the Institutional Manufacturing Margin in the Context of the Commission's Proposals on Agricultural Prices

- Clarke, C. J. (1987): "Acquisitions - Techniques for Measuring Strategic Fit", in: Long Range Planing, Vol. 20, No. 3, S. $12-18$

- Coenenberg, A. G. (1994): Jahresabschluss und Jahresabschlussanalyse: Betriebswirtschaftliche, handels- und steuerrechtliche Grundlagen, 15. Aufl., Landsberg am Lech

- Coley, S.C., Reinton, S.E. (1988): "The Hunt for Value", in: The McKinsey Quarterly, o. Jg., o. Nr., Spring, S. 29-39

- Conner, J.M.; Rogers, R.; Bhagavan, V. (1996): "Concentration and Countervailing Power in the US Food Manufacturing Industries", in: Review of Industrial Organization, Vol. 11, S. 473-492

- Cosh, A.D.; Hughes, A. (1995): Failures, Acquisitiones and Post Merger Success: the Comperative Financial Characteristics of Large and Small Companies. Working Paper 18, ESRC Center for Business research, University of Cambridge, Cambridge

- Cotterill, R.W. (1986): "Market Power in the Retail Food Industry: Evidence from Vermont", in: The Review of Economics and Statistics, Vol. 58, S. 379-386

- CotTeRILl, R. W. (1989): Food and Mergers: Paper and comments presented at the industrial organization society sessions of the Southern Economic Association's fifty-ninth annual conference, 
Orlando, Florida, November 19 - 21, 1989 and American Economic Association's hundred and second annual meeting, Atlanta, Georgia, December $28-30,1989$

- Cowling, K.; Stoneman, P.; Cubbin, J.; Cable, J.; Hall, G.; Domberger, S.; Dutton, P. (1989): Mergers and Economic Performance, cambridge University Press, Cambridge

- $\quad$ Cramon-Taubadel, S. von; Loy, J. P.; Musfeldt, E. (1995): "Empirische Methoden zur Analyse der Marktintegration am Beispiel des EU-Schweinefleischmarktes", in: GeWiSoLa-Tagungsband Nr. 31, S. 119-132, Landwirtschaftsverlag, Münster-Hiltrup

CRAMPTON, P. (1994): "Alternative Approaches to Competition Law: Consumers surplus, Total Surplus, Total Welfare and non-efficiency goals", in: World Competition 17, S. 55-86

- Cullen, B.; Whelan, A. (1997): "Concentration of the Retail Sector and Trapped Brands", in: Long Range Planning, Vol. 30, S. 906-916

- Demmer, C. (2002): "Was sind eigentlich Synergieeffekte”, in Süddeutsche Zeitung vom 13.05.2002

- Demsetz, H. (1973): „Industry Structure, Market Rivalry and Public Policy”, in: Journal of Law and Economics 16, S. 1-9

- DeneCKere, R.; Davidson, C. (1985): „Incentives to form Coalations with Bertrand Competition”, in: Rand Journal of Economics 16, S. 473-486

- Dertouzous, J.N.; ThORPE, K.E. (1982): Newspaper Groups: Economics of Scale, Tax Laws and Merger Incentives, Publication R-2878-SBS, Rand Corporation

- Deutsche GenossenschaftSbank (2000): Die Europäische Ernährungsindustrie, Frankfurt am Main

- Deutsche Genossenschaftsbank ResearCh (1999): Deutscher Lebensmitteleinzelhandel: Strukturen - Probleme - Strategien, Frankfurt am Main

- Deutsche Milchwirtschaft Spezial (2000): Die größten Molkereiunternehmen in Europa, Thomas Mann Verlag, Gelsenkirchen

- Deutsche Milchwirtschaft Spezial (2001): Die umsatzstärksten Mopro- Anbieter in Deutschland und Kerneuropa 2001, Thomas Mann Verlag, Gelsenkirchen

- DeWeY, D. (1961): „Mergers and Cartels: Some Reservations About Policy”, in: American Economic Review, Papers and Proceedings, Vol. 51, No. 2, S. 255-262

- DEWING, A.S. (1922): "A Statistical Test of the Success of Consolidations", in: Quarterly Journal of Economics, Vol. 36, S. 84-101

- Die AkTiengesellschaft (AG): Zeitschrift für das gesamte Aktienwesen, für deutsches, europäisches und internationales Unternehmens- und Kapitalmarktrecht, Otto-Schmidt-Verlag, Köln

- DLZ-Agrarmagazin, Fachzeitschrift der deutschen Landwirtschaft, Deutscher Landwirtschaftsverlag, Hannover

- Dobson Consulting (1999): Buyer Power and its impact on competition in the food retail distribution sector of the European Union, Nottingham, internet: http://europa.eu.int/comm/competition/publications/studies/bpifrs/chap01.pdf

- DOCKNER, E.J. (1992): "A dynamic theory of conjectural variations", in: Journal of Industrial Economics XL (4), S. 377-395

- Dockner, J. Engelbrecht; Gaunersdorfer, A. (2001): "On the profitability of horizontal industries with dynamic competition", in: Japan and the World Economy 13, S. 195-216

- DoHmS, H. (2002): Ein interregionaler Vergleich der Regelungen zur zwischenbetrieblichen Übertragung von Rübenlieferrechten sowie der Pacht- und Kaufpreise dieser Rechte in Deutschland, Diplomarbeit, Universität Göttingen

- Drauz, G.; SChroeder, D. (1995): Praxis der Europäischen Fusionskontrolle, RWS-Verlag, Köln

- DRESCHER, K. (1999): „Preisbildung und Konzentration im deutschen Lebensmitteleinzelhandel”, in: Agrarwirtschaft, Vol. 48, Heft 6, S. 230-239

- Drews, M. (1982): "Ursachen und Prognosen des Molkeriestrukturwandels", in: Deutsche Milchwirtschaft Teil 1: Nr. 16, S. 557-562- Teil 2: Nr. 17, S. 588-592

- DREXLER, U. (1999): Bundesverband des Deutschen Lebensmitteleinzelhandels, mündliche Mitteilung

- DUMSTORF, H. (1999): „Die deutsche Milchwirtschaft im Wettbewerb”, in: Welt der Milch, S. 558563 
- DuTz, M. A. (1989): „Horizontal mergers in declining industries: Theory and evidence”, in: International Journal of Industrial Organization 7, S. 11-33

- ECKво, B. E. (1983): "Horizontal Mergers, Collusion and Stockholder Wealth", in: Journal of Financial Economics, Vol. 11, S. 241-273

- EDWARDS, C.D. (1955): "Conglomerate bigness as a source of power", in: Stigler, G.J. (1955): Business Concentration and Price Policy, Princton University Press, Princton, New York, S. 331-359

- Elzinger, K. G. (1981): "Defining geographic market boundaries", in. Antitrust Bulletin, S. 739-752

- Elzinga, K. G.; HogaRTY, T. F. (1973): "The Problem of Geographic Delineation in Antimerger Suits", in: Antitrust Bulletin, S. 45-81

- EMmerich, V. (1991): Kartellrecht - 6. Auflage, Verlag Beck, München

- EMmerich, V. (1995): Kartellrecht - 7. Auflage, Verlag Beck, München

- $\quad$ EMmerich, V. (1999): Kartellrecht - 8. Auflage, Verlag Beck, München

- Epstein, R. J.; Rubinfeld, D. (2001): Merger Simulation: A Simplfied Approach with New Applications, Working Paper No. E01-309, University of Berkeley-Economic Departement

- EUROPÄISCHE-KOMMISSION - Generaldirektion Wettbewerb (laufende Jahrgänge): Bericht über die Wettbewerbspolitik, Amt für Amtliche Veröffentlichungen der Europäischen Gemeinschaften, Luxemburg

- EUROPÄISCHE-KOMMISSION (1995): Amtsblatt L 211, Brüssel

- EUROPÄISCHE-KOMmISSION - Generaldirektion Wettbewerb (2001), Beschluss Comp/M.2399, Friesland Coberco/ Nutricia, Luxemburg

- EUROPÄISCHE-KOMMISSION - Generaldirektion Wirtschaft und Finanzen (2000a): Europäische Wirtschaft - Wirtschaftsanalysen, Beiheft A, No. 5/6, Fusionen und Übernahmen, internet: www.europa.eu.int/comm/economy_finance

- EUROPÄISCHE-KOMMISSION (1999): Fact Sheet: Reform der GAP: Milch und Milcherzeugnisse, Brüssel

- EUROPÄISCHE-KOMmISSION (1999): Die Wettbewerbspolitik der Europäischen Gemeinschaft: XXIX Bericht über die Wettbewerbspolitik 1999, Brüssel

- EuRopÄISCHE-Kommission (2001), Fall Nr. COMP/ M.2530 - Südzucker/ Saint Louis Sucre, Brüssel

- $\quad$ EUROPÄISCHE-Kommission (2001): GRÜNBUCH über die Revision der Verordnung (EWG) Nr. 4064/89 des Rates, Brüssel

- EUROPÄISCHE Kommission - Amtsblatt C 50 (15. Feb. 2001), Brüssel

- $\quad$ EUROPÄISCHE KOMMISSION (1997), Official Journal 372, 9.12.1997

- EUROPÄISCHE KOMMISSION (2000): Die Wettbewerbspolitik der Europäischen Union 1999, Amt für amtliche Veröffentlichungen der Europäischen Gemeinschaft, Luxemburg

- EUROPÄISCHE KOMMISSION (27.10.2000): Newsletter: Vorschlag für eine Überarbeitung der Zuckermarktordnung, Brüssel

- EUROPÄISCHE KOMMISSION (4.10.2000): Vorschlag für eine Verordnung des Rates über die gemeinsame Marktorganisation für Zucker, Brüssel

- $\quad$ European Commission (1996): Competition Letters, No. $2+3$, Brüssel

- $\quad$ EuRopean Commission (1997): Competition Letters, No. 1, Brüssel

- EuROPEAN COMmission (1997): Commission Notice on the definition of the relevant market for the purpose of Community law, internet: http://europa.eu.int/comm/competition/antitrust/relevma_en.html (Stand: 2.12.2002)

- EuRopeAn COMmission - Directorate General for Economics and Financial Affairs (2001): European Economy - Economic Trends, Supplement A, No. 12, Mergers and Acquisitions, internet: www.europa.eu.int/comm/economy_finance

- EUROPÄISCHER-RECHNUNGSHOF (2001): Sonderbericht Nr. 6/2001 über Milchquoten, zusammen mit Antworten der Kommission, Official Journal C 305/1, Brüssel

- EUROPÄISCHER ReCHNUNGSHOF (2000): Sonderbericht Nr. 20/2000 über die Verwaltung der gemeinsamen Marktorganisation für Zucker, zusammen mit den Antworten der Kommission, Luxemburg

- $\quad$ F.O. LICHTs Europäisches Zuckerjournal, verschiedene Jahrgänge, Verlag F.O. Licht, Ratzeburg 
- FarRell, J.; Shapiro, C. 1990: "Horizontal Mergers: An Equilibrium Analysis", in: American economic Review, Vol 80, No. 1, S. 107-126

FARRIS, P.W.; AILAWADI, K.L. (1992): "Retail Power: Monster or Mouse?", in: Journal of Retailing, Vol. 68 , S. 351-369

- FathmanN, B. (1998): "Exportschlager", in: Agrarmarkt Nr. 8, S. 6-8

FAULLI-OLLER, R.; MotTA, M. (1996): Managerial Incentives for Takeover: A discussion, Instituto Valenciano de Investigaciones Economicas 22, Valencia

FeHL, U.; Oberender, P. (1989): Grundlagen der Mikrotheorie, 3. Aufl., Vahlen, München

FEINBERG, R. M. (1985): "Sales at risk": A test of the mutural forbearance theory of conglomerate behaviour", in: Journal of Business, Vol. 58, S. 225-241

- $\quad$ Financial Times DeutSCHLAND (FTD): Verlag Financial Times Deutschland, Hamburg

- $\quad$ Frankfurter Allgemeine Zeitung (FAZ): Verlag Frankfurter Allgemeine Zeitung, online: www.faz.net, Frankfurt am Main

- LeberT, R. (2002): „Weniger Fusionen und Übernahmen”, in Financial Times Deutschland vom 10.01.2002, www.ftd.de/fusionen

- FIRTH, M. (1980): "Takeovers, Shareholder Returns and the Theory of the Firm", in The Quarterly Journal of Economics 94 (2), S. 235-260

- Fleischer, H.; KÖRBeR, T. (2001): Der Einfluß des US-amerikanischen Antitrustrechts auf das Europäische Wettbewerbsrecht in WuW 1/2001, S.6ff., Verlagsgruppe Handelsblatt, Düsseldorf

- FRANCOISE, M. H. (1997) : Kontingentierung als produktionslenkende Maßnahme landwirtschaftlicher Produkte, Dissertation Universität Bonn

- FrANKE, G. (1993): "Neuere Entwicklungen auf dem Gebiet der Finanzmarktlehre", in: Das Wirtschaftsstudium Heft 8, S. 389-398

- $\quad$ Frankfurter Kommentar zum Gesetz gegen Wettbewerbsbeschränkungen (1998): Glassen, H. u.a., Köln

- FRESE, E. (1993): "Geschäftssegmentierung als organisationstheoretisches Konzept zur Leitbildfunktion mittelständischer Strukturen für Großunternehmungen", in: Zeitschrift für betriebswirtschaftliche Forschung, Nr. 12, S. 999-1024

- Fridolfsson, S.-O.; STeneK, J. (1999): Why Mergers Reduce Profits and Rise Share Prices - A Theory of Preemptive Mergers, Research Institute for Industrial Economics

- GASSNER, U. M. (1999): Grundzüge des Kartellrechts, Verlag Vahlen, München

- Gaudet, G.; SAlent, S. W. (1993): Towards a Theory of Horizontal Mergers", in: Norman, G.; LaManna, M., The New Industrial Economic Review, recent Developments in Industrial Organization, Oligopoly and Game Theory, Aldershot, S. 137-158

- Geithmann, F. E.; Marvel, H. P.; Weiss, L. W. (1981): "Concentration, Price and Critical Concentration Ratios", in: Review of Economics and Statistics, Vol. 63, No. 3, S. 346-353

- GERPOTT, T. J. (1993): Integrationsgestaltungen und Erfolg von Unternehmensakquisitionen, Schäffer-Poeschel, Stuttgart

- GiMPEL-ISKE, E. (1973): Untersuchung zur Vorteilhaftigkeit von Unternehmenszusammenschlüssen, Dissertation, Universität Bonn

- Glassen, H. u.a.. (2000): Frankfurter Kommentar zum Kartellrecht. Mit Kommentierung des GWB, des EG-Kartellrechts und einer Darstellung ausländischer Kartellrechtsordnungen, Otto-SchmidtVerlag, Köln

- GLoY, D. (1995): Wettbewerbs- und Unternehmensentwicklungsstrategien in der europäischen Molkereiwirtschaft, Dissertation Universität Kiel

- Goldberg, L. (1973): The Effect of Conglomerat Mergers on Competition", in: Journal of Law and Economics, Vol. 16, S. 137-158

- GoRT, M. (1969): "An economic Disturbance Theory of Mergers", in: Quarterly Journal of economic, Vol. 13, S. 624-642

- Gowrisankaran, G. (1999): "A dynamic model of endogenous horizontal mergers", in: Rand Journal of Economics 30 (1), p. 56-83 
- Gracia, A.; Albisu, L.M. (2001): "Food Consumption in the European Union: Main Determinants and Country Differences", in: Agribusiness, Vol. 17, S. 469-488

- $\quad$ GrÄFER, H. (1997): Bilanzanalyse, 7.Aufl, Nwb-Verlag, Berlin

- Grant, R.; JAMmine, A. (1988) : Performance Differences between the Wrigley/Rumelt Strategic Categories », in : Strategic management Journal, p. 333-346

- Greer, D. F. (1980): Industrial Organization and Public Policy, Macmillan-Verlag, New York

- $\quad$ GrünBerger, H. (2001): Praxis der Bilanzierung, 7. Auflage, Springer-Verlag, Wien

- GÜNTHER, W.A. (1996): „Technische Logistik in der Milchwirtschaft - Anforderungen, Möglichkeiten, Trends", in: DMW Nr. 2, S. 53-57

GUtenBerg, E. (1975): Grundlagen der Betriebswirtschaftslehre, Dritter Band: Finanzen, 7. Aufl., Berlin u.a.

- HAISCH, K.H.; BETZ, J. (1983): "Zeitaufwand bei der Milchübernahme mit Tanksammelwagen", in: Deutsche Milchwirtschaft Nr. 14, S. 450-454

- $\quad$ HAISCH, K.H.; STÖCKL, J.P. (1986): „Der Konzentrationsdruck in der Milchverarbeitung unter besonderer Beachtung der Milch-Garantiemengen-Regelung, Alta

- HAMBLOCH, C. (2002): Unterschiedliche Entwicklungen am Zuckermarkt - ZMP Marktkommentar vom 09.11.2001, Bonn

- HANDELSBLATT: Wirtschafts- und Finanzzeitung, online- Version: www.handelsblatt.com, Verlag economy one, Düsseldorf

- HANNAH, A.C. (1997): "The world sugar market and reform", in: F.O.Licht's International Sugar and Sweetener Report, Vol. 129, No. 31, S. 595-599

- HARRIS, W.; RAVIV, A. (1979): "Optimal Incentive Contracts with Imperfect Information", in: The Journal of Economic Theory, S. $231 \mathrm{ff}$.

- Hartmann, R. S. (1996): "Predicting the Efficiency Effect of Mergers", in: Journal of Forensic Economics 9 (3), S. 295-323

- $\quad H A X, H$. (1987): "Handlungsbedingungen der Unternehmen in der BRD", in: Deutscher Bundestag: Materialen zur Lage der Nation im geteilten Deutschland, Drucksache 11/ 11, S. 172-175

- HAY, G. A.; Werden, G. J. (1993): „Horizontal Mergers: Law, Policy and economics”, in: American Economic review, Papers and Proceedings, Vol. 83, No. 2, S. 173-177

Healy, P. M.; Palepy, K. G.; Ruback, R. S. (1992): "Does Corporate Performance Improve after Mergers?", in: Journal of Financial Economics, Vol. 31, No. 2, S. 135-175

- HeRdZina, K. (1999): Wettbewerbspolitik, 5. Aufl., Lucius-Verlag, Stuttgart

- Hermes, O. (1988): Die Erfassung von Nachfragemacht im Handel - Ökonomische Grundlagen und kartellrechtliche Konsequenzen, GHS-Reihe, Band 12, Göttingen

- Herrmann, R.; Möser, A.; ElKe, W. (2002): "Neue empirische Befunde zur Preissetzung und zum Verbraucherverhalten im Lebensmitteleinzelhandel", in Agrarwirtschaft, Vol. 51, Heft 2,

- HeRRY, M. (2001): Transportpreise und Transportkosten der verschiedenen Verkehrsträger im Güterverkehr, Arbeiterkammer Wien Bericht Nr. 14, Wien

- HewITT, G. (1998): Roundtable on Buying Power - Background Paper by the Secretariat, DAFFE/CLP(98)23, OECD, Paris

- Higgins, R.C.; SCHALL, L.D. (1975): "Corporate bankruptcy and conglomerat merger", in: Journal of Finance, Vol. 30, No.1, S. 93-113

Hiltmair, J. (1996): „Aktuelle Logistikprobleme im Molkeriebereich”, in: Deutsche Milchwirtschaft Nr. 2, S. $58 \mathrm{f}$.

- HiRSHleifer, J.; RileY, J.G. (1979): "The Analytics of Uncertainty and Information - An Expository Survey", in: The Journal of Economic Literature, S. $1375 \mathrm{ff}$.

- HoffmanN, F. (1989): „So wird Diversifikation zum Erfolg", in: Harvard Manager, No. 4, S. 52-57

HOFFMANN, J.; VENEMA, J. (2001): „Unternehmenskäufe und Unternehmenszusammenschlüsse Branchenanalys Nordmilch", Seminararbeit SS01, Universität Göttingen

HogaRTY, T.F. (1970): "The Profitability of Corporate Mergers", in: Journal of Business, Vol. 43, S. 317-327 
HOLDERNESS, C. G.; SHEEHAN, D. P. (1985): "Raiders or saviors? The evidence on six controversial investors", in: Journal of Financial Economics Vol. 14, S. 555-579

HöPer, U.; Jürgensen, M.; HaRgens, R.; Groß, K.-U., HülSemeYer, F. (2000): "UnternehmensgröBenabhängige ökonomische und ökologische Auswirkungen bei Erfassung, Be- und Verarbeitung und Distribution von Milch und Milchprodukten", in: Regionale Vermarktungssysteme in der Land-, Ernährungs- und Forstwirtschaft, DLG-Verlag, Frankfurt am Main

HoPkINS, H.D. (1987): "Acquisitions Strategy and the market Position of acquiring Firms", in: Strategic Management Journal, Vol. 8, S. 535-547

- HORN, H.; PERSON, L. (1996): Endogenous Mergers in an International Oligopoly, mimeo, IIES, Stockholm University

- HoRowitz, I. (1982): "Market Definition in Antitrust Analysis: Reply”, in: Southern Economic Journal, S. 564-566

- IDF (International Dairy Federation) (2001): "Milchquote - Ja oder Nein?", in: Welt der Milch, S. 532

- $\quad$ ILO (International Labour Organization) (verschiedene Jahrgänge): Yearbook, Genf

- IMMENGA, U. (1995): Konzepte einer grenzüberschreitenden und international koordinierten Wettbewerbspolitik, Kieler Arbeitspapiere Nr. 692, Institut für Weltwirtschaft an der Universität Kiel

- IMmengA, U.; MeSTMÄCKER, E.-J. (1997): EG- Wettbewerbsrecht Kommentar Band 1, Beck- Verlag, München

- IRSCH, N. (1988): "Zum Zusammenhang von Unternehmensgröße und Rentabilität in der BRD in der Mitte der 80er Jahre", in: Jahrbuch für Nationalökonomie und Statistik, Bd. 205/5, Fischer, Stuttgart u.a.O., S. 519-537

- IVALDi, M.; Verboven, F. (2001): "Quantifying the effects of horizonatal mergers in European Competition Policy" CEPR- Discussion Paper No. 2697

- IZZ (Informationszentrum Zuckerverwender) (2001): IZZ-Vorschläge für eine wirksame Reform der EU-Marktordnung für Zucker, Bonn

- JANSEN; MÜLLER-STEWENS (2001): „Pre- und Post Merger-Integration bei grenzüberschreitenden Zusammenschlüssen", in: Jansen/ Picot/ Schiereck: Internationales Fusionsmanagement - Erfolgsfaktoren grenzüberschreitender Unternehmenskäufe, Stuttgart, S. 3-4

- JANSIK, C. (2002): Determinants and Influence of Foreign Direct Investmensts in the Hungarian Food Industry in a Central and Eastern European Contextm, MTLL, Finnland

- JENSEN, M. C. (1984): "Takeovers: folklore and science”, in: Harvard Business Review, 11/12, S. 109-124

- Jensen, M. C. (1986): "Agency Costs of Free Cash-Flow, Corporate Finance and takeovers", in: American Economic Review Papers and Proceedings, Vol. 76, No. 2, S. 323-329

- Jensen, M. C. (1988): Takeovers: Their Causes and Consequences", in: Journal of Economic Perspectives, Vol. 2, No. 1, S. 21-48

- Jensen, M. C.; RUBACK, R. S. (1983): "The market for corporate control" in: Journal of Financial Economics 11, S. 5-50

- Jensen; M. C.; MeCKLing, William, H. (1976): „Theory of Firm: Managerial Behaviour, Agency Costs and Ownership Structure", in: Journal of Financial Economics 3, S. 305-360

- KAAS, K.P.; Gegenmantel, R. (1995): „Ökonomische Determinanten der Macht auf dem Lebensmittelmarkt", in: Zeitschrift für Betriebswirtschaft, Jg. 65, S. 885-904

KAHNeman, D; TVersky, A. (1979): „Prospect Theory: An Analysis of Decision Making under Risk”, in: Econometrica, Vol. 47, No. 2, S. 263-237

KALLFASS, H. H. (1996): Konzepte und Indikatoren zur Abgrenzung räumlicher Märkte in der europäischen Zusammenschlusskontrolle, Institut für Volkswirtschaftslehre, Diskussionspapier Nr. 7, Universität IImenau

- Kallfass, H. H. (1997): „Konzepte und Indikatoren zur Abgrenzung räumlicher Märkte in der europäischen Zusammenschlusskontrolle", in: Jörn Kruse, Kurt Stockmann, Lothar Vollmer (Hrsg.): Wettbewerbspolitik im Spannungsfeld nationaler und internationaler Kartellrechtsordnungen, Nomos-Verlag, Baden-Baden, S. 111-129

KAMIEN, L.M.; ZANG, I. (1990): "The limits of Monopolization through acquisition", in: Quartely Journal of Economics 105, S. 465-500 
- KANTZENBACH, E.; KRÜGER, R. (1990): „Zur Frage der richtigen Abgrenzung des sachlich relevanten Marktes bei der wettbewerbspolitischen Beurteilung von Unternehmenszusammenschlüssen", in: Wirtschaft und Wettbewerb, S. 472-481

- KANTZENBACH, E. (1984): "Internationaler Wettbewerb und nationale Zusammenschlusskontrolle", in: Jahrbuch für Sozialwissenschaften, Bd. 35, S. 443-458

- KantZenbach, E.; KotTmAnN, E.; KRÜGER, R. (1996): Kollektive Marktbeherrschung: Neue Industrieökonomik und Erfahrung aus der Europäischen Fusionskontrolle, Nomos-Verlag, Baden-Baden

KANTZENBACH, E.; KRÜGER, R. (1993): Wettbewerbspolitische Leitbilder für Europa, HWWADiskussionspapier Nr. 15, Hamburg

KANTZenBACH, E.; KRUSE, J. (1989): Kollektive Marktbeherrschung, Göttingen

- Kaplan, S. (1987): Management Buyouts: Efficiency Gains or Value Transfer, Harvard Business School Working Paper

KAUFER, E. (1980): Industrieökonomik, München

- KEANE, M. (1998): Structural change, economies of scale and international competitivness in dairying, IDF congress Aarhus, Dänemark

- KELLY, E.M. (1967): The profitability of Growth through Mergers, Pennsylvania University

- KERBER, W. (1994): Die Europäische Fusionskontrollpraxis und die Wettbewerbskonzeption der EG, Verlag PCO, Bayreuth

- KERBER, W. (1999): Europäische Fusionskontrolle: Entwicklungslinien und Perspektiven, Volkswirtschaftliche Beiträge Nr. 12, Universität Marburg

- KERBER, W. (2000): „Europäische Fusionskontrolle: Entwicklungslinien und Perspektiven", in: Die Europäische Fusionskontrolle, Hrsg.: Oberender, Peter, Duncker u. Humblodt, Berlin

- KIEfer, J. (1990): „Marketing und Absatzstrategien", in: Deutscher Raiffeisenverband e.V.: 1. Fachtagung der genossenschaftlichen Milchwirtschaft vom 14. bis 15.3 in Hannover, Bonn, S. 145-165

- $\quad$ Kim, B-D.; Blattberg, R.; Rossi, P. (1995): "Modelling the Distribution of Price Sensitivity and Implications for Optimal Retail Prices", in: Journal of Business and Economic Statistics, Cornwall

- KIM, E.; SingaL, V. (1993): "Mergers and Market Power: Evidence from the Airline-Industry", in: American Economic Review, Vol. 83, No. 3, S. 549-569

- KINNE, K. (1997): Internationale Wettbewerbsfähigkeit von Unternehmen in der Europäischen Zusammenschlusskontrolle, HWWA- Report 168, Hamburg

- KInNE, K. (2000): Effizienzvorteile in der Zusammenschlusskontrolle - Eine vergleichende Analyse der deutschen, europäischen und US-amerikanischen Wettbewrebspolitik, HWWA-Studien Band 57, Nomos-Verlag, Baden-Baden

- Kitching, J. (1967): "Why do Mergers Miscarry?", in: Harvard Business Review, Vol. 45, No. 6, S. 84-101

- Kitching, J. (1974): "Winning and Losing with European Acquisitions", in: Harvard Business Review, Vol. 52, No. 2, p. 124-136

- KLEINERT, J.; KLODT, H. (2000): Megafusionen: Trends, Ursachen und Implikationen, Mohr Siebeck, Tübingen

- KNITTEL, Michael (1999): Die marktbeherrschende Stellung von Unternehmen nach der Fusionskontrollverordnung Nr. 4046/89, Universität Köln

- Kogeler, R. (1992): Synergiemanagement im Akquisitions- und Integrationsprozess von Unternehmungen, GBI-Verlag, München

- KoopmanN, G. (2000): Internationalisierung der Wettbewerbspolitik: Korrelat zur internationalen Handelspolitik, HWWA Diskussionspapier 108, Hamburg

- Kottmann, E. (2000): Die räumliche Abgrenzung des relevanten Marktes, Nomos-Verlag, BadenBaden

- KPMG (2000): Der Handel im Wandel - Der Kunde ist König, Köln

- $\quad$ KPMG (2002): Pressemitteilung: „M\&A- Markt am Boden", 24.06.2002, www.kpmg.de

KPMG (Corporate Finance) (2000): European`s recipe for success...innovate and consolidate - A survey of Europeans Food Processors, London 
- $\quad$ KPMG 1998 Management Consulting (1998): Colouring the Map: Mergers \& Acquisitions in Europe. www. kpmg.co.uk/uk/service/manage/research/mergers

KPMG/ EHI (2001): Status Quo und Perspektiven im deutschen Lebensmitteleinzelhandel - Eine Marktanalyse, Köln

Krebs, D. M.; SCheinkman, J. A. (1983): "Quantity Precommitment and Bertrand Competition Yield Cournot Outcomes", in: The Bell Journal of Economics, Vol. 14, No. 2, S. 326-337

- Krell, E.; Wietbrauk, H. (1993): „Die Kosten der Modellabteilung Schnittkäserei am Beispiel der Herstellung von Gouda-Käse", in: Kieler Milchwirtschaftliche Forschungsberichte - Teil 1: Grundlagen und modellspezifischer Faktoreinsatz. In Nr. 2, S. 145-187 - Teil 2: Ergebnisse und Interpretation der Modellkalkulationen . In Nr. 3, S. 245-271

- KRELL, E.; SCHMIDT, E.; WIDERA, H.; HÖPER, U. (1997): "Ökonomie der Milchbe- und -verarbeitung", in: Betriebs- und Marktwirtschaftliche Studien zur Ernährungswirtschaft, Heft Nr. 10, Bundesanstalt für Milchforschung, Kiel

- KRICK, A. (2000): "Die Konzentration in der europäischen Zuckerindustrie”, in: Zuckerindustrie Nr. 6, S. $424-430$

- KRICK, A. (2001): Die Struktur der Zuckerindustrie in Europa, internet: www.vsz.de/dzz/0102/struktureu.html, Stand: 19.12.2001

- KRUGMAN, P. R.: (1996): „Making Sense of the Competiveness Debate" ,in: Oxford Review of economic Policy 12 (3), S. 17-25

- KRUSE, J. (1985): Ökonomie der Monopolregulierung, Vandenhoek \& Ruprecht, Göttingen

- KRUSE, J. (1994): Kollusion, Diskussionsbeitrag aus dem Institut für VWL Universität Hohenheim, Nr. 10, Hohenheim

- KUMAR, M.S. (1981): "Do Mergers Reduce Corporative Investment? Evidence from the United Kingdom", in: Cambridge Journal of Economics, Vol. 5, S. 107-118

- KUMPS, A.M. (1975): Conglomerate Mergers: The Case of Great Britain, The Second Conference of Economics of Industrial Structure, Seminar Proceeding, IIM Berlin, Vol. 1, Berlin, S. 12-33

- KURANDT, D. (1972): Fusionen Deutscher Aktiengesellschaften. Eine empirische Untersuchung über Gründe und Wirkungen, Dissertation, Saarbrücken

- LADEMANN, R. (1988): "Methodologische und erfahrungswissenschaftliche Probleme bei der Abgrenzung des relevanten Marktes" in: Wirtschaft und Wettbewerb 1988, S. 575- 586

- LADEMANN, R. (1988): Machtverteilung zwischen Industrie und Handel, GHS-Reihe, Band 13, Göttingen

- LADEMANN, R. (1996): Marktstruktur und Wettbewerb in der Ernährungswirtschaft, GHS-Reihe, Band 44, Göttingen

- LADEMANN, R. (2001): „Der Konditionenpoker nach Fusionen - Missbrauch von Nachfragemacht oder Leistungswettbewerb - Wettbewerbsanalytische Tatsachen zum Fall Metro/ Allkauf", in: Lebensmittelzeitung, Nr. 2 v. 12.01.2001, S. 44 f.

- LADEMANN, R. (1986): Nachfragemacht von Handelsunternehmen, GHS-Reihe, Band 7, Göttingen

- LADEMANN, R. (2000): „Erfahrungswissenschaftliche Ansatzpunkte bei der Marktabgrenzung in Kartellverfahren", in: FIW- Schriftenreihe, Heft 181, Köln, Berlin u.a., S. 67-91

- $\quad$ Lahmann, M. (2001): Prognose der Nachfrage nach Milch und Milcherzeugnissen in Deutschland und Frankreich bis zum Jahr 2005, FAL Braunschweig

- Lamber, A.; LARCKer, D.F.; Weigelt, K. (1993): "The Structure of Organizational Incentives", in: Administrative Science Quarterly, Vol. 38, S. 438-461

- LAMM, R.M. (1981): "Prices and Concentration in the Food Retailing Industry", in: Journal of Industrial Economics, Vol. 30, S. 67-78

- LANCASter, K. (1966): "A New Approach to Consumer Theory", in: Journal of Political Economy, Vol. 74 , S. $132-157$

- $\quad$ LANCASTER, K. (1971): Consumer Demand: A New Approach, New York

- $\quad$ Landes, W. M.; Posner, R. A. (1981): "Market Power in Antitrust Cases”, in: Harvard Law Review, Vol. 94, No. 5, S. 937-983 
- $\quad$ LLM SCHWÄBISCH GMÜND (LANDESSTELle FÜR LANDWiRTSCHAFTLICHE MARKTKUNDE) (LLM) (2001 und 2002a): Agrarmärkte - Milch, internet: $\underline{\text { www.landwirtschaft-mlr.baden- }}$ wuerttemberg.de/la/lel/llm/meb/kap2.htm

- $\quad$ LLM SCHWÄBISCH GMÜND (LANDESSTELLE FÜR LANDWIRTSCHAFTLICHE MARKTKUNDE) (2002b): Loseblattsammlung 2.1.8: Marktordnung für Zucker: www.landwirtschaft-mlr.baden-wuertemberg.de

- $\quad$ LAND- UND ForstWiRTSChAFtLiChe Zeitung: Mitteilungsblatt der Landwirtschaftskammer Hannover, Landbuch Verlag, Hannover

- $\quad$ LANGEN, E.; BUNTE, H.-J. (2001): Kommentar zum deutschen und europäischen Kartellrecht, Verlag Luchterhand, Neuwied u.a.

- $\quad$ LebensmitTelzeitung, diverse Artikel, Deutscher Fach-Verlag, Frankfurt am Main

- Lev, B. Mandelker, G. (1972): „The Microeconomic Consequences of Corporate Mergers", in: Journal of Business, Vol. 45, S. 85-104

LeVIN, D. (1990): "Horizontal Mergers: The 50 Percent benchmark", in: American Economic Review 80 , p. $1238-1245$

- LeWellen, W. G. (1971): The ownership income of management, Columbia University Press, New York

- LEXECON (2001): Competition Memo vom 29.06.2001: Marktdefinition: Wie Stationaritätstests die Genauigkeit verbessern können, internet: www.lexecon.co.uk/publications/media/media_GR/stationarity-gr.pdf

- $\quad$ LIVERMORE, S. (1935): "The Success of Industrial Mergers", in: Quarterly Journal of Economics, Vol. 50, S. $68-96$

- LONDON BUSINESS SCHOOL; ZeHNDER, E. (1987): International: Acquisitions - the human factor, London

- LoRIE, J.H., HALPERn P. (1979): "Conglomerates: The Rhetoric and the Evidence", in: Journal of Business Research, Vol. 15, S. 303-312

- LORIE, J. H.; HALPERN, P. (1970): "Conglomerates: The Rhetoric and the Evidence", in: The Journal of Law and Economics, Vol. 13, S. 149-166

- $\quad$ LUSTgarTen, S.H. (1975): "The Impact of Buyer Concentration in Manufacturing Industries", in: Review of Economics and Statistics, Vol. 47, S. 125-132

- $\quad$ LZ-NEWS: Wöchentlicher Newsletter (e-mail) der Lebensmittelzeitung, Deutscher Fachverlag, Frankfurt am Main

- MADAKOM-Datensatz: Lebensmitteleinzelhandeldscanneratensatz der Firma MADAKOM GmbH, Köln

- M+M-EURODATA: internet: www.mm-eurodata.de, diverse Pressemeldungen

- MAHLeR, P. (1994): "Efficiency losses as a result of insufficient structural adjustments due to the EC sugar regime: The case of Germany", in: European Review of Agricultural Economics, Vol. 21, pp. 199-218

- MAHLER, P. (1992): "Effizienzverluste in der deutschen Zuckerwirtschaft durch strukturkonservierende Wirkungen der EG-Zuckermarktordnung", in. Agrarwirtschaft 41, Heft 4/5, S. 117-131

- Malatesta, P.H. (1983): "The Wealth Effect of Merger Activity and Objective Function of Merging Firms", in: Journal of Financial Economics, Vol. 11, S. 155-181

- MANN, H.M. (1966): "Seller Concentration, Barriers to Entry and Rates of Returns in Thirty Industries 1950-1960", in: Review of Economics and Statistics 48, p. 296-307

- MAnne, H.G. (1965): "Mergers and the Market for Corporate Control", in. Journal of Political Economy, April, S. 110-120

- MÄNNEL, W. (1995): „Ausrichtung der Kostenrechnung, Kostencontrolling und Kostenmanagement auf marktorientierte segmentierte Unternehmen", in: Bühner, R. et al.: Die Dimensionierung des Unternehmens, Schäffer-Poeschel, Stuttgart, S. 405-424

- MARION, B.W. (1989): "The Concentration-Price Relationship in Food Retailing", in: Weiss, L.W., (Hrsg.), Concentration and Price, Cambridge, England, S. 185-193

- Marion, B.W., Mueller, W.F, Cotterill, R.W., Geithman, F.E. und Schmelzer, J.R. (1979): The Food Retailing Industry: Market Structure, Profits and Prices, Praeger, New York 
- Marn, M. V.; Rosiello, R. L. (1993): „Balanceakt auf der Preistreppe”, in: Harvard Business Manager, Nr. 2/1993, S. 46-56

- MARRIS, R. (1963): "A model of the Managerial Enterprise", in: Quarterly Journal of economics 77, S. $185-209$

- MARRIS, R. (1964): The Economic Theory of Managerial Capitalism, London

- MARSChALL, B. (2002): „Deutsches Vorbild für Europa”, in: Financial Times Deutschland, 24. Juli 2002, S. 26

- MARTIN, S. (1982): "Industrial Demand Characteristics and the Structure-Perfomance Relationship", in: Journal of Economcs and Business, vol. 34, S. 59-65

- MARTIN, S. (1983): "Vertical Relationships and Industrial Performance", in: Quarterly Review of Economics and Business, Vol. 34, S. 6-18

- Martin, S. (1994): Industrial Economics, Economic Analysis and Public policy, Second Edition, New York

- MARTIN, S. (2002): Industrial Organization: A European Perspective, Oxford University Press, New York

- Marvel, H. P. (1978): "Competition and Price Levels in the Retail Gasoline Market", in: Review of Economics and Statistics, Vol. 6, No. 2, S. 445-450

- MATSCHUK, H.J.; VIETH, R. (1989): „Die Konzentration im Lebensmittelhandel: Entwicklungen, Ursachen und Folgen", in: Forschungsstelle für den Handel (Hrsg.), Forschungsbericht 1989, Berlin, S. $135-151$

- Maucher H. (1993): Corporate Size: A View from the Top. - esprit d enterprise et technologies nouvelles. Hommage à Pierre Goetschin. Universität Lausanne, S. 53-62

- McAffe, P. R., Williams, M. A. (1992): "Horizontal Merger and Antitrust Policy", in: The Journal of Industrial Economics 2, S. 181-187

- McAffe, P. R.; Williams, M. A. (1988): "Can Event Studies Detect Anticompetitive Mergers?", Economics Letters, Vol. 28, No. 2, S. 199-203

- McElRoY, W. F. (1991): "Price and Welfare Effects of Oligopolistic Mergers", in: Bulletin of Economic Research, Vol. 43, S. 331-354

- Meeks, G. (1977): Disappointing Marriage: A Study of the Gains from Mergers, Cambridge u.a.

- Meffert, H. (2000): Marketing - Grundlagen marktorientierter Unternehmensführung, 9. Aufl., Gabler, Wiesbaden

- Mergers-and-Acquisitions: www.mergers-and-acquisitions.de: Informationsplattform C.M. Picot finance GbR, Köln

- Messinger, P.R.; NARASimhan, C. (1995): "Has power shifted in the grocery channel?", in: Marketing Science, Vol. 14, S. 189-223

- MöLleR, W. P. (1983): Der Erfolg von Unternehmenszusammenschlüssen. Eine empirische Untersuchung, München.

- MonOPOLKOMmission (1984): Hauptgutachten V, Ökonomische Kriterien für die Rechtsanwendung, Nomos-Verlag, Baden-Baden

- MonOPOLKOMmission (1992): Wettbewerbspolitik oder Industriepolitik, Hauptgutachten 1990/1991, Nomos-Verlag, Baden-Baden

- MONOPOLKOMMISSION (1994): Sondergutachten 23: Marktstruktur und Wettbewerb im Handel, Nomos-Verlag, Baden-Baden

- MONOPOLKOMMISSION (1998): 12. Hauptgutachten 1996/97: Marktöffnung umfassend verwirklichen, Nomos-Verlag, Baden-Baden

- Monopolkommission (2000): Wettbewerbspolitik in Netzwerkstrukturen, 13. Hauptgutachten, Nomos-verlag, Baden-Baden

- MORCK, R.; YeUnG, B. (1997) : Why Investors value Multinationality, Discussion Paper 282. University of Michigan, Ann Arbor, Michigan

- MösCHEL, W. (1983): Recht der Wettbewerbsbeschränkungen, Verlag Carl Heymann, Köln, Bonn u.a. 
- MotTA, M. (1996): Multinationals Without Advantages. Working Paper 464. Industrial Institute for Economic and Social Research, Stockholm

- MotTA, M. (2000): Economic Analysis and Merger Policy, European University Institute, EUIWorking Paper RSC No. 2000/33, Florence

- Mueller, D.C. (1983): Mergers and Market Share, Internationales Institut für Management und Verwaltung, Wissenschaftszentrum Berlin, Discussion Paper No. 33, Berlin

- $\quad$ Mueller, D.C. (1986): Profits in the Long Run, Cambridge u.a.

- Mueller, D. C. (1969): „A Theory of Conglomerate Merger" ,in: Quarterly Journal of Economics, Vol. 83 , S. 643-659

- Mueller, D. C. (1979): Hypothesen über Unternehmenszusammenschlüsse. Discussion Paper Series, International Institute of Management, Wissenschaftszentrum Berlin

- MuelleR, D. C. (1995): The United State's Antitrust Policy: Lessons for Europe, Ludwig-BoltzmannInstitut zur Analyse Wirtschaftspolitischer Aktivitäten, Wien

- Müller, J.; Hochreiter, R. (1975): Stand und Entwicklungstendenzen der Konzentration in der Bundesrepublik Deutschland, Verlag Schwartz, Göttingen

- $\quad$ MÜLLER-URI, R. (1989): Kartellrecht, Verlag Heymann, Köln

- NARVER, J.C. (1967): Conglomerate Mergers and Market Competition, Berkeley, Los Angeles

- National Industrial Conference Board (NICB) (1929): Mergers in Industry. A Study of Certain Economic Aspects of Industrial Consolidation, New York

- Needham, D. (1978): "The problem of market and industry delineation", in: Needham, D.: The Economics of Industrial Structure, Conduct and Performance, New York

- Nelson, R.L. (1959): Merger Movements in American Industry 1895-1956, Princeton, New York

- $\quad$ Netherlands Economic Institute (NEI): van der Linde, Martin; Minne, Veerle; Wooning, André; van der Zee, Frans (2000): Evaluation of the Common Organisation of Markets in the Sugar Sector, NEI, Rotterdam

- NeumAnN, G. (im Druck): Wettbewerbsmonitor der deutschen Ernährungsindustrie, Universität Kiel

- Neumann, M. (2000): Wettbewerbspolitik - Geschichte, Theorie und Praxis, Gabler-Verlag, Wiesbaden

- Neven, D.; Nuttal, R.; Seabright, P. (1993): Merger in Daylight- The Economics and Politics of European Merger Control, Centre of Economic Policy Research, London

- Niederhut-BollmanN, C.; SChrader, H.-J. (2001): „Fallbeispiel Nordzucker AG", Seminararbeit: Unternehmenszusammenschlüsse und Unternehmenskäufe SS 01, Universität Göttingen

- NOEL, P. E. (1997): "Efficiency considerations in the assessment of horizontal mergers under European and US antitrust law", in: European Competition Law Review 18, S. 507

- $\quad$ NORDMILCH (1998a): Die Milchwirtschaft des Nordens gestaltet die Zukunft, Zeller Druck, Zeven

- $\quad$ NORDMILCH (1998b): Der Norden - die Milch - die neue Nordmilch, Zeller Druck, Zeven

- $\quad$ NORDMILCH (1999): Informationen für Milcherzeuger, Jahrgang 1999, Ausgabe 10, Bremen

- NORDMILCH (laufende Jahrgänge): Geschäftsbericht

- $\quad$ NORDZUCKER (verschiedene Jahrgänge): Geschäftsbericht/ Umweltbericht

- NORDZUCKER, Pressemitteilungen: www.nordzucker.de/presse

- NUSSKERN, W. (1999): Möglichkeiten und Grenzen der Anwendung von statistischen Konzentrationsmaßen in der Fusionskontrollverordnung, Lang-Verlag, Frankfurt am Main u.a.

- $\quad$ o.V.: "Das Geschäft mit Fusionen lahmt", in: Welt (online-Ausgabe vom 25.06.2002: www.welt.de)

- $\quad$ o.V. (1992): „Nur wer den Markt hat, hat auch die Zukunft”, in: Welt der Milch Nr. 13, S. 337-339

- OECD (1981): Buying Power: The exercise of market power by dominant buyers, Report of the Committee of Experts on Restrictive Business Practices, OECD, Paris

- OECD (1996): Antitrust and Market Access - The Scope and Coverage of Competition Laws and Implications for Trade, OECD Publications, Paris 
- $\quad$ OECD (1999): Trade and Competition - Policies for Tomorrow, OECD Publications, Paris

- OECD (1999): Trade and Competition Policies - Exploring the Ways Forward, OECD Publications, Paris

- $\quad$ OECD (1999): A framework for the design and implementation of competition law and policy, World Bank, Washington

- Olbermann, S. (1993): Renditeunterschiede, Marktstruktur und dynamischer Wettbewerb, EulVerlag, Bergisch Gladbach

- OlBRICH, R. (1998): Unternehmenswachstum, Verdrängung und Konzentration im Konsumgüterhandel, Schäffer-Poeschel, Stuttgart

- OLSEN, M. E. (1970): Power as societal process, New York

- Ordover, J. A. (1990): „Economic Foundations of Competition Policy”, in: Comanor, W. S. et al., Competition Policy in Europe and North America: Economic Issues and Institutions, Chur, London, S. 7-42

- Osmers, K. (2001): "Guter Marktverlauf am Milchmarkt 2000 und 2001”, in: Welt der Milch, S. 633637

- O.V. (1992c): „Nur wer den Markt hat, hat auch die Zukunft”, in: Welt der Milch, Nr. 13, S. 337339

- Panzar, J. C.; Willig, R. D. (1981): "Economies of Scope", in: American Economic Review, Vol. 71, No. 2 , S. $268-272$

- PAPROTTKA, S. (1996): Synergiepotentiale und ihre Umsetzungsmöglichkeiten durch Integration, Gabler, Wiesbaden

- Penrose, E. T. (1959): The Theory of the Growth of the Firm, Wiley, New York

- PerlitZ, M.; LÖBleR, H. (1989): „Das Innovationsverhalten in der mittelständischen Industrie", in: Schriften zur Mittelstandsforschung, Nr. 27, Stuttgart

- PerRy, M. (1984): "Sustainable Positive Profit Multiple-Price Strategies in Contestable Markets”, in: Journal of Economic Theory 32, S. 246-265

- Perry, M. K.; PORTeR, R.H. (1985): "Oligopoply and the incentive for horizontal merger", in: American Economic Review 75 (1), S. 219-227

- Petersen, TH. (1990): "Das Deligationsproblem zwischen Prinzipalen und Agenten", in: Albach, H.: Organisation, Wiesbaden, S. 109-132

- Picot, A. (1982): "Transaktionskostenansatz in der Organisationstheorie", in: Die Betriebswirtschaft, S. $267 \mathrm{ff}$.

- $\quad$ PIEPER, U. (1998): Wirkungen auf Unternehmensakquisitionen auf Forschung und Entwicklung: Eine empirische Untersuchung aus der Perspektive des erwerbenden Unternehmens, Wiesbaden

- Polasky, A. S.; MASON, C. F. (1997): "On the Welfare Effects of Mergers: Short Run versus Long Run", in: Quartely Review of Economics and Finance (38), No.1, S. 1-24

- PORTER, M. E. (1987): „Diversifikation - Konzerne ohne Konzept”, in: Harvard Manager 4, S. 30-49

- PORTER, M. E. (1989): Wettbewerbsvorteile: Spitzenleistungen erreichen und behaupten, Sonderausgabe, Campus, Frankfurt/ Main

- PORTER, M. E. (1990): Wettbewerbsstrategie: Methode zur Analyse von Branchen und Konkurrenten, 6. Aufl. , Campus, Frankfurt/ Main

- Pusnik, K. (2001) „Market Power versus Efficiency - The Theoretical concepts of Welfare Effects of Mergers" in: Nase Gospordatsvo, Maribor

- PÜTZ, P. ; MEYERHÖFER, W. (1982): „Hemmnisse und Hilfen für Unternehmensgründungen”, in: Untersuchungen zur Wirtschaftspolitik, Band 52, Institut für Wirtschaftspolitik an der Universität Köln

- PÜTZ, P.; WiLLGERODT, H. (1985): Gleiches Recht für Beteiligungskapital, Baden-Baden

- Rabobank (2000): Die Milchmärkte Europas 1999 - Eine Analyse der Rabobank International, Frankfurt am Main

- Rabobank (2000): Pressemitteilung: „Neuordnung der Molkeriebranche 1999- Jeden zweiten Tag eine Fusion oder Übernahme", Frankfurt am Main

- RABOBANK (1995): The world of sugar and sweeteners, Rabobank International, Frankfurt am Main 
- Ravenscraft, D. J.; SCherer F.M. (1989): „The Profitability of Merger”, in: International Journal of Industrial Organization 7, S. 101-116

Ravenscraft, D. J.; Scherer, F. M. (1987): Mergers, Sells-Offs \& Economic Efficiency, Washington D.C.

- ReHKUgleR, H. (1989): "Die Unternehmensgröße als Klassifikationsmerkmal in der Betriebswirtschaftslehre", in: Kirsch, W.; Picot, A.: Die Betriebswirtschaftslehre im Spannungsfeld zwischen Generalisierung und Spezialisierung, Wiesbaden

- $\quad$ ReID, S. R. (1968): Mergers, Managers and the Economy, McGraw-Hill, New York

- RENDER, H. (1989): Ein Strukturkonzept zur Verbesserung der Wettbewerbsstellung der norddeutschen Zuckerwirtschaft, Verlag Peter Lang, Frankfurt am Main

- RITTNER, F. (1989): Wettbewerbs- und Kartellrecht: eine systematische Darstellung für Studium und Praxis, Müller - Juristischer Verlag, Heidelberg

RoberTS, G. L.; SALOPP, S. C. (1994): Efficiency Benefits in Dynamic Merger Analysis (mineo)

- $\quad$ RoBinson, J. (1961): The Economies of Imperfect Competition, London

- RODRIGUES, V. (2001): „Endogenous mergers and market structure”, in: International Journal of Industrial Organization 19, Elsevier, Amsterdam, S. 1245-1261

- RoLl, R. (1988): "Empirical Evidence on Takeover Activity and Shareholder Wealth", in: Coffee, J.C.; Rose-Ackerman, S.; Lowenstein, L.: Knights, Raiders and Targets, Oxford University Press, Oxford, S. 241-252

- RolL, R. (1986): "The Hubris Hypothesis of Corporate Takeovers”, in: Journal of Business, Vol. 59, No. 2, S.197-216

- $\quad$ Röller, L.-H.; StenneK, J.; Verboven, F. (2000): Efficiency Gains from Mergers, The Research Insitute of Industrial Economics, Working Paper No. 543, Stockholm

- $\quad$ RomeISER, T. (1998): Marktzutrittsschranken im Handel, GHS-Reihe Band 54, Göttingen

- Rumelt, R. (1982): "Diversification strategy and profitability", in: Strategic Management Journal, No. 3, S. 359-369

- RWI (RHEINISCH WeSTFÄLISCHES INSTITUT FÜR WIRTSCHAFTSFORSCHUNG) (1998): CO2-Emissionen und wirtschaftliche Entwicklung: Monitoring Bericht 1998, Essen

- RYDEN, B. (1972): Mergers in Swedish Industry. An Empirical Analysis of Conglomerate Mergers, Stockholm

- Salant, S. W., Switzer, S.; Reynolds, R. J. (1983): "Losses from Horizontal Merger: The Effects of an Exogenous Change in Industry Structure on Cournot-Nash-Equilibrium", in: The Quarterly Journal of Economics Vol. 98, No. 2, S. 185-199

- Schengber, R. A. (1996): Marktabgrenzung und Machtmessung. Eine Analyse von Methoden und Indikatoren für das Koordinationsmängel-Diagnosekonzept, Verlag Josef Eul, Köln u.a.

- SCHENK, H. (1996): „Bandwagon Mergers, International Competiveness and Government Policy”, in: Empirica Vol 23, No. 3, S. 255-278

- Scherer, F.M.; Ross, D. (1990): Industrial Market Structure and Economic Performance, 3. Aufl., Boston

- SCherer, F. M. (1975): The economics of multi-plant operation: an international comparison study, Harvard University Press, Cambridge/Mass.

- SCHERER, F. M. (1988): "Corporate Takeovers: The Efficiency Arguments", in: Journal of Economic Perspectives, Vol. 2, No. 1, S. 69-82

- SChierenBeCK, H. (1989): Grundzüge der Betriebswirtschaftslehre, 9. Aufl., Oldenbourg, München

SchmalenseE, R. (1985): „Do Markets Differ Much?”, in: American Economic Review 75, p. 341-351

- SChmalensee, R. (1987): "Collusion vs. Differential Efficiency: testing Alternative Hypotheses", in: Journal of Industrial Economics 35, S. 399-425

- SChmalensee, R.; Willig, R.D. (1989): Handbook of Industrial Organization, Amsterdam

- Schmidt, A. G. (1995): Der Einfluss der Unternehmensgröße auf die Rentabilität von Industrieunternehmen, Gabler Wiesbaden 
- SCHMIDT, C.; HöPER, U. (1997): „Efficient Consumer Response als neues Konzept für den Vertriebsbereich in der Ernährungsindustrie - Darstellung von Funktionsweise, Bedeutung und Konsequenzen des ECR an Fallbeispielen der Molkereiwirtschaft", in: Berichte über Landwirtschaft Nr. 3, S. 274-401

- SCHMIDT, E. (1991): "Analyse der Beschaffungspolitik von Zuckerfabriken in der BRD”, in: Agrarwirtschaft 40, Heft 11, S. 340-349

- SCHMIDT, I. (1996): Wettbewerbspolitik und Kartellrecht - Eine Einführung, 5. Auflage, Verlag Lucius $\&$ Lucius, Stuttgart

- Schmidt, I. (1999): Wettbewerbspolitik und Kartellrecht: eine Einführung- 6. Auflage, Verlag Lucius \& Lucius, München

- SCHMIDT, I.; SCHMIDT A. (1997): Europäische Wettbewerbspolitik - Eine Einführung, Verlag Vahlen, München

- SchmiTZ, R. (1989): „Die optimale Eigentümerstruktur”, in: Albach, H. : Organisation. Mikroökonomische Theorie und ihre Anwendung, Wiesbaden, S. 133-153

- Schmusch, M. (2002): „Die Goodwill Blase”, in: Frankfurter Allgemeine Zeitung, 4. Juni 2002, Beilage Nr. 126, S. 1

- SCHNEIDER, D. (1993): Investion, Finanzierung und Besteuerung, 7. Auflage, Wiesbaden

- $\quad$ SCHULT, E. (1999): Bilanzanalyse - Möglichkeiten und Grenzen externer Unternehmensbeurteilung, 10. Auflage, Erich-Schmidt-Verlag, Berlin

- SCHULZE, M. (1998): Nachfragemacht im Lebensmitteleinzelhandel- Ökonomische und kartellrechtliche Aspekte, Gabler, Wiesbaden

- SchüTz, J. (2000): Die EG-Fusionskontrolle aus Sicht der Industrie, Schriften des Vereins für Sozialpolitik, Gesellschaft für Wirtschafts- und Sozialwissenschaften, band 270, Duncker \& Humblot, Berlin

- SChwalbaCH, J. (1991): "Profitability and Market Share: a Reflection on the Functional Relationship", in: Strategic Management Journal 12, S. 299-306

- SCHWARZ, D. (1987): "Wettbewerbspolitische Problematik des Predatory Pricing", in: Wirtschaft und Wettbewerb 1987, S. $93-99$

- $\quad$ SeTH, R. (1990): Explaining LBOs and acquisitions, Ferderal Reserve Bank of New York, New York

- ShaviLl, S. (1979): "Risk Sharing and Incentives in the Principal and Agent Relationship", in: The Bell Journal of Economics, S. $55 \mathrm{ff}$.

- Shepherd, W.G. (1975): The Treatment of market Power: Antitrust, Regulation and Public Enterprise, New York, London

- ShePherd, W.G. (1986): "Tobin's Q and the Structure-Performance-Relationship: Comment", in: American Economic Review 76, S. 1205-1210

- Shrieves, R. E. (1978): "Geographic Markets Areas and Market Structure in the Bituminous Coal Industry", in: Antitrust Bulletin, S. 589-625

- Singh, A. (1971): Takeovers: The Relevance to the Stock Market and the Theory of the Firm, Cambridge

- SMith, K.W.; HeRshman, S.E. (1997): Making Mergers Work for Profitable Growth: the Importance of Pre-Deal Planning Post-Deal Management, www.mercermc.com/news/mercerandhoredart.html

- $\quad$ SOMmer, U. (2002): „Der Markt für Zucker”, in: Agrarwirtschaft 51, S. 35-41

- SOMmer, U. (1998): „Der Markt für Zucker”, in: Agrarwirtschaft 47, S. 38

- Statistisches Bundesamt (2000): Produzierendes Gewerbe, Reihe 4/4/3/2, Metzler-Poeschel, Wiesbaden

- STEgemAnN, K. (1974): "Cross Elasticity and the Relevant Market", in: Zeitschrift für Wirtschaft- und Sozialwissenschaften, S. 151-165

STEHLE, R. (1995): „Der Einfluss der Unternehmensgrösse auf die Kapitalstruktur börsennotierter Aktiengesellschaften", in: Bühner, R. et al. Die Dimensionierung des Unternehmens, SchäfferPoeschel, Stuttgart, S. 405-424 
- SteinmanN, H.; SChreyögG, G. (1991): Management - Grundlagen der Unternehmensführung, Gabler. 2. Auflage, Wiesbaden

- StenneK, J. (2002): Lectures Notes on Antitrust Economics, Research Institute for Industrial Economocs, Stockholm, internet. www.iui.se

- STEWART, J.F.; KIM, S.K. (1993): "Mergers and Social Welfare in US manifacturing 1985-1986", in: Southern Economic Journal, Vol. 59, No. 4, S. 701-720

- STICH, R.S. (1974): "Have U.S. Mergers been Profitable?", in: Management International Review, Vol. 14 , No. $2-3$, S. 33-45

- $\quad$ Stigler, G.J. (1947): The Theory of Price, New York

- Stigler, G.J.; Sherwin, R.A. (1985): "The Extent of the Market", in: Journal of Law and Economics, S. $555-585$

- Stigler, G. J. (1950): "Monopoly and Oligopoly by Merger", in: American Economic Review, Proceedings, Vol. 40, S. 23-34

- Stillman, R. (1983): "Examining Antitrust Policy towards Horizontal Mergers”, in: Journal of Financial Economics 11, S. 225-240

- $\quad$ Stockum, S. (1990): The Market Power-Efficiency Tradeoff, Residiual Demand Elasticity Estimation and USAir/Piedmont Merger, Dissertation university of Pennsylvanier

- Stockum, S. (1993): „The Effencies Defense For Horizontal Mergers: What is Government's Standard", in: Antitrust Law Journal, Vol. 61, S. 829-855

- SÜDZUCKER Geschäftsbericht (laufende Jahrgänge); Mannheim/ Ochsenfurt

- Thomson FinANCIAL (2002): Internationaler Finanzdienstleister: www.thomsonfinancial.com (newsletter)

- TieTZ, B. (1995): „Systemdynamik und Konzentration im Handel”, in: Bühner, R. et al: Die Dimensionierung des Unternehmens, Schäffer-Poeschel, Stuttgart, S. 405-424

- TIROLE, J. (1995): Industrieökonomik, München

- $\quad$ TOP AGRAR: Das Magazin für moderne Landwirtschaft, Landwirtschaftsverlag, Münster

- Trautwein, F. (1990): „Merger Motives and Merger Prescriptions”, in: Strategic Manager Journal 11, S. $293-295$

- Trumm, S. (2001): Das Prinzip des Weltfreihandels und der europäische Agraraußenhandel am Beispiel der Gemeinsamen Marktordnungen für Getreide, Zucker und Bananen, Duncker \& Humblot, Berlin

- UhLig, T. (1996): Zusagen, Auflagen und Bedingungen im Fusionskontrollverfahren: Eine Untersuchung zum deutschen und europäischen Recht, Pro Universitate Verlag, Sinzheim

- $\quad$ ULMER, P. (1987): "Brauchen wir eine Kartellgesetznovelle", in: Markenartikel, Heft 5, S. 326-334

- United nations Conference on Trade And Development (UNCTAD): www.unctad.org

- UtTON, M. A. (1974): "On Measuring the Effects of Industrial Mergers", in: Scottish Journal of Political Economics, Vol. 21, p. 13-28

- $\quad$ UtTON, M. A. (1995): Market Dominance and Antitrust Policy, Elgar, Aldershot

- VAN MieRT (1995): Eurpäische Wettbewerbspolitik, Brüssel

- VARIAN, H. R. (1991): Grundzüge der Mikroökonomik, 2. Aufl. Oldenbourg-Verlag, München

- VARIAN, H. R. (1995): Grundzüge der Mikroökonomik, 3. Aufl. Oldenbourg-Verlag, München

Verboven, F. (1996): "Corporate Restructing in a Collusive Oligopoly", in: International Journal of Industrial Organization, Vol. 13, No. 3, S. 240-268

- $\quad$ VIK (Verband der Industriellen Energie- und Kraftwirtschaft e.V.): Jahrbuch 2000/2001, Essen

- WALKENHORST, P. (1997): Agro-industrial restructuring in East-Central Europe: The case of sugarbeet processing in Poland, paper, S. $19 \mathrm{ff}$.

Waterson, M. (1980): "Price-Cost Margins and Successive Market Power", in: Quarterly Journal of Economics, Vol. 94, S. 135-150 
- WATERSON, M. (1983): „Economies of Scope Within Market Frameworks", in: International Journal of Industrial Organization, Vol. 1, S. 223-237

- Weichenrieder, A. J. (1998): „Foreign Profits and Domestic Investment”, in: Journal of Public Economics Vol. 69, No. 3, S. 451-463

- Weindlaier, H. U.A. (1999): "Milcherfassung in Deutschland und Österreich im Jahr 1998 - Rahmenbedingungen, Durchführung und Kosten", in Deutsche Milchwirtschaft, Teil 1: Nr. 19, S. 818821 - Teil 2: Nr. 20, S. 879-883

Weindlmaier, H.; BetZ, J. (1997): „Erhebliches Einsparungspotential - Kostenstruktur und Kosteneinsparungsmöglichkeiten bei der Milcherfassung", in: Österreichische Milchwirtschaft Nr. 12, S. $19-25$

- Weindlmaier, H. (1998): „Molkereisstruktur in Deutschland: Entwicklungstendenzen und Anpassungserfordernisse", in: Agrarwirtschaft Nr. 47, Heft 6, S. 242-250

- Weindlmaier, H. (2001): „Rahmenbedingungen der Ernährungsindustrie”, Vortragsunterlagen zum Agrarökonomischen Seminar - Universität Göttingen (WS 2001)

- WeindlMAier, H.; FLORIOT, J.-L. (2001): "Strategien der deutschen und französischen Ernährungsindustrie und deren Auswirkungen auf die Wettbewerbsposition", in: Agrarwirtschaft, Vol 5, Heft 3

- WelleRT, K. (1994): Zur Wettbewerbsfähigkeit von Molkerei- und Schlachtunternehmen im vereinten Deutschland - Analysiert anhand ausgewählter Regionen, Dissertation Universität Göttingen

- Welt: Tageszeitung, Online-Ausgabe: www.welt.de, Axel-Springer-Verlag, Berlin

- Werden, G. (1997): "An Economic Perspective on the Analysis of Merger Efficiencies", in: Antitrust, Summer, S. $12-16$

- Werden, G.; Froeb, L. M. (1994): "The Effects of Mergers in Differentiated Products Industries: Logit Demand and Merger Policy", in: Journal of law, Economics and Organization, Vol. 10, No. 2, S. $407-426$

- Werden, G.; Froeb, L. M. (1998): "The Entry Inducing Effects of Horizontal Mergers: An Exploraty Analysis", in: Journal of Industrial Economics, Vol. 46, No. 4, S. 525-543

- Weston, J. F.; ChUnG, S. (1983): "Some aspects of merger Theory", in: Journal of Midwest Finance Association 12, S. 1-33

- Weston, J. F.; MAnsinghKA, S.K. (1971): "Test of Effiency Performance of Conglomerate Firms", in: The Journal of Finance, Vol. 26, S. 919-936

- Wickelgren, A. L. (2001): Moral Hazard, Mergers and Market Power, Working Paper No. 239, Bureau of Economics-Federal Trade Commission, Washington

- Wied-Nebbeling, S. (1997): Markt- und Preistheorie, 3. Aufl., Springer-Verlag, Berlin u.a.O.

- $\quad$ Wied-Nebbeling, S. (1993): Markt- und Preistheorie, Heidelberg

- WiLD, S. (1999): „Logistik als Wettbewerbsfaktor - Gemini-Studie: Im Trend liegen Konzepte zur Zentrallagerbelieferung", in: Lebensmittelzeitung 8/1999, S. 879

- Wille, M. (2001): „Die Entwicklung der deutschen Milchwirtschaft im Hinblick auf die Neuausrichtung der Agrarpolitik", in: Welt der Mich, S. 597-599

- Williamson, O.E. (1975): Markets and Hierachies: Analysis and Antitrust Implications, New York

- Williamson, O. E. (1964): The Economics of Discretionary Behaviour: Managerial Objectives in a Theory of the Firm, Prentice Hall, Englewood Cliffs, New York

- Williamson, O. E. (1968): "Economies as an Antitrust Defense: The Welfare Trade-Offs", in: American Economic Review 58, S. 18-36

- Williamson, O. E. (1969): „Economies as an Antitrust Defense: Reply”, in American Economic Review Vol. 59, Issue 5, S. 954-959

- Williamson, O. E. (1977): "Economies as an Antitrust Defense Revisted", in: Jaquemin, A.P.; de Jong, H.W.: Welfare Aspects of Industrial Markets, Leiden, S. 237-271

- Williamson, O. E. (1979): "Transaction-cost economic: The governance of contractual relations", in: The Journal of law and Economics, No. 12,S. 233-261

- Williamson, O. E. (1989): "Transaction Cost Economies", in Schmalensee, R.; Willig, R. D.: Handbook of Industrial Organization, Amsterdam, Vol. 1, S. 135-179 
- WiLlig, R. D. (1991): „Merger Analysis, Industrial Organization Theory and Merger Guidelines”, in: Brookings Papers on Economic Activity, Microeconomics, S. 281-332

- WinkLeR, W. (1981) in IMmEngA; MeStMÄCKER (1981): Gesetz gegen Wettbewerbsbeschränkungen Kommentar, Beck-Verlag, München

- WinkLer, W. (1997) in IMmengA; MestMÄCKER (1997): EG-Wettbewerbsrecht, Beck-Verlag, München

- WirTSChAFT UND WetTBEWERB (WUW): Zeitschrift für Deutsches und Europäisches Wettbewerbsrecht, Verlagsgruppe Handelsblatt, Düsseldorf

- Wirtschaftliche Vereinigung ZuCKer (WVZ) (2001): Jahresbericht 2000/01, Bonn

- WiRTSCHAFTSWOCHE vom 01.02.2001, S. 47-53: „Ende des Wahns”; Verlag Economy One, Düsseldorf

- WissensChAftLicher Beirat Beim BMELF (1983): Milchmarktpolitik - Kontingentierung oder marktorientierte Preispolitik? Schriftenreihe A: Angewandte Wissenschaft, H. 286, Münster-Hiltrup

- WissenschaftLiCHER BEIRAT BEIM BUNDESMINISTERIUM FÜR ERNÄHRUNG, LANDWIRTSCHAFT UND FORSTEN (2000): Zur Wettbewerbsfähigkeit der deutschen Milchwirtschaft, Bonn

- WiSSENSCHAFTLICHER BEIRAT: Schriftenreihe des Bundesministeriums für Ernährung, Landwirtschaft und Forsten (1993): Vorschläge für eine grundlegende Reform der EG-Zuckermarktpolitik, Reihe A: Angewandte Wissenschaft, Heft 430, Landwirtschaftsverlag, Münster

- WÖHE, G. (1996): Einführung in die allgemeine Betriebswirtschaftslehre, 19. Aufl., Vahlen, München

- WORLD BANK; OECD (1999): A Framework for the Design and Implementation of Competition Law and Policy, project director: R. Shyam Khemani, Paris, Washington

- Wossidlo, P.R. (1990): "Finanzierung", in: Pfohl, H.C.: Betriebswirtschaftslehre der Mittel- und Kleinbetriebe, Berlin, S. 239-287

- $\quad$ WRONG, D.H. (1979): Power, Its Forms, Bases and Uses, Oxford

- You, V.; CAVES, R.; SMITH, M.; HenRY, J. (1986): "Mergers and Bidders Wealth: Managerial and Strategic Factors", in: Thomas, Lacy Glenn: The Economics of Strategic Planning, Lexington Books, Lexington/ Mass

- Zentes, J. HURTH, J. (1996): Status und Folgen der Handelskonzentration, Gutachten im Auftrag des Markenverbandes Wiesbaden, (Zentes-Studie)

- Zentrale MarkT- Und PreisberichtSstelle (ZMP) (laufende Jahrgängeg): ZMP-Marktbilanz Milch, ZMP, Bonn

- ZiegleR, M. (1997): Synergieeffekte bei Unternehmenskäufen - Identifikation im Beschaffungs- und Produktionsbereich von Industriebetrieben, Dissertation, Wiesbaden

- ZimmermanN, B.; WieSeR, H.; Zeddies, J. (2000): Internationale Wettbewerbsfähigkeit der Zuckererzeugung - Komparative Kostenunterschiede und Wettbewerbsverzerrungen, Schriften der GeWiSoLa, Band 36, S. 109-116

- ZUCKERINDUSTRIE: Zeitschrift für die Zuckerindustrie, Verlag Bartens, Bonn

- $\quad$ ZUCKERINDUSTRIE (1998): „Die Größten in der EU-Rübenzuckerindustrie” , Nr. 12, S. 998

- ZUCKERINDUSTRIE (1998): "Norddeutsche Gesamtlösung am Zuckermarkt bleibt das Ziel", Nr. 11, S. 914

- $\quad$ ZUCKERINDUSTRIE (1997): „Union Zucker verlässt Nordzucker AG”: Nr. 12, S. 980

- $\quad$ ZUCKERINDUSTRIE (1999): „Unterstützung der Fusion von Union und Nordzucker”, Nr. 10, S. 768

- ZUCKERINDUSTRIE (1997): „Verhandlung über Bildung der Nordzucker AG abgeschlossen”, Nr. 8, S. 666

- ZUCKERINDUSTRIE (1999): "Zuckerfabrik Harsum erhöht Dividende und Bonuszahlung und schließt sich Union-Zucker an", Nr. 11, S. 900

- $\quad$ ZVN (laufende Jahre), Geschäftsbericht, Uelzen-Braunschweig 


\section{B. Konzentration}

\section{Begriffe und Formen der Unternehmenskonzentrationen}

Wirtschaftliche Konzentration kann als „die Ballung von wirtschaftlichen oder wirtschaftlich relevanten Größen einschließlich der Verfügungsmacht über Produktionsfaktoren" 816 verstanden werden. Konzentration von Unternehmen wird auf drei Ursachen zurückgeführt ${ }^{817}$ (vgl. Abbildung 78):

- $\quad$ internes Wachstum: einige Unternehmen wachsen schneller als ihre Mitbewerber

- externes Wachstum: Unternehmen schließen sich zusammen

- Verdrängung: Unternehmen scheiden endgültig aus

Abbildung 78: Ursachen von Konzentration

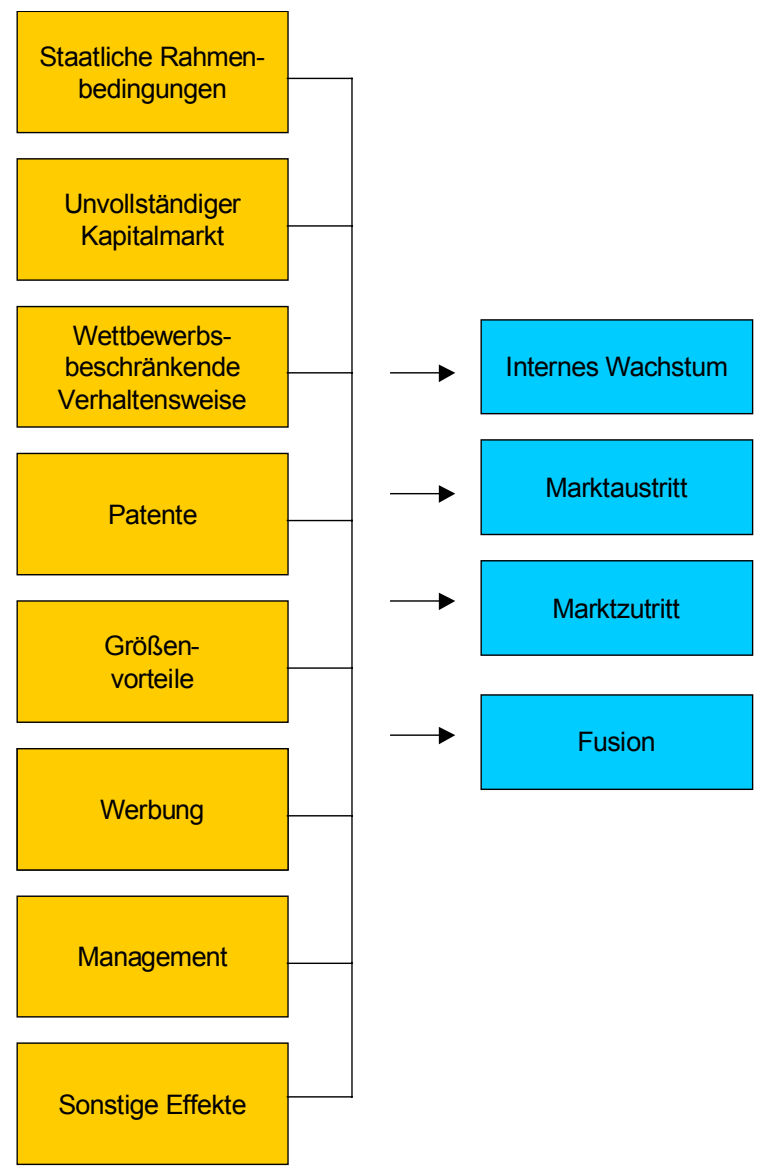

Quelle: eigene Darstellung

${ }^{816}$ Vgl. MÜLLER/HOCHREITER 1975, S. 18

${ }^{817}$ Vgl. SCHMIDT 1999, S.134 und die dort zitierte Literatur 
Die Zusammenschlusskontrolle erfasst unterschiedliche Fälle von Unternehmenszusammenschlüssen. Im folgenden wird ein Überblick über mögliche Formen vermittelt ${ }^{818}$.

Fusion: Verschmelzung von Unternehmen durch Vermögenserwerb; die Verschmelzung kann zur "Einverleibung" des übernommenen Unternehmens oder auch zur Bildung eines neuen Unternehmens führen.

Anteilserwerb: Erwerb von (stimmberechtigten) Kapitalanteilen. Je nach Umfang der Kapitalbeteiligung variiert die Kontrolle. Beispielsweise genügen $25 \%$ des stimmberechtigten Kapitals, um Gesellschafterbeschlüsse zu blockieren. Selbst bei geringeren Beteiligungen kann bei ansonsten stark gestreutem Anteilseigentum wesentlicher Einfluss gesichert werden.

Konzernbildung: Vertraglicher Zusammenschluss von rechtlich selbständigen Unternehmen unter einer gemeinsamen Leitung, wobei der Vertrag eine gemeinsame Leitung oder Gleichrangigkeit oder die Unterordnung von Unternehmen beinhalten kann. In den meisten Fällen kommen solche Verträge erst unter Kapitalbeteiligungen zustande.

Gemeinschaftsunternehmen (joint venture): Gründung eines gemeinschaftlichen Unternehmens durch zwei oder mehr Unternehmen mit prinzipiell beliebigen Beteiligungsverhältnissen.

Sonstige Unternehmensverträge: Verträge über Gewinnabführung (im Ganzen oder in Teilen des Gewinns), Betriebspacht- oder Betriebsüberlassungsverträge etc. Auch diesen Verträgen geht oftmals ein Anteilserwerb voraus.

Personelle Verflechtungen: Bei weiter Fassung des Begriffs "Unternehmenszusammenschluss" ist es sinnvoll, personelle Verflechtungen zwischen Unternehmen hinzuzuziehen. Einfluss auf unternehmerische Entscheidungen und Koordination von Verhaltensweisen läßt sich dadurch erreichen, dass Personen in wichtigen Positionen und Organe (z.B. Aufsichtsräte) anderer Unternehmen entsandt werden. Derartige personelle Verflechtungen sind keineswegs auf jene Fälle beschränkt, bei denen Kapitalbeteiligungen bestehen: Auch über Kreditbeziehungen lassen sich derartige Einflussmöglichkeiten begründen (beispielsweise das Phänomen der Besetzung von Aufsichtsräten in Nicht-Banken-Aktiengesellschaften mit zahlreichen Bankenvertretern).

\section{Messung von Konzentration}

Zur regelmäßigen Begutachtung der Unternehmenskonzentration in Deutschland wurde 1973 die Monopolkommission gebildet. Von einer Vielzahl möglicher Verfahren werden von der Monopolkommission vornehmlich drei Konzentrationsmaße verwendet: Konzentrationsraten, der Hirschmann-Herfindahl-Index und der Variationskoeffizient ${ }^{819}$. Darüber hinaus wird der LindaIndex vorgestellt, der eine theoretische Beziehung zwischen Konzentration und Marktmacht

${ }^{818}$ In Anlehnung an AHRNS/FESER 1997, S. 57

${ }^{819} \mathrm{Vgl.} \mathrm{BLEYMÜLLER/GeHLERT} 1989$ 
wiedergibt, allerdings aufgrund von Problemen der empirischen Datenermittlung kaum Anwendung findet ${ }^{820}$.

Die Konzentrationsrate (CR) weist die Relation des Umsatzes der größten Unternehmen eines Marktes zum erzielten Gesamtumsatz des betrachteten Marktes aus ${ }^{821}$.

$\sum_{j=1}^{n} x_{j}=x$ entspricht dem Gesamtumsatz und $s_{i}=x_{i} / x$ dem Umsatzanteil von Unternehmen $i$.

Die Konzentrationsrate wird formal folgendermaßen definiert:

$C R_{i}=\sum_{j=1}^{i} S_{j}$

Konzentrationsraten werden häufig verwendet, weil sie einfach zu errechnen sind und weil die verfügbaren Daten zumeist ausreichen, um die Ermittlung des Umsatzanteils der führenden Anbieter zuzulassen. Die Aussagefähigkeit dieses Konzentrationsmaßes ist jedoch begrenzt. Es werden keine Informationen darüber geboten, wie der Umsatzanteil, den die größten Unternehmen insgesamt bestreiten, sich auf einzelne Mitglieder dieser Gruppe aufteilt. Verschiebungen und Verteilungen im Zeitablauf bleiben unberücksichtigt. Ebenso wenig wird eine Aussage über die konkrete Verteilung der Umsatzanteile auf die einzelnen Unternehmen der außer Betracht gelassenen Unternehmen bzw. deren Anzahl geboten ${ }^{822}$.

Dieses Problem tritt beim sog. "Hirschmann-Herfindahl-Index" nicht auf. Der HirschmannHerfindahl-Index ( $\mathrm{HHI})$ ist das arithmetische Mittel der mit sich selbst gewogenen Marktanteile. Die Marktanteile der Anbieter werden quadriert und summiert:

$\mathrm{HHI}=\sum_{i=1}^{n} s_{i}^{2}$

Die Marktanteile der größten Anbieter werden also stärker gewichtet als die der kleineren. Die errechneten Werte werden zumeist mit 10000 multipliziert, da sich anderenfalls für stark besetzte Märkte sehr geringe Werte ergäben. Die maximale Konzentration liegt dann vor, wenn ein Anbieter den gesamten Marktanteil auf sich vereint, also den Anteil eins hat und somit einen Index von 10000. Ein höherer Hirschmann-Herfindahl-Index signalisiert eine höhere Konzentration des Umsatzes auf wenige Unternehmen. Der Index wird von zwei Größen positiv beeinflusst:

- $\quad$ einer sinkenden Anzahl von Unternehmen in einem Markt und

- $\quad$ einer größeren Varianz der Umatzverteilung bei gegebener Anbieterzahl.

\footnotetext{
${ }^{820} \mathrm{Vgl.}$ MONOPOLKOMMISSION 1992, S. 63

${ }^{821}$ Vgl. BERG 1999, S. 332

822 Vgl. Berg 1999, S. 333
} 
Wettbewerbspolitische Bedeutung erlangte der HHI 1982 in den vom US Departement of Justice veröffentlichten „Merger Guidelines". Danach wird ein Markt als niedrig konzentriert angesehen, wenn der HHI Werte aufweist, die unter 1000 liegen. Als „mäßig konzentriert” gelten Märkte, wenn dieser Index Werte zwischen 1000 und 1800 annimmt; hoch konzentrierte Märkte sind schließlich durch HHI Werte über 1800 gekennzeichnet ${ }^{823}$.

Um die in der Berechnung des HHI enthaltene Verteilung der Marktanteile sichtbar zu machen, wird von der Monopolkommission zusätzlich der Variationskoeffizient berechnet, der die Standardabweichung der Marktanteile in Prozent ihres Mittelwertes misst ${ }^{824}$.

Mit Hilfe der Linda-Indexes kann die Disparität innerhalb einer Unternehmensgruppe ermittelt werden und eine dominierende Unternehmensgruppe von unbedeutenden Unternehmen getrennt werden. Grundbaustein für beide Aufgaben ist die sog. Dominanz, die aussagt, in welchem Maße die führenden i Unternehmen die folgenden k-i Unternehmen dominieren. Hierzu wird der durchschnittliche Marktanteil der dominierenden i Unternehmen in Relation gesetzt zum durchschnittlichen Marktanteil der k-i Unternehmen.

$\mathrm{v}_{i, k}=\frac{C R_{i}}{C R_{k}-C R_{i}}=\frac{\frac{C R_{i}}{i}}{\frac{C R_{k}-C R_{i}}{k-i}}$

Errechnet man nun für $\mathrm{v}_{i, k}$ für $\mathrm{i}=1$ bis $\mathrm{i}=\mathrm{k}-1$, summiert diese Werte auf und dividiert sie durch k-1, so erhält man das arithmetische Mittel aller Dominanzen bzw. den einfach gewichteten Linda-Index $L^{*}$ :

$\mathrm{L}_{k}^{*}=\frac{1}{k-1} \sum_{i=1}^{k-1} v_{i, k} \quad 1 \leq L_{k}^{*}<\propto$

Diese sog. mittlere Dominanz gibt an, wie stark die Disparität bzw. die Ungleichheit der k Unternehmen ist. Nimmt L* den Wert eins an, so liegt ein vollkommen symmetrisches Oligopol vor. Ist $L^{*}$ dagegen deutlich größer als eins, so kann von einem asymmetrischen Oligopol ausgegangen werden. Bei Multiplikation $L^{*}$ mit $1 / k$ gelangt man zur doppelt gemittelten Dominanz bzw. zum doppelt gemittelten Linda-Index $\mathrm{L}_{k}$ :

$\mathrm{L}_{k}=\frac{1}{k} * \frac{1}{(k-1)} \sum_{i=1}^{k-1} v_{i, k} \quad \mathrm{i}=1,2, \ldots, \mathrm{k}-1 ; \mathrm{k}=2,3, \ldots, \mathrm{n}$

Mit Hilfe von $L_{k}$ kann die Grenze zwischen der dominierenden Unternehmensgruppe und den unbedeutenden kleinen Unternehmen ermittelt werden. Entsprechend der Konzeption des Linda-

${ }^{823}$ Vgl. BERG 1999, S. 333

${ }^{824} \mathrm{Vgl}$. BERG 1999, S. 333 
Indexes ist sie dort gegeben, wo der Quotient der Marktanteile zweier aufeinander folgenden Unternehmen den größten Wert annimmt ${ }^{825}$.

Der Hirschmann-Herfindahl-Index weist bei homogenen Märkten einen engen Zusammenhang mit einem Marktmacht-Index, dem Lerner-Index, auf. Zur Verdeutlichung dieser Beziehungen wird ein homogener Markt mit konstanten Grenzkosten $c_{i}$ für alle Unternehmen $i$ betrachtet ${ }^{826}$. Die Bedingung erster Ordnung für ein Cournot-Gleichgewicht lautet:

$p-c_{i}=\frac{\partial P}{\partial Q} q$

oder

$$
L_{i} \equiv \frac{p-c_{i}}{p}=\frac{\partial P}{\partial Q} \frac{Q}{P} \frac{q_{i}}{Q}=\frac{s_{i}}{\varepsilon_{Q P}}
$$

wobei $L_{i}=\left(p-c_{i}\right) / p$ der Lerner-Index für Unternehmen $i$ ist, $s_{i}$ der Marktanteil und $\varepsilon_{Q P}$ die Preiselastizität der Nachfrage. Ein naheliegender Index für die gesamte Branche ist dann

$$
L=\sum_{i=1}^{n} s_{i} L_{i}
$$

Wird die obige Beziehung genutzt, ergibt sich:

$$
L=\sum_{i=1}^{n} s_{i}^{2} / \varepsilon_{Q P}=H / \varepsilon_{Q P}
$$

Der Marktmachtindex $L$ ist demnach proportional zum Hirschmann-Herfindahl-Index. Außerdem lässt sich aufgrund der als konstant angenommenen Grenzkosten $L$ wie folgt umformen:

$L=\sum_{i=1}^{n} s_{i} L_{i}=\sum_{1=1}^{n} \frac{\left(p-c_{i}\right) q_{i}}{p Q}$.

Damit lässt sich $L$ durch das Verhältnis von variablem Gewinn der Branche zum Branchenumsatz messen. Neben diesen positiven Eigenschaften des Hirschmann-Herfindahl-Index ist jedoch auch daran zu erinnern, dass eine Erhöhung des Hirschmann-Herfindahl-Index (und entsprechend eine Erhöhung des Lerner-Index) nicht mit der Erhöhung der allokativen Ineffizienz einhergehen muss. Eine höhere Konzentration gem. $H$ oder eine höhere Marktmacht gem. $L$ kann schlichtweg den größeren Preisspielraum eines besonders effizienten Unternehmens widerspiegeln. Eine Erhöhung der Konzentration kann daher mit Effizienzsteigerungen einhergehen.

\footnotetext{
${ }^{825}$ Vgl. SCHMIDT 1999, S. 137

${ }^{826}$ Formelle Darstellung in Anlehnung an BOFInGER 2001, S. 9 ff.
} 


\section{Marktdaten der Zuckerproduktion}

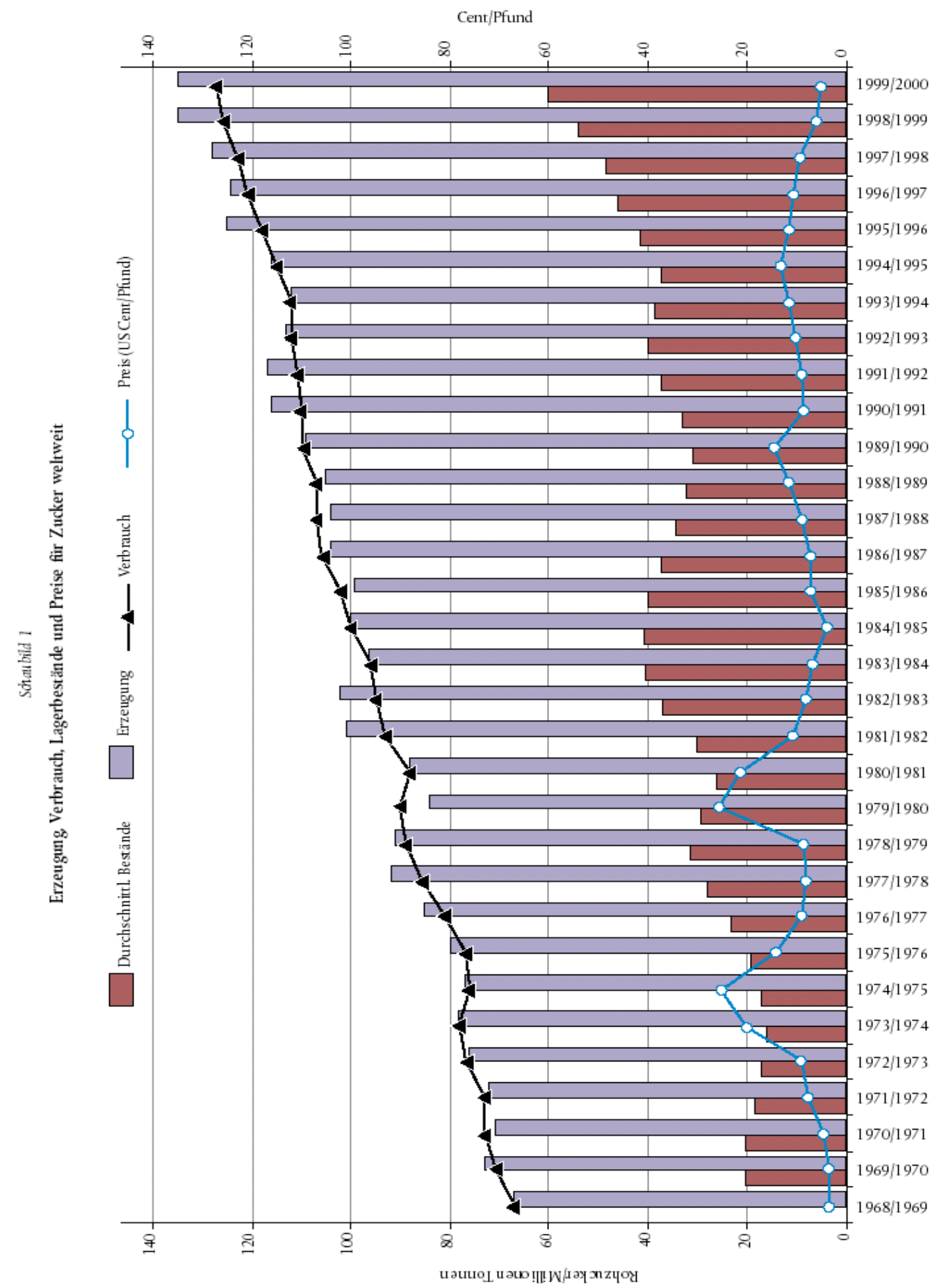

Quelle: EUROPÄISCHER-RECHNUNGSHOF, S. 5 ff. 


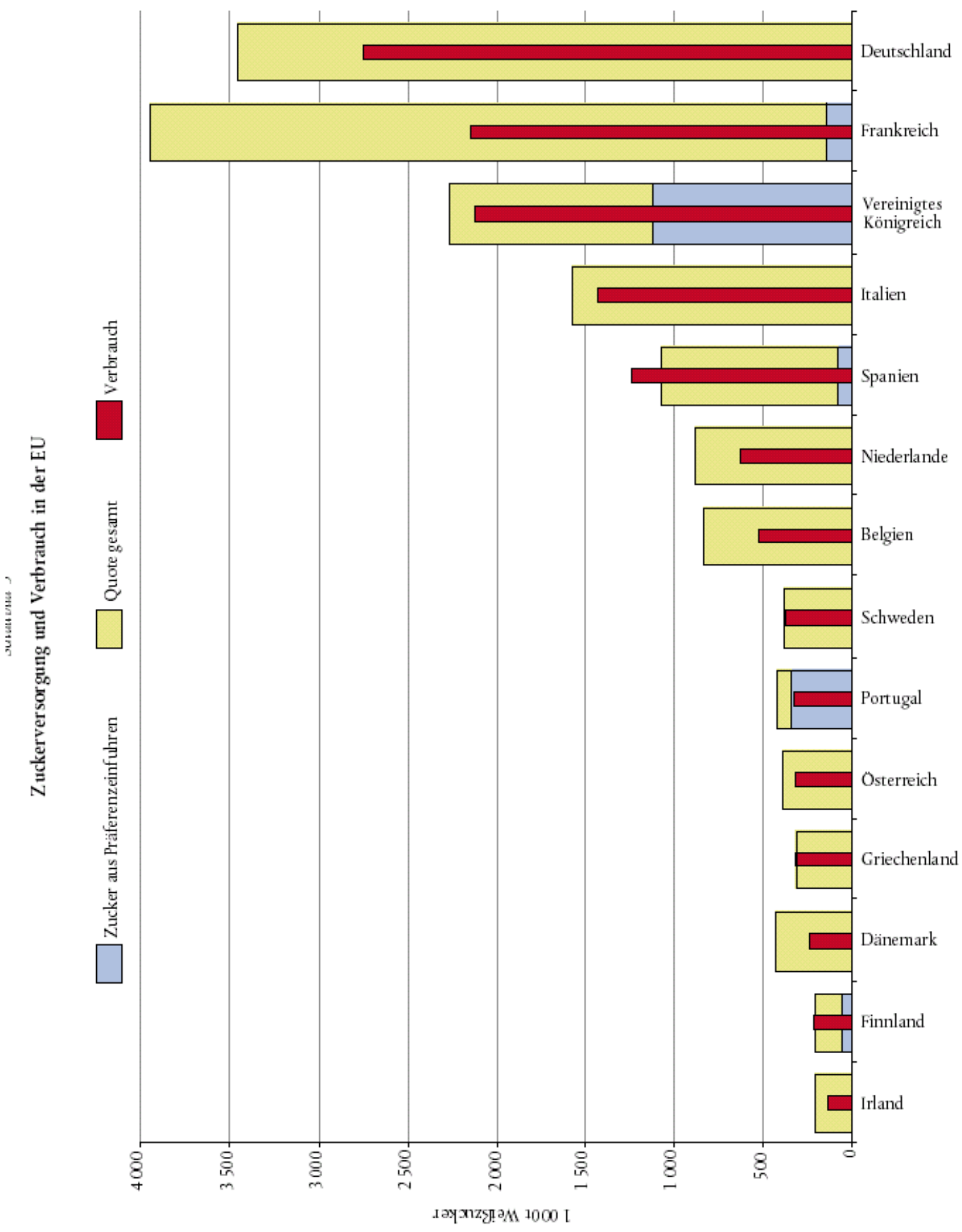

Quelle: EUROPÄISCHER-RECHNUNGSHOF, S. 5 ff. 


\section{Zuckerfabrikkapazitäten}

Table 14.2 Number and capacity of beet processing plants in the EU member states

\begin{tabular}{|l|r|r|l|r|r|r|}
\hline $\begin{array}{l}\text { Member } \\
\text { State }\end{array}$ & $\begin{array}{c}\text { Total } \\
\text { number } \\
\text { of plants }\end{array}$ & $\begin{array}{c}\text { Total capacity } \\
\text { of the plants } \\
\text { (tonnes of beet } \\
\text { per day) }\end{array}$ & Largest company & $\begin{array}{c}\text { Number of } \\
\text { plants of the } \\
\text { company }\end{array}$ & $\begin{array}{c}\text { Capacity } \\
\text { tonnes of beet } \\
\text { per day) }\end{array}$ & $\begin{array}{c}\text { Capacity largest } \\
\text { company as \% of } \\
\text { total capacity }\end{array}$ \\
\hline A & 3 & 30,500 & Agrana & 3 & 30,500 & $100 \%$ \\
B & 34 & 33,000 & Tirlemont & 5 & 59,000 & $71 \%$ \\
D & 312,200 & Südzucker & 14 & 129,200 & $41 \%$ \\
DK & 17 & 39,200 & Danisco & 4 & 39,200 & $100 \%$ \\
E & 109,800 & Az.EbroAgrí & 14 & 88,100 & $80 \%$ \\
ELL & 3 & 32,100 & HIS & 5 & 32,100 & $100 \%$ \\
F & 41 & 402,000 & Béghin-Say & 10 & 125,500 & $31 \%$ \\
IRL & 2 & 16,000 & Greencore & 2 & 16,000 & $100 \%$ \\
ITA & 23 & 235,500 & Eridania & 7 & 73,000 & $31 \%$ \\
NL & 5 & 69,000 & Cosun & 3 & 43,000 & $62 \%$ \\
P & 1 & 5,000 & DAI & 1 & 5,000 & $100 \%$ \\
S & 4 & 28,300 & Danisco & 4 & 28,300 & $100 \%$ \\
SF & 3 & 16,000 & Danisco & 3 & 16,000 & $100 \%$ \\
UK & 9 & 67,900 & British Sugar & 97,900 & $100 \%$ \\
\hline
\end{tabular}

\section{Veränderung der Kosten der Zuckerindustrie}

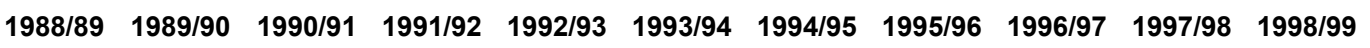

Prozentuale Anteile der Kosten

\begin{tabular}{|c|c|c|c|c|c|c|c|c|c|c|}
\hline Labour & 28 & 29 & 28 & 28 & 26 & 28 & 27 & 25 & 24 & 25 \\
\hline Energy & 32 & 28 & 30 & 30 & 31 & 28 & 27 & 30 & 33 & 28 \\
\hline $\begin{array}{l}\text { Deprecia- } \\
\text { tion }\end{array}$ & 13 & 14 & 14 & 14 & 14 & 14 & 15 & 14 & 13 & 14 \\
\hline Other & 4 & 5 & 5 & 5 & 5 & 5 & 5 & 5 & 5 & 6 \\
\hline $\begin{array}{l}\text { Distribu- } \\
\text { tion/Adminis } \\
\text { tration }\end{array}$ & 22 & 24 & 23 & 24 & 24 & 25 & 26 & 26 & 25 & 28 \\
\hline
\end{tabular}

Quelle: CIUS, Ifd. Jgg.

\section{Daten Stromverbrauch (kwh/kg Zucker)}

\begin{tabular}{|c|c|c|c|c|c|c|c|}
\hline Jahr & 1978 & 1982 & 1985 & 1989 & 1993 & 1997 & 2000 \\
\hline Verbrauch & 0,2835 & 0,2698 & 0,2684 & 0,2573 & 0,2534 & 2394 & 0,2225 \\
\hline
\end{tabular}

Quelle: Statistisches Bundesamt, Fachserie Produzierendes Gewerbe, laufende Jahrgänge, eigene Berechnungen 


\section{Energieeinsatz in der Zuckerindustrie}

\section{Brennstoffeinsatz in der Zuckerindustrie \\ 1999/2000}

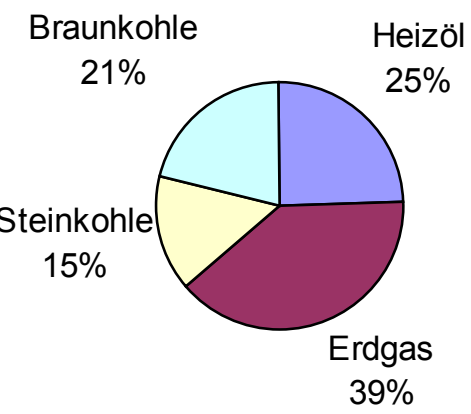

Quelle: Wirtschaftliche Vereinigung ZUCKER 2001, S. 52

\section{Industriezuckerpreise in der EU (100kg)}

\begin{tabular}{|c|c|c|c|c|c|c|c|c|c|c|c|c|c|c|}
\hline \multirow[b]{2}{*}{ Land } & \multicolumn{14}{|c|}{ in ECU } \\
\hline & $A$ & B & $\mathrm{D}$ & DK & $E$ & $\mathrm{~F}$ & GR & I & IRL & NL & $P$ & S & FIN & UK \\
\hline Schnitt 1989 & & 66,70 & 68,85 & 67,90 & 86,50 & 65,90 & 44,70 & 64,50 & 69,75 & 69,00 & 63,45 & & & 58,70 \\
\hline Schnitt 1990 & & 65,80 & 68,10 & 67,20 & 86,90 & 66,50 & 47,65 & 68,30 & 71,35 & 67,15 & 73,30 & & & 65,70 \\
\hline Schnitt 1991 & & 66,20 & 66,70 & 67,40 & 87,80 & 67,50 & 60,75 & 72,00 & 73,50 & 66,50 & 74,70 & & & 68,15 \\
\hline Schnitt 1992 & & 69,72 & 68,06 & 68,22 & 83,82 & 69,74 & 71,70 & 31,64 & 74,08 & 68,89 & & & & 68,36 \\
\hline Schnitt 1993 & & 70,29 & 70,90 & 71,49 & 78,25 & 72,12 & 77,60 & 72,52 & 72,11 & 72,03 & 86,67 & 48,18 & & 74,80 \\
\hline Schnitt 1994 & & 72,97 & 70,57 & 74,09 & 78,31 & 73,60 & 77,56 & 72,87 & 76,41 & 74,00 & 75,40 & 48,09 & & 74,47 \\
\hline Schnitt 1995 & 75,79 & 72,42 & 72,89 & 73,42 & 78,25 & 75,39 & 61,56 & 72,78 & 75,33 & 73,58 & 76,34 & 75,64 & 84,88 & 72,68 \\
\hline Schnitt 1996 & 74,30 & 70,10 & 73,05 & 71,60 & 78,39 & 76,96 & 78,58 & 73,46 & 74,14 & 75,82 & 77,10 & 74,35 & 77,45 & 2,38 \\
\hline Schnitt 1997 & 72,44 & 69,54 & 71,53 & 70,86 & 77,72 & 76,65 & 76,94 & 71,43 & 51,06 & 73,04 & 77,13 & 73,20 & 76,05 & 77,38 \\
\hline Schnitt 1998 & 73,08 & & 71,94 & 70,46 & 77,28 & 76,44 & 71,34 & 72,58 & 74,27 & 72,06 & 75,74 & 75,20 & & 78,96 \\
\hline Schnitt 1999 & 0,00 & & 72,09 & 70,58 & 77,60 & 77,29 & 74,33 & 72,91 & 75,50 & 73,15 & 76,17 & 73,09 & & 81,68 \\
\hline Schnitt 2000 & 0,00 & & 72,06 & 70,54 & 77,60 & 77,71 & 73,20 & 72,47 & 75,50 & 73,15 & 76,47 & 72,95 & & 81,89 \\
\hline Jan 0 & 73,76 & & 73,38 & 71,70 & 77,60 & 77,97 & 73,07 & 74,64 & 75,50 & 74,11 & 78,56 & & & 78,33 \\
\hline
\end{tabular}

Quelle: CaObisco 


\section{Zuckerdurchschnittsverarbeitung}

Tabelle 59: Durchschnittliche Verarbeitung pro Zuckerfabrik:

\begin{tabular}{|c|c|c|c|c|c|c|c|c|c|c|c|c|c|}
\hline & 1985 & 1990 & 1991 & 1992 & 1993 & 1994 & 1995 & 1996 & 1997 & 1998 & 1999 & 2000 & 2001 \\
\hline A & 5935 & 5581 & 8475 & 8697 & 9142 & 9627 & 9948 & 10158 & 10143 & 10497 & 10730 & 11430 & 11430 \\
\hline B & 5530 & 7051 & 7619 & 7415 & 8795 & 8904 & 8821 & 8979 & 9717 & 9895 & 8781 & 10220 & 10650 \\
\hline DK & 6700 & 7467 & 8240 & 8240 & 9125 & 9381 & 9478 & 9143 & 8909 & 9448 & 9658 & 9970 & 11000 \\
\hline$E$ & 3667 & 4342 & 4346 & 4375 & 4723 & 4952 & 5190 & 5400 & 5667 & 6118 & 7000 & 7000 & 7320 \\
\hline GR & 5300 & 5392 & 4919 & 5322 & 5387 & 5320 & 5325 & 6251 & 6347 & 6049 & 6049 & 6070 & 6300 \\
\hline D & 5570 & 7193 & 7251 & 5380 & 6157 & 7270 & 7733 & 8365 & 8669 & 9274 & 9232 & 9620 & 10160 \\
\hline IRL & 4125 & 8090 & 7574 & 7784 & 7667 & 7698 & 8172 & 8115 & 8298 & 8410 & 8735 & 8800 & 8800 \\
\hline ITA & 4031 & 6733 & 6661 & 6414 & 6560 & 6670 & 7304 & 7043 & 7261 & 8435 & 8000 & 8230 & 10500 \\
\hline NL & 8000 & 10500 & 11736 & 11738 & 12700 & 12396 & 12500 & 11667 & 13298 & 13200 & 10826 & 14100 & 14100 \\
\hline$S$ & 4343 & 4494 & 4882 & 4967 & 5620 & 6300 & 6525 & 6750 & 6681 & 6998 & 8516 & 9170 & 9410 \\
\hline SF & 3748 & 4064 & 4137 & 4697 & 4647 & 4913 & 1913 & 5425 & 4698 & 5477 & 5357 & 6750 & 7000 \\
\hline UK & 6235 & 6118 & 6084 & 6650 & 6864 & 6791 & 7635 & 7722 & 7466 & 7935 & 7915 & 7980 & 8050 \\
\hline$F$ & 7804 & 8480 & 8460 & 7242 & 6790 & 7167 & 7761 & 7621 & 9289 & 8333 & 8375 & 11080 & 11770 \\
\hline EU & 5461 & 6950 & 7072 & 6315 & 6630 & 7095 & 7534 & 7659 & 8277 & 8432 & 8400 & 9380 & 10050 \\
\hline-15 & & & & & & & & & & & & & \\
\hline
\end{tabular}

Quelle: CEFS, sugar statistics, BARTENS: Zuckerwirtschaft 


\section{T-Test-Ergebnisse der MADAKOM-Datensatz Preisuntersuchung Preisuntersuchungen}

Anmerkung: Variable 1 = Preisbeobachtungen im Gesamten Erhebungsgebiet; Variable $2=$ Preisbeobachtungen in den Überschneidungsgebieten; Signifikanzniveau $\alpha=0,05$

\section{Nordzucker}

\begin{tabular}{|lrr|}
\hline \multicolumn{1}{|c}{ Nordzucker Würfel 500g } & Variable 1 & Variable 2 \\
\hline Mittelwert & 1,428571429 & 1,30454545 \\
Varianz & 0,036925346 & 0,01672727 \\
Beobachtungen & 63 & 11 \\
Hypothetische Differenz der Mittelwerte & 0 & \\
Freiheitsgrade (df) & 19 & \\
t-Statistik & 2,702111616 \\
P(T<=t) einseitig & 0,007062393 \\
Kritischer t-Wert bei einseitigem t-Test & 1,729131327 \\
P(T<=t) zweiseitig & 0,014124786 \\
Kritischer t-Wert bei zweiseitigem t-Test & 2,093024705 & \\
\hline
\end{tabular}

\begin{tabular}{|lrr|}
\hline \multicolumn{1}{|c}{ Nordzucker Raffinade 1000g HH } & Variable 1 & Variable 2 \\
\hline Mittelwert & 1,8194 & 1,76090909 \\
Varianz & 0,016703714 & 0,00474909 \\
Beobachtungen & 50 & 11 \\
Hypothetische Differenz der Mittelwerte & 0 & \\
Freiheitsgrade (df) & 28 & \\
t-Statistik & 2,113624844 \\
P(T<=t) einseitig & 0,021793709 & \\
Kritischer t-Wert bei einseitigem t-Test & 1,701130259 & \\
P(T<=t) zweiseitig & 0,043587418 & \\
Kritischer t-Wert bei zweiseitigem t-Test & 2,048409442 & \\
\hline
\end{tabular}

\begin{tabular}{|lrr|}
\hline \multicolumn{1}{|c}{ Nordzucker Zuckerhut 250g } & Variable 1 & Variable 2 \\
\hline Mittelwert & 1,629285714 & 1,4 \\
Varianz & 0,038268571 & 0,0007 \\
Beobachtungen & 56 & 7 \\
Hypothetische Differenz der Mittelwerte & 0 & \\
Freiheitsgrade (df) & 60 & \\
t-Statistik & 8,1920815 & \\
P(T<=t) einseitig & $1,15289 \mathrm{E}-11$ & \\
Kritischer t-Wert bei einseitigem t-Test & 1,670648544 & \\
P(T<=t) zweiseitig & $2,30578 \mathrm{E}-11$ & \\
Kritischer t-Wert bei zweiseitigem t-Test & 2,000297172 & \\
\hline
\end{tabular}




\begin{tabular}{|lrr|}
\hline \multicolumn{1}{|c}{ Nordzucker Würfel 1000g } & Variable 1 & Variable 2 \\
\hline Mittelwert & 2,144545455 & 1,97 \\
Varianz & 0,020727273 & 0,047 \\
Beobachtungen & 11 & 5 \\
Hypothetische Differenz der Mittelwerte & 0 & \\
Freiheitsgrade (df) & 6 & \\
t-Statistik & 1,643127558 & \\
P(T<=t) einseitig & 0,075731183 & \\
Kritischer t-Wert bei einseitigem t-Test & 1,943180905 & \\
P(T<=t) zweiseitig & 0,151462365 & \\
Kritischer t-Wert bei zweiseitigem t-Test & 2,446913641 & \\
\hline
\end{tabular}

\begin{tabular}{|lrr|}
\hline \multicolumn{1}{|c}{ Nordzucker Raffinade 1000g } & Variable 1 & Variable 2 \\
\hline Mittelwert & 2,05875 & 1,95 \\
Varianz & 0,018346774 & 0,02266667 \\
Beobachtungen & 32 & 10 \\
Hypothetische Differenz der Mittelwerte & 0 & \\
Freiheitsgrade (df) & 14 & \\
t-Statistik & 2,04065618 & \\
P(T<=t) einseitig & 0,030305033 & \\
Kritischer t-Wert bei einseitigem t-Test & 1,76130925 & \\
$\quad$ Nordzucker Raffinade 1000g (Fortsetzung) & Variable 1 & Variable 2 \\
P(T<=t) zweiseitig & 0,060610066 & \\
Kritischer t-Wert bei zweiseitigem t-Test & 2,144788596 & \\
\hline
\end{tabular}

\begin{tabular}{|lrr|}
\hline \multicolumn{1}{|c}{ Nordzucker Einmachzucker 1000g } & Variable 1 & Variable 2 \\
\hline Mittelwert & 2,106842105 & 2,05571429 \\
Varianz & 0,034767251 & 0,03022857 \\
Beobachtungen & 19 & 7 \\
Hypothetische Differenz der Mittelwerte & 0 & \\
Freiheitsgrade (df) & 11 \\
t-Statistik & 0,652052369 \\
P(T<=t) einseitig & 0,263878574 \\
Kritischer t-Wert bei einseitigem t-Test & 1,795883691 & \\
P(T<=t) zweiseitig & 0,527757148 & \\
Kritischer t-Wert bei zweiseitigem t-Test & 2,200986273 & \\
\hline
\end{tabular}




\begin{tabular}{|lrr|}
\hline \multicolumn{1}{|c}{ Nordzucker Gelierzucker 1000g } & Variable 1 & Variable 2 \\
\hline Mittelwert & 2,679180328 & 2,57181818 \\
Varianz & 0,043620984 & 0,04963636 \\
Beobachtungen & 61 & 11 \\
Hypothetische Differenz der Mittelwerte & 0 & \\
Freiheitsgrade (df) & 13 & \\
t-Statistik & 1,484924524 & \\
P(T<=t) einseitig & 0,080702246 & \\
Kritischer t-Wert bei einseitigem t-Test & 1,770931704 & \\
P(T<=t) zweiseitig & 0,161404492 & \\
Kritischer t-Wert bei zweiseitigem t-Test & 2,16036824 & \\
\hline
\end{tabular}

\begin{tabular}{|lrr|}
\hline \multicolumn{1}{|c}{ Nordzucker Glückswürfel 500g Variable 2 } & \multicolumn{1}{c|}{ Variable 1 } \\
\hline Mittelwert & 1,665 & 1,822592593 \\
Varianz & 0,045 & 0,034212251 \\
Beobachtungen & 8 & 27 \\
Hypothetische Differenz der Mittelwerte & 0 & \\
Freiheitsgrade (df) & 10 & \\
t-Statistik & $-1,898276068$ \\
P(T<=t) einseitig & 0,043434523 & \\
Kritischer t-Wert bei einseitigem t-Test & 1,812461505 & \\
P(T<=t) zweiseitig & 0,086869046 & \\
Kritischer t-Wert bei zweiseitigem t-Test & 2,228139238 & \\
\hline
\end{tabular}

\section{Südzucker (=SZ)}

\begin{tabular}{|lrr|}
\hline \multicolumn{1}{|c}{ SZ-Einmachzucker $500 \mathrm{~g}$} & Variable 1 & Variable 2 \\
\hline Mittelwert & 4,930740741 & 5,05 \\
Varianz & 0,072444444 & 0,24933333 \\
Beobachtungen & 81 & 10 \\
Hypothetische Differenz der Mittelwerte & 0 & \\
Freiheitsgrade (df) & 10 & \\
t-Statistik & $-0,742077329$ & \\
P(T<=t) einseitig & 0,237554574 & \\
Kritischer t-Wert bei einseitigem t-Test & 1,812461505 & \\
P(T<=t) zweiseitig & 0,475109148 & \\
Kritischer t-Wert bei zweiseitigem t-Test & 2,228139238 & \\
\hline
\end{tabular}




\begin{tabular}{|lrr|}
\hline \multicolumn{1}{|c}{ SZ-Feinzucker 1000g } & Variable 1 & \multicolumn{1}{c|}{ Variable 2 } \\
\hline Mittelwert & 2,103173077 & 1,90818182 \\
Varianz & 0,063724785 & 0,01763636 \\
Beobachtungen & 104 & 11 \\
Hypothetische Differenz der Mittelwerte & 0 & \\
Freiheitsgrade (df) & 19 & \\
t-Statistik & 4,142151154 & \\
P(T<=t) einseitig & 0,000276902 & \\
Kritischer t-Wert bei einseitigem t-Test & 1,729131327 & \\
P(T<=t) zweiseitig & 0,000553805 & \\
Kritischer t-Wert bei zweiseitigem t-Test & 2,093024705 & \\
\hline
\end{tabular}

\begin{tabular}{|lrr|}
\hline \multicolumn{1}{|c}{ SZ_Gelierzucker (2) $1000 \mathrm{~g}$} & Variable 1 & \multicolumn{1}{c|}{ Variable 2 } \\
\hline Mittelwert & 1,844545455 & 1,93 \\
Varianz & 0,056335944 & 0,08933333 \\
Beobachtungen & 66 & 10 \\
Hypothetische Differenz der Mittelwerte & 0 & \\
Freiheitsgrade (df) & 11 & \\
t-Statistik & $-0,86379843$ & \\
P(T<=t) einseitig & 0,20306999 & \\
Kritischer t-Wert bei einseitigem t-Test & 1,795883691 & \\
P(T<=t) zweiseitig & 0,40613998 & \\
Kritischer t-Wert bei zweiseitigem t-Test & 2,200986273 & \\
\hline
\end{tabular}

\begin{tabular}{|lrr|}
\hline \multicolumn{1}{|c}{ SZ-Gelierzucker (1) 1000g } & Variable 1 & Variable 2 \\
\hline Mittelwert & 2,566637931 & 2,515 \\
Varianz & 0,03002425 & 0,05295455 \\
Beobachtungen & 116 & 12 \\
Hypothetische Differenz der Mittelwerte & 0 & \\
Freiheitsgrade (df) & 12 & \\
t-Statistik & 0,755493902 & \\
P(T<=t) einseitig & 0,232262145 & \\
\multicolumn{1}{|c|}{ SZ-Gelierzucker (1) 1000g (Fortsetzung) } & Variable 1 & Variable 2 \\
Kritischer t-Wert bei einseitigem t-Test & 1,782286745 & \\
P(T<=t) zweiseitig & 0,464524291 & \\
Kritischer t-Wert bei zweiseitigem t-Test & 2,178812792 & \\
\hline
\end{tabular}




\begin{tabular}{|lrr|}
\hline \multicolumn{1}{|c}{ SZ-Glückswürfel 500g } & Variable 1 & Variable 2 \\
\hline Mittelwert & 1,831222222 & 1,85363636 \\
Varianz & 0,028267029 & 0,02854545 \\
Beobachtungen & 90 & 11 \\
Hypothetische Differenz der Mittelwerte & 0 & \\
Freiheitsgrade (df) & 13 & \\
t-Statistik & $-0,415567268$ \\
P(T<=t) einseitig & 0,342252163 & \\
Kritischer t-Wert bei einseitigem t-Test & 1,770931704 & \\
P(T<=t) zweiseitig & 0,684504326 & \\
Kritischer t-Wert bei zweiseitigem t-Test & 2,16036824 & \\
\hline
\end{tabular}

\begin{tabular}{|lrr|}
\hline \multicolumn{1}{|c}{ SZ-Raffinade 1000g } & Variable 1 & Variable 2 \\
\hline Mittelwert & 1,944382022 & 1,92636364 \\
Varianz & 0,014390807 & 0,00854545 \\
Beobachtungen & 89 & 11 \\
Hypothetische Differenz der Mittelwerte & 0 & \\
Freiheitsgrade (df) & 15 & \\
t-Statistik & 0,588147513 & \\
P(T<=t) einseitig & 0,282592099 & \\
Kritischer t-Wert bei einseitigem t-Test & 1,753051038 & \\
P(T<=t) zweiseitig & 0,565184198 & \\
Kritischer t-Wert bei zweiseitigem t-Test & 2,131450856 & \\
\hline
\end{tabular}

\begin{tabular}{|lrr|}
\hline \multicolumn{1}{|c}{ SZ-Würfel 500g } & Variable 1 & \multicolumn{1}{c|}{ Variable 2 } \\
\hline Mittelwert & 1,380194175 & 1,20833333 \\
Varianz & 0,030452903 & 0,01721667 \\
Beobachtungen & 103 & 6 \\
Hypothetische Differenz der Mittelwerte & 0 & \\
Freiheitsgrade (df) & 6 & \\
t-Statistik & 3,054801267 \\
P(T<=t) einseitig & 0,011186833 \\
Kritischer t-Wert bei einseitigem t-Test & 1,943180905 & \\
P(T<=t) zweiseitig & 0,022373666 & \\
Kritischer t-Wert bei zweiseitigem t-Test & 2,446913641 & \\
\hline
\end{tabular}




\begin{tabular}{|lrr|}
\hline \multicolumn{1}{|c}{ SZ-Zuckerhut 250g } & Variable 1 & \multicolumn{1}{c|}{ Variable 2 } \\
\hline Mittelwert & 1,629452055 & 1,5025 \\
Varianz & 0,047099696 & 0,02696429 \\
Beobachtungen & 73 & 8 \\
Hypothetische Differenz der Mittelwerte & 0 & \\
\multicolumn{1}{|c}{ SZ-Zuckerhut 250g (Fortsetzung) } & Variable 1 & Variable 2 \\
Freiheitsgrade (df) & 10 & \\
t-Statistik & 2,003351254 & \\
P(T<=t) einseitig & 0,036489641 & \\
Kritischer t-Wert bei einseitigem t-Test & 1,812461505 & \\
P(T<=t) zweiseitig & 0,072979282 & \\
Kritischer t-Wert bei zweiseitigem t-Test & 2,228139238 & \\
\hline
\end{tabular}

\section{Pfeifer \& Langen/Diamant (=PL):}

\begin{tabular}{|lrr|}
\hline \multicolumn{1}{|c}{ Diamant Extra Fein } & Variable 1 & Variable 2 \\
\hline Mittelwert & 2,19780488 & 2,19 \\
Varianz & 0,05221756 & 0 \\
Beobachtungen & 41 & 3 \\
Hypothetische Differenz der Mittelwerte & 0 & \\
Freiheitsgrade (df) & 40 & \\
t-Statistik & 0,21870049 & \\
P(T<=t) einseitig & 0,41399771 & \\
Kritischer t-Wert bei einseitigem t-Test & 1,68385213 & \\
P(T<=t) zweiseitig & 0,82799542 & \\
Kritischer t-Wert bei zweiseitigem t-Test & 2,02107458 & \\
\hline
\end{tabular}

\begin{tabular}{|lrr|}
\hline \multicolumn{1}{|c}{ Diamant Würfel 500g } & Variable 1 & \multicolumn{1}{c|}{ Variable 2 } \\
\hline Mittelwert & 1,3905 & 1,233125 \\
Varianz & 0,020015 & 0,04304958 \\
Beobachtungen & 60 & 16 \\
Hypothetische Differenz der Mittelwerte & 0 & \\
Freiheitsgrade (df) & 19 & \\
t-Statistik & 2,861750452 & \\
P(T<=t) einseitig & 0,004991073 & \\
Kritischer t-Wert bei einseitigem t-Test & 1,729131327 & \\
P(T<=t) zweiseitig & 0,009982147 & \\
Kritischer t-Wert bei zweiseitigem t-Test & 2,093024705 & \\
\hline
\end{tabular}




\begin{tabular}{|lrr|}
\hline \multicolumn{1}{|c}{ Diamant 1000g } & Variable 1 & Variable 2 \\
\hline Mittelwert & 1,828529412 & 1,815 \\
Varianz & 0,012752317 & 0,005 \\
Beobachtungen & 34 & 8 \\
Hypothetische Differenz der Mittelwerte & 0 & \\
Freiheitsgrade (df) & 17 & \\
t-Statistik & 0,427822987 & \\
P(T<=t) einseitig & 0,337075248 & \\
Kritischer t-Wert bei einseitigem t-Test & 1,739606432 & \\
P(T<=t) zweiseitig & 0,674150496 & \\
Kritischer t-Wert bei zweiseitigem t-Test & 2,109818524 & \\
\hline
\end{tabular}

\begin{tabular}{|lrr|}
\hline \multicolumn{1}{|c}{ Diamant Einmachzucker } & Variable 2 & Variable 1 \\
\hline Mittelwert & 4,69 & 5,01 \\
Varianz & 0,12222222 & 0,04921519 \\
Beobachtungen & 10 & 80 \\
Hypothetische Differenz der Mittelwerte & 0 & \\
Freiheitsgrade (df) & 10 & \\
t-Statistik & $-2,82430267$ & \\
P(T<=t) einseitig & 0,00901359 & \\
Kritischer t-Wert bei einseitigem t-Test & 1,81246151 & \\
P(T<=t) zweiseitig & 0,01802719 & \\
Kritischer t-Wert bei zweiseitigem t-Test & 2,22813924 & \\
\hline
\end{tabular}

\begin{tabular}{|lrr|}
\hline \multicolumn{1}{|c}{ PL Gelier 1000g } & Variable 1 & Variable 2 \\
\hline Mittelwert & 2,601452991 & 2,45333333 \\
Varianz & 0,029059078 & 0,01337647 \\
Beobachtungen & 117 & 18 \\
Hypothetische Differenz der Mittelwerte & 0 & \\
Freiheitsgrade (df) & 30 & \\
t-Statistik & 4,703976577 & \\
P(T<=t) einseitig & $2,68393 \mathrm{E}-05$ & \\
Kritischer t-Wert bei einseitigem t-Test & 1,697260359 & \\
P(T<=t) zweiseitig & $5,36785 \mathrm{E}-05$ & \\
Kritischer t-Wert bei zweiseitigem t-Test & 2,042270353 & \\
\hline
\end{tabular}




\begin{tabular}{|lrr|}
\hline \multicolumn{1}{|c}{ PL-Gelier 500g } & Variable 1 & Variable 2 \\
\hline Mittelwert & 1,7582 & 1,68285714 \\
Varianz & 0,020953837 & 0,00840659 \\
Beobachtungen & 50 & 14 \\
Hypothetische Differenz der Mittelwerte & 0 & \\
Freiheitsgrade (df) & 33 & \\
t-Statistik & 2,359599592 & \\
P(T<=t) einseitig & 0,012181413 & \\
Kritischer t-Wert bei einseitigem t-Test & 1,692360456 & \\
P(T<=t) zweiseitig & 0,024362826 & \\
Kritischer t-Wert bei zweiseitigem t-Test & 2,03451691 & \\
\hline
\end{tabular}

\begin{tabular}{|lrr|}
\hline \multicolumn{1}{|c}{$P L$ Würfel $500 g$} & Variable 1 & Variable 2 \\
\hline Mittelwert & 1,397317073 & 1,13375 \\
Varianz & 0,034612466 & 0,04896964 \\
Beobachtungen & 82 & 8 \\
Hypothetische Differenz der Mittelwerte & 0 & \\
Freiheitsgrade (df) & 8 & \\
t-Statistik & 3,258310382 & \\
P(T<=t) einseitig & 0,005778231 & \\
Kritischer t-Wert bei einseitigem t-Test & 1,85954832 & \\
P(T<=t) zweiseitig & 0,011556461 & \\
\multicolumn{1}{|c|}{$P L$ Würfel 500g (Fortsetzung) } & Variable 1 & Variable 2 \\
Kritischer t-Wert bei zweiseitigem t-Test & 2,306005626 & \\
\hline
\end{tabular}

\begin{tabular}{|lrr|}
\hline \multicolumn{1}{|c}{ PL Zuckerhüte } & Variable 1 & Variable 2 \\
\hline Mittelwert & 1,604761905 & 1,51307692 \\
Varianz & 0,033893088 & 0,00358974 \\
Beobachtungen & 63 & 13 \\
Hypothetische Differenz der Mittelwerte & 0 & \\
Freiheitsgrade (df) & 60 & \\
t-Statistik & 3,213320812 & \\
P(T<=t) einseitig & 0,001056135 & \\
Kritischer t-Wert bei einseitigem t-Test & 1,670648544 & \\
P(T<=t) zweiseitig & 0,002112269 & \\
Kritischer t-Wert bei zweiseitigem t-Test & 2,000297172 & \\
\hline
\end{tabular}




\begin{tabular}{|lrr|}
\hline \multicolumn{1}{|c}{ Diamant Raffinade $1000 \mathrm{~g}$} & Variable 1 & Variable 2 \\
\hline Mittelwert & 1,908378378 & 1,97333333 \\
Varianz & 0,020763964 & 0,00966667 \\
Beobachtungen & 37 & 6 \\
Hypothetische Differenz der Mittelwerte & 0 & \\
Freiheitsgrade (df) & 9 & \\
t-Statistik & $-1,393645934$ & \\
P(T<=t) einseitig & 0,098438669 & \\
Kritischer t-Wert bei einseitigem t-Test & 1,833113856 & \\
P(T<=t) zweiseitig & 0,196877338 & \\
Kritischer t-Wert bei zweiseitigem t-Test & 2,262158887 & \\
\hline
\end{tabular}

\section{Gesamtauswertung}

Nullhypothese: „Es gibt keine signifikante Preisabweichung in den Überschneidungsgebieten gegenüber dem Gesamtgebiet"

\begin{tabular}{|l|c|c|c|}
\hline & $\begin{array}{l}\text { Nullhypothese nicht } \\
\text { verworfen }\end{array}$ & $\begin{array}{l}\text { Nullhypothese ver- } \\
\text { worfen }\end{array}$ & 9 \\
\hline Nordzucker & 6 & 2 & 8 \\
\hline Südzucker & 6 & 5 & 9 \\
\hline Pfeifer \& Langen & 4 & 10 & 26 \\
\hline Gesamt & 16 & 3 & 2 \\
\hline
\end{tabular}

Anm.: Signifikanzniveau $\alpha=0,05$ 


\section{Lebensmitteleinzelhandel (LEH) Charakteristika}

\section{Exkurs: Wichtige Formen des Handels und ihre Merkmale:}

\section{Definition der Einzelhandelstypen}

SB-Warenhäuser Einzelhandelsgeschäfte mit mindestens $5.000 \mathrm{~m}^{2}$ Verkaufsfläche, die ein breites, warenhausähnliches Sortiment des Lebensmittel- und Nichtlebensmittelbereichs in Selbstbedienung anbieten.

Die Umsatzangaben beziehen sich auf das vom Lebensmitteleinzelhandel üblicherweise definierte FMCG-Sortiment (Fast Moving Consumer Goods).
Große
Einzelhandelsgeschäfte mit einer Verkaufsfläche zwischen 1.500 und $4.999 \mathrm{~m}^{2}$, Verbrauchermärkte die ein breites, warenhausähnliches Sortiment des Lebensmittel- und Nichtlebensmittelbereiches in Selbstbedienung anbieten.
Die Umsatzangaben beziehen sich auf das vom Lebensmitteleinzelhandel üblicherweise definierte FMCG-Sortiment (Fast Moving Consumer Goods).
Kleine
Lebensmittel-Einzelhandeisgeschäfte mit einer Verkaufsfläche zwischen
Supermärkte 800 und $1.499 \mathrm{~m}^{2}$.
Lebensmittel-Einzelhandelsgeschäfte mit einer Verkaufsfläche zwischen 400 und $799 \mathrm{~m}^{2}$.
Discountmärkte
Lebensmittel-Einzelhandelsgeschăfte, für deren Absatzpolitik das Discount- Prinzip (Niedrigpreise, begrenztes Sortiment) maßgebend ist, unabhängig von der Größe der Verkaufsfläche.
Restliche Geschäfte Lebensmittel-Einzelhandelsgeschäfte mit einer Verkaufsfläche unter $400 \mathrm{~m}^{2}$. (unter $\mathbf{4 0 0} \mathrm{m}^{2}$ )

Quelle: AC NIELSEN

\section{E. Daten zur Molkereiwirtschaft}

\section{Entwicklung der Molkereistruktur in Deutschland und Europa}

Anzahl der Unternehmen und Betriebe (Milchindustrie) in Deutschland

\begin{tabular}{|l|l|l|l|l|l|l|l|l|l|l|}
\hline Gebiet & 1973 & 1976 & 1979 & 1982 & 1985 & 1988 & 1991 & 1994 & 1997 & 2000 \\
\hline
\end{tabular}

a) Anzahl Betriebe

\begin{tabular}{|c|c|c|c|c|c|c|c|c|c|c|}
\hline a. BL & 1024 & 890 & 780 & 687 & 617 & 519 & 412 & 353 & 305 & 273 \\
\hline n. BL & & & & & & 267 & 96 & 61 & 51 & 51 \\
\hline$D$ & & & & & & 786 & 508 & 414 & 356 & 324 \\
\hline \multicolumn{11}{|c|}{ b) AnzahI Unternehmen } \\
\hline a. BL & 812 & 708 & 624 & 562 & 515 & 431 & 315 & 262 & 229 & 214 \\
\hline n. BL & & & & & & 120 & 64 & 52 & 40 & 36 \\
\hline $\mathrm{D}$ & & & & & & 551 & 379 & 314 & 269 & 250 \\
\hline
\end{tabular}

Anm.: a. BL = alte Bundesländer; $\mathrm{n} . \mathrm{BL}=$ neue Bundesländer

Quelle : ZMP Marktbilanz Milch, Ifd. Jgg. 
Entwicklung der durchschnittlichen (jährlichen) Milchanlieferung sowie Milchbe- und verarbeitung in 1000 Tonnen in Deutschland

\begin{tabular}{|c|c|c|c|c|c|c|c|c|c|c|}
\hline & 1973 & 1976 & 1979 & 1982 & 1985 & 1988 & 1991 & 1994 & 1997 & 2000 \\
\hline Ailchanlieferung & 18812 & 20046 & 22050 & 23670 & 23637 & 29207 & 26409 & 25862 & 26987 & 27211 \\
\hline be & 25435 & 27292 & 28096 & 30863 & 30903 & 37050 & 34462 & 32931 & 32591 & 3361 \\
\hline
\end{tabular}

Anm.: ab 1988 neue Bundesländer miterfasst

Quelle : ZMP Marktbilanz Milch, Ifd. Jgg.

Anzahl der Betriebe

\begin{tabular}{|c|c|c|c|c|c|c|}
\hline & 1982 & 1985 & 1988 & 1991 & 1994 & 1997 \\
\hline Belgien & 71 & 83 & 77 & 88 & 88 & 111 \\
\hline Dänemark & 167 & 90 & 65 & 52 & 42 & 30 \\
\hline Deutschland & 665 & 609 & 528 & 358 & 284 & 240 \\
\hline Griechenland & . & . & . & 1019 & 1010 & 835 \\
\hline Spanien & . & . & 462 & 497 & 836 & 645 \\
\hline Frankreich & 1497 & 1332 & 1143 & 966 & 815 & 564 \\
\hline Irland & 93 & 90 & 84 & 46 & 71 & 76 \\
\hline Italien & 3115 & 2816 & 2625 & 2430 & 2182 & 1806 \\
\hline Luxemburg & 2 & 2 & 2 & 2 & . & . \\
\hline Niederlande & 49 & 38 & 33 & 22 & 19 & 11 \\
\hline Österreich & . & . & . & . & 133 & 91 \\
\hline Portugal & . & . & 97 & 93 & 113 & 77 \\
\hline Finnland & . & . & . & . & 61 & 26 \\
\hline Schweden & . & . & . & . & 9 & 7 \\
\hline Vereinigtes Königreich & 374 & 336 & $3783)$ & $3403)$ & $2913)$ & 1063 \\
\hline Vereinigtes Königreich & 5914 & 5276 & 4815 & 4242 & 3790 & 5582 \\
\hline
\end{tabular}

Quelle: ZMP, BML, EUROSTAT, Ifd. Jgg.

Durchschnittliche Milchanlieferungsmenge je Molkerei in Europa: (in 1000 t)

$\begin{array}{lcccccc} & 1982 & 1985 & 1988 & 1991 & 1994 & 1997 \\ \text { Belgien } & 43,6 & 37 & 39,8 & 33,7 & 33,9 & 26,6576577 \\ \text { Dänemark } & 30 & 54,4 & 69,8 & 85,4 & 105,5 & 145,633333 \\ \text { Deutschland (a.BL.) } & 43,4 & 48,3 & 53 & 72,5 & 90,4 & . \\ \text { Deutschland gesamt } & . & . & . & . & 91,7 & 110,7125 \\ \text { Griechenland } & . & . & . & 1,1 & 1,2 & . \\ \text { Spanien } & \cdot & . & 9,5 & 7,2 & 5,3 & . \\ \text { Frankreich } & 17,3 & 19,5 & 21,4 & 24,6 & 29,1 & 40,836578 \\ \text { Irland } & 53,2 & 63,1 & 61,8 & 105,5 & 74,2 & 69,1710526 \\ \text { Italien } & 2,5 & 3 & 3,2 & 4 & 4,5 & 7,47951274\end{array}$


Niederlande

Österreich

Fortsetzung

Portugal

Finnland

Schweden

Vereinigtes König-

reich

EU-9

Quelle: ZMP , BML
252,6

321,9

334

478,9

552,4

949,090909

12,2

15,4

12,8

43,9

45,4

55,3

16,8

19,1

19,3
41,5

24
16,5

39,1

91,2

258,2

468

26,6

$47,2 \quad 14,1683913$

\section{Kostenstruktur Milchprodukte}

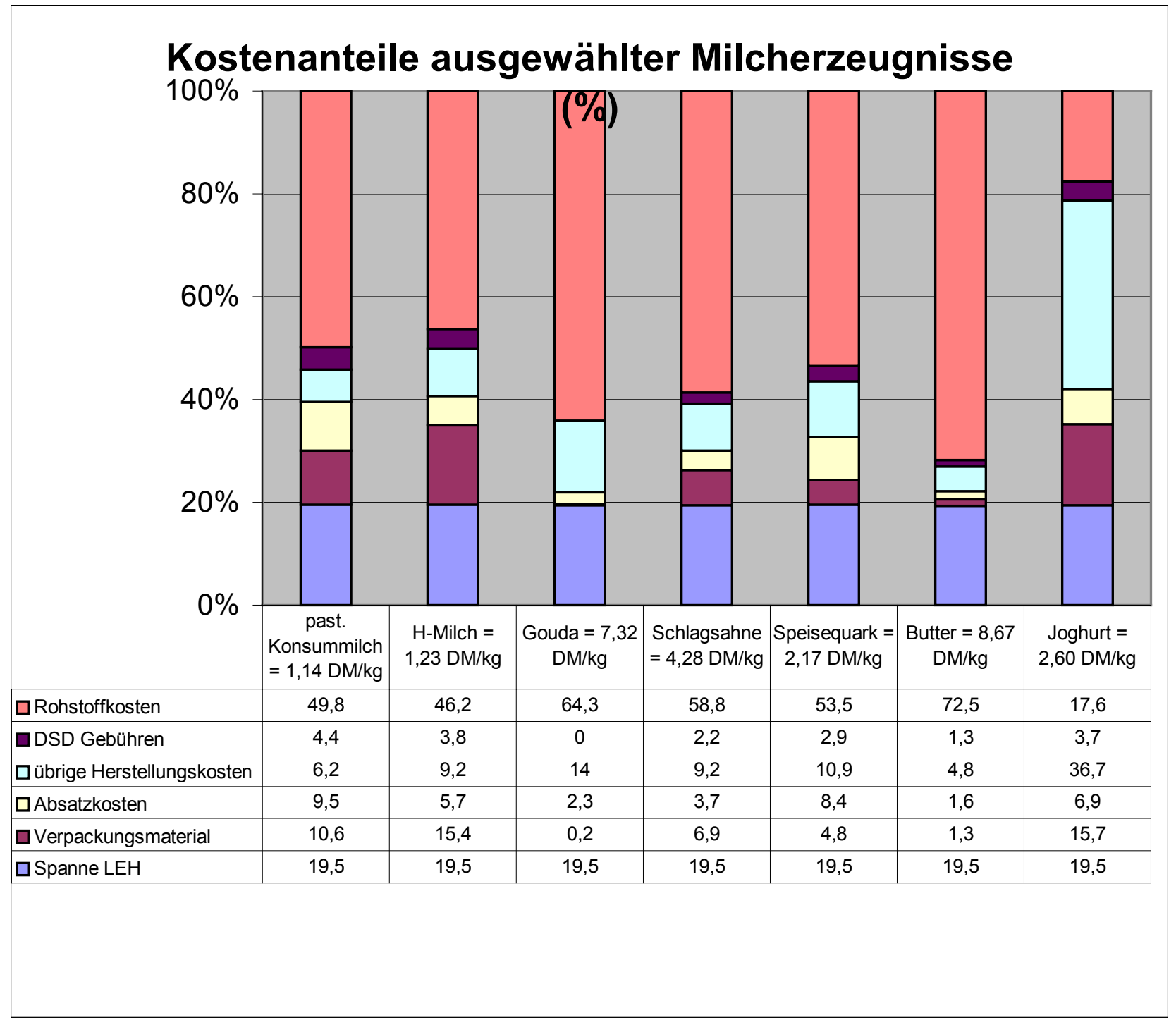

Quelle: WissenschaftLiCher Beirat 2000, Anhang 1, S. 56 


\section{Genossenschaftlicher Korrekturfaktor}

Veränderung wichtiger Jahresabschlusspositionen und Kennzahlen durch Aufbereitungsmaßnahmen (auf Basis 22 Jahresabschlüsse deutscher Molkereiunternehmen für das Jahr 1997)

\begin{tabular}{|l|l|l|l|l|}
\hline Kennzahl & Definition & Min. & Max. & $\begin{array}{l}\text { Durchschnitt } \\
\text { (gew.) }\end{array}$ \\
\hline $\begin{array}{l}\text { Veränderung des Eigenkapi- } \\
\text { tals }\end{array}$ & $\begin{array}{l}\text { (korr. EK - unkorr. EK) / } \\
\text { unkorr. EK }\end{array}$ & $-4,7 \%$ & $0,5 \%$ & $-0,4 \%$ \\
\hline $\begin{array}{l}\text { Veränderung der Bilanzsum- } \\
\text { me }\end{array}$ & $\begin{array}{l}\text { (korr. Bilanzsumme - unkorr. } \\
\text { Bilanzsumme)/unkorr. Bilanz- } \\
\text { summe }\end{array}$ & $-0,9 \%$ & $1,7 \%$ & $0,1 \%$ \\
\hline $\begin{array}{l}\text { Veränderung der Eigenkapi- } \\
\text { talquote }\end{array}$ & $\begin{array}{l}\text { Korr. EK-Quote /unkorr. EK- } \\
\text { Quote }\end{array}$ & $-2,4 \%$ Punkte & $0,2 \%$ Punkte & $-0,2 \%$ Punkte \\
\hline $\begin{array}{l}\text { Genossenschaftlicher Korrek- } \\
\text { turbetrag pro kg Eigenmilch } \\
\text { (nur Genossenschaften) }\end{array}$ & $\begin{array}{l}\text { (genossenschaftl. Korrektur- } \\
\text { betrag/ Eigenmilchmenge) } * \\
100\end{array}$ & $-2,5$ Pf & 5,5 Pf & 0,0 Pf \\
\hline $\begin{array}{l}\text { Veränderung des ordentlichen } \\
\text { Betriebserfolges pro kg Roh- } \\
\text { stoffeingang }\end{array}$ & $\begin{array}{l}\text { [(korr. Ordentlicher Betriebs- } \\
\text { erfolg - ordentlicher Be- } \\
\text { triebserfolg) / Rohstoffein- } \\
\text { gang] } * 100\end{array}$ & $-1,9$ Pf & 5,4 Pf & $-0,1$ Pf \\
\hline $\begin{array}{l}\text { Veränderung des ordentlichen } \\
\text { Betriebserfolges pro kg Roh- } \\
\text { stoffeingang }\end{array}$ & $\begin{array}{l}\text { Veränderung des ordentlichen } \\
\text { Betriebserfolges pro kg Roh- } \\
\text { stoffeingang - Genossen- } \\
\text { schaftlicher Korrekturbetrag } \\
\text { pro kg Eigenmilch }\end{array}$ & $-1,1$ Pf & 0,6 Pf & $-0,1$ Pf \\
\hline $\begin{array}{l}\text { Veränderung der } \\
\text { Gesamtkapitalrentabilität } \\
\text { tät - unkorr. Gesamtkapital- } \\
\text { rentabilität }\end{array}$ & $-9,8 \%$ Punkte & $15,7 \%$ Punkte & $0,7 \%$ Punkte \\
\hline
\end{tabular}

Quelle: BRAUn 2000b, S. 16

\section{ZMP-Vergleichspreise}

\begin{tabular}{|l|r|r|r|r|r|r|}
\hline \multicolumn{7}{|c|}{ Auszahlungspreise für Betriebe mit 500000 kg in Pfennig } \\
\cline { 2 - 7 } & pro kg Milch (3,7\% Fett/3,4\% Protein) \\
\hline Deutschland & $\mathbf{5 7 , 6 6}$ & $\mathbf{5 8 , 4 2 6 5 8 7}$ & $\mathbf{6 0 , 8 6 0 2 1 2}$ & $\mathbf{5 7 , 5 3 4 8 7 4}$ & $\mathbf{6 1 , 5 1 6 9 3 4}$ & $\mathbf{6 6 , 7 1 9 8 2 8}$ \\
\hline Norddeutschland: & $\mathbf{5 7 , 3 3}$ & $\mathbf{5 8 , 1 9 5}$ & $\mathbf{6 1 , 1 9 2 5}$ & $\mathbf{5 7 , 9 0 7 5}$ & $\mathbf{6 1 , 6 8 2 5}$ & $\mathbf{6 6 , 1}$ \\
\hline Mecklenburg-Vorpommern & 56,28 & 57,88 & 61,23 & 57,6 & 62,13 & 67,04 \\
Schleswig-Holstein & 57,99 & 58,69 & 61,3 & 57,32 & 62,36 & 65,17 \\
Niedersachsen & 57,11 & 58,01 & 60,89 & 57,43 & 61 & 65,85 \\
Nordrhein-Westfalen & 57,94 & 58,2 & 61,35 & 59,28 & 61,24 & 66,34 \\
\hline Nordmilch & $\mathbf{5 7 , 1}$ & $\mathbf{5 7 , 6}$ & $\mathbf{6 1 , 7}$ & $\mathbf{5 7}$ & $\mathbf{5 9 , 3}$ & $\mathbf{6 4 , 9 3}$ \\
\hline Bremerland & 57,5 & 57,2 & 60,9 & 56,4 Fusion & Fusion \\
Hansano & 56,4 & 56,3 & 59,8 & 56,5 Fusion & Fusion \\
MzO & 55,1 & 57,5 & 60,5 & 57,3 Fusion & Fusion \\
\hline Jahr: & $\mathbf{1 9 9 6}$ & $\mathbf{1 9 9 7}$ & $\mathbf{1 9 9 8}$ & $\mathbf{1 9 9 9}$ & $\mathbf{2 0 0 0}$ & $\mathbf{2 0 0 1}$ \\
\hline
\end{tabular}

Quelle: eigene Darstellung auf Grundlage ZMP 


\section{Erfasste Molkereien:}

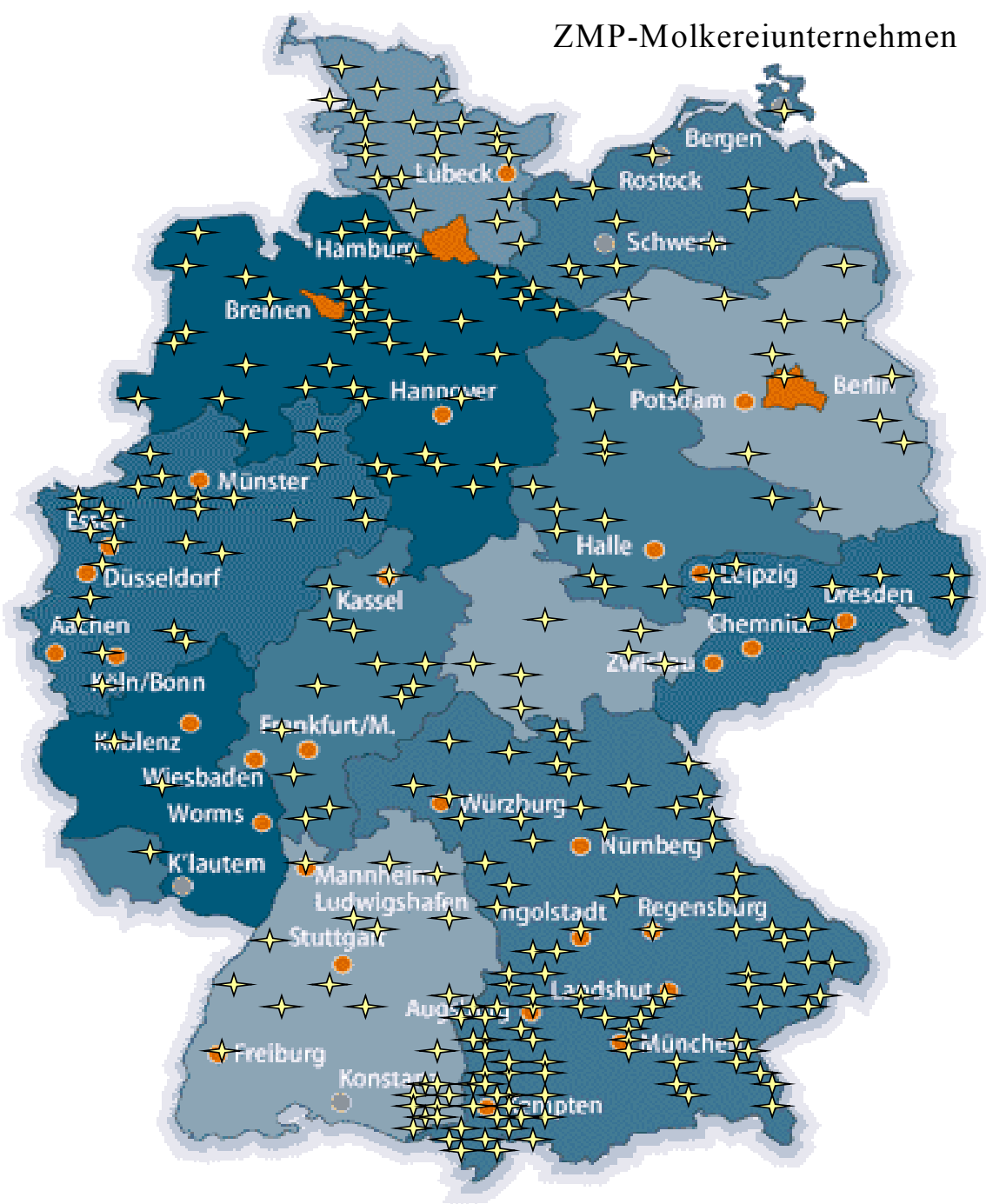

Quelle: eigene Darstellung auf Grundlage ZMP

\section{F. Veröffentlichungspflicht}

Kriterien für die Einordnung in eine Größenklasse bzw. für die Publizitätspflicht

\begin{tabular}{|c|c|c|c|}
\hline & Bilanzsumme in Mio. $€$ & Umsatz in Mio. $€$ & Arbeitnehmer \\
\hline \multicolumn{4}{|c|}{ Kapitalgesellschaften und Genossenschaften } \\
\hline kleine & $\leq 3,125$ & $\leq 6,25$ & $\leq 50$ \\
\hline mittelgroße & $\leq 12,5$ & $\leq 25$ & $\leq 250$ \\
\hline große & $>12,5$ & $>25$ & $>250$ \\
\hline \multicolumn{4}{|c|}{ Einzel- und Personengesellschaften } \\
\hline Publizitätspflicht & > 125 Mio. DM & > 250 Mio. DM & $>5000$ \\
\hline
\end{tabular}

Quelle: Grünberger 2001; Braun 2000b, S. 4 (in Anlehnung an Coenenberg 1994, S. 21 f) 
Größen- und rechtsformabhängige Publizitätspflicht

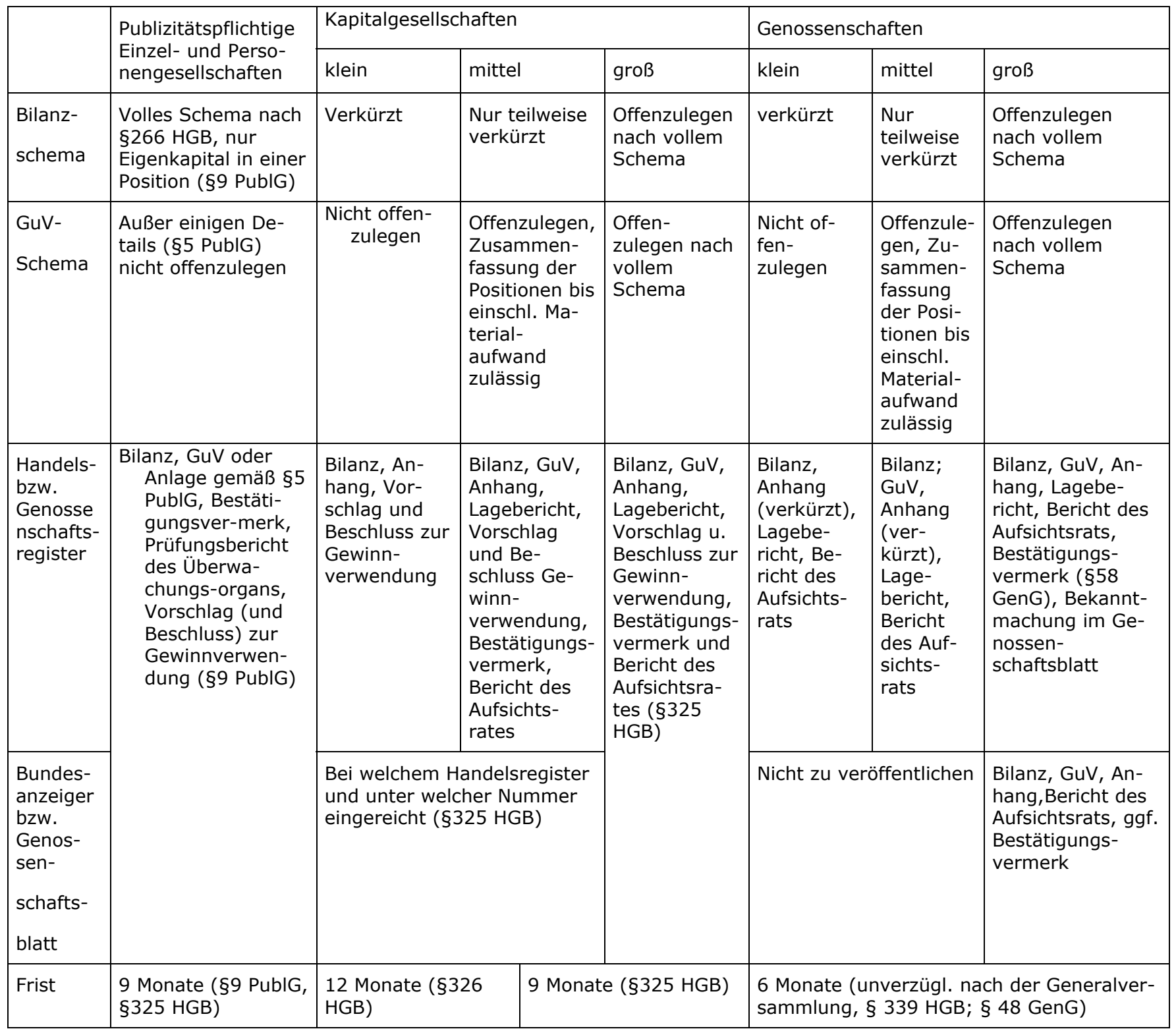

Anm.: GenG = Genossenschaftsgesetz; PublG = Publizitätsgesetz; HGB = Handelsgesetzbuch; GuV = Gewinn- und Verlustrechnung

Quelle: BRAUn 2000b, S. 5; vgl. dazu BIENERT 1986

\section{G. Definition von Erfolgskennziffern}

Folgende Definitionen der Erfolgskennziffern werden zur Bilanzanalyse herangezogen ${ }^{827}$ (selbsterklärende Kennziffern werden nicht erläutert):

Cash- flow: gibt Auskunft über das Selbstfinanzierungspotential eines Unternehmens. Offizielle Definition: Jahresaufwand + auszahlungslose Aufwendungen - einzahlungslose Erträge. Hier

827 Definitionen in Anlehnung an: GRÜNBERGER 2001; WöHE 1996; SCHULT 1997; SCHULT 1999; GRÄFER 1997 
wird die vereinfachte Definition verwendet: Cash-flow einfach = Jahresüberschuss+Abschreibungen

Schuldentilgungsdauer $=$ Fremdkapital ${ }^{828} /$ Cash flow: innerhalb so vieler Jahre kann Unternehmen Schulden tilgen, wenn Cash flow gleich bleibt

Goldene Bilanzregel = Eigenkapital/ Anlagevermögen: Anlagevermögen sollte durch Eigenkapital gedeckt sein.

Deckungsgrad A = Eigenkapital/ Anlagevermögen (*100)

Selbstfinanzierungsgrad $=$ Gewinnrücklage/ Gesamtkapital $(* 100)$

Wachstumsmöglichkeit $=$ Cash-flow einfach $(* 100) /$ Gesamtkapital

EK-Rentabilität nach Steuer = Jahresüberschuss/ EK $(* 100)$

EK-Rentabilität vor Steuer = (Jahresüberschuss + Steuern vom Einkommen u. Ertrag)*100/ Eigenkapital

Umsatzrentabilität a) = Betriebsergebnis/ Umsatzerlöse $(* 100)$

Umsatzrentabilität b) = Jahresüberschuss/ Umsatzerlöse (*100)

Gesamtkapitalrentabilität $=(\mathrm{JÜ}+$ Steueraufwand + Fremdkapitalzinsen $) /$ GK $(* 100)$

Personalintensität $=$ Personalaufwand $/$ Betriebsergebnis $(* 100)$

Kapitalintensität $=$ Abschreibungen/ Betriebsergebnis $(* 100)$

Relativer Reingewinn = Jahresüberschuss/ Gesamtkapital (*100)

Arbeitsintensität (Umlaufintensität) = Umlaufvermögen/ Gesamtvermögen $(* 100)$

Anlagenintensität $=$ Anlagevermögen/ Gesamtvermögen $(* 100)$

\section{H. Ergebnisse der Bilanzanalyse ausgewählter Zusammenschlüs- se und Vergleichsunternehmen:}

${ }^{828}$ Fremdkapital $=$ Fremdkapital $+\left(0,5^{*}\right.$ Sonderposten mit Rücklagenanteil $)$ 


\section{Milchindustrie:}

\section{Unterstellte Fusion}

\section{Nordmilch Betrieb}

Umsatz DM

Jahresüberschuss/-unterschuss

Betriebsergebnis

Eigenkapital (=EK)

Fremdkapital (=FK)

Gesamtkapital

Anlagevermögen

Umlaufvermögen

Abschreibungen

Personalaufwand (Mio. DM)

Mitarbeiter

Milchverarbeitung $\mathrm{kg}$

FK-Quote

EK-Quote

EK-Rentabilität vor Steuer

EK-Rentabilität nach Steuer

Gesamtkapitalrentabilität

Umsatzrentabilität

a) Betriebsergebnis (=BE)

b) Jahresüberschuss (=JÜ)

Cash-flow einfach

Relativer Reingewinn

\begin{tabular}{|c|c|c|c|c|c|c|c|c|}
\hline 1994 & 1995 & 1996 & 1997 & 1998 (errechnet) & 1998 & 1999 & 2000 & 2001 \\
\hline 3942012767 & 4226179909 & 4150087361 & 4119260105 & 4337756143 & 4208942321 & 4035518816 & 4131540421 & 4385057900 \\
\hline$-1059899,8$ & 1480735,27 & $-22278711,7$ & $-2867161,08$ & 1635865,53 & 1687428,45 & 3013034,67 & 3604427,61 & -24889075 \\
\hline 7547392,05 & 7191990,53 & $-16106560,9$ & 2089353,78 & 6976144,21 & 7229392,25 & 8604837,49 & 5390539,29 & $-22690717,4$ \\
\hline 313387763 & 346487719 & 342723090 & 356658241 & 372951050 & 392577172 & 377563528 & 387732446 & 369063657 \\
\hline 673544052 & 764818915 & 725226934 & 691481475 & 682112211 & 662073897 & 618300801 & 711163047 & 803943248 \\
\hline 986931816 & 1111306634 & 1067950024 & 1048139716 & 1055063261 & 1054651070 & 995864328 & 1098895493 & 1173006906 \\
\hline 437723436 & 470369901 & 465532341 & 431407668 & 432140842 & 432361449 & 414617798 & 417169100 & 436098290 \\
\hline 547304334 & 640150622 & 601547626 & 615993350 & 622405633 & 1054099960 & 995391300 & 1098060691 & 1172851618 \\
\hline 94678160 & 86579909,1 & 97732520,2 & 85681162,1 & 76852859,7 & 77964047,7 & 82672708,5 & 81332979,1 & 79983536,6 \\
\hline 283548521 & 304347082 & 312610431 & 300425328 & 296468792 & 304759400 & 304475112 & 314149620 & 247695526 \\
\hline 3670 & 4303 & 4249 & 4001 & - & 4051 & 3995 & 3909 & 3684 \\
\hline 3641159200 & 3896414000 & 3863194000 & 3715344000 & 3757462000 & 3651693000 & 3671647000 & 3642293000 & 3789482000 \\
\hline 68,24626 & 68,8215918 & 67,9083213 & 65,9722616 & 64,6513092 & 62,7765824 & 62,0868509 & 64,7161675 & 68,5369578 \\
\hline \multirow[t]{2}{*}{31,75374} & 31,1784082 & 32,0916787 & 34,0277384 & 35,3486908 & 37,2234176 & 37,9131491 & 35,2838325 & 31,4630422 \\
\hline & 0,92516202 & $-5,95269796$ & $-0,31995067$ & 1,45073166 & 1,43775736 & 0,79045029 & 0,9294884 & $-6,74384384$ \\
\hline$-0,33820714$ & 0,42735577 & $-6,5004992$ & $-0,80389593$ & 0,43862741 & 0,42983356 & 0,79802058 & 0,92961723 & $-6,74384338$ \\
\hline 2,35518132 & 1,96130079 & $-0,1897477$ & 1,12235034 & 1,46582399 & 1,48857783 & 41,9336488 & 1,20874455 & 1019,01266 \\
\hline 0,19146037 & 0,1701771 & $-0,38810173$ & 0,05072158 & 0,1608238 & 0,17176268 & 0,21322754 & 0,13047287 & $-0,51745537$ \\
\hline$-0,02688727$ & 0,0350372 & $-0,53682513$ & $-0,06960379$ & 0,03771225 & 0,04009151 & 0,07466288 & 0,08724174 & $-0,56758829$ \\
\hline 93618260,2 & 88060644,4 & 75453808,5 & 82814001 & 78488725,2 & 79651476,1 & 85685743,1 & 84937406,7 & 55094461,6 \\
\hline$-0,10739342$ & 0,13324273 & $-2,08611931$ & $-0,2735476$ & 0,15504905 & 0,15999874 & 0,30255473 & 0,32800459 & $-2,12181829$ \\
\hline
\end{tabular}




\section{Cashflow*100/Umsatz \\ JÜ/Umsatz ${ }^{*} 100$ \\ Umsatz DM pro Mitarbeiter \\ JÜ DM pro Mitarbeiter}

Aufwand DM pro Mitarbeiter

Milch kg pro Mitarbeiter

Personalintensität

Umsatz je kg Milch

JÜ je kg Milch

Anlageintensität

Kapitalintensität

Arbeitsintensität

Selbstfinanzierungsgrad

Deckungsgrad A:

Wachstumsmögl.keit:

Schuldentilgungsdauer

Goldene Bilanzregel

Kosten=Umsatz-JÜ

vor Steuer: U-BE

Anlagevermögen pro $t$ Milch

\section{Unterstellung Fusion}

\section{Nordmilch Konzern}

Umsatz DM

Jahresüberschuss/-unterschuss

Betriebsergebnis

Eigenkapital

\begin{tabular}{|c|c|c|c|c|c|c|c|c|}
\hline 2,37488475 & 2,0836937 & 1,81812579 & 2,01040961 & 1,80943148 & 1,89243449 & 2,1232894 & 2,05582901 & 1,25641355 \\
\hline$-0,02688727$ & 0,0350372 & $-0,53682513$ & $-0,06960379$ & 0,03771225 & 0,04009151 & 0,07466288 & 0,08724174 & $-0,56758829$ \\
\hline 1074117,92 & 982147,318 & 976720,961 & 1029557,64 & - & 1038988,48 & 1010142,38 & 1056930,27 & 1190298,02 \\
\hline$-288,801035$ & 344,116958 & $-5243,28353$ & $-716,611117$ & - & 416,546149 & 754,201419 & 922,084321 & $-6755,99214$ \\
\hline 77261,1775 & 70729,0453 & 73572,7067 & 75087,56 & - & 75230,6591 & 76214,0455 & 80365,7254 & 67235,4848 \\
\hline 992141,471 & 905511,039 & 909200,753 & 928603,849 & - & 901430,017 & 919060,576 & 931771,041 & 1028632,46 \\
\hline 3756,90728 & 4231,75031 & $-1940,88877$ & 14378,8635 & 4249,75149 & 4215,56044 & 3538,41792 & 5827,79576 & $-1091,61611$ \\
\hline 1,08262577 & 1,08463318 & 1,07426326 & 1,10871567 & 1,15443779 & 1,15260027 & 1,09910316 & 1,13432402 & 1,15716552 \\
\hline$-0,00029109$ & 0,00038003 & $-0,00576692$ & $-0,00077171$ & 0,00043536 & 0,00046209 & 0,00082062 & 0,0009896 & $-0,00656794$ \\
\hline 44,351943 & 42,325843 & 43,5912103 & 41,1593666 & 40,9587613 & 40,9956868 & 41,6339642 & 37,9625818 & 37,1778109 \\
\hline 1254,44868 & 1203,83792 & $-606,787017$ & 4100,8451 & 1101,65239 & 1078,43156 & 960,770132 & 1508,80969 & $-352,494525$ \\
\hline 55,4551313 & 57,6034194 & 56,3273198 & 58,7701563 & 58,9922573 & 99,9477448 & 99,9525007 & 99,9240327 & 99,9867615 \\
\hline 13,2803443 & 12,2436493 & 10,7412811 & 10,5709496 & 10,5955307 & 11,6828741 & 12,6468616 & 11,8323926 & 11,2369378 \\
\hline 71,5949244 & 73,662817 & 73,6196091 & 82,6731343 & 86,3031246 & 90,7983755 & 91,0630296 & 92,9437117 & 84,6285496 \\
\hline 9,48578805 & 7,92406359 & 7,06529395 & 7,90104599 & 7,43924351 & 7,55240083 & 8,60415829 & 7,72934344 & 4,69685739 \\
\hline 7,19457989 & 8,68513876 & 9,61153518 & 8,34981364 & 8,69057574 & 8,31213594 & 7,21591221 & 8,37278974 & 14,5920883 \\
\hline 0,71594924 & 0,73662817 & 0,73619609 & 0,82673134 & 0,86303125 & 0,90798376 & 0,9106303 & 0,92943712 & 0,8462855 \\
\hline 3943072667 & 4224699173 & 4172366073 & 4122127266 & 4336120277 & 4207254892 & 4032505781 & 4127935993 & 4409946975 \\
\hline 3934465375 & 4218987918 & 4166193922 & 4117170751 & 4330779999 & 4201712928 & 4026913978 & 4126149881 & 4407748617 \\
\hline 120,215407 & 120,718666 & 120,504521 & 116,115134 & 115,008706 & 118,400273 & 112,924199 & 114,534745 & 115,081241 \\
\hline
\end{tabular}

1994

1995

4168089085

4506585650

$-1040350,47$

1601895,17

7674819,17

7328007,85

313542342
346594006
1996

19971998 (errechnet)

1998

4403590412

4682166832

4729652074

4849380,81

$-15986772$

34278210
649370,36

7026856,01

341800883
11886125

363300970

4575086714
4900943,73
13015850,9
382382836

2000

4371723356

4316304471

5238250,72

8704114,58

8129537,96

386058399
2001

4679640501

$-23924559,9$

$-21163137,1$

368368326 
Fremdkapital

Gesamtkapital

Anlagevermögen

Umlaufvermögen

Abschreibungen

Personalaufwand (Mio. DM)

Mitarbeiter

Milchverarbeitung $\mathrm{kg}$

FK-Quote

EK-Quote

EK-Rentabilität vor Steuer

EK-Rentabilität nach Steuer

Gesamtkapitalrentabilität

Umsatzrentabilität

a) $B E$

b) JÜ

Cash-flow einfach

Relativer Reingewinn

Cashflow ${ }^{*} 100 /$ msatz

JÜ/Umsatz ${ }^{\star} 100$

Umsatz DM pro Mitarbeite

JÜ DM pro Mitarbeiter

Aufwand DM pro Mitarbeiter

Milch kg pro Mitarbeite

Personalintensität

Umsatz je kg Milch

\begin{tabular}{|c|c|c|c|c|c|c|c|c|}
\hline 724798684 & 795169695 & 763776506 & 808601972 & 780729363 & 768006680 & 714021219 & 781879240 & 883759928 \\
\hline 1038341026 & 1141763701 & 1106558607 & 1150402855 & 1144030333 & 1150389516 & 1086999534 & 1167937639 & 1252128255 \\
\hline 466955638 & 492482012 & 489158482 & 485437163 & 477331920 & 479585862 & 454403620 & 452556961 & 481976069 \\
\hline 569396511 & 648489661 & 616523167 & 663356044 & 665850045 & 1149509993 & 1086257648 & 1167035490 & 1251830586 \\
\hline 101484652 & 104248523 & 103989893 & 107943582 & 93138609,7 & 95119951,7 & 96217799 & 94390612,8 & 93810192,4 \\
\hline 301882167 & 323273154 & 331538784 & 339735565 & 334837402 & 345695664 & 341276634 & 348677257 & 355923875 \\
\hline 4036 & 4651 & 4586 & - & - & 4587 & 4592 & 4446 & 4436 \\
\hline 3912959200 & 4198614000 & 4178194000 & - & - & 4132000000 & 4132000000 & 3967000000 & 3894000000 \\
\hline 69,8035295 & 69,643981 & 69,022689 & 70,2885923 & 68,2437642 & 66,7605772 & 65,6873528 & 66,9452901 & 70,5806234 \\
\hline 30,1964705 & 30,356019 & 30,977311 & 29,7114077 & 31,7562358 & 33,2394228 & 34,3126472 & 33,0547099 & 29,4193766 \\
\hline 1,97339214 & 0,95300341 & $-5,95023425$ & 1,09046402 & 2,68669693 & 2,74304983 & 2,70550565 & 1,58956632 & $-6,41520872$ \\
\hline$-0,33180542$ & 0,46218202 & $-6,49858057$ & 0,18998499 & 1,33481086 & 1,28168507 & 2,33367845 & 1,35685449 & $-6,49473861$ \\
\hline 2,41360885 & 2,03490389 & $-0,09643027$ & 1,81751869 & 1,99286423 & 2,06937666 & 1,75799614 & 1,5197664 & $-0,74817014$ \\
\hline 0,1841328 & 0,16260665 & $-0,3630395$ & 0,15007701 & 0,25131077 & 0,28449408 & 0,36442028 & 0,18834487 & $-0,45223852$ \\
\hline$-0,02495989$ & 0,03554565 & $-0,50585929$ & 0,01386901 & 0,10253145 & 0,10712242 & 0,19910031 & 0,12135962 & $-0,51124782$ \\
\hline 100444302 & 105850418 & 81713922,2 & 108592953 & 97987990,5 & 100020895 & 104921914 & 99628863,5 & 69885632,5 \\
\hline$-0,10019352$ & 0,14030006 & $-2,01308551$ & 0,05644721 & 0,42388568 & 0,42602472 & 0,80074685 & 0,44850432 & $-1,91071161$ \\
\hline 2,40984056 & 2,34879411 & 1,8556204 & 2,31928841 & 2,0717801 & 2,18620764 & 2,40001265 & 2,30819823 & 1,49339746 \\
\hline$-0,02495989$ & 0,03554565 & $-0,50585929$ & 0,01386901 & 0,10253145 & 0,10712242 & 0,19910031 & 0,12135962 & $-0,51124782$ \\
\hline 1032727,72 & 968949,828 & 960224,686 & - & - & 997402,815 & 952030,348 & 970828,716 & 1054923,47 \\
\hline$-257,767708$ & 344,419516 & $-4857,38574$ & - & - & 1068,44206 & 1895,49534 & 1178,19404 & $-5393,2732$ \\
\hline 74797,3656 & 69506,1609 & 72293,673 & - & - & 75364,2171 & 74319,8245 & 78424,9342 & 80235,3191 \\
\hline 969514,172 & 902733,606 & 911075,883 & - & - & 900806,627 & 899825,784 & 892262,708 & 877817,854 \\
\hline 3933,41082 & 4411,47391 & $-2073,83187$ & 4834,81609 & 2817,04427 & 2655,95901 & 2142,15726 & 4289,01691 & $-1681,81056$ \\
\hline 1,06520126 & 1,07335079 & 1,05394589 & - & - & 1,10723299 & 1,0580163 & 1,08805255 & 1,20175668 \\
\hline
\end{tabular}




\section{JÜ je kg Milch \\ Anlageintensität \\ Kapitalintensität \\ Arbeitsintensität \\ Selbstfinanzierungsgrad \\ Deckungsgrad A: \\ Wachstumsmögl.keit: \\ Schuldentilgungsdauer \\ Goldene Bilanzrege \\ Kosten=Umsatz-JÜ \\ vor Steuer: U-BE}

Anlagevermögen pro $\mathrm{t}$ Milch

\section{Einzelergebnisse vor der Fusion}

\section{Nordmilch Betrieb}

Umsatz DM

Jahresüberschuss/-unterschuss

Betriebsergebnis

Eigenkapital

Fremdkapital

Gesamtkapital

Anlagevermögen

Umlaufvermögen

Abschreibungen

Personalaufwand (Mio. DM)

Mitarbeiter

Milchverarbeitung kg

$\begin{array}{rrrllllll}-0,00026587 & 0,00038153 & -0,00533148 & - & - & 0,00118609 & 0,00210651 & 0,00132046 & -0,00614395 \\ 44,9713173 & 43,1334445 & 44,2053841 & 42,1971452 & 41,7237119 & 41,688998 & 41,8034788 & 38,7483839 & 38,4925479 \\ 1322,30675 & 1422,60387 & -650,474591 & 1536,15759 & 783,591034 & 730,800872 & 603,948927 & 1161,08213 & -443,271675 \\ 54,837139 & 56,7971867 & 55,715365 & 57,6629344 & 58,2021321 & 99,9235457 & 99,9317492 & 99,9227571 & 99,976227 \\ 12,6231044 & 11,9170444 & 10,3710463 & 8,01943469 & 8,45579678 & 9,57577831 & 10,8346615 & 10,7701147 & 10,1980276 \\ 67,1460662 & 70,3769879 & 70,0758779 & 70,4109427 & 76,1107638 & 79,7318824 & 82,0808416 & 85,3060349 & 76,4287586 \\ 9,67353683 & 9,27078153 & 7,3845092 & 9,43955868 & 8,56515668 & 8,69452425 & 9,65243409 & 8,5303239 & 5,58134778 \\ 7,21592637 & 7,51220173 & 9,34695687 & 7,44617356 & 7,96760255 & 7,67846236 & 6,80526302 & 7,84791889 & 12,6458028 \\ 0,67146066 & 0,70376988 & 0,70075878 & 0,70410943 & 0,76110764 & 0,79731882 & 0,82080842 & 0,85306035 & 0,76428759 \\ 4169129436 & 4504983755 & 4425866383 & 4681517461 & 4724802693 & 4570185770 & 4363019242 & 4311066221 & 4703565061 \\ 4160414266 & 4499257642 & 4419577185 & 4675139976 & 4717765949 & 4562070863 & 4355791910 & 4308174933 & 4700803638 \\ 119,335678 & 117,29633 & 117,074143 & - & - & 116,066278 & 109,971834 & 114,080403 & 123,774029\end{array}$

1990

1991

1992

1993

1994

1995

1996

1997

1998

871415062

926044921

1015719222

1478232891

1423456752
1487604397

1490442340

1507417247

502275,5

2766305,19

108392757

219169746

327562503

119258679

208134892

28774336

105156228

1434

1471000000

1710954203

502089,93

4914582,27

112297278

211708756

324006033

116640822

207212618

25917370

105345802

1477

1506000000
1474

1512000000
1492600000 


\begin{tabular}{|c|c|c|c|c|c|c|c|c|c|}
\hline FK-Quote & 65,0240137 & 65,234565 & 69,8120142 & 73,0812728 & 73,0137994 & 70,4895897 & 66,9927515 & 66,9092902 & 65,3409918 \\
\hline EK-Quote & 34,9759863 & 34,765435 & 30,1879858 & 26,9187272 & 26,9862006 & 29,5104103 & 33,0072485 & 33,0907098 & 34,6590082 \\
\hline EK-Rentabilität vor Steuer & 10,0336152 & 10,6288516 & 3,13043391 & 2,27993658 & 5,69436806 & 1,77407814 & 1,36419959 & 1,76994739 & 3,979846 \\
\hline EK-Rentabilität nach Steuer & 4,01904791 & 3,65452495 & 1,02426309 & 0,6551723 & 1,81849954 & 1,03778091 & 0,38620568 & 0,46338474 & 0,44710784 \\
\hline Gesamtkapitalrentabilität & 3,54929235 & 4,26957549 & 1,48457727 & 2,25271436 & 3,10027191 & 1,69534348 & 1,60205246 & 1,56327694 & 1,89438565 \\
\hline \multicolumn{10}{|l|}{ Umsatzrentabilität } \\
\hline a) $\mathrm{BE}$ & 0,88802088 & 0,96892425 & 0,40293538 & 0,20334001 & 0,39260319 & 0,17812096 & 0,17687306 & 0,18351291 & 0,28724219 \\
\hline b) JÜ & 0,29470951 & 0,27597232 & 0,07397559 & 0,03397818 & 0,10191751 & 0,06750231 & 0,02699822 & 0,03332027 & 0,02934561 \\
\hline Cash-flow einfach & 21734130,1 & 23781293,6 & 25746128,1 & 38022070,7 & 34801882,7 & 33703447,3 & 31875760 & 29276611,5 & 26419459,9 \\
\hline Relativer Reingewinn & 1,40570164 & 1,2705115 & 0,30920439 & 0,17636404 & 0,49074394 & 0,30625341 & 0,12747587 & 0,1533373 & 0,15496314 \\
\hline Cashflow*100/Umsatz & 2,49411917 & 2,56804968 & 2,53476822 & 2,57212994 & 2,44488515 & 2,26561896 & 2,13867783 & 1,9421704 & 1,54413601 \\
\hline JÜ/Umsatz ${ }^{*} 100$ & 0,29470951 & 0,27597232 & 0,07397559 & 0,03397818 & 0,10191751 & 0,06750231 & 0,02699822 & 0,03332027 & 0,02934561 \\
\hline Umsatz DM pro Mitarbeiter & 872287,349 & 829789,356 & 888643,239 & 1068859,65 & 976977,867 & 1007179,69 & 1011154,91 & 1051197,52 & \\
\hline JÜ DM pro Mitarbeiter & 2570,71379 & 2289,98891 & 657,379064 & 363,179009 & 995,711558 & 679,869546 & 272,993867 & 350,261855 & \\
\hline Aufwand DM pro Mitarbeiter & 56135,2667 & 57152,5838 & 61771,597 & 63068,3466 & 65148,5999 & 67144,7435 & 69870,5712 & 73330,7026 & \\
\hline Milch kg pro Mitarbeiter & 828528,529 & 723476,703 & 689763,78 & 1046131,6 & 972065,889 & 1019634,39 & 1025780,19 & 1025801,95 & \\
\hline Personalintensität & 724,691289 & 710,850352 & 1725,14652 & 2901,80299 & 1698,50386 & 3742,74324 & 3906,74358 & 3801,32416 & 2143,53523 \\
\hline Umsatz je kg Milch & 1,0528151 & 1,14694689 & 1,28832981 & 1,0217258 & 1,00505313 & 0,98778512 & 0,98574229 & 1,0247568 & 1,14629117 \\
\hline JÜ je kg Milch & 0,00310275 & 0,00316526 & 0,00095305 & 0,00034716 & 0,00102433 & 0,00066678 & 0,00026613 & 0,00034145 & 0,00033639 \\
\hline Anlageintensität & 40,7325204 & 48,1431428 & 44,8688917 & 47,3361552 & 43,3877188 & 37,6601392 & 39,434975 & 36,4079153 & 35,9995834 \\
\hline Kapitalintensität & 247,675445 & 236,558983 & 610,716448 & 1248,23037 & 596,777534 & 1234,05839 & 1193,89556 & 1040,17214 & 527,356519 \\
\hline Arbeitsintensität & 58,6938898 & 51,3077068 & 54,7179569 & 52,2067255 & 56,5351132 & 62,2990852 & 60,5270653 & 63,540512 & 63,9533208 \\
\hline Selbstfinanzierungsgrad & 14,0382143 & 14,0239684 & 12,2889185 & 10,7059883 & 10,6441097 & 9,97145706 & 10,5800964 & 10,3338275 & 10,6022777 \\
\hline Deckungsgrad A: & 85,8674738 & 72,2126413 & 67,2804355 & 56,8671601 & 62,1977864 & 78,3598014 & 83,7004423 & 90,8887792 & 96,2761367 \\
\hline Wachstumsmögl.keit: & 11,8964175 & 11,8226954 & 10,5948664 & 13,3506648 & 11,7723884 & 10,2789598 & 10,0980648 & 8,93771761 & 8,15400246 \\
\hline Schuldentilgungsdauer & 5,46584831 & 5,51774047 & 6,58923025 & 5,47398003 & 6,2021229 & 6,85765789 & 6,63421683 & 7,48617188 & 8,01336426 \\
\hline
\end{tabular}




\section{Goldene Bilanzregel}

Kosten=Umsatz-JÜ

vor Steuer: U-BE

Anlagevermögen pro $t$ Milch

Nordmilch Konzern einzeln

Umsatz DM

Jahresüberschuss/-unterschuss

Betriebsergebnis

Eigenkapital

Fremdkapital

Gesamtkapital

Anlagevermögen

Umlaufvermögen

Abschreibungen

Personalaufwand (Mio. DM)

Mitarbeiter

Milchverarbeitung kg

FK-Quote

EK-Quote

EK-Rentabilität vor Steuer

EK-Rentabilität nach Steuer

Gesamtkapitalrentabilität

Umsatzrentabilität

a) $B E$

b) JÜ

$\begin{array}{rrrrrrrrr}0,85867474 & 0,72212641 & 0,67280436 & 0,5686716 & 0,62197786 & 0,78359801 & 0,83700442 & 0,90888779 & 0,96276137 \\ 868846919 & 923489294 & 1014967838 & 1477730614 & 1422006001 & 1486600229 & 1490039947 & 1506914971 & 1710452113 \\ 863676714 & 917072247 & 1011626530 & 1475227052 & 1417868216 & 1484954661 & 1487806149 & 1504650942 & 1706039621 \\ 89,9071833 & 119,940172 & 138,297767 & 93,1788514 & 90,5627747 & 81,9940062 & 82,3288734 & 81,0732011 & 78,14606888\end{array}$

1990

1992

1993

1994

968337976

2653062,86

7983372,54

64631568,8

132794764

197426333

77285066,6

119013274

21345539,4

65510654,4

1183

948800000

67,2629441

32,7370559

10,0734251

4,10490247

3,84465283

0,82444072

0,27398108
1991

1104929262

6365270,8

13076895,3

70691763,3

179559902

250251665

115641050

133425182

26098795

82535413,3

1608

1031500000

71,7517311

28,2482689

15,9912684

9,0042609

5,6315575

1,1835052

0,57607948
770142,34

4294522,35

74097954,7

228448440

302546394

135766755

165689768

31003775,4

90610825,

1608

1018400000

75,5085646

24,4914354

3,08815986

1,03935708

1,43512897

0,35332533

0,0633623
1215458383
1712601454

816640,7

3561072,33

77156745,6

275056563

352213308

166686357

184223833

44975961,5

109581892

1847

1698700000

78,093745

21,906255

2,72974524

1,05841776

2,44073111

0,20793351

0,04768422
1649533070

1470301,07

5715963,74

79931983,2

267100185

347032168

157496259

189222952

40157623

113255156

1823

1688100000

76,9669816

23,0330184

5,70712621

1,83944025

3,16471315

0,34652011

0,08913438
1995

1768010138

1125327,22

2785752,55

96867299,2

261477491

358344790

145595084

212610092

50367894

11809885

1825

1808200000

72,9681296

27,0318704

1,87276396

1,16172045

1,9524635

0,0636493
1996

1743945391

405133,68

2755979,33

104250373

250020275

354270649

148107398

206036526

37730740

121917575

1811

1827000000

70,5732401

29,4267599

1,36815871

0,38861605

1,6982567

0,15803129

0,02323087
1997

1776374808

502275,5

2847011,89

108442949

251895173

360338122

135979940

224187531

42099425, 1

124042407

1769

1781000000

69,9052245

30,0947755

1,76920198

0,46317027

1,66415241

0,1602709

0,02827531
1998

1805940278

502089,93

4992506,49

114345334

233123582

347468915

130096626

217219696

33131139,8

123935962

1730

67,0919243

32,9080757

3,90865322

0,43909962

1,85240659

0,27644915

0,02780213 
Cash-flow einfach

Relativer Reingewinn

Cashflow*100/Umsatz

JÜ/Umsatz ${ }^{*} 100$

Umsatz DM pro Mitarbeiter

JÜ DM pro Mitarbeiter

Aufwand DM pro Mitarbeiter

Milch kg pro Mitarbeite

Personalintensität

Umsatz je kg Milch

JÜ je kg Milch

Anlageintensität

Kapitalintensität

Arbeitsintensität

Selbstfinanzierungsgrad

Deckungsgrad A:

Wachstumsmögl.keit:

Schuldentilgungsdauer

Goldene Bilanzregel

Kosten=Umsatz-JÜ

vor Steuer: U-BE

Anlagevermögen pro $t$ Milch

\section{MZO}

Umsatz DM

Jahresüberschuss/-unterschuss

\begin{tabular}{|c|c|c|c|c|c|c|c|c|}
\hline 23998602,3 & 32464065,8 & 31773917,7 & 45792602,2 & 41627924,1 & 51493221,2 & 38135873,7 & 42601700,6 & 33633229,7 \\
\hline 1,34382421 & 2,54354783 & 0,25455347 & 0,23185969 & 0,42367861 & 0,31403477 & 0,11435711 & 0,13939005 & 0,14449924 \\
\hline 2,47832914 & 2,93811259 & 2,61415102 & 2,67386216 & 2,52361864 & 2,91249581 & 2,18675848 & 2,39823827 & 1,86236666 \\
\hline 0,27398108 & 0,57607948 & 0,0633623 & 0,04768422 & 0,08913438 & 0,06364936 & 0,02323087 & 0,02827531 & 0,02780213 \\
\hline 818544,359 & 687145,064 & 755882,079 & 927234,139 & 904845,349 & 968772,678 & 962973,711 & 1004168,91 & 1043896,11 \\
\hline 2242,65669 & 3958,50174 & 478,944241 & 442,144396 & 806,528289 & 616,617655 & 223,707167 & 283,931882 & 290,225393 \\
\hline 55376,7154 & 51327,9934 & 56350,0156 & 59329,6652 & 62125,7028 & 64711,7034 & 67320,5828 & 70120,0717 & 71639,2842 \\
\hline 802028,74 & 641480,1 & 633333,333 & 919707,634 & 926001,097 & 990794,521 & 1008834,9 & 1006783,49 & 0 \\
\hline 820,588718 & 631,154502 & 2109,91625 & 3077,21612 & 1981,38339 & 4239,38798 & 4423,74782 & 4356,93322 & 2482,43967 \\
\hline 1,0205923 & 1,07118688 & 1,19349802 & 1,00818358 & 0,97715365 & 0,97777355 & 0,95454044 & 0,99740304 & \#DIV/O! \\
\hline 0,00279623 & 0,00617089 & 0,00075623 & 0,00048074 & 0,00087098 & 0,00062235 & 0,00022175 & 0,00028202 & \#DIV/0! \\
\hline 39,1462808 & 46,2099024 & 44,8746893 & 47,3254 & 45,3837637 & 40,6298872 & 41,8062852 & 37,7367621 & 37,4412272 \\
\hline 267,374963 & 199,579445 & 721,937688 & 1262,98927 & 702,552095 & 1808,05341 & 1369,05018 & 1478,72319 & 663,61736 \\
\hline 60,2823707 & 53,3164012 & 54,7650779 & 52,3046203 & 54,5260553 & 59,3311518 & 58,1579441 & 62,2158794 & 62,5148572 \\
\hline 12,9908189 & 11,2723915 & 9,87047659 & 8,65673005 & 9,06813921 & 9,12394557 & 9,4412401 & 9,40781268 & 9,90080248 \\
\hline 83,6274997 & 61,1303367 & 54,5773926 & 46,2885788 & 50,7516709 & 66,5319848 & 70,3883634 & 79,7492255 & 87,8926205 \\
\hline 12,1557251 & 12,9725673 & 10,5021637 & 13,0013833 & 11,9954079 & 14,3697418 & 10,7646157 & 11,8227015 & 9,6794931 \\
\hline 5,53343742 & 5,53103555 & 7,18981026 & 6,00657201 & 6,41637053 & 5,07790122 & 6,55603901 & 5,91279619 & 6,93134688 \\
\hline 0,836275 & 0,61130337 & 0,54577393 & 0,46288579 & 0,50751671 & 0,66531985 & 0,70388363 & 0,79749226 & 0,8789262 \\
\hline 965684913 & 1098563992 & 1214688240 & 1711784814 & 1648062769 & 1766884811 & 1743540257 & 1775872533 & 1805438189 \\
\hline 960354604 & 1091852367 & 1211163860 & 1709040382 & 1643817107 & 1765224386 & 1741189411 & 1773527796 & 1800947772 \\
\hline 81,4555929 & 112,109598 & 133,313781 & 98,1258356 & 93,297944 & 80,5193475 & 81,065899 & 76,3503311 & \#DIV/0! \\
\hline
\end{tabular}

1455202388

1122881,57
1993

1420066593

919467,21
1994

1752862000

433000
1996

1867512397

431637,89
1813130774

$-22717519,1$
1997

1781386488

1663609,1
1998

1795861899

1103401,3 


\begin{tabular}{|c|c|c|c|c|c|c|c|c|c|}
\hline Betriebsergebnis & 2676376 & 3151812,68 & 3404560,61 & 1704350 & 3481000 & 2177907,65 & $-20723473,2$ & 3333205,7 & 1502828,21 \\
\hline Eigenkapital & 41707793 & 41959139,4 & 40824284,9 & 38184977,2 & 123419000 & 129971237 & 116718936 & 129577928 & 140811074 \\
\hline Fremdkapital & 171166699 & 175244334 & 174767119 & 180025799 & 361914000 & 422231225 & 402734270 & 364910236 & 364943789 \\
\hline Gesamtkapital & 212874492 & 217203474 & 215591404 & 218210776 & 485333000 & 552202462 & 519453206 & 494488164 & 505754863 \\
\hline Anlagevermögen & 53307218 & 71288202,1 & 56360300 & 50854764,2 & 218958000 & 240040284 & 233375676 & 208861121 & 207183970 \\
\hline Umlaufvermögen & 53307218 & 71288202,1 & 56360300 & 50854764,2 & 218958000 & 240040284 & 233375676 & 208861121 & 207183970 \\
\hline Abschreibungen & 5889999 & 7081553,73 & 17821092,7 & 5682760,73 & 32214000 & 32523099,6 & 43230169,7 & 31703419 & 30514218,8 \\
\hline Personalaufwand (Mio. DM) & 24104237 & 23542322,5 & 24577489,1 & 25179316,9 & 119663000 & 131731772 & 140399797 & 128268695 & 129211534 \\
\hline Mitarbeiter & 399 & 346 & 355 & 345 & 1851 & 1838 & 1862 & 1709 & 1674 \\
\hline Milchverarbeitung kg & - & - & - & - & 1466159200 & 1510714000 & 1466794000 & 1369244000 & 1401762000 \\
\hline FK-Quote & 80,4073318 & 80,6821048 & 81,0640479 & 82,5008747 & 74,5702435 & 76,4631189 & 77,5304233 & 73,7955451 & 72,1582362 \\
\hline EK-Quote & 19,5926682 & 19,3178952 & 18,9359521 & 17,4991253 & 25,4297565 & 23,5368811 & 22,4695767 & 26,2044549 & 27,8417638 \\
\hline EK-Rentabilität vor Steuer & 4,05390187 & 4,69802319 & 6,34192507 & 3,09432098 & 2,82047335 & 0,60180929 & $-19,2762809$ & 1,51770377 & 0,59118816 \\
\hline EK-Rentabilität nach Steuer & 1,29591849 & 2,60721501 & 2,75052355 & 2,40792919 & 0,35083739 & 0,33210262 & $-19,4634391$ & 1,28386765 & 0,78360406 \\
\hline Gesamtkapitalrentabilität & 7,506269 & 7,10743324 & 7,26257924 & 5,59174528 & 3,1576258 & 2,67801573 & $-1,61694031$ & 2,24576262 & 1,7526534 \\
\hline \multicolumn{10}{|l|}{ Umsatzrentabilität } \\
\hline a) BE & 0,18096883 & 0,20879835 & 0,23395788 & 0,12001902 & 0,19858951 & 0,11662079 & $-1,14296627$ & 0,187113 & 0,08368284 \\
\hline b) JÜ & 0,03654699 & 0,07247197 & 0,07716326 & 0,06474818 & 0,02470246 & 0,02311299 & $-1,25294433$ & 0,09338844 & 0,06144132 \\
\hline Cash-flow einfach & 6430498 & 8175518,71 & 18943974,3 & 6602227,94 & 32647000 & 32954737,5 & 20512650,6 & 33367028,1 & 31617620,1 \\
\hline Relativer Reingewinn & 0,25390501 & 0,50365906 & 0,52083782 & 0,42136655 & 0,08921709 & 0,0781666 & $-4,37335238$ & 0,33643052 & 0,21816919 \\
\hline Cashflow¹00/Umsatz & 0,43481175 & 0,54160414 & 1,30181028 & 0,46492383 & 1,86249688 & 1,76463287 & 1,13133872 & 1,87309314 & 1,76058193 \\
\hline JÜ/Umsatz ${ }^{*} 100$ & 0,03654699 & 0,07247197 & 0,07716326 & 0,06474818 & 0,02470246 & 0,02311299 & $-1,25294433$ & 0,09338844 & 0,06144132 \\
\hline Umsatz DM pro Mitarbeiter & 3706554,93 & 4362719,04 & 4099161,66 & 4116135,05 & 946981,091 & 1016056,8 & 973754,444 & 1042356,05 & 1072796,83 \\
\hline JÜ DM pro Mitarbeiter & 1354,63409 & 3161,7485 & 3163,04668 & 2665,12235 & 233,927607 & 234,841072 & $-12200,601$ & 973,440082 & 659,140562 \\
\hline Aufwand DM pro Mitarbeiter & 60411,6216 & 68041,3946 & 69232,3635 & 72983,5271 & 64647,758 & 71671,2577 & 75402,6837 & 75054,8245 & 77187,296 \\
\hline Milch kg pro Mitarbeiter & - & - & - & - & 792090,33 & 821933,624 & 787751,88 & 801196,021 & 837372,76 \\
\hline
\end{tabular}




Personalintensität
Umsatz je kg Milch
JÜ je kg Milch
Anlageintensität
Kapitalintensität
Arbeitsintensität
Selbstinanzierungsgrad
Deckungsgrad A:
Wachstumsmögl.keit:
Schuldentilgungsdauer
Goldene Bilanzregel
Kosten=Umsatz-JÜ
vor Steuer: U-BE
Anlagevermögen pro t Milch
Hansano
Umsatz DM
Jahresüberschuss/-unterschuss
Betriebsergebnis
Eigenkapital
Gremdkapital
Gesamtkapital
Abschreibungen

Personalaufwand (Mio. DM)

$\begin{array}{ccc}900,629695 & 746,945485 & 721,899002 \\ - & - & - \\ - & - & -\end{array}$$$
\text { - }
$$

100

100

$$
-
$$

$$
220,073674
$$

224,681935

523,447657

100

$7,69283245 \quad 10,1387346$

78,2404233

3,02079312

26,6179539

0,78240423

1478374918

1476239041

$-$

1990

244657237

10000,82

784437,43

19541480,5

32651824,9

52193305,4

14657544,9

37017743,4

3943490,9

16597533,4

58,8584621

3,76399077

0,58858462

1508406824

1506348976

1991

261735657

10000

875836,93

20705178,2

30872399,2

51577577,4

17901429,7

33370599,8

4387221,17

18068774,5
21,4352557
72,4344705

8,78698037

9,22547275

0,7243447

1454079506

1451797827

-

1992

342509072

10000,3

1142371,09

38408955,8

40742763

79151718,8

32628388,8

45539196,6

9431839,84

26444058
10,6543852

$\begin{array}{rr}3437,60414 & 6048,54717 \\ 1,19554684 & 1,23617865 \\ 0,00029533 & 0,00028572 \\ 100 & 100\end{array}$

$-677,491635$

3848,2082

8597,89114

1,23611821

1,30100003

1,28114609

$-0,01548787$

0,00121498

0,00078715

$\begin{array}{lll}333,426862 & 925,423729 & 1493,31858\end{array}$

100

100

10,9370695

75,0863322

3,02561957

27,2674316

0,75086332

1419147126

1418362243

$$
\text { - }
$$

12,723223

56,3665178

6,72672165

11,085674

0,56366518

1752429000

1749381000

149,341218

$$
100
$$

$-208,604848$

951,138989

100

2030,45289

11,259817

54,1455937

5,96787226

12,812459

0,54145594

1867080759

1865334489

158,891944

1993

1994

1995

315896039

304862246

$-3021469,11$

1128656,74

$-3071602,43$

38590757,2

37231404,4

$35885204,3 \quad 33043228,2$

$74475961,6 \quad 70274632,7$

34551586,3 28275647,2

$38918999,3 \quad 41355332,4$

8009747,06

25461625,6
382126235

10000,14

550838,9

44442450,2

43081102,1

87523552,2

30272296,4

56933884,6

9138342,19

30344410,8
100

100

100

$7,71521204 \quad 8,24022076$

8,1456293

$50,0133255 \quad 62,0402338$

67,9642708

$3,94889286 \quad 6,74779106$

6,25157015

$19,6334583 \quad 10,9362522$

11,5424181

$0,50013325 \quad 0,62040234$

0,67964271

$1835848293 \quad 1779722879$

1794758498

$1833854247 \quad 1778053282$

1794359071

$159,105966 \quad 152,537547$

147,80253

1996

368437871

1997

1998

10000,34

361354324

358818442

10000,59

627727,65

$-5061999,25$

131519,43

44899162,7

40067107

43053741,9

83120848,9

32046298,2

$59366472,6 \quad 58338101,9$

51045408,3

$8316559,55 \quad 10753266,4$

6466419,54

26650087,3

26127707,9

26203358,2

22416910,5 


\section{Mitarbeiter}

Milchverarbeitung kg

FK-Quote

EK-Quote

EK-Rentabilität vor Steuer

EK-Rentabilität nach Steuer

Gesamtkapitalrentabilität

Umsatzrentabilität

a) $B E$

b) JÜ

Cash-flow einfach

Relativer Reingewinn

Cashflow*100/Umsatz

JÜ/Umsatz ${ }^{\star} 100$

Umsatz DM pro Mitarbeiter

JÜ DM pro Mitarbeiter

Aufwand DM pro Mitarbeiter

Milch kg pro Mitarbeiter

Personalintensität

Umsatz je kg Milch

JÜ je kg Milch

Anlageintensität

Kapitalintensität

Arbeitsintensität

Selbstfinanzierungsgrad

Deckungsgrad A:
179500000

62,5594119

37,4405881

1,61848935

0,05117739

1,13925839

0,32062711

0,00408769

3953491,72

0,01916112

1,61593083

0,00408769

967024,651

39,5289328

65602,8988

709486,166

2115,85179

1,36299296

5,57E-05

28,0831897

502,7158

70,9243132

22,2094221

133,320284

\section{0}

198500000

59,8562414

40,1437586

1,82581848

0,0482971

0,96417481

0,33462652

0,00382065

4397221,17

0,01938827

1,68002374

0,00382065

1006675,61

38,4615385

69495,2864

763461,538

2063,02953

1,31856754

5,04E-05

34,7077753

500,917582

64,6998201

23,9358728

115,662149
376

290200000

51,4742619

48,5257381

1,1005703

0,02603638

0,74823579

0,33353017

0,00291972

9441840,14

0,01263434

2,75666863

0,00291972

910928,383

26,5965426

70329,9415

771808,511

2314,83957

1,1802518

3,45E-05

41,2225904

825,637126

57,534059

34,7701729

117,716373
345

266800000

48,1836066

51,8163934

1,15443555

0,02591294

0,78374717

0,3572874

0,0031656

8019747,06

0,01342715

2,53872985

0,0031656

915640,694

28,9855072

73801,8132

773333,333

2255,92287

1,18401814

3,75E-05

46,3929375

709,670777

52,257129

36,9665449

111,690262

\section{2}

265900000

47,0201366

52,9798634

$-6,49345666$

$-8,11537775$

$-3,31043651$

$-1,00753782$

$-0,99109324$

9210011,59

$-4,29951605$

3,02104039

$-0,99109324$

842160,902

$-8346,59975$

73619,0256

734530,387

$-867,628147$

1,1465297

$-0,01136318$

40,2359231

$-398,211715$

58,8481658

36,5719479

131,673041
364

366300000

49,2222962

50,7777038

$-0,06796392$

0,02250132

0,40679595

0,14415103

0,00261697

9148342,33

0,01142566

2,39406288

0,00261697

1049797,35

27,4729121

83363,7658

1006318,68

5508,76322

1,04320566

2,73E-05

34,5876003

1658,98636

65,0497873

33,0925665

146,808982
347

365300000

48,3174376

51,6825624

0,02074533

0,02227289

0,10468468

0,17037544

0,0027142

8326559,8

0,0115112

2,25996309

0,00271425

1061780,6

28,8194236

75295,9883

1052737,7

4162,26813

1,00858985

2,74E-05

31,3350979

1324,86749

68,3356045

33,351172

164,93506
308

266

352000000

51,7965619

48,2034381

0,02662181

0,0249596

0,0829905

0,03665348

0,00278709

6476420,13

0,01203139

1,80492956

0,00278709

1348941,51

37,596203

84274,0995

1323308,27

17044,5618

1,01937057

2,84E-05

38,553863

4916,70283

61,4110767

28,7795316

125,028815 
Wachstumsmögl.keit:

Schuldentilgungsdauer

Goldene Bilanzrege

Kosten=Umsatz-JÜ

vor Steuer: U-BE

Anlagevermögen pro $t$ Milch

\section{Bremerland/ Nordheide}

Umsatz DM

Jahresüberschuss/-unterschuss

Betriebsergebnis

Eigenkapital

Fremdkapital

Gesamtkapital

Anlagevermögen

Umlaufvermögen

Abschreibungen

Personalaufwand (Mio. DM)

Mitarbeiter

Milchverarbeitung kg

FK-Quote

EK-Quote

EK-Rentabilität vor Steuer

EK-Rentabilität nach Steuer

Gesamtkapitalrentabilität

Umsatzrentabilität

\begin{tabular}{|c|c|c|c|c|}
\hline 7,57471038 & 8,52545116 & 11,9287872 & 10,7682357 & 13,1057413 \\
\hline 8,258984 & 7,02088843 & 4,31512951 & 4,4746055 & 3,58775099 \\
\hline 1,33320284 & 1,15662149 & 1,17716373 & 1,11690262 & 1,31673041 \\
\hline 244647236 & 261725657 & 342499072 & 315886039 & 307883715 \\
\hline 243872799 & 260859821 & 341366701 & 314767383 & 307933849 \\
\hline 81,6576319 & 90,1835247 & 112,434145 & 129,503697 & 106,339403 \\
\hline 1994 & 1995 & 1996 & 1997 & 1998 \\
\hline 460831768 & 488936880 & 478076376 & 469102046 & 472121599 \\
\hline 77817,57 & 34929,92 & 26414,13 & 28953,57 & 20373,71 \\
\hline 1549457,86 & 1813508,75 & 1352993,65 & 641689,1 & 427214,3 \\
\hline 72959954 & 75313019,4 & 76913628,4 & 78682590,6 & 79775590,9 \\
\hline 62741270,4 & 68379877,1 & 69046246,8 & 63254816,9 & 62405924,9 \\
\hline 135701224 & 143692896 & 145959875 & 141937407 & 142181516 \\
\hline 62225731,1 & 76574347,1 & 80453080,6 & 77644093,2 & 76269751,9 \\
\hline 73259227,1 & 66917205,2 & 65162463,3 & 64062246,2 & 65729306,7 \\
\hline 16881548,3 & 12219187,3 & 14712423,9 & 14450140,7 & 13954851,4 \\
\hline 42313924 & 43098113,3 & 43093703,7 & 40797046,8 & 39494546,1 \\
\hline- & 624 & 566 & 550 & 530 \\
\hline 492800000 & 513400000 & 519100000 & 519100000 & 511100000 \\
\hline 46,2348595 & 47,5875139 & 47,3049506 & 44,5652897 & 43,8917285 \\
\hline 53,7651405 & 52,4124861 & 52,6950494 & 55,4347103 & 56,1082715 \\
\hline 0,77056776 & 0,97855944 & 0,86734565 & 0,04528425 & 0,12301413 \\
\hline 0,10665792 & 0,04637966 & 0,03434259 & 0,03679794 & 0,02553878 \\
\hline 0,79609845 & 0,76074159 & 0,83914919 & 0,37590723 & 0,27734572 \\
\hline
\end{tabular}


Anhang

\begin{tabular}{|c|c|c|c|c|c|}
\hline a) $\mathrm{BE}$ & 0,3362307 & 0,37090856 & 0,28300784 & 0,13679094 & 0,09048819 \\
\hline b) JÜ & 0,01688633 & 0,00714406 & 0,00552509 & 0,00617213 & 0,00431535 \\
\hline Cash-flow einfach & 16959365,9 & 12254117,2 & 14738838 & 14479094,3 & 13975225,1 \\
\hline Relativer Reingewinn & 0,05734478 & 0,02430873 & 0,01809684 & 0,02039883 & 0,01432937 \\
\hline Cashflow*100/Umsatz & 3,68016422 & 2,50627794 & 3,08294632 & 3,08655535 & 2,96009018 \\
\hline JÜ/Umsatz ${ }^{*} 100$ & 0,01688633 & 0,00714406 & 0,00552509 & 0,00617213 & 0,00431535 \\
\hline Umsatz DM pro Mitarbeiter & - & 783552,692 & 844657,909 & 852912,81 & 890795,469 \\
\hline JÜ DM pro Mitarbeiter & - & 55,9774359 & 46,6680742 & 52,6428545 & 38,4409623 \\
\hline Aufwand DM pro Mitarbeiter & - & 69067,4892 & 76137,2857 & 74176,4487 & 74518,0115 \\
\hline Milch kg pro Mitarbeiter & - & 822756,41 & 917137,809 & 943818,182 & 964339,623 \\
\hline Personalintensität & 2730,88576 & 2376,50429 & 3185,06326 & 6357,75904 & 9244,66857 \\
\hline Umsatz je kg Milch & 0,9351294 & 0,95235076 & 0,92097164 & 0,90368339 & 0,92373625 \\
\hline JÜ je kg Milch & 0,00015791 & $6,80 \mathrm{E}-05$ & $5,09 \mathrm{E}-05$ & $5,58 \mathrm{E}-05$ & $3,99 \mathrm{E}-05$ \\
\hline Anlageintensität & 45,8549518 & 53,2902802 & 55,1199982 & 54,7030515 & 53,6425227 \\
\hline Kapitalintensität & 1089,51322 & 673,787058 & 1087,39785 & 2251,89125 & 3266,47572 \\
\hline Arbeitsintensität & 53,9856787 & 46,5695987 & 44,6440936 & 45,1341527 & 46,2291503 \\
\hline Selbstfinanzierungsgrad & 8,95401612 & 8,51018205 & 8,40193717 & 8,65865549 & 8,66415345 \\
\hline Deckungsgrad A: & 117,250457 & 98,3528064 & 95,6006007 & 101,33751 & 104,596631 \\
\hline Wachstumsmögl.keit: & 12,4975776 & 8,52799093 & 10,0978697 & 10,2010418 & 9,82914342 \\
\hline Schuldentilgungsdauer & 3,69950568 & 5,58015531 & 4,68464655 & 4,36869984 & 4,46546832 \\
\hline Goldene Bilanzregel & 1,17250457 & 0,98352806 & 0,95600601 & 1,0133751 & 1,04596631 \\
\hline Kosten=Umsatz-JÜ & 460753951 & 488901950 & 478049962 & 469073092 & 472101225 \\
\hline vor Steuer: U-BE & 459282311 & 487123371 & 476723383 & 468460356 & 471694384 \\
\hline Anlagevermögen pro t Milch & 126,269747 & 149,151436 & 154,985707 & 149,574443 & 149,226672 \\
\hline
\end{tabular}




\begin{tabular}{|c|c|c|c|c|c|c|c|c|c|c|}
\hline Humana & 1996 WM & 1997 WM & 1996 MWW & 1997 MWW & 1996 & 1997 & 1998 & 1999 & 2000 & 2001 \\
\hline Umsatz DM & 406896544 & 415817350 & 871771396 & 830465519 & 1278667939 & 1246282868 & 1260202130 & 1212320422 & 1450313101 & 1652693951 \\
\hline Jahresüberschuss/-unterschuss & 111241,29 & 366525,66 & 38474,74 & $-21711557,4$ & 149716,03 & $-21345031,7$ & 1080412,95 & 1152277,26 & 1408375,28 & 10088054 \\
\hline Betriebsergebnis & 654744,47 & 1402025,65 & 571572,6 & $-6050503,96$ & 1226317,07 & $-4648478,31$ & 1840879,73 & 931022,22 & 3676357,69 & 10295915,8 \\
\hline Eigenkapital & 37409547,6 & 40494738,3 & 78609233 & 57828689,5 & 116018781 & 98323427,8 & 112967936 & 122308045 & 121210902 & 132353879 \\
\hline Fremdkapital & 95986580 & 84162041,4 & 133926270 & 142556180 & 229912850 & 226718221 & 255126307 & 258810761 & 318604341 & 343501688 \\
\hline Gesamtkapital & 133396128 & 124656780 & 212535503 & 200384870 & 345931631 & 325041649 & 368094243 & 381118805 & 439815243 & 475855567 \\
\hline Anlagevermögen & 47685686 & 45520133,4 & 100882538 & 88882265,3 & 148568224 & 134402399 & 134390348 & 139703936 & 159345034 & 147884034 \\
\hline Umlaufvermögen & 71270183 & 56776102,6 & 105513260 & 74141814,5 & 176783443 & 130917917 & 233681950 & 241394220 & 280361957 & 327162859 \\
\hline Abschreibungen & 7721928,53 & 7266210,05 & 18879685,1 & 19334787,8 & 26601613,6 & 26600997,9 & 20885822,6 & 17816008,6 & 18129656,6 & 18044017,9 \\
\hline Personalaufwand (Mio. DM) & 26058783 & 26072320,5 & 96946176 & 77476420,5 & 123004959 & 103548741 & 96510875 & 86064433,6 & 86837541,9 & 62660372 \\
\hline Mitarbeiter & 0 & 308 & 1009 & 968 & 0 & 1276 & 1121 & 911 & 920 & 616 \\
\hline Milchverarbeitung kg & 406361351 & 381994027 & 555487889 & 534448157 & 961849240 & 916442184 & 872928805 & 1046969740 & 1071270000 & 1078646000 \\
\hline FK-Quote & 71,9560468 & 67,5150133 & 63,0135992 & 71,1411896 & 66,4619334 & 69,7505141 & 69,3100508 & 67,908158 & 72,4404955 & 72,1861237 \\
\hline EK-Quote & 28,0439532 & 32,4849867 & 36,9864008 & 28,8588104 & 33,5380666 & 30,2494859 & 30,6899492 & 32,091842 & 27,5595045 & 27,8138763 \\
\hline EK-Rentabilität vor Steuer & 0,73846057 & 2,67651294 & $-0,48751946$ & $-36,4811265$ & $-0,09220968$ & $-20,3539593$ & 1,3702178 & $-43,8333544$ & 1,17215404 & 7,62202263 \\
\hline EK-Rentabilität nach Steuer & 0,29736069 & 0,90511922 & 0,0489443 & $-37,5446124$ & 0,12904465 & $-21,7089987$ & 0,95638903 & 0,94211077 & 1,1619213 & 7,62203121 \\
\hline Gesamtkapitalrentabilität & 2,42341306 & 3,23395369 & 0,95479935 & $-9,15785356$ & 1,52111756 & $-4,4054694$ & 1,8484414 & $-12,4702088$ & 2,36659569 & 3,72619632 \\
\hline \multicolumn{11}{|l|}{ Umsatzrentabilität } \\
\hline a) $B E$ & 0,16091178 & 0,33717344 & 0,0655645 & $-0,72856775$ & 0,09590583 & $-0,37298742$ & 0,14607813 & 0,07679671 & 0,25348717 & 0,62297776 \\
\hline b) JÜ & 0,02733896 & 0,08814583 & 0,0044134 & $-2,61438396$ & 0,01170875 & $-1,71269559$ & 0,08573331 & 0,09504725 & 0,09710836 & 0,61040061 \\
\hline Cash-flow einfach & 7833169,82 & 7632735,71 & 18918159,8 & $-2376769,52$ & 26751329,7 & 5255966,19 & 21966235,6 & 18968285,9 & 19538031,9 & 28132071,9 \\
\hline Relativer Reingewinn & 0,08339169 & 0,29402786 & 0,01810274 & $-10,8349285$ & 0,04327908 & $-6,56686051$ & 0,29351531 & 0,3023407 & 0,32021975 & 2,11998233 \\
\hline Cashflow*100/Umsatz & 1,9251011 & 1,83559818 & 2,17008265 & $-0,28619726$ & 2,09212485 & 0,4217314 & 1,7430724 & 1,56462644 & 1,34715958 & 1,70219488 \\
\hline
\end{tabular}




\begin{tabular}{|c|c|c|c|c|c|c|c|c|c|c|}
\hline JÜ/Umsatz*100 & 0,02733896 & 0,08814583 & 0,0044134 & $-2,61438396$ & 0,01170875 & $-1,71269559$ & 0,08573331 & 0,09504725 & 0,09710836 & 0,61040061 \\
\hline Umsatz DM pro Mitarbeiter & - & 1350056,33 & 863995,437 & 857918,924 & - & 976710,712 & 1124176,74 & 1330757,87 & 1576427,28 & 2682944,73 \\
\hline JÜ DM pro Mitarbeiter & - & 1190,01838 & 38,131556 & $-22429,2948$ & - & $-16728,0813$ & 963,793889 & 1264,8488 & 1530,8427 & 16376,711 \\
\hline Aufwand DM pro Mitarbeiter & - & 84650,3912 & 96081,443 & 80037,6245 & - & 81151,051 & 86093,5549 & 94472,4847 & 94388,6324 & 101721,383 \\
\hline Milch kg pro Mitarbeiter & - & 1240240,35 & 550533,091 & 552115,865 & - & 718214,878 & 778705,446 & 1149253,28 & 1164423,91 & 1751048,7 \\
\hline Personalintensität & 3979,99284 & 1859,61794 & 16961,3057 & $-1280,49533$ & 10030,4368 & $-2227,58361$ & 5242,64967 & 9244,07944 & 2362,05367 & 608,594448 \\
\hline Umsatz je kg Milch & 1,00131704 & 1,08854411 & 1,56937966 & 1,55387479 & 1,32938499 & 1,35991434 & 1,44364824 & 1,15793263 & 1,35382593 & 1,53219309 \\
\hline JÜ je kg Milch & 0,00027375 & 0,00095951 & $6,93 \mathrm{E}-05$ & $-0,04062425$ & 0,00015565 & $-0,02329119$ & 0,00123769 & 0,00110058 & 0,00131468 & 0,00935252 \\
\hline Anlageintensität & 35,747429 & 36,516372 & 47,4662053 & 44,3557767 & 42,9472794 & 41,3492852 & 36,5097663 & 36,6562694 & 36,2299936 & 31,077504 \\
\hline Kapitalintensität & 1179,38049 & 518,265129 & 3303,11234 & $-319,556651$ & 2169,22803 & $-572,251737$ & 1134,55661 & 1913,5965 & 493,141803 & 175,254132 \\
\hline Arbeitsintensität & 53,4274752 & 45,5459404 & 49,6450044 & 36,9997069 & 51,1035786 & 40,2772744 & 63,4842719 & 63,3383125 & 63,7453934 & 68,7525544 \\
\hline Selbstfinanzierungsgrad & 11,6132944 & 12,5969296 & 13,5535671 & 14,3832172 & 12,8053705 & 13,6981577 & 13,7584706 & 13,2882814 & 11,9265262 & 11,0401438 \\
\hline Deckungsgrad A: & 78,4502662 & 88,9600607 & 77,9215456 & 65,0621238 & 78,0912481 & 73,1560067 & 84,0595607 & 87,5480307 & 76,0682015 & 89,4984235 \\
\hline Wachstumsmögl.keit: & 5,87211185 & 6,12300088 & 8,90117629 & $-1,18610229$ & 7,73312622 & 1,61701314 & 5,96755749 & 4,97700078 & 4,44232714 & 5,91189298 \\
\hline Schuldentilgungsdauer & 12,2538617 & 11,0264582 & 7,07924405 & $-59,9789668$ & 8,59444571 & 43,1354033 & 11,6144756 & 13,6443937 & 16,30688 & 12,2103231 \\
\hline Goldene Bilanzregel & 0,78450266 & 0,88960061 & 0,77921546 & 0,65062124 & 0,78091248 & 0,73156007 & 0,84059561 & 0,87548031 & 0,76068202 & 0,89498424 \\
\hline Kosten=Umsatz-JÜ & 406785302 & 415450824 & 871732921 & 852177076 & 1278518223 & 1267627900 & 1259121717 & 1211168144 & 1448904726 & 1642605897 \\
\hline vor Steuer: U-BE & 406241799 & 414415324 & 871199823 & 836516023 & 1277441622 & 1250931347 & 1258361250 & 1211389399 & 1446636744 & 1642398035 \\
\hline Anlagevermögen pro t Milch & 117,347986 & 119,164516 & 181,610689 & 166,306618 & 154,46103 & 146,656713 & 153,953389 & 133,43646 & 148,744046 & 137,101545 \\
\hline
\end{tabular}

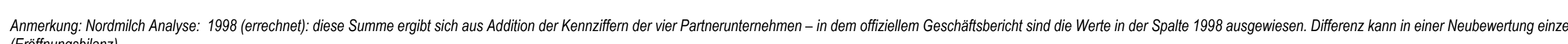
(Eröffnungsbilanz). 


\section{Zuckerindustrie:}

\section{Unterstellte Fusion}

jeweils in $1000 \mathrm{DM}$

\section{ZVN+ZAG-Konzern}

\section{Nordzucker}

Umsatz 1000 DM

Jahresüberschuss/-unterschuss

Betriebsergebnis

Eigenkapital

Fremdkapital

Gesamtkapital

Anlagevermögen

Umlaufvermögen

Abschreibungen

Personalaufwand (Mio. DM)

Mitarbeiter

Mengen Rüben Tonnen

Menge Zuckererzeugung

FK-Quote

EK-Quote

EK-Rentabilität vor Steuer

EK-Rentabilität nach Steuer

Gesamtkapitalrentabilität

Umsatzrentabilität

a) $B E$

b) JÜ

\begin{tabular}{|c|c|c|c|c|c|c|c|c|c|c|}
\hline & \multicolumn{10}{|c|}{ Nordzucker AG } \\
\hline 1991 & 1992 & 1993 & 1994 & 1995 & 1996 & 1997 & 1998 & 1999 & 2000 & 2001 \\
\hline - & 2526135,42 & 2765653,58 & 2777982,16 & 2578427,43 & 2643756,81 & 1336012 & 1366400 & 1255546 & 1338805 & 1472904,28 \\
\hline 16450,8101 & 14468,213 & $-34795,774$ & 27200,372 & 17781,613 & 38247,97 & 17487 & 40920 & 46782 & 31736 & 116602,673 \\
\hline 29033,7354 & 58382,2788 & 59734,385 & 77179,212 & 37862,994 & 77119,809 & 32136 & 88090 & 91514 & 83246 & 177098,451 \\
\hline 393872,004 & 588232,666 & 557587,523 & 611506,686 & 637154,961 & 661698,385 & 426111 & 452255 & 525975 & 529057 & 608141,869 \\
\hline 9354,996 & 1499966,35 & 1532493,33 & 1671588,72 & 1633059,9 & 1433142,08 & 921232 & 981335 & 901969,5 & 1117374 & 1035741,07 \\
\hline 1363227 & 2088199,02 & 2090080,86 & 2283095,41 & 2270214,86 & 2094840,46 & 1347343 & 1433590 & 1427945 & 1646431 & 1643882,94 \\
\hline 673536 & 891456,978 & 1050687,6 & 1210712,22 & 1253245,58 & 1149478,15 & 516114 & 564927 & 661661 & 841431 & 835098,338 \\
\hline 688407 & 1066629,42 & 942040,43 & 1020240,39 & 980523,297 & 916995,613 & 1446546 & 868111 & 765942 & 804514 & 808403,214 \\
\hline 92096,1611 & 128298,843 & 141495,775 & 146384,55 & 169105,236 & 170788,055 & 116181 & 112644 & 94315 & 91429 & 81546,376 \\
\hline 179511 & 252457,063 & 258261,629 & 251391,314 & 224188,275 & 215648,56 & 162457 & 176056 & 152087 & 158401 & 143818,047 \\
\hline 2601 & 4429 & 3758 & 3189 & 2657 & 2307 & 1956 & 1908 & 1994 & 1620 & 1307 \\
\hline 7688539 & 9556700 & 7978616 & 8683000 & 7243800 & 7817100 & 8017000 & 8056200 & 8044600 & 7889300 & 7619030 \\
\hline - & 1095938 & 1215369 & 1166261 & 975364 & 1127581 & 1244000 & 1230700 & 1294100 & 1286500 & 1121906 \\
\hline 71,1073795 & 71,8306224 & 73,3222032 & 73,2158944 & 71,934156 & 68,4129461 & 68,3739775 & 68,4529747 & 63,1655631 & 67,8664335 & 63,005768 \\
\hline 28,8926205 & 28,1693776 & 26,6777968 & 26,7841056 & 28,065844 & 31,5870539 & 31,6260225 & 31,5470253 & 36,8344019 & 32,1335665 & 36,994232 \\
\hline 6,61098271 & 5,09084377 & $-4,37648961$ & 5,56858687 & 2,81486312 & 7,82692994 & 7,30842433 & 19,3631911 & 16,9532772 & 15,4758372 & 28,7989889 \\
\hline 4,17668936 & 2,4596072 & $-6,24041474$ & 4,4480907 & 2,7907831 & 5,78027253 & 4,10386026 & 9,04799284 & 8,89433908 & 5,9985975 & 19,1735973 \\
\hline 2,50712538 & 1,83440309 & $-0,80079653$ & 2,08147806 & 1,52487117 & 3,25031072 & 2,65552276 & 6,5022775 & 6,85488587 & 5,89839477 & 12,1613936 \\
\hline 3779331 & 2,31113021 & 2,15986505 & 2,77824721 & 1,46845296 & 2,91705382 & 2,40536762 & 6,44686768 & 7,28878114 & 6,21793316 & 12,0237583 \\
\hline 1,15463443 & 0,57274099 & $-1,25813928$ & 0,97914135 & 0,68963015 & 1,446728 & 1,30889543 & 2,99473068 & 3,72602836 & 2,37047217 & 7,91651396 \\
\hline
\end{tabular}


Cash-flow einfach

Relativer Reingewinn

Cashflow*100/Umsatz

JÜ/Umsatz ${ }^{*} 100$

Umsatz DM pro Mitarbeiter

JÜ DM pro Mitarbeiter

Aufwand DM pro Mitarbeiter

Rüben t pro Mitarbeiter

Zucker t pro Mitarbeiter

Personalintensität

Umsatz je t. Rübe

JÜ je t. Rübe

Umsatz/Zucker kg

JÜ/kg Zucker

Anlageintensität

Kapitalintensität

Arbeitsintensität

Selbstfinanzierungsgrad

Deckungsgrad A:

Wachstumsmögl.keit:

Schuldentilgungsdauer

Goldene Bilanzregel

Rüben/Zucker

Kosten=Umsatz-JÜ

Vor Steuer: U-BE

\begin{tabular}{|c|c|c|c|c|c|c|c|c|c|c|}
\hline 108546,971 & 142767,056 & 106700,001 & 173584,922 & 186886,849 & 209036,025 & 133668 & 153564 & 141097 & 123165 & 198149,049 \\
\hline 20675501 & 0,69285604 & $-1,66480516$ & 1,19138131 & 0,78325683 & 1,8258178 & 1,29788777 & 2,85437259 & 3,2761766 & 1,92756332 & 7,09312508 \\
\hline 7,61859568 & 5,65159946 & 3,85803927 & 6,24859743 & 7,24809421 & 7,90677964 & 10,005 & 11,2385831 & 11,2378997 & 9,19962205 & 13,4529481 \\
\hline 1,15463443 & 0,57274099 & $-1,25813928$ & 0,97914135 & 0,68963015 & 1,446728 & 1,30889543 & 2,99473068 & 3,72602836 & 2,37047217 & 7,91651396 \\
\hline 47775,282 & 570362,48 & 735937,621 & 871113,88 & 970428,087 & 1145971,74 & 683032,72 & 716142,558 & 629661,986 & 826422,84 & 1126935,18 \\
\hline 6324,80203 & 3266,69971 & $-9259,12028$ & 8529,43619 & 6692,3647 & 16579,0941 & 8940,18405 & 21446,5409 & 23461,3842 & 19590,1235 & 89213,9808 \\
\hline 9016,1476 & 57000,9173 & 68723,1583 & 78830,7664 & 84376,4678 & 93475,7521 & 83055,726 & 92272,5367 & 76272,317 & 97778,3951 & 110036,762 \\
\hline \multirow[t]{2}{*}{2955,99346} & 2157,7557 & 2123,10165 & 2722,79712 & 2726,30787 & 3388,42653 & 4098,67076 & 4222,32704 & 4034,40321 & 4869,93827 & 5829,40321 \\
\hline & 247,445925 & 323,408462 & 365,713703 & 367,092209 & 488,765063 & 635,99182 & 645,020964 & 648,996991 & 794,135802 & 858,382555 \\
\hline 8,284204 & 432,420706 & 432,350026 & 325,724126 & 592,103929 & 279,627975 & 505,529624 & 199,859235 & 166,189873 & 190,280614 & 81,2079647 \\
\hline 85,310045 & 264,331351 & 346,633248 & 319,933452 & 355,949561 & 338,201738 & 166,647374 & 169,6085 & 156,073142 & 169,698833 & 193,319134 \\
\hline 13965359 & 1,51393399 & $-4,36112905$ & 3,13260071 & 2,4547355 & 4,89285924 & 2,18123987 & 5,07931779 & 5,81532954 & 4,02266361 & 15,3041362 \\
\hline- & 2,30499848 & 2,27556699 & 2,38195581 & 2,64355402 & 2,34462696 & 1,07396463 & 1,11026245 & 0,97020787 & 1,04065682 & 1,3128589 \\
\hline- & 0,01320167 & $-0,0286298$ & 0,02332271 & 0,01823075 & 0,03392037 & 0,01405707 & 0,03324937 & 0,03615022 & 0,02466848 & 0,10393266 \\
\hline 9,4074721 & 42,6902307 & 50,2701893 & 53,0294185 & 55,203831 & 54,8718707 & 38,3060587 & 39,4064551 & 46,3365886 & 51,1063628 & 50,8003531 \\
\hline 17,203969 & 219,756483 & 236,874917 & 189,668366 & 446,624 & 221,458089 & 361,529126 & 127,873765 & 103,060734 & 109,829902 & 157874 \\
\hline 50,4983396 & 51,0789161 & 45,0719611 & 44,6867171 & 43,1907708 & 43,7740071 & 107,362862 & 60,5550401 & 53,6394609 & 48,8641188 & 49,1764465 \\
\hline 2,91213422 & 3,37950178 & 4,11337407 & 4,4874557 & 4,01652088 & 5,31564943 & 0,4453209 & 1,81362872 & 3,43150471 & 2,97613444 & 6,50954665 \\
\hline 58,4782408 & 65,985536 & 53,068821 & 50,5080131 & 50,8403918 & 57,565112 & 82,5614109 & 80,0554762 & 79,4931241 & 62,8758627 & 72,8227852 \\
\hline 7,96250156 & 6,83685102 & 5,10506571 & 7,60305161 & 8,23212165 & 9,97861312 & 9,92085905 & 10,7118493 & 9,88112287 & 7,48072649 & 12,0537201 \\
\hline 8,93028139 & 10,5063899 & 14,3626365 & 9,62980368 & 8,73822802 & 6,85595737 & 6,89194123 & 6,39039749 & 6,3925491 & 9,07217148 & 5,2270807 \\
\hline 0,58478241 & 0,65985536 & 0,53068821 & 0,50508013 & 0,50840392 & 0,57565112 & 0,82561411 & 0,80055476 & 0,79493124 & 0,62875863 & 0,72822785 \\
\hline - & 8,72011008 & 6,5647684 & 7,44516022 & 7,4267658 & 6,93299195 & 6,44453376 & 6,54603071 & 6,21636659 & 6,13237466 & 6,79114828 \\
\hline 1408312,7 & 2511667,21 & 2800449,35 & 2750781,79 & 2560645,81 & 260550 & 1318525 & 1325480 & 1208764 & 1307069 & 1356301,61 \\
\hline 1395729,77 & 2467753,14 & 2705919,19 & 2700802,95 & 2540564,43 & 2566637 & 1303876 & 1278310 & 1164032 & 1255559 & 5805,8 \\
\hline
\end{tabular}

LXXVI 


\section{Einzeldaten der Zuckerindustrie}

\begin{tabular}{|c|c|c|c|c|c|c|c|}
\hline \multicolumn{8}{|c|}{ ZVN+ZAG-Betrieb } \\
\hline & 1990 & 1991 & 1992 & 1993 & 1994 & 1995 & 1996 \\
\hline Umsatz $1000 \mathrm{DM}$ & - & 1402391,51 & 1351812,56 & 1412996,09 & 1455207,69 & 1362624 & 1344172,83 \\
\hline Jahresüberschuss/-unterschuss & 16450,8101 & 7615,52512 & 47587,5043 & 15921,961 & 28085,07 & 29819,248 & \\
\hline Betriebsergebnis & 28873,7354 & 27388,6426 & 60661,8015 & 54758,183 & 43363,745 & 60798,564 & \\
\hline Eigenkapital & & 394325,004 & 427685,571 & 363424,313 & 387737,509 & 416552 & 429044,836 \\
\hline Fremdkapital & & 966477,996 & 1036163,37 & 1043383,23 & 978346,953 & 1016733 & 861440,434 \\
\hline Gesamtkapital & 1360803 & 1463848,94 & 1406807,54 & 1366084,46 & 1433285 & 1290485,27 & \\
\hline Anlagevermögen & 659895 & 766272,305 & 724313,309 & 654373,214 & 614272 & 564941,398 & \\
\hline Umlaufvermögen & 699735 & 695763,595 & 681110,847 & 710901,582 & 817949 & 725114,873 & \\
\hline Abschreibungen & 90282,1611 & 94658,7425 & 104109,664 & 105646,416 & 104775 & 102549,712 & \\
\hline Personalaufwand (Mio. DM) & 173571 & 192882,122 & 206453,854 & 209584,91 & 192389 & 183266,299 & \\
\hline Mitarbeiter & - & 2398 & 3639 & 3027 & 2542 & 2816 & 2498 \\
\hline Mengen Rüben Tonnen & 7688539 & 4592900 & 4600600 & 4998200 & 3916200 & 7854600 & \\
\hline Menge Zucker & - & 1095938 & 1215369 & 1166261 & 975364 & 1127581 & \\
\hline FK-Quote & & 71,0226238 & 70,78349 & 74,1667355 & 71,6168714 & 70,9372525 & 66,7532171 \\
\hline EK-Quote & & 28,9773762 & 29,21651 & 25,8332645 & 28,3831286 & 29,0627475 & 33,2467829 \\
\hline EK-Rentabilität & 6,60338802 & 4,91295031 & 15,620393 & 5,82765363 & 6,74227227 & 10,0062863 & \\
\hline EK-Rentabilität nach Steuer & 4,17189118 & 1,78063644 & 13,0941994 & 4,10637625 & 6,74227227 & 6,95014728 & \\
\hline Gesamtkapitalrentabilität & 2,51159132 & 1,76901784 & 4,35212227 & 2,16503846 & 2,25984853 & 3,64399724 & \\
\hline Umsatzrentabilität & & & & & & & \\
\hline a) $B E$ & & 2,05889263 & 2,02606807 & 4,29313301 & 3,76291189 & 3,18237056 & 4,52312102 \\
\hline
\end{tabular}


b) JÜ

Cash-flow einfach

Relativer Reingewinn

Cashflow*100/Umsatz

JÜ/Umsatz*100

Umsatz DM pro Mitarbeiter

JÜ DM pro Mitarbeiter

Aufwand DM pro Mitarbeiter

Rüben t pro Mitarbeiter

Zucker t pro Mitarbeiter

Personalintensität

Umsatz je t Rüben

JÜ je t. Rübe

Umsatz/Zucker kg

JÜ/kg Zucker

Anlageintensität

Kapitalintensität

Arbeitsintensität

Selbstfinanzierungsgrad

Deckungsgrad A:

Wachstumsmögl.keit:

Schuldentilgungsdauer

Goldene Bilanzregel

Rüben/Zucker

Kosten=Umsatz-JÜ

\begin{tabular}{|c|c|c|c|c|c|c|}
\hline & 1,17305403 & 0,56335659 & 3,36784402 & 1,09413668 & 2,06110196 & 2,21840877 \\
\hline 106732,971 & 102274,268 & 151697,169 & 121568,377 & 132860,07 & 132368,96 & \\
\hline 1,2089046 & 0,52023982 & 3,38265917 & 1,16551805 & 1,95948956 & 2,31070038 & \\
\hline 7,61078276 & 7,56571365 & 10,735852 & 8,35402246 & 9,75031043 & 9,84761456 & \\
\hline 1,17305403 & 0,56335659 & 3,36784402 & 1,09413668 & 2,06110196 & 2,21840877 & \\
\hline 584817,143 & 371479,132 & 466797,519 & 572465,652 & 483886,364 & 538099,614 & \\
\hline 6860,22105 & 2092,75216 & 15721,0123 & 6263,55665 & 9973,39134 & 11937,249 & \\
\hline 72381,568 & 53004,1554 & 68204,1144 & 82448,8238 & 68319,9574 & 73365,2116 & \\
\hline 3206,22977 & 1262,13245 & 1519,85464 & 1966,24705 & 1390,69602 & 3144,35548 & \\
\hline- & 301,164606 & 401,509415 & 419,354839 & 392,755682 & 487,990392 & \\
\hline 601,138015 & 704,241259 & 340,335844 & 382,746283 & 443,663249 & 301,431953 & \\
\hline \multirow[t]{4}{*}{182,400259} & 294,326583 & 307,133002 & 291,14635 & 347,945457 & 171,131927 & \\
\hline & 2,13965359 & 1,65810819 & 10,3437604 & 3,18553899 & 7,17151065 & 3,79640567 \\
\hline & 1,2334754 & 1,16260666 & 1,24775474 & 1,39704151 & 1,19208539 & \\
\hline & - & 0,00694886 & 0,03915478 & 0,01365214 & 0,02879445 & 0,02644533 \\
\hline 48,4930589 & 52,3464058 & 51,4863113 & 47,901373 & 42,8576312 & 43,7774387 & \\
\hline 312,679186 & 345,61312 & 171,6231 & 192,932654 & 241,618892 & 168,671273 & \\
\hline 51,420742 & 47,52974 & 48,4153538 & 52,0393579 & 57,0681337 & 56,1893181 & \\
\hline 2,95061078 & 2,79072512 & 2,9038798 & 2,99044467 & 2,85023565 & 3,72356052 & \\
\hline 59,7557193 & 55,8137843 & 50,1750152 & 59,2532672 & 67,8123046 & 75,9450161 & \\
\hline 7,84338153 & 6,98666816 & 10,783079 & 8,89903812 & 9,26961979 & 10,2573011 & \\
\hline 9,05510252 & 10,1312225 & 6,87806658 & 8,04770926 & 7,65266043 & 6,50787341 & \\
\hline 0,59755719 & 0,55813784 & 0,50175015 & 0,59253267 & 0,67812305 & 0,75945016 & \\
\hline- & - & 8,38167853 & 6,33134135 & 7,88949343 & 6,02188065 & 5,72822806 \\
\hline 1385940,7 & 1344197,04 & 1365408,58 & 1439285,73 & 1334538,93 & 1314353,59 & \\
\hline
\end{tabular}




\section{ZVN Betrieb}

Umsatz 1000 DM

Jahresüberschuss/-unterschuss

Betriebsergebnis

Eigenkapital

Fremdkapital

Gesamtkapital

Anlagevermögen

Umlaufvermögen

Abschreibungen

Personalaufwand (Mio. DM)

\section{Mitarbeiter}

Mengen Rüben Tonnen

Menge Zuckererzeugung

\section{FK-Quote}

EK-Quote

EK-Rentabilität vor Steuer

EK-Rentabilität nach Steuer

Gesamtkapitalrentabilität

Umsatzrentabilität

\begin{tabular}{rrrrrr}
1991 & 1992 & 1993 & 1994 & 1995 & 1996 \\
964521,508 & 923180,563 & 939657,089 & 917055,686 & 858139 & 907928,835 \\
12247,8101 & 4663,52512 & 4476,50429 & 5501,961 & 5355,07 & 5366,248 \\
21645,7354 & 20211,6426 & 36261,8015 & 43332,183 & 19765,745 & 32297,564 \\
264350,004 & 261701,571 & 249928,313 & 250065,009 & 248486,5 & 237050,836 \\
638912,996 & 579949,366 & 541568,226 & 545615,453 & 594035,5 & 477856,434 \\
903263 & 841650,937 & 791496,539 & 795680,462 & 842522 & 714907,27 \\
406845 & 404992,305 & 379553,309 & 338632,214 & 322260 & 312028,398 \\
495813 & 436455,595 & 411787,847 & 456928,582 & 519707 & 402782,873 \\
63699,161 & 62610,7425 & 63447,6643 & 60890,416 & 58458 & 56134,7118 \\
118505 & 131614,122 & 132227,854 & 136194,91 & 123687 & 117668,299 \\
1741 & 1692 & 1671 & 1582 & 1408 & 1249 \\
5301000 & 4592900 & 4600600 & 4998200 & 3916200 & 3927300 \\
- & - & - & - & - & - \\
70,7338833 & 68,9061629 & 68,4233221 & 68,5721818 & 70,5068236 & 66,8417366 \\
29,2661167 & 31,0938371 & 31,5766779 & 31,4278182 & 29,4931764 & 33,1582634 \\
7,78354823 & 6,22349324 & 5,42447778 & 4,86633418 & 2,15507482 & 6,59708895 \\
4,63317945 & 1,78200119 & 1,79111531 & 2,20021227 & 2,15507482 & 2,26375409 \\
3,17889807 & 2,5152647 & 2,27593973 & 2,4065256 & 1,14644721 & 2,759973 \\
2,24419417 & 2,18934881 & 3,85904624 & 4,72514196 & 2,30332673 & 3,55727925 \\
& & & & & \\
& & & & & \\
\hline
\end{tabular}




\begin{tabular}{|c|c|c|c|c|c|c|}
\hline b) JÜ & 1,26983276 & 0,5051585 & 0,47639765 & 0,59995931 & 0,62403294 & 0,5910428 \\
\hline Cash-flow einfach & 75946,9711 & 67274,2676 & 67924,1686 & 66392,377 & 63813,07 & 61500,9598 \\
\hline Relativer Reingewinn & 1,35595171 & 0,55409255 & 0,56557471 & 0,69147871 & 0,63560002 & 0,75062155 \\
\hline Cashflow¹00/Umsatz & 7,87405677 & 7,28722747 & 7,22861237 & 7,23973233 & 7,43621604 & 6,77376436 \\
\hline JÜ/Umsatz*100 & 1,26983276 & 0,5051585 & 0,47639765 & 0,59995931 & 0,62403294 & 0,5910428 \\
\hline Umsatz DM pro Mitarbeiter & 554004,313 & 545614,99 & 562332,19 & 579681,218 & 609473,722 & 726924,607 \\
\hline JÜ DM pro Mitarbeiter & 7034,92824 & 2756,22052 & 2678,93734 & 3477,85145 & 3803,31676 & 4296,43555 \\
\hline Aufwand DM pro Mitarbeiter & 68067,2028 & 77786,1239 & 79130,972 & 86090,335 & 87845,8807 & 94210,007 \\
\hline Rüben t pro Mitarbeiter & 3044,80184 & 2714,47991 & 2753,20168 & 3159,41846 & 2781,39205 & 3144,35548 \\
\hline Zucker t pro Mitarbeiter & - & - & - & - & - & - \\
\hline Personalintensität & 547,475046 & 651,179738 & 364,64778 & 314,30429 & 625,764422 & 364,325615 \\
\hline Umsatz je t. Rübe & 181,95086 & 201,001668 & 204,246639 & 183,477189 & 219,125428 & 231,183977 \\
\hline JÜ je Tonne Rübe & 2,31047162 & 1,01537702 & 0,97302619 & 1,10078848 & 1,36741484 & 1,36639625 \\
\hline Umsatz/Zucker kg & - & - & - & - & - & - \\
\hline JÜ/kg Zucker & - & - & - & - & - & - \\
\hline Anlageintensität & 45,0416988 & 48,1187968 & 47,9538811 & 42,5588198 & 38,2494463 & 43,6459959 \\
\hline Kapitalintensität & 294,280419 & 309,775626 & 174,971076 & 140,520075 & 295,754094 & 173,804785 \\
\hline Arbeitsintensität & 54,8913218 & 51,8570794 & 52,0264874 & 57,4261407 & 61,68468 & 56,3405759 \\
\hline Selbstfinanzierungsgrad & 0,58676155 & 0,71288461 & 0,75805764 & 0,75407155 & 0,71214758 & 0,83926969 \\
\hline Deckungsgrad A: & 64,975606 & 64,6188997 & 65,8480132 & 73,8456055 & 77,1074598 & 75,9709172 \\
\hline Wachstumsmögl.keit: & 8,40806843 & 7,99313166 & 8,58173918 & 8,34410045 & 7,57405385 & 8,60264854 \\
\hline Schuldentilgungsdauer & 8,41261984 & 8,62067157 & 7,97312999 & 8,21804366 & 9,30899422 & 7,76990205 \\
\hline Goldene Bilanzregel & 0,64975606 & 0,646189 & 0,65848013 & 0,73845606 & 0,7710746 & 0,75970917 \\
\hline Rüben/Zucker & - & - & - & - & - & - \\
\hline Kosten=Umsatz-JÜ & 952273,698 & 918517,037 & 935180,585 & 911553,725 & 852783,93 & 902562,587 \\
\hline
\end{tabular}


Vor Steuer: U-BE

Anlagevermögen pro Tonne Rüben

\section{ZVN Konzern}

Umsatz 1000 DM

Jahresüberschuss/-unterschuss

Betriebsergebnis

Eigenkapital

Fremdkapital

Gesamtkapital

Anlagevermögen

Umlaufvermögen

Abschreibungen

Personalaufwand (Mio. DM)

Mitarbeiter

Mengen Rüben Tonnen

Menge Zuckererzeugung

FK-Quote

EK-Quote

EK-Rentabilität vor Steuer

EK-Rentabilität nach Steuer

Gesamtkapitalrentabilität

Umsatzrentabilität

a) $\mathrm{BE}$

b) JÜ

$\begin{array}{rrrrrr}942875,773 & 902968,92 & 903395,288 & 873723,503 & 838373,255 & 875631,271 \\ 76,7487267 & 88,1779061 & 82,5008279 & 67,7508331 & 82,2889536 & 79,4511236\end{array}$

1991

1992

1993

1994

1995

1996

\section{7,8101}

964521,508

1879288,42

6647,226

1663923,58

1608173,16

1497094,43

21645,7354

46888,2788

48247,385

264350,004

343641,166

638912,996

827028,852

1098272,86

903263

1170670,02

494389,602

495813

428615,978

654532,425

541332,43

63699,161

79723,8429

76443,775

147699,629

1741

2463

5301000

757700

4963800

4884300

629500

619200

70,7338833

70,6457703

29,2661167

29,3542297

7,78354823

7,51889139

4,79500209

1,88152367

3,53077984

2,92114659

2,24030894

11785,372

5702,613

13307,97

53468,212

22455,994

45193,809

353289,523

370909,186

377313,461

$744983,333 \quad 871430,22$

941869,4

1242339,41

1319182,86

1174690,46

617810,218

702666,575

662220,15

600978,386

606349,297

507762,613

93675,055

$72892,55 \quad 94281,236$

138930,275

134052,56

1856

1628

5271000

662900

67,8322631

32,1677369

5,0228686

3,17742792

2,58376494

4499800

4762100

570100

567600

$70,1442952 \quad 71,3979409$

$29,8557048 \quad 28,6020591$

$1,5520358 \quad 6,2974322$

$1,51137279 \quad 3,51069038$

3,17889807

2,24419417

2,49500174

2,89961544

0,39949106

3,32477952

1,49997179

1,26983276

0,64562804

0,73284223

0,38091205 
Anhang

\begin{tabular}{|c|c|c|c|c|c|c|}
\hline Cash-flow einfach & 75946,9711 & 91857,0559 & 83091,001 & 84677,922 & 99983,849 & 106983,025 \\
\hline Relativer Reingewinn & 1,35595171 & 1,03643322 & 0,60524358 & 0,9486435 & 0,43228374 & 1,13289164 \\
\hline Cashflow*100/Umsatz & 7,87405677 & 4,88786366 & 4,99367892 & 5,2654729 & 6,67852656 & 6,66540218 \\
\hline JÜ/Umsatz¹00 & 1,26983276 & 0,64562804 & 0,39949106 & 0,73284223 & 0,38091205 & 0,82913128 \\
\hline Umsatz DM pro Mitarbeiter & 554004,313 & 763007,886 & 825358,918 & 866472,609 & 919591,171 & 1100102,68 \\
\hline JÜ DM pro Mitarbeiter & 7034,92824 & 4926,19285 & 3297,23512 & 6349,87716 & 3502,83354 & 9121,29541 \\
\hline Aufwand DM pro Mitarbeiter & 68067,2028 & 62135,2264 & 73263,7049 & 81479,695 & 85338,0068 & 91879,7533 \\
\hline Rüben t pro Mitarbeiter & 3044,80184 & 2015,34714 & 2422,76786 & 2839,97845 & 2764,00491 & 3263,94791 \\
\hline Zucker t pro Mitarbeiter & 435,20965 & 255,582623 & 307,142857 & 357,165948 & 350,184275 & 389,033585 \\
\hline Personalintensität & 547,475046 & 326,390873 & 306,129812 & 282,834058 & 618,677913 & 296,617088 \\
\hline Umsatz je t Rübe & 181,95086 & 378,59874 & 340,667768 & 305,098304 & 332,702437 & 337,046641 \\
\hline JÜ je t Rübe & & 2,31047162 & 2,44433962 & 1,36093729 & 2,23588921 & 1,26730366 \\
\hline Umsatz/Zucker kg & 1,27295963 & 2,98536684 & 2,68721508 & 2,42596645 & 2,62602075 & 2,82778331 \\
\hline JÜ/kg Zucker & 0,01616446 & 0,01927437 & 0,01073518 & 0,01777851 & 0,01000283 & 0,02344604 \\
\hline Anlageintensität & 45,0416988 & 36,6128774 & 45,015189 & 49,729584 & 53,2652899 & 56,3740127 \\
\hline Kapitalintensität & 294,280419 & 170,029365 & 158,441281 & 136,328759 & 419,848865 & 207,274087 \\
\hline Arbeitsintensität & 54,8913218 & 55,9109241 & 49,2894299 & 48,3747342 & 45,9640066 & 43,2252265 \\
\hline Selbstfinanzierungsgrad & 0,58676155 & 3,14270652 & 4,23117477 & 4,66498082 & 4,34637651 & 5,94372537 \\
\hline Deckungsgrad A: & 64,975606 & 80,1746046 & 71,4597398 & 60,0361041 & 53,6973686 & 57,2422759 \\
\hline Wachstumsmögl.keit: & 8,40806843 & 7,84653699 & 7,56560636 & 6,81600548 & 7,57922589 & 9,10733751 \\
\hline Schuldentilgungsdauer & 8,41261984 & 9,00343304 & 8,96587265 & 10,2911148 & 9,42021546 & 7,43688614 \\
\hline Goldene Bilanzregel & 0,64975606 & 0,80174605 & 0,7145974 & 0,60036104 & 0,53697369 & 0,57242276 \\
\hline Rüben/Zucker & 6,99617263 & 7,8853058 & 7,8880814 & 7,95142555 & 7,89300123 & 8,38988724 \\
\hline Kosten=Umsatz-JÜ & 952273,698 & 1867155,21 & 1657276,35 & 1596387,79 & 1491391,81 & 1591741,84 \\
\hline Vor Steuer: U-BE & 942875,773 & 1832400,14 & 1615676,19 & 1554704,95 & 1474638,43 & 1559856 \\
\hline
\end{tabular}


Anhang

\begin{tabular}{|c|c|c|c|c|c|c|}
\hline Anlagevermögen pro T Rüben & 76,7487267 & 86,3483577 & 101,220155 & 117,2093 & 156,155068 & 139,06053 \\
\hline Menge Zucker: & 757700 & 759100 & 755100 & 662900 & 570100 & 698400 \\
\hline Zucker pro Mitarbeiter & 435,20965 & 308,20138 & 374,553571 & 357,165948 & 350,184275 & 478,68403 \\
\hline Umsatz kg Zucker & 1,27295963 & 2,47567965 & 2,20358042 & 2,42596645 & 2,62602075 & 2,29818128 \\
\hline JÜ/kg Zucker & 0,01616446 & 0,01598368 & 0,00880311 & 0,01777851 & 0,01000283 & 0,01905494 \\
\hline Rüben/Zucker & 6,99617263 & 6,53905941 & 6,46841478 & 7,95142555 & 7,89300123 & 6,81858534 \\
\hline ZAG Betrieb & 1991 & 1992 & 1993 & 1994 & 1995 & 1996 \\
\hline Umsatz $1000 \mathrm{DM}$ & 437870 & 428632 & 473339 & 538152 & 504485 & 436244 \\
\hline Jahresüberschuss/-unterschuss & 4203 & 2952 & -43111 & 10420 & 22730 & 24453 \\
\hline Betriebsergebnis & 7228 & 7177 & 24400 & 11426 & 23598 & 28501 \\
\hline Eigenkapital & 129975 & 165984 & 113496 & 137672,5 & 168065,5 & 191994 \\
\hline Fremdkapital & 327565 & 456214 & 501815 & 432731,5 & 422697,5 & 383584 \\
\hline Gesamtkapital & 457540 & 622198 & 615311 & 570404 & 590763 & 575578 \\
\hline Anlagevermögen & 253050 & 361280 & 344760 & 315741 & 292012 & 252913 \\
\hline Umlaufvermögen & 203922 & 259308 & 269323 & 253973 & 298242 & 322332 \\
\hline Abschreibungen & 26583 & 32048 & 40662 & 44756 & 46317 & 46415 \\
\hline Personalaufwand (Mio. DM) & 55066 & 61268 & 74226 & 73390 & 68702 & 65598 \\
\hline Mitarbeiter & 657 & 1947 & 1356 & 960 & 1408 & 1249 \\
\hline Mengen Rüben Tonnen & 2387539 & 4592900 & 3094316 & 3412000 & 2744000 & 3055410 \\
\hline Menge Zuckererzeugung & - & - & - & - & - & - \\
\hline FK-Quote & 71,5926476 & 73,3229615 & 81,5546935 & 75,8640367 & 71,5511127 & 66,6432699 \\
\hline EK-Quote & 28,4073524 & 26,6770385 & 18,4453065 & 24,1359633 & 28,4488873 & 33,3567301 \\
\hline EK-Rentabilität vor Steuer & 4,20311598 & 2,84665992 & $-37,8964897$ & 7,57377109 & 13,524489 & 14,2155484 \\
\hline EK-Rentabilität nach Steuer & 3,23369879 & 1,77848467 & $-37,9845986$ & 7,56868656 & 13,524489 & 12,7363355 \\
\hline
\end{tabular}




\begin{tabular}{|c|c|c|c|c|c|c|}
\hline Gesamtkapitalrentabilität & 1,19421253 & 0,75956528 & $-6,989966117$ & 1,82817792 & 3,84773589 & 4,74201585 \\
\hline \multicolumn{7}{|l|}{ Umsatzrentabilität } \\
\hline a) $B E$ & 1,65071825 & 1,67439669 & 5,15486786 & 2,12319196 & 4,67764156 & 6,53327037 \\
\hline b) JÜ & 0,95987394 & 0,68870266 & $-9,10784871$ & 1,93625593 & 4,5055849 & 5,6053493 \\
\hline Cash-flow einfach & 30786 & 35000 & -2449 & 55176 & 69047 & 70868 \\
\hline Relativer Reingewinn & 0,91860821 & 0,47444704 & $-7,00637564$ & 1,82677541 & 3,84756662 & 4,24842506 \\
\hline Cashflow*100/Umsatz & 7,03085391 & 8,16551261 & $-0,51738817$ & 10,2528654 & 13,6866309 & 16,2450372 \\
\hline JÜ/Umsatz¹00 & 0,95987394 & 0,68870266 & $-9,10784871$ & 1,93625593 & 4,5055849 & 5,6053493 \\
\hline Umsatz DM pro Mitarbeiter & 666468,798 & 220149,974 & 349070,059 & 560575 & 358299,006 & 349274,62 \\
\hline JÜ DM pro Mitarbeiter & 6397,26027 & 1516,17874 & $-31792,7729$ & 10854,1667 & 16143,4659 & 19578,0624 \\
\hline Aufwand DM pro Mitarbeiter & 83814,3075 & 31467,8993 & 54738,9381 & 76447,9167 & 48794,0341 & 52520,4163 \\
\hline Rüben t pro Mitarbeiter & 3634,00152 & 2358,96251 & 2281,94395 & 3554,16667 & 1948,86364 & 2446,28503 \\
\hline Zucker t pro Mitarbeiter & - & - & - & - & - & - \\
\hline Personalintensität & 761,842833 & 853,67145 & 304,204918 & 642,307019 & 291,134842 & 230,160345 \\
\hline Umsatz je t Rübe & 183,398051 & 93,3249145 & 152,970479 & 157,723329 & 183,850219 & 142,777565 \\
\hline JÜ je t Rübe & 1,76039009 & 0,64273117 & $-13,9323198$ & 3,05392732 & 8,2835277 & 8,00318124 \\
\hline Umsatz/Zucker kg & - & - & - & - & - & - \\
\hline JÜ/kg Zucker & - & - & - & - & - & - \\
\hline Anlageintensität & 55,3066399 & 58,0651175 & 56,0302026 & 55,3539246 & 49,4296359 & 43,9406996 \\
\hline Kapitalintensität & 367,778085 & 446,537551 & 166,647541 & 391,703133 & 196,275108 & 162,853935 \\
\hline Arbeitsintensität & 44,569218 & 41,6761224 & 43,7702235 & 44,5251085 & 50,4842043 & 56,0014455 \\
\hline Selbstfinanzierungsgrad & 7,61725751 & 5,60143234 & 5,66412757 & 6,11005533 & 5,8994893 & 7,30604714 \\
\hline Deckungsgrad A: & 51,3633669 & 45,9433127 & 32,9202924 & 43,6029847 & 57,5543128 & 75,913061 \\
\hline Wachstumsmögl.keit: & 6,72859204 & 5,62521898 & $-0,39801011$ & 9,67314395 & 11,6877665 & 12,3124928 \\
\hline Schuldentilgungsdauer & 10,6400637 & 13,0346857 & $-204,906084$ & 7,84274866 & 6,12188075 & 5,41265451 \\
\hline
\end{tabular}


Anhang

\begin{tabular}{|c|c|c|c|c|c|c|c|}
\hline Goldene Bilanzregel & 0,51363367 & 0,45943313 & 0,32920292 & 0,43602985 & 0,57554313 & 0,75913061 & \\
\hline Rüben/Zucker & \#DIV/0! & \#DIV/0! & \#DIV/0! & \#DIV/0! & \#DIV/0! & \#DIV/0! & \\
\hline Kosten=Umsatz-JÜ & 433667 & 425680 & 516450 & 527732 & 481755 & 411791 & \\
\hline Vor Steuer: U-BE & 430642 & 421455 & 448939 & 526726 & 480887 & 407743 & \\
\hline Anlagevermögen pro Tonne Rüben & 105,987797 & 78,6605413 & 111,417192 & 92,5383939 & 106,418367 & 82,7754704 & \\
\hline ZAG Konzern & & 1991 & 1992 & 1993 & 1994 & 1995 & 1996 \\
\hline Umsatz 1000 DM & & 460242 & 646847 & 1101730 & 1169809 & 1081333 & 1038707 \\
\hline Jahresüberschuss/-unterschuss & & 4203 & 2335 & -41443 & 15415 & 12079 & 24940 \\
\hline Betriebsergebnis & & 7388 & 11494 & 11487 & 23711 & 15407 & 31926 \\
\hline Eigenkapital & & 129522 & 244591,5 & 204298 & 240597,5 & 259841,5 & 282628,5 \\
\hline Fremdkapital & & 330442 & 672937,5 & 787510 & 800158,5 & 691190,5 & 637521,5 \\
\hline Gesamtkapital & & 459964 & 917529 & 991808 & 1040756 & 951032 & 920150 \\
\hline Anlagevermögen & & 266691 & 462841 & 556298 & 592902 & 550579 & 487258 \\
\hline Umlaufvermögen & & 192594 & 412097 & 400708 & 419262 & 374174 & 409233 \\
\hline Abschreibungen & & 28397 & 48575 & 65052 & 73492 & 74824 & 77113 \\
\hline Personalaufwand (Mio. DM) & & 61006 & 99418 & 110562 & 100165 & 85258 & 81596 \\
\hline Mitarbeiter & & 860 & 1966 & 1742 & 1333 & 1029 & 848 \\
\hline Mengen Rüben Tonnen & & 2387539 & 4592900 & 3094316 & 3412000 & 2744000 & 3055410 \\
\hline Menge Zuckererzeugung & & 0 & 336838 & 460269 & 503361 & 405264 & 429181 \\
\hline FK-Quote & & 71,8408397 & 73,3423685 & 79,4014567 & 76,8824297 & 72,6779435 & 69,2845188 \\
\hline EK-Quote & & 28,1591603 & 26,6576315 & 20,5985433 & 23,1175703 & 27,3220565 & 30,7154812 \\
\hline EK-Rentabilität vor Steuer & & 4,21781628 & 1,67953506 & $-20,2366151$ & 6,40987541 & 4,64860309 & 9,87833853 \\
\hline EK-Rentabilität nach Steuer & & 3,24500857 & 0,95465296 & $-20,2855632$ & 6,40696599 & 4,64860309 & 8,8243047 \\
\hline Gesamtkapitalrentabilität & & 1,18791905 & 0,44783326 & $-4,1683471$ & 1,48190354 & 1,27019911 & 3,03428789 \\
\hline
\end{tabular}


Umsatzrentabilität

a) $B E$

b) JÜ

Cash-flow einfach

Relativer Reingewinn

Cashflow*100/Umsatz

JÜ/Umsatz ${ }^{\star} 100$

Umsatz DM pro Mitarbeiter

JÜ DM pro Mitarbeiter

Aufwand DM pro Mitarbeiter

Rüben $t$ pro Mitarbeiter

Zucker t pro Mitarbeiter

Personalintensität

Umsatz je t Rübe

JÜ je t Rübe

Umsatz/Zucker kg

JÜ/kg Zucker

Anlageintensität

Kapitalintensität

Arbeitsintensität

Selbstfinanzierungsgrad

Deckungsgrad A:

Wachstumsmögl.keit:

Schuldentilgungsdauer

Goldene Bilanzregel

\begin{tabular}{|c|c|c|c|c|c|}
\hline 1,60524246 & 1,77692716 & 1,04263295 & 2,02691209 & 1,42481548 & 3,07362904 \\
\hline 0,91321522 & 0,36098181 & $-3,76162944$ & 1,31773649 & 1,1170472 & 2,40106209 \\
\hline 32600 & 50910 & 23609 & 88907 & 86903 & 102053 \\
\hline 0,91376716 & 0,25448787 & $-4,17853052$ & 1,48113487 & 1,27009396 & 2,71042765 \\
\hline 7,08323013 & 7,8704856 & 2,14290253 & 7,60012959 & 8,03665476 & 9,82500359 \\
\hline 0,91321522 & 0,36098181 & $-3,76162944$ & 1,31773649 & 1,1170472 & 2,40106209 \\
\hline 535165,116 & 329016,785 & 632451,206 & 877576,144 & 1050858,11 & 1224890,33 \\
\hline 4887,2093 & 1187,69074 & $-23790,4707$ & 11564,141 & 11738,5811 & 29410,3774 \\
\hline 70937,2093 & 50568,6673 & 63468,4271 & 75142,5356 & 82855,1992 & 96221,6981 \\
\hline 2776,20814 & 2336,1648 & 1776,3008 & 2559,63991 & 2666,66667 & 3603,07783 \\
\hline - & 171,331638 & 264,218714 & 377,615154 & 393,842566 & 506,10967 \\
\hline 825,74445 & 864,955629 & 962,496735 & 422,441061 & 553,371844 & 255,578525 \\
\hline 192,768369 & 140,836291 & 356,049608 & 342,851407 & 394,071793 & 339,956667 \\
\hline 1,76039009 & 0,50839339 & $-13,3932669$ & 4,51787808 & 4,40196793 & 8,16257065 \\
\hline - & 1,92035044 & 2,39366544 & 2,3239961 & 2,66821874 & 2,42020733 \\
\hline - & 0,00693212 & $-0,09004082$ & 0,03062414 & 0,02980526 & 0,05811068 \\
\hline 57,980842 & 50,4442911 & 56,0892834 & 56,9683961 & 57,8927944 & 52,9541923 \\
\hline 384,36654 & 422,611797 & 566,309741 & 309,948969 & 485,64938 & 241,536679 \\
\hline 41,8715378 & 44,9137847 & 40,4017713 & 40,2843702 & 39,3439968 & 44,4745965 \\
\hline 7,47862876 & 3,6816275 & 3,98292815 & 4,27554585 & 3,55897593 & 4,51382927 \\
\hline 48,5663183 & 52,8456857 & 36,7245613 & 40,5796405 & 47,1942264 & 58,0038706 \\
\hline 7,0875112 & 5,54859846 & 2,38040024 & 8,54254023 & 9,13775772 & 11,0909091 \\
\hline 10,1362577 & 13,2181791 & 33,3563472 & 8,99994939 & 7,95358618 & 6,24696481 \\
\hline 0,48566318 & 0,52845686 & 0,36724561 & 0,4057964 & 0,47194226 & 0,58003871 \\
\hline
\end{tabular}




\section{Rüben/Zucker}

Kosten=Umsatz-Jü

Vor Steuer: U-BE

Anlagevermögen pro Tonne Rüben

jeweils DM

Jülich

Umsatz 1000 DM

Jahresüberschuss/-unterschuss

Betriebsergebnis

Eigenkapital

Fremdkapital

Gesamtkapital

Anlagevermögen

Umlaufvermögen

Abschreibungen

Personalaufwand (Mio. DM)

Mitarbeiter

Mengen Rüben Tonnen

Menge Zuckererzeugung

FK-Quote

EK-Quote

EK-Rentabilität vor Steuer

EK-Rentabilität nach Steuer

Gesamtkapitalrentabilität

$\begin{array}{rrrrrr}-13,6353381 & 6,72284251 & 6,77843536 & 6,77089502 & 7,11916418 \\ 456039 & 644512 & 1143173 & 1154394 & 1069254 & 1013767 \\ 452854 & 635353 & 1090243 & 1146098 & 1065926 & 1006781 \\ 111,701212 & 100,77315 & 179,780604 & 173,769637 & 200,648324 & 159,473851\end{array}$

\begin{tabular}{rrrrrr}
1995 & 1996 & 1997 & 1998 & 1999 & 2000 \\
148481000 & 197394667 & 245054607 & 246639147 & 222019406 & 248457819 \\
8074000 & 12113431,9 & 7764716,51 & 9257356,15 & 10636698 & 14293783 \\
17993000 & 12634965,1 & 15430626,9 & 24426670,7 & 23769467 & 23849453 \\
88041000 & 100984341 & 105503470 & 111993941 & 119630723 & 130140320 \\
83724000 & 138934440 & 144590690 & 147278311 & 143272789 & 143359277 \\
171765000 & 239918781 & 250094160 & 259272253 & 262903511 & 273499597 \\
108749000 & 130785743 & 143701531 & 164782744 & 159192182 & 155584686 \\
62869000 & 108714803 & 106058239 & 94194334,5 & 103452396 & 117564554 \\
13103000 & 21708643,5 & 26693634,7 & 25244145,5 & & 225 \\
20103000 & 27134186,5 & 32411342,9 & 25594941,5 & 25001894 & 25757788 \\
& 351 & 268 & 214 & & 220 \\
1074098 & 1101121 & 1103917 & 1073870 & 1079917 & 1240438 \\
163564 & 163145 & 171019 & 159304 & 153975 & 185126 \\
48,7433412 & 57,908947 & 57,8145007 & 56,8045018 & 54,496339 & 52,4166319 \\
1,2566588 & 42,091053 & 42,1854993 & 43,1954982 & 45,503661 & 47,5833681 \\
19,6942334 & 12,2131657 & 14,2656441 & 20,5736256 & 19,6771243 & 18,3386379 \\
\hline 17072727 & 11,9953567 & 7,35967877 & 8,26594371 & 8,89127624 & 10,9833624 \\
10,1702908 & 5,47263928 & 6,38681157 & 9,57507235 & 8,95381196 & 8,72614156
\end{tabular}


Anhang

\begin{tabular}{|c|c|c|c|c|c|c|}
\hline \multicolumn{7}{|l|}{ Umsatzrentabilität } \\
\hline a) $\mathrm{BE}$ & 12,1180488 & 6,40086447 & 6,2968116 & 9,90380925 & 10,7060313 & 9,59899475 \\
\hline b) JÜ & 5,43773277 & 6,13665614 & 3,16856582 & 3,75340097 & 4,79088661 & 5,75300188 \\
\hline Cash-flow einfach & 21177000 & 33822075,4 & 34458351,2 & 34501501,6 & 10636698 & 14293783 \\
\hline Relativer Reingewinn & 4,70060839 & 5,04897195 & 3,10471724 & 3,57051557 & 4,0458562 & 5,22625377 \\
\hline Cashflow*100/Umsatz & 14,2624309 & 17,1342397 & 14,061499 & 13,9886559 & 4,79088661 & 5,75300188 \\
\hline JÜ/Umsatz¹00 & 5,43773277 & 6,13665614 & 3,16856582 & 3,75340097 & 4,79088661 & 5,75300188 \\
\hline Umsatz DM pro Mitarbeiter & - & 562377,968 & 914382,862 & 1152519,38 & 1009179,12 & 1104256,97 \\
\hline JÜ DM pro Mitarbeiter & - & 34511,2021 & 28972,8228 & 43258,6736 & 48348,6273 & 63527,9244 \\
\hline Aufwand DM pro Mitarbeiter & - & 77305,3746 & 120937,847 & 119602,53 & 113644,973 & 114479,058 \\
\hline Rüben t pro Mitarbeiter & - & 3137,09687 & 4119,09328 & 5018,08411 & 4908,71364 & 5513,05778 \\
\hline Zucker t pro Mitarbeiter & - & 464,80057 & 638,130597 & 744,411215 & 699,886364 & 822,782222 \\
\hline Personalintensität & 111,726783 & 214,75474 & 210,045535 & 104,782767 & 105,184916 & 108,001588 \\
\hline Umsatz je t Rübe & 138,237852 & 179,267008 & 221,986442 & 229,673189 & 205,589324 & 200,298458 \\
\hline JÜ je t. Rübe & 7,51700497 & 11,0009998 & 7,03378652 & 8,6205557 & 9,8495514 & 11,5231741 \\
\hline Umsatz/Zucker kg & 0,90778533 & 1,2099339 & 1,43290867 & 1,54822947 & 1,44191853 & 1,34210116 \\
\hline JÜ/kg Zucker & 0,04936294 & 0,07424948 & 0,04540265 & 0,05811126 & 0,06908068 & 0,0772111 \\
\hline Anlageintensität & 63,3126656 & 54,5125075 & 57,4589709 & 63,5558733 & 60,5515618 & 56,8866235 \\
\hline Kapitalintensität & 72,8227644 & 171,814036 & 172,991252 & 103,346648 & - & - \\
\hline Arbeitsintensität & 36,6017524 & 45,3131694 & 42,4073234 & 36,3302797 & 39,3499484 & 42,985275 \\
\hline Selbstfinanzierungsgrad & 43,1193782 & 30,9088475 & 31,1524667 & 32,5021282 & 34,5894962 & 36,0817771 \\
\hline Deckungsgrad A: & 80,9579858 & 77,2135697 & 73,4184735 & 67,964605 & 75,1486166 & 83,6459701 \\
\hline Wachstumsmögl.keit: & 12,3290542 & 14,0973021 & 13,7781511 & 13,3070551 & 4,0458562 & 5,22625377 \\
\hline Schuldentilgungsdauer & 3,95353449 & 4,1078035 & 4,19610007 & 4,26875076 & 13,4696678 & 10,0294846 \\
\hline Goldene Bilanzregel & 0,80957986 & 0,7721357 & 0,73418473 & 0,67964605 & 0,75148617 & 0,8364597 \\
\hline
\end{tabular}




\begin{tabular}{|c|c|c|c|c|c|c|c|c|c|}
\hline Rüben/Zucker & 6,56683622 & 6,74933954 & 6,45493776 & 6,7410109 & 7,01358662 & 6,70050668 & & & \\
\hline Kosten=Umsatz-JÜ & 140407000 & 185281235 & 237289890 & 237381791 & 211382708 & 234164036 & & & \\
\hline Vor steuer: U-BE & 130488000 & 184759702 & 229623980 & 222212477 & 198249939 & 224608366 & & & \\
\hline Anlagevermögen pro Tonne Rüben & 101,246814 & 118,775088 & 130,174217 & 153,447572 & 147,411497 & 125,427217 & & & \\
\hline jeweils in $D M$ & & 1994 & 1995 & 1996 & 1997 & 1998 & 1999 & 2000 & 2001 \\
\hline \multicolumn{10}{|l|}{ Union-Zucker } \\
\hline Umsatz 1000 DM & & 168011799 & 172986232 & 160247609 & 197001478 & 192017913 & 249388289 & 307037744 & 268911129 \\
\hline Jahresüberschuss/-unterschuss & & 3247949,66 & 1850971,55 & 4885278,68 & 3853785,91 & 3060842,01 & 3493695,24 & 7195556,96 & 7604285,31 \\
\hline Betriebsergebnis & & 4406664,12 & 4722376,1 & 10094072,3 & 7532694,95 & 6687501,04 & 13554701,3 & 14104506,6 & 11706073,1 \\
\hline Eigenkapital & & 53765056 & 59201796,6 & 65063926,7 & 68482744,2 & 70050259,3 & 73617078,4 & 114958982 & 117936503 \\
\hline Fremdkapital & & 116561609 & 104310847 & 118277550 & 124809945 & 113808641 & 106812431 & 105377529 & 103792103 \\
\hline Gesamtkapital & & 170326665 & 163512644 & 183341476 & 193292689 & 183858900 & 180429510 & 220336511 & 221728606 \\
\hline Anlagevermögen & & 91125224,5 & 91925888,9 & 88651304,4 & 97497581,3 & 89975925 & 85607566,7 & 110258397 & 120148884 \\
\hline Umlaufvermögen & & 78668524,6 & 71332139 & 94486447,4 & 95723569,9 & 98853639,5 & 94799716 & 110078114 & 101579,722 \\
\hline Abschreibungen & & 20819048,3 & 17960539,6 & 19471904,5 & 22328719 & 20433709,5 & 20748939,4 & 16932901,3 & 19153879,1 \\
\hline Personalaufwand (Mio. DM) & & 21100000 & 20800000 & 20000000 & 18600000 & 19771939,5 & 25474637,5 & 23073158,5 & 23004001 \\
\hline Mitarbeiter & & 261 & 250 & 224 & 213 & 211 & 242 & 225 & 216 \\
\hline Mengen Rüben Tonnen & & 748000 & 793400 & 752000 & 796800 & 771900 & 1058700 & 966800 & 930700 \\
\hline Menge Zuckererzeugung & & 130300 & 130500 & 131600 & 135200 & 119100 & 169600 & 155100 & 138700 \\
\hline FK-Quote & & 68,4341522 & 63,7937501 & 64,5121617 & 64,5704427 & 61,8999901 & 59,1989811 & 47,8257226 & 46,8104251 \\
\hline EK-Quote & & 31,5658478 & 36,2062499 & 35,4878383 & 35,4295573 & 38,1000099 & 40,8010189 & 52,1742774 & 53,1895749 \\
\hline EK-Rentabilität vor Steuer & & 7,23391472 & 7,10197576 & 14,7315029 & 11,0065954 & 9,44461104 & 8,06664346 & 10,8998101 & 9,87495582 \\
\hline EK-Rentabilität nach Steuer & & 6,04100489 & 3,12654625 & 7,50842891 & 5,62738243 & 4,36949419 & 4,74576731 & 6,25923857 & 6,44777918 \\
\hline Gesamtkapitalrentabilität & & 3,75010787 & 3,83421461 & 5,87801266 & 4,40966131 & 4,22046677 & 3,7372326 & 6,38198486 & 5,48800547 \\
\hline
\end{tabular}


Umsatzrentabilität

a) $B E$

b) JÜ

Cash-flow einfach

Relativer Reingewinn

Cashflow*100/Umsatz

JÜ/Umsatz ${ }^{\star} 100$

Umsatz DM pro Mitarbeiter

JÜ DM pro Mitarbeiter

Aufwand DM pro Mitarbeiter

Rüben $t$ pro Mitarbeiter

Zucker t pro Mitarbeiter

Personalintensität

Umsatz je t Rübe

JÜ je t Rübe

Umsatz/Zucker kg

JÜ/kg Zucker

Anlageintensität

Kapitalintensität

Arbeitsintensität

Selbstfinanzierungsgrad

Deckungsgrad A:

Wachstumsmögl.keit:

Schuldentilgungsdauer

Goldene Bilanzregel

\begin{tabular}{|c|c|c|c|c|c|c|c|}
\hline 2,62283015 & 2,72991443 & 6,29904706 & 3,82367434 & 3,48274853 & 5,43517956 & 4,59373704 & 4,35313819 \\
\hline 1,9331676 & 1,07001091 & 3,04858133 & 1,95622183 & 1,59403983 & 1,40090589 & 2,3435415 & 2,8278061 \\
\hline 24066998 & 19811511,1 & 24357183,2 & 26182504,9 & 23494551,5 & 24242634,7 & 24128458,2 & 26758164,4 \\
\hline 1,90689441 & 1,13200515 & 2,66457911 & 1,99375668 & 1,66477772 & 1,93632142 & 3,26571249 & 3,42954634 \\
\hline 14,324588 & 11,452652 & 15,1997171 & 13,2905119 & 12,235604 & 9,72083924 & 7,85846649 & 9,95056043 \\
\hline 1,9331676 & 1,07001091 & 3,04858133 & 1,95622183 & 1,59403983 & 1,40090589 & 2,3435415 & 2,8278061 \\
\hline 643723,368 & 691944,928 & 715391,11 & 924889,566 & 910037,501 & 1030530,12 & 1364612,2 & 1244958,93 \\
\hline 12444,2516 & 7403,8862 & 21809,2798 & 18092,8916 & 14506,3602 & 14436,7572 & 31980,2532 & 35205,0246 \\
\hline 80842,9119 & 83200 & 89285,7143 & 87323,9437 & 93705,8743 & 105267,097 & 102547,371 & 106500,004 \\
\hline 2865,90038 & 3173,6 & 3357,14286 & 3740,84507 & 3658,29384 & 4374,79339 & 4296,88889 & 4308,7963 \\
\hline 499,233716 & 522 & 587,5 & 634,741784 & 564,454976 & 700,826446 & 689,333333 & 642,12963 \\
\hline 478,820246 & 440,456236 & 198,136089 & 246,923579 & 295,655124 & 187,939497 & 163,587137 & 196,51339 \\
\hline 224,614705 & 218,03155 & 213,095224 & 247,24081 & 248,760089 & 235,560866 & 317,581448 & 288,934275 \\
\hline 4,34217869 & 2,33296137 & 6,49638122 & 4,8365787 & 3,9653349 & 3,29998606 & 7,44265304 & 8,17050103 \\
\hline 1,28942286 & 1,325565 & 1,217687 & 1,45711152 & 1,61224108 & 1,47044982 & 1,9796115 & 1,9387969 \\
\hline 0,0249267 & 0,01418369 & 0,03712218 & 0,02850433 & 0,02569976 & 0,02059962 & 0,04639302 & 0,05482542 \\
\hline 53,5002692 & 56,2194376 & 48,3531093 & 50,4403874 & 48,9374867 & 47,446544 & 50,0409109 & 54,1873627 \\
\hline 472,444637 & 380,328445 & 192,90435 & 296,424044 & 305,550748 & 153,075593 & 120,053128 & 163,623437 \\
\hline 46,1868518 & 43,6248459 & 51,5357732 & 49,5226024 & 53,7660343 & 52,5411371 & 49,9590891 & 0,04581264 \\
\hline 11,6700752 & 12,5381863 & 12,8352408 & 13,2104427 & 14,5434569 & 14,2437897 & 15,8377901 & 18,2149248 \\
\hline 59,0012878 & 64,401658 & 73,3930844 & 70,2404544 & 77,8544475 & 85,9936583 & 104,263245 & 98,1586337 \\
\hline 14,1299061 & 12,1161952 & 13,2851462 & 13,5455226 & 12,7785772 & 13,4360697 & 10,9507308 & 12,0679802 \\
\hline 4,8432135 & 5,26516361 & 4,85596174 & 4,76692148 & 4,84404401 & 4,40597455 & 4,36735441 & 3,87889474 \\
\hline 0,59001288 & 0,64401658 & 0,73393084 & 0,70240454 & 0,77854447 & 0,85993658 & 1,04263245 & 0,98158634 \\
\hline
\end{tabular}




\section{Rüben/Zucker}

Kosten=Umsatz-Jü

Vor Steuer: U-BE

Anlagevermögen pro Tonne Rüben

$\begin{array}{rrrrrrrr}5,74059862 & 6,07969349 & 5,71428571 & 5,89349112 & 6,48110831 & 6,24233491 & 6,23339781 & 6,71016583 \\ 164763849 & 171135260 & 155362330 & 193147692 & 188957071 & 245894594 & 299842187 & 261306844 \\ 163605135 & 168263856 & 150153536 & 189468783 & 185330412 & 235833588 & 292933237 & 257205056 \\ 121,825166 & 115,863233 & 117,887373 & 122,361422 & 116,564225 & 80,8610246 & 114,04468 & 129,09518\end{array}$

